Supporting Information for

\title{
NiH-Catalyzed Hydroamination/Cyclization Cascade: Rapid Access to Quinolines
}

Yang Gao, ${ }^{* 1}$ Simin Yang, ${ }^{1}$ Yanping Huo, ${ }^{1}$ Qian Chen, ${ }^{1}$ Xianwei Li ${ }^{1}$ and Xiao-Qiang Hu*2

${ }^{1}$ School of Chemical Engineering and Light Industry, Guangdong University of Technology, Guangzhou, 510006, China.

${ }^{2}$ Key Laboratory of Catalysis and Energy Materials Chemistry of Ministry of Education \& Hubei Key Laboratory of Catalysis and Materials Science, School of Chemistry and Materials Science, South-Central University for Nationalities, Wuhan 430074, China.

*Correspondence: gaoyang@gdut.edu.cn (Y. G.), huxiaoqiang@mail.scuec.edu.cn (X.-Q. H.) 


\section{Table of Contents}

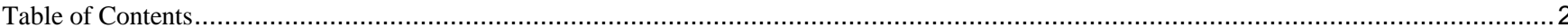

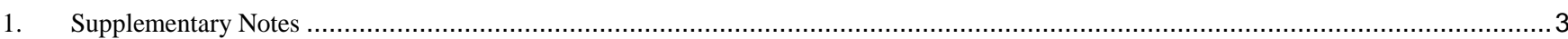

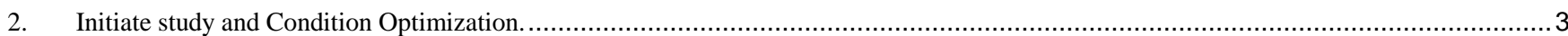

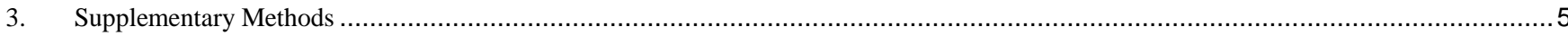

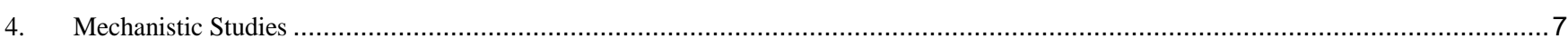

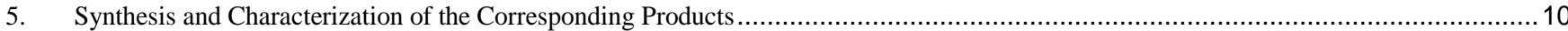

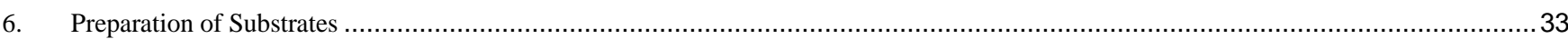

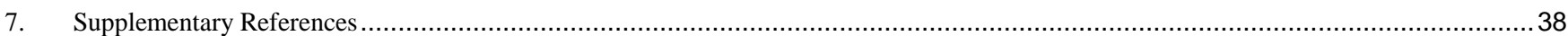

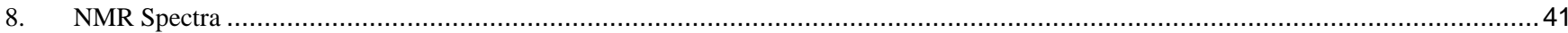




\section{Supplementary Notes}

\section{General analytical information:}

All reactions were performed in oven-dried glassware containing a Teflon-coated stirring bar and dry septum under argon atmosphere. All optimization reactions were monitored by ${ }^{1} \mathrm{H}$ NMR using 1,3,5-trimethoxybenzene as an internal standard. NMR spectra were recorded at ambient temperature using $\mathrm{CDCl}_{3}$ as solvent, with proton, carbon, and fluorine resonances at 400, 100 and $375 \mathrm{MHz}$, respectively. All NMR data are reported in ppm relative to the solvent signal. Data are reported as follows: chemical shift, multiplicity $(\mathrm{s}=$ singlet, $\mathrm{d}=$ doublet, $\mathrm{t}=$ triplet, $\mathrm{q}=$ quartet, $\mathrm{m}=$ multiplet), coupling constants (Hz) and integration. Column chromatography was performed with 200-300 mesh silica gel plates $\left(\mathrm{GF}_{254}\right)$, and visualization was effected at $254 \mathrm{~nm}$. TLC was performed using commercially prepared $100-$ 400 mesh silica gel plates $\left(\mathrm{GF}_{254}\right)$. Mass spectral data were acquired on a Varian GC-MS Saturn $2100 \mathrm{~T}$. The ionization was achieved by EI AGC. HRMS analyses were carried out on a Waters GCT Premier CAB163 with a TOF mass analyzer. The MS ionization was achieved by $\mathrm{EI}^{+}$. Melting points were measured on a Mettler FP 61 and are uncorrected. Parallel heating mantle were used in our experiments.

\section{General reagent information:}

All solvents were purified and dried by passage through alumina and Q5 reactant-packed columns on a solvent purification system. Commercial reagents were purchased from Aldrich Chemical, Alfa Aesar, TCI, Acros, Innochem, Adamas-beta, Aladdin, Bide Pharmatech, and were used as received.

Anthranils were synthesized according to the literature procedure, and were reported in our previous works. ${ }^{1}$

Some of the alkynes $(\mathbf{1 g}, 1 \mathbf{h}, 1 \mathrm{~m}, 1 \mathrm{~g}, 1 \mathbf{r}, 1 \mathrm{~s}, 1 \mathrm{an}, 1 \mathbf{a p}, \mathbf{1 a v}, 1 \mathbf{a y}, 1 \mathbf{a z}, 1 \mathbf{b c}-1 \mathbf{b i})$ were synthesized according to the literature procedures, please see the "Preparation of Substrates" section for detail.

\section{Initiate study and Condition Optimization.}

Table S1. Initiate study. ${ }^{\mathrm{a}}$

\begin{tabular}{|c|c|c|c|c|c|c|c|}
\hline $1 a$ & + & $\begin{array}{r}\mathrm{Cu}(\mathrm{OAc})_{2}(5 \\
\mathrm{L}(5.5 \mathrm{~mol} \%), \mathrm{h} \\
\text { solvent, } \mathrm{Ar}, \mathrm{r}\end{array}$ & $\begin{array}{l}\mathrm{Ol} \%) \\
\underset{12 \mathrm{~h}}{\stackrel{\text { rosilane }}{\longrightarrow}}\end{array}$ & $3 a$ & & $\begin{array}{r}H \\
\end{array}$ & $7 a$ \\
\hline entry & catalyst & ligand & solvent & hydrosilane & $\begin{array}{c}\text { yield } \\
6(\%)^{b}\end{array}$ & $\begin{array}{c}\text { yield } \\
7 \mathbf{a}(\%)^{\mathrm{b}}\end{array}$ & $\begin{array}{c}\text { yield } \\
\mathbf{3 a}(\%)^{\mathrm{b}}\end{array}$ \\
\hline $1^{\mathrm{c}}$ & $\mathrm{Cu}(\mathrm{OAc})_{2}$ & 6,6'-dimethyl-2,2'-bipyridine & DMA & $\mathrm{Me}(\mathrm{OEt})_{2} \mathrm{SiH}$ & 12 & trace & 0 \\
\hline $2^{\mathrm{d}}$ & $\mathrm{Cu}(\mathrm{OAc})_{2}$ & 6,6'-dimethyl-2,2'-bipyridine & Cyclohexane & $\mathrm{PhSiH}_{3}$ & 13 & trace & 0 \\
\hline $3^{\mathrm{d}}$ & $\mathrm{Cu}(\mathrm{OAc})_{2}$ & Xantphos & Cyclohexane & $\mathrm{PhSiH}_{3}$ & 10 & 38 & 8 \\
\hline $4^{\mathrm{d}}$ & $\mathrm{Cu}(\mathrm{OAc})_{2}$ & Xantphos & DMA & $\mathrm{PhSiH}_{3}$ & 15 & trace & 0 \\
\hline $5^{\mathrm{d}}$ & $\mathrm{Cu}(\mathrm{OAc})_{2}$ & Xantphos & THF & $\mathrm{PhSiH}_{3}$ & 23 & 30 & trace \\
\hline $6^{\mathrm{d}}$ & $\mathrm{Cu}(\mathrm{OAc})_{2}$ & Xantphos & Toluene & $\mathrm{PhSiH}_{3}$ & 16 & 20 & trace \\
\hline $7^{\mathrm{d}}$ & $\mathrm{NiBr}_{2}$ & Xantphos & DMA & $\mathrm{PhSiH}_{3}$ & 5 & trace & 16 \\
\hline $8^{\mathrm{d}}$ & $\mathrm{NiBr}_{2}$ & 2,2-bipyridine & DMA & $\mathrm{PhSiH}_{3}$ & 5 & trace & 30 \\
\hline
\end{tabular}

a The reactions were conducted with 1a $(0.2 \mathrm{mmol}), \mathbf{2 a}(0.22 \mathrm{mmol})$, catalyst $(10 \mathrm{~mol} \%)$, ligand $(11 \mathrm{~mol} \%), \mathrm{PhSiH}_{3}(1.0 \mathrm{equiv})$ in $\mathrm{DMA}(1 \mathrm{~mL})$ as solvent under argon at room temperature.

${ }^{\mathrm{b}}$ Yields determined by ${ }^{1} \mathrm{H}$ NMR spectroscopy using 1,3,5-trimethoxybenzene as the internal standard.

${ }^{\mathrm{c}} \mathrm{Me}(\mathrm{OEt})_{2} \mathrm{SiH} 3.0$ equivalent.

${ }^{\mathrm{d}} \mathrm{PhSiH}_{3} 1.0$ equivalent.

Initially, the reaction of $\mathbf{1 a}$ and $\mathbf{2 a}$ was conducted with commonly used $\mathrm{Cu}(\mathrm{OAc})_{2}$ as a catalyst (entries 1-6). However, the desired product 3a was not detected when 6,6'-dimethyl-2,2'-bipyridine was used as a ligand, and most of the starting materials 1a and 2a were recovery with a slight 2-aminobenzaldehyde (6) and styrene (7a) detected as byproducts (entries 1 and 2). In the case of using xantphose as a ligand (entries 3-6), the desired quinoline 3a was detected in low yields ( $8 \%$ in $\mathrm{CyH},<5 \%$ in THF, $<5 \%$ in Toluene), meanwhile 2-aminobenzaldehyde and styrene were also detected. It should be noted that there are some other compounds that have not been identified in the reaction systems. To our delight, when $\mathrm{NiBr}_{2}$ was tested as a catalyst, a significant increase in the yield of $\mathbf{3 a}$ was observed (entries 5 and 6). Therefore, the research continues by using nickel salt as a catalyst.

Table S2. Optimization of Solvent. ${ }^{\mathrm{a}}$ 


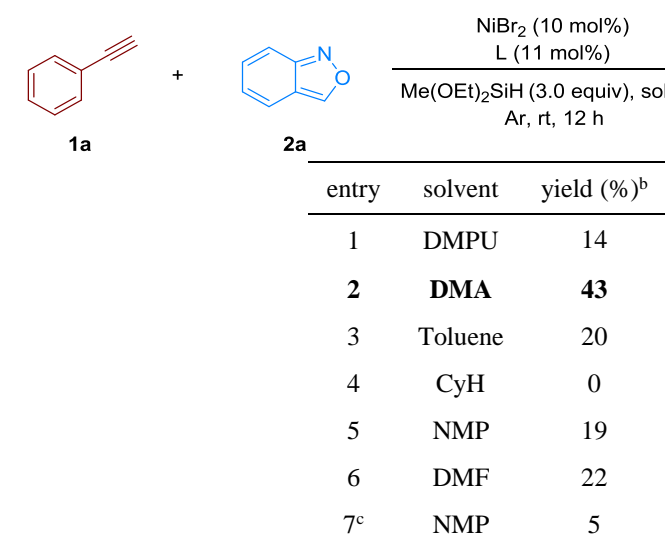

${ }^{a}$ Different solvents were screening with $1 \mathbf{a}(0.2 \mathrm{mmol}), \mathbf{2 a}(0.22 \mathrm{mmol}), \mathrm{NiBr}_{2}(10 \mathrm{~mol} \%), \mathrm{Me}(\mathrm{OEt}){ }_{2} \mathrm{SiH}(3.0$ equiv), 6,6'-dimethyl-2,2'-bipyridine $(11 \mathrm{~mol} \%)$ as ligand under argon at room temperature.

${ }^{\mathrm{b}}$ Yields determined by ${ }^{1} \mathrm{H}$ NMR spectroscopy using 1,3,5-trimethoxybenzene as the internal standard.

${ }^{\mathrm{c}}$ Under air atmosphere.

The results show that DMA is the best solvent for this transformation (entry 2). Beside, control experiment indicated that the yield of 3a decreased when the reaction was conducted under air atmosphere (entry 7).

Table S3. Optimization of Ligand. ${ }^{\mathrm{a}}$

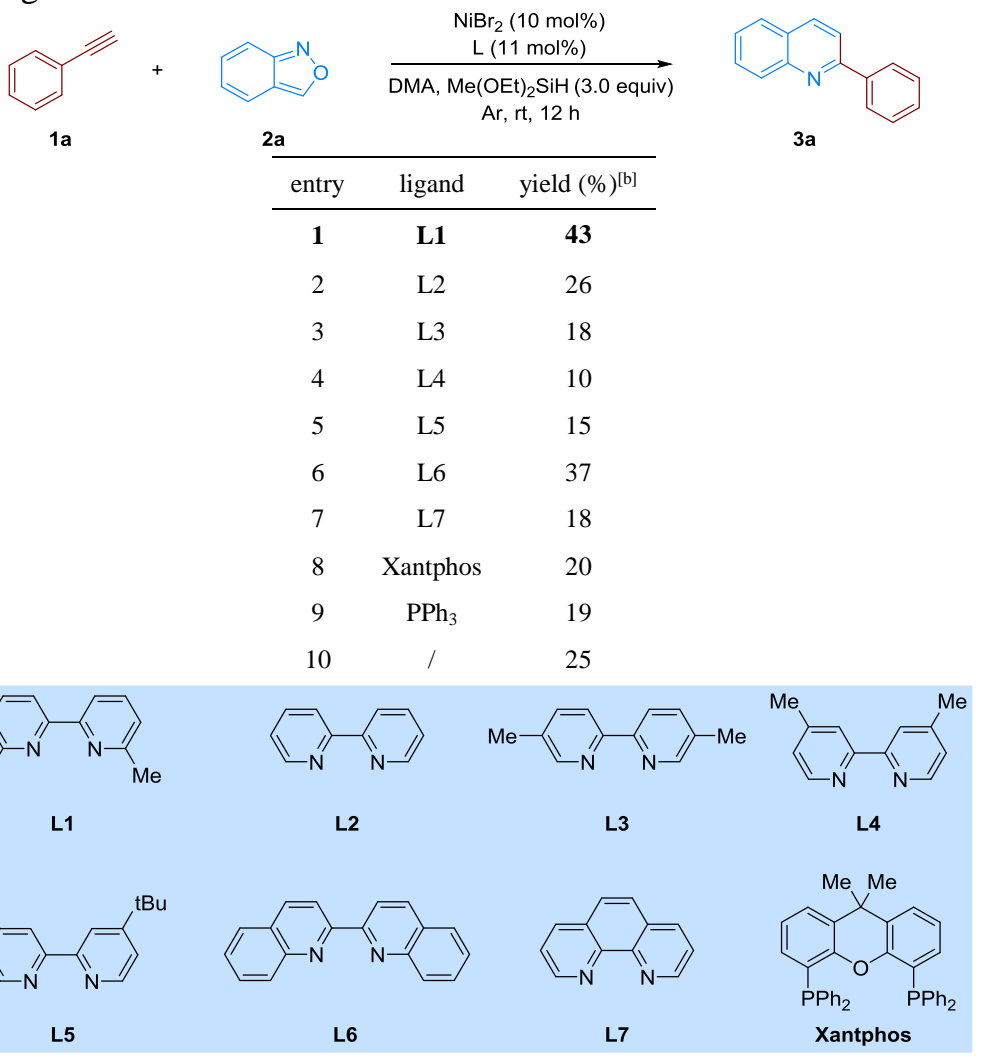

${ }^{\text {a }}$ Different ligands were screening with $\mathbf{1 a}(0.2 \mathrm{mmol}), \mathbf{2 a}(0.22 \mathrm{mmol}), \mathrm{NiBr}_{2}(10 \mathrm{~mol} \%), \mathrm{Me}(\mathrm{OEt})_{2} \mathrm{SiH}(3.0$ equiv) in $\mathrm{DMA}(1 \mathrm{~mL})$ as solvent under argon at room temperature.

${ }^{b}$ Yields determined by ${ }^{1} \mathrm{H}$ NMR spectroscopy using 1,3,5-trimethoxybenzene as the internal standard.

The results show that ligand is not essential for this transformation to proceed, however, the use of 6,6'-dimethyl-2,2'bipyridine as a ligand can increase the yield to $43 \%$ (entry 1 ).

Table S4. Optimization of Silane. ${ }^{a}$

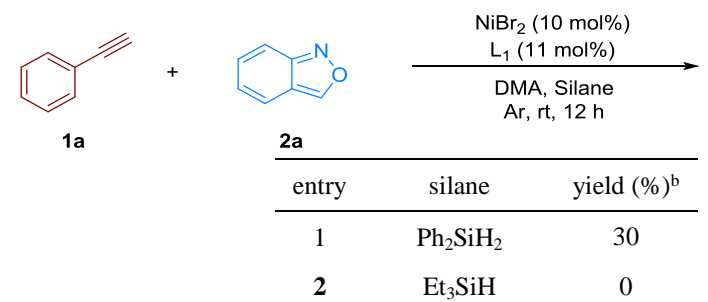

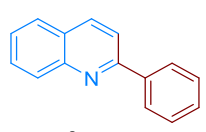

3a 


$\begin{array}{ccc}3 & \mathrm{PhSiH}_{3} & 13 \\ 4 & / & 0 \\ \mathbf{5} & \mathbf{M e}(\mathbf{O E t})_{2} \mathbf{S i H} & \mathbf{4 3} \\ 6 & (\mathrm{EtO})_{3} \mathrm{SiH} & 25 \\ 7 & \mathrm{PMHS} & 42\end{array}$

${ }^{\text {a }}$ A series of silanes were screening with $\mathbf{1 a}(0.2 \mathrm{mmol}), \mathbf{2 a}(0.22 \mathrm{mmol}), \mathrm{NiBr}_{2}(10 \mathrm{~mol} \%), 6,6$ '-dimethyl-2,2'-bipyridine (11 mol\%) in DMA (1 mL) under argon at room temperature.

${ }^{b}$ Yields determined by ${ }^{1} \mathrm{H}$ NMR spectroscopy using 1,3,5-trimethoxybenzene as the internal standard.

Control experiment indicated that silane as reductant is essential for this transformation to proceed (entry 4). Among the silanes tested, both $\mathrm{Me}(\mathrm{OEt})_{2} \mathrm{SiH}$ and $\mathrm{PMHS}$ are suitable for this reaction, and $\mathrm{Me}(\mathrm{OEt})_{2} \mathrm{SiH}$ gave the highest yield (entry 6).

Table S5. Optimization of Nickel Catalyst. ${ }^{\mathrm{a}}$

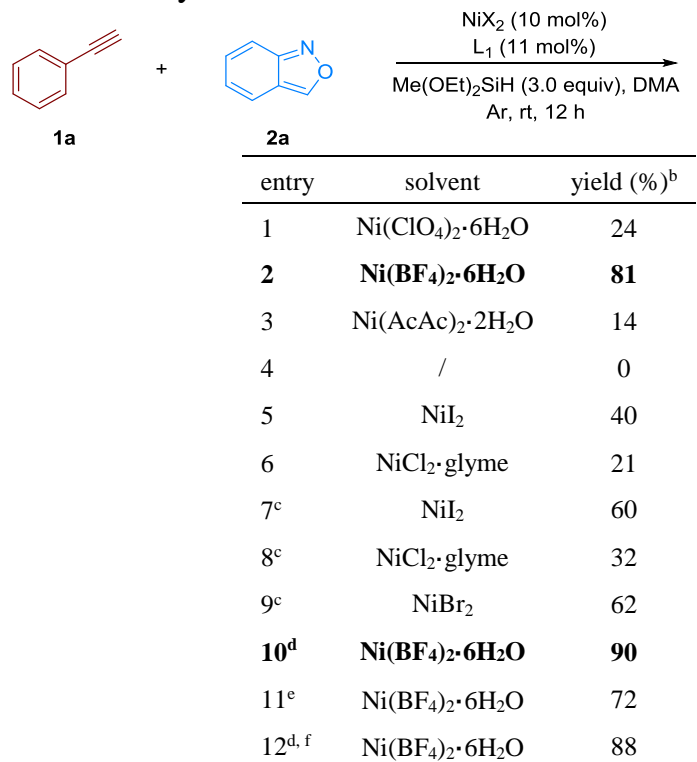

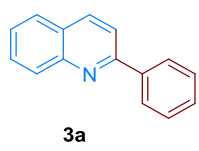

3

, $2 \mathrm{a}(0.22 \mathrm{mmol}), \mathrm{Me}(\mathrm{OEt})_{2} \mathrm{SiH}$ (3.0 equiv), 6,6'-dimethyl-2,2'-bipyridine (11 mol\%) as ligand in DMA

${ }^{a}$ Different nickel catalysts were screening with $\mathbf{1 a}(0.2 \mathrm{mmol}), \mathbf{2 a}(0.22 \mathrm{mmol}), \mathrm{Me}(\mathrm{OEt})_{2} \mathrm{SiH}(3.0 \mathrm{equi}$
$(1 \mathrm{~mL})$ under argon at room temperature.

${ }^{b}$ Yields determined by ${ }^{1} \mathrm{H}$ NMR spectroscopy using 1,3,5-trimethoxybenzene as the internal standard.

c 1.0 equivent of $\mathrm{H}_{2} \mathrm{O}$ was added.

${ }^{\mathrm{d}} \mathrm{Ni}\left(\mathrm{BF}_{4}\right)_{2} \cdot 6 \mathrm{H}_{2} \mathrm{O}(5 \mathrm{~mol} \%), \mathrm{L}_{2}(5.5 \mathrm{~mol} \%)$.

e $\mathrm{Ni}\left(\mathrm{BF}_{4}\right)_{2} \cdot 6 \mathrm{H}_{2} \mathrm{O}(15 \mathrm{~mol} \%), \mathrm{L}_{2}(16.5 \mathrm{~mol} \%)$.

${ }^{\mathrm{f}} \mathrm{PMHS}$ as reductant.

Nickel catalysts were found to have significant impact on this reaction and the yield of 3a increased to $81 \%$ when 10 mol\% $\mathrm{Ni}\left(\mathrm{BF}_{4}\right)_{2} \cdot 6 \mathrm{H}_{2} \mathrm{O}$ was used as catalyst (entry 2). In contrast, other nickel catalysts such as $\mathrm{Ni}_{2}\left(\mathrm{ClO}_{4}\right)_{2} \cdot 6 \mathrm{H}_{2} \mathrm{O}$, $\mathrm{Ni}(\mathrm{AcAc})_{2} \cdot 2 \mathrm{H}_{2} \mathrm{O}, \mathrm{NiI}_{2}$ and $\mathrm{NiCl}_{2} \cdot$ glyme gave decreased yields (entries 1-6 ). Considering $\mathrm{H}_{2} \mathrm{O}$ may have an impact on the reaction, control experiments with the addition of 1.0 equivent of $\mathrm{H}_{2} \mathrm{O}$ in the reaction system was conducted by using $\mathrm{NiI}_{2}, \mathrm{NiCl}_{2} \cdot$ glyme and $\mathrm{NiBr}_{2}$ as a catalyst. As a result, it is found that $\mathrm{H}_{2} \mathrm{O}$ can significantly improve the yield of 3a. Finally, the best result was obtained by adjusting the amount of the catalyst and ligand (entries 10 and 11).

\section{Supplementary Methods}

\section{General Procedure (A) for nickel-catalysed annulation of alkynes and anthranils}
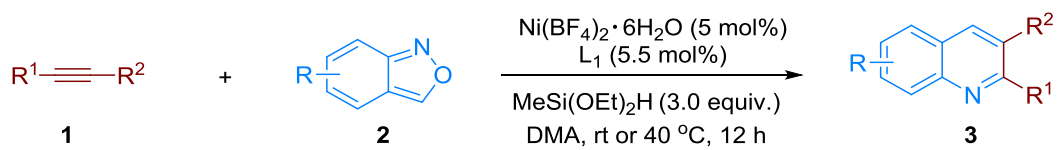

An oven-dried $20 \mathrm{~mL}$ vial was charged with $\mathrm{Ni}\left(\mathrm{BF}_{4}\right)_{2} \cdot 6 \mathrm{H}_{2} \mathrm{O}(5 \mathrm{~mol} \%)$ and 6,6'-dimethyl-2,2'-bipyridin (5.5 mol\%), and closed with a septum cap. After it was evacuated and back-filled with argon 3 times, DMA (1.0 mL), $\mathrm{Me}(\mathrm{OEt})_{2} \mathrm{SiH}(0.9 \mathrm{mmol}, 3.0$ equiv) and alkyne $(0.3 \mathrm{mmol}, 1$ equiv) were successively added via syringe, and the mixture was stirred at room temperature for $5 \mathrm{~min}$. Then, anthranil $(0.33 \mathrm{mmol}, 1.1$ equiv) was added and the mixture was stirred under an argon atmosphere at room temperature or $40{ }^{\circ} \mathrm{C}$ for $12 \mathrm{~h}$. After completion of the reaction, the resulting mixture was diluted with $1 \mathrm{M} \mathrm{LiCl}$ aqueous solution water $(10 \mathrm{~mL})$. Following phase separation, the aqueous layer was extracted 3 times with diethyl ether $(5 \mathrm{~mL})$. The combined organic phases were washed with brine $(10 \mathrm{~mL})$, 
dried over anhydrous $\mathrm{MgSO}_{4}$, filtered, and the organic phase was evaporated under reduced pressure (rotary evaporator). The residue was purified by column chromatography $\left(\mathrm{SiO}_{2}\right.$, ethyl acetate/petroleum ether gradient).

\section{Gram Scale Synthesis of 3cd from 2-bromo-9H-fluoren-9-one.}

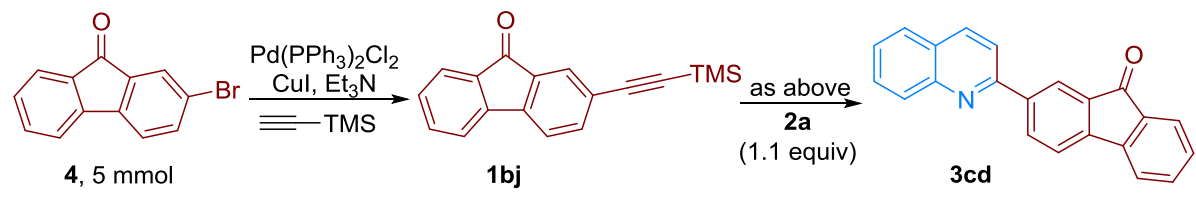

To a solution of 2-bromo-9H-fluoren-9-one $(5.0 \mathrm{mmol})$ in triethylamine $(5 \mathrm{~mL})$ and $\mathrm{DMF}(8 \mathrm{~mL})$ were added ethynyltrimethylsilane $(588.3 \mathrm{mg}, 6.0 \mathrm{mmol})$ and $\mathrm{Pd}\left(\mathrm{PPh}_{3}\right)_{2} \mathrm{Cl}_{2}(6 \mathrm{~mol} \%)$ and $\mathrm{CuI}(5 \mathrm{~mol} \%)$ at room temperature. Then the mixture was stirred at $80^{\circ} \mathrm{C}$ for $12 \mathrm{~h}$ under argon. After the starting material was consumed, the reaction mixture was quenched saturated $\mathrm{NH}_{4} \mathrm{Cl}$ solution and extracted with ethyl acetate. The combined organic extracts were washed with water and brine, and dried over anhydrous $\mathrm{Na}_{2} \mathrm{SO}_{4}$ and concentrated in vacuo. The crude product was purified by flash column chromatography on silica gel to give the corresponding alkynes $(1.1 \mathrm{~g}, 80 \%)$.

An oven-dried vial was charged with $\mathrm{Ni}\left(\mathrm{BF}_{4}\right)_{2} .6 \mathrm{H}_{2} \mathrm{O}(67.7 \mathrm{mg}, 0.199 \mathrm{mmol})$ and 6,6'-dimethyl-2,2'-bipyridin $(40 \mathrm{mg}$, $0.219 \mathrm{mmol})$, and closed with a septum cap. After it was evacuated and back-filled with argon 3 times, DMA (10 mL), $\mathrm{Me}(\mathrm{OEt})_{2} \mathrm{SiH}(8.0 \mathrm{mmol})$ and the obtained alkynes $(1.1 \mathrm{~g}, 3.98 \mathrm{mmol})$ were successively added, and the mixture was stirred at room temperature for $5 \mathrm{~min}$. Then, anthranil $(0.476 \mathrm{mg}, 4.0 \mathrm{mmol})$ was added and the mixture was stirred under an argon atmosphere at room temperature for $16 \mathrm{~h}$. After completion of the reaction, the resulting mixture was diluted with $1 \mathrm{M} \mathrm{LiCl}$ aqueous solution water $(15 \mathrm{~mL})$. Following phase separation, the aqueous layer was extracted 3 times with diethyl ether $(10 \mathrm{~mL})$. The combined organic phases were washed with brine $(10 \mathrm{~mL})$, dried over anhydrous $\mathrm{MgSO}_{4}$, filtered, and the organic phase was evaporated under reduced pressure (rotary evaporator). The residue was purified by column chromatography $\left(\mathrm{SiO}_{2}\right.$, ethyl acetate/petroleum ether gradient) to give 3cd $(0.92 \mathrm{~g})$ in $75 \%$ yield.

\section{Synthesis of 3ce from acetophenone.}

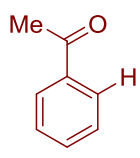

5

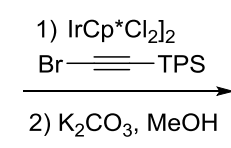

2) $\mathrm{K}_{2} \mathrm{CO}_{3}, \mathrm{MeOH}$

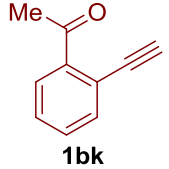

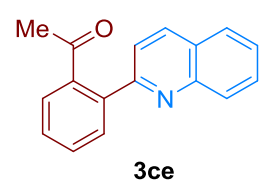

$3 \mathrm{ce}$

The C-H alkylation of acetophenone was conducted according to the reported method. ${ }^{2}$ An oven-dried vial was charged with $\left[\mathrm{Cp}^{*} \mathrm{IrCl}_{2}\right]_{2}(32 \mathrm{mg}, 4 \mathrm{~mol} \%), \operatorname{AgNTf}_{2}(62 \mathrm{mg}, 16 \mathrm{~mol} \%), \operatorname{AgOAc}(334 \mathrm{mg}, 0.2 \mathrm{mmol})$, and NaOAc $(216 \mathrm{mg}, 0.15 \mathrm{mmol})$, and then acetophenone $(1.0 \mathrm{mmol})$, (bromoethynyl)triisopropylsilane $(2.0 \mathrm{mmol})$ in DCE $(5.0$ $\mathrm{mL}$ ) was injected into the system via syringe. Then the reaction mixture was stirred at $120^{\circ} \mathrm{C}$ for $24 \mathrm{~h}$. After cooling to room temperature, the mixture was passed through a pad of Celite with $\mathrm{CH}_{2} \mathrm{Cl}_{2}$ as the eluent to remove the insoluble precipitate. The resulting solution was concentrated under reduced pressure (rotary evaporator). Then, the crude product was dissolved in methanol and $\mathrm{K}_{2} \mathrm{CO}_{3}(1.0 \mathrm{mmol})$ was added. The mixture was stirred at room temperature for $2 \mathrm{~h}$. After completion of the reaction, the solvent was evaporated under reduced pressure (rotary evaporator). The residue was purified by column chromatography $\left(\mathrm{SiO}_{2}\right.$, ethyl acetate/petroleum ether gradient) to give 1-(2ethynylphenyl)ethan-1-one (72.2 mg, 50\%).

The following the procedure A described above, the obtained 1-(2-ethynylphenyl)ethan-1-one $(0.3 \mathrm{mmol})$ was reacted with anthranils under the standard reaction conditions, giving the desired quinoline product 3ce $(38.5 \mathrm{mg}, 52 \%)$.

Synthesis of Graveolinine (3cg) from $30 .{ }^{3}$
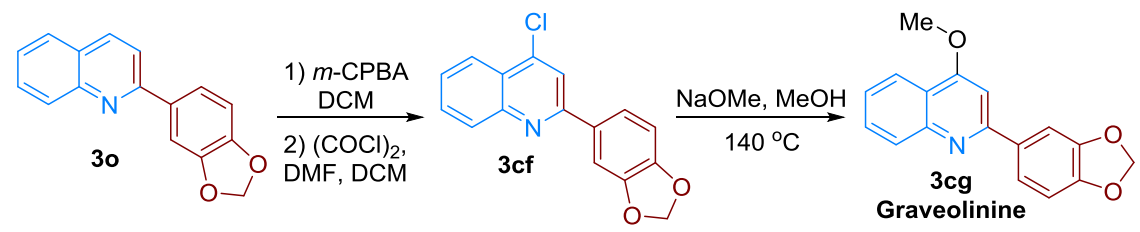

The synthesis of quinoline $N$-oxide was conducted according to the reported methods. 3-Chloroperbenzoic acid (1.2 mmol) was added to a solution of $\mathbf{3 o}(1.0 \mathrm{mmol})$ in dichloromethane $(5 \mathrm{~mL})$. The reaction mixture was stirred overnight at room temperature. The product mixture was concentrated under vacuum and the obtained residue was purified by flash-column chromatography 9:1 (EtOAc/MeOH) to yield the corresponding quinoline $N$-oxide.

The obtained quinoline $N$-oxide $(220 \mathrm{mg})$ was dissolved in DCM $(1.0 \mathrm{~mL})$ and added to a solution of DMF (2.0 mmol) and $(\mathrm{COCl})_{2}(2.0 \mathrm{mmol})$ in $\mathrm{DCM}(1.0 \mathrm{~mL})$ at $0{ }^{\circ} \mathrm{C}$. The reaction mixture was stirred for $12 \mathrm{~h}$. The resulting mixture was 
evaporated under reduced pressure (rotary evaporator) and then purified by silica gel column chromatography to give 2-(benzo[d][1,3]dioxol-5-yl)-4-chloroquinoline (189 mg, 67\%).

The obtained 2-(benzo[d][1,3]dioxol-5-yl)-4-chloroquinoline $(56.6 \mathrm{mg}, 0.2 \mathrm{mmol})$ was dissolved in $\mathrm{MeOH}(2.0 \mathrm{~mL})$, and $\mathrm{NaOMe}\left(0.24 \mathrm{mmol}, 1.2\right.$ equiv.) was added. The mixture was reacted at $140{ }^{\circ} \mathrm{C}$ for 10 hours under argon atmosphere. The resulting solution was concentrated under reduced pressure (rotary evaporator), and then purified by silica gel column chromatography to give Graveolinine (33.5 mg, 86\%).

\section{Synthesis of 3ci from $3 \mathrm{v}$.}

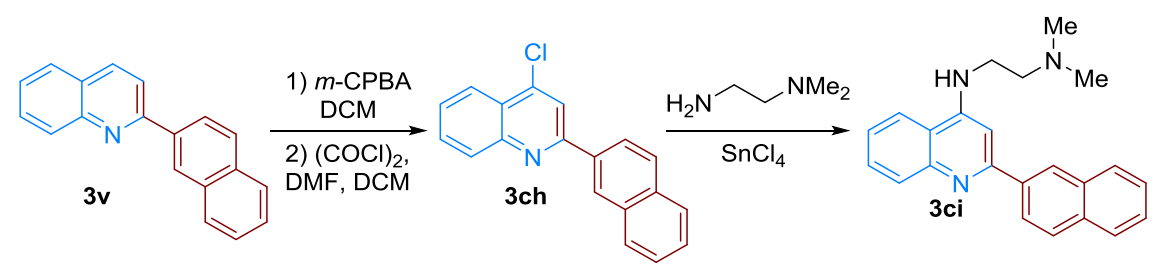

The synthesis of $\mathbf{3 c g}$ was conducted following the literature procedure. ${ }^{4} 3$-Chloroperbenzoic acid (1.2 mmol) was added to a solution of $3 \mathbf{v}(1.0 \mathrm{mmol})$ in dichloromethane $(5 \mathrm{~mL})$. The reaction mixture was stirred overnight at room temperature. The product mixture was concentrated under vacuum and the obtained residue was purified by flashcolumn chromatography 9:1 (EtOAc/MeOH) to yield the corresponding quinoline $N$-oxide.

The obtained quinoline $N$-oxide $(217 \mathrm{mg})$ was dissolved in DCM $(1.0 \mathrm{~mL})$ and added to a solution of DMF (2.0 mmol) and $(\mathrm{COCl})_{2}(2.0 \mathrm{mmol})$ in DCM $(1.0 \mathrm{~mL})$ at $0{ }^{\circ} \mathrm{C}$. The reaction mixture was stirred for $12 \mathrm{~h}$. The resulting mixture was evaporated under reduced pressure (rotary evaporator) and then purified by silica gel column chromatography to give 4-chloro-2-(naphthalen-2-yl)quinoline (188 mg, 65\%).

The obtained 4-chloro-2-(naphthalen-2-yl)quinoline $(57.8 \mathrm{mg}, 0.2 \mathrm{mmol})$ was dissolved in $N^{l}, N^{l}$-dimethylethane-1,2diamine $(1.0 \mathrm{~mL})$, and $\mathrm{SnCl}_{4}(0.02 \mathrm{mmol})$ was added. The mixture was reacted at $130{ }^{\circ} \mathrm{C}$ for 4 hours under argon atmosphere. The resulting solution was concentrated under reduced pressure (rotary evaporator), and then purified by silica gel column chromatography to give 3ci $(60.7 \mathrm{mg}, 89 \%)$.

\section{Mechanistic Studies}

\section{Hydroamination of styrene}

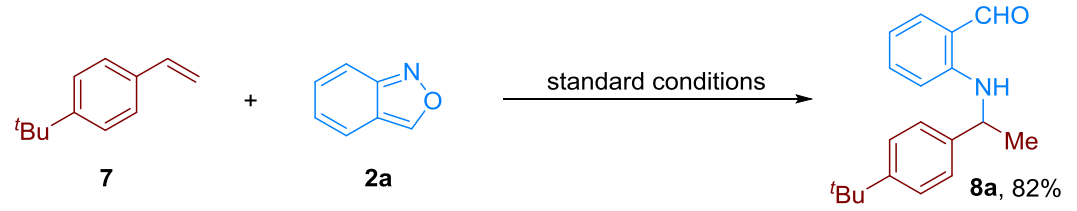

The hydroamination of styrene 7 was conducted fellowing the general procedure A under the standard reaction conditions. An oven-dried $20 \mathrm{~mL}$ vial was charged with $\mathrm{Ni}\left(\mathrm{BF}_{4}\right)_{2} \cdot 6 \mathrm{H}_{2} \mathrm{O}(5 \mathrm{~mol} \%)$ and 6,6'-dimethyl-2,2'-bipyridin (5.5 mol\%), and closed with a septum cap. After it was evacuated and back-filled with argon 3 times, DMA (1.0 mL), $\mathrm{Me}(\mathrm{OEt})_{2} \mathrm{SiH}$ (3.0 equiv) and styrene $(0.3 \mathrm{mmol}, 1$ equiv) were successively added. After stirring at room temperature for $5 \mathrm{~min}$, anthranil $2 \mathrm{a}(0.33 \mathrm{mmol}, 1.1$ equiv) was added via syringe and the mixture was stirred under an argon atmosphere at room temperature for $12 \mathrm{~h}$. After completion of the reaction, the resulting mixture was diluted with $1 \mathrm{M}$ $\mathrm{LiCl}$ aqueous solution water $(10 \mathrm{~mL})$. Following phase separation, the aqueous layer was extracted 3 times with diethyl ether $(5 \mathrm{~mL})$. The combined organic phases were washed with brine $(10 \mathrm{~mL})$, dried over anhydrous $\mathrm{MgSO}_{4}$, filtered, and the organic phase was evaporated under reduced pressure (rotary evaporator). The residue was purified by column chromatography $\left(\mathrm{SiO}_{2}\right.$, ethyl acetate/petroleum ether gradient). The desired hydroamination product 8a was obtained in $82 \%$ yield as light yellow oil.

\section{Deuteration with $\mathrm{Ph}_{2} \mathrm{SiD}_{2}$}

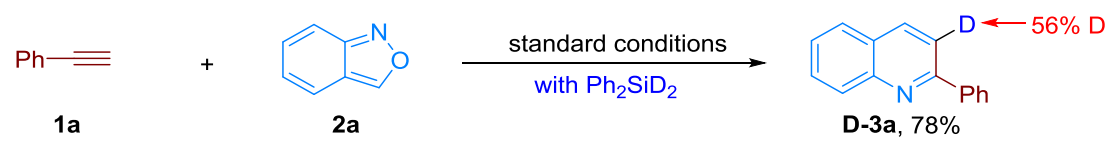

An isotope labeling experiment was conducted with $\mathrm{Ph}_{2} \mathrm{SiD}_{2}$ under the standard reaction conditions. An oven-dried 20 $\mathrm{mL}$ vial was charged with $\mathrm{Ni}\left(\mathrm{BF}_{4}\right)_{2} \cdot 6 \mathrm{H}_{2} \mathrm{O}(5 \mathrm{~mol} \%)$ and 6,6'-dimethyl-2,2'-bipyridin $(5.5 \mathrm{~mol} \%)$, and closed with a septum cap. After it was evacuated and back-filled with argon 3 times, DMA (1.0 mL), $\mathrm{Ph}_{2} \mathrm{SiD}_{2}(0.45 \mathrm{mmol}, 1.5$ equiv) and alkyne ( $0.3 \mathrm{mmol}, 1$ equiv) were successively added. After stirring at room temperature for $5 \mathrm{~min}$, anthranil (0.33 mmol, 1.1 equiv) was added via syringe and the mixture was stirred under an argon atmosphere at room temperature 
for $12 \mathrm{~h}$. After completion of the reaction, the resulting mixture was diluted with $1 \mathrm{M} \mathrm{LiCl}$ aqueous solution water (10 $\mathrm{mL})$. Following phase separation, the aqueous layer was extracted 3 times with diethyl ether $(5 \mathrm{~mL})$. The combined organic phases were washed with brine $(10 \mathrm{~mL})$, dried over anhydrous $\mathrm{MgSO}_{4}$, filtered, and the organic phase was evaporated under reduced pressure (rotary evaporator). The residue was purified by column chromatography $\left(\mathrm{SiO}_{2}\right.$, ethyl acetate/petroleum ether gradient). Deuterated 3a was obtained in 78\% yield with 56\% deuterium incorporation.
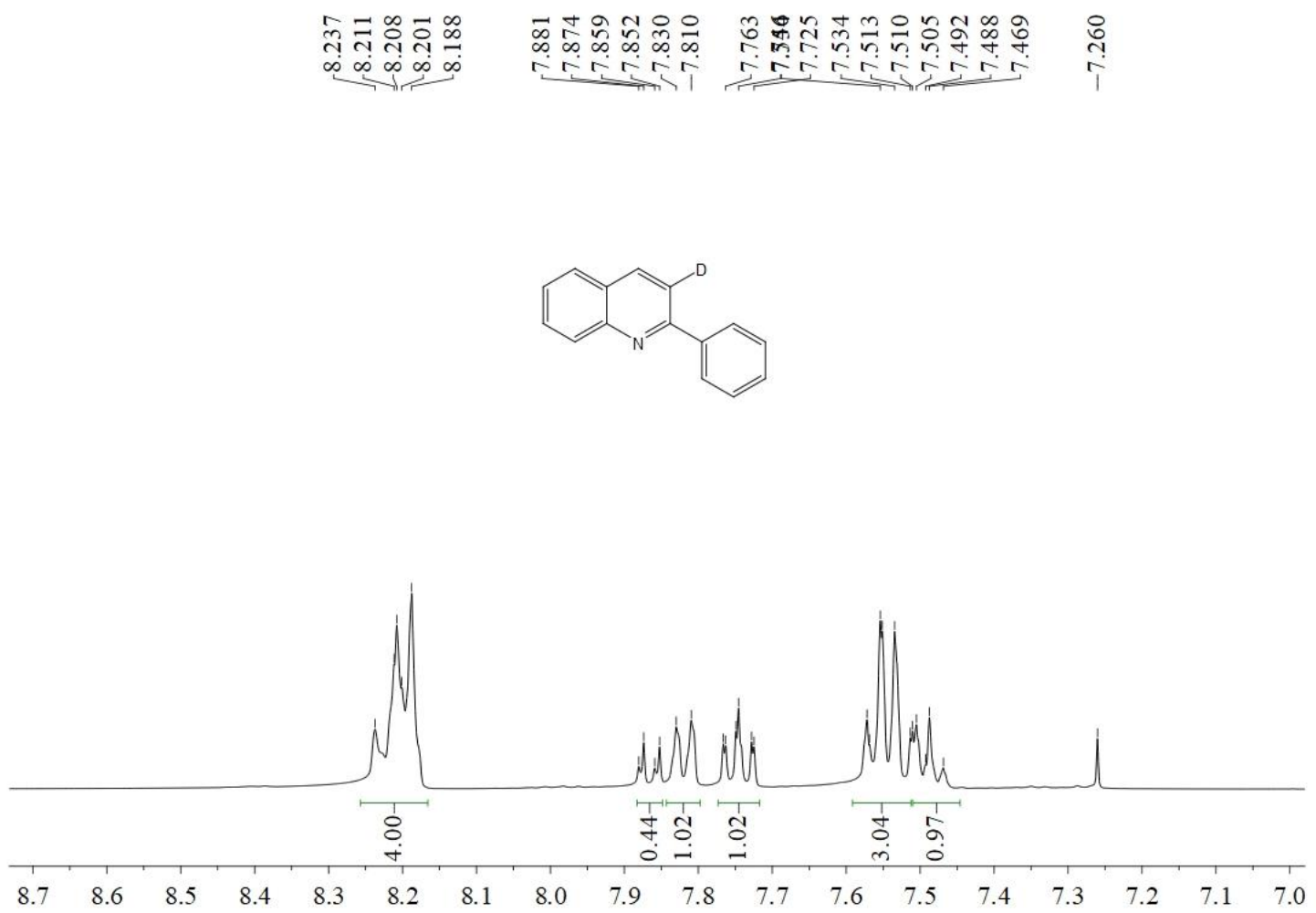

Kinetic isotope effects (KIE) experiments

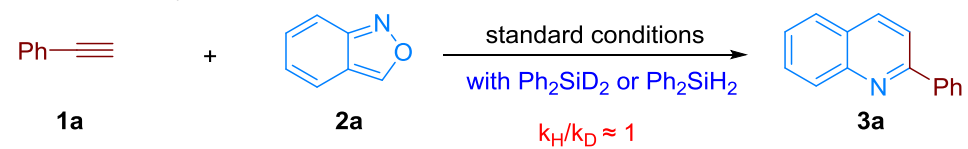

An oven-dried $20 \mathrm{~mL}$ vial equipped with a Teflon-coated stirring bar was charged with $\mathrm{Ni}\left(\mathrm{BF}_{4}\right)_{2} \cdot 6 \mathrm{H}_{2} \mathrm{O}(5 \mathrm{~mol} \%)$ and 6,6'-dimethyl-2,2'-bipyridin (5.5 mol\%), 1,3,5-trimethoxybenzene $(16.8 \mathrm{mg}, 0.1 \mathrm{mmol})$ as the internal standard and closed with a septum cap. After it was evacuated and back-filled with argon 3 times, DMA (1.0 mL), $\mathrm{Ph}_{2} \mathrm{SiD}_{2}(0.3$ mmol, 1.0 equiv), alkyne $(0.3 \mathrm{mmol}, 1$ equiv) and anthranil $(0.33 \mathrm{mmol}, 1.1$ equiv) were successively added via syringe. The resulting mixture was stirred at room temperature and samples $(50 \mathrm{uL})$ were taken with syringe at $5 \mathrm{~min}$, $10 \mathrm{~min}$ and $15 \mathrm{~min}$. The obtained sample was diluted with $1 \mathrm{M} \mathrm{LiCl}$ aqueous solution water $(1 \mathrm{~mL})$ and extracted 3 times with diethyl ether $(5 \mathrm{~mL})$. After evaporating the solvent under reduced pressure (rotary evaporator), the residue was analyzed with ${ }^{1} \mathrm{H}$ NMR and the yields was collected in the Table S6.

The parallel experiments with $\mathrm{Ph}_{2} \mathrm{SiH}_{2}$ ( 0.3 mmol, 1.0 equiv) were conducted fellowing the same procedure described above and the ${ }^{1} \mathrm{H}$ NMR yields were collected in $5 \mathrm{~min}, 10 \mathrm{~min}$ and $15 \mathrm{~min}$.

Table S6. NMR yields detected in the parallel KIE experiments

\begin{tabular}{|c|c|c|c|}
\hline Yields Time (min) & 5 & 10 & 15 \\
\hline D-3a & $16 \%$ & $23 \%$ & $40 \%$ \\
\hline 3a & $18 \%$ & $28 \%$ & $41 \%$ \\
\hline
\end{tabular}




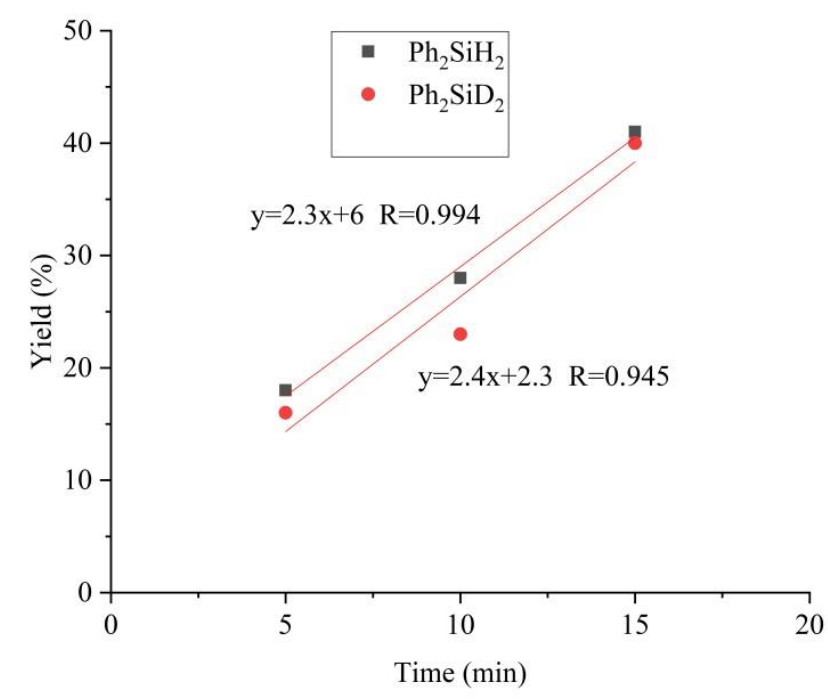

\section{Stepwise stoichiometric reaction}

$$
\begin{gathered}
\mathrm{Ni}\left(\mathrm{BF}_{4}\right)_{2} \cdot 6 \mathrm{H}_{2} \mathrm{O} \\
0.1 \mathrm{mmol}
\end{gathered}
$$
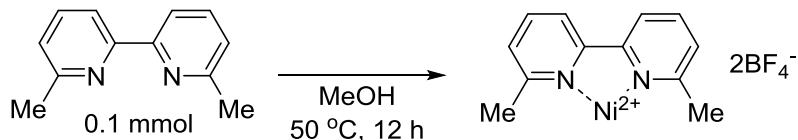

6,6'-Dimethyl-2,2'-bipyridine $(0.1 \mathrm{mmol})$ and $\mathrm{Ni}\left(\mathrm{BF}_{4}\right)_{2} \cdot 6 \mathrm{H}_{2} \mathrm{O}(0.1 \mathrm{mmol})$ were dissolved in $\mathrm{MeOH}(2 \mathrm{~mL})$, and the resulting mixture was stirred at room temperature for $12 \mathrm{~h}$. After that, the solvent was removed through the rotary evaporator, and then continue to use oil pump to remove the traces amount of water in the system. The obtained complex was analyzed by ${ }^{1} \mathrm{H}$ NMR with acetone- $\mathrm{d}_{6}$ as solvent. Comparing the ${ }^{1} \mathrm{H}$ NMR spectrum of ligand and the obtained complex, a significant shift of the corresponding hydrogen can be observed.
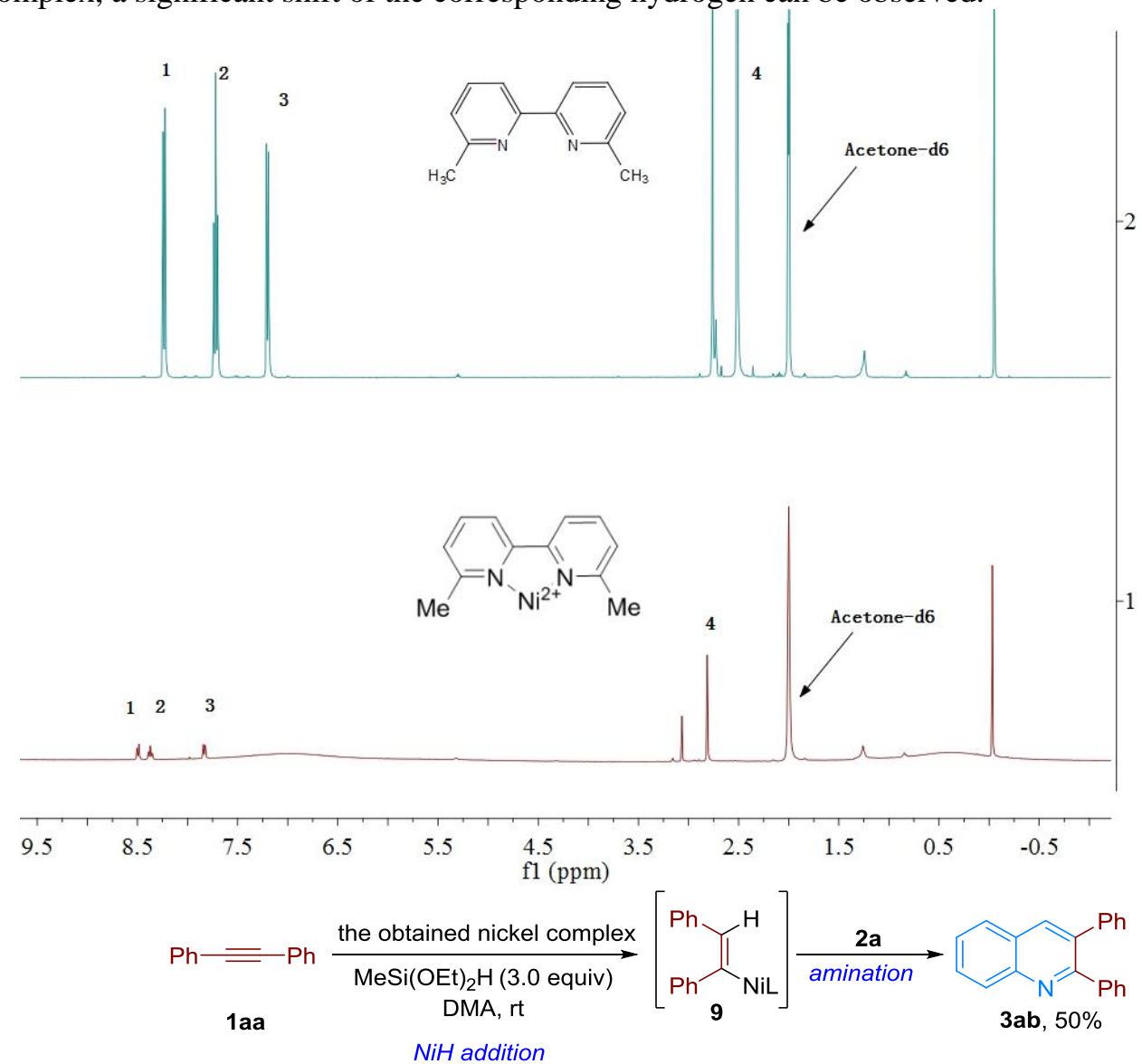

The obtained complex was transferred to an oven-dried vial equipped with a Teflon-coated stirring bar, and sealed with a crimp cap. After evacuated and back-filled with argon 3 times, DMA $(1 \mathrm{~mL})$ and $\mathrm{Me}(\mathrm{OEt})_{2} \mathrm{SiH}$ (3.0 equiv) were successively added and the mixture was stirred under an argon atmosphere for $5 \mathrm{~min}$. Then, 1,2-diphenylethyne $(0.1$ 
mmol) was added in one pot, and the mixture continue to react at room temperature and was monitored by GC. After consumption of 1,2-diphenylethyne (about 2 hours), anthranil $\mathbf{1 a}(0.11 \mathrm{mmol})$ was added via syringe and the mixture continue to react at room temperature for another 3 hours. Then, 1,3,5-trimethoxybenzene $(16.8 \mathrm{mg}, 0.1 \mathrm{mmol}) \mathrm{was}$ added as an internal standard, and a sample was taken from the resulting mixture. The obtained sample was diluted with $1 \mathrm{M} \mathrm{LiCl}$ aqueous solution water $(1 \mathrm{~mL})$ and extracted 3 times with diethyl ether $(5 \mathrm{~mL})$. After evaporating the solvent under reduced pressure (rotary evaporator), the residue was analyzed with ${ }^{1} \mathrm{H}$ NMR and the desired product $\mathbf{3 a}$ was detected in $50 \%$ yield.

\section{The reaction of $\mathrm{CH}_{2}=\mathrm{CHMgBr}$ and anthranil $2 a$.}

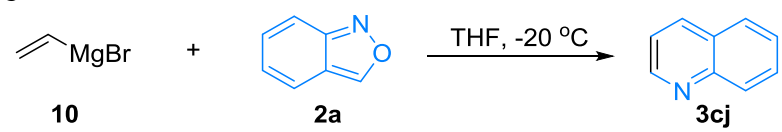

To an oven-dried vial equipped with a Teflon-coated stirring bar, anthranil $\mathbf{2 a}(0.3 \mathrm{mmol})$ was added and then the vial was sealed with a crimp cap. After evacuated and back-filled with argon 3 times, dry THF (1.0 mL) was added via syringe. The resulting mixture was cooled to $-20^{\circ} \mathrm{C}$, and $\mathrm{CH}_{2}=\mathrm{CHMgBr}(0.3 \mathrm{~mL}, 1 \mathrm{M}$ THF solution) was added dropwise. After strring at $-20^{\circ} \mathrm{C}$ for $2 \mathrm{~h}$, the reaction mixture was quenched by the addition of saturated $\mathrm{NH}_{4} \mathrm{Cl}$ aqueous solution $(5 \mathrm{~mL})$ and extracted 3 times with diethyl ether $(5 \mathrm{~mL})$. The combined organic phases were washed with brine $(5 \mathrm{~mL})$, dried over anhydrous $\mathrm{MgSO}_{4}$, filtered, and the organic phase was evaporated under reduced pressure (rotary evaporator). The residue was analyzed with ${ }^{1} \mathrm{H}$ NMR and GC-MS, however, the desired quinoline was not detected and most $\mathbf{2 a}$ was recovery. This result indicates that the direct nucleophilic attack of organometallic reagent to the nitrogen of anthranil is not feasible.

\section{Synthesis and Characterization of the Corresponding Products}

\section{2-Phenylquinoline (3a) ${ }^{5}$ [cas: 612-96-4]}<smiles>c1ccc(-c2ccc3ccccc3n2)cc1</smiles>

Compound 3a was prepared following the general procedure A from phenylacetylene $1 \mathbf{a}(0.30 \mathrm{mmol})$ and benzo[c]isoxazole $\mathbf{2 a}(0.33 \mathrm{mmol})$ and isolated as a white solid $(52.9 \mathrm{mg}, 86 \%)$, m.p 77-79 ${ }^{\circ} \mathrm{C}$.

${ }^{1} \mathbf{H}$ NMR $\left(400 \mathrm{MHz} \mathrm{CDCl}_{3}\right) \delta 8.21(\mathrm{~m}, 4 \mathrm{H}), 7.86(\mathrm{~d}, J=8.6 \mathrm{~Hz}, 1 \mathrm{H}), 7.81(\mathrm{~d}, J=8.1 \mathrm{~Hz}, 1 \mathrm{H}), 7.75(\mathrm{t}, J=7.6 \mathrm{~Hz}, 1 \mathrm{H})$, $7.53(\mathrm{~m}, 4 \mathrm{H}) \mathrm{ppm}$.

${ }^{13} \mathrm{C}$ NMR $\left(100 \mathrm{MHz}, \mathrm{CDCl}_{3}\right) \delta$ 157.2, 148.2, 139.6, 136.6, 129.6, 129.5, 129.2, 128.7, 127.5, 127.4, 127.1, 126.2, $118.8 \mathrm{ppm}$.

MS (EI) m/z C ${ }_{15} \mathrm{H}_{11} \mathrm{~N}: 205.1[\mathrm{M}]^{+}, 176.1,128.0,102.2,75.1$.

\section{2-(4-Fluorophenyl)quinolone $(3 b)^{6}$ [cas: 323-91-1]}<smiles>Fc1ccc(-c2ccc3ccccc3n2)cc1</smiles>

Compound $\mathbf{3 b}$ was prepared following the general procedure A from 1-ethynyl-4-fluorobenzene $\mathbf{1 b}(0.30 \mathrm{mmol})$ and benzo[c]isoxazole $2 \mathbf{a}(0.33 \mathrm{mmol})$ and isolated as white solid $(56.9 \mathrm{mg}, 85 \%)$, m.p 86-88 ${ }^{\circ} \mathrm{C}$.

${ }^{1} \mathbf{H}$ NMR $\left(400 \mathrm{MHz}, \mathrm{CDCl}_{3}\right) \delta 8.23-8.14(\mathrm{~m}, 4 \mathrm{H}), 7.82(\mathrm{~d}, J=8.5 \mathrm{~Hz}, 2 \mathrm{H}), 7.73(\mathrm{t}, J=7.6 \mathrm{~Hz}, 1 \mathrm{H}), 7.53(\mathrm{t}, J=7.4$ $\mathrm{Hz}, 1 \mathrm{H}), 7.21(\mathrm{t}, J=8.6 \mathrm{~Hz}, 2 \mathrm{H}) \mathrm{ppm}$.

${ }^{13}$ C NMR $\left(100 \mathrm{MHz}, \mathrm{CDCl}_{3}\right) \delta 163.8(\mathrm{~d}, J=248.9 \mathrm{~Hz}), 156.2,148.2,136.8,135.8(\mathrm{~d}, J=3.1 \mathrm{~Hz}), 129.7,129.6,129.4$ $(\mathrm{d}, J=8.4 \mathrm{~Hz}), 127.4,127.0,126.3,118.6,115.7(\mathrm{~d}, J=21.6 \mathrm{~Hz}) \mathrm{ppm}$.

${ }^{19} \mathbf{F}$ NMR $\left(375 \mathrm{MHz}, \mathrm{CDCl}_{3}\right) \delta-112.47(\mathrm{~s}, 1 \mathrm{H}) \mathrm{ppm}$.

MS (EI) m/z C ${ }_{15} \mathrm{H}_{10} \mathrm{FN}: 223.1[\mathrm{M}]^{+}, 194.0,111.2,75.1$. 
<smiles>Clc1ccc(-c2ccc3ccccc3n2)cc1</smiles>

Compound $3 \mathbf{c}$ was prepared following the general procedure A from 1-chloro-4-ethynylbenzene $1 \mathbf{c}(0.30 \mathrm{mmol})$ and benzo[c]isoxazole $2 \mathbf{a}(0.33 \mathrm{mmol})$ and isolated as white solid $(55.4 \mathrm{mg}, 78 \%)$, m.p $106-108{ }^{\circ} \mathrm{C}$.

${ }^{1} \mathbf{H}$ NMR $\left(400 \mathrm{MHz}, \mathrm{CDCl}_{3}\right) \delta 8.12(\mathrm{t}, J=9.3 \mathrm{~Hz}, 2 \mathrm{H}), 8.06(\mathrm{~d}, J=8.6 \mathrm{~Hz}, 2 \mathrm{H}), 7.71(\mathrm{~m}, 2 \mathrm{H}), 7.51-7.40(\mathrm{~m}, 2 \mathrm{H})$ ppm.

${ }^{13}$ C NMR $\left(100 \mathrm{MHz}, \mathrm{CDCl}_{3}\right) \delta 155.8,148.1,137.9,136.8,135.4,129.7,129.6,128.9,128.7,127.4,127.1,126.4$, $118.4 \mathrm{ppm}$.

MS (EI) m/z C $15 \mathrm{H}_{10} \mathrm{ClN}: 239.1[\mathrm{M}]^{+}, 204.1,176.1,102.1,75.1$.

2-(4-Bromophenyl)quinolone (3d) ${ }^{6}$ [cas: 24641-31-4]<smiles>Brc1ccc(-c2ccc3ccccc3n2)cc1</smiles>

Compound 3d was prepared following the general procedure A from 1-bromo-4-ethynylbenzene $1 \mathbf{d}(0.30 \mathrm{mmol})$ and benzo[c]isoxazole $2 \mathrm{a}(0.33 \mathrm{mmol})$ and isolated as a white solid $(70.2 \mathrm{mg}, 83 \%)$, m.p $111-113{ }^{\circ} \mathrm{C}$.

${ }^{1} \mathbf{H}$ NMR $\left(400 \mathrm{MHz}, \mathrm{CDCl}_{3}\right) \delta 8.17(\mathrm{~m}, 2 \mathrm{H}), 8.04(\mathrm{~d}, J=8.4 \mathrm{~Hz}, 2 \mathrm{H}), 7.80(\mathrm{~m}, 2 \mathrm{H}), 7.73(\mathrm{t}, J=7.6 \mathrm{~Hz}, 1 \mathrm{H}), 7.64(\mathrm{~d}, J$ $=8.4 \mathrm{~Hz}, 2 \mathrm{H}), 7.53(\mathrm{t}, J=7.5 \mathrm{~Hz}, 1 \mathrm{H}) \mathrm{ppm}$.

${ }^{13} \mathrm{C}$ NMR $\left(100 \mathrm{MHz}, \mathrm{CDCl}_{3}\right) \delta 155.9,148.2,138.4,136.9,131.9,129.8,129.6,129.0,127.4,127.2,126.4,123.9$, $118.4 \mathrm{ppm}$.

HRMS (ESI) $\mathrm{m} / \mathrm{z}$ calcd. for $\mathrm{C}_{15} \mathrm{H}_{11} \mathrm{BrN}[\mathrm{M}+\mathrm{H}]^{+} 284.00694$, found 284.00656 .

2-(4-Methoxyphenyl)quinolone $(3 e)^{6}$ [cas: 16032-40-9]<smiles>COc1ccc(-c2ccc3ccccc3n2)cc1</smiles>

Compound 3e was prepared following the general procedure A from 1-ethynyl-4-methoxybenzene $1 \mathbf{e}(0.30 \mathrm{mmol})$ and benzo[c]isoxazole $2 \mathrm{a}(0.33 \mathrm{mmol})$ and isolated as a white solid $(56.4 \mathrm{mg}, 80 \%)$, m.p $113-115{ }^{\circ} \mathrm{C}$.

${ }^{1} \mathbf{H}$ NMR $\left(400 \mathrm{MHz}, \mathrm{CDCl}_{3}\right) \delta 8.16(\mathrm{~m}, 4 \mathrm{H}), 7.81(\mathrm{~m}, 2 \mathrm{H}), 7.71(\mathrm{t}, J=7.7 \mathrm{~Hz}, 1 \mathrm{H}), 7.50(\mathrm{t}, J=7.5 \mathrm{~Hz}, 1 \mathrm{H}), 7.05(\mathrm{~d}, J$ $=6.9 \mathrm{~Hz}, 2 \mathrm{H}), 3.88(\mathrm{~s}, 3 \mathrm{H}) \mathrm{ppm}$.

${ }^{13} \mathrm{C}$ NMR $\left(100 \mathrm{MHz}, \mathrm{CDCl}_{3}\right) \delta 160.8,156.9,148.2,136.6,132.2,129.5,128.8,128.3,127.4,126.9,125.9,118.5$, $114.2,55.3 \mathrm{ppm}$.

MS (EI) m/z C C $16 \mathrm{H}_{13} \mathrm{NO}: 235.1[\mathrm{M}]^{+}, 220.0,204.1,126.1,89.0,75.1$.

\section{2-(p-Tolyl)quinolone (3f $)^{5}$ [cas: 24667-94-5]}<smiles>Cc1ccc(-c2ccc3ccccc3n2)cc1</smiles>

Compound $\mathbf{3 f}$ was prepared following the general procedure A from 1-ethynyl-4-methylbenzene $\mathbf{1 f}(0.30 \mathrm{mmol})$ and benzo[c]isoxazole $2 \mathrm{a}(0.33 \mathrm{mmol})$ and isolated as a white solid $(58.5 \mathrm{mg}, 89 \%)$, m.p $78-80{ }^{\circ} \mathrm{C}$.

${ }^{1} \mathbf{H}$ NMR $\left(400 \mathrm{MHz}, \mathrm{CDCl}_{3}\right) \delta 8.19(\mathrm{~m}, 2 \mathrm{H}), 8.10(\mathrm{~d}, J=8.1 \mathrm{~Hz}, 2 \mathrm{H}), 7.86(\mathrm{~d}, J=8.6 \mathrm{~Hz}, 1 \mathrm{H}), 7.81(\mathrm{~d}, J=8.1 \mathrm{~Hz}$, $1 \mathrm{H}), 7.78-7.69(\mathrm{~m}, 1 \mathrm{H}), 7.52(\mathrm{~m}, 1 \mathrm{H}), 7.35(\mathrm{~d}, J=8.1 \mathrm{~Hz}, 2 \mathrm{H}) \mathrm{ppm}$.

${ }^{13} \mathrm{C}$ NMR $\left(100 \mathrm{MHz}, \mathrm{CDCl}_{3}\right) \delta 157.3,148.2,139.3,136.8,136.6,129.6,129.5,127.4,127.0,126.0,118.8,21.3 \mathrm{ppm}$. MS (EI) m/z C ${ }_{16} \mathrm{H}_{13} \mathrm{~N}: 219.1[\mathrm{M}]^{+}, 189.0,140.1,108.7,75.1$.

2-(4-(Methylthio)phenyl)quinolone (3g) ${ }^{7}$ [cas: 1380751-78-9]<smiles>CSc1ccc(-c2ccc3ccccc3n2)cc1</smiles> 
Compound $\mathbf{3 g}$ was prepared following the general procedure A from (4-ethynylphenyl)(methyl)sulfane $\mathbf{1 g}(0.30 \mathrm{mmol})$ and benzo[c] isoxazole $\mathbf{2 a}(0.33 \mathrm{mmol})$ and isolated as a yellow solid $(57.2 \mathrm{mg}, 76 \%)$, m.p 141-143 ${ }^{\circ} \mathrm{C}$.

${ }^{1} \mathbf{H}$ NMR $\left(400 \mathrm{MHz}, \mathrm{CDCl}_{3}\right) \delta 8.22-8.08(\mathrm{~m}, 4 \mathrm{H}), 7.82(\mathrm{~m}, 2 \mathrm{H}), 7.73(\mathrm{~m}, 1 \mathrm{H}), 7.51(\mathrm{t}, J=7.4 \mathrm{~Hz}, 1 \mathrm{H}), 7.39(\mathrm{~d}, J=$ $8.4 \mathrm{~Hz}, 2 \mathrm{H}), 2.55(\mathrm{~s}, 3 \mathrm{H}) \mathrm{ppm}$.

${ }^{13} \mathrm{C}$ NMR $\left(100 \mathrm{MHz}, \mathrm{CDCl}_{3}\right) \delta 156.6,148.3,140.4,136.7,136.2,129.6,129.5,127.8,127.4,127.1,126.4,126.1$, $118.5,15.5 \mathrm{ppm}$.

MS (EI) m/z C16H13NS: $251.1[\mathrm{M}]^{+}, 204.2,128.0,75.0$.

$N, N$-Dimethyl-4-(quinolin-2-yl)aniline (3h) ${ }^{5}$ [cas:16032-41-0]<smiles>CN(C)c1ccc(-c2ccc3ccccc3n2)cc1</smiles>

Compound $\mathbf{3 h}$ was prepared following the general procedure A from 4-ethynyl-N,N-dimethylaniline $\mathbf{1 h}(0.30 \mathrm{mmol})$ and benzo[c] isoxazole $2 \mathbf{a}(0.33 \mathrm{mmol})$ and isolated as a yellow solid $(60.3 \mathrm{mg}, 81 \%)$, m.p $153-155^{\circ} \mathrm{C}$.

${ }^{1} \mathbf{H}$ NMR $\left(400 \mathrm{MHz}, \mathrm{CDCl}_{3}\right) \delta 8.10(\mathrm{~m}, 4 \mathrm{H}), 7.81(\mathrm{~d}, J=8.7 \mathrm{~Hz}, 1 \mathrm{H}), 7.75(\mathrm{~d}, J=8.1 \mathrm{~Hz}, 1 \mathrm{H}), 7.70-7.63(\mathrm{~m}, 1 \mathrm{H})$, $7.48-7.41(\mathrm{~m}, 1 \mathrm{H}), 6.83(\mathrm{t}, J=5.9 \mathrm{~Hz}, 2 \mathrm{H}), 3.03(\mathrm{~s}, 6 \mathrm{H}) \mathrm{ppm}$.

${ }^{13}$ C NMR $\left(100 \mathrm{MHz}, \mathrm{CDCl}_{3}\right) \delta 157.3,151.3,148.3,136.3,129.3,129.2,128.4,127.3,127.2,126.6,125.3,118.2$, $112.2,40.3 \mathrm{ppm}$.

MS (EI) $\mathrm{m} / \mathrm{z} \mathrm{C}_{17} \mathrm{H}_{16} \mathrm{~N}_{2}: 248.1[\mathrm{M}]^{+}, 204.1,139.0,74.9$.

4-(4-(Quinolin-2-yl)benzyl)morpholine (3i)<smiles>c1ccc2nc(-c3ccc(CN4CCOCC4)cc3)ccc2c1</smiles>

Compound $\mathbf{3 i}$ was prepared following the general procedure A from 4-(4-ethynylbenzyl)morpholine $\mathbf{1 i}(0.30 \mathrm{mmol})$ and benzo[c]isoxazole 2a $(0.33 \mathrm{mmol})$ and isolated as a white solid $(68.4 \mathrm{mg}, 75 \%)$, m.p $76-78{ }^{\circ} \mathrm{C}$.

${ }^{1} \mathbf{H}$ NMR $\left(400 \mathrm{MHz}, \mathrm{CDCl}_{3}\right) \delta 8.19(\mathrm{~m}, 2 \mathrm{H}), 8.12(\mathrm{~d}, J=8.2 \mathrm{~Hz}, 2 \mathrm{H}), 7.86(\mathrm{~d}, J=8.6 \mathrm{~Hz}, 1 \mathrm{H}), 7.81(\mathrm{~d}, J=8.1 \mathrm{~Hz}$, $1 \mathrm{H}), 7.72(\mathrm{~m}, 1 \mathrm{H}), 7.56-7.46(\mathrm{~m}, 3 \mathrm{H}), 3.77-3.70(\mathrm{~m}, 4 \mathrm{H}), 3.58(\mathrm{~s}, 2 \mathrm{H}), 2.53-2.43(\mathrm{~m}, 4 \mathrm{H}) \mathrm{ppm}$.

${ }^{13}$ C NMR $\left(100 \mathrm{MHz}, \mathrm{CDCl}_{3}\right) \delta 157.1,148.2,139.1,138.6,136.7,129.6,129.6,129.6,127.4,127.4,127.1,126.2$, $118.9,67.0,63.1,53.6 \mathrm{ppm}$.

HRMS (ESI) $\mathrm{m} / \mathrm{z}$ calcd. for $\mathrm{C}_{20} \mathrm{H}_{21} \mathrm{~N}_{2} \mathrm{O}[\mathrm{M}+\mathrm{H}]^{+} 305.16484$, found 305.16445 .

\section{4-(4-(6-Fluoroquinolin-2-yl)benzyl)morpholine (3j)}<smiles>Fc1ccc2nc(-c3ccc(CN4CCOCC4)cc3)ccc2c1</smiles>

Compound $\mathbf{3} \mathbf{j}$ was prepared following the general procedure A from 4-(4-ethynylbenzyl)morpholine $\mathbf{1 i}(0.30 \mathrm{mmol})$ and 5-fluorobenzo[c]isoxazole $\mathbf{2 b}(0.33 \mathrm{mmol})$ and isolated as a white solid $(70.5 \mathrm{mg}, 73 \%)$, m.p 136-138 ${ }^{\circ} \mathrm{C}$.

${ }^{1} \mathbf{H}$ NMR $\left(400 \mathrm{MHz}, \mathrm{CDCl}_{3}\right) \delta 8.20-8.05(\mathrm{~m}, 4 \mathrm{H}), 7.84(\mathrm{~s}, 1 \mathrm{H}), 7.46(\mathrm{~m}, 3 \mathrm{H}), 7.38(\mathrm{~m}, 1 \mathrm{H}), 3.78-3.68(\mathrm{~m}, 4 \mathrm{H})$, $3.56(\mathrm{~s}, 2 \mathrm{H}), 2.47(\mathrm{~d}, J=3.3 \mathrm{~Hz}, 4 \mathrm{H}) \mathrm{ppm}$.

${ }^{13}$ C NMR $\left(100 \mathrm{MHz}, \mathrm{CDCl}_{3}\right) \delta 160.2(\mathrm{~d}, J=248.5 \mathrm{~Hz}), 156.5,145.3,139.3,138.3,136.0(\mathrm{~d}, J=5.1 \mathrm{~Hz}), 132.1(\mathrm{~d}, J=$ 9.1 Hz), 129.6, $127.6(\mathrm{~d}, J=10.2 \mathrm{~Hz}), 127.3,119.8(\mathrm{~d}, J=25.6 \mathrm{~Hz}), 119.6(\mathrm{~d}, J=1.6 \mathrm{~Hz}), 110.4(\mathrm{~d}, J=21.7 \mathrm{~Hz}), 67.0$, 63.1, $53.6 \mathrm{ppm}$.

${ }^{19}$ F NMR $\left(375 \mathrm{MHz}, \mathrm{CDCl}_{3}\right) \delta-113.77 \mathrm{ppm}$.

HRMS (ESI) m/z calcd. for $\mathrm{C}_{20} \mathrm{H}_{20} \mathrm{FN}_{2} \mathrm{O}[\mathrm{M}+\mathrm{H}]^{+} 323.15542$, found 323.15495 .

Ethyl 4-(quinolin-2-yl)benzoate $(3 k)^{8}$ [cas: 1079883-97-8]<smiles>CCOC(=O)c1ccc(-c2ccc3ccccc3n2)cc1</smiles> 
Compound 3k was prepared following the general procedure A from ethyl 4-ethynylbenzoate $\mathbf{1 j}$ ( $0.30 \mathrm{mmol})$ and benzo[c]isoxazole $2 \mathrm{a}(0.33 \mathrm{mmol})$ and isolated as a white solid $(58.2 \mathrm{mg}, 70 \%)$, m.p $76-77^{\circ} \mathrm{C}$.

${ }^{1}$ H NMR $\left(400 \mathrm{MHz}, \mathrm{CDCl}_{3}\right) \delta 8.27-8.17(\mathrm{~m}, 6 \mathrm{H}), 7.88(\mathrm{~d}, J=8.6 \mathrm{~Hz}, 1 \mathrm{H}), 7.82(\mathrm{~d}, J=8.1 \mathrm{~Hz}, 1 \mathrm{H}), 7.74(\mathrm{~m}, 1 \mathrm{H})$, $7.57-7.50(\mathrm{~m}, 1 \mathrm{H}), 4.42(\mathrm{q}, J=7.1 \mathrm{~Hz}, 2 \mathrm{H}), 1.43(\mathrm{t}, J=7.1 \mathrm{~Hz}, 3 \mathrm{H}) \mathrm{ppm}$.

${ }^{13} \mathrm{C}$ NMR $\left(100 \mathrm{MHz}, \mathrm{CDCl}_{3}\right) \delta 166.4,156.0,148.2,143.6,137.0,131.0,130.0,129.9,129.8,127.5,127.4,126.7$, $119.0,61.1,14.3 \mathrm{ppm}$.

MS (EI) m/z C ${ }_{18} \mathrm{H}_{15} \mathrm{NO}_{2}: 277.1[\mathrm{M}]^{+}, 232.1,204.1,176.1,102.1,75.0$.

2-(4-(Trifluoromethyl)phenyl)quinolone (31) ${ }^{6}$ [cas: 506421-62-1]<smiles>FC(F)(F)c1ccc(-c2ccc3ccccc3n2)cc1</smiles>

Compound $\mathbf{3 l}$ was prepared following the general procedure A from 1-ethynyl-4-(trifluoromethyl)benzene $\mathbf{1 k}(0.30$ $\mathrm{mmol})$ and benzo[c]isoxazole $\mathbf{2 a}(0.33 \mathrm{mmol})$ and isolated as a white solid $(68.8 \mathrm{mg}, 84 \%), \mathrm{m} . \mathrm{p} 118-120^{\circ} \mathrm{C}$.

${ }^{1} \mathbf{H}$ NMR $\left(400 \mathrm{MHz}, \mathrm{CDCl}_{3}\right) \delta 8.28(\mathrm{~d}, J=8.1 \mathrm{~Hz}, 2 \mathrm{H}), 8.23(\mathrm{~d}, J=8.6 \mathrm{~Hz}, 1 \mathrm{H}), 8.19(\mathrm{~d}, J=8.5 \mathrm{~Hz}, 1 \mathrm{H}), 7.85(\mathrm{t}, J=$ $8.5 \mathrm{~Hz}, 2 \mathrm{H}), 7.76(\mathrm{t}, J=9.0 \mathrm{~Hz}, 3 \mathrm{H}), 7.56(\mathrm{t}, J=7.4 \mathrm{~Hz}, 1 \mathrm{H}) \mathrm{ppm}$.

${ }^{13}$ C NMR $\left(100 \mathrm{MHz}, \mathrm{CDCl}_{3}\right) \delta 155.6,148.2,142.9,137.0,131.1(\mathrm{q}, J=32.5 \mathrm{~Hz}), 129.9,129.8,127.8,127.5,127.4$, $126.8,125.7(\mathrm{q}, J=3.8 \mathrm{~Hz}), 124.3(\mathrm{q}, J=272.1 \mathrm{~Hz}), 118.7 \mathrm{ppm}$.

${ }^{19} \mathbf{F}$ NMR $\left(376 \mathrm{MHz}, \mathrm{CDCl}_{3}\right) \delta-62.5 \mathrm{ppm}$.

MS (EI) m/z C $\mathrm{C}_{16} \mathrm{H}_{10} \mathrm{~F}_{3} \mathrm{~N}: 273.1[\mathrm{M}]^{+}$, 204.2, 136.6, 101.1, 75.1.

4-(Quinolin-2-yl)benzonitrile (3m) ${ }^{7}$ [cas: 181867-59-4]<smiles>N#Cc1ccc(-c2ccc3ccccc3n2)cc1</smiles>

Compound $\mathbf{3 m}$ was prepared following the general procedure A from 4-ethynylbenzonitrile $\mathbf{1 l}(0.30 \mathrm{mmol})$ and benzo[c]isoxazole $2 \mathbf{a}(0.33 \mathrm{mmol})$ and isolated as a white solid $(59.3 \mathrm{mg}, 86 \%)$, m.p $119-121{ }^{\circ} \mathrm{C}$.

${ }^{1} \mathbf{H}$ NMR $\left(400 \mathrm{MHz}, \mathrm{CDCl}_{3}\right) \delta 8.28(\mathrm{~m}, 3 \mathrm{H}), 8.17(\mathrm{~d}, J=8.5 \mathrm{~Hz}, 1 \mathrm{H}), 7.90-7.84(\mathrm{~m}, 2 \mathrm{H}), 7.83-7.73(\mathrm{~m}, 3 \mathrm{H}), 7.61-$ $7.55(\mathrm{~m}, 1 \mathrm{H}) \mathrm{ppm}$.

${ }^{13} \mathbf{C}$ NMR $\left(100 \mathrm{MHz}, \mathrm{CDCl}_{3}\right) \delta 154.9,148.2,143.6,137.2,132.6,130.1,129.8,128.0,127.5,127.5,127.1,118.8$, $118.6,112.7 \mathrm{ppm}$.

MS (EI) $\mathrm{m} / \mathrm{z} \mathrm{C}_{16} \mathrm{H}_{10} \mathrm{~N}_{2}: 230.1[\mathrm{M}]^{+}, 201.1,176.0,101.0,76.0$.

\section{2-(4-(Methylsulfonyl)phenyl)quinolone (3n) ${ }^{9}$ [cas: 1671064-38-2]}<smiles>CS(=O)(=O)c1ccc(-c2ccc3ccccc3n2)cc1</smiles>

Compound 3n was prepared following the general procedure A from 1-ethynyl-4-(methylsulfonyl)benzene $\mathbf{1 m}(0.30$ $\mathrm{mmol})$ and benzo[c]isoxazole $\mathbf{2 a}(0.33 \mathrm{mmol})$ and isolated as a yellow solid $(62.8 \mathrm{mg}, 74 \%)$, m.p $155-157^{\circ} \mathrm{C}$.

${ }^{1} \mathbf{H}$ NMR $\left(400 \mathrm{MHz}, \mathrm{CDCl}_{3}\right) \delta 8.41-8.34(\mathrm{~m}, 2 \mathrm{H}), 8.29(\mathrm{~d}, J=8.6 \mathrm{~Hz}, 1 \mathrm{H}), 8.19(\mathrm{~d}, J=8.5 \mathrm{~Hz}, 1 \mathrm{H}), 8.10(\mathrm{~d}, J=8.4$ $\mathrm{Hz}, 2 \mathrm{H}), 7.91(\mathrm{~d}, J=8.6 \mathrm{~Hz}, 1 \mathrm{H}), 7.87(\mathrm{~d}, J=8.0 \mathrm{~Hz}, 1 \mathrm{H}), 7.81-7.74(\mathrm{~m}, 1 \mathrm{H}), 7.59(\mathrm{~m}, 1 \mathrm{H}), 3.10(\mathrm{~s}, 3 \mathrm{H}) \mathrm{ppm}$.

${ }^{13}$ C NMR $\left(101 \mathrm{MHz}, \mathrm{CDCl}_{3}\right) \delta 155.0,148.2,144.7,140.8,137.3,130.1,129.9,128.4,127.9,127.5,127.1,118.8,44.6$ ppm.

MS (EI) m/z C C $16 \mathrm{H}_{13} \mathrm{NO}_{2} \mathrm{~S}: 283.1[\mathrm{M}]^{+}, 204.1,127.8,76.8$.

2-(Benzo[d][1,3]dioxol-5-yl)quinolone $(30)^{9}$ [cas: 6808-65-7]<smiles>c1ccc2nc(-c3ccc4c(c3)OCO4)ccc2c1</smiles>

Compound 30 was prepared following the general procedure A from 5-ethynylbenzo[d][1,3]dioxole $1 \mathbf{n}(0.30 \mathrm{mmol})$ and benzo[c]isoxazole $2 \mathrm{a}(0.33 \mathrm{mmol})$ and isolated as a white solid $(54.5 \mathrm{mg}, 73 \%)$, m.p $91-93{ }^{\circ} \mathrm{C}$. 
${ }^{1} \mathbf{H}$ NMR $\left(400 \mathrm{MHz}, \mathrm{CDCl}_{3}\right) \delta 8.19-8.11(\mathrm{~m}, 2 \mathrm{H}), 7.79(\mathrm{~m}, 2 \mathrm{H}), 7.75(\mathrm{~d}, J=1.6 \mathrm{~Hz}, 1 \mathrm{H}), 7.74-7.69(\mathrm{~m}, 1 \mathrm{H}), 7.66$ $(\mathrm{m}, 1 \mathrm{H}), 7.50(\mathrm{t}, J=7.5 \mathrm{~Hz}, 1 \mathrm{H}), 6.95(\mathrm{~d}, J=8.1 \mathrm{~Hz}, 1 \mathrm{H}), 6.04(\mathrm{~s}, 2 \mathrm{H}) \mathrm{ppm}$.

${ }^{13}$ C NMR $\left(100 \mathrm{MHz}, \mathrm{CDCl}_{3}\right) \delta 156.6,148.8,148.4,148.1,136.6,134.1,129.6,129.5,127.4,126.9,126.0,121.7$, $118.6,108.4,107.9,101.3 \mathrm{ppm}$.

MS (EI) m/z C C16 $\mathrm{H}_{10} \mathrm{~F}_{3} \mathrm{~N}: 273.1[\mathrm{M}]^{+}, 204.2,136.6,101.1,75.1$.

2-(Pyridin-3-yl)quinolone (3p) ${ }^{6}$ [cas: 6294-65-1]<smiles>c1cncc(-c2ccc3ccccc3n2)c1</smiles>

Compound 3p was prepared following the general procedure A from 3-ethynylpyridine $10(0.30 \mathrm{mmol})$ and benzo[c]isoxazole 2a $(0.33 \mathrm{mmol})$ and isolated as a yellow solid $(50.7 \mathrm{mg}, 82 \%)$, m.p $59-61{ }^{\circ} \mathrm{C}$.

${ }^{1} \mathbf{H}$ NMR $\left(400 \mathrm{MHz}, \mathrm{CDCl}_{3}\right) \delta 9.34(\mathrm{~s}, 1 \mathrm{H}), 8.68(\mathrm{~s}, 1 \mathrm{H}), 8.46(\mathrm{~d}, J=7.9 \mathrm{~Hz}, 1 \mathrm{H}), 8.16(\mathrm{~m}, 2 \mathrm{H}), 7.80(\mathrm{t}, J=8.4 \mathrm{~Hz}$, $2 \mathrm{H}), 7.74-7.68(\mathrm{~m}, 1 \mathrm{H}), 7.55-7.48(\mathrm{~m}, 1 \mathrm{H}), 7.40(\mathrm{~m}, 1 \mathrm{H}) \mathrm{ppm}$.

${ }^{13}$ C NMR $\left(100 \mathrm{MHz}, \mathrm{CDCl}_{3}\right) \delta 154.4,150.0,148.7,148.2,137.0,134.9,134.8,129.8,129.6,127.4,127.2,126.6$, $123.6,118.3 \mathrm{ppm}$.

MS (EI) m/z C C $16 \mathrm{H}_{11} \mathrm{NO}_{2}: 249.1[\mathrm{M}]^{+}, 191.1,163.1,128.1,63.1$.

2-(Thiophen-3-yl)quinolone (3q) ${ }^{11}$ [cas: 104967-53-5]<smiles>c1ccc2nc(-c3ccsc3)ccc2c1</smiles>

Compound $\mathbf{3 q}$ was prepared following the general procedure A from 3-ethynylthiophene $\mathbf{1 p}(0.30 \mathrm{mmol})$ and benzo[c]isoxazole $2 \mathrm{a}(0.33 \mathrm{mmol})$ and isolated as a white solid $(39.9 \mathrm{mg}, 63 \%), \mathrm{m} . \mathrm{p} 121-122{ }^{\circ} \mathrm{C}$.

${ }^{1} \mathbf{H}$ NMR $\left(400 \mathrm{MHz}, \mathrm{CDCl}_{3}\right) \delta 8.16(\mathrm{~d}, J=8.6 \mathrm{~Hz}, 1 \mathrm{H}), 8.12(\mathrm{~d}, J=8.5 \mathrm{~Hz}, 1 \mathrm{H}), 8.05(\mathrm{~d}, J=1.9 \mathrm{~Hz}, 1 \mathrm{H}), 7.89(\mathrm{~m}$, $1 \mathrm{H}), 7.78(\mathrm{t}, J=8.1 \mathrm{~Hz}, 2 \mathrm{H}), 7.74-7.68(\mathrm{~m}, 1 \mathrm{H}), 7.50(\mathrm{t}, J=7.4 \mathrm{~Hz}, 1 \mathrm{H}), 7.45(\mathrm{~m}, 1 \mathrm{H}) \mathrm{ppm}$.

${ }^{13} \mathbf{C}$ NMR $\left(100 \mathrm{MHz}, \mathrm{CDCl}_{3}\right) \delta 153.3,148.2,142.7,136.6,129.6,129.5,127.4,127.1,126.8,126.4,126.1,124.6$, $119.0 \mathrm{ppm}$.

HRMS (ESI) $\mathrm{m} / \mathrm{z}$ calcd. for $\mathrm{C}_{13} \mathrm{H}_{10} \mathrm{NS}[\mathrm{M}+\mathrm{H}]^{+} 212.05285$, found 212.05264 .

2-(Benzo[b]thiophen-3-yl)quinolone $(3 \mathrm{r})^{11}$ [cas: 855762-22-0]<smiles>c1ccc2nc(-c3csc4ccccc34)ccc2c1</smiles>

Compound $\mathbf{3 r}$ was prepared following the general procedure A from (benzo[b]thiophen-3-ylethynyl)trimethylsilane 1q $(0.30 \mathrm{mmol})$ and benzo[c]isoxazole $\mathbf{2 a}(0.33 \mathrm{mmol})$ and isolated as a yellow solid $(59.5 \mathrm{mg}, 76 \%)$, m.p $82-84{ }^{\circ} \mathrm{C}$.

${ }^{1} \mathbf{H}$ NMR $\left(400 \mathrm{MHz}, \mathrm{CDCl}_{3}\right) \delta 8.83(\mathrm{~d}, J=8.2 \mathrm{~Hz}, 1 \mathrm{H}), 8.20(\mathrm{t}, J=8.8 \mathrm{~Hz}, 2 \mathrm{H}), 7.92(\mathrm{~d}, J=10.3 \mathrm{~Hz}, 2 \mathrm{H}), 7.80(\mathrm{t}, J=$ $9.4 \mathrm{~Hz}, 2 \mathrm{H}), 7.74(\mathrm{t}, J=7.6 \mathrm{~Hz}, 1 \mathrm{H}), 7.57-7.46(\mathrm{~m}, 2 \mathrm{H}), 7.42(\mathrm{t}, J=7.5 \mathrm{~Hz}, 1 \mathrm{H}) \mathrm{ppm}$.

${ }^{13}$ C NMR $\left(100 \mathrm{MHz}, \mathrm{CDCl}_{3}\right) \delta 154.4,148.1,140.9,137.4,136.6,136.5,129.7,129.6,127.5,127.5,126.9,126.3$, 124.9, 124.8, 124.7, 122.6, $120.8 \mathrm{ppm}$.

MS (EI) m/z C ${ }_{17} \mathrm{H}_{11} \mathrm{NS}: 261.1[\mathrm{M}]^{+}, 216.2,159.0,108.6$.

\section{2-(Imidazo[1,2-a]pyridin-3-yl)quinolone (3s)}<smiles>c1ccc2nc(-c3cnc4ccccn34)ccc2c1</smiles>

Compound $3 \mathbf{s}$ was prepared following the general procedure A from 3-((trimethylsilyl)ethynyl)imidazo[1,2-a]pyridine 1r $(0.30 \mathrm{mmol})$ and benzo[c]isoxazole $2 \mathbf{a}(0.33 \mathrm{mmol})$ and isolated as a red solid $(58.8 \mathrm{mg}, 80 \%)$, m.p $143-144{ }^{\circ} \mathrm{C}$. ${ }^{1} \mathbf{H}$ NMR $\left(400 \mathrm{MHz}, \mathrm{CDCl}_{3}\right) \delta 10.43(\mathrm{~d}, J=7.0 \mathrm{~Hz}, 1 \mathrm{H}), 8.33(\mathrm{~s}, 1 \mathrm{H}), 8.12(\mathrm{t}, J=9.1 \mathrm{~Hz}, 2 \mathrm{H}), 7.84(\mathrm{~d}, J=8.7 \mathrm{~Hz}$, $1 \mathrm{H}), 7.80-7.69(\mathrm{~m}, 3 \mathrm{H}), 7.50(\mathrm{t}, J=7.5 \mathrm{~Hz}, 1 \mathrm{H}), 7.40-7.33(\mathrm{~m}, 1 \mathrm{H}), 7.04(\mathrm{t}, J=6.8 \mathrm{~Hz}, 1 \mathrm{H}) \mathrm{ppm}$.

${ }^{13}$ C NMR $\left(100 \mathrm{MHz}, \mathrm{CDCl}_{3}\right) \delta 150.0,147.9,147.4,136.3,136.2,129.7,128.8,127.5,126.2,126.1,125.9,118.8$, $117.5,113.2 \mathrm{ppm}$.

HRMS (ESI) m/z calcd. for $\mathrm{C}_{16} \mathrm{H}_{12} \mathrm{~N}_{3}[\mathrm{M}+\mathrm{H}]^{+} 246.10257$, found 246.10224 . 
<smiles>Cc1noc(C)c1-c1ccc2ccccc2n1</smiles>

Compound $3 \mathbf{t}$ was prepared following the general procedure A from 3,5-dimethyl-4-((trimethylsilyl)ethynyl)isoxazole 1s $(0.30 \mathrm{mmol})$ and benzo[c]isoxazole $2 \mathbf{a}(0.33 \mathrm{mmol})$ and isolated as a yellow solid $(48.4 \mathrm{mg}, 72 \%)$, m.p $102-103{ }^{\circ} \mathrm{C}$. ${ }^{1} \mathbf{H}$ NMR $\left(400 \mathrm{MHz}, \mathrm{CDCl}_{3}\right) \delta 8.22(\mathrm{~d}, J=8.5 \mathrm{~Hz}, 1 \mathrm{H}), 8.10(\mathrm{~d}, J=8.5 \mathrm{~Hz}, 1 \mathrm{H}), 7.84(\mathrm{~d}, J=8.1 \mathrm{~Hz}, 1 \mathrm{H}), 7.74(\mathrm{t}, 1 \mathrm{H})$, $7.56(\mathrm{t}, 1 \mathrm{H}), 7.47(\mathrm{~d}, J=8.5 \mathrm{~Hz}, 1 \mathrm{H}), 2.66(\mathrm{~s}, 3 \mathrm{H}), 2.52(\mathrm{~s}, 3 \mathrm{H}) \mathrm{ppm}$.

${ }^{13}$ C NMR $\left(100 \mathrm{MHz}, \mathrm{CDCl}_{3}\right) \delta 168.0,158.9,150.9,148.3,136.6,129.9,129.3,127.5,126.6,126.6,120.8,116.5,12.6$, $11.6 \mathrm{ppm}$.

HRMS (ESI) $\mathrm{m} / \mathrm{z}$ calcd. for $\mathrm{C}_{14} \mathrm{H}_{13} \mathrm{~N}_{2} \mathrm{O}[\mathrm{M}+\mathrm{H}]^{+} 225.10224$, found 225.10205 .

\section{2-Ferrocenylquinoline $(3 \mathbf{u})^{9}$}

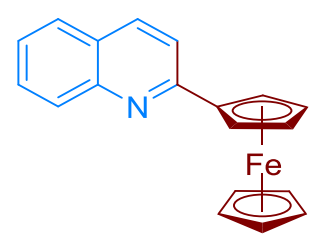

Compound $3 \mathbf{u}$ was prepared following the general procedure A from 2-ferrocenylacetylene $1 \mathbf{t}(0.30 \mathrm{mmol})$ and benzo[c]isoxazole 2a $(0.33 \mathrm{mmol})$ and isolated as a red solid $(56.3 \mathrm{mg}, 60 \%)$, m.p $133-135^{\circ} \mathrm{C}$.

${ }^{1} \mathbf{H}$ NMR $\left(400 \mathrm{MHz}, \mathrm{CDCl}_{3}\right) \delta 8.03(\mathrm{~m}, 2 \mathrm{H}), 7.74(\mathrm{~d}, J=8.0 \mathrm{~Hz}, 1 \mathrm{H}), 7.68-7.63(\mathrm{~m}, 1 \mathrm{H}), 7.56(\mathrm{~d}, J=8.6 \mathrm{~Hz}, 1 \mathrm{H})$, $7.46(\mathrm{~m}, 1 \mathrm{H}), 5.11-5.03(\mathrm{~m}, 2 \mathrm{H}), 4.51-4.43(\mathrm{~m}, 2 \mathrm{H}), 4.05(\mathrm{~s}, 5 \mathrm{H}) \mathrm{ppm}$.

${ }^{13}$ C NMR (100 MHz, $\left.\mathrm{CDCl}_{3}\right) \delta 159.5,148.2,135.4,129.4,128.9,127.5,126.7,125.4,119.5,83.9,70.4,69.6,67.9$ ppm.

MS (EI) m/z C ${ }_{19} \mathrm{H}_{15} \mathrm{FeN}: 313.1[\mathrm{M}]^{+}, 248.1,222.0,191.0,165.1,121.0,75.1$.

\section{2-(Naphthalen-2-yl)quinolone (3v) ${ }^{6}$ [cas: 47077-29-2]}<smiles>c1ccc2cc(-c3ccc4ccccc4n3)ccc2c1</smiles>

Compound $\mathbf{3 v}$ was prepared following the general procedure A from 2-ethynylnaphthalene $1 \mathbf{u}(0.30 \mathrm{mmol})$ and benzo[c]isoxazole $2 \mathbf{a}(0.33 \mathrm{mmol})$ and isolated as a white solid $(65.8 \mathrm{mg}, 86 \%)$, m.p $155-157{ }^{\circ} \mathrm{C}$.

${ }^{1} \mathbf{H}$ NMR $\left(400 \mathrm{MHz}, \mathrm{CDCl}_{3}\right) \delta 8.63(\mathrm{~s}, 1 \mathrm{H}), 8.38(\mathrm{~m}, 1 \mathrm{H}), 8.25(\mathrm{~m}, 2 \mathrm{H}), 8.08-7.97(\mathrm{~m}, 3 \mathrm{H}), 7.91(\mathrm{~m}, 1 \mathrm{H}), 7.86(\mathrm{~d}, J$ $=8.1 \mathrm{~Hz}, 1 \mathrm{H}), 7.76(\mathrm{t}, J=7.6 \mathrm{~Hz}, 1 \mathrm{H}), 7.62-7.47(\mathrm{~m}, 3 \mathrm{H}) \mathrm{ppm}$.

${ }^{13}$ C NMR $\left(100 \mathrm{MHz}, \mathrm{CDCl}_{3}\right) \delta 157.1,148.3,136.9,136.8,133.8,133.5,129.7,129.7,128.8,128.5,127.7,127.5$, $127.2,127.1,126.7,126.3,126.3,125.0,119.1 \mathrm{ppm}$.

MS (EI) $\mathrm{m} / \mathrm{z} \mathrm{C}_{19} \mathrm{H}_{13} \mathrm{~N}: 255.1[\mathrm{M}]^{+}, 226.1,127.2,75.0$.

2-Pentylquinoline (3w) ${ }^{13}$ [cas: 93005-16-4]<smiles>CCCCCc1ccc2ccccc2n1</smiles>

Compound 3w was prepared following the general procedure A from hept-1-yne $\mathbf{1 v}(0.30 \mathrm{mmol})$ and benzo[c]isoxazole $\mathbf{2 a}(0.33 \mathrm{mmol})$ and isolated as a light yellow oil $(31.6 \mathrm{mg}, 53 \%)$.

${ }^{1} \mathbf{H}$ NMR $\left(400 \mathrm{MHz}, \mathrm{CDCl}_{3}\right) \delta 8.05(\mathrm{~d}, J=8.4 \mathrm{~Hz}, 2 \mathrm{H}), 7.76(\mathrm{~d}, J=7.9 \mathrm{~Hz}, 1 \mathrm{H}), 7.67(\mathrm{t}, J=7.7 \mathrm{~Hz}, 1 \mathrm{H}), 7.47(\mathrm{t}, J=$ $7.5 \mathrm{~Hz}, 1 \mathrm{H}), 7.29(\mathrm{~d}, J=8.4 \mathrm{~Hz}, 1 \mathrm{H}), 3.00-2.93(\mathrm{~m}, 2 \mathrm{H}), 1.82(\mathrm{~m}, 2 \mathrm{H}), 1.46-1.35(\mathrm{~m}, 4 \mathrm{H}), 0.90(\mathrm{t}, J=6.9 \mathrm{~Hz}, 3 \mathrm{H})$ ppm.

${ }^{13}$ C NMR $\left(100 \mathrm{MHz}, \mathrm{CDCl}_{3}\right) \delta 163.1,147.9,136.1,129.3,128.8,127.4,126.7,125.6,121.3,39.3,31.7,29.7,22.5$, $14.0 \mathrm{ppm}$.

MS (EI) m/z C ${ }_{14} \mathrm{H}_{17} \mathrm{~N}: 199.1[\mathrm{M}]^{+}, 143.1,115.1,83.5,50.9$. 
<smiles>CCCCc1ccc2ccccc2n1</smiles>

Compound $\mathbf{3} \mathbf{x}$ was prepared following the general procedure A from hex-1-yne $\mathbf{1 w}(0.30 \mathrm{mmol})$ and benzo[c]isoxazole $2 \mathrm{a}(0.33 \mathrm{mmol})$ and isolated as a light yellow oil $(31.2 \mathrm{mg}, 56 \%)$.

${ }^{1} \mathbf{H}$ NMR $\left(400 \mathrm{MHz}, \mathrm{CDCl}_{3}\right) \delta 8.05(\mathrm{~d}, J=8.4 \mathrm{~Hz}, 2 \mathrm{H}), 7.76(\mathrm{~d}, J=8.1 \mathrm{~Hz}, 1 \mathrm{H}), 7.67(\mathrm{~m}, 1 \mathrm{H}), 7.47(\mathrm{~m}, 1 \mathrm{H}), 7.29(\mathrm{~m}$, $1 \mathrm{H}), 3.01-2.93(\mathrm{~m}, 2 \mathrm{H}), 1.80(\mathrm{~m}, 2 \mathrm{H}), 1.43(\mathrm{~m}, 2 \mathrm{H}), 0.96(\mathrm{t}, J=7.4 \mathrm{~Hz}, 3 \mathrm{H}) \mathrm{ppm}$.

${ }^{13} \mathbf{C}$ NMR $\left(101 \mathrm{MHz}, \mathrm{CDCl}_{3}\right) \delta 163.1,147.9,136.1,129.2,128.8,127.4,126.7,125.6,121.3,39.06,32.1,22.6,13.9$ ppm.

MS (EI) m/z C ${ }_{13} \mathrm{H}_{15} \mathrm{~N}: 185.1[\mathrm{M}]^{+}, 170.1,156.1,143.1,75.0$.

2-Cyclopropylquinoline $(3 y)^{6}$ [cas: 58173-64-1]<smiles>c1ccc2nc(C3CC3)ccc2c1</smiles>

Compound $\mathbf{3 y}$ was prepared following the general procedure A from ethynylcyclopropane $\mathbf{1 x}(0.30 \mathrm{mmol})$ and benzo[c]isoxazole $2 \mathbf{a}(0.33 \mathrm{mmol})$ and isolated as a colorless oil $(30.4 \mathrm{mg}, 60 \%)$.

${ }^{1} \mathbf{H}$ NMR $\left(400 \mathrm{MHz}, \mathrm{CDCl}_{3}\right) \delta 7.98(\mathrm{t}, J=8.2 \mathrm{~Hz}, 2 \mathrm{H}), 7.73(\mathrm{~d}, J=7.9 \mathrm{~Hz}, 1 \mathrm{H}), 7.68-7.61(\mathrm{~m}, 1 \mathrm{H}), 7.43(\mathrm{t}, J=7.4$ $\mathrm{Hz}, 1 \mathrm{H}), 7.16(\mathrm{~d}, J=8.5 \mathrm{~Hz}, 1 \mathrm{H}), 2.25$ (ddd, $J=13.3,8.2,4.9 \mathrm{~Hz}, 1 \mathrm{H}), 1.19-1.13(\mathrm{~m}, 2 \mathrm{H}), 1.10$ (ddt, $J=10.6,5.0$, $2.6 \mathrm{~Hz}, 2 \mathrm{H}) \mathrm{ppm}$.

${ }^{13}$ C NMR $\left(100 \mathrm{MHz}, \mathrm{CDCl}_{3}\right) \delta 163.4,147.9,135.8,129.2,128.6,127.4,126.7,125.1,119.3,18.1,10.2 \mathrm{ppm}$.

MS (EI) m/z C ${ }_{12} \mathrm{H}_{11} \mathrm{~N}: 169.1[\mathrm{M}]^{+}, 128.0,75.1$.

3-(Quinolin-2-yl)propyl benzoate (3z)<smiles>O=C(OCCCc1ccc2ccccc2n1)c1ccccc1</smiles>

Compound $\mathbf{3 z}$ was prepared following the general procedure A from pent-4-yn-1-yl benzoate $\mathbf{1 y}(0.30 \mathrm{mmol})$ and benzo[c]isoxazole 2a $(0.33 \mathrm{mmol})$ and isolated as a light yellow oil $(56.7 \mathrm{mg}, 65 \%)$.

${ }^{1} \mathbf{H}$ NMR $\left(400 \mathrm{MHz}, \mathrm{CDCl}_{3}\right) \delta 8.07-8.03(\mathrm{~m}, 2 \mathrm{H}), 8.01-7.96(\mathrm{~m}, 2 \mathrm{H}), 7.76(\mathrm{dd}, J=8.1,0.9 \mathrm{~Hz}, 1 \mathrm{H}), 7.68(\mathrm{ddd}, J=$ $8.4,6.9,1.4 \mathrm{~Hz}, 1 \mathrm{H}), 7.54-7.45(\mathrm{~m}, 2 \mathrm{H}), 7.42-7.35(\mathrm{~m}, 2 \mathrm{H}), 7.31(\mathrm{~d}, J=8.4 \mathrm{~Hz}, 1 \mathrm{H}), 4.45(\mathrm{t}, J=6.4 \mathrm{~Hz}, 2 \mathrm{H}), 3.19$ $-3.12(\mathrm{~m}, 2 \mathrm{H}), 2.40-2.31(\mathrm{~m}, 2 \mathrm{H}) \mathrm{ppm}$.

${ }^{13}$ C NMR $\left(100 \mathrm{MHz}, \mathrm{CDCl}_{3}\right) \delta 166.5,161.4,147.9,136.3,132.8,130.2,129.5,129.4,128.8,128.2,127.4,126.7$, $125.8,121.3,64.5,35.6,28.5 \mathrm{ppm}$.

HRMS (ESI) $\mathrm{m} / \mathrm{z}$ calcd. for $\mathrm{C}_{19} \mathrm{H}_{18} \mathrm{NO}_{2}[\mathrm{M}+\mathrm{H}]^{+} 292.13321$, found 292.13328 .

Ethyl quinoline-2-carboxylate (3aa) [cas: 4491-33-2]<smiles>CCOC(=O)c1ccc2ccccc2n1</smiles>

Compound 3aa was prepared following the general procedure A from ethyl propiolate $\mathbf{1 z}(0.30 \mathrm{mmol})$ and benzo[c]isoxazole $\mathbf{2 a}(0.33 \mathrm{mmol})$ and isolated as a light yellow oil $(27.7 \mathrm{mg}, 46 \%)$.

${ }^{1}$ H NMR $\left(400 \mathrm{MHz}, \mathrm{CDCl}_{3}\right) \delta 8.35-8.27(\mathrm{~m}, 2 \mathrm{H}), 8.18(\mathrm{~d}, J=8.5 \mathrm{~Hz}, 1 \mathrm{H}), 7.87(\mathrm{~d}, J=8.1 \mathrm{~Hz}, 1 \mathrm{H}), 7.81-7.75(\mathrm{~m}$, $1 \mathrm{H}), 7.64(\mathrm{t}, J=7.5 \mathrm{~Hz}, 1 \mathrm{H}), 4.56(\mathrm{q}, J=7.1 \mathrm{~Hz}, 2 \mathrm{H}), 1.49(\mathrm{t}, J=7.1 \mathrm{~Hz}, 3 \mathrm{H}) \mathrm{ppm}$.

${ }^{13} \mathrm{C}$ NMR $\left(100 \mathrm{MHz}, \mathrm{CDCl}_{3}\right) \delta 165.4,148.2,147.6,137.2,130.8,130.2,129.3,128.5,127.5,121.0,62.2,14.4 \mathrm{ppm}$.

HRMS (ESI) $\mathrm{m} / \mathrm{z}$ calcd. for $\mathrm{C}_{12} \mathrm{H}_{12} \mathrm{NO}_{2}[\mathrm{M}+\mathrm{H}]^{+} 202.08626$, found 202.08609 .

2,3-Diphenylquinoline (3ab) ${ }^{9}$ [cas: 22514-82-5]<smiles>c1ccc(-c2cc3ccccc3nc2-c2ccccc2)cc1</smiles> 
Compound 3ab was prepared following the general procedure A from 1,2-diphenylethyne 1aa $(0.30 \mathrm{mmol})$ and benzo[c]isoxazole 2a $(0.33 \mathrm{mmol})$ and isolated as a white solid $(77.6 \mathrm{mg}, 92 \%)$, m.p $78-79^{\circ} \mathrm{C}$.

${ }^{1} \mathbf{H}$ NMR $\left(400 \mathrm{MHz}, \mathrm{CDCl}_{3}\right) \delta 8.20(\mathrm{~d}, J=8.5 \mathrm{~Hz}, 1 \mathrm{H}), 8.16(\mathrm{~s}, 1 \mathrm{H}), 7.85(\mathrm{~d}, J=8.1 \mathrm{~Hz}, 1 \mathrm{H}), 7.72(\mathrm{~m}, J=7.7 \mathrm{~Hz}$, $1 \mathrm{H}), 7.55(\mathrm{~m}, J=7.5 \mathrm{~Hz}, 1 \mathrm{H}), 7.45(\mathrm{~m}, J=6.2,2.5 \mathrm{~Hz}, 2 \mathrm{H}), 7.31-7.21(\mathrm{~m}, 8 \mathrm{H}) \mathrm{ppm}$.

${ }^{13}$ C NMR $\left(100 \mathrm{MHz}, \mathrm{CDCl}_{3}\right) \delta 158.4,147.3,140.4,140.0,137.5,134.5,130.0,129.7,129.6,129.4,128.2,128.0$, $127.9,127.4,127.2,127.1,126.7 \mathrm{ppm}$.

MS (EI) m/z C ${ }_{21} \mathrm{H}_{15} \mathrm{~N}: 280.1[\mathrm{M}]^{+}$, 207.0, 139.1, 91.1, 75.0.

2,3-bis(4-Fluorophenyl) quinolone (3ac)<smiles>Fc1ccc(-c2cc3ccccc3nc2-c2ccc(F)cc2)cc1</smiles>

Compound 3ac was prepared following the general procedure A from 1,2-bis(4-fluorophenyl) ethyne 1ab (0.30 mmol) and benzo[c]isoxazole 2a $(0.33 \mathrm{mmol})$ and isolated as a light yellow solid $(85.6 \mathrm{mg}, 90 \%)$, m.p 116-118 ${ }^{\circ} \mathrm{C}$.

${ }^{1} \mathrm{H}$ NMR $\left(400 \mathrm{MHz}, \mathrm{CDCl}_{3}\right) \delta 8.19(\mathrm{~d}, J=8.5 \mathrm{~Hz}, 1 \mathrm{H}), 8.14(\mathrm{~s}, 1 \mathrm{H}), 7.86(\mathrm{~d}, J=8.1 \mathrm{~Hz}, 1 \mathrm{H}), 7.78-7.72(\mathrm{~m}, 1 \mathrm{H})$, $7.57(\mathrm{~m}, J=8.0,7.0 \mathrm{~Hz}, 1 \mathrm{H}), 7.47-7.39(\mathrm{~m}, 2 \mathrm{H}), 7.20(\mathrm{~m}, J=5.2,4.3,1.8 \mathrm{~Hz}, 2 \mathrm{H}), 7.00(\mathrm{~m}, J=9.8,1.2 \mathrm{~Hz}, 4 \mathrm{H})$ ppm.

${ }^{13}$ C NMR $\left(100 \mathrm{MHz}, \mathrm{CDCl}_{3}\right) \delta 162.7(\mathrm{~d}, J=248.2 \mathrm{~Hz}), 161.2(\mathrm{~d}, J=247.7 \mathrm{~Hz}), 157.1,147.3,137.6,136.3(\mathrm{~d}, J=3.4$ $\mathrm{Hz}), 135.8(\mathrm{~d}, J=3.4 \mathrm{~Hz}), 133.3,131.8(\mathrm{~d}, J=8.3 \mathrm{~Hz}), 131.3(\mathrm{~d}, J=8.0 \mathrm{~Hz}), 129.8,129.4,127.4,127.1,126.9,115.4$ $(\mathrm{d}, J=21.5 \mathrm{~Hz}), 115.0(\mathrm{~d}, J=21.6 \mathrm{~Hz}) \mathrm{ppm}$.

${ }^{19}$ F NMR $\left(375 \mathrm{MHz}, \mathrm{CDCl}_{3}\right) \delta-113.5,-114.6 \mathrm{ppm}$.

HRMS (ESI) $\mathrm{m} / \mathrm{z}$ calcd. for $\mathrm{C}_{21} \mathrm{H}_{14} \mathrm{~F}_{2} \mathrm{~N}[\mathrm{M}+\mathrm{H}]^{+} 318.10888$, found 318.10895 .

\section{2,3-bis(4-Chlorophenyl) quinolone (3ad)}<smiles>Clc1ccc(-c2cc3ccccc3nc2-c2ccc(Cl)cc2)cc1</smiles>

Compound 3ad was prepared following the general procedure A from 1,2-bis(4-chlorophenyl) ethyne 1ac (0.30 mmol) and benzo[c]isoxazole $2 \mathbf{a}(0.33 \mathrm{mmol})$ and isolated as a light yellow solid $(85.9 \mathrm{mg}, 82 \%)$, m.p $157-159{ }^{\circ} \mathrm{C}$.

${ }^{1} \mathbf{H}$ NMR $\left(400 \mathrm{MHz}, \mathrm{CDCl}_{3}\right) \delta 8.18(\mathrm{~d}, J=8.5 \mathrm{~Hz}, 1 \mathrm{H}), 8.14(\mathrm{~s}, 1 \mathrm{H}), 7.86(\mathrm{~d}, J=8.1 \mathrm{~Hz}, 1 \mathrm{H}), 7.79-7.73(\mathrm{~m}, 1 \mathrm{H})$, $7.59(\mathrm{~m}, J=11.1,3.9 \mathrm{~Hz}, 1 \mathrm{H}), 7.39(\mathrm{~d}, J=8.5 \mathrm{~Hz}, 2 \mathrm{H}), 7.29(\mathrm{~m}, J=7.9 \mathrm{~Hz}, 4 \mathrm{H}), 7.17(\mathrm{~d}, J=8.4 \mathrm{~Hz}, 2 \mathrm{H}) \mathrm{ppm}$. ${ }^{13}$ C NMR $\left(100 \mathrm{MHz}, \mathrm{CDCl}_{3}\right) \delta 156.7,147.4,138.5,138.1,137.7,134.4,133.6,133.1,131.3,130.9,130.0,129.4$, $128.7,128.3,127.5,127.1,127.1 \mathrm{ppm}$.

HRMS (ESI) $\mathrm{m} / \mathrm{z}$ calcd. for $\mathrm{C}_{21} \mathrm{H}_{14} \mathrm{Cl}_{2} \mathrm{~N}[\mathrm{M}+\mathrm{H}]^{+} 350.04978$, found 350.04984 .

\section{2,3-bis(4-Bromophenyl) quinolone (3ae)}<smiles>Brc1ccc(-c2cc3ccccc3nc2-c2ccc(Br)cc2)cc1</smiles>

Compound 3ae was prepared following the general procedure A from 1,2-bis(4-bromophenyl) ethyne 1ad (0.30 mmol) and benzo[c]isoxazole $\mathbf{2 a}(0.33 \mathrm{mmol})$ and isolated as a yellow solid $(113.3 \mathrm{mg}, 86 \%)$, m.p $120-121{ }^{\circ} \mathrm{C}$.

${ }^{1} \mathbf{H}$ NMR $\left(400 \mathrm{MHz}, \mathrm{CDCl}_{3}\right) \delta 8.13(\mathrm{~d}, J=9.0 \mathrm{~Hz}, 1 \mathrm{H}), 8.07(\mathrm{~s}, 1 \mathrm{H}), 7.84(\mathrm{~d}, J=2.3 \mathrm{~Hz}, 1 \mathrm{H}), 7.66(\mathrm{~m}, J=9.0,2.3 \mathrm{~Hz}$, $1 \mathrm{H}), 7.45-7.41(\mathrm{~m}, 2 \mathrm{H}), 7.31-7.27(\mathrm{~m}, 5 \mathrm{H}), 7.25-7.20(\mathrm{~m}, 2 \mathrm{H}) \mathrm{ppm}$.

${ }^{13}$ C NMR $\left(100 \mathrm{MHz}, \mathrm{CDCl}_{3}\right) \delta 158.7,145.6,140.0,139.5,136.5,135.5,132.4,131.0,130.5,129.9,129.7,128.3$, $128.2,128.0,127.8,127.4,126.0 \mathrm{ppm}$.

HRMS (ESI) m/z calcd. for $\mathrm{C}_{21} \mathrm{H}_{14} \mathrm{Br}_{2} \mathrm{~N}[\mathrm{M}+\mathrm{H}]^{+} 437.94875$, found 437.94882 . 


\section{2,3-bis(4-(Trifluoromethyl) phenyl) quinolone (3af)}<smiles>FC(F)(F)c1ccc(-c2cc3ccccc3nc2-c2ccc(C(F)(F)F)cc2)cc1</smiles>

Compound 3af was prepared following the general procedure A from 1,2-bis(4-(trifluoromethyl) phenyl) ethyne 1ae $(0.30 \mathrm{mmol})$ and benzo[c] isoxazole $\mathbf{2 a}(0.33 \mathrm{mmol})$ and isolated as a light yellow solid $(100.1 \mathrm{mg}, 80 \%)$, m.p $102-$ $103^{\circ} \mathrm{C}$.

${ }^{1} \mathbf{H}$ NMR $\left(400 \mathrm{MHz}, \mathrm{CDCl}_{3}\right) \delta 8.23(\mathrm{~d}, J=7.6 \mathrm{~Hz}, 2 \mathrm{H}), 7.91(\mathrm{~d}, J=8.1 \mathrm{~Hz}, 1 \mathrm{H}), 7.80(\mathrm{~m}, J=7.7 \mathrm{~Hz}, 1 \mathrm{H}), 7.66-7.57$ $(\mathrm{m}, 7 \mathrm{H}), 7.38(\mathrm{~d}, J=7.9 \mathrm{~Hz}, 2 \mathrm{H}) \mathrm{ppm}$.

${ }^{13}$ C NMR $\left(100 \mathrm{MHz}, \mathrm{CDCl}_{3}\right) \delta 156.3,147.5,143.4,143.1,143.1,138.1,132.8,130.4,130.3,130.3(\mathrm{q}, J=32.0 \mathrm{~Hz})$, $130.0,129.5,129.7(\mathrm{q}, J=32.7 \mathrm{~Hz}), 127.6,127.5,127.2,125.4(\mathrm{q}, J=3.7 \mathrm{~Hz}), 125.1(\mathrm{q}, J=3.7 \mathrm{~Hz}), 122.7,122.6$ ppm.

${ }^{19}$ F NMR $\left(376 \mathrm{MHz}, \mathrm{CDCl}_{3}\right) \delta-62.5,-62.6 \mathrm{ppm}$.

HRMS (ESI) $\mathrm{m} / \mathrm{z}$ calcd. for $\mathrm{C}_{23} \mathrm{H}_{14} \mathrm{~F}_{6} \mathrm{~N}[\mathrm{M}+\mathrm{H}]^{+} 418.10250$, found 418.10269 .

\section{2,3-Di-p-tolylquinoline (3ag) [cas: 860718-97-4]}<smiles>Cc1ccc(-c2cc3ccccc3nc2-c2ccc(C)cc2)cc1</smiles>

Compound 3ag was prepared following the general procedure A from 1,2-di-p-tolylethyne 1af $(0.30 \mathrm{mmol})$ and benzo[c]isoxazole 2a $(0.33 \mathrm{mmol})$ and isolated as a light yellow solid $(86.2 \mathrm{mg}, 93 \%)$, m.p 104-106 ${ }^{\circ} \mathrm{C}$.

${ }^{1} \mathbf{H}$ NMR $\left(400 \mathrm{MHz}, \mathrm{CDCl}_{3}\right) \delta 8.21(\mathrm{~d}, J=8.5 \mathrm{~Hz}, 1 \mathrm{H}), 8.13(\mathrm{~s}, 1 \mathrm{H}), 7.84(\mathrm{~d}, J=8.1 \mathrm{~Hz}, 1 \mathrm{H}), 7.72(\mathrm{~m}, J=7.7 \mathrm{~Hz}$, $1 \mathrm{H}), 7.54(\mathrm{~m}, J=7.5 \mathrm{~Hz}, 1 \mathrm{H}), 7.40(\mathrm{~d}, J=8.0 \mathrm{~Hz}, 2 \mathrm{H}), 7.17(\mathrm{~d}, J=8.0 \mathrm{~Hz}, 2 \mathrm{H}), 7.12(\mathrm{~m}, J=7.8,4.1 \mathrm{~Hz}, 4 \mathrm{H}), 2.37(\mathrm{~d}$, $J=7.5 \mathrm{~Hz}, 6 \mathrm{H}) \mathrm{ppm}$.

${ }^{13}$ C NMR $\left(100 \mathrm{MHz}, \mathrm{CDCl}_{3}\right) \delta 158.4,147.2,137.7,137.6,137.4,137.2,136.8,134.4,129.9,129.5,129.3,128.9$, 128.6, 127.3, 127.1, 126.4, 21.2, $21.1 \mathrm{ppm}$.

HRMS (ESI) $\mathrm{m} / \mathrm{z}$ calcd. for $\mathrm{C}_{23} \mathrm{H}_{20} \mathrm{~N}[\mathrm{M}+\mathrm{H}]^{+} 310.15903$, found 310.15890 .

\section{2,3-bis(4-Methoxyphenyl) quinolone (3ah) [cas: 5818-05-3]}<smiles>COc1ccc(-c2cc3ccccc3nc2-c2ccc(OC)cc2)cc1</smiles>

Compound 3ah was prepared following the general procedure A from 1,2-bis(4-methoxyphenyl) ethyne $\mathbf{1 a g}$ (0.30 $\mathrm{mmol})$ and benzo[c]isoxazole $\mathbf{2 a}(0.33 \mathrm{mmol})$ and isolated as a light yellow oil $(81.8 \mathrm{mg}, 80 \%)$.

${ }^{1}$ H NMR $\left(400 \mathrm{MHz}, \mathrm{CDCl}_{3}\right) \delta 8.17(\mathrm{~d}, J=8.5 \mathrm{~Hz}, 1 \mathrm{H}), 8.10(\mathrm{~s}, 1 \mathrm{H}), 7.83(\mathrm{~d}, J=8.1 \mathrm{~Hz}, 1 \mathrm{H}), 7.73-7.67(\mathrm{~m}, 1 \mathrm{H})$, $7.52(\mathrm{~m}, J=11.0,4.0 \mathrm{~Hz}, 1 \mathrm{H}), 7.42(\mathrm{~d}, J=8.8 \mathrm{~Hz}, 2 \mathrm{H}), 7.21-7.15(\mathrm{~m}, 2 \mathrm{H}), 6.84(\mathrm{~m}, J=11.2,8.7 \mathrm{~Hz}, 4 \mathrm{H}), 3.82(\mathrm{~d}, J$ $=6.9 \mathrm{~Hz}, 6 \mathrm{H}) \mathrm{ppm}$.

${ }^{13}$ C NMR $\left(100 \mathrm{MHz}, \mathrm{CDCl}_{3}\right) \delta 159.6,158.9,158.1,147.3,137.4,134.1,133.0,132.6,131.4,130.8,129.4,129.2$, $127.4,127.2,126.5,113.8,113.5,55.3 \mathrm{ppm}$.

HRMS (ESI) $\mathrm{m} / \mathrm{z}$ calcd. for $\mathrm{C}_{23} \mathrm{H}_{20} \mathrm{NO}_{2}[\mathrm{M}+\mathrm{H}]^{+} 342.14886$, found 342.14881 .

\section{Diethyl 4,4'-(quinoline-2,3-diyl)dibenzoate (3ai)}<smiles>CCOC(=O)c1ccc(-c2cc3ccccc3nc2-c2ccc(C(=O)OCC)cc2)cc1</smiles> 
Compound 3ai was prepared following the general procedure A from diethyl 4,4'-(ethyne-1,2-diyl)dibenzoate 1ah $(0.30 \mathrm{mmol})$ and benzo[c]isoxazole $\mathbf{2 a}(0.33 \mathrm{mmol})$ and isolated as a light yellow solid $(110.9 \mathrm{mg}, 87 \%)$, m.p 107 $109^{\circ} \mathrm{C}$.

${ }^{1} \mathbf{H}$ NMR $\left(400 \mathrm{MHz}, \mathrm{CDCl}_{3}\right) \delta 8.20(\mathrm{~d}, J=10.5 \mathrm{~Hz}, 2 \mathrm{H}), 7.97(\mathrm{~m}, 4 \mathrm{H}), 7.87(\mathrm{~d}, J=8.1 \mathrm{~Hz}, 1 \mathrm{H}), 7.79-7.71(\mathrm{~m}, 1 \mathrm{H})$, $7.58(\mathrm{t}, J=7.5 \mathrm{~Hz}, 1 \mathrm{H}), 7.50(\mathrm{~d}, J=7.8 \mathrm{~Hz}, 2 \mathrm{H}), 7.31-7.26(\mathrm{~m}, 2 \mathrm{H}), 4.37(\mathrm{~m}, 4 \mathrm{H}), 1.44-1.32(\mathrm{~m}, 6 \mathrm{H})$.

${ }^{13} \mathrm{C}$ NMR $\left(100 \mathrm{MHz}, \mathrm{CDCl}_{3}\right) \delta 166.2,166.1,156.8,147.3,144.3,144.0,137.7,133.3,130.1,129.9,129.9,129.6$, $129.5,129.4,129.3,129.2,127.5,127.2,127.0,61.0,60.9,14.2,14.2$.

HRMS (ESI) $\mathrm{m} / \mathrm{z}$ calcd. for $\mathrm{C}_{27} \mathrm{H}_{24} \mathrm{NO}_{4}[\mathrm{M}+\mathrm{H}]^{+} 426.16998$, found 426.16953 .

\section{2,3-Di-o-tolylquinoline (3aj)}<smiles>Cc1ccccc1-c1cc2ccccc2nc1-c1ccccc1C</smiles>

Compound 3aj was prepared following the general procedure A from 1,2-di-o-tolylethyne 1ai $(0.30 \mathrm{mmol})$ and benzo[c]isoxazole $2 \mathbf{a}(0.33 \mathrm{mmol})$ and isolated as a light yellow oil $(55.6 \mathrm{mg}, 60 \%)$.

${ }^{1} \mathbf{H}$ NMR $\left(400 \mathrm{MHz}, \mathrm{CDCl}_{3}\right) \delta 8.21(\mathrm{~d}, J=8.5 \mathrm{~Hz}, 1 \mathrm{H}), 8.11(\mathrm{~s}, 1 \mathrm{H}), 7.88(\mathrm{~d}, J=8.1 \mathrm{~Hz}, 1 \mathrm{H}), 7.76(\mathrm{t}, J=7.6 \mathrm{~Hz}, 1 \mathrm{H})$, $7.60(\mathrm{t}, J=7.5 \mathrm{~Hz}, 1 \mathrm{H}), 7.17-7.10(\mathrm{~m}, 4 \mathrm{H}), 7.06(\mathrm{~m}, J=11.3,5.7 \mathrm{~Hz}, 3 \mathrm{H}), 7.02-6.97(\mathrm{~m}, 1 \mathrm{H}), 2.23(\mathrm{~s}, 3 \mathrm{H}), 2.09(\mathrm{~s}$, 3H) ppm.

${ }^{13}$ C NMR $\left(100 \mathrm{MHz}, \mathrm{CDCl}_{3}\right) \delta 159.7,146.9,139.7,138.8,137.6,136.1,135.8,134.9,130.7,130.4,130.0,129.9$, $129.5,129.4,127.8,127.5,127.4,126.8,126.7,125.2,124.9,20.2,20.0 \mathrm{ppm}$.

HRMS (ESI) $\mathrm{m} / \mathrm{z}$ calcd. for $\mathrm{C}_{23} \mathrm{H}_{20} \mathrm{~N}[\mathrm{M}+\mathrm{H}]^{+} 310.15903$, found 310.15912 .

\section{2,3-bis(3-Chlorophenyl) quinolone (3ak)}<smiles>Clc1cccc(-c2cc3ccccc3nc2-c2cccc(Cl)c2)c1</smiles>

Compound 3ak was prepared following the general procedure A from 1,2-bis(3-chlorophenyl) ethyne 1aj (0.30 mmol) and benzo[c]isoxazole $\mathbf{2 a}(0.33 \mathrm{mmol})$ and isolated as a light yellow solid $(85.9 \mathrm{mg}, 82 \%)$, m.p $93-95{ }^{\circ} \mathrm{C}$.

${ }^{1} \mathbf{H}$ NMR $\left(400 \mathrm{MHz}, \mathrm{CDCl}_{3}\right) \delta 8.24-8.17(\mathrm{~d}, 1 \mathrm{H}), 8.16(\mathrm{~s}, J=2.8 \mathrm{~Hz}, 1 \mathrm{H}), 7.90-7.84(\mathrm{~d}, 1 \mathrm{H}), 7.81-7.73(\mathrm{~m}, 1 \mathrm{H})$, $7.63-7.55(\mathrm{~m}, 2 \mathrm{H}), 7.36-7.27(\mathrm{~m}, 3 \mathrm{H}), 7.24-7.14(\mathrm{~m}, 3 \mathrm{H}), 7.04(\mathrm{~d}, J=7.6 \mathrm{~Hz}, 1 \mathrm{H}) \mathrm{ppm}$.

${ }^{13} \mathrm{C}$ NMR $\left(100 \mathrm{MHz}, \mathrm{CDCl}_{3}\right) \delta 156.4,147.3,141.7,141.3,137.8,134.3,134.2,132.9,130.2,130.0,129.5,129.4$, $129.1,128.4,128.2,128.1,127.6,127.5,127.3,127.1 \mathrm{ppm}$.

HRMS (ESI) m/z calcd. for $\mathrm{C}_{21} \mathrm{H}_{14} \mathrm{Cl}_{2} \mathrm{~N}[\mathrm{M}+\mathrm{H}]^{+} 350.04978$, found 350.04990 .

\section{2,3-Di(naphthalen-1-yl) quinolone (3al)}<smiles>c1ccc2nc(-c3cccc4ccccc34)c(-c3cccc4ccccc34)cc2c1</smiles>

Compound 3al was prepared following the general procedure A from 1,2-di(naphthalen-1-yl) ethyne 1ak (0.30 mmol) and benzo[c] isoxazole $2 \mathbf{a}(0.33 \mathrm{mmol})$ and isolated as a yellow solid $(89.2 \mathrm{mg}, 78 \%)$, m.p 190-192 ${ }^{\circ} \mathrm{C}$.

${ }^{1} \mathrm{H}$ NMR $\left(400 \mathrm{MHz}, \mathrm{CDCl}_{3}\right) \delta 8.34(\mathrm{~m}, J=9.1 \mathrm{~Hz}, 2 \mathrm{H}), 7.95(\mathrm{~d}, J=8.2 \mathrm{~Hz}, 1 \mathrm{H}), 7.90(\mathrm{~d}, J=8.2 \mathrm{~Hz}, 1 \mathrm{H}), 7.85(\mathrm{~m}, J$ $=8.2,7.0 \mathrm{~Hz}, 1 \mathrm{H}), 7.75(\mathrm{~m}, J=18.8,9.6 \mathrm{~Hz}, 3 \mathrm{H}), 7.69-7.60(\mathrm{~m}, 3 \mathrm{H}), 7.38(\mathrm{~m}, J=14.9,7.3 \mathrm{~Hz}, 4 \mathrm{H}), 7.23-7.06(\mathrm{~m}$, 4H) $\mathrm{ppm}$.

${ }^{13} \mathbf{C}$ NMR $\left(100 \mathrm{MHz}, \mathrm{CDCl}_{3}\right) \delta 159.5,147.2,138.7,137.6,136.7,134.3,133.5,133.3,132.0,131.8,129.9,129.5$, $128.1,128.0,127.7,127.6,127.0,126.9,125.9,125.9,125.7,125.6,125.5,124.7,124.4 \mathrm{ppm}$.

HRMS (ESI) m/z calcd. for $\mathrm{C}_{29} \mathrm{H}_{20} \mathrm{~N}[\mathrm{M}+\mathrm{H}]^{+} 382.15903$, found 382.15915 . 
<smiles>c1cc(-c2cc3ccsc3nc2-c2ccsc2)cs1</smiles>

Compound 3am was prepared following the general procedure A from 1,2-di(thiophen-3-yl) ethyne 1al ( $0.30 \mathrm{mmol})$ and benzo[c]isoxazole 2a $(0.33 \mathrm{mmol})$ and isolated as a light yellow solid $(58.9 \mathrm{mg}, 67 \%)$, m.p $63-65^{\circ} \mathrm{C}$.

${ }^{1} \mathrm{H}$ NMR $\left(400 \mathrm{MHz}, \mathrm{CDCl}_{3}\right) \delta 8.13(\mathrm{~d}, J=8.6 \mathrm{~Hz}, 1 \mathrm{H}), 8.11(\mathrm{~s}, J=1.3 \mathrm{~Hz}, 1 \mathrm{H}), 7.78(\mathrm{~d}, J=8.1 \mathrm{~Hz}, 1 \mathrm{H}), 7.68(\mathrm{~m}, J=$ $7.7 \mathrm{~Hz}, 1 \mathrm{H}), 7.54-7.47(\mathrm{~m}, 1 \mathrm{H}), 7.40-7.35(\mathrm{~m}, 1 \mathrm{H}), 7.29-7.26(\mathrm{~m}, 1 \mathrm{H}), 7.25-7.18(\mathrm{~m}, 3 \mathrm{H}), 6.93-6.86(\mathrm{~m}, 1 \mathrm{H})$ ppm.

${ }^{13}$ C NMR $\left(100 \mathrm{MHz}, \mathrm{CDCl}_{3}\right) \delta 153.4,147.2,141.6,140.4,137.1,129.6,129.3,129.2,128.8,128.8,127.3,126.9$, 126.6, 126.3, 125.5, 124.7, $123.6 \mathrm{ppm}$.

MS (EI) m/z C ${ }_{17} \mathrm{H}_{11} \mathrm{NS}_{2}: 293.0[\mathrm{M}]^{+}, 260.0,248.1,146.4,75.0$.

\section{2,3-Dipropylquinoline (3an) ${ }^{16}$ [cas: 1642809-12-8]}<smiles>CCCc1cc2ccccc2nc1CCC</smiles>

Compound 3an was prepared following the general procedure A from oct-4-yne 1am $(0.30 \mathrm{mmol})$ and benzo[c] isoxazole 2a $(0.33 \mathrm{mmol})$ and isolated as a light yellow oil $(50.5 \mathrm{mg}, 29 \%)$.

${ }^{1} \mathbf{H}$ NMR $\left(400 \mathrm{MHz}, \mathrm{CDCl}_{3}\right) \delta 8.01(\mathrm{~d}, J=8.5 \mathrm{~Hz}, 1 \mathrm{H}), 7.83(\mathrm{~s}, 1 \mathrm{H}), 7.70(\mathrm{~d}, J=8.1 \mathrm{~Hz}, 1 \mathrm{H}), 7.60(\mathrm{ddd}, J=8.4,6.9$, $1.4 \mathrm{~Hz}, 1 \mathrm{H}), 7.47-7.40(\mathrm{~m}, 1 \mathrm{H}), 2.99-2.92(\mathrm{~m}, 2 \mathrm{H}), 2.80-2.73(\mathrm{~m}, 2 \mathrm{H}), 1.88-1.80(\mathrm{~m}, 2 \mathrm{H}), 1.71(\mathrm{dt}, J=14.9,7.4$ $\mathrm{Hz}, 2 \mathrm{H}), 1.05$ (dt, $J=10.7,7.3 \mathrm{~Hz}, 6 \mathrm{H}) \mathrm{ppm}$.

${ }^{13}$ C NMR $\left(100 \mathrm{MHz}, \mathrm{CDCl}_{3}\right) \delta 162.1,146.4,134.8,133.9,128.4,128.9,127.2,126.8,125.5,37.78,34.4,23.5,23.0$, $14.3,14.0 \mathrm{ppm}$.

MS (EI) m/z C ${ }_{15} \mathrm{H}_{19} \mathrm{~N}: 213.2[\mathrm{M}]^{+}, 198.2,184.2,157.1,115.1,51.1$.

6,7,8,9,10,11,12,13,14,15-Decahydrocyclododeca[b]quinoline (3ao) ${ }^{17}$ [cas: 13225-95-1]

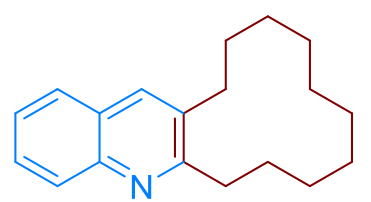

Compound 3ao was prepared following the general procedure A from cyclododecyne 1 an $(0.30 \mathrm{mmol})$ and benzo[c]isoxazole $2 \mathbf{a}(0.33 \mathrm{mmol})$ and isolated as a white solid $(64.1 \mathrm{mg}, 80 \%)$, m.p $72-77^{\circ} \mathrm{C}$.

${ }^{1} \mathbf{H}$ NMR $\left(400 \mathrm{MHz}, \mathrm{CDCl}_{3}\right) \delta 7.99(\mathrm{~d}, J=8.5 \mathrm{~Hz}, 1 \mathrm{H}), 7.79(\mathrm{~d}, J=3.2 \mathrm{~Hz}, 1 \mathrm{H}), 7.67-7.61(\mathrm{~m}, 1 \mathrm{H}), 7.59-7.52(\mathrm{~m}$, $1 \mathrm{H}), 7.40-7.33(\mathrm{~m}, 1 \mathrm{H}), 2.98-2.94(\mathrm{~m}, 2 \mathrm{H}), 2.72-2.69(\mathrm{~m}, 2 \mathrm{H}), 1.92-1.91(\mathrm{~m}, 2 \mathrm{H}), 1.71(\mathrm{brs}, 2 \mathrm{H}), 1.51-1.40(\mathrm{~m}$, $8 \mathrm{H}), 1.36$ (brs, 4H) ppm.

${ }^{13}$ C NMR $\left(100 \mathrm{MHz}, \mathrm{CDCl}_{3}\right) \delta 162.4,146.4,135.5,134.6,128.2,128.2,126.9,126.6,125.3,32.5,29.5,29.4,28.3$, 26.5, 26.3, 25.8, 25.2, 22.9, $22.8 \mathrm{ppm}$.

MS (EI) m/z C ${ }_{19} \mathrm{H}_{25} \mathrm{~N}: 267.2[\mathrm{M}]^{+}, 238.1,157.1,115.1,55.1$.

2-Chloro-6,7,8,9,10,11,12,13,14,15-decahydrocyclododeca[b]quinoline (3ap)<smiles>CCCCCCCCCCCCc1nc2ccc(Cl)cc2cc1CCCCC</smiles>

Compound 3ap was prepared following the general procedure A from cyclododecyne 1an $(0.30 \mathrm{mmol})$ and 5chlorobenzo[c]isoxazole $2 \mathbf{c}(0.33 \mathrm{mmol})$ and isolated as a white solid $(74.0 \mathrm{mg}, 82 \%)$, m.p $84-85^{\circ} \mathrm{C}$.

${ }^{1} \mathrm{H}$ NMR $\left(400 \mathrm{MHz}, \mathrm{CDCl}_{3}\right) \delta 7.89(\mathrm{~d}, J=9.0 \mathrm{~Hz}, 1 \mathrm{H}), 7.72(\mathrm{~s}, 1 \mathrm{H}), 7.61(\mathrm{~d}, J=2.0 \mathrm{~Hz}, 1 \mathrm{H}), 7.49(\mathrm{dd}, J=8.9,2.1 \mathrm{~Hz}$, $1 \mathrm{H}), 2.95(\mathrm{t}, J=7.7 \mathrm{~Hz}, 2 \mathrm{H}), 2.78-2.70(\mathrm{t}, J=7.6 \mathrm{~Hz}, 2 \mathrm{H}), 1.95-1.86(\mathrm{~m}, 2 \mathrm{H}), 1.78-1.70(\mathrm{~m}, 2 \mathrm{H}), 1.52-1.42(\mathrm{~m}$, $8 \mathrm{H}), 1.37$ (brs, 4H) ppm.

${ }^{13}$ C NMR $\left(100 \mathrm{MHz}, \mathrm{CDCl}_{3}\right) \delta 162.9,144.8,135.8,134.6,131.0,130.0,129.1,127.6,125.3,32.6,29.6,28.3,26.6$, 26.3, 25.9, 25.3, 23.0, $22.9 \mathrm{ppm}$.

HRMS (ESI) $\mathrm{m} / \mathrm{z}$ calcd. for $\mathrm{C}_{19} \mathrm{H}_{25} \mathrm{ClN}[\mathrm{M}+\mathrm{H}]^{+} 302.16700$, found 302.16666 . 
<smiles>Cc1cc2ccccc2nc1-c1ccccc1</smiles>

Compound 3aq was prepared following the general procedure A from prop-1-yn-1-ylbenzene 1ao $(0.30 \mathrm{mmol})$ and benzo[c]isoxazole $\mathbf{2 a}(0.33 \mathrm{mmol})$ and isolated as a viscous oil $(49.3 \mathrm{mg}, 75 \%)$.

${ }^{1} \mathbf{H}$ NMR $\left(400 \mathrm{MHz}, \mathrm{CDCl}_{3}\right) \delta 8.13(\mathrm{~d}, J=8.5 \mathrm{~Hz}, 1 \mathrm{H}), 8.02(\mathrm{~s}, 1 \mathrm{H}), 7.78(\mathrm{~d}, J=8.1 \mathrm{~Hz}, 1 \mathrm{H}), 7.69-7.64(\mathrm{~m}, 1 \mathrm{H})$, $7.62-7.58(\mathrm{~m}, 2 \mathrm{H}), 7.54-7.42(\mathrm{~m}, 4 \mathrm{H}), 2.47(\mathrm{~s}, 3 \mathrm{H}) \mathrm{ppm}$.

${ }^{13}$ C NMR $\left(100 \mathrm{MHz}, \mathrm{CDCl}_{3}\right) \delta 160.5,146.6,140.8,136.7,129.3,129.2,128.8,128.7,128.3,128.1,127.6,126.7$, $126.4,20.6 \mathrm{ppm}$.

MS (EI) m/z C ${ }_{16} \mathrm{H}_{13} \mathrm{~N}: 219.1[\mathrm{M}]^{+}, 165.0,128.1,75.1$.

3-Pentyl-2-phenylquinoline (3ar) ${ }^{12}$ [cas: 136800-94-7]<smiles>CCCCCc1cc2ccccc2nc1-c1ccccc1</smiles>

Compound 3ar was prepared following the general procedure A from hept-1-yn-1-ylbenzene 1ap (0.30 mmol) and benzo[c]isoxazole 2a $(0.33 \mathrm{mmol})$ and isolated as a yellow oil $(66.0 \mathrm{mg}, 80 \%)$.

${ }^{1} \mathbf{H}$ NMR $\left(400 \mathrm{MHz}, \mathrm{CDCl}_{3}\right) \delta 8.14(\mathrm{~d}, J=8.4 \mathrm{~Hz}, 1 \mathrm{H}), 8.04(\mathrm{~s}, 1 \mathrm{H}), 7.80(\mathrm{~d}, J=8.1 \mathrm{~Hz}, 1 \mathrm{H}), 7.67$ (ddd, $J=8.4,6.9$, $1.4 \mathrm{~Hz}, 1 \mathrm{H}), 7.58-7.51(\mathrm{~m}, 3 \mathrm{H}), 7.51-7.43(\mathrm{~m}, 3 \mathrm{H}), 2.77(\mathrm{t}, J=8.0 \mathrm{~Hz}, 2 \mathrm{H}), 1.58-1.50(\mathrm{~m}, 2 \mathrm{H}), 1.26-1.19(\mathrm{~m}$, $4 \mathrm{H}), 0.82(\mathrm{t}, J=6.9 \mathrm{~Hz}, 1 \mathrm{H}) \mathrm{ppm}$.

${ }^{13} \mathrm{C}$ NMR $\left(100 \mathrm{MHz}, \mathrm{CDCl}_{3}\right) \delta 160.7,146.3,140.9,135.6,134.1,129.2,128.7,128.7,128.2,128.0,127.6,126.9$, $126.3,32.8,31.4,30.2,22.3,13.9 \mathrm{ppm}$.

MS (EI) m/z C ${ }_{20} \mathrm{H}_{22} \mathrm{~N}: 275.2[\mathrm{M}]^{+}, 232.2,217.2,204.1,176.1,128.1,102.1,75.1$.

Phenylquinolin-3-yl)methanol (3as) [cas: 871022-77-4]<smiles>OCc1cc2ccccc2nc1-c1ccccc1</smiles>

Compound 3as was prepared following the general procedure A from 3-phenylprop-2-yn-1-ol 1aq (0.30 mmol) and benzo[c]isoxazole $2 \mathbf{a}(0.33 \mathrm{mmol})$ and isolated as a yellow solid $(45.8 \mathrm{mg}, 65 \%)$, m.p $117-119^{\circ} \mathrm{C}$.

${ }^{1} \mathbf{H}$ NMR $\left(400 \mathrm{MHz}, \mathrm{CDCl}_{3}\right) \delta 8.28(\mathrm{~s}, 1 \mathrm{H}), 8.13(\mathrm{~d}, J=8.5 \mathrm{~Hz}, 1 \mathrm{H}), 7.78(\mathrm{~d}, J=8.1 \mathrm{~Hz}, 1 \mathrm{H}), 7.68(\mathrm{ddd}, J=8.4,6.9$, $1.4 \mathrm{~Hz}, 1 \mathrm{H}), 7.52(\mathrm{dt}, J=10.9,1.9 \mathrm{~Hz}, 1 \mathrm{H}), 7.50-7.44(\mathrm{~m}, 2 \mathrm{H}), 7.41-7.35(\mathrm{~m}, 3 \mathrm{H}), 4.62(\mathrm{~s}, 2 \mathrm{H}) \mathrm{ppm}$.

${ }^{13} \mathrm{C}$ NMR $\left(100 \mathrm{MHz}, \mathrm{CDCl}_{3}\right) \delta 158.7,146.7,139.5,135.1,132.5,129.5,128.8,128.6,128.5,128.3,127.4,127.3$, $126.6,61.9 \mathrm{ppm}$.

HRMS (ESI) $\mathrm{m} / \mathrm{z}$ calcd. for $\mathrm{C}_{16} \mathrm{H}_{14} \mathrm{NO}[\mathrm{M}+\mathrm{H}]^{+} 236.10699$, found 236.10665 .

((4-Methoxyphenoxy)methyl)-2-phenylquinoline (3at)<smiles>COc1ccc(OCc2cc3ccccc3nc2-c2ccccc2)cc1</smiles>

Compound 3at was prepared following the general procedure A from 1-methoxy-4-((3-phenylprop-2-yn-1yl)oxy)benzene $1 \mathbf{a r}(0.30 \mathrm{mmol})$ and benzo[c]isoxazole $\mathbf{2 a}(0.33 \mathrm{mmol})$ and isolated as a viscous oil $(72.6 \mathrm{mg}, 71 \%)$.

${ }^{1} \mathbf{H}$ NMR $\left(400 \mathrm{MHz}, \mathrm{CDCl}_{3}\right) \delta 8.42(\mathrm{~s}, 1 \mathrm{H}), 8.20(\mathrm{~d}, J=8.5 \mathrm{~Hz}, 1 \mathrm{H}), 7.87(\mathrm{~d}, J=8.1 \mathrm{~Hz}, 1 \mathrm{H}), 7.74(\mathrm{t}, J=7.4 \mathrm{~Hz}, 1 \mathrm{H})$, $7.68(\mathrm{~d}, J=6.4 \mathrm{~Hz}, 2 \mathrm{H}), 7.56(\mathrm{t}, J=7.5 \mathrm{~Hz}, 1 \mathrm{H}), 7.53-7.45(\mathrm{~m}, 3 \mathrm{H}), 6.90-6.79(\mathrm{~m}, 4 \mathrm{H}), 5.08(\mathrm{~s}, 2 \mathrm{H}), 3.75(\mathrm{~s}, 3 \mathrm{H})$ ppm.

${ }^{13}$ C NMR $\left(100 \mathrm{MHz}, \mathrm{CDCl}_{3}\right) \delta 159.0,154.2,152.3,147.3,139.7,136.5,129.8,129.3,128.8,128.6,128.5,128.4$, $127.5,127.1,126.6,115.9,114.6,68.3,55.6 \mathrm{ppm}$.

HRMS (ESI) $\mathrm{m} / \mathrm{z}$ calcd. for $\mathrm{C}_{23} \mathrm{H}_{20} \mathrm{NO}_{2}[\mathrm{M}+\mathrm{H}]^{+} 342.14886$, found 342.14835 . 
<smiles>CCOC(=O)c1cc2ccccc2nc1-c1ccccc1</smiles>

Compound 3au was prepared following the general procedure A from ethyl 3-phenylpropiolate 1as $(0.30 \mathrm{mmol})$ and benzo[c]isoxazole 2a $(0.33 \mathrm{mmol})$ and isolated as a light yellow oil $(66.5 \mathrm{mg}, 80 \%)$.

${ }^{1} \mathbf{H}$ NMR $\left(400 \mathrm{MHz}, \mathrm{CDCl}_{3}\right) \delta 8.65(\mathrm{~s}, 1 \mathrm{H}), 8.19(\mathrm{~d}, J=8.5 \mathrm{~Hz}, 1 \mathrm{H}), 7.92(\mathrm{~d}, J=8.1 \mathrm{~Hz}, 1 \mathrm{H}), 7.84-7.79(\mathrm{~m}, 1 \mathrm{H})$, $7.65-7.57(\mathrm{~m}, 3 \mathrm{H}), 7.50-7.43(\mathrm{~m}, 3 \mathrm{H}), 4.19(\mathrm{q}, J=7.1 \mathrm{~Hz}, 2 \mathrm{H}), 1.08(\mathrm{t}, J=7.1 \mathrm{~Hz}, 3 \mathrm{H})$.

${ }^{13}$ C NMR $\left(100 \mathrm{MHz}, \mathrm{CDCl}_{3}\right) \delta 168.0,158.1,148.3,140.8,139.0,131.5,129.5,128.6,128.5,128.2,128.2,127.2$, $125.8,125.5,61.5,13.6$.

MS (EI) m/z C ${ }_{18} \mathrm{H}_{15} \mathrm{NO}_{2}: 277.1[\mathrm{M}]^{+}, 248.1,232.1,207.0,176.1,101.9,74.9$.

\section{4-(tert-Butyl)phenyl 2-phenylquinoline-3-carboxylate (3av)}<smiles>CC(C)(C)c1ccc(OC(=O)c2cc3ccccc3nc2-c2ccccc2)cc1</smiles>

Compound 3av was prepared following the general procedure A from 4-(tert-butyl)phenyl 3-phenylpropiolate 1at $(0.30 \mathrm{mmol})$ and benzo[c]isoxazole $2 \mathbf{a}(0.33 \mathrm{mmol})$ and isolated as a light yellow oil $(96.0 \mathrm{mg}, 84 \%)$.

${ }^{1}$ H NMR $\left(400 \mathrm{MHz}, \mathrm{CDCl}_{3}\right) \delta 8.83(\mathrm{~s}, 1 \mathrm{H}), 8.23(\mathrm{~d}, J=8.5 \mathrm{~Hz}, 1 \mathrm{H}), 7.98(\mathrm{~d}, J=8.1 \mathrm{~Hz}, 1 \mathrm{H}), 7.86(\mathrm{t}, J=7.6 \mathrm{~Hz}, 1 \mathrm{H})$, $7.75(\mathrm{~d}, J=6.8 \mathrm{~Hz}, 2 \mathrm{H}), 7.64(\mathrm{t}, J=7.5 \mathrm{~Hz}, 1 \mathrm{H}), 7.55-7.46(\mathrm{~m}, 3 \mathrm{H}), 7.35(\mathrm{~d}, J=8.7 \mathrm{~Hz}, 2 \mathrm{H}), 6.85(\mathrm{~d}, J=8.7 \mathrm{~Hz}$, $2 \mathrm{H}), 1.30(\mathrm{~s}, 9 \mathrm{H}) \mathrm{ppm}$.

${ }^{13}$ C NMR (100 MHz, $\left.\mathrm{CDCl}_{3}\right) \delta 166.7,158.1,148.9,148.6,148.1,140.7,139.8,131.9,129.6,128.8,128.7,128.4$, $128.3,127.4,126.3,125.8,124.8,120.5,34.5,31.4 \mathrm{ppm}$.

HRMS (ESI) $\mathrm{m} / \mathrm{z}$ calcd. for $\mathrm{C}_{26} \mathrm{H}_{24} \mathrm{NO}_{2}[\mathrm{M}+\mathrm{H}]^{+} 382.18016$, found 382.17963 .

\section{$N$-Methyl-N,2-diphenylquinoline-3-carboxamide (3aw)}<smiles>CN(C(=O)c1cc2ccccc2nc1-c1ccccc1)c1ccccc1</smiles>

Compound 3aw was prepared following the general procedure A from $N$-methyl- $N, 3$-diphenylpropiolamide 1au $(0.30$ $\mathrm{mmol})$ and benzo[c]isoxazole $\mathbf{2 a}(0.33 \mathrm{mmol})$ and isolated as a light yellow solid $(73.0 \mathrm{mg}, 72 \%)$, m.p $157-158^{\circ} \mathrm{C}$. ${ }^{1} \mathbf{H}$ NMR $\left(400 \mathrm{MHz}, \mathrm{CDCl}_{3}\right) \delta 8.42(\mathrm{~s}, 1 \mathrm{H}), 8.02(\mathrm{~d}, J=8.5 \mathrm{~Hz}, 1 \mathrm{H}), 7.85(\mathrm{~d}, J=8.1 \mathrm{~Hz}, 1 \mathrm{H}), 7.68(\mathrm{t}, J=7.2 \mathrm{~Hz}, 1 \mathrm{H})$, $7.54-7.47(\mathrm{~m}, 3 \mathrm{H}), 7.45-7.37(\mathrm{~m}, 3 \mathrm{H}), 6.90(\mathrm{t}, J=7.3 \mathrm{~Hz}, 1 \mathrm{H}), 6.82(\mathrm{t}, J=7.6 \mathrm{~Hz}, 2 \mathrm{H}), 6.19(\mathrm{~d}, J=7.7 \mathrm{~Hz}, 2 \mathrm{H})$, $3.33(\mathrm{~s}, 3 \mathrm{H}) \mathrm{ppm}$.

${ }^{13} \mathbf{C}$ NMR $\left(100 \mathrm{MHz}, \mathrm{CDCl}_{3}\right) \delta 169.8,154.8,147.7,142.0,139.5,137.5,130.3,130.3,129.4,128.9,128.9,128.3$, $128.1,127.6,126.8,126.3,126.1,125.8,37.3 \mathrm{ppm}$.

HRMS (ESI) $\mathrm{m} / \mathrm{z}$ calcd. for $\mathrm{C}_{23} \mathrm{H}_{19} \mathrm{~N}_{2} \mathrm{O}[\mathrm{M}+\mathrm{H}]^{+} 339.14919$, found 339.14903 .

\section{Phenyl(2-phenylquinolin-3-yl)methanone (3ax) ${ }^{14}$ [cas: 113059-25-9]}<smiles>O=C(c1ccccc1)c1cc2ccccc2nc1-c1ccccc1</smiles>

Compound 3ax was prepared following the general procedure A from 1,3-diphenylprop-2-yn-1-one 1av (0.30 mmol) and benzo[c]isoxazole $2 \mathbf{a}(0.33 \mathrm{mmol})$ and isolated as a yellow solid $(71.4 \mathrm{mg}, 77 \%)$, m.p $118-120^{\circ} \mathrm{C}$. 
${ }^{1} \mathbf{H}$ NMR $\left(400 \mathrm{MHz}, \mathrm{CDCl}_{3}\right) \delta 8.35(\mathrm{~s}, 1 \mathrm{H}), 8.26(\mathrm{~d}, J=8.5 \mathrm{~Hz}, 1 \mathrm{H}), 7.91(\mathrm{~d}, J=8.1 \mathrm{~Hz}, 1 \mathrm{H}), 7.84(\mathrm{ddd}, J=8.4,7.0$, $1.3 \mathrm{~Hz}, 1 \mathrm{H}), 7.75-7.70(\mathrm{~m}, 2 \mathrm{H}), 7.65-7.60(\mathrm{~m}, 3 \mathrm{H}), 7.48(\mathrm{t}, J=7.4 \mathrm{~Hz}, 1 \mathrm{H}), 7.36-7.27(\mathrm{~m}, 5 \mathrm{H}) \mathrm{ppm}$.

${ }^{13}$ C NMR $\left(100 \mathrm{MHz}, \mathrm{CDCl}_{3}\right) \delta 196.9,157.4,148.3,139.6,137.6,137.0,133.3,132.8,131.2,129.9,129.6,129.2$, $128.8,128.4,128.4,128.1,127.3,125.8 \mathrm{ppm}$.

MS (EI) m/z C ${ }_{22} \mathrm{H}_{15} \mathrm{NO}: 309.1[\mathrm{M}]^{+}, 280.1,232.1,204.1,176.1,125.8,77.1$.

\section{(2-Phenylquinolin-3-yl)ethan-1-one (3ay) [cas: 1350934-84-7]}<smiles>CC(=O)c1cc2ccccc2nc1-c1ccccc1</smiles>

Compound 3ay was prepared following the general procedure A from 4-phenylbut-3-yn-2-one 1aw $(0.30 \mathrm{mmol})$ and benzo[c]isoxazole $2 \mathbf{a}(0.33 \mathrm{mmol})$ and isolated as a light yellow oil $(46.7 \mathrm{mg}, 63 \%)$.

${ }^{1} \mathbf{H}$ NMR $\left(400 \mathrm{MHz}, \mathrm{CDCl}_{3}\right) \delta 8.38(\mathrm{~s}, 1 \mathrm{H}), 8.19(\mathrm{~d}, J=8.5 \mathrm{~Hz}, 1 \mathrm{H}), 7.93(\mathrm{~d}, J=8.1 \mathrm{~Hz}, 1 \mathrm{H}), 7.84-7.79(\mathrm{~m}, 1 \mathrm{H})$, $7.70-7.66(\mathrm{~m}, 2 \mathrm{H}), 7.60(\mathrm{t}, J=7.5 \mathrm{~Hz}, 1 \mathrm{H}), 7.54-7.48(\mathrm{~m}, 3 \mathrm{H}), 2.15(\mathrm{~s}, 3 \mathrm{H}) \mathrm{ppm}$.

${ }^{13}$ C NMR $\left(100 \mathrm{MHz}, \mathrm{CDCl}_{3}\right) \delta 203.1,156.8,148.3,140.2,136.9,134.8,131.4,129.5,129.3,129.1,128.8,128.4$, $127.3,126.1,30.4 \mathrm{ppm}$.

HRMS (ESI) m/z calcd. for $\mathrm{C}_{17} \mathrm{H}_{14} \mathrm{NO}[\mathrm{M}+\mathrm{H}]^{+} 248.10699$, found 248.10672 .

2-Phenylquinoline-3-carbaldehyde (3az) ${ }^{15}$ [cas: 227803-21-6]<smiles>O=Cc1cc2ccccc2nc1-c1ccccc1</smiles>

Compound 3az was prepared following the general procedure A from 3-phenylpropiolaldehyde 1ax $(0.30 \mathrm{mmol})$ and benzo[c]isoxazole $2 \mathbf{a}(0.33 \mathrm{mmol})$ and isolated as a yellow solid $(41.9 \mathrm{mg}, 60 \%)$, m.p $115-117^{\circ} \mathrm{C}$.

${ }^{1}$ H NMR $\left(400 \mathrm{MHz}, \mathrm{CDCl}_{3}\right) \delta 10.18(\mathrm{~s}, 1 \mathrm{H}), 8.85(\mathrm{~s}, 1 \mathrm{H}), 8.22(\mathrm{~d}, J=8.5 \mathrm{~Hz}, 1 \mathrm{H}), 8.02(\mathrm{~d}, J=8.2 \mathrm{~Hz}, 1 \mathrm{H}), 7.88(\mathrm{ddd}$, $J=8.4,7.0,1.3 \mathrm{~Hz}, 1 \mathrm{H}), 7.69(\mathrm{dd}, J=7.8,1.6 \mathrm{~Hz}, 2 \mathrm{H}), 7.64(\mathrm{t}, J=7.5 \mathrm{~Hz}, 1 \mathrm{H}), 7.60-7.51(\mathrm{~m}, 3 \mathrm{H}) \mathrm{ppm}$.

${ }^{13} \mathrm{C}$ NMR $\left(100 \mathrm{MHz}, \mathrm{CDCl}_{3}\right) \delta 191.5,160.3,149.6,138.2,137.8,132.6,130.2,129.6,129.4,129.4,128.7,127.7$, $127.5,126.4 \mathrm{ppm}$.

MS (EI) m/z C $16 \mathrm{H}_{11} \mathrm{NO}: 233.1[\mathrm{M}]^{+}, 204.1,175.9,127.0,101.1,74$.

3-Ethoxy-2-phenylquinoline (3ba) [cas: 83463-80-3]<smiles>CCOc1cc2ccccc2nc1-c1ccccc1</smiles>

Compound 3ba was prepared following the general procedure A from (ethoxyethynyl)benzene 1ay $(0.30 \mathrm{mmol})$ and benzo[c]isoxazole (2a, $0.33 \mathrm{mmol})$ and isolated as a colorless oil $(48.7 \mathrm{mg}, 65 \%)$.

${ }^{1} \mathbf{H}$ NMR $\left(400 \mathrm{MHz}, \mathrm{CDCl}_{3}\right) \delta 8.11(\mathrm{~d}, J=8.3 \mathrm{~Hz}, 1 \mathrm{H}), 8.08-7.98(\mathrm{~m}, 2 \mathrm{H}), 7.73(\mathrm{~d}, J=8.0 \mathrm{~Hz}, 1 \mathrm{H}), 7.59-7.54(\mathrm{~m}$, $1 \mathrm{H}), 7.52-7.42(\mathrm{~m}, 5 \mathrm{H}), 4.19(\mathrm{q}, J=7.0 \mathrm{~Hz}, 2 \mathrm{H}), 1.49(\mathrm{t}, J=7.0 \mathrm{~Hz}, 3 \mathrm{H})$.

${ }^{13}$ C NMR $\left(100 \mathrm{MHz}, \mathrm{CDCl}_{3}\right) \delta 151.9,150.9,143.0,137.9,129.8,129.3,128.6,127.9,126.7,126.7,126.1,113.6,64.0$, 14.5 .

HRMS (ESI) $\mathrm{m} / \mathrm{z}$ calcd. for $\mathrm{C}_{17} \mathrm{H}_{16} \mathrm{NO}[\mathrm{M}+\mathrm{H}]^{+} 250.12264$, found 250.12245 .

$N$-Butyl-4-methyl-N-(2-phenylquinolin-3-yl)benzenesulfonamide (3bb)<smiles>CC(C)(C)c1cc2ccccc2nc1-c1ccccc1</smiles>

Compound 3bb was prepared following the general procedure A from $N$-Butyl-4-methyl- $N$ (phenylethynyl)benzenesulfonamide $\mathbf{1 a z}(0.30 \mathrm{mmol})$ and benzo[c] isoxazole $(\mathbf{2 a}, 0.33 \mathrm{mmol})$ and isolated as a yellow solid (74.8 mg, 58\%), m.p $148-150{ }^{\circ} \mathrm{C}$. 
${ }^{1} \mathbf{H}$ NMR $\left(400 \mathrm{MHz}, \mathrm{CDCl}_{3}\right) \delta 8.17(\mathrm{~d}, J=8.4 \mathrm{~Hz}, 1 \mathrm{H}), 7.92(\mathrm{~s}, 1 \mathrm{H}), 7.74(\mathrm{ddd}, J=9.9,9.4,5.4 \mathrm{~Hz}, 4 \mathrm{H}), 7.57(\mathrm{dd}, J=$ $11.5,7.9 \mathrm{~Hz}, 3 \mathrm{H}), 7.46-7.40(\mathrm{~m}, 3 \mathrm{H}), 7.29(\mathrm{~d}, J=8.1 \mathrm{~Hz}, 2 \mathrm{H}), 3.44-3.19(\mathrm{~m}, 2 \mathrm{H}), 2.47(\mathrm{~s}, 3 \mathrm{H}), 1.29-1.24(\mathrm{~m}, 2 \mathrm{H})$, $1.05(\mathrm{dt}, J=14.9,7.4 \mathrm{~Hz}, 2 \mathrm{H}), 0.70(\mathrm{t}, J=7.3 \mathrm{~Hz}, 3 \mathrm{H}) \mathrm{ppm}$.

${ }^{13} \mathrm{C}$ NMR $\left(100 \mathrm{MHz}, \mathrm{CDCl}_{3}\right) \delta 159.9,146.9,143.8,138.9,136.3,132.1,130.4,129.6,129.4,129.4,128.5,128.1$, $128.1,127.3,127.1,127.0,51.6,29.8,21.6,19.8,13.5 \mathrm{ppm}$.

HRMS (ESI) m/z calcd. for $\mathrm{C}_{26} \mathrm{H}_{27} \mathrm{~N}_{2} \mathrm{O}_{2} \mathrm{~S}[\mathrm{M}+\mathrm{H}]^{+} 431.17878$, found 431.17840 .

1,3-Di(quinolin-2-yl)benzene (3bc) [cas: 189694-27-7]<smiles>c1ccc2nc(-c3ccc(-c4ccc5ccccc5n4)cc3)ccc2c1</smiles>

Compound 3bc was prepared following the general procedure A from 1,3-diethynylbenzene $\mathbf{1 b a}(0.30 \mathrm{mmol})$ and benzo[c]isoxazole $2 \mathbf{a}(0.66 \mathrm{mmol})$ and isolated as a white solid $(79.7 \mathrm{mg}, 80 \%)$, m.p $137-139^{\circ} \mathrm{C}$.

${ }^{1} \mathbf{H}$ NMR $\left(400 \mathrm{MHz}, \mathrm{CDCl}_{3}\right) \delta 8.98(\mathrm{~s}, 1 \mathrm{H}), 8.30(\mathrm{dd}, J=7.7,1.8 \mathrm{~Hz}, 2 \mathrm{H}), 8.24(\mathrm{~d}, J=8.5 \mathrm{~Hz}, 4 \mathrm{H}), 8.01(\mathrm{dd}, J=8.6$, $1.5 \mathrm{~Hz}, 2 \mathrm{H}), 7.84(\mathrm{~d}, J=8.1 \mathrm{~Hz}, 2 \mathrm{H}), 7.78-7.72(\mathrm{~m}, 2 \mathrm{H}), 7.70(\mathrm{t}, J=7.7 \mathrm{~Hz}, 1 \mathrm{H}), 7.54(\mathrm{t}, J=7.5 \mathrm{~Hz}, 2 \mathrm{H}) \mathrm{ppm}$.

${ }^{13} \mathbf{C}$ NMR $\left(100 \mathrm{MHz}, \mathrm{CDCl}_{3}\right) \delta 157.0,148.2,140.2,136.8,129.7,129.6,129.4,128.5,127.4,127.2,126.7,126.3$, $119.1 \mathrm{ppm}$.

HRMS (ESI) m/z calcd. for $\mathrm{C}_{24} \mathrm{H}_{17} \mathrm{~N}_{2}[\mathrm{M}+\mathrm{H}]^{+} 333.13863$, found 333.13810.

\section{1,3,5-Tri(quinolin-2-yl)benzene (3bd) [cas: 3886-42-8]}<smiles>c1ccc2nc(-c3cc(-c4ccc5ccccc5n4)cc(-c4ccc5ccccc5n4)c3)ccc2c1</smiles>

Compound 3bd was prepared following the general procedure A from 1,3,5-triethynylbenzene $\mathbf{1 b b}(0.30 \mathrm{mmol})$ and benzo[c]isoxazole $2 \mathbf{a}(0.99 \mathrm{mmol})$ and isolated as a white solid $(92.4 \mathrm{mg}, 67 \%)$, m.p 205-206 ${ }^{\circ} \mathrm{C}$.

${ }^{1} \mathbf{H}$ NMR $\left(400 \mathrm{MHz}, \mathrm{CDCl}_{3}\right) \delta 9.12(\mathrm{~s}, 3 \mathrm{H}), 8.29(\mathrm{t}, J=8.8 \mathrm{~Hz}, 6 \mathrm{H}), 8.18(\mathrm{~d}, J=8.5 \mathrm{~Hz}, 3 \mathrm{H}), 7.87(\mathrm{~d}, J=8.0 \mathrm{~Hz}, 3 \mathrm{H})$, $7.77(\mathrm{t}, J=8.2 \mathrm{~Hz}, 3 \mathrm{H}), 7.57(\mathrm{t}, J=7.5 \mathrm{~Hz}, 3 \mathrm{H}) \mathrm{ppm}$.

${ }^{13}$ C NMR $\left(100 \mathrm{MHz}, \mathrm{CDCl}_{3}\right) \delta 156.8,148.2,140.7,136.9,129.7,127.7,127.5,127.4,126.4,119.3$ ppm.

HRMS (ESI) m/z calcd. for $\mathrm{C}_{33} \mathrm{H}_{22} \mathrm{~N}_{3}[\mathrm{M}+\mathrm{H}]^{+} 460.18082$, found 460.18040 .

6-Fluoro-2,3-diphenylquinoline (3be) ${ }^{21}$ [cas: 1306601-47-7]<smiles>Fc1ccc2nc(-c3ccccc3)c(-c3ccccc3)cc2c1</smiles>

Compound 3be was prepared following the general procedure A from 1,2-diphenylethyne 1aa $(0.30 \mathrm{mmol})$ and 5 fluorobenzo[c]isoxazole $\mathbf{2 b}(0.33 \mathrm{mmol})$ and isolated as a yellow solid $(68.1 \mathrm{mg}, 76 \%)$, m.p $150-151{ }^{\circ} \mathrm{C}$.

${ }^{1} \mathbf{H}$ NMR $\left(400 \mathrm{MHz} \mathrm{CDCl}_{3}\right) \delta 8.20(\mathrm{~m}, J=9.0,5.3 \mathrm{~Hz}, 1 \mathrm{H}), 8.10(\mathrm{~s}, 1 \mathrm{H}), 7.52-7.43(\mathrm{~m}, 4 \mathrm{H}), 7.28(\mathrm{~m}, J=4.7 \mathrm{~Hz}$, $6 \mathrm{H}), 7.24(\mathrm{~m}, J=3.5,2.7 \mathrm{~Hz}, 2 \mathrm{H}) \mathrm{ppm}$.

${ }^{13}$ C NMR $\left(100 \mathrm{MHz}, \mathrm{CDCl}_{3}\right) \delta 160.6(\mathrm{~d}, J=248.4 \mathrm{~Hz}), 157.8(\mathrm{~d}, J=2.8 \mathrm{~Hz}), 144.4,140.1,139.6,136.8(\mathrm{~d}, J=5.4$ $\mathrm{Hz}), 135.3,131.9(\mathrm{~d}, J=9.3 \mathrm{~Hz}), 129.9,129.7,128.2,128.1,127.9,127.8(\mathrm{~d}, J=10.2 \mathrm{~Hz}), 127.4,119.8(\mathrm{~d}, J=25.7$ $\mathrm{Hz}), 110.3(\mathrm{~d}, J=21.6 \mathrm{~Hz}) \mathrm{ppm}$.

${ }^{19} \mathbf{F}$ NMR $\left(375 \mathrm{MHz}, \mathrm{CDCl}_{3}\right) \delta-113.1 \mathrm{ppm}$

MS (EI) m/z C ${ }_{21} \mathrm{H}_{14} \mathrm{FN}: 299.1[\mathrm{M}]^{+}, 281.0,207.0,149.4$.

6-Chloro-2,3-diphenylquinoline (3bf) ${ }^{20}$ [cas: 1909244-83-2]<smiles>Clc1ccc2nc(-c3ccccc3)c(-c3ccccc3)cc2c1</smiles>

Compound 3bf was prepared following the general procedure A from 1,2-diphenylethyne 1aa (0.30 mmol) and 5chlorobenzo[c]isoxazole $2 \mathrm{c}(0.33 \mathrm{mmol})$ and isolated as a yellow solid $(73.7 \mathrm{mg}, 78 \%)$, m.p $136-138{ }^{\circ} \mathrm{C}$. 
${ }^{1} \mathbf{H}$ NMR $\left(400 \mathrm{MHz}, \mathrm{CDCl}_{3}\right) \delta 8.13(\mathrm{~d}, J=9.0 \mathrm{~Hz}, 1 \mathrm{H}), 8.08(\mathrm{~s}, 1 \mathrm{H}), 7.85(\mathrm{~d}, J=2.1 \mathrm{~Hz}, 1 \mathrm{H}), 7.66(\mathrm{~m}, J=9.0,2.2 \mathrm{~Hz}$, $1 \mathrm{H}), 7.43(\mathrm{~m}, J=5.3,2.4 \mathrm{~Hz}, 2 \mathrm{H}), 7.29(\mathrm{~m}, J=9.1,5.6 \mathrm{~Hz}, 6 \mathrm{H}), 7.23(\mathrm{~m}, J=6.2,3.1 \mathrm{~Hz}, 2 \mathrm{H}) \mathrm{ppm}$.

${ }^{13}$ C NMR $\left(100 \mathrm{MHz}, \mathrm{CDCl}_{3}\right) \delta 158.7,145.6,140.0,139.5,136.5,135.4,132.4,131.0,130.5,129.9,129.7,128.3$, $128.2,128.0,127.8,127.4,126.0 \mathrm{ppm}$.

MS (EI) m/z C ${ }_{21} \mathrm{H}_{14} \mathrm{ClN}: 315.1[\mathrm{M}]^{+}, 308.1,278.1,138.9$.

6-Bromo-2,3-diphenylquinoline (3bg) [cas: 1909244-84-3]<smiles>Brc1ccc2nc(-c3ccccc3)c(-c3ccccc3)cc2c1</smiles>

Compound $3 \mathbf{b g}$ was prepared following the general procedure A from 1,2-diphenylethyne 1aa $(0.30 \mathrm{mmol})$ and 5bromobenzo[c]isoxazole $2 \mathbf{d}(0.33 \mathrm{mmol})$ and isolated as a yellow solid $(91.5 \mathrm{mg}, 85 \%)$, m.p $132-133{ }^{\circ} \mathrm{C}$.

${ }^{1} \mathbf{H}$ NMR $\left(400 \mathrm{MHz}, \mathrm{CDCl}_{3}\right) \delta 8.05(\mathrm{t}, J=4.4 \mathrm{~Hz}, 2 \mathrm{H}), 8.01(\mathrm{~d}, J=2.2 \mathrm{~Hz}, 1 \mathrm{H}), 7.78(\mathrm{~m}, J=9.0,2.2 \mathrm{~Hz}, 1 \mathrm{H}), 7.45-$ $7.41(\mathrm{~m}, 2 \mathrm{H}), 7.28(\mathrm{~m}, J=6.9,3.9 \mathrm{~Hz}, 6 \mathrm{H}), 7.24-7.19(\mathrm{~m}, 2 \mathrm{H}) \mathrm{ppm}$.

${ }^{13}$ C NMR $\left(100 \mathrm{MHz}, \mathrm{CDCl}_{3}\right) \delta 158.8,145.8,140.0,139.5,136.4,135.4,133.0,131.2,129.9,129.7,129.4,128.3$, $128.2,128.0,127.4,120.5 \mathrm{ppm}$.

HRMS (ESI) $\mathrm{m} / \mathrm{z}$ calcd. for $\mathrm{C}_{21} \mathrm{H}_{15} \mathrm{BrN}[\mathrm{M}+\mathrm{H}]^{+} 360.03824$, found 360.03818 .

6-Methoxy-2,3-diphenylquinoline (3bh) ${ }^{21}$ [cas: 847025-52-9]<smiles>COc1ccc2nc(-c3ccccc3)c(-c3ccccc3)cc2c1</smiles>

Compound 3bh was prepared following the general procedure A from 1,2-diphenylethyne 1aa $(0.30 \mathrm{mmol})$ and 5methoxybenzo[c]isoxazole $2 \mathbf{e}(0.33 \mathrm{mmol})$ and isolated as a yellow solid $(76.5 \mathrm{mg}, 82 \%)$, m.p $179-181^{\circ} \mathrm{C}$.

${ }^{1} \mathbf{H}$ NMR $\left(400 \mathrm{MHz}, \mathrm{CDCl}_{3}\right) \delta 8.10(\mathrm{~d}, J=9.2 \mathrm{~Hz}, 1 \mathrm{H}), 8.06(\mathrm{~s}, 1 \mathrm{H}), 7.43(\mathrm{~m}, J=6.6,3.0 \mathrm{~Hz}, 2 \mathrm{H}), 7.39(\mathrm{~m}, J=9.2$, $2.8 \mathrm{~Hz}, 1 \mathrm{H}), 7.30-7.26(\mathrm{~m}, 4 \mathrm{H}), 7.24(\mathrm{~m}, J=7.5,3.5 \mathrm{~Hz}, 4 \mathrm{H}), 7.12(\mathrm{~d}, J=2.7 \mathrm{~Hz}, 1 \mathrm{H}), 3.94(\mathrm{~s}, 3 \mathrm{H}) \mathrm{ppm}$.

${ }^{13} \mathrm{C}$ NMR $\left(100 \mathrm{MHz}, \mathrm{CDCl}_{3}\right) \delta 158.0,155.9,143.4,140.5,140.1,136.4,134.7,130.9,130.0,129.7,128.2,128.2$, $127.9,127.7,127.1,122.4,104.8,55.6 \mathrm{ppm}$.

MS (EI) m/z C $22 \mathrm{H}_{17} \mathrm{NO}: 311.1[\mathrm{M}]^{+}, 281.0,267.0,207.0,132.9$.

\section{6-(Benzyloxy)-2,3-diphenyl (3bi)}<smiles>c1ccc(COc2ccc3nc(-c4ccccc4)c(-c4ccccc4)cc3c2)cc1</smiles>

Compound 3bi was prepared following the general procedure A from 1,2-diphenylethyne 1aa $(0.30 \mathrm{mmol})$ and 5(benzyloxy) benzo[c]isoxazole $2 \mathbf{f}(0.33 \mathrm{mmol})$ and isolated as a yellow solid $(102.2 \mathrm{mg}, 88 \%)$, m.p $157-158^{\circ} \mathrm{C}$.

${ }^{1} \mathbf{H}$ NMR $\left(400 \mathrm{MHz}, \mathrm{CDCl}_{3}\right) \delta 8.11(\mathrm{~d}, J=9.2 \mathrm{~Hz}, 1 \mathrm{H}), 8.02(\mathrm{~s}, 1 \mathrm{H}), 7.49(\mathrm{~d}, J=7.1 \mathrm{~Hz}, 2 \mathrm{H}), 7.45(\mathrm{~d}, J=2.8 \mathrm{~Hz}, 1 \mathrm{H})$, $7.41(\mathrm{~m}, J=11.1,4.9 \mathrm{~Hz}, 4 \mathrm{H}), 7.35(\mathrm{~d}, J=7.2 \mathrm{~Hz}, 1 \mathrm{H}), 7.28-7.21(\mathrm{~m}, 8 \mathrm{H}), 7.18(\mathrm{~d}, J=2.7 \mathrm{~Hz}, 1 \mathrm{H}), 5.20(\mathrm{~s}, 2 \mathrm{H})$ ppm.

${ }^{13}$ C NMR $\left(100 \mathrm{MHz}, \mathrm{CDCl}_{3}\right) \delta 157.1,156.0,143.5,140.5,140.1,136.5,136.4,134.7,131.0,130.0,129.7,128.7$, $128.2,128.1,127.9,127.7,127.5,127.1,122.7,106.2,70.3 \mathrm{ppm}$.

HRMS (ESI) $\mathrm{m} / \mathrm{z}$ calcd. for $\mathrm{C}_{28} \mathrm{H}_{22} \mathrm{NO}[\mathrm{M}+\mathrm{H}]^{+} 388.16959$, found 388.16974 .

\section{2,3-Diphenyl-7-(trifluoromethyl)quinolone (3bj)}<smiles>FC(F)(F)c1ccc2cc(-c3ccccc3)c(-c3ccccc3)nc2c1</smiles>

Compound 3bj was prepared following the general procedure A from 1,2-diphenylethyne 1aa $(0.30 \mathrm{mmol})$ and 5(trifluoromethyl) benzo[c]isoxazole $2 \mathbf{g}(0.33 \mathrm{mmol})$ and isolated as a yellow solid $(66.0 \mathrm{mg}, 63 \%)$, m.p $135-137^{\circ} \mathrm{C}$. ${ }^{1} \mathbf{H}$ NMR $\left(400 \mathrm{MHz}, \mathrm{CDCl}_{3}\right) \delta 8.52(\mathrm{~s}, 1 \mathrm{H}), 8.20(\mathrm{~s}, 1 \mathrm{H}), 7.96(\mathrm{~d}, J=8.5 \mathrm{~Hz}, 1 \mathrm{H}), 7.72(\mathrm{~m}, J=8.5,1.5 \mathrm{~Hz}, 1 \mathrm{H}), 7.47$ $-7.43(\mathrm{~m}, 2 \mathrm{H}), 7.30(\mathrm{~m}, J=9.2,5.4 \mathrm{~Hz}, 6 \mathrm{H}), 7.26-7.23(\mathrm{~m}, 2 \mathrm{H}) \mathrm{ppm}$.

${ }^{13}$ C NMR $\left(100 \mathrm{MHz}, \mathrm{CDCl}_{3}\right) \delta 159.9,146.2,139.8,139.3,137.3,136.5,131.2(\mathrm{q}, J=32.4 \mathrm{~Hz}), 129.9,129.6,128.6$, $128.4,128.3,128.0,127.6,127.3(\mathrm{q}, J=4.2 \mathrm{~Hz}), 127.2(\mathrm{q}, J=271.1 \mathrm{~Hz}), 122.3(\mathrm{q}, J=2.8 \mathrm{~Hz}) \mathrm{ppm}$.

${ }^{19} \mathbf{F}$ NMR $\left(375 \mathrm{MHz}, \mathrm{CDCl}_{3}\right) \delta-62.6 \mathrm{ppm}$.

HRMS (ESI) $\mathrm{m} / \mathrm{z}$ calcd. for $\mathrm{C}_{22} \mathrm{H}_{15} \mathrm{~F}_{3} \mathrm{~N}[\mathrm{M}+\mathrm{H}]^{+} 350.11511$, found 350.11519 . 
<smiles>Clc1ccc2cc(-c3ccccc3)c(-c3ccccc3)nc2c1</smiles>

Compound 3bk was prepared following the general procedure A from 1,2-diphenylethyne 1aa (0.30 mmol) and 6chlorobenzo[c] isoxazole $2 \mathbf{h}(0.33 \mathrm{mmol})$ and isolated as a yellow solid $(64.2 \mathrm{mg}, 68 \%), \mathrm{m} . \mathrm{p} 150-152{ }^{\circ} \mathrm{C}$.

${ }^{1} \mathbf{H}$ NMR $\left(400 \mathrm{MHz}, \mathrm{CDCl}_{3}\right) \delta 8.21(\mathrm{~s}, 1 \mathrm{H}), 8.14(\mathrm{~s}, 1 \mathrm{H}), 7.79(\mathrm{~d}, J=8.7 \mathrm{~Hz}, 1 \mathrm{H}), 7.51(\mathrm{dd}, J=8.7,1.6 \mathrm{~Hz}, 1 \mathrm{H}), 7.46$ $-7.42(\mathrm{~m}, 2 \mathrm{H}), 7.32-7.27(\mathrm{~m}, 6 \mathrm{H}), 7.24(\mathrm{dd}, J=6.3,3.2 \mathrm{~Hz}, 2 \mathrm{H}) \mathrm{ppm}$.

${ }^{13}$ C NMR $\left(100 \mathrm{MHz}, \mathrm{CDCl}_{3}\right) \delta 159.4,147.6,140.0,139.6,137.3,135.3,134.8,130.0,129.6,128.7,128.4,128.3$, $128.2,127.9,127.7,127.3,125.5 \mathrm{ppm}$.

HRMS (ESI) m/z calcd. for C21H15ClN [M+H] ${ }^{+} 316.08875$, found 316.08882 .

6,7-Dimethoxy-2,3-diphenylquinoline (3bl) [cas: 175697-59-3]<smiles>COc1cc2cc(-c3ccccc3)c(-c3ccccc3)nc2cc1OC</smiles>

Compound 3bl was prepared following the general procedure A from 1,2-diphenylethyne 1aa $(0.30 \mathrm{mmol})$ and 5,6dimethoxybenzo[c]isoxazole $2 \mathbf{i}(0.33 \mathrm{mmol})$ and isolated as a yellow solid $(84.9 \mathrm{mg}, 83 \%)$, m.p 125-127 ${ }^{\circ} \mathrm{C}$.

${ }^{1} \mathrm{H}$ NMR $\left(400 \mathrm{MHz}, \mathrm{CDCl}_{3}\right) \delta 8.02(\mathrm{~s}, 1 \mathrm{H}), 7.55(\mathrm{~s}, 1 \mathrm{H}), 7.45-7.41(\mathrm{~m}, 2 \mathrm{H}), 7.30-7.26(\mathrm{~m}, 6 \mathrm{H}), 7.25-7.22(\mathrm{~m}$, $2 \mathrm{H}), 7.10(\mathrm{~s}, 1 \mathrm{H}), 4.06(\mathrm{~s}, 3 \mathrm{H}), 4.04(\mathrm{~s}, 3 \mathrm{H}) \mathrm{ppm}$.

${ }^{13} \mathrm{C}$ NMR $\left(100 \mathrm{MHz}, \mathrm{CDCl}_{3}\right) \delta 156.0,152.6,150.0,144.2,140.6,140.2,135.9,132.7,129.9,129.7,128.1,127.8$, 127.6, 126.8, 122.7, 108.0, 104.7, 56.1, $56.0 \mathrm{ppm}$.

HRMS (ESI) $\mathrm{m} / \mathrm{z}$ calcd. for $\mathrm{C}_{23} \mathrm{H}_{20} \mathrm{NO}_{2}[\mathrm{M}+\mathrm{H}]^{+} 342.14886$, found 342.14881 .

\section{6,7-Diphenyl- [1,3] dioxolo[4,5-g] quinolone (3bm) [cas: 854255-27-9]}<smiles>c1ccc(-c2cc3cc4c(cc3nc2-c2ccccc2)OCO4)cc1</smiles>

Compound 3bm was prepared following the general procedure A from 1,2-diphenylethyne 1aa $(0.30 \mathrm{mmol})$ and $[1,3]$ dioxolo [4',5':4,5] benzo[1,2-c] isoxazole $2 \mathbf{j}(0.33 \mathrm{mmol})$ and isolated as a yellow solid ( $82.9 \mathrm{mg}, 85 \%)$, m.p 153$155^{\circ} \mathrm{C}$.

${ }^{1} \mathbf{H}$ NMR $\left(400 \mathrm{MHz}, \mathrm{CDCl}_{3}\right) \delta 7.98(\mathrm{~s}, 1 \mathrm{H}), 7.51(\mathrm{~s}, 1 \mathrm{H}), 7.46-7.41(\mathrm{~m}, 2 \mathrm{H}), 7.30-7.26(\mathrm{~m}, 6 \mathrm{H}), 7.23(\mathrm{~m}, J=7.1$, $2.6 \mathrm{~Hz}, 2 \mathrm{H}), 7.10(\mathrm{~s}, 1 \mathrm{H}), 6.14-6.09(\mathrm{~m}, 2 \mathrm{H}) \mathrm{ppm}$.

${ }^{13}$ C NMR $\left(100 \mathrm{MHz}, \mathrm{CDCl}_{3}\right) \delta 155.9,150.8,148.0,145.5,140.5,140.1,136.5,132.7,130.0,129.7,128.1,127.8$, 127.7, 126.9, 124.2, 105.8, 102.3, 101.6 ppm.

HRMS (ESI) $\mathrm{m} / \mathrm{z}$ calcd. for $\mathrm{C}_{22} \mathrm{H}_{16} \mathrm{NO}_{2}[\mathrm{M}+\mathrm{H}]^{+} 326.11756$, found 326.11751 .

4-Methyl-2,3-diphenylquinoline (3bn) ${ }^{22}$ [cas: 6829-12-5]<smiles>Cc1c(-c2ccccc2)c(-c2ccccc2)nc2ccccc12</smiles>

Compound 3bn was prepared following the general procedure A from 1,2-diphenylethyne 1aa (0.30 mmol) and 3methylbenzo[c]isoxazole $2 \mathbf{k}(0.33 \mathrm{mmol})$ and isolated as a yellow solid $(62.0 \mathrm{mg}, 70 \%)$, m.p $114-115^{\circ} \mathrm{C}$.

${ }^{1} \mathbf{H}$ NMR $\left(400 \mathrm{MHz}, \mathrm{CDCl}_{3}\right) \delta 8.18(\mathrm{~d}, J=8.1 \mathrm{~Hz}, 1 \mathrm{H}), 8.04(\mathrm{~d}, J=8.4,0.7 \mathrm{~Hz}, 1 \mathrm{H}), 7.70(\mathrm{~m}, J=8.3,6.9,1.3 \mathrm{~Hz}$, $1 \mathrm{H}), 7.56(\mathrm{~m}, J=8.2,6.9,1.2 \mathrm{~Hz}, 1 \mathrm{H}), 7.30-7.26(\mathrm{~m}, 2 \mathrm{H}), 7.26-7.20(\mathrm{~m}, 3 \mathrm{H}), 7.18-7.12(\mathrm{~m}, 3 \mathrm{H}), 7.08(\mathrm{~m}, J=4.9$, $3.0 \mathrm{~Hz}, 2 \mathrm{H}), 2.51(\mathrm{~s}, 3 \mathrm{H}) \mathrm{ppm}$.

${ }^{13}$ C NMR (100 MHz, $\left.\mathrm{CDCl}_{3}\right) \delta 158.8,146.7,142.2,141.3,139.0,133.9,130.7,130.2,129.7,129.1,127.9,127.5$, 127.4, 127.1, 126.9, 126.5, 124.1, $16.2 \mathrm{ppm}$.

MS (EI) m/z C $22 \mathrm{H}_{17} \mathrm{~N}: 295.1[\mathrm{M}]^{+}, 280.0,207.0,140.0$.

\section{2,3,4-Triphenylquinoline (3bo) $)^{22}$ [cas: 10380-73-1]}<smiles>c1ccc(-c2nc3ccccc3c(-c3ccccc3)c2-c2ccccc2)cc1</smiles> 
Compound 3bo was prepared following the general procedure A from 1,2-diphenylethyne 1aa $(0.30 \mathrm{mmol})$ and 3phenylbenzo[c]isoxazole $2 \mathrm{l}(0.33 \mathrm{mmol})$ and isolated as a yellow solid $(84.6 \mathrm{mg}, 79 \%)$, m.p $194-195{ }^{\circ} \mathrm{C}$.

${ }^{1} \mathbf{H}$ NMR $\left(400 \mathrm{MHz}, \mathrm{CDCl}_{3}\right) \delta 8.28(\mathrm{~d}, J=8.4 \mathrm{~Hz}, 1 \mathrm{H}), 7.77-7.72(\mathrm{~m}, 1 \mathrm{H}), 7.61(\mathrm{~d}, J=7.8 \mathrm{~Hz}, 1 \mathrm{H}), 7.49-7.44(\mathrm{~m}$, $1 \mathrm{H}), 7.40(\mathrm{~m}, J=7.4,3.3 \mathrm{~Hz}, 2 \mathrm{H}), 7.31-7.27(\mathrm{~m}, 3 \mathrm{H}), 7.25-7.21(\mathrm{~m}, 3 \mathrm{H}), 7.18-7.14(\mathrm{~m}, 2 \mathrm{H}), 7.04-6.99(\mathrm{~m}, 3 \mathrm{H})$, $6.91(\mathrm{~m}, J=6.6,3.0 \mathrm{~Hz}, 2 \mathrm{H}) \mathrm{ppm}$.

${ }^{13}$ C NMR $\left(100 \mathrm{MHz}, \mathrm{CDCl}_{3}\right) \delta 159.0,147.6,147.3,141.1,138.3,136.9,132.9,131.3,130.3,129.9,129.7,129.3$, $127.8,127.6,127.6,127.3,127.2,126.6,126.6,126.5,126.3 \mathrm{ppm}$.

MS (EI) m/z C ${ }_{27} \mathrm{H}_{19} \mathrm{~N}: 357.1[\mathrm{M}]^{+}, 280.0,207.0,191.0,177.0,133.0,95.8$.

\section{6-Chloro-2,3,4-triphenylquinoline (3bp) ${ }^{22}$ [cas: 1208258-68-7]}<smiles>Clc1ccc2nc(-c3ccccc3)c(-c3ccccc3)c(-c3ccccc3)c2c1</smiles>

Compound 3bp was prepared following the general procedure A from 1,2-diphenylethyne 1aa $(0.30 \mathrm{mmol})$ and 5chloro-3-phenylbenzo[c]isoxazole $\mathbf{2 m}(0.33 \mathrm{mmol})$ and isolated as a yellow solid $(95.1 \mathrm{mg}, 81 \%)$, m.p $200-202{ }^{\circ} \mathrm{C}$. ${ }^{1} \mathbf{H}$ NMR $\left(400 \mathrm{MHz}, \mathrm{CDCl}_{3}\right) \delta 8.21(\mathrm{~d}, J=8.9 \mathrm{~Hz}, 1 \mathrm{H}), 7.67(\mathrm{~m}, J=8.9,2.3 \mathrm{~Hz}, 1 \mathrm{H}), 7.59(\mathrm{~d}, J=2.1 \mathrm{~Hz}, 1 \mathrm{H}), 7.40$ $(\mathrm{m}, J=6.6,2.9 \mathrm{~Hz}, 2 \mathrm{H}), 7.33-7.28(\mathrm{~m}, 3 \mathrm{H}), 7.25-7.20(\mathrm{~m}, 3 \mathrm{H}), 7.16-7.11(\mathrm{~m}, 2 \mathrm{H}), 7.05-6.99(\mathrm{~m}, 3 \mathrm{H}), 6.91(\mathrm{~m}$, $J=6.5,3.0 \mathrm{~Hz}, 2 \mathrm{H}) \mathrm{ppm}$.

${ }^{13} \mathbf{C}$ NMR $\left(100 \mathrm{MHz}, \mathrm{CDCl}_{3}\right) \delta 159.2,146.9,145.6,140.7,137.9,136.2,133.7,132.4,131.3,131.2,130.2,130.1$, $129.8,127.9,127.7,127.6,127.5,127.4,126.5,125.3 \mathrm{ppm}$.

MS (EI) $\mathrm{m} / \mathrm{z} \mathrm{C}_{27} \mathrm{H}_{18} \mathrm{ClN}: 391.1[\mathrm{M}]^{+}, 354.0,281.0,207.0,176.1,133.0,95.7$.

\section{3-(Benzyloxy)-2-phenylquinoline (3bq)}<smiles>c1ccc(COc2ccc3nc(-c4ccccc4)ccc3c2)cc1</smiles>

Compound 3bq was prepared following the general procedure A from phenylacetylene 1a $(0.30 \mathrm{mmol})$ and 5(benzyloxy) benzo[c]isoxazole $2 \mathbf{f}(0.33 \mathrm{mmol})$ and isolated as a yellow solid $(78.4 \mathrm{mg}, 84 \%)$, m.p $142-143{ }^{\circ} \mathrm{C}$.

${ }^{1} \mathbf{H}$ NMR $\left(400 \mathrm{MHz}, \mathrm{CDCl}_{3}\right) \delta 8.14(\mathrm{~d}, J=7.2 \mathrm{~Hz}, 2 \mathrm{H}), 8.10(\mathrm{dd}, J=8.9,2.4 \mathrm{~Hz}, 2 \mathrm{H}), 7.83(\mathrm{~d}, J=8.6 \mathrm{~Hz}, 1 \mathrm{H}), 7.58-$ $7.47(\mathrm{~m}, 5 \mathrm{H}), 7.46-7.41(\mathrm{~m}, 3 \mathrm{H}), 7.37(\mathrm{t}, J=7.2 \mathrm{~Hz}, 1 \mathrm{H}), 7.18(\mathrm{~d}, J=2.7 \mathrm{~Hz}, 1 \mathrm{H}), 5.21(\mathrm{~s}, 2 \mathrm{H}) \mathrm{ppm}$.

${ }^{13} \mathrm{C}$ NMR $\left(100 \mathrm{MHz}, \mathrm{CDCl}_{3}\right) \delta 156.8,155.2,144.4,139.7,136.5,135.6,131.2,128.9,128.8,128.7,128.1,128.0$, $127.5,127.3,122.6,119.2,106.4,70.3 \mathrm{ppm}$.

HRMS (ESI) $\mathrm{m} / \mathrm{z}$ calcd. for $\mathrm{C}_{22} \mathrm{H}_{18} \mathrm{NO}[\mathrm{M}+\mathrm{H}]^{+} 312.13829$, found 312.13791 .

\section{2-Phenyl-7-(trifluoromethyl)quinoline (3br) ${ }^{14}$ [cas: 887237-11-8]}<smiles>FC(F)(F)c1ccc2ccc(-c3ccccc3)nc2c1</smiles>

Compound 3br was prepared following the general procedure A from phenylacetylene 1a $(0.30 \mathrm{mmol})$ and benzo[c]isoxazole $2 \mathrm{~g}(0.33 \mathrm{mmol})$ and isolated as a white solid $(50.0 \mathrm{mg}, 61 \%)$, m.p $82-84{ }^{\circ} \mathrm{C}$.

${ }^{1} \mathbf{H}$ NMR $\left(400 \mathrm{MHz}, \mathrm{CDCl}_{3}\right) \delta 8.49(\mathrm{~s}, 1 \mathrm{H}), 8.28(\mathrm{~d}, J=8.7 \mathrm{~Hz}, 1 \mathrm{H}), 8.20(\mathrm{dd}, J=5.2,3.3 \mathrm{~Hz}, 2 \mathrm{H}), 8.00(\mathrm{~d}, J=8.7 \mathrm{~Hz}$, $1 \mathrm{H}), 7.95(\mathrm{~d}, J=8.5 \mathrm{~Hz}, 1 \mathrm{H}), 7.70(\mathrm{dd}, J=8.5,1.5 \mathrm{~Hz}, 1 \mathrm{H}), 7.59-7.46(\mathrm{~m}, 3 \mathrm{H}) \mathrm{ppm}$.

${ }^{13}$ C NMR $\left(100 \mathrm{MHz}, \mathrm{CDCl}_{3}\right) \delta 158.6,147.3,138.9,136.6,131.4(\mathrm{q}, J=32.8 \mathrm{~Hz}), 129.9,129.0,128.6,128.5(\mathrm{q}, J=$ $5.6 \mathrm{~Hz}), 127.6,127.5(\mathrm{q}, J=4.3 \mathrm{~Hz}), 124.1(\mathrm{q}, J=272.6 \mathrm{~Hz}), 121.9(\mathrm{q}, J=3.0 \mathrm{~Hz}), 120.7 \mathrm{ppm}$.

${ }^{19} \mathbf{F}$ NMR $\left(375 \mathrm{MHz}, \mathrm{CDCl}_{3}\right) \delta-62.6 \mathrm{ppm}$.

MS (EI) m/z C $\mathrm{C}_{16} \mathrm{H}_{10} \mathrm{~F}_{3} \mathrm{~N}: 273.1[\mathrm{M}]^{+}, 204.1,176.0,136.5,75.0$.

\section{2-Phenylquinolin-6-yl benzoate (3bs) [cas: 5855-53-8]}<smiles>O=C(Oc1ccc2nc(-c3ccccc3)ccc2c1)c1ccccc1</smiles>

Compound 3bs was prepared following the general procedure A from phenylacetylene 1a $(0.30 \mathrm{mmol})$ and benzo[c]isoxazol-5-yl benzoate $2 \mathrm{o}(0.33 \mathrm{mmol})$ and isolated as a yellow solid $(78.2 \mathrm{mg}, 80 \%)$, m.p 131-133 ${ }^{\circ} \mathrm{C}$. ${ }^{1} \mathbf{H}$ NMR $\left(400 \mathrm{MHz}, \mathrm{CDCl}_{3}\right) \delta 8.27-8.16(\mathrm{~m}, 6 \mathrm{H}), 7.89(\mathrm{~d}, J=8.6 \mathrm{~Hz}, 1 \mathrm{H}), 7.70(\mathrm{~d}, J=2.4 \mathrm{~Hz}, 1 \mathrm{H}), 7.66(\mathrm{t}, J=7.4$ $\mathrm{Hz}, 1 \mathrm{H}), 7.60(\mathrm{dd}, J=9.1,2.6 \mathrm{~Hz}, 1 \mathrm{H}), 7.57-7.50(\mathrm{~m}, 4 \mathrm{H}), 7.48-7.45(\mathrm{~m}, 1 \mathrm{H}) \mathrm{ppm}$. 
${ }^{13}$ C NMR $\left(100 \mathrm{MHz}, \mathrm{CDCl}_{3}\right) \delta 165.2,157.2,148.6,146.4,139.4,136.5,133.8,131.3,130.2,129.4,129.3,128.9$, $128.6,127.5,127.4,124.9,119.5,118.3 \mathrm{ppm}$.

HRMS (ESI) $\mathrm{m} / \mathrm{z}$ calcd. for $\mathrm{C}_{22} \mathrm{H}_{16} \mathrm{NO}_{2}[\mathrm{M}+\mathrm{H}]^{+} 326.11756$, found 326.11716 .

6,7-Dimethoxy-2-phenylquinoline (3bt) [cas: 57179-07-4]<smiles>COc1cc2ccc(-c3ccccc3)nc2cc1OC</smiles>

Compound 3bt was prepared following the general procedure A from phenylacetylene 1a $(0.30 \mathrm{mmol})$ and 5,6dimethoxybenzo[c]isoxazole $\mathbf{2 j}(0.33 \mathrm{mmol})$ and isolated as a white solid $(69.4 \mathrm{mg}, 87 \%)$, m.p $124-126^{\circ} \mathrm{C}$.

${ }^{1} \mathbf{H}$ NMR $\left(400 \mathrm{MHz}, \mathrm{CDCl}_{3}\right) \delta 8.11(\mathrm{~d}, J=7.3 \mathrm{~Hz}, 2 \mathrm{H}), 8.03(\mathrm{~d}, J=8.5 \mathrm{~Hz}, 1 \mathrm{H}), 7.72(\mathrm{~d}, J=8.5 \mathrm{~Hz}, 1 \mathrm{H}), 7.51(\mathrm{t}, J=$ $7.5 \mathrm{~Hz}, 3 \mathrm{H}), 7.43(\mathrm{t}, J=7.3 \mathrm{~Hz}, 1 \mathrm{H}), 7.05(\mathrm{~s}, 1 \mathrm{H}), 4.06(\mathrm{~s}, 3 \mathrm{H}), 4.01(\mathrm{~s}, 3 \mathrm{H}) \mathrm{ppm}$.

${ }^{13} \mathrm{C}$ NMR $\left(100 \mathrm{MHz}, \mathrm{CDCl}_{3}\right) \delta 155.3,152.5,149.6,145.2,139.9,134.8,128.8,128.7,127.2,122.6,117.2,108.3$, $104.9,56.1,56.0 \mathrm{ppm}$.

HRMS (ESI) $\mathrm{m} / \mathrm{z}$ calcd. for $\mathrm{C}_{17} \mathrm{H}_{17} \mathrm{NO}_{2}[\mathrm{M}+\mathrm{H}]^{+} 266.11756$, found 266.11725 .

6-Chloro-2,4-diphenylquinoline (3bu) ${ }^{15}$ [cas: 21923-40-0]<smiles>Clc1ccc2nc(-c3ccccc3)cc(-c3ccccc3)c2c1</smiles>

Compound 3bu was prepared following the general procedure A from phenylacetylene 1a $(0.30 \mathrm{mmol})$ and 5-chloro3-phenylbenzo[c]isoxazole $\mathbf{2 n}(0.33 \mathrm{mmol})$ and isolated as a yellow solid $(71.8 \mathrm{mg}, 76 \%)$, m.p 126-128 ${ }^{\circ} \mathrm{C}$.

${ }^{1} \mathbf{H}$ NMR $\left(400 \mathrm{MHz}, \mathrm{CDCl}_{3}\right) \delta 8.19(\mathrm{~m}, 3 \mathrm{H}), 7.88(\mathrm{~d}, J=1.9 \mathrm{~Hz}, 1 \mathrm{H}), 7.84(\mathrm{~s}, 1 \mathrm{H}), 7.67(\mathrm{~m}, 1 \mathrm{H}), 7.61-7.45(\mathrm{~m}, 8 \mathrm{H})$ ppm.

${ }^{13}$ C NMR $\left(101 \mathrm{MHz}, \mathrm{CDCl}_{3}\right) \delta 157.0,148.4,147.2,139.2,137.7,132.2,131.7,130.4,129.6,129.4,128.9,128.8$, $128.7,127.5,126.5,124.5,120.0 \mathrm{ppm}$.

MS (EI) m/z C $21 \mathrm{H}_{14} \mathrm{ClN}: 315.1[\mathrm{M}]^{+}, 280.1,201.1,238.0,139.4,77.1$.

2-Methyl-2-phenylquinoline (3bv) ${ }^{2}$ [cas: 4789-76-8]<smiles>Cc1cc(-c2ccccc2)nc2ccccc12</smiles>

Compound $\mathbf{3 b v}$ was prepared following the general procedure A from phenylacetylene $\mathbf{1 a}(0.30 \mathrm{mmol})$ and 3methylbenzo[c]isoxazole $2 \mathbf{2}(0.33 \mathrm{mmol})$ and isolated as a light yellow oil $(53.9 \mathrm{mg}, 82 \%)$.

${ }^{1} \mathrm{H}$ NMR $\left(400 \mathrm{MHz}, \mathrm{CDCl}_{3}\right) \delta 8.16(\mathrm{~s}, 3 \mathrm{H}), 8.02-7.97(\mathrm{~m}, 1 \mathrm{H}), 7.76-7.70(\mathrm{~m}, 2 \mathrm{H}), 7.54(\mathrm{ddd}, J=11.7,7.1,3.6 \mathrm{~Hz}$, $3 \mathrm{H}), 7.50-7.43(\mathrm{~m}, 1 \mathrm{H}), 2.76(\mathrm{~s}, 3 \mathrm{H}) \mathrm{ppm}$.

${ }^{13} \mathrm{C}$ NMR (100 MHz, $\left.\mathrm{CDCl}_{3}\right) \delta 157.0,148.1,144.7,139.8,130.3,129.3,129.1,128.7,127.5,127.2,126.0,123.6$, $119.7,19.0 \mathrm{ppm}$.

MS (EI) m/z C ${ }_{16} \mathrm{H}_{13} \mathrm{~N}: 219.1[\mathrm{M}]^{+}, 204.1,115.1,75.1$.

13-Methyl-3-(quinolin-2-yl)-6,7,8,9,11,12,13,14,15,16-decahydro-17H-cyclopenta[a]phenanthren-17-one (3bw)<smiles>CC12CCC3c4ccc(-c5ccc6ccccc6n5)cc4CCC3C1CCC2=O</smiles>

Compound 3bw was prepared following the general procedure A from 13-Methyl-3-((trimethylsilyl)ethynyl)6,7,8,9,11,12,13,14,15,16-decahydro-17H-cyclopenta[a]phenanthren-17-one $\mathbf{1 b c}(0.30 \mathrm{mmol})$ and benzo[c]isoxazole 2a $(0.33 \mathrm{mmol})$ and isolated as a white solid $(98.4 \mathrm{mg}, 86 \%)$, m.p $202-204{ }^{\circ} \mathrm{C}$.

${ }^{1} \mathbf{H}$ NMR $\left(400 \mathrm{MHz}, \mathrm{CDCl}_{3}\right) \delta 8.18(\mathrm{dd}, J=12.5,8.6 \mathrm{~Hz}, 2 \mathrm{H}), 7.95(\mathrm{~s}, 1 \mathrm{H}), 7.94-7.89(\mathrm{~m}, 1 \mathrm{H}), 7.86(\mathrm{~d}, J=8.6 \mathrm{~Hz}$, $1 \mathrm{H}), 7.82(\mathrm{~d}, J=7.5 \mathrm{~Hz}, 1 \mathrm{H}), 7.72(\mathrm{ddd}, J=8.4,6.9,1.4 \mathrm{~Hz}, 1 \mathrm{H}), 7.54-7.49(\mathrm{~m}, 1 \mathrm{H}), 7.45(\mathrm{~d}, J=8.1 \mathrm{~Hz}, 1 \mathrm{H}), 3.06$ $(\mathrm{dd}, J=9.3,6.3 \mathrm{~Hz}, 2 \mathrm{H}), 2.59-2.47(\mathrm{~m}, 2 \mathrm{H}), 2.38(\mathrm{td}, J=11.0,3.6 \mathrm{~Hz}, 1 \mathrm{H}), 2.17(\mathrm{dd}, J=18.4,9.2 \mathrm{~Hz}, 1 \mathrm{H}), 2.12-$ $2.05(\mathrm{~m}, 2 \mathrm{H}), 2.03-1.98(\mathrm{~m}, 1 \mathrm{H}), 1.72-1.60(\mathrm{~m}, 3 \mathrm{H}), 1.59-1.50(\mathrm{~m}, 3 \mathrm{H}), 0.94(\mathrm{~s}, 3 \mathrm{H}) \mathrm{ppm}$. 
${ }^{13} \mathrm{C}$ NMR $\left(100 \mathrm{MHz}, \mathrm{CDCl}_{3}\right) \delta 157.2,148.3,141.2,137.1,137.0,136.6,129.6,129.6,128.0,127.4,127.1,126.1$, $125.8,124.8,118.9,50.5,48.0,44.5,38.1,35.8,31.6,29.5,26.5,25.7,21.6,13.8 \mathrm{ppm}$.

HRMS (ESI) $\mathrm{m} / \mathrm{z}$ calcd. for $\mathrm{C}_{27} \mathrm{H}_{27} \mathrm{NO}[\mathrm{M}+\mathrm{H}]^{+} 382.21654$, found 382.21606 .

\section{2-Phenyl-3-(((2,5,7,8-tetramethyl-2-(4,8,12-trimethyltridecyl)chroman-6-yl)oxy)methyl)quinoline (3bx)}<smiles>Cc1c(C)c2c(c(C)c1OCc1cc3ccccc3nc1-c1ccccc1)CCC(C)(CCCC(C)CCCC(C)CCCC(C)C)O2</smiles>

Compound 3bx was prepared following the general procedure A from 2,5,7,8-tetramethyl-6-((3-phenylprop-2-yn-1yl)oxy)-2-(4,8,12-trimethyltridecyl)chromane $\mathbf{1 b d}(0.30 \mathrm{mmol})$ and benzo[c]isoxazole $\mathbf{2 a}(0.33 \mathrm{mmol})$ and isolated as a light yellow oil $(116.5 \mathrm{mg}, 60 \%)$.

${ }^{1} \mathbf{H}$ NMR $\left(400 \mathrm{MHz}, \mathrm{CDCl}_{3}\right) \delta 8.65(\mathrm{~s}, 1 \mathrm{H}), 8.20(\mathrm{~d}, J=8.5 \mathrm{~Hz}, 1 \mathrm{H}), 7.94(\mathrm{~d}, J=8.0 \mathrm{~Hz}, 1 \mathrm{H}), 7.75(\mathrm{t}, J=7.7 \mathrm{~Hz}, 1 \mathrm{H})$, $7.62-7.56(\mathrm{~m}, 3 \mathrm{H}), 7.50-7.42(\mathrm{~m}, 3 \mathrm{H}), 4.85(\mathrm{~s}, 2 \mathrm{H}), 2.56(\mathrm{t}, J=6.7 \mathrm{~Hz}, 2 \mathrm{H}), 2.11(\mathrm{~s}, 3 \mathrm{H}), 2.09(\mathrm{~s}, 3 \mathrm{H}), 2.04(\mathrm{~s}, 3 \mathrm{H})$, $1.84-1.74(\mathrm{~m}, 3 \mathrm{H}), 1.60-1.49(\mathrm{~m}, 3 \mathrm{H}), 1.45-1.36(\mathrm{~m}, 4 \mathrm{H}), 1.30-1.27(\mathrm{~m}, 6 \mathrm{H}), 1.25$ (brs, $4 \mathrm{H}), 1.19-1.08(\mathrm{~m}, 6 \mathrm{H})$, $0.89-0.85(\mathrm{~m}, 12 \mathrm{H}) \mathrm{ppm}$.

${ }^{13}$ C NMR $\left(100 \mathrm{MHz}, \mathrm{CDCl}_{3}\right) \delta 158.7,148.1,147.8,147.1,139.9,136.7,135.3,129.8,129.5,129.3,128.7,128.4$, 128.3 , 127.8, 127.6, 127.4, 126.6, 125.9, 123.0, 117.6, 74.8, 71.5, 40.0, 40.0, 39.3, 37.4, 37.4, 37.4, 37.3, 32.8, 32.8, $32.7,32.6,31.2,31.2,28.0,24.8,24.8,24.4,23.8,22.7,22.6,21.0,20.6,19.7,19.7,12.8,11.9,11.8$ ppm.

HRMS (ESI) $\mathrm{m} / \mathrm{z}$ calcd. for $\mathrm{C}_{45} \mathrm{H}_{62} \mathrm{NO}_{2}[\mathrm{M}+\mathrm{H}]^{+} 648.47751$, found 648.47722 .

(E)-3,7-dimethylocta-2,6-dien-1-yl 2-phenylquinoline-3-carboxylate (3by)<smiles>CC(C)=CCCC(C)=CCOC(=O)c1cc2ccccc2nc1-c1ccccc1</smiles>

Compound 3by was prepared following the general procedure A from (E)-3,7-dimethylocta-2,6-dien-1-yl 3phenylpropiolate 1be $(0.30 \mathrm{mmol})$ and benzo[c]isoxazole $\mathbf{2 a}(0.33 \mathrm{mmol})$ and isolated as a colorless oil $(86.6 \mathrm{mg}$, $75 \%)$.

${ }^{1} \mathbf{H}$ NMR $\left(400 \mathrm{MHz}, \mathrm{CDCl}_{3}\right) \delta 8.63(\mathrm{~s}, 1 \mathrm{H}), 8.18(\mathrm{~d}, J=8.4 \mathrm{~Hz}, 1 \mathrm{H}), 7.91(\mathrm{~d}, J=7.5 \mathrm{~Hz}, 1 \mathrm{H}), 7.81$ (ddd, $J=8.4,6.9$, $1.4 \mathrm{~Hz}, 1 \mathrm{H}), 7.66-7.62(\mathrm{~m}, 2 \mathrm{H}), 7.62-7.57(\mathrm{~m}, 1 \mathrm{H}), 7.49-7.43(\mathrm{~m}, 3 \mathrm{H}), 5.14(\mathrm{td}, J=7.3,1.2 \mathrm{~Hz}, 1 \mathrm{H}), 5.08-5.03$ $(\mathrm{m}, 1 \mathrm{H}), 4.66(\mathrm{~d}, J=7.3 \mathrm{~Hz}, 2 \mathrm{H}), 2.07-2.01(\mathrm{~m}, 4 \mathrm{H}), 1.73(\mathrm{~d}, J=0.9 \mathrm{~Hz}, 3 \mathrm{H}), 1.66(\mathrm{~s}, 3 \mathrm{H}), 1.58(\mathrm{~s}, 3 \mathrm{H}) \mathrm{ppm}$.

${ }^{13}$ C NMR $\left(100 \mathrm{MHz}, \mathrm{CDCl}_{3}\right) \delta 167.9,158.1,148.4,143.0,140.6,138.9,132.2,131.5,129.6,128.7,128.6,128.2$, $127.2,125.8,125.6,123.6,118.4,62.1,32.1,26.6,25.7,23.5,17.7 \mathrm{ppm}$.

HRMS (ESI) $\mathrm{m} / \mathrm{z}$ calcd. for $\mathrm{C}_{26} \mathrm{H}_{28} \mathrm{NO}_{2}[\mathrm{M}+\mathrm{H}]^{+} 386.21146$, found 386.21101 .

(1R,2S,5R)-2-Isopropyl-5-methylcyclohexyl 2-phenylquinoline-3-carboxylat (3bz)<smiles>CC1CC[C@@H](C(C)C)[C@H](OC(=O)c2cc3ccccc3nc2-c2ccccc2)C1</smiles>

Compound 3bz was prepared following the general procedure A from 2-isopropyl-5-methylcyclohexyl 3phenylpropiolate $\mathbf{1 b f}(0.30 \mathrm{mmol})$ and benzo[c]isoxazole $\mathbf{2 a}(0.33 \mathrm{mmol})$ and isolated as a light yellow oil $(70.8 \mathrm{mg}$, $61 \%)$.

${ }^{1} \mathbf{H}$ NMR $\left(400 \mathrm{MHz}, \mathrm{CDCl}_{3}\right) \delta 8.59(\mathrm{~s}, 1 \mathrm{H}), 8.18(\mathrm{~d}, J=8.5 \mathrm{~Hz}, 1 \mathrm{H}), 7.93(\mathrm{~d}, J=8.0 \mathrm{~Hz}, 1 \mathrm{H}), 7.81$ (ddd, $J=8.4,7.0$, $1.2 \mathrm{~Hz}, 1 \mathrm{H}), 7.61(\mathrm{dt}, J=8.8,5.2 \mathrm{~Hz}, 3 \mathrm{H}), 7.49-7.42(\mathrm{~m}, 3 \mathrm{H}), 4.80(\mathrm{td}, J=10.9,4.4 \mathrm{~Hz}, 1 \mathrm{H}), 1.99(\mathrm{dd}, J=7.0,4.6$ $\mathrm{Hz}, 1 \mathrm{H}), 1.72-1.55(\mathrm{~m}, 4 \mathrm{H}), 1.45(\mathrm{ddd}, J=15.2,7.6,4.3 \mathrm{~Hz}, 1 \mathrm{H}), 1.30-1.20(\mathrm{~m}, 2 \mathrm{H}), 1.03(\mathrm{dd}, J=12.9,3.2 \mathrm{~Hz}$, $1 \mathrm{H}), 0.89(\mathrm{~d}, J=6.5 \mathrm{~Hz}, 3 \mathrm{H}), 0.81(\mathrm{~d}, J=7.0 \mathrm{~Hz}, 3 \mathrm{H}), 0.73(\mathrm{~d}, J=6.9 \mathrm{~Hz}, 3 \mathrm{H}) \mathrm{ppm}$. 
${ }^{13} \mathrm{C}$ NMR $\left(100 \mathrm{MHz}, \mathrm{CDCl}_{3}\right) \delta 167.4,158.1,148.2,140.8,138.5,131.4,129.5,128.6,128.5,128.2,128.1,127.1$, $126.2,125.8,75.8,46.8,40.3,34.1,31.3,25.9,23.1,22.0,20.8,16.1 \mathrm{ppm}$.

HRMS (ESI) $\mathrm{m} / \mathrm{z}$ calcd. for $\mathrm{C}_{26} \mathrm{H}_{30} \mathrm{NO}_{2}[\mathrm{M}+\mathrm{H}]^{+} 388.22711$, found 388.22661 .

10,13-Dimethyl-17-(6-methylheptan-2-yl)-2,3,4,7,8,9,10,11,12,13,14,15,16,17-tetradecahydro-1Hcyclopenta[a]phenanthren-3-yl 2-phenylquinoline-3-carboxylate (3ca)<smiles>CC(C)CCCC(C)C1CCC2C3CC=C4CC(OC(=O)c5cc6ccccc6nc5-c5ccccc5)CCC4(C)C3CCC12C</smiles>

Compound 3ca was prepared following the general procedure A from Cholesterol phenylpropiolate $1 \mathbf{b g}(0.30 \mathrm{mmol})$ and benzo[c]isoxazole $2 \mathbf{a}(0.33 \mathrm{mmol})$ and isolated as a white solid $(98.0 \mathrm{mg}, 53 \%)$, m.p $85-86^{\circ} \mathrm{C}$.

${ }^{1} \mathbf{H}$ NMR $\left(400 \mathrm{MHz}, \mathrm{CDCl}_{3}\right) \delta 8.64(\mathrm{~s}, 1 \mathrm{H}), 8.18(\mathrm{~d}, J=8.5 \mathrm{~Hz}, 1 \mathrm{H}), 7.92(\mathrm{~d}, J=8.1 \mathrm{~Hz}, 1 \mathrm{H}), 7.83-7.77(\mathrm{~m}, 1 \mathrm{H})$, $7.66-7.60(\mathrm{~m}, 2 \mathrm{H}), 7.59(\mathrm{t}, J=7.5 \mathrm{~Hz}, 1 \mathrm{H}), 7.47(\mathrm{q}, J=5.4 \mathrm{~Hz}, 3 \mathrm{H}), 5.38-5.29(\mathrm{~m}, 1 \mathrm{H}), 4.76-4.61(\mathrm{~m}, 1 \mathrm{H}), 2.21-$ $2.14(\mathrm{~m}, 1 \mathrm{H}), 2.09-1.93(\mathrm{~m}, 3 \mathrm{H}), 1.87-1.78(\mathrm{~m}, 2 \mathrm{H}), 1.72(\mathrm{~d}, J=12.2 \mathrm{~Hz}, 1 \mathrm{H}), 1.59-1.25(\mathrm{~m}, 12 \mathrm{H}), 1.18-1.00$ $(\mathrm{m}, 8 \mathrm{H}), 0.92(\mathrm{~d}, J=8.4 \mathrm{~Hz}, 7 \mathrm{H}), 0.88(\mathrm{~d}, J=1.6 \mathrm{~Hz}, 3 \mathrm{H}), 0.86(\mathrm{~d}, J=1.6 \mathrm{~Hz}, 3 \mathrm{H}), 0.67(\mathrm{~s}, 3 \mathrm{H}) \mathrm{ppm}$.

${ }^{13}$ C NMR $\left(100 \mathrm{MHz}, \mathrm{CDCl}_{3}\right) \delta 167.3,158.1,148.2,140.9,139.4,138.9,131.4,129.5,128.6,128.4,128.1,127.1$, $125.9,125.8,122.7,75.4,56.6,56.1,49.9,42.2,39.6,39.4,37.4,36.8,36.5,36.1,35.7,31.8,31.8,28.2$, 27.9, 27.1, $24.2,23.8,22.8,22.5,20.9,19.1,18.7,11.8 \mathrm{ppm}$.

HRMS (ESI) $\mathrm{m} / \mathrm{z}$ calcd. for $\mathrm{C}_{43} \mathrm{H}_{56} \mathrm{NO}_{2}[\mathrm{M}+\mathrm{H}]^{+}$618.43056, found 618.43009 .

4-(Quinolin-2-yl)phenyl 2-(4-isobutylphenyl)propanoate (3cb)<smiles>CC(C)Cc1ccc(C(C)C(=O)Oc2ccc(-c3ccc4ccccc4n3)cc2)cc1</smiles>

Compound 3cb was prepared following the general procedure A from 4-((trimethylsilyl)ethynyl)phenyl 2-(4isobutylphenyl)propanoate $\mathbf{1 b h}(0.30 \mathrm{mmol})$ and benzo[c]isoxazole $\mathbf{2 a}(0.33 \mathrm{mmol})$ and isolated as a yellow solid (104.3 mg, 85\%), m.p $82-83^{\circ} \mathrm{C}$.

${ }^{1} \mathbf{H}$ NMR $\left(400 \mathrm{MHz}, \mathrm{CDCl}_{3}\right) \delta 8.21(\mathrm{~d}, J=8.6 \mathrm{~Hz}, 1 \mathrm{H}), 8.16(\mathrm{~d}, J=8.6 \mathrm{~Hz}, 3 \mathrm{H}), 7.83(\mathrm{~m}, 2 \mathrm{H}), 7.73(\mathrm{t}, J=7.7 \mathrm{~Hz}, 1 \mathrm{H})$, $7.52(\mathrm{t}, J=7.5 \mathrm{~Hz}, 1 \mathrm{H}), 7.34(\mathrm{~d}, J=8.0 \mathrm{~Hz}, 2 \mathrm{H}), 7.17(\mathrm{~d}, J=8.3 \mathrm{~Hz}, 4 \mathrm{H}), 3.98(\mathrm{q}, J=7.1 \mathrm{~Hz}, 1 \mathrm{H}), 2.49(\mathrm{~d}, J=7.2 \mathrm{~Hz}$, $2 \mathrm{H}), 1.89(\mathrm{~m}, 1 \mathrm{H}), 1.64(\mathrm{~d}, J=7.1 \mathrm{~Hz}, 4 \mathrm{H}), 0.93(\mathrm{~d}, J=6.6 \mathrm{~Hz}, 6 \mathrm{H}) \mathrm{ppm}$.

${ }^{13} \mathbf{C}$ NMR $\left(100 \mathrm{MHz}, \mathrm{CDCl}_{3}\right) \delta 173.1,156.4,151.9,148.2,140.8,137.2,137.1,136.8,129.7,129.6,129.5,128.6$, $127.4,127.2,127.1,126.3,121.8,118.7,45.3,45.0,30.2,22.4,18.6 \mathrm{ppm}$.

HRMS (ESI) $\mathrm{m} / \mathrm{z}$ calcd. for $\mathrm{C}_{28} \mathrm{H}_{28} \mathrm{NO}_{2}[\mathrm{M}+\mathrm{H}]^{+} 410.21146$, found 410.21100 .

(2,2,7,7-tetra-Methyl-tetra-hydro-5H-bis([1,3]dioxolo)[4,5-b:4',5'-d]pyran-5-yl)methyl 4-(3-butylquinolin-2yl)benzoate (3cc)<smiles>CCCCc1cc2ccccc2nc1-c1ccc(C(=O)OCC2OC3OC(C)(C)OC3C3OC(C)(C)OC23)cc1</smiles> 
Compound 3cc was prepared following the general procedure A from $(2,2,7,7$-tetra-methyltetrahydro- $5 \mathrm{H}$ bis([1,3]dioxolo)[4,5-b:4',5'-d]pyran-5-yl)methyl 4-(hex-1-yn-1-yl)benzoate $\mathbf{1 b i}(0.30 \mathrm{mmol})$ and benzo[c]isoxazole $\mathbf{2 a}$ $(0.33 \mathrm{mmol})$ and isolated as a viscous oil $(142.8 \mathrm{mg}, 87 \%)$.

${ }^{1}$ H NMR $\left(400 \mathrm{MHz}, \mathrm{CDCl}_{3}\right) \delta 8.16(\mathrm{~d}, J=8.3 \mathrm{~Hz}, 2 \mathrm{H}), 8.09(\mathrm{~d}, J=8.4 \mathrm{~Hz}, 1 \mathrm{H}), 8.02(\mathrm{~s}, 1 \mathrm{H}), 7.77(\mathrm{~d}, J=8.0 \mathrm{~Hz}, 1 \mathrm{H})$, $7.66-7.58(\mathrm{~m}, 3 \mathrm{H}), 7.52-7.47(\mathrm{~m}, 1 \mathrm{H}), 5.57(\mathrm{~d}, J=4.9 \mathrm{~Hz}, 1 \mathrm{H}), 4.65(\mathrm{~m}, 1 \mathrm{H}), 4.57(\mathrm{~m}, 1 \mathrm{H}), 4.45(\mathrm{~m}, 1 \mathrm{H}), 4.35-$ $4.31(\mathrm{~m}, 2 \mathrm{H}), 4.24-4.19(\mathrm{~m}, 1 \mathrm{H}), 2.78-2.67(\mathrm{~m}, 2 \mathrm{H}), 1.51(\mathrm{~s}, 3 \mathrm{H}), 1.49-1.43(\mathrm{~m}, 5 \mathrm{H}), 1.34(\mathrm{~s}, 3 \mathrm{H}), 1.31(\mathrm{~s}, 3 \mathrm{H})$, $1.23(\mathrm{~m}, 2 \mathrm{H}), 0.79(\mathrm{t}, J=7.3 \mathrm{~Hz}, 3 \mathrm{H})$.

${ }^{13}$ C NMR $\left(100 \mathrm{MHz}, \mathrm{CDCl}_{3}\right) \delta 166.1,159.4,146.2,145.4,135.8,133.6,129.6,129.5,129.1,128.9,128.8,127.6$, 126.8, 126.6, 109.6, 108.7, 96.2, 71.0, 70.6, 70.4, 66.1, 63.9, 32.6, 32.3, 25.9, 25.8, 24.8, 24.4, 22.2, 13.6.

HRMS (ESI) $\mathrm{m} / \mathrm{z}$ calcd. for $\mathrm{C}_{32} \mathrm{H}_{38} \mathrm{NO}_{7}[\mathrm{M}+\mathrm{H}]^{+} 548.26428$, found 548.26410.

\section{2-(Quinolin-2-yl)-9H-fluoren-9-one (3cd)}<smiles>O=C1c2ccccc2-c2ccc(-c3ccc4ccccc4n3)cc21</smiles>

Yellow solid (0.92 g, 75\%), m.p $165-167^{\circ} \mathrm{C}$.

${ }^{1} \mathbf{H}$ NMR $\left(400 \mathrm{MHz}, \mathrm{CDCl}_{3}\right) \delta 8.41(\mathrm{~d}, J=1.2 \mathrm{~Hz}, 1 \mathrm{H}), 8.37(\mathrm{~m}, 1 \mathrm{H}), 8.18(\mathrm{~d}, J=8.6 \mathrm{~Hz}, 1 \mathrm{H}), 8.15(\mathrm{~d}, J=8.5 \mathrm{~Hz}$, $1 \mathrm{H}), 7.84(\mathrm{~d}, J=8.6 \mathrm{~Hz}, 1 \mathrm{H}), 7.79(\mathrm{~d}, J=8.1 \mathrm{~Hz}, 1 \mathrm{H}), 7.75-7.69(\mathrm{~m}, 1 \mathrm{H}), 7.67(\mathrm{~d}, J=7.3 \mathrm{~Hz}, 1 \mathrm{H}), 7.61(\mathrm{~d}, J=7.8$ $\mathrm{Hz}, 1 \mathrm{H}), 7.54-7.45(\mathrm{~m}, 3 \mathrm{H}), 7.30(\mathrm{t}, J=7.3 \mathrm{~Hz}, 1 \mathrm{H}) \mathrm{ppm}$.

${ }^{13}$ C NMR $\left(100 \mathrm{MHz}, \mathrm{CDCl}_{3}\right) \delta 193.5,155.5,148.1,145.0,144.0,140.5,136.9,134.7,134.7,134.6,133.9,129.8$, $129.7,129.2,127.4,127.3,126.5,124.4,123.2,120.7,120.6,118.3 \mathrm{ppm}$.

HRMS (ESI) $\mathrm{m} / \mathrm{z}$ calcd. for $\mathrm{C}_{22} \mathrm{H}_{14} \mathrm{NO}[\mathrm{M}+\mathrm{H}]^{+} 308.10699$, found 308.10647 .

\section{1-(2-(Quinolin-2-yl)phenyl)ethan-1-one (3ce) [cas: 1125420-46-3]}<smiles>CC(=O)c1ccccc1-c1ccc2ccccc2n1</smiles>

Compound 3cc was prepared following the general procedure A from 1-(2-ethynylphenyl)ethan-1-one (0.30 mmol) and benzo[c]isoxazole $(0.33 \mathrm{mmol})$ and isolated as a yellow oil $(38.5 \mathrm{mg}, 52 \%)$.

${ }^{1} \mathbf{H}$ NMR $\left(400 \mathrm{MHz}, \mathrm{CDCl}_{3}\right) \delta 8.24-8.16(\mathrm{~m}, 1 \mathrm{H}), 8.12(\mathrm{~d}, J=8.4 \mathrm{~Hz}, 1 \mathrm{H}), 7.87(\mathrm{~s}, 1 \mathrm{H}), 7.78-7.73(\mathrm{~m}, 1 \mathrm{H}), 7.71(\mathrm{~d}$, $J=1.7 \mathrm{~Hz}, 1 \mathrm{H}), 7.64-7.55(\mathrm{~m}, 2 \mathrm{H}), 6.93(\mathrm{~d}, J=8.1 \mathrm{~Hz}, 1 \mathrm{H}), 6.04(\mathrm{~s}, 2 \mathrm{H}) \mathrm{ppm}$.

${ }^{13} \mathrm{C}$ NMR $\left(100 \mathrm{MHz}, \mathrm{CDCl}_{3}\right) \delta 203.8,156.5,149.2,148.9,148.4,143.0,132.9,130.5,129.8,126.9,125.1,123.9$, $121.8,118.6,108.5,107.8,101.4 \mathrm{ppm}$.

HRMS (ESI) $\mathrm{m} / \mathrm{z}$ calcd. for $\mathrm{C}_{17} \mathrm{H}_{14} \mathrm{NO}[\mathrm{M}+\mathrm{H}]^{+} 248.10699$, found 248.10699 .

\section{2-(Benzo[d][1,3]dioxol-5-yl)-4-chloroquinoline (3cf) ${ }^{3}$ [cas: 97440-40-9]}<smiles>Clc1cc(-c2ccc3c(c2)OCO3)nc2ccccc12</smiles>

White solid (189 mg, 67\%), m.p $112-113^{\circ} \mathrm{C}$.

${ }^{1} \mathbf{H}$ NMR $\left(400 \mathrm{MHz}, \mathrm{CDCl}_{3}\right) \delta 8.19(\mathrm{~d}, J=8.3 \mathrm{~Hz}, 1 \mathrm{H}), 8.13(\mathrm{~d}, J=8.4 \mathrm{~Hz}, 1 \mathrm{H}), 7.88(\mathrm{~s}, 1 \mathrm{H}), 7.80-7.70(\mathrm{~m}, 2 \mathrm{H})$, $7.65-7.55(\mathrm{~m}, 2 \mathrm{H}), 6.94(\mathrm{~d}, J=8.1 \mathrm{~Hz}, 1 \mathrm{H}), 6.05(\mathrm{~s}, 2 \mathrm{H}) \mathrm{ppm}$.

${ }^{13} \mathrm{C}$ NMR $\left(100 \mathrm{MHz}, \mathrm{CDCl}_{3}\right) \delta 156.5,149.2,148.9,148.5,143.0,133.0,130.5,129.8,126.9,125.1,123.9,121.8$, $118.6,108.5,107.8,101.5 \mathrm{ppm}$.

MS (EI) m/z C ${ }_{16} \mathrm{H}_{10} \mathrm{ClNO}_{2}: 283.0[\mathrm{M}]^{+}, 253.0,248.1,115.1,75.1$. 
<smiles>COc1cc(-c2ccc3c(c2)OCO3)nc2ccccc12</smiles>

White solid (33.5 mg, 86\%), m.p 104-106 ${ }^{\circ} \mathrm{C}$.

${ }^{1} \mathbf{H}$ NMR $\left(400 \mathrm{MHz}, \mathrm{CDCl}_{3}\right) \delta 8.16(\mathrm{~d}, J=8.2 \mathrm{~Hz}, 1 \mathrm{H}), 8.06(\mathrm{~d}, J=8.4 \mathrm{~Hz}, 1 \mathrm{H}), 7.72-7.65(\mathrm{~m}, 2 \mathrm{H}), 7.61(\mathrm{~m}, 1 \mathrm{H})$, $7.46(\mathrm{t}, J=7.6 \mathrm{~Hz}, 1 \mathrm{H}), 7.08(\mathrm{~s}, 1 \mathrm{H}), 6.94(\mathrm{~d}, J=8.1 \mathrm{~Hz}, 1 \mathrm{H}), 6.03(\mathrm{~s}, 2 \mathrm{H}), 4.09(\mathrm{~s}, 3 \mathrm{H}) \mathrm{ppm}$.

${ }^{13} \mathrm{C}$ NMR $\left(100 \mathrm{MHz}, \mathrm{CDCl}_{3}\right) \delta 162.7,158.1,149.0,148.7,148.2,134.8,129.9,129.0,125.1,121.6,121.6,120.2$, 108.3, 108.0, 101.3, 97.5, 55.6 ppm.

MS (EI) m/z C ${ }_{17} \mathrm{H}_{13} \mathrm{NO}_{3}: 279.1[\mathrm{M}]^{+}, 249.0,191.0,178.0,151.0,75.0$.

4-Chloro-2-(naphthalen-2-yl)quinoline (3ch) ${ }^{4}$ [cas: 194919-90-9]<smiles>Clc1cc(-c2ccc3ccccc3c2)nc2ccccc12</smiles>

White solid (188 mg, 65\%), m.p 109-110 ${ }^{\circ} \mathrm{C}$.

${ }^{1} \mathbf{H}$ NMR $\left(400 \mathrm{MHz}, \mathrm{CDCl}_{3}\right) \delta 8.60(\mathrm{~s}, 1 \mathrm{H}), 8.34(\mathrm{dd}, J=8.6,1.7 \mathrm{~Hz}, 1 \mathrm{H}), 8.24(\mathrm{t}, J=7.6 \mathrm{~Hz}, 2 \mathrm{H}), 8.13(\mathrm{~s}, 1 \mathrm{H}), 8.03$ $-7.97(\mathrm{~m}, 2 \mathrm{H}), 7.90(\mathrm{dq}, J=6.7,3.4 \mathrm{~Hz}, 1 \mathrm{H}), 7.83-7.77(\mathrm{~m}, 1 \mathrm{H}), 7.64(\mathrm{t}, J=7.6 \mathrm{~Hz}, 1 \mathrm{H}), 7.57-7.52(\mathrm{~m}, 2 \mathrm{H}) \mathrm{ppm}$. ${ }^{13}$ C NMR $\left(100 \mathrm{MHz}, \mathrm{CDCl}_{3}\right) \delta 157.1,149.1,143.2,135.8,134.0,133.4,130.6,130.0,128.9,128.7,127.7,127.3$, $127.0,126.5,125.3,124.7,124.0,119.2 \mathrm{ppm}$.

MS (EI) m/z C ${ }_{19} \mathrm{H}_{12} \mathrm{ClN}: 289.1[\mathrm{M}]^{+}, 254.1,162.1,127.0,75.0$.

$N^{I}, N^{1}$-dimethyl- $N^{2}$-(2-(naphthalen-2-yl)quinolin-4-yl)ethane-1,2-diamine (3ci) ${ }^{4}$ [cas: 150314-42-4]<smiles>CN(C)CCNc1cc(-c2ccc3ccccc3c2)nc2ccccc12</smiles>

Yellow solid (60.7 mg, 89\%), m.p 146-148 ${ }^{\circ} \mathrm{C}$.

${ }^{1} \mathbf{H}$ NMR $\left(400 \mathrm{MHz}, \mathrm{CDCl}_{3}\right) \delta 8.57(\mathrm{~s}, 1 \mathrm{H}), 8.30(\mathrm{~d}, J=8.6 \mathrm{~Hz}, 1 \mathrm{H}), 8.14(\mathrm{~d}, J=8.4 \mathrm{~Hz}, 1 \mathrm{H}), 7.98(\mathrm{~m}, 2 \mathrm{H}), 7.92-$ $7.86(\mathrm{~m}, 1 \mathrm{H}), 7.84(\mathrm{~d}, J=8.2 \mathrm{~Hz}, 1 \mathrm{H}), 7.71-7.63(\mathrm{t}, 1 \mathrm{H}), 7.57-7.48(\mathrm{~m}, 2 \mathrm{H}), 7.43(\mathrm{t}, J=7.4 \mathrm{~Hz}, 1 \mathrm{H}), 6.98(\mathrm{~s}, 1 \mathrm{H})$, $5.98(\mathrm{~s}, 1 \mathrm{H}), 3.41(\mathrm{~m}, 2 \mathrm{H}), 2.71(\mathrm{t}, J=5.8 \mathrm{~Hz}, 2 \mathrm{H}), 2.32(\mathrm{~s}, 6 \mathrm{H}) \mathrm{ppm}$.

${ }^{13} \mathrm{C}$ NMR $\left(100 \mathrm{MHz}, \mathrm{CDCl}_{3}\right) \delta 158.1,150.4,148.6,138.2,133.6,133.4,130.0,129.2,128.7,128.1,127.6,126.8$, $126.3,126.0,125.3,124.3,119.7,118.1,96.9,57.0,45.0,40.0 \mathrm{ppm}$.

MS (EI) $\mathrm{m} / \mathrm{z} \mathrm{C}_{23} \mathrm{H}_{23} \mathrm{~N}_{3}: 341.2[\mathrm{M}]^{+}, 297.1,269.1,127.0,75.1$.

\section{3-((1-(4-(tert-Butyl)phenyl)ethyl)amino)benzaldehyde (8a)}<smiles>CC(Nc1ccccc1C=O)c1ccc(C(C)(C)C)cc1</smiles>

Compound 8a was prepared following the general procedure A from 1-(tert-butyl)-4-vinylbenzene 7 (0.30 mmol) and benzo[c]isoxazole $2 \mathbf{a}(0.33 \mathrm{mmol})$ and isolated as a light yellow oil $(69.1 \mathrm{mg}, 82 \%)$.

${ }^{1} \mathbf{H}$ NMR $\left(400 \mathrm{MHz}, \mathrm{CDCl}_{3}\right) \delta 9.86(\mathrm{~s}, 1 \mathrm{H}), 8.72(\mathrm{~d}, J=5.6 \mathrm{~Hz}, 1 \mathrm{H}), 7.45(\mathrm{dd}, J=7.7,1.5 \mathrm{~Hz}, 1 \mathrm{H}), 7.34-7.30(\mathrm{~m}$, $2 \mathrm{H}), 7.26-7.20(\mathrm{~m}, 3 \mathrm{H}), 6.67-6.60(\mathrm{~m}, 1 \mathrm{H}), 6.48(\mathrm{t}, J=8.0 \mathrm{~Hz}, 1 \mathrm{H}), 4.60(\mathrm{~m}, 1 \mathrm{H}), 1.58(\mathrm{~d}, J=6.8 \mathrm{~Hz}, 3 \mathrm{H}), 1.29(\mathrm{~s}$, 7H) $\mathrm{ppm}$. 
${ }^{13}$ C NMR $\left(100 \mathrm{MHz}, \mathrm{CDCl}_{3}\right) \delta 194.1,149.8,149.8,141.1,136.6,135.6,125.6,125.3,118.4,114.9,112.2,52.1,34.4$, 31.3, $24.7 \mathrm{ppm}$.

HRMS (ESI) m/z calcd. for $\mathrm{C}_{19} \mathrm{H}_{24} \mathrm{NO}[\mathrm{M}+\mathrm{H}]^{+} 282.18524$, found 282.18529 .

\section{Preparation of Substrates}

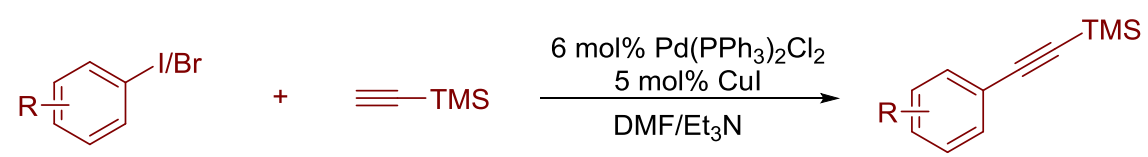

General procedure B for the synthesis of trimethyl(arylethynyl)silane: To a solution of Aryl iodo/bromide( 2.4 mmol) in triethylamine $(2 \mathrm{~mL})$ and DMF $(5 \mathrm{~mL})$ were added ethynyltrimethylsilane $(282.4 \mathrm{mg}, 2.88 \mathrm{mmol})$ and $\mathrm{Pd}\left(\mathrm{PPh}_{3}\right)_{2} \mathrm{Cl}_{2}(6 \mathrm{~mol} \%)$ and $\mathrm{CuI}(5 \mathrm{~mol} \%)$ at room temperature. Then the mixture was stirred at $50{ }^{\circ} \mathrm{C}$ (or $80{ }^{\circ} \mathrm{C}$ for aryl bromdie) for $12 \mathrm{~h}$ under $\mathrm{N}_{2}$. After the starting material was consumed, the reaction mixture was quenched saturated $\mathrm{NH}_{4} \mathrm{Cl}$ solution and extracted with ethyl acetate. The combined organic extracts were washed with water and brine, and dried over anhydrous $\mathrm{Na}_{2} \mathrm{SO}_{4}$ and concentrated in vacuo. The crude product was purified by flash column chromatography on silica gel to give the corresponding alkynes.

Trimethyl((4-(methylthio)phenyl)ethynyl)silane $(1 \mathrm{~g})^{23}$ [cas: 134317-21-8]<smiles>CSCC#Cc1ccc(SC)cc1</smiles>

Prepared following general procedure B from (4-bromophenyl)(methyl)sulfane and ethynyltrimethylsilane. The product was purified by column chromatography. ${ }^{1} \mathbf{H}$ NMR $\left(400 \mathrm{MHz}, \mathrm{CDCl}_{3}\right) \delta 7.38-7.34(\mathrm{~m}, 2 \mathrm{H}), 7.14(\mathrm{~m}, 2 \mathrm{H})$, $2.46(\mathrm{~s}, 3 \mathrm{H}), 0.24(\mathrm{~s}, 9 \mathrm{H})$. Spectral data matched those reported in the literature. ${ }^{23}$

$N, N$-dimethyl-4-((trimethylsilyl)ethynyl)aniline (1h) ${ }^{23}$ [cas: 40230-97-5]<smiles>CN(C)CC#Cc1ccc(N(C)C)cc1</smiles>

Prepared following general procedure B from 4-bromo- $N, N$-dimethylaniline and ethynyltrimethylsilane. The product was purified by column chromatography. ${ }^{1} \mathbf{H}$ NMR $\left(400 \mathrm{MHz}, \mathrm{CDCl}_{3}\right) \delta 7.35(\mathrm{~m}, J=17.9,8.9 \mathrm{~Hz}, 2 \mathrm{H}), 6.62(\mathrm{~m}, J=$ $9.1,2.8 \mathrm{~Hz}, 2 \mathrm{H}), 2.99(\mathrm{t}, J=15.6 \mathrm{~Hz}, 6 \mathrm{H}), 0.30-0.25(\mathrm{~m}, 9 \mathrm{H})$. Spectral data matched those reported in the literature. ${ }^{23}$

Trimethyl((4-(methylsulfonyl)phenyl)ethynyl)silane (1m) ${ }^{24}$ [cas: 113854-19-6]<smiles>CC#Cc1ccc(S(=O)(=O)O)cc1</smiles>

Prepared following general procedure B from 1-bromo-4-(methylsulfonyl)benzene and ethynyltrimethylsilane. The product was purified by column chromatography. ${ }^{1} \mathbf{H}$ NMR $\left(400 \mathrm{MHz}, \mathrm{CDCl}_{3}\right) \delta 7.89-7.85(\mathrm{~m}, 2 \mathrm{H}), 7.64-7.60(\mathrm{~m}$, $2 \mathrm{H}), 3.04(\mathrm{~s}, 3 \mathrm{H}), 0.26(\mathrm{~m}, 9 \mathrm{H})$. Spectral data matched those reported in the literature. ${ }^{24}$

(Benzo[b]thiophen-3-ylethynyl)trimethylsilane (1q) ${ }^{25}$ [cas: 866863-45-8]<smiles>CC#Cc1csc2ccccc12</smiles>

Prepared following general procedure B from 3-bromobenzo[b]thiophene and ethynyltrimethylsilane. The product was purified by column chromatography. ${ }^{1} \mathbf{H}$ NMR $\left(400 \mathrm{MHz}, \mathrm{CDCl}_{3}\right) \delta 7.97(\mathrm{~d}, J=8.0 \mathrm{~Hz}, 1 \mathrm{H}), 7.85(\mathrm{~d}, J=8.0 \mathrm{~Hz}, 1 \mathrm{H})$, $7.66(\mathrm{~s}, 1 \mathrm{H}), 7.49-7.46(\mathrm{~m}, 1 \mathrm{H}), 7.42-7.38(\mathrm{~m}, 1 \mathrm{H}), 0.34(\mathrm{~s}, 9 \mathrm{H})$. Spectral data matched those reported in the literature. ${ }^{25}$ 
(1)

Prepared following general procedure B from 3-bromoimidazo[1,2-a]pyridine and ethynyltrimethylsilane. The product was purified by column chromatography. ${ }^{1} \mathbf{H}$ NMR $\left(400 \mathrm{MHz}, \mathrm{CDCl}_{3}\right) \delta 8.26(\mathrm{~d}, J=6.8 \mathrm{~Hz}, 1 \mathrm{H}), 7.85(\mathrm{~s}, 1 \mathrm{H}), 7.69-$ $7.61(\mathrm{~m}, 2 \mathrm{H}), 7.28(\mathrm{~s}, 1 \mathrm{H}), 0.32-0.29(\mathrm{~s}, 9 \mathrm{H})$. Spectral data matched those reported in the literature. ${ }^{26}$

\section{3,5-Dimethyl-4-((trimethylsilyl)ethynyl)isoxazole (1s) ${ }^{26}$ [cas: 1201657-82-0]}<smiles>CC#Cc1c(C)noc1C</smiles>

Prepared following general procedure B from 4-bromo-3,5-dimethylisoxazole and ethynyltrimethylsilane. The product was purified by column chromatography. ${ }^{1} \mathrm{H}$ NMR $\left(400 \mathrm{MHz}, \mathrm{CDCl}_{3}\right) \delta 2.45(\mathrm{~s}, 3 \mathrm{H}), 2.28(\mathrm{~s}, 3 \mathrm{H}), 0.25(\mathrm{~s}, 3 \mathrm{H})$. Spectral data matched those reported in the literature. ${ }^{26}$

\section{Diarylacetylene $^{27}$}

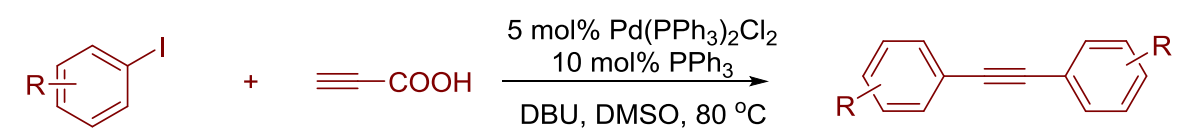

$\mathrm{Pd}\left(\mathrm{PPh}_{3}\right)_{2} \mathrm{Cl}_{2}$ (35.0 mg, $\left.0.05 \mathrm{mmol}\right), \mathrm{PPh}_{3}(26.2 \mathrm{mg}, 0.1 \mathrm{mmol})$, aryl bromide $(2.0 \mathrm{mmol})$, propiolic acid $(62 \mu \mathrm{L}, 1.0$ $\mathrm{mmol})$ and $\mathrm{DBU}(0.3 \mathrm{~mL}, 2 \mathrm{mmol})$ were added successively into a $50 \mathrm{~mL}$ round bottom flask under $\mathrm{N}_{2}$ atmosphere. Then DMSO $(15 \mathrm{~mL})$ was added and the flask was sealed with a septum. The resulting mixture was placed in an oil bath at $80{ }^{\circ} \mathrm{C}$ and allowed to stir for 3 hours. The reaction mixture was cooled to room temperature before poured into saturated aqueous $\mathrm{NH}_{4} \mathrm{Cl}(25 \mathrm{~mL})$ and extracted with diethyl ether $(20 \mathrm{~mL} \times 4)$. The combined organic layer was washed with brine $(90 \mathrm{~mL})$ and dried over $\mathrm{Na}_{2} \mathrm{SO}_{4}$. The solvent was removed under reduced pressure and the residue was purified by flash chromatography on silica gel using hexanes/ethyl acetate as the eluent.

\section{Cyclododecyne (1an) ${ }^{28}$ [cas: 1129-90-4]}

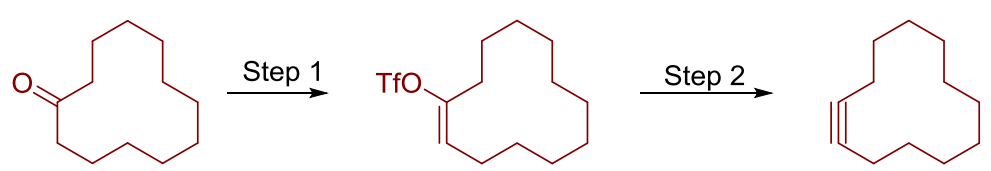

Step 1: To a stirred solution of cyclodocecanone $(1.0 \mathrm{~g}, 5.5 \mathrm{mmol})$ and $\operatorname{PhNTf}_{2}(2.15 \mathrm{~g}, 6.0 \mathrm{mmol})$ in THF $(20 \mathrm{~mL})$ was added $\mathrm{KN}(\mathrm{TMS})_{2}(6.5 \mathrm{~mL}, 1 \mathrm{M}, 6.5 \mathrm{mmol})$ at $-78^{\circ} \mathrm{C}$. After stirring at this temperature for 1 hour, the reaction was quenched with saturated aqueous $\mathrm{NH}_{4} \mathrm{Cl}$ and extracted with ethyl acetate. The enol triflate was purified by column chromatography (hexanes/EA = 200/1).

Step 2: The enol triflate $(0.94 \mathrm{~g}, 3.0 \mathrm{mmol})$ was dissolved in $10 \mathrm{~mL}$ of DMF and treated with $\mathrm{LiCl}(508 \mathrm{mg}, 12.0$ $\mathrm{mmol}$ ) for 20 minutes. Then the reaction mixture was directly subjected to column chromatography (hexanes) to give cyclododecyne as colorless liquid $(350 \mathrm{mg})$.

${ }^{1} \mathbf{H}$ NMR $\left(400 \mathrm{MHz}, \mathrm{CDCl}_{3}\right) \delta 2.15-2.06(\mathrm{~m}, 4 \mathrm{H}), 1.55-1.26(\mathrm{~m}, 16 \mathrm{H})$. Spectral data matched those reported in the literature. ${ }^{28}$

Oct-1-yn-1-ylbenzene (1ap) ${ }^{27}$ [cas: 1129-65-3]

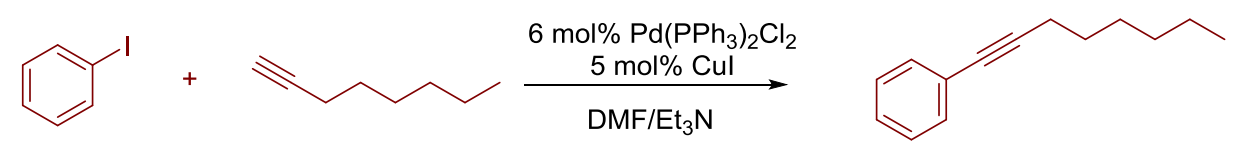

To a solution of iodobenzene $(5.0 \mathrm{mmol})$ and 1-octyne $(6.0 \mathrm{mmol})$ in $\mathrm{Et}_{3} \mathrm{~N}(25 \mathrm{~mL})$ was added $\mathrm{PdCl}_{2}\left(\mathrm{PPh}_{3}\right)_{2}(70.0 \mathrm{mg}$, $0.1 \mathrm{mmol})$. After $5 \mathrm{~min}$, CuI $(38.0 \mathrm{mg}, 0.2 \mathrm{mmol})$ was added to the reaction mixture. The resulting mixture was warmed to $60{ }^{\circ} \mathrm{C}$ under $\mathrm{N}_{2}$ atmosphere for $22 \mathrm{~h}$ when full conversion of the starting material was determined by GCMS. The solution was allowed to cool to room temperature and filtered through a short celite pad. After removal of solvent under reduced pressure, the product was obtained through flash chromatography as yellow oil $(0.80 \mathrm{~g}, 86 \%)$. ${ }^{1} \mathbf{H}$ NMR $\left(400 \mathrm{MHz}, \mathrm{CDCl}_{3}\right) \delta 7.46-7.37(\mathrm{~m}, 2 \mathrm{H}), 7.32-7.26(\mathrm{~m}, 3 \mathrm{H}), 2.41(\mathrm{t}, J=7.1 \mathrm{~Hz}, 2 \mathrm{H}), 1.71-1.56(\mathrm{~m}, 2 \mathrm{H})$, $1.52-1.41(\mathrm{~m}, 2 \mathrm{H}), 1.39-1.24(\mathrm{~m}, 4 \mathrm{H}), 0.92(\mathrm{t}, J=7.2 \mathrm{~Hz}, 3 \mathrm{H})$. Spectral data matched those reported in the literature. ${ }^{27}$ 

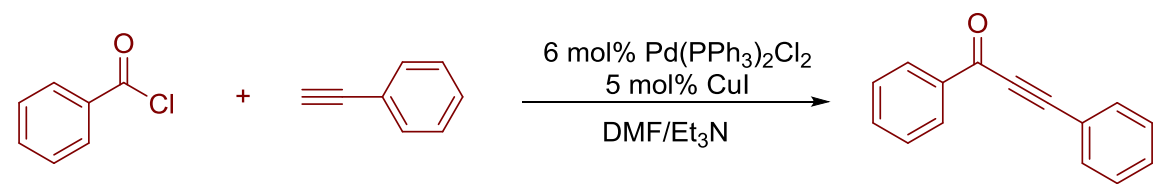

In a flask under nitrogen, $\mathrm{Pd}\left(\mathrm{PPh}_{3}\right)_{2} \mathrm{Cl}_{2}(6 \mathrm{~mol} \%)$ and $\mathrm{CuI}(5 \mathrm{~mol} \%)$ were added to dry THF $(0.30 \mathrm{M})$. $\mathrm{Et}_{3} \mathrm{~N}(1.00$ equiv.), the ethynylbenzene (1.10 equiv.) and acyl chloride (1.00 equiv.) were then added to the solution. The reaction mixture was stirred at room temperature until total consumption of the starting material, as monitored by TLC. The solution was diluted with $\mathrm{Et}_{2} \mathrm{O}$ and washed with water. The organic layers were combined, dried over $\mathrm{MgSO}_{4}$, filtered and the solvents were removed under vacuum. The crude product was purified on flash chromatography.

${ }^{1} \mathbf{H}$ NMR $\left(400 \mathrm{MHz}, \mathrm{CDCl}_{3}\right) \delta 8.27-8.17(\mathrm{~m}, 2 \mathrm{H}), 7.71-7.65(\mathrm{~m}, 2 \mathrm{H}), 7.64-7.59(\mathrm{~m}, 1 \mathrm{H}), 7.50(\mathrm{t}, J=7.6 \mathrm{~Hz}, 2 \mathrm{H})$, $7.46(\mathrm{~d}, J=7.4 \mathrm{~Hz}, 1 \mathrm{H}), 7.40(\mathrm{t}, J=7.3 \mathrm{~Hz}, 2 \mathrm{H})$. Spectral data matched those reported in the literature. ${ }^{29}$

(Ethoxyethynyl)benzene (1ay) ${ }^{30}$ [cas: 32569-84-9]<smiles>ClC#Cc1ccccc1</smiles><smiles>CCO[N+](=O)OCCOc1ccccc1</smiles><smiles>CCOC#Cc1ccccc1</smiles>

A dry reaction tube was charged with sodium alkoxide $(10.0 \mathrm{mmol})$. The tube was sealed, evacuated, and backfilled with nitrogen. Then, a solution of chlorinated alkyne $(5 \mathrm{mmol})$ in dry DMSO $(5 \mathrm{~mL})$ was added dropwise at $0{ }^{\circ} \mathrm{C}$ and the progress of the reaction was monitored by TLC. Upon completion, ice water was added to quench the reaction. The mixture was then extracted with ether $(3 \times 10 \mathrm{~mL})$ and the combined organic layer was dried over $\mathrm{MgSO}_{4}$, filtered and concentrated in vacuum. The residue was purified by flash chromatography on silica gel to afford the alkynyl ether (290 mg, 41\%).

${ }^{1} \mathbf{H}$ NMR $\left(400 \mathrm{MHz}, \mathrm{CDCl}_{3}\right) \delta 7.34(\mathrm{~m}, 2 \mathrm{H}), 7.27-7.20(\mathrm{~m}, 3 \mathrm{H}), 4.21(\mathrm{q}, J=7.1 \mathrm{~Hz}, 2 \mathrm{H}), 1.44(\mathrm{t}, J=7.1 \mathrm{~Hz}$, $3 \mathrm{H})$.Spectral data matched those reported in the literature. ${ }^{30}$

$N$-Butyl-4-methyl- $N$-(phenylethynyl)benzenesulfonamide (1az) ${ }^{31}$ [cas: 1007597-67-2]

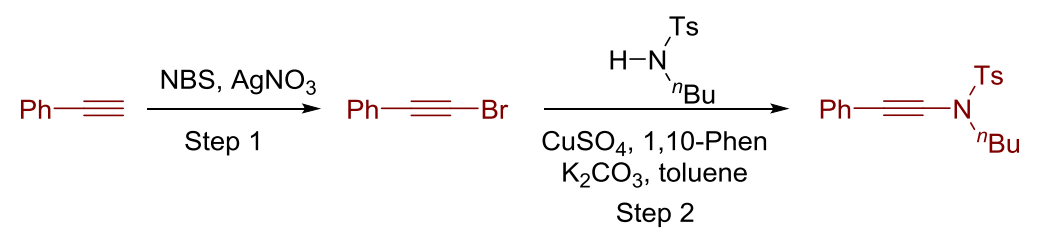

Step 1: In the dark, to a solution of terminal alkyne $(5.5 \mathrm{mmol})$ in $25 \mathrm{~mL}$ acetone was added NBS $(1.1 \mathrm{~g}, 6.05 \mathrm{mmol})$ and $\mathrm{AgNO}_{3}(94 \mathrm{mg}, 0.55 \mathrm{mmol})$. The resulting mixture was stirred at room temperature for $3 \mathrm{~h}$. The mixture was filtered and washed with petroleum ether; the filtrate was evaporated. Then $20 \mathrm{~mL}$ petroleum ether was added to the residue, filtered again and the filtrate was evaporated under vacuum to give the corresponding alkynyl bromide which was used directly.

Step 2: Amide (5.0 mmol), alkynyl bromide (5.5 mmol), copper sulfate pentahydrate (187.0 mg, $0.75 \mathrm{mmol}), 1,10-$ phenanthroline ( $270.0 \mathrm{mg}, 1.5 \mathrm{mmol}), \mathrm{K}_{2} \mathrm{CO}_{3}(1.38 \mathrm{~g}, 10.0 \mathrm{mmol})$, and toluene $(25 \mathrm{~mL})$ were added to a flask. The reaction flask was evacuated under vacuum and flushed with nitrogen for three times. Then, the reaction mixture was heated to $80^{\circ} \mathrm{C}$ and stirred overnight. The reaction mixture was cooled down to room temperature, filtered through a pad of silica gel, the filtrate was evaporated and purified by column chromatography (silica gel, EtOAc: Petroleum ether $=1: 20$ to $1: 10)$ to give the desired ynamide.

${ }^{1} \mathbf{H}$ NMR $\left(400 \mathrm{MHz}, \mathrm{CDCl}_{3}\right) \delta 7.91-7.79(\mathrm{~d}, J=8.3 \mathrm{~Hz}, 2 \mathrm{H}), 7.45-7.33(\mathrm{td}, J=6.6,3.6 \mathrm{~Hz}, 4 \mathrm{H}), 7.32-7.27(\mathrm{~m}$, $3 \mathrm{H}), 3.43-3.37(\mathrm{t}, J=7.2 \mathrm{~Hz}, 2 \mathrm{H}), 2.47-2.43(\mathrm{~s}, 3 \mathrm{H}), 1.75-1.65(\mathrm{~m}, 1 \mathrm{H}), 1.45-1.33(\mathrm{~m}, 1 \mathrm{H}), 0.93(\mathrm{t}, \mathrm{J}=7.4 \mathrm{~Hz}$, $2 \mathrm{H})$. Spectral data matched those reported in the literature. ${ }^{31}$

13-Methyl-3-((trimethylsilyl)ethynyl)-6,7,8,9,11,12,13,14,15,16-decahydro-17H-cyclopenta[a]phenanthren-17one (1bc) $)^{27}$ [cas: 1052063-00-9]
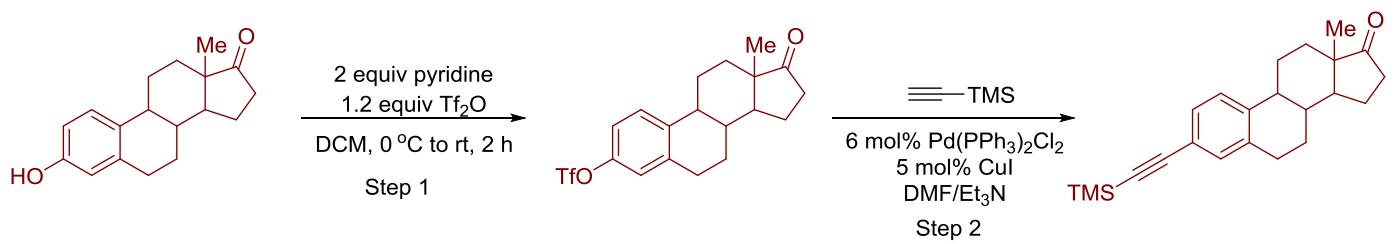
Step 1 To a solution of 1,3,5(10)-estratrien-3-ol-17-one (1.35 g, $5 \mathrm{mmol})$ in DCM (20 mL) was added pyridine (0.8 $\mathrm{mL}, 10 \mathrm{mmol})$. Then $\mathrm{Tf}_{2} \mathrm{O}(1 \mathrm{~mL}, 6 \mathrm{mmol}$, diluted with $20 \mathrm{~mL}$ DCM$)$ was added dropwise to the mixture at $0{ }^{\circ} \mathrm{C}$. The resulting solution was warmed up to room temperature and stirred for $2 \mathrm{~h}$. Then the mixture was quenched with saturated $\mathrm{NH}_{4} \mathrm{Cl}$ solution and extracted with dichloromethane. The combined organic extracts were washed with water and brine, and dried over anhydrous $\mathrm{Na}_{2} \mathrm{SO}_{4}$ and concentrated in vacuo. The crude product was purified by flash column chromatography on silica gel to give the (8R,9S,13S,14S)-13-methyl-17-oxo-7,8,9,11,12,13,14,15,16,17decahydro-6H-cyclopenta[a]phenanthren-3-yl trifluoromethanesulfonate $(0.5 \mathrm{~g})$ as a white solid.

Step 2 To a solution of the above compound $(0.482 \mathrm{~g}, 1.2 \mathrm{mmol})$ in triethylamine $(1 \mathrm{~mL})$ and DMF $(2.5 \mathrm{~mL})$ were added ethynyltrimethylsilane (141.2 mg, $1.44 \mathrm{mmol})$ and $\mathrm{Pd}\left(\mathrm{PPh}_{3}\right)_{2} \mathrm{Cl}_{2}(50.5 \mathrm{mg}, 0.072 \mathrm{mmol})$ at room temperature. Then the mixture was stirred at $50{ }^{\circ} \mathrm{C}$ for $12 \mathrm{~h}$ under $\mathrm{N}_{2}$. After the starting material was consumed, the reaction mixture was quenched saturated $\mathrm{NH}_{4} \mathrm{Cl}$ solution and extracted with dichloromethane. The combined organic extracts were washed with water and brine, and dried over anhydrous $\mathrm{Na}_{2} \mathrm{SO}_{4}$ and concentrated in vacuo. The crude product was purified by flash column chromatography on silica gel to give the (8R,9S,13S,14S)-13-methyl-3((trimethylsilyl)ethynyl)-7,8,9,11,12,13,15,16-octahydro-6H-cyclopenta[a]phenanthren-17(14H)-one $(0.30$ g) as a withe solid.

${ }^{1}$ H NMR (400 MHz, CDCl $): \delta 7.37-7.14(\mathrm{~m}, 3 \mathrm{H}), 2.89$ (br s, 2H), $2.53(\mathrm{dd}, \mathrm{J}=18.8,8.5 \mathrm{~Hz}, 1 \mathrm{H}), 2.43-2.40(\mathrm{~m}, 1 \mathrm{H})$, 2.32-2.25 (m, 1H), 2.22-1.95 (m, 4H), 1.70-1.41 (m, 6H), $0.93(\mathrm{~s}, 3 \mathrm{H}), 0.27$ (s, 9H). Spectral data matched those reported in the literature. ${ }^{27}$

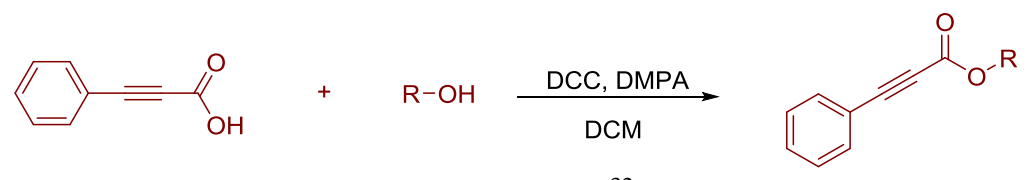

General procedure (C) for the synthesis of phenylpropiolate: ${ }^{32}$ To a solution of alcohol (3.0 mmol, 1.0 equiv.) in $\mathrm{CH}_{2} \mathrm{Cl}_{2}(12 \mathrm{~mL})$ was added phenylpropiolic acid $\left(482.4 \mathrm{mg}, 3.3 \mathrm{mmol}, 1.1\right.$ equiv) at $0{ }^{\circ} \mathrm{C}$. Then, a mixture of DCC ( $873.0 \mathrm{mg}, 4.4 \mathrm{mmol}, 1.5$ equiv.) and DMAP (36.0 mg, $1.5 \mathrm{mmol}, 0.1$ equiv.) in $\mathrm{CH}_{2} \mathrm{Cl}_{2}(6 \mathrm{~mL})$ was added dropwise. The resulting mixture was stirred at room temperature for $12 \mathrm{~h}$. The reaction mixture was filtered through a pad of silica gel. The filtrate was concentrated, and the crude residue was purified by silica gel column to give the corresponding propiolate.

\section{Cholesterol Phenylpropiolate (1bd) ${ }^{32}$ [cas: 373389-75-4]}

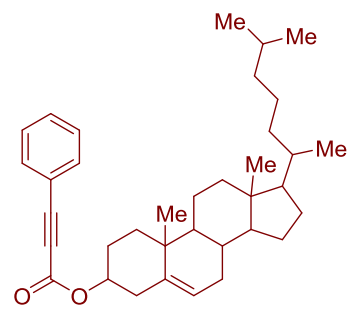

Prepared following general procedure $\mathrm{C}$ from Cholesterol and 3-phenylpropiolic acid. The product was purified by column chromatography. ${ }^{1} \mathbf{H}$ NMR $\left(400 \mathrm{MHz}, \mathrm{CDCl}_{3}\right) \delta 7.59(\mathrm{~d}, J=7.4 \mathrm{~Hz}, 2 \mathrm{H}), 7.44(\mathrm{~m}, J=8.5,6.3 \mathrm{~Hz}, 1 \mathrm{H}), 7.36(\mathrm{t}$, $J=7.6 \mathrm{~Hz}, 2 \mathrm{H}), 5.41(\mathrm{~d}, J=4.7 \mathrm{~Hz}, 1 \mathrm{H}), 4.78(\mathrm{~m}, J=16.3,9.2,4.6 \mathrm{~Hz}, 1 \mathrm{H}), 2.43(\mathrm{~d}, J=6.9 \mathrm{~Hz}, 2 \mathrm{H}), 2.07-1.98(\mathrm{~m}$, $2 \mathrm{H}), 1.96(\mathrm{~d}, J=5.0 \mathrm{~Hz}, 1 \mathrm{H}), 1.92-1.87(\mathrm{~m}, 1 \mathrm{H}), 1.86-1.79(\mathrm{~m}, 1 \mathrm{H}), 1.75-1.02(\mathrm{~m}, 25 \mathrm{H}), 0.92(\mathrm{~d}, J=6.5 \mathrm{~Hz}, 3 \mathrm{H})$, $0.87(\mathrm{~m}, J=6.6,1.5 \mathrm{~Hz}, 6 \mathrm{H}), 0.68(\mathrm{~s}, 3 \mathrm{H})$. Spectral data matched those reported in the literature. ${ }^{32}$

\section{2-Isopropyl-5-methylcyclohexyl 3-phenylpropiolate (1be) ${ }^{33}$ [cas: 59078-31-8]}<smiles>CC(C)C1C(C)CCC(C)C1OC(=O)C=Cc1ccccc1</smiles>

Prepared following general procedure $\mathrm{C}$ from Menthol and 3-phenylpropiolic acid. The product was purified by column chromatography. ${ }^{1} \mathbf{H}$ NMR $\left(400 \mathrm{MHz}, \mathrm{CDCl}_{3}\right) \delta 7.61-7.56(\mathrm{~m}, 2 \mathrm{H}), 7.46-7.40(\mathrm{~m}, 1 \mathrm{H}), 7.39-7.33(\mathrm{~m}, 2 \mathrm{H})$, $4.85(\mathrm{td}, J=10.9,4.4 \mathrm{~Hz}, 1 \mathrm{H}), 2.13-2.03(\mathrm{~m}, 1 \mathrm{H}), 2.01-1.93(\mathrm{~m}, 1 \mathrm{H}), 1.74-1.64(\mathrm{~m}, 2 \mathrm{H}), 1.53-1.44(\mathrm{~m}, 2 \mathrm{H})$, $1.14-1.03(\mathrm{~m}, 2 \mathrm{H}), 0.97-0.84(\mathrm{~m}, 6 \mathrm{H}), 0.89-0.86(\mathrm{~m}, 1 \mathrm{H}), 0.80(\mathrm{~d}, J=7.0 \mathrm{~Hz}, 3 \mathrm{H})$. Spectral data matched those reported in the literature. ${ }^{33}$ 
<smiles>CC(C)=CCCC(C)=CCOC(=O)C#Cc1ccccc1</smiles>

Prepared following general procedure $\mathrm{C}$ from Nerol and 3-phenylpropiolic acid. The product was purified by column chromatography. ${ }^{1} \mathbf{H}$ NMR $\left(400 \mathrm{MHz}, \mathrm{CDCl}_{3}\right) \delta 7.59-7.53(\mathrm{~m}, 2 \mathrm{H}), 7.46-7.40(\mathrm{~m}, 1 \mathrm{H}), 7.35(\mathrm{t}, J=7.4 \mathrm{~Hz}, 2 \mathrm{H})$, $5.43(\mathrm{t}, J=7.3 \mathrm{~Hz}, 1 \mathrm{H}), 5.11(\mathrm{t}, J=6.3 \mathrm{~Hz}, 1 \mathrm{H}), 4.72(\mathrm{~d}, J=7.4 \mathrm{~Hz}, 2 \mathrm{H}), 2.19-2.06(\mathrm{~m}, 4 \mathrm{H}), 1.79$ (s, 3H), 1.69 (s, $3 \mathrm{H}), 1.61(\mathrm{~s}, 3 \mathrm{H})$.

${ }^{13} \mathrm{C}$ NMR $\left(100 \mathrm{MHz}, \mathrm{CDCl}_{3}\right) \delta 154.0,143.8,132.9,132.2,130.5,128.5,123.4,119.6,118.2,86.0,80.6,62.4,32.1$, $26.5,25.6,23.4,17.6$.

\section{2,5,7,8-Tetramethyl-6-((3-phenylprop-2-yn-1-yl)oxy)-2-(4,8,12-trimethyltridecyl)chromane $(1 \mathrm{bg})^{34}$ [cas: 2452012-80-3]}

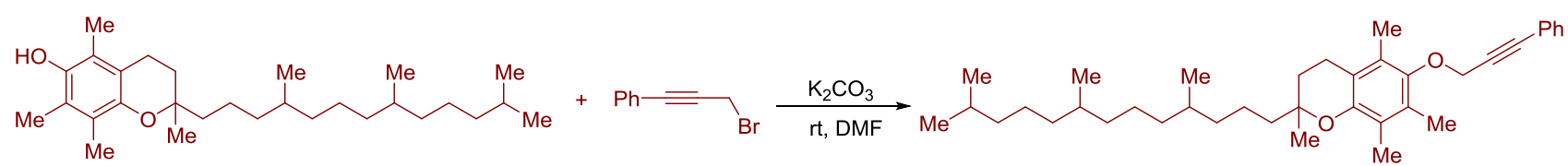

The title compound was prepared according to previously reported literature procedure. ${ }^{[12]}$ To a solution of Tocopherol ( $2.0 \mathrm{mmol}, 1.0$ equiv.) in dry THF $(10 \mathrm{~mL})$ was added $n$-BuLi $\left(2.0 \mathrm{mmol}, 2.5 \mathrm{M}\right.$ THF solution) at $-20^{\circ} \mathrm{C}$. The mixture was stirred at $-20^{\circ} \mathrm{C}$ for $30 \mathrm{~min}$. Then, a solution of (3-bromoprop-1-yn-1-yl)benzene (2.2 mmol) in THF was added drop-wise. The resulting mixture was stirred at room temperature for $12 \mathrm{~h}$. The reaction was quenched with saturated $\mathrm{NH}_{4} \mathrm{Cl}$ solution and extracted with ethyl acetate. The combined organic extracts were washed with water and brine, and dried over anhydrous $\mathrm{Na}_{2} \mathrm{SO}_{4}$ and concentrated in vacuo. The crude product was purified by flash column chromatography on silica gel to give the desired product $(0.81 \mathrm{~g}$, white solid).

${ }^{1} \mathbf{H}$ NMR $\left(400 \mathrm{MHz}, \mathrm{CDCl}_{3}\right) \delta 7.44(\mathrm{~m}, J=6.7,3.0 \mathrm{~Hz}, 2 \mathrm{H}), 7.35-7.28(\mathrm{~m}, 3 \mathrm{H}), 4.60(\mathrm{~s}, 2 \mathrm{H}), 2.58(\mathrm{t}, J=6.6 \mathrm{~Hz}, 2 \mathrm{H})$, $2.25(\mathrm{~s}, 3 \mathrm{H}), 2.21(\mathrm{~s}, 3 \mathrm{H}), 2.09(\mathrm{~s}, 3 \mathrm{H}), 1.84-1.74(\mathrm{~m}, 2 \mathrm{H}), 1.56-1.48(\mathrm{~m}, 3 \mathrm{H}), 1.36-1.21(\mathrm{~m}, 15 \mathrm{H}), 1.15-1.05(\mathrm{~m}$, $6 \mathrm{H}), 0.88-0.83(\mathrm{~m}, 12 \mathrm{H})$.

\section{4-((Trimethylsilyl)ethynyl)phenyl 2-(4-isobutylphenyl)propanoate (1bh)}

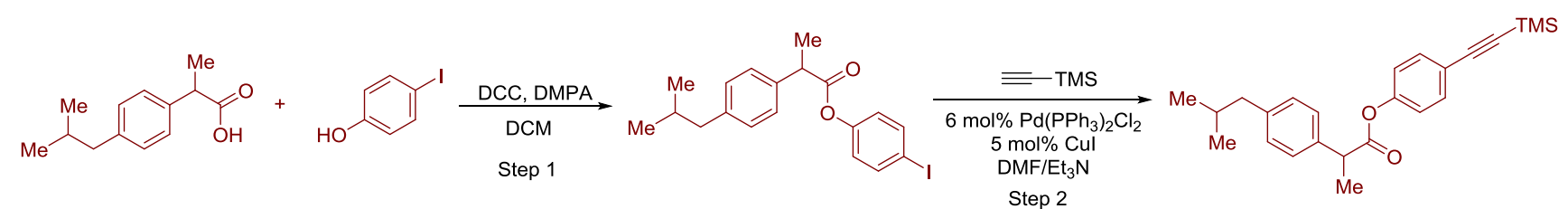

Step 1 To a solution of 4-iodophenol $(660.0 \mathrm{mg}, 3.0 \mathrm{mmol})$ in DCM (12 mL) was added Ibuprofen (680.0 mg, 3.3 $\mathrm{mmol})$ at $0{ }^{\circ} \mathrm{C}$. Then, a mixture of DCC (873.0 $\left.\mathrm{mg}, 4.4 \mathrm{mmol}\right)$ and DMAP $(36.0 \mathrm{mg}, 1.5 \mathrm{mmol})$ in DCM (6 mL) was added dropwise. The resulting mixture was stirred at room temperature for $12 \mathrm{~h}$. The reaction mixture was filtered through a pad of silica gel. The filtrate was concentrated, and the crude residue was purified by silica gel column to give the ester intermediate.

Step 2 To a solution of the above compound $(490.0 \mathrm{mg}, 1.2 \mathrm{mmol})$ in triethylamine $(1 \mathrm{~mL})$ and DMF $(2.5 \mathrm{~mL})$ were added ethynyltrimethylsilane (141.2 mg, $1.44 \mathrm{mmol}), \mathrm{Pd}\left(\mathrm{PPh}_{3}\right)_{2} \mathrm{Cl}_{2}(50.5 \mathrm{mg}, 0.072 \mathrm{mmol})$ and $\mathrm{CuI}(13.7 \mathrm{mg}, 0.06$ $\mathrm{mmol}$ ) at room temperature. Then the mixture was stirred at $50{ }^{\circ} \mathrm{C}$ for $12 \mathrm{~h}$ under $\mathrm{N}_{2}$. After the starting material was consumed, the reaction mixture was quenched saturated $\mathrm{NH}_{4} \mathrm{Cl}$ solution and extracted with dichloromethane. The combined organic extracts were washed with water and brine, and dried over anhydrous $\mathrm{Na}_{2} \mathrm{SO}_{4}$ and concentrated in vacuo. The crude product was purified by flash column chromatography on silica gel to give the desired product.

${ }^{1} \mathbf{H}$ NMR $\left(400 \mathrm{MHz}, \mathrm{CDCl}_{3}\right) \delta 7.44-7.40(\mathrm{~m}, 2 \mathrm{H}), 7.28(\mathrm{~d}, J=8.1 \mathrm{~Hz}, 2 \mathrm{H}), 7.14(\mathrm{~d}, J=8.1 \mathrm{~Hz}, 2 \mathrm{H}), 6.96-6.91(\mathrm{~m}$, $2 \mathrm{H}), 3.91(\mathrm{~d}, J=7.1 \mathrm{~Hz}, 1 \mathrm{H}), 2.47(\mathrm{~d}, J=7.2 \mathrm{~Hz}, 2 \mathrm{H}), 1.86(\mathrm{~s}, 1 \mathrm{H}), 1.59(\mathrm{~d}, J=7.1 \mathrm{~Hz}, 3 \mathrm{H}), 0.91(\mathrm{~d}, J=6.6 \mathrm{~Hz}, 6 \mathrm{H})$, $0.23(\mathrm{~s}, 9 \mathrm{H})$.

\section{(2,2,7,7-tetra-Methyltetrahydro-5H-bis([1,3]dioxolo)[4,5-b:4',5'-d]pyran-5-yl)methyl 4-(hex-1-yn-1-yl)benzoate} (1bi)

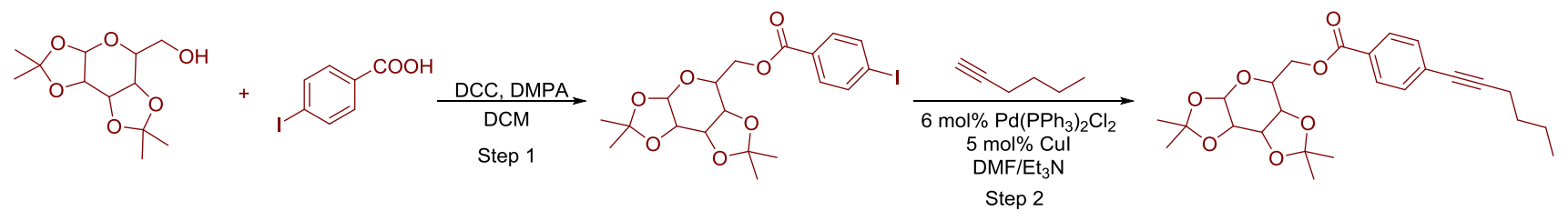


The title compound was prepared according to previously reported literature procedure..$^{35}$

Step 1 To a solution of 4-iodobenzoic acid $(744.0 \mathrm{mg}, 3.0 \mathrm{mmol})$ in DCM $(12 \mathrm{~mL})$ was added 2,2,7,7tetramethyltetrahydro-3aH-bis([1,3]dioxolo)[4,5-b:4',5'-d]pyran-5-yl)methanol $(858.0 \mathrm{mg}, 3.3 \mathrm{mmol})$ at $0{ }^{\circ} \mathrm{C}$. Then, a mixture of DCC (873.0 mg, $4.4 \mathrm{mmol})$ and DMAP $(36.0 \mathrm{mg}, 1.5 \mathrm{mmol})$ in DCM $(6 \mathrm{~mL})$ was added dropwise. The resulting mixture was stirred at room temperature for $12 \mathrm{~h}$. The reaction mixture was filtered through a pad of silica gel. The filtrate was concentrated, and the crude residue was purified by silica gel column to give the ester intermediate $(0.9 \mathrm{~g})$.

Step 2 To a solution of the above compound $(588.0 \mathrm{mg}, 1.2 \mathrm{mmol})$ in triethylamine $(1 \mathrm{~mL})$ and DMF $(2.5 \mathrm{~mL})$ were added hex-1-yne $(118.0 \mathrm{mg}, 1.44 \mathrm{mmol}), \mathrm{Pd}\left(\mathrm{PPh}_{3}\right)_{2} \mathrm{Cl}_{2}(50.5 \mathrm{mg}, 0.072 \mathrm{mmol})$ and $\mathrm{CuI}(13.7 \mathrm{mg}, 0.06 \mathrm{mmol})$ at room temperature. Then the mixture was stirred at $50{ }^{\circ} \mathrm{C}$ for $12 \mathrm{~h}$ under $\mathrm{N}_{2}$. After the starting material was consumed, the reaction mixture was quenched saturated $\mathrm{NH}_{4} \mathrm{Cl}$ solution and extracted with dichloromethane. The combined organic extracts were washed with water and brine, and dried over anhydrous $\mathrm{Na}_{2} \mathrm{SO}_{4}$ and concentrated in vacuo. The crude product was purified by flash column chromatography on silica gel to give the desired product.

${ }^{1} \mathbf{H}$ NMR $\left(400 \mathrm{MHz}, \mathrm{CDCl}_{3}\right) \delta 7.95(\mathrm{~d}, J=8.4 \mathrm{~Hz}, 2 \mathrm{H}), 7.42(\mathrm{~d}, J=8.3 \mathrm{~Hz}, 2 \mathrm{H}), 5.55(\mathrm{~d}, J=5.0 \mathrm{~Hz}, 1 \mathrm{H}), 4.64(\mathrm{dd}, J$ $=7.9,2.5 \mathrm{~Hz}, 1 \mathrm{H}), 4.51(\mathrm{dd}, J=11.5,4.9 \mathrm{~Hz}, 1 \mathrm{H}), 4.41(\mathrm{dd}, J=11.5,7.5 \mathrm{~Hz}, 1 \mathrm{H}), 4.32(\mathrm{ddd}, J=9.9,6.4,2.1 \mathrm{~Hz}, 2 \mathrm{H})$, $4.19-4.13(\mathrm{~m}, 1 \mathrm{H}), 2.42(\mathrm{t}, J=7.0 \mathrm{~Hz}, 2 \mathrm{H}), 1.62-1.57(\mathrm{~m}, 2 \mathrm{H}), 1.50(\mathrm{~s}, 3 \mathrm{H}), 1.49-1.41(\mathrm{~m}, 5 \mathrm{H}), 1.35(\mathrm{~s}, 3 \mathrm{H}), 1.32$ $(\mathrm{s}, 3 \mathrm{H}), 0.95(\mathrm{t}, J=7.3 \mathrm{~Hz}, 3 \mathrm{H})$.

\section{Supplementary References}

(1) (a) Gao, Y.; Nie, J.; Li, Y.; Li, X.; Chen, Q.; Huo, Y.; Hu, X.-Q. Rh-Catalyzed C-H Amination/Annulation of Acrylic Acids and Anthranils by Using $-\mathrm{COOH}$ as a Deciduous Directing Group: An Access to Diverse Quinolines. Org. Lett. 2020, 22 (7), 2600-2605. (b) Gao, Y.; Nie, J.; Li, Y.; Liao, G.; Huo, Y.; Hu, X. Rh(III) - Catalyzed Selective Ortho - C-H Amination of Benzoic Acids with Anthranils: A Facile Access to Anthranilic Acid Derivatives (AAs). ChemCatChem 2020, 12 (10), 2721-2725. (c) Gao, Y.; Yang, S.; Li, Y.; Huo, Y.; Huang, Z.; Chen, Z.; Hu, X.-Q. Copper-Catalyzed Electrophilic Amination of Arylboronic Acids with Anthranils: An Access to N-Aryl-2-Aminophenones. J. Org. Chem. 2020, 85 (15), 10222-10231.

(2) Li, X.; Wu, G.; Liu, X.; Zhu, Z.; Huo, Y.; Jiang, H. Regioselective C-H Bond Alkynylation of Carbonyl Compounds through Ir(III) Catalysis. J. Org. Chem. 2017, 82 (24), 13003-13011.

(3) Luo, W.; Lv, J.-W.; Wang, T.; Zhang, Z.-Y.; Guo, H.-Y.; Song, Z.-Y.; Wang, C.-J.; Ma, J.; Chen, Y. Synthesis, in Vitro and in Vivo Biological Evaluation of Novel Graveolinine Derivatives as Potential Anti-Alzheimer Agents. Bioorg. Med. Chem. 2020, 28 (1), 115190.

(4) Chaires, J. B.; Ren, J.; Henary, M.; Zegrocka, O.; Bishop, G. R.; Strekowski, L. Triplex Selective 2-(2-Naphthyl)Quinoline Compounds: Origins of Affinity and New Design Principles. J. Am. Chem. Soc. 2003, 125 (24), 7272-7283.

(5) Iglesias, M. J.; Prieto, A.; Nicasio, M. C. Kumada-Tamao-Corriu Coupling of Heteroaromatic Chlorides and Aryl Ethers Catalyzed by (IPr)Ni(Allyl)Cl. Org. Lett. 2012, 14 (17), 4318-4321.

(6) Guo, B.; Yu, T.; Li, H.; Zhang, S.; Braunstein, P.; Young, D. J.; Li, H.; Lang, J. Phosphine Ligand - Free Ruthenium Complexes as Efficient Catalysts for the Synthesis of Quinolines and Pyridines by Acceptorless Dehydrogenative Coupling Reactions. ChemCatChem 2019, 11 (10), $2500-2510$

(7) Liu, Y.; Hu, Y.; Cao, Z.; Zhan, X.; Luo, W.; Liu, Q.; Guo, C. Copper - Catalyzed Aerobic Oxidative Cyclization of Anilines, Aryl Methyl Ketones and DMSO: Efficient Assembly of 2 - Arylquinolines. Adv. Synth. Catal. 2018, 360 (14), 2691-2695.

(8) Takahashi, F.; Nogi, K.; Yorimitsu, H. Intramolecular Desulfitative Coupling: Nickel-Catalyzed Transformation of Diaryl Sulfones into Biaryls via Extrusion of $\mathrm{SO}_{2}$. Org. Lett. 2018, 20 (20), 6601-6605.

(9) Xi, L.-Y.; Zhang, R.-Y.; Zhang, L.; Chen, S.-Y.; Yu, X.-Q. An Efficient Synthesis of Quinolines via Copper-Catalyzed C-N Cleavage. Org. Biomol. Chem. 2015, 13 (13), 3924-3930.

(10) Kuriyama, M.; Matsuo, S.; Shinozawa, M.; Onomura, O. Ether-Imidazolium Carbenes for Suzuki-Miyaura Cross-Coupling of Heteroaryl Chlorides with Aryl/Heteroarylboron Reagents. Org. Lett. 2013, 15 (11), 2716-2719.

(11) Zhuo, F.-F.; Xie, W.-W.; Yang, Y.-X.; Zhang, L.; Wang, P.; Yuan, R.; Da, C.-S. TMEDA-Assisted Effective Direct Ortho Arylation of Electron-Deficient $N$-Heteroarenes with Aromatic Grignard Reagents. J. Org. Chem. 2013, 78 (7), 3243-3249.

(12) Tanaka, S.; Yasuda, M.; Baba, A. Practical and Simple Synthesis of Substituted Quinolines by an HCl-DMSO System on a Large Scale: Remarkable Effect of the Chloride Ion. J. Org. Chem. 2006, 71 (2), 800-803.

(13) Genç, S.; Arslan, B.; Gülcemal, S.; Günnaz, S.; Çetinkaya, B.; Gülcemal, D. Iridium(I)-Catalyzed C-C and C-N Bond Formation Reactions via the Borrowing Hydrogen Strategy. J. Org. Chem. 2019, 84 (10), 6286-6297. 
(14) Cai, S.; Zeng, J.; Bai, Y.; Liu, X.-W. Access to Quinolines through Gold-Catalyzed Intermolecular Cycloaddition of 2-Aminoaryl Carbonyls and Internal Alkynes. J. Org. Chem. 2012, 77 (1), 801-807.

(15) Zhang, X.; Song, X.; Li, H.; Zhang, S.; Chen, X.; Yu, X.; Wang, W. An Organocatalytic Cascade Approach toward Polysubstituted Quinolines and Chiral 1,4-Dihydroquinolines-Unanticipated Effect of N-Protecting Groups. Angew. Chem. Int. Ed. 2012, 51 (29), $7282-7286$.

(16) Kong, L.; Zhou, Y.; Huang, H.; Yang, Y.; Liu, Y.; Li, Y. Copper-Catalyzed Synthesis of Substituted Quinolines via C-N Coupling/Condensation from Ortho -Acylanilines and Alkenyl Iodides. J. Org. Chem. 2015, 80 (2), 1275-1278.

(17) McNaughton, B. R.; Miller, B. L. A Mild and Efficient One-Step Synthesis of Quinolines. Org. Lett. 2003, 5 (23), 4257-4259.

(18) Rubio-Presa, R.; Suárez-Pantiga, S.; Pedrosa, M. R.; Sanz, R. Molybdenum-Catalyzed Sustainable Friedländer Synthesis of Quinolines. Adv. Synth. Catal. 2018, 360 (11), 2216-2220.

(19) Li, J.; Tan, E.; Keller, N.; Chen, Y.-H.; Zehetmaier, P. M.; Jakowetz, A. C.; Bein, T.; Knochel, P. Cobalt-Catalyzed Electrophilic Aminations with Anthranils: An Expedient Route to Condensed Quinolines. J. Am. Chem. Soc. 2019, 141 (1), 98-103.

(20) Saunthwal, R. K.; Patel, M.; Verma, A. K. Metal- and Protection-Free [4 + 2] Cycloadditions of Alkynes with Azadienes: Assembly of Functionalized Quinolines. Org. Lett. 2016, 18 (9), 2200-2203.

(21) Tseng, C.-H.; Chen, Y.-L.; Chung, K.-Y.; Wang, C.-H.; Peng, S.-I.; Cheng, C.-M.; Tzeng, C.-C. Synthesis and Antiproliferative Evaluation of 2,3-Diarylquinoline Derivatives. Org. Biomol. Chem. 2011, 9 (9), 3205.

(22) Zhou, W.; Lei, J. Palladium-Catalyzed Synthesis of Polysubstituted Quinolines from 2-Amino Aromatic Ketones and Alkynes. Chem. Commun. 2014, 50 (42), 5583-5585.

(23) Feng, L.; Hu, T.; Zhang, S.; Xiong, H.-Y.; Zhang, G. Copper-Mediated Deacylative Coupling of Ynones via C-C Bond Activation under Mild Conditions. Org. Lett. 2019, 21 (23), 9487-9492.

(24) Bao, H.; Zhou, B.; Luo, S.-P.; Xu, Z.; Jin, H.; Liu, Y. P/N Heteroleptic Cu(I)-Photosensitizer-Catalyzed Deoxygenative Radical Alkylation of Aromatic Alkynes with Alkyl Aldehydes Using Dipropylamine as a Traceless Linker Agent. ACS Catal. 2020, 10 (14), $7563-7572$.

(25) Ou, Y.-P.; Zhang, J.; Kuang, D.; Zhang, F.; Yu, J.; Zhu, X.; Liu, S. H.; Hartl, F. Mononuclear Piano-Stool Iron 2Ethynylbenzo[ $b$ ]Thiophene Complex: Crystal Structure and Reversible Oxidation Studied by Spectro-Electrochemical and DFT Methods. $J$. Coord. Chem. 2017, 70 (4), 722-733.

(26) Huang, W.-S.; Metcalf, C. A.; Sundaramoorthi, R.; Wang, Y.; Zou, D.; Thomas, R. M.; Zhu, X.; Cai, L.; Wen, D.; Liu, S.; Romero, J.; Qi, J.; Chen, I.; Banda, G.; Lentini, S. P.; Das, S.; Xu, Q.; Keats, J.; Wang, F.; Wardwell, S.; Ning, Y.; Snodgrass, J. T.; Broudy, M. I.; Russian, K.; Zhou, T.; Commodore, L.; Narasimhan, N. I.; Mohemmad, Q. K.; Iuliucci, J.; Rivera, V. M.; Dalgarno, D. C.; Sawyer, T. K.; Clackson, T.; Shakespeare, W. C. Discovery of 3-[2-(Imidazo[1,2-b]Pyridazin-3-Yl)Ethynyl]-4-Methyl- $N$-\{4-[(4-Methylpiperazin-1-Yl)Methyl]-3(Trifluoromethyl)Phenyl benzamide (AP24534), a Potent, Orally Active Pan-Inhibitor of Breakpoint Cluster Region-Abelson (BCR-ABL) Kinase Including the T315I Gatekeeper Mutant. J. Med. Chem. 2010, 53 (12), 4701-4719.

(27) Li, W.; Yu, S.; Li, J.; Zhao, Y. Nickel - Catalyzed Allylmethylation of Alkynes with Allylic Alcohols and AlMe 3 : Facile Access to Skipped Dienes and Trienes. Angew. Chem. Int. Ed. 2020, 59 (34), 14404-14408.

(28) Le Fouler, V.; Chen, Y.; Gandon, V.; Bizet, V.; Salomé, C.; Fessard, T.; Liu, F.; Houk, K. N.; Blanchard, N. Activating Pyrimidines by PreDistortion for the General Synthesis of 7-Aza-Indazoles from 2-Hydrazonylpyrimidines via Intramolecular Diels-Alder Reactions. J. Am. Chem. Soc. 2019, 141 (40), 15901-15909.

(29) Shen, W.-B.; Xiao, X.-Y.; Sun, Q.; Zhou, B.; Zhu, X.-Q.; Yan, J.-Z.; Lu, X.; Ye, L.-W. Highly Site Selective Formal [5+2] and [4+2] Annulations of Isoxazoles with Heterosubstituted Alkynes by Platinum Catalysis: Rapid Access to Functionalized 1,3-Oxazepines and 2,5Dihydropyridines. Angew. Chem. Int. Ed. 2017, 56 (2), 605-609.

(30) Pan, F.; Li, X.-L.; Chen, X.-M.; Shu, C.; Ruan, P.-P.; Shen, C.-H.; Lu, X.; Ye, L.-W. Catalytic Ynamide Oxidation Strategy for the Preparation of $\alpha$-Functionalized Amides. ACS Catal. 2016, 6 (9), 6055-6062.

(31) Su, L.; Ren, T.; Dong, J.; Liu, L.; Xie, S.; Yuan, L.; Zhou, Y.; Yin, S.-F. Cu(I)-Catalyzed 6- Endo-Dig Cyclization of Terminal Alkynes, 2Bromoaryl Ketones, and Amides toward 1-Naphthylamines: Applications and Photophysical Properties. J. Am. Chem. Soc. 2019, 141 (6), 25352544.

(32) Murayama, H.; Nagao, K.; Ohmiya, H.; Sawamura, M. Phosphine-Catalyzed Vicinal Acylcyanation of Alkynoates. Org. Lett. 2016, 18 (7), 1706-1709.

(33) Kumar, R.; Dwivedi, V.; Sridhar Reddy, M. Metal-Free Iodosulfonylation of Internal Alkynes: Stereodefined Access to Tetrasubstituted Olefins. Adv. Synth. Catal. 2017, 359 (16), 2847-2856.

(34) Yu, L.; Lv, L.; Qiu, Z.; Chen, Z.; Tan, Z.; Liang, Y.; Li, C. Palladium - Catalyzed Formal Hydroalkylation of Aryl - Substituted Alkynes with Hydrazones. Angew. Chem. Int. Ed. 2020, 59 (33), 14009-14013. 
(35) Cheung, C. W.; Zhurkin, F. E.; Hu, X. Z -Selective Olefin Synthesis via Iron-Catalyzed Reductive Coupling of Alkyl Halides with Terminal Arylalkynes. J. Am. Chem. Soc. 2015, 137 (15), 4932-4935. 


\section{NMR Spectra}

${ }^{1} \mathrm{H}$ NMR $\left(400 \mathrm{MHz}, \mathrm{CDCl}_{3}\right)$

\section{2-Phenylquinoline (3a)}

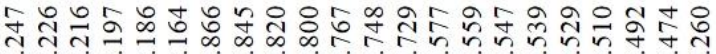

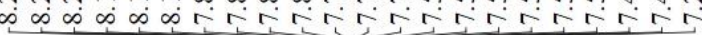
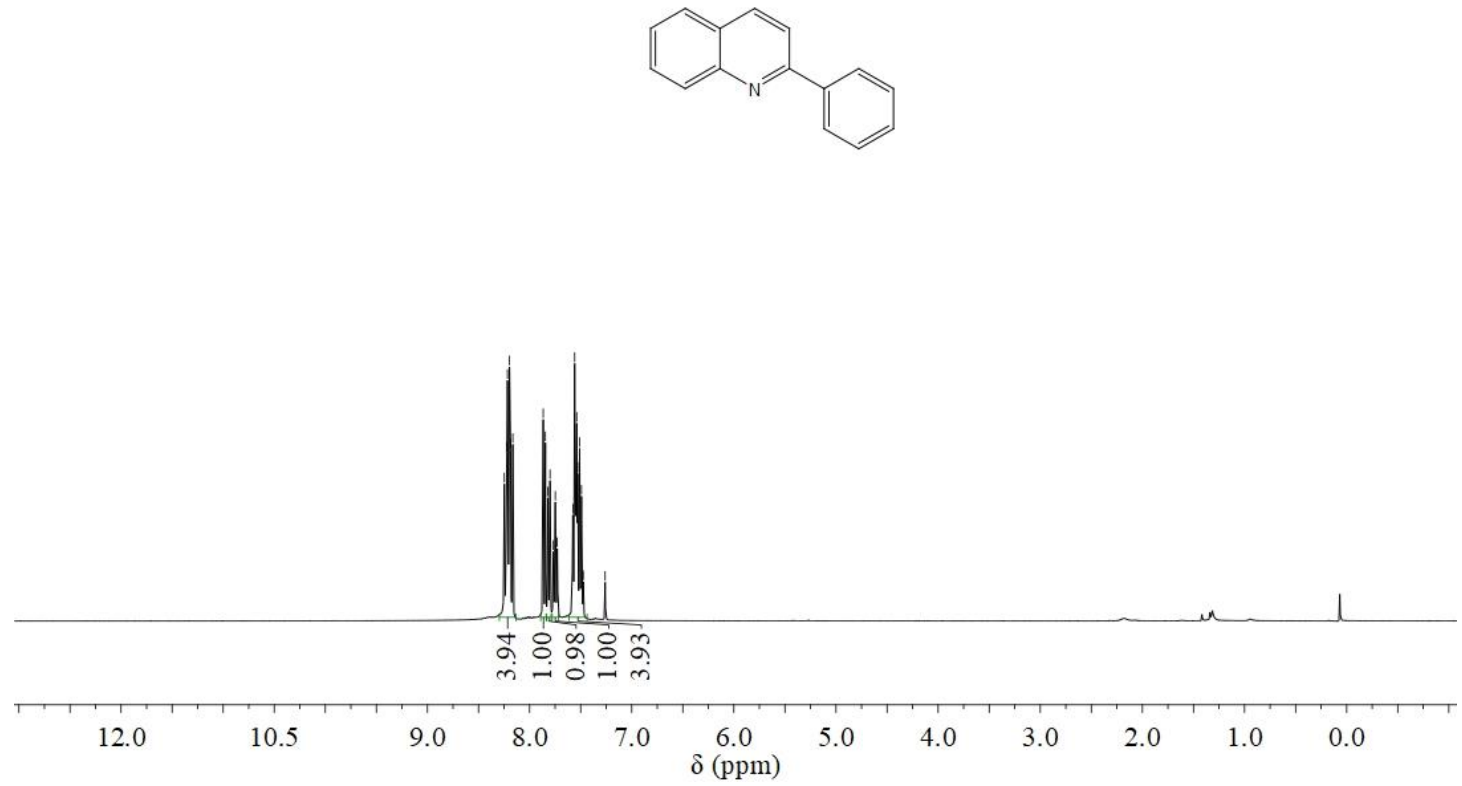

${ }^{13} \mathrm{C}$ NMR $\left(100 \mathrm{MHz}, \mathrm{CDCl}_{3}\right)$

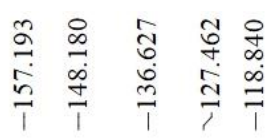

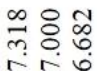

논
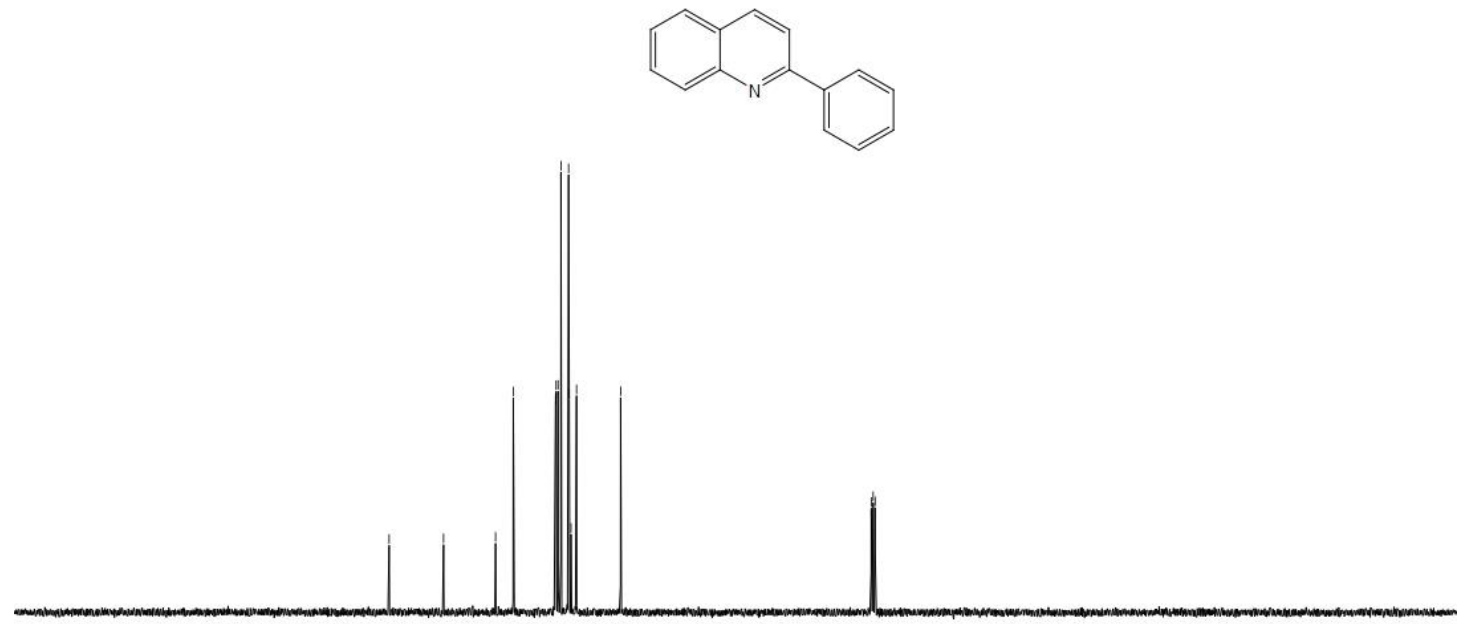

$\begin{array}{lllllllllllllll}210 & 190 & 170 & 150 & 130 & 110_{\delta(\mathrm{ppm})}^{90} & 80 & 70 & 60 & 50 & 40 & 30 & 20 & 10 & 0\end{array}$ 
${ }^{1} \mathrm{H}$ NMR (400 MHz, $\mathrm{CDCl}_{3}$ )

2-(4-Fluorophenyl)quinolone (3b)

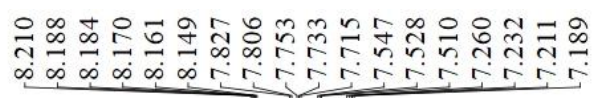
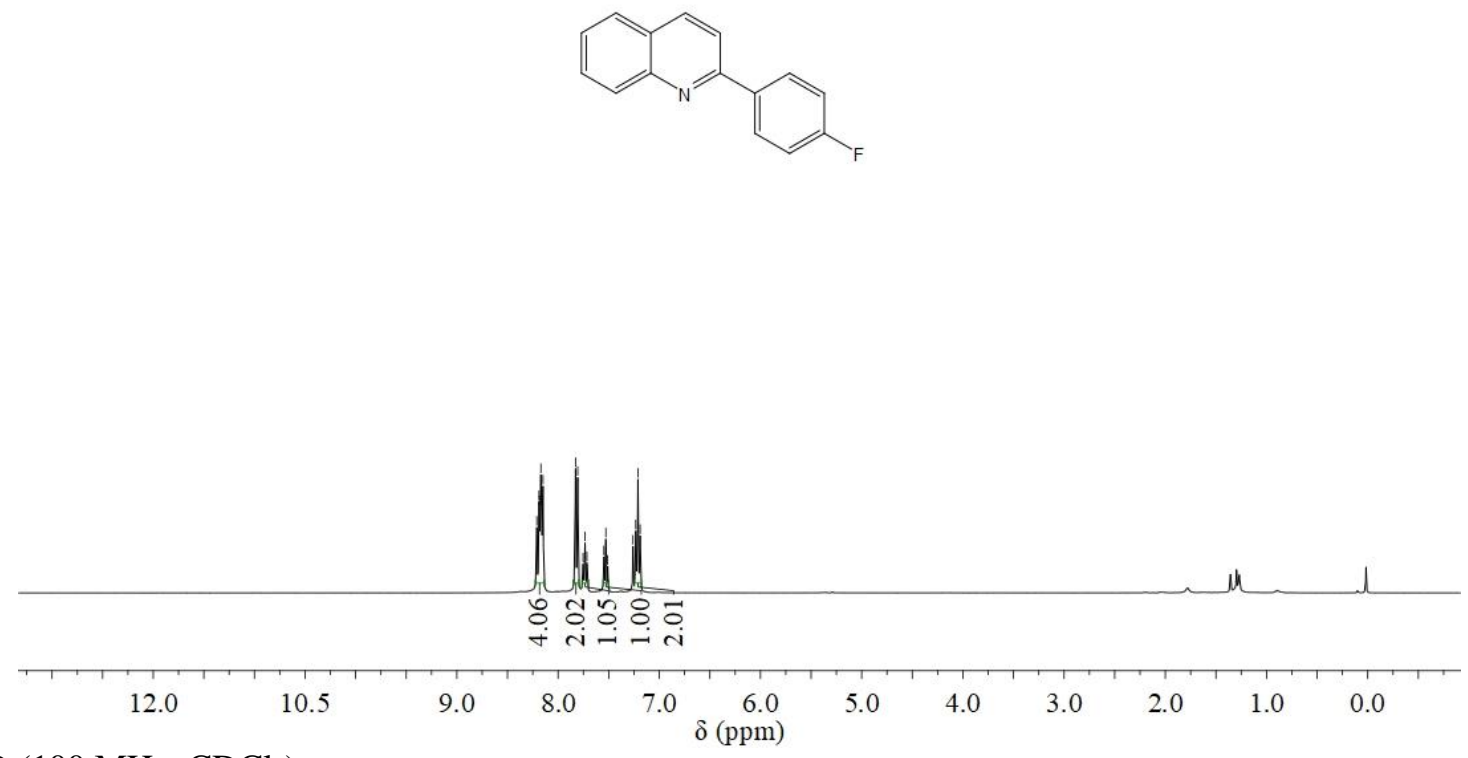

${ }^{13} \mathrm{C}$ NMR $\left(100 \mathrm{MHz}, \mathrm{CDCl}_{3}\right)$
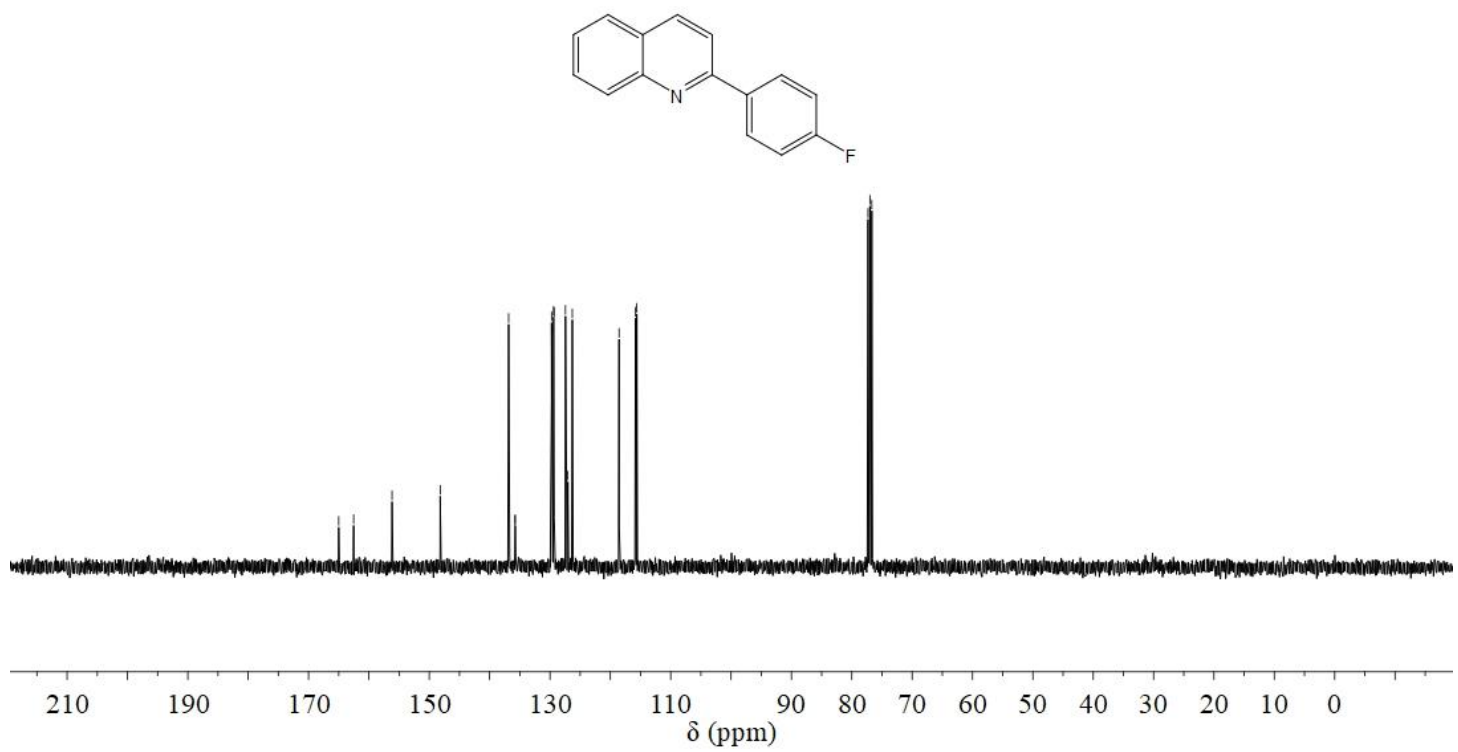

42 
${ }^{19} \mathrm{~F}$ NMR (376 MHz, $\left.\mathrm{CDCl}_{3}\right)$

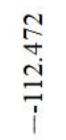

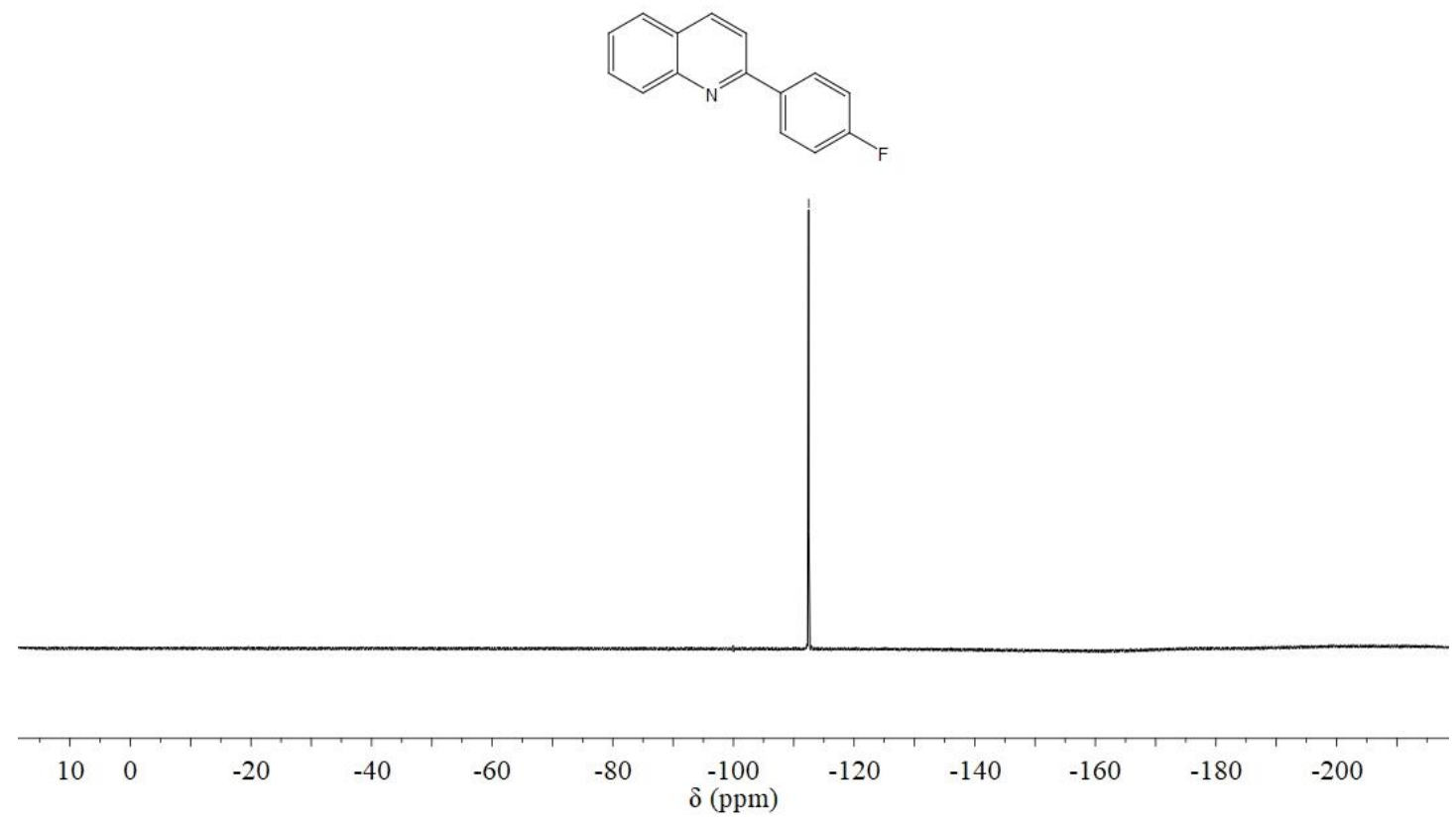


${ }^{1} \mathrm{H} \mathrm{NMR}\left(400 \mathrm{MHz}, \mathrm{CDCl}_{3}\right)$

2-(4-Chlorophenyl)quinolone (3c)

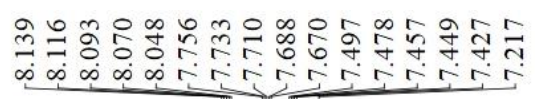
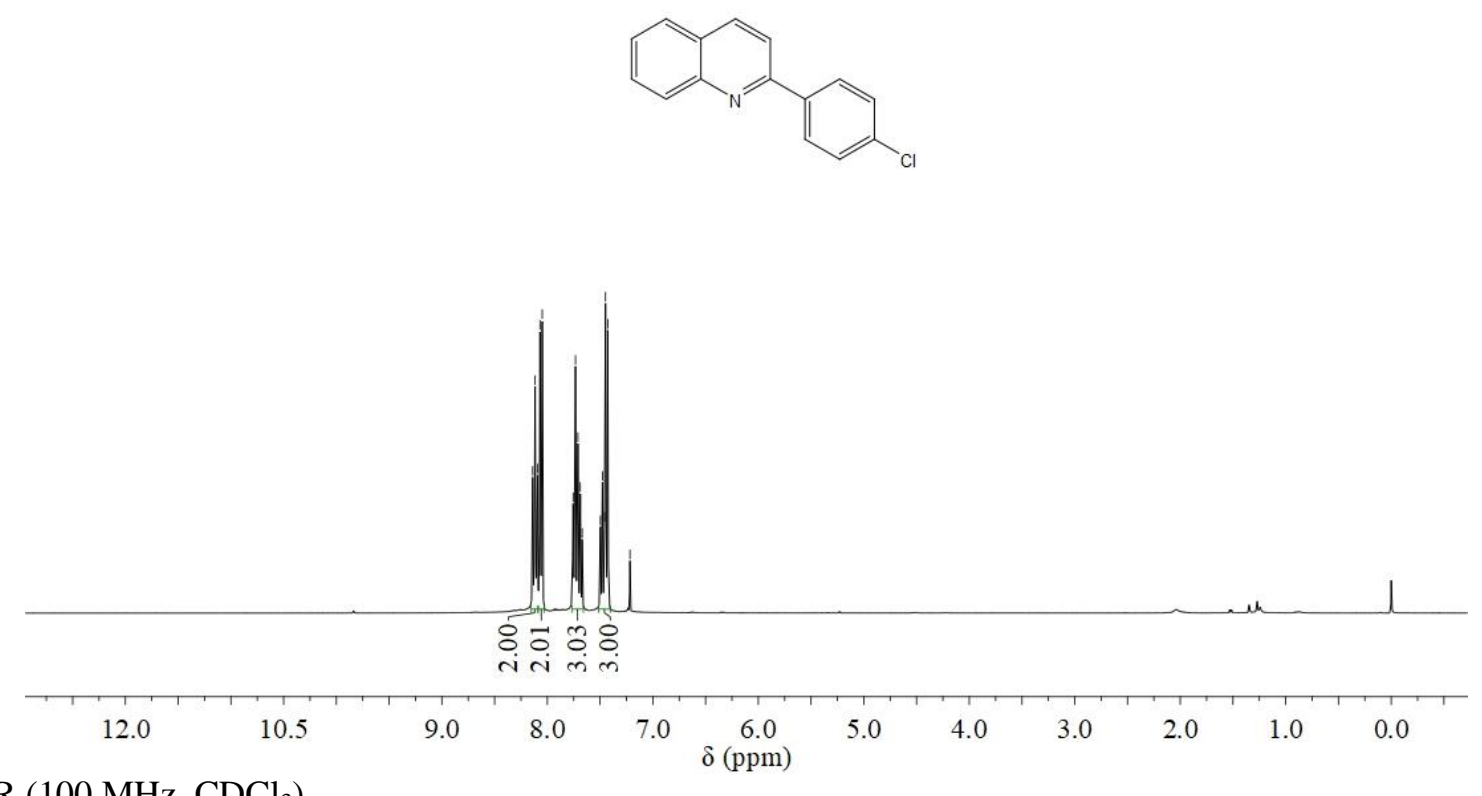

${ }^{13} \mathrm{C}$ NMR (100 MHz, $\mathrm{CDCl}_{3}$ )
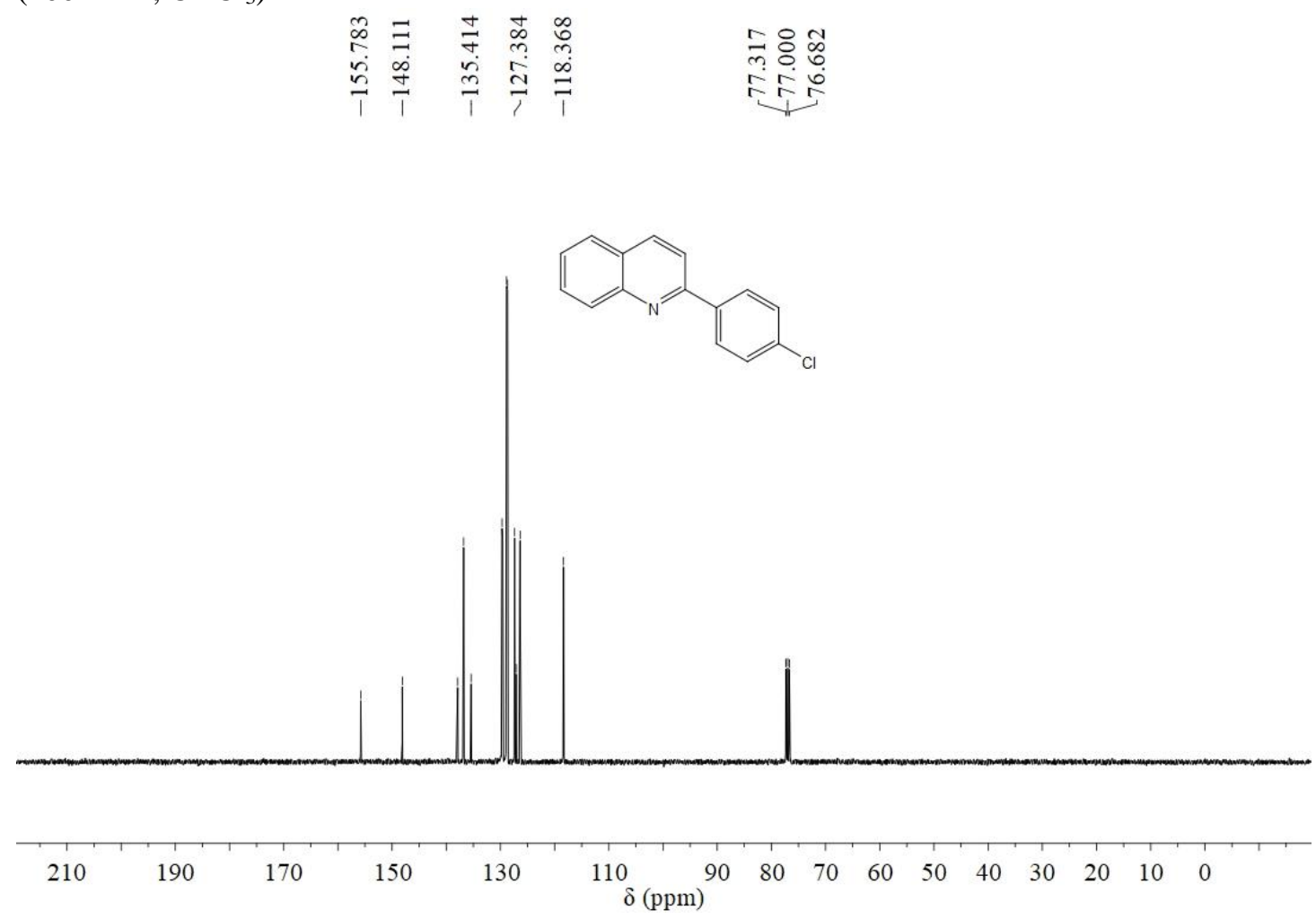

44 
${ }^{1} \mathrm{H}$ NMR (400 MHz, $\mathrm{CDCl}_{3}$ )

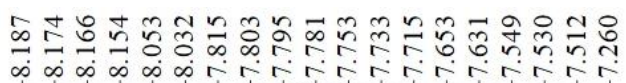
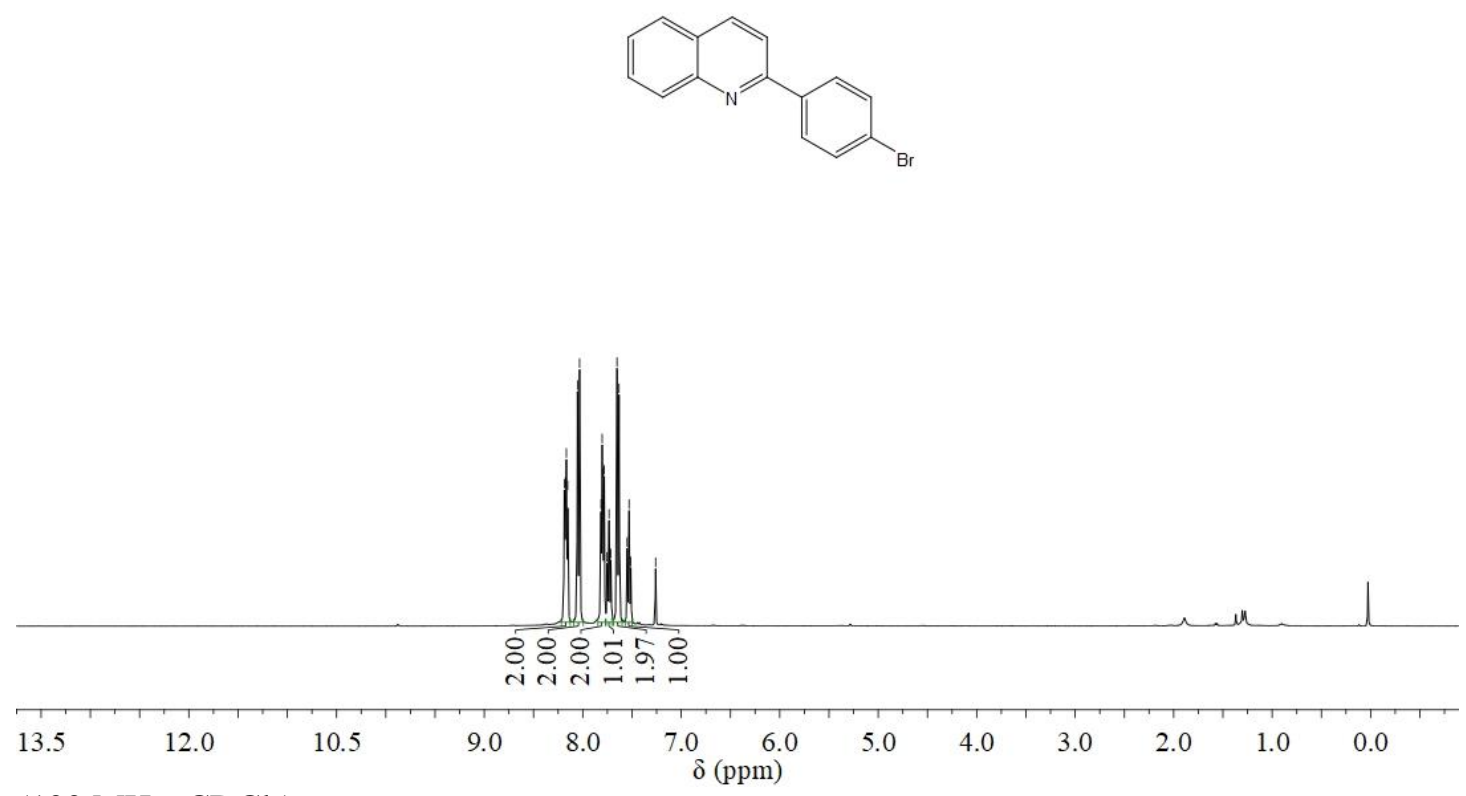

${ }^{13} \mathrm{C}$ NMR (100 MHz, $\left.\mathrm{CDCl}_{3}\right)$
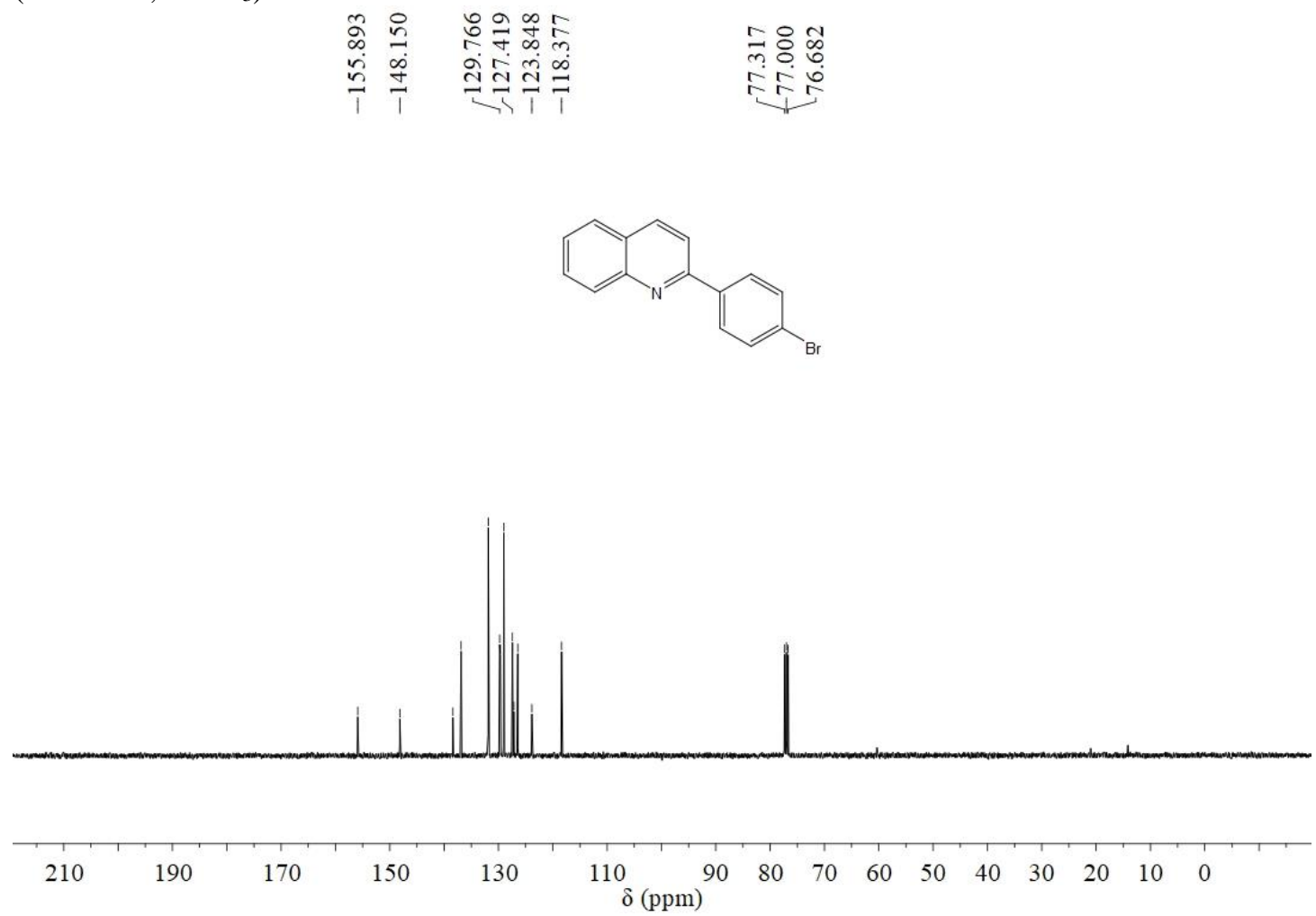
${ }^{1} \mathrm{H}$ NMR (400 MHz, $\mathrm{CDCl}_{3}$ )

2-(4-Methoxyphenyl)quinolone (3e)

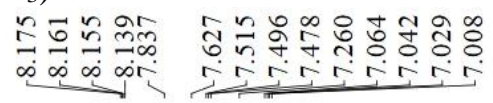

$\underset{\substack{+\infty}}{\substack{\infty \\ i}}$
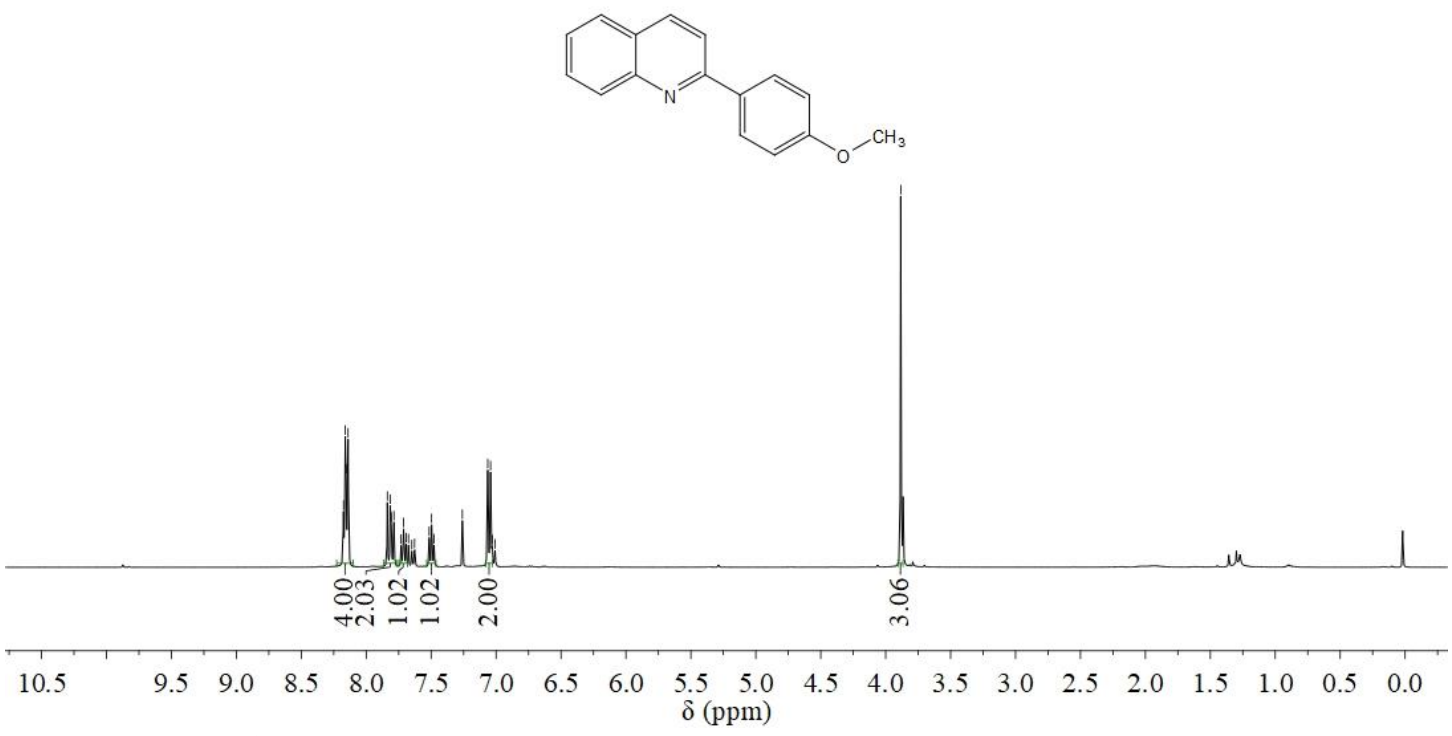

${ }^{13} \mathrm{C} \mathrm{NMR}\left(100 \mathrm{MHz}, \mathrm{CDCl}_{3}\right)$

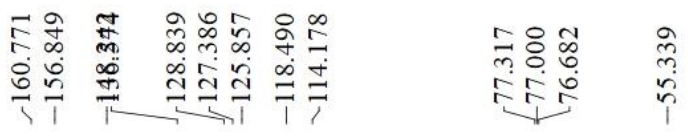
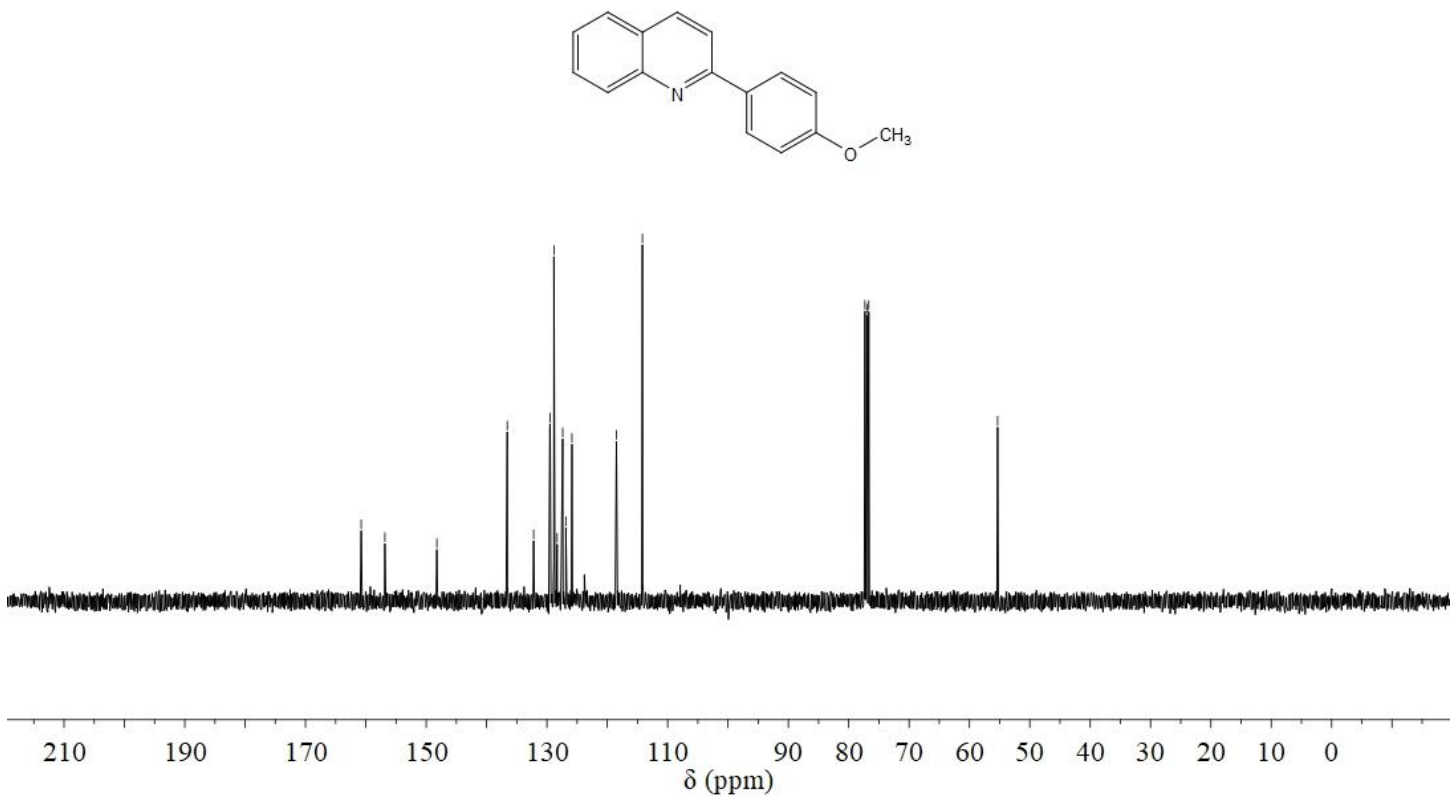

46 
${ }^{1} \mathrm{H}$ NMR (400 MHz, $\left.\mathrm{CDCl}_{3}\right)$

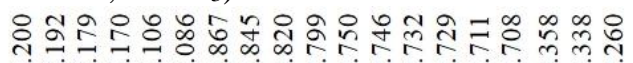

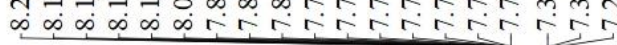

$\underset{\substack{n \\ \text { i }}}{i}$
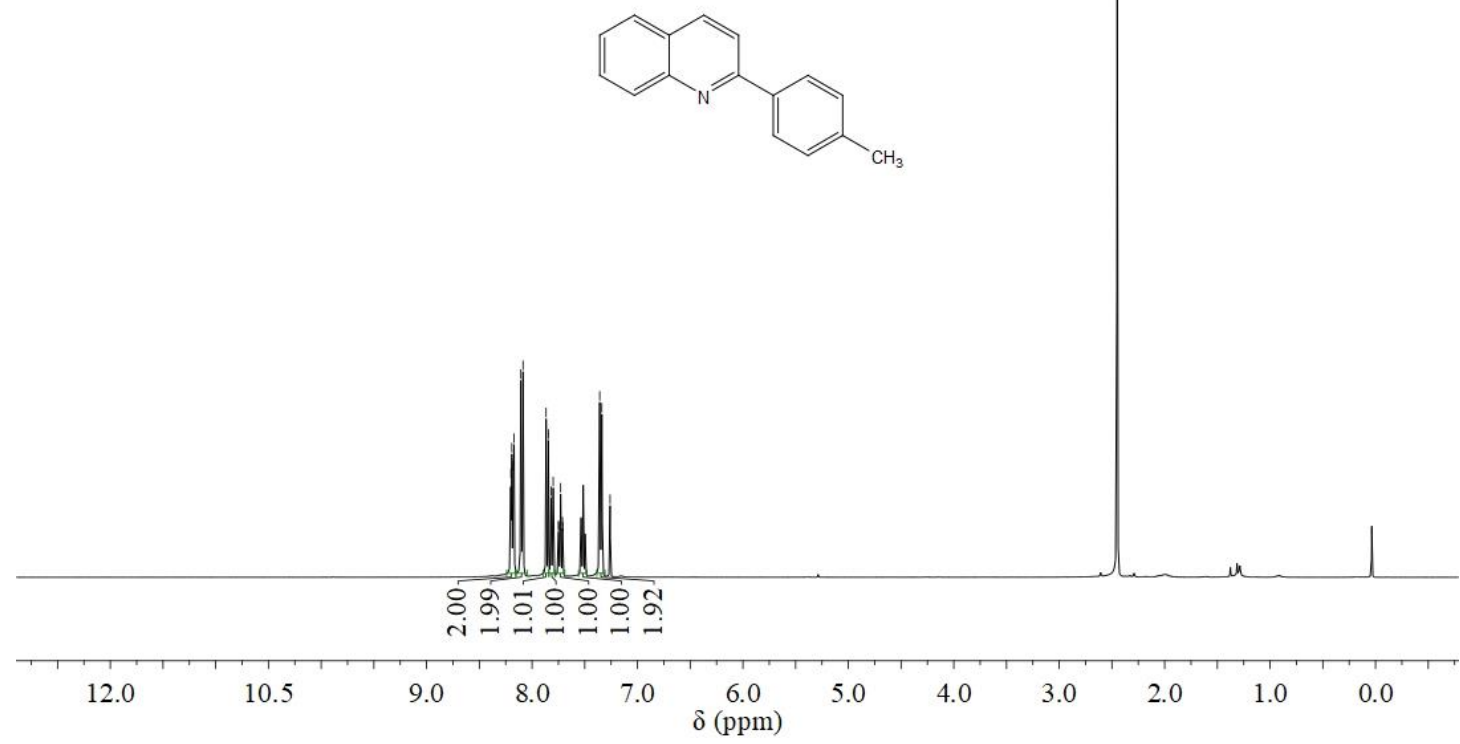

${ }^{13} \mathrm{C}$ NMR (100 MHz, $\left.\mathrm{CDCl}_{3}\right)$

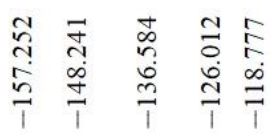

m.

둔

$\underset{\substack{\sim \\ i}}{\stackrel{\infty}{T}}$
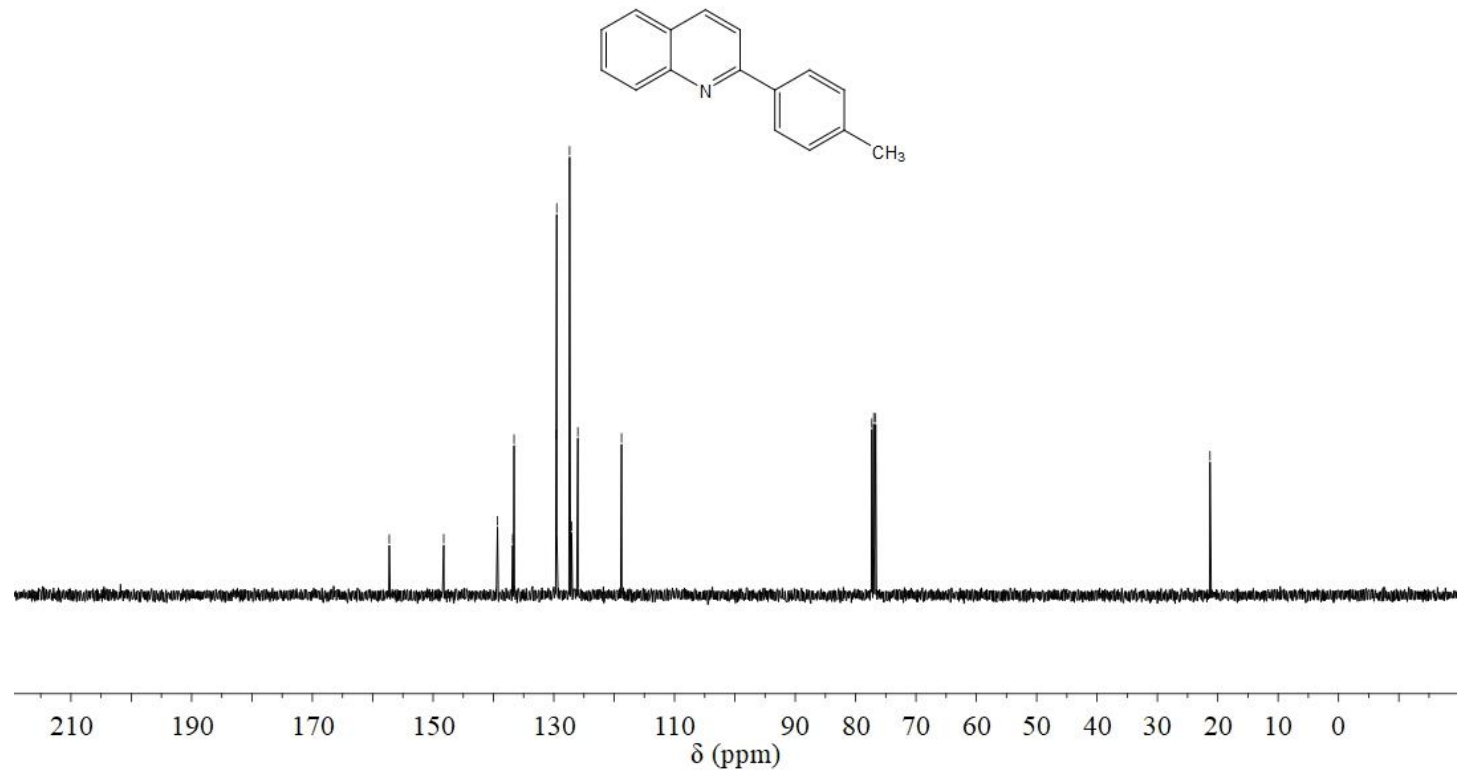
${ }^{1} \mathrm{H}$ NMR (400 MHz, $\mathrm{CDCl}_{3}$ )

2-(4-(Methylthio)phenyl)quinolone (3g)

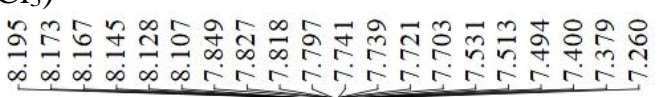

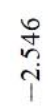
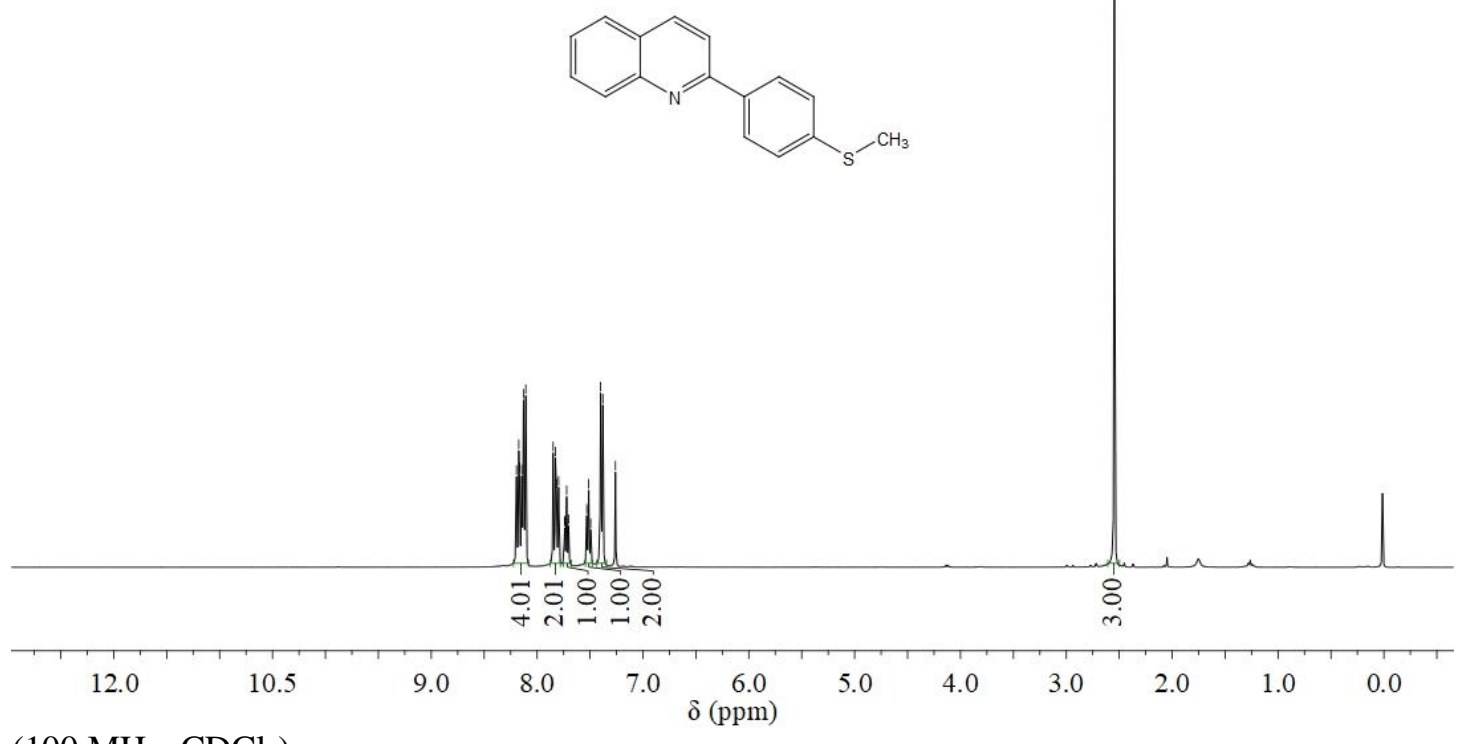

${ }^{13} \mathrm{C} \mathrm{NMR}\left(100 \mathrm{MHz}, \mathrm{CDCl}_{3}\right)$

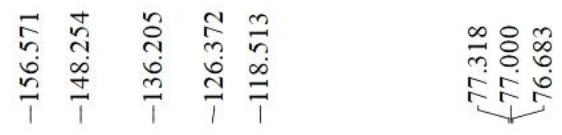

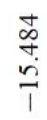
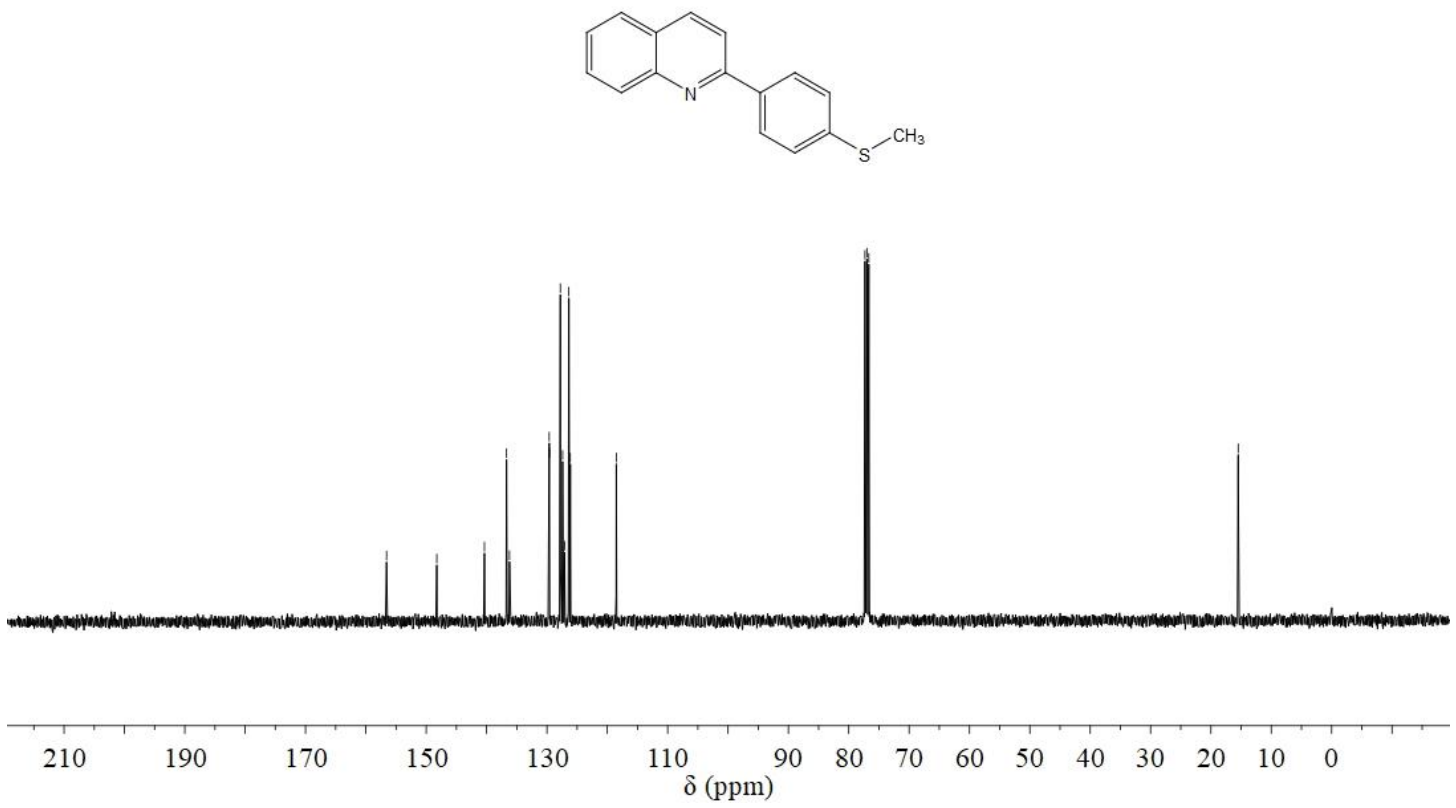

48 
${ }^{1} \mathrm{H}$ NMR (400 MHz, $\left.\mathrm{CDCl}_{3}\right)$

N,N-dimethyl-4-(quinolin-2-yl)aniline (3h)

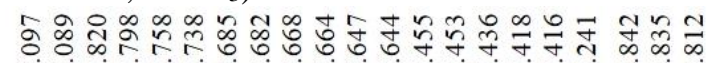

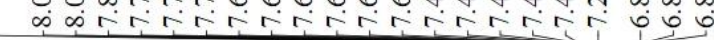
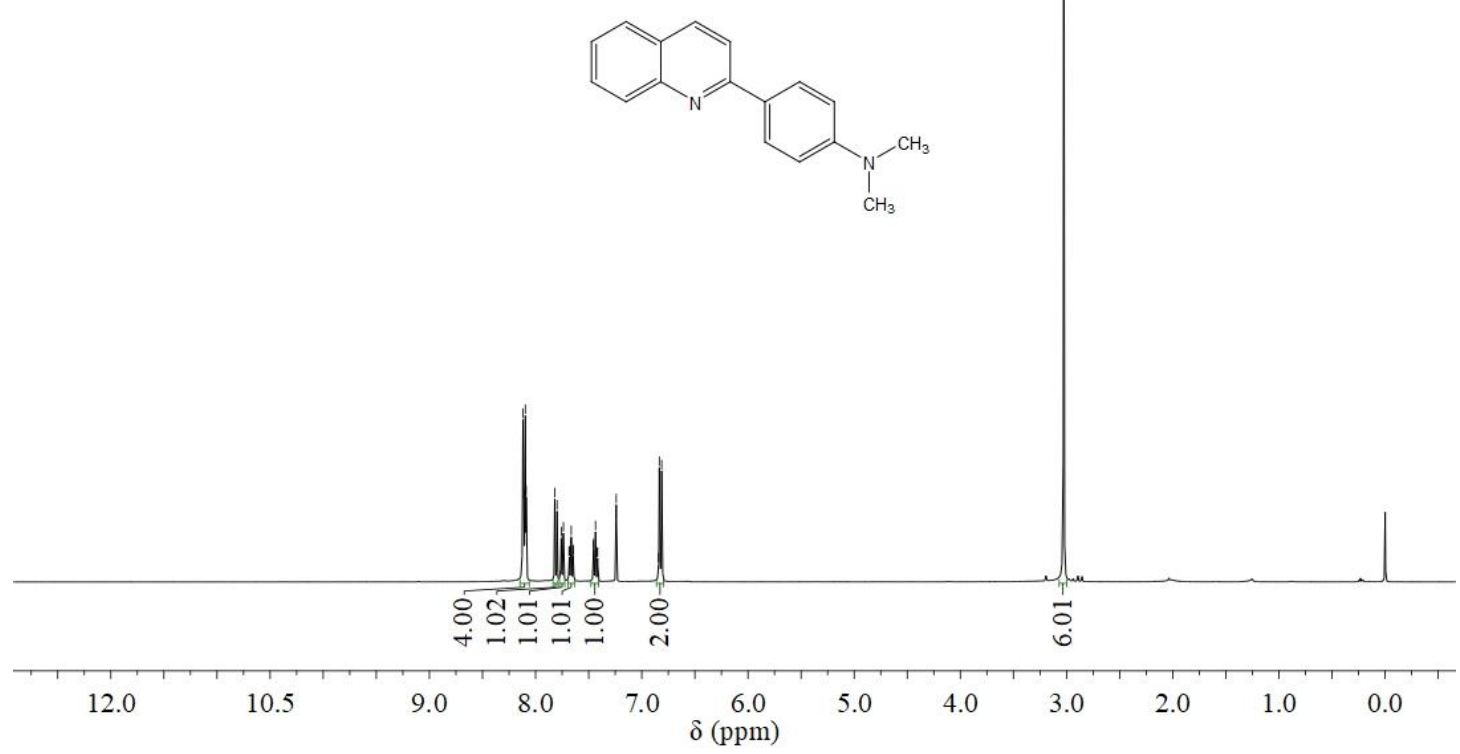

${ }^{13} \mathrm{C}$ NMR (100 MHz, $\left.\mathrm{CDCl}_{3}\right)$

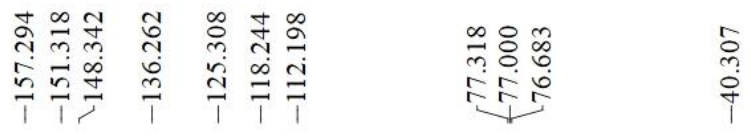
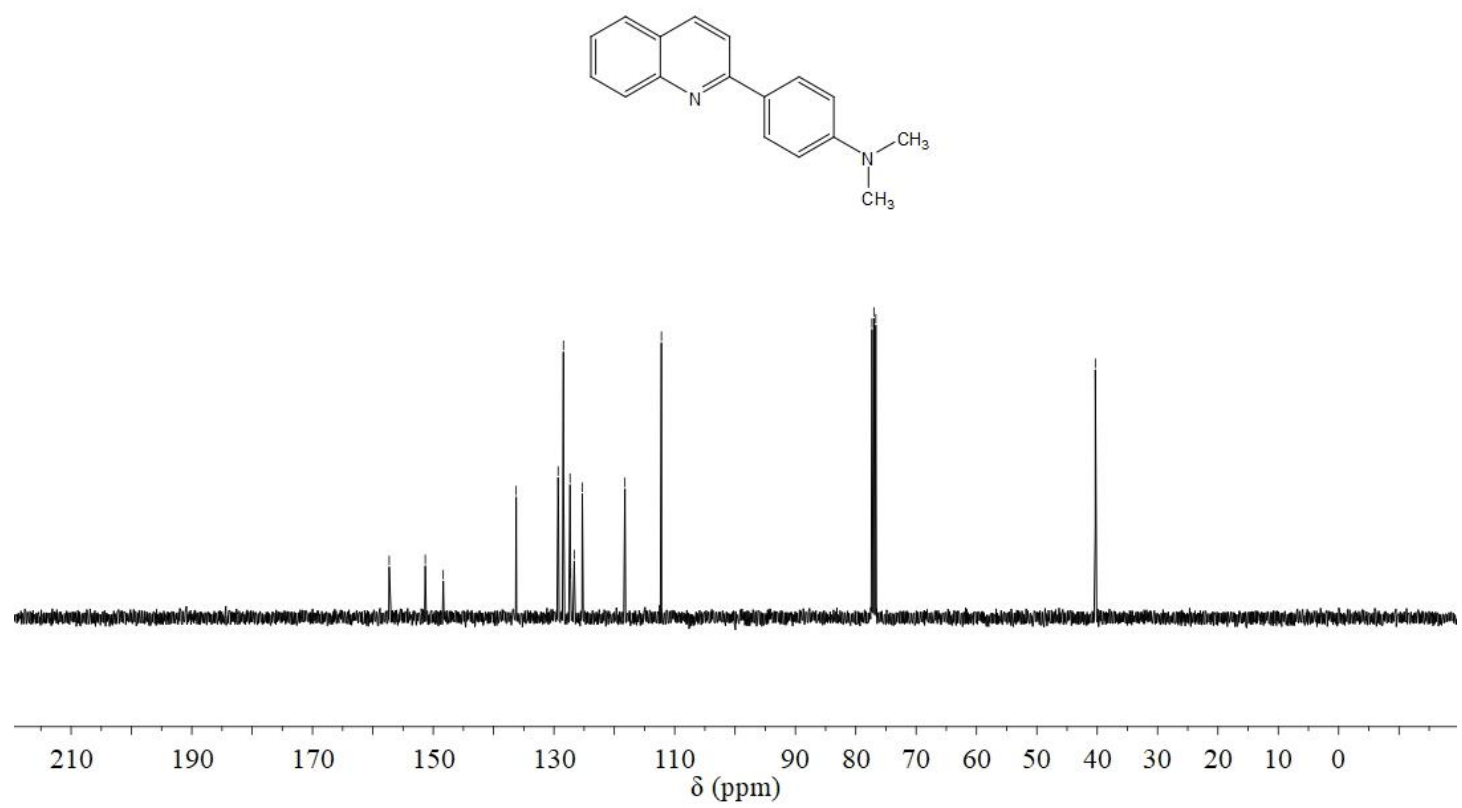
${ }^{1} \mathrm{H}$ NMR (400 MHz, $\left.\mathrm{CDCl}_{3}\right)$

\section{4-(4-(Quinolin-2-yl)benzyl)morpholine (3i)}

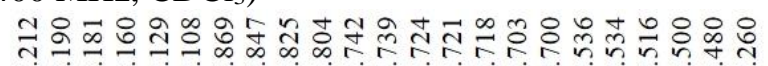

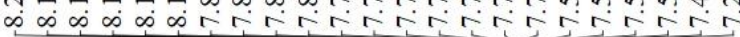

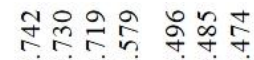

लुखिक तin
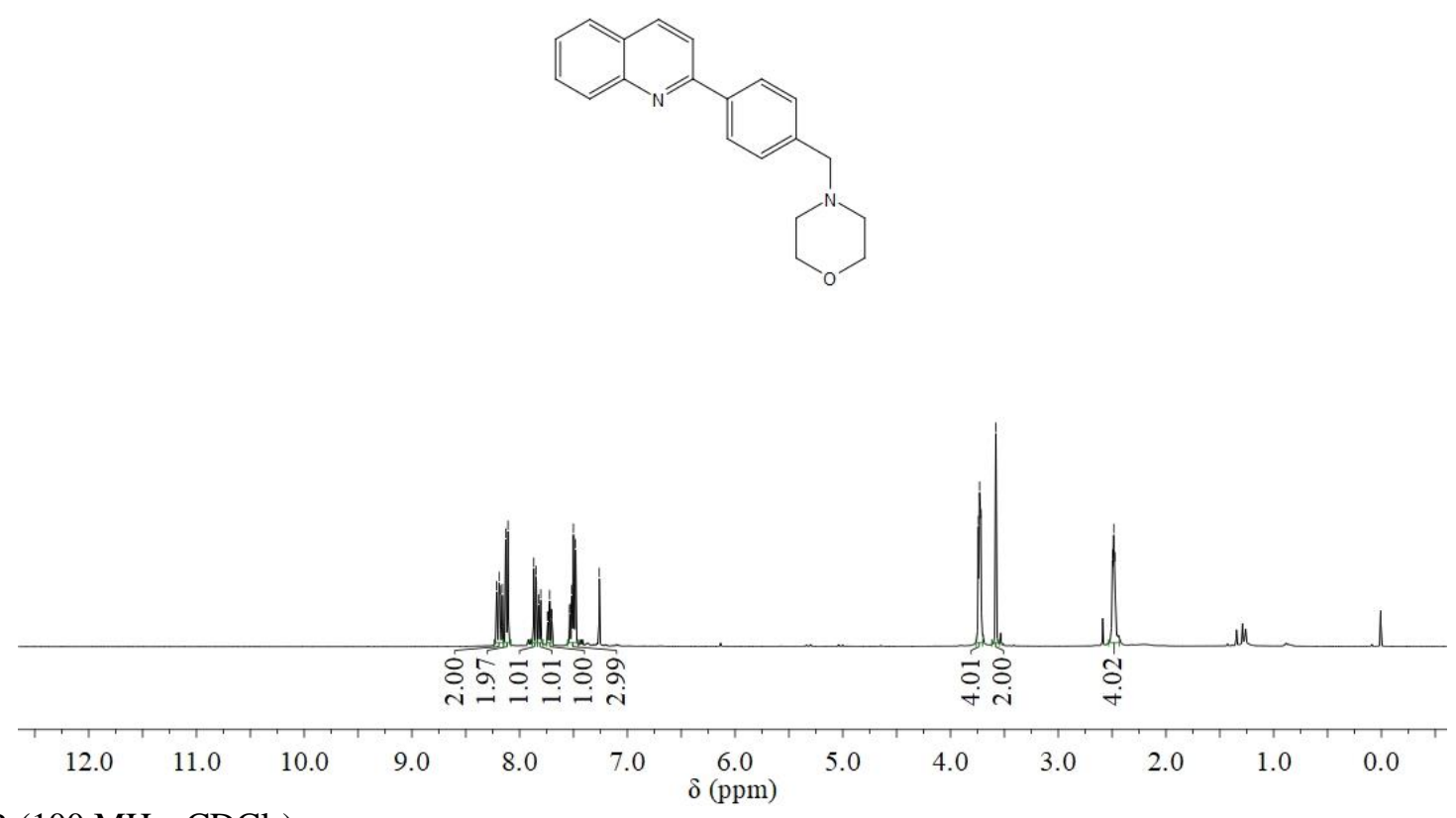

${ }^{13} \mathrm{C}$ NMR $\left(100 \mathrm{MHz}, \mathrm{CDCl}_{3}\right)$

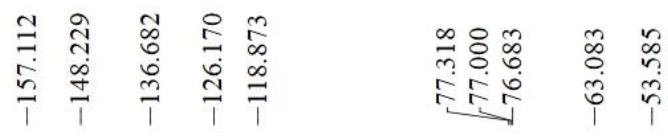

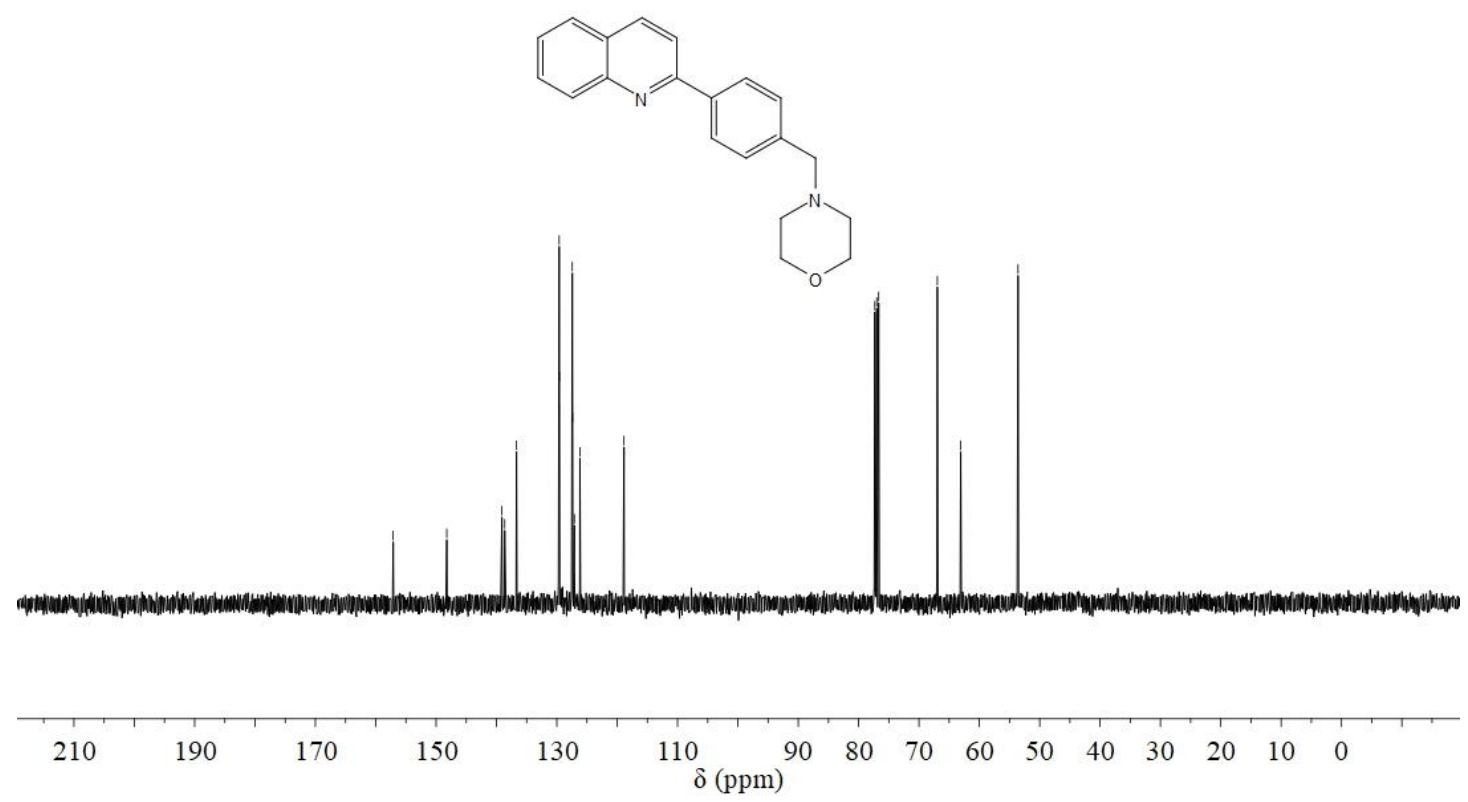


${ }^{1} \mathrm{H}$ NMR $\left(400 \mathrm{MHz}, \mathrm{CDCl}_{3}\right)$

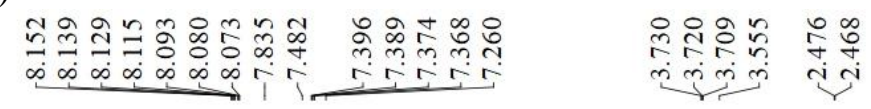<smiles>Fc1ccc2nc(-c3ccc(CN4CCOCC4)cc3)ccc2c1</smiles>

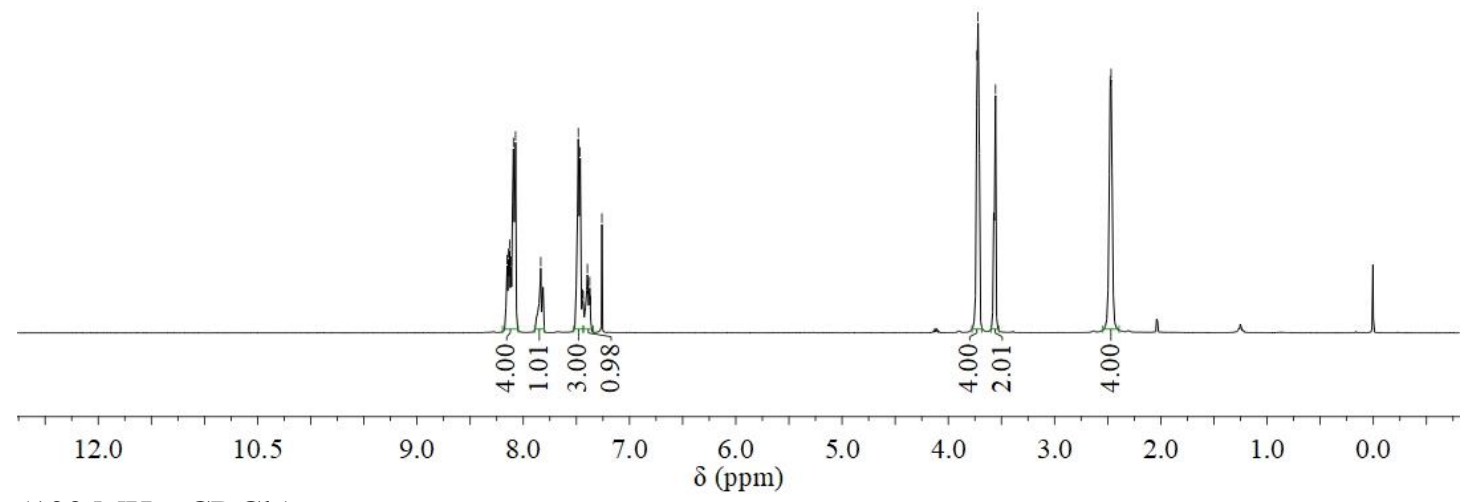

${ }^{13} \mathrm{C} \mathrm{NMR}\left(100 \mathrm{MHz}, \mathrm{CDCl}_{3}\right)$

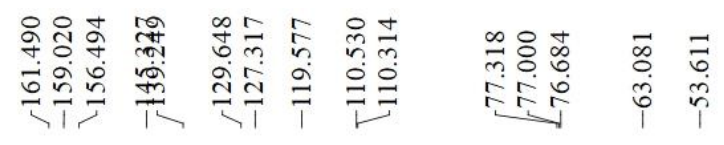
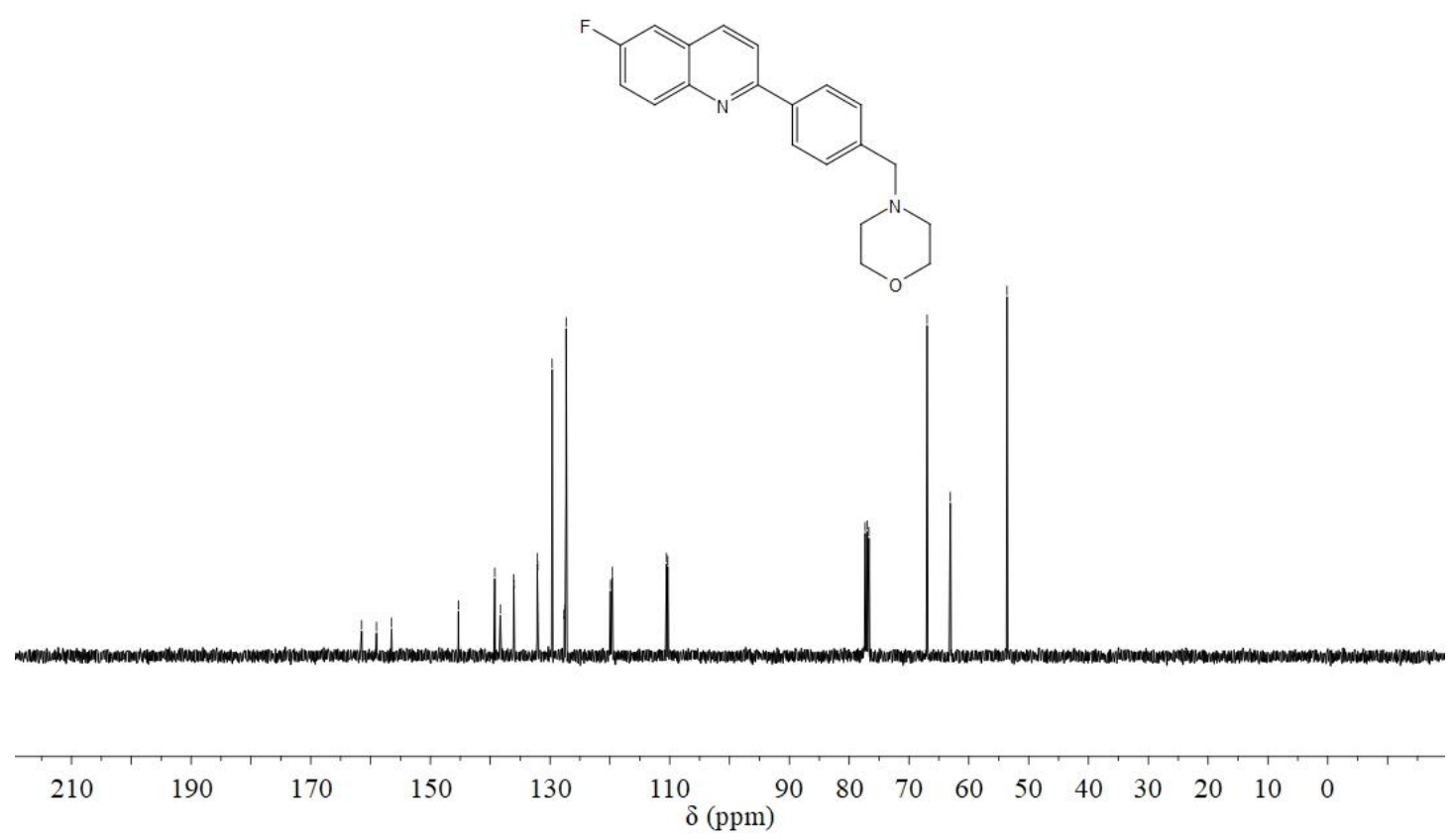

${ }^{19} \mathrm{~F}$ NMR (376 MHz, $\left.\mathrm{CDCl}_{3}\right)$ 


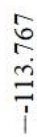

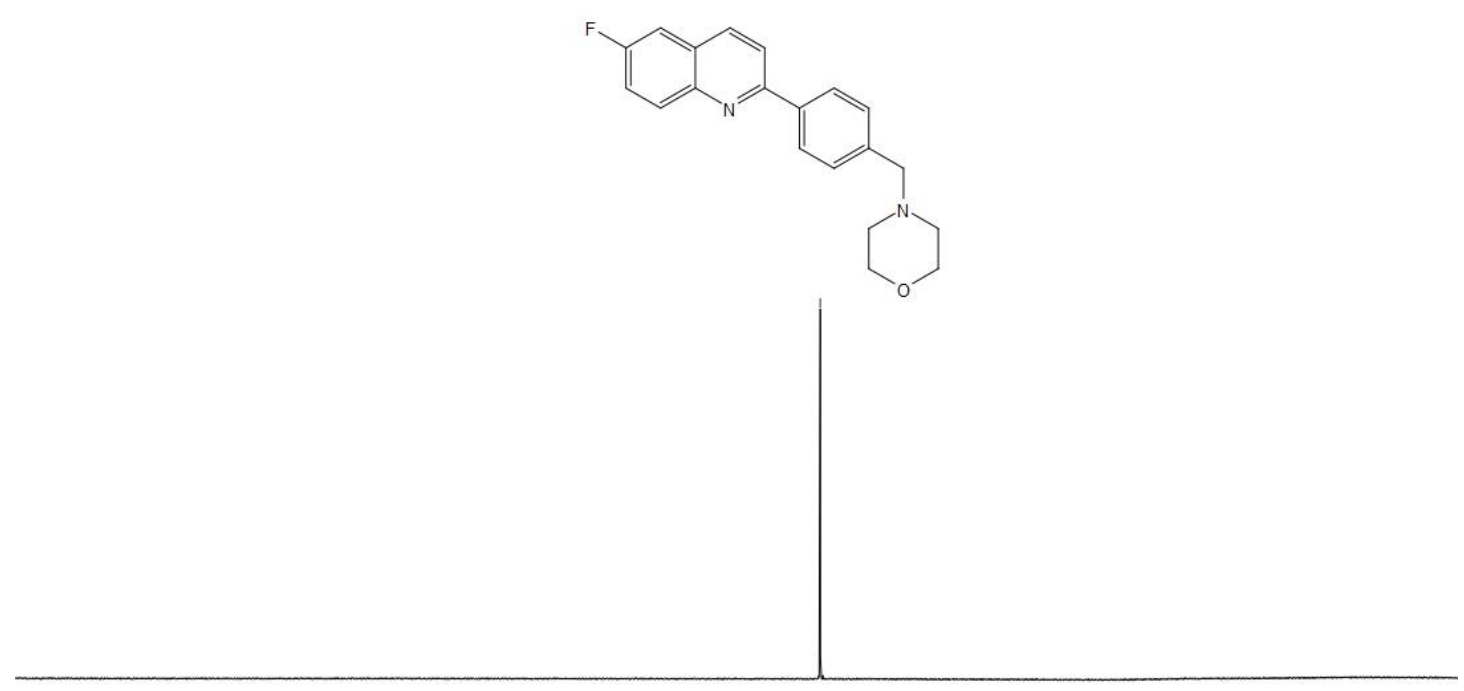

\begin{tabular}{|c|c|c|c|c|c|c|c|c|c|c|c|}
\hline 10 & 0 & -20 & -40 & -60 & -80 & $\begin{array}{c}-100 \\
\delta(\mathrm{ppm})\end{array}$ & -120 & -140 & -160 & -180 & -200 \\
\hline
\end{tabular}


${ }^{1} \mathrm{H}$ NMR (400 MHz, $\left.\mathrm{CDCl}_{3}\right)$

Ethyl 4-(quinolin-2-yl)benzoate (3k)

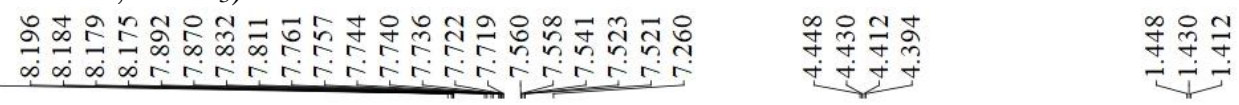<smiles>CCOC(=O)c1ccc(-c2ccc3ccccc3n2)cc1</smiles>

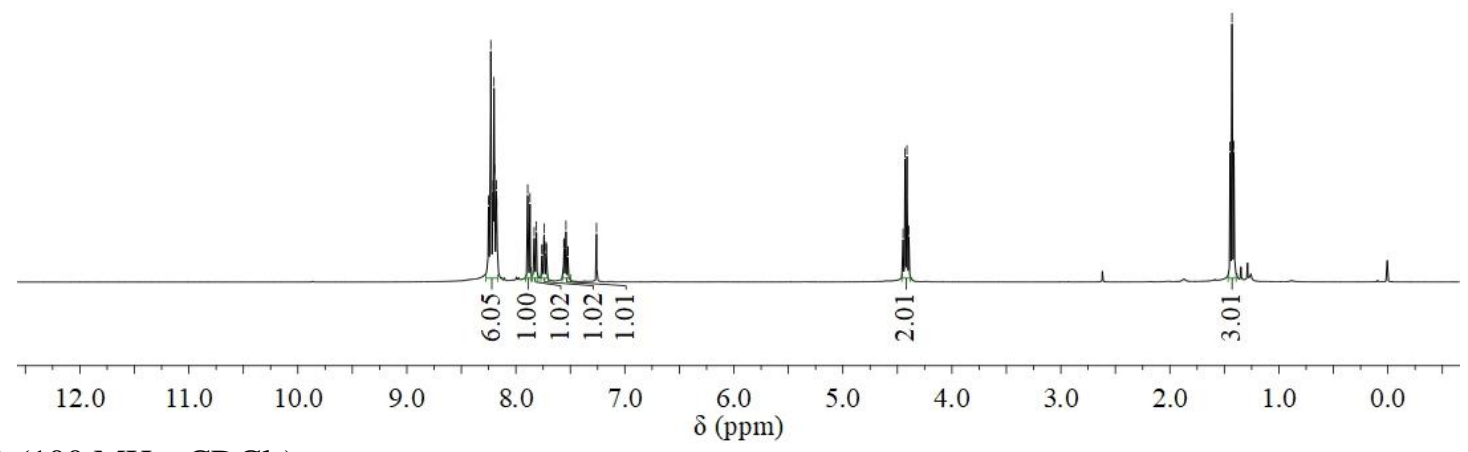

${ }^{13} \mathrm{C}$ NMR $\left(100 \mathrm{MHz}, \mathrm{CDCl}_{3}\right)$
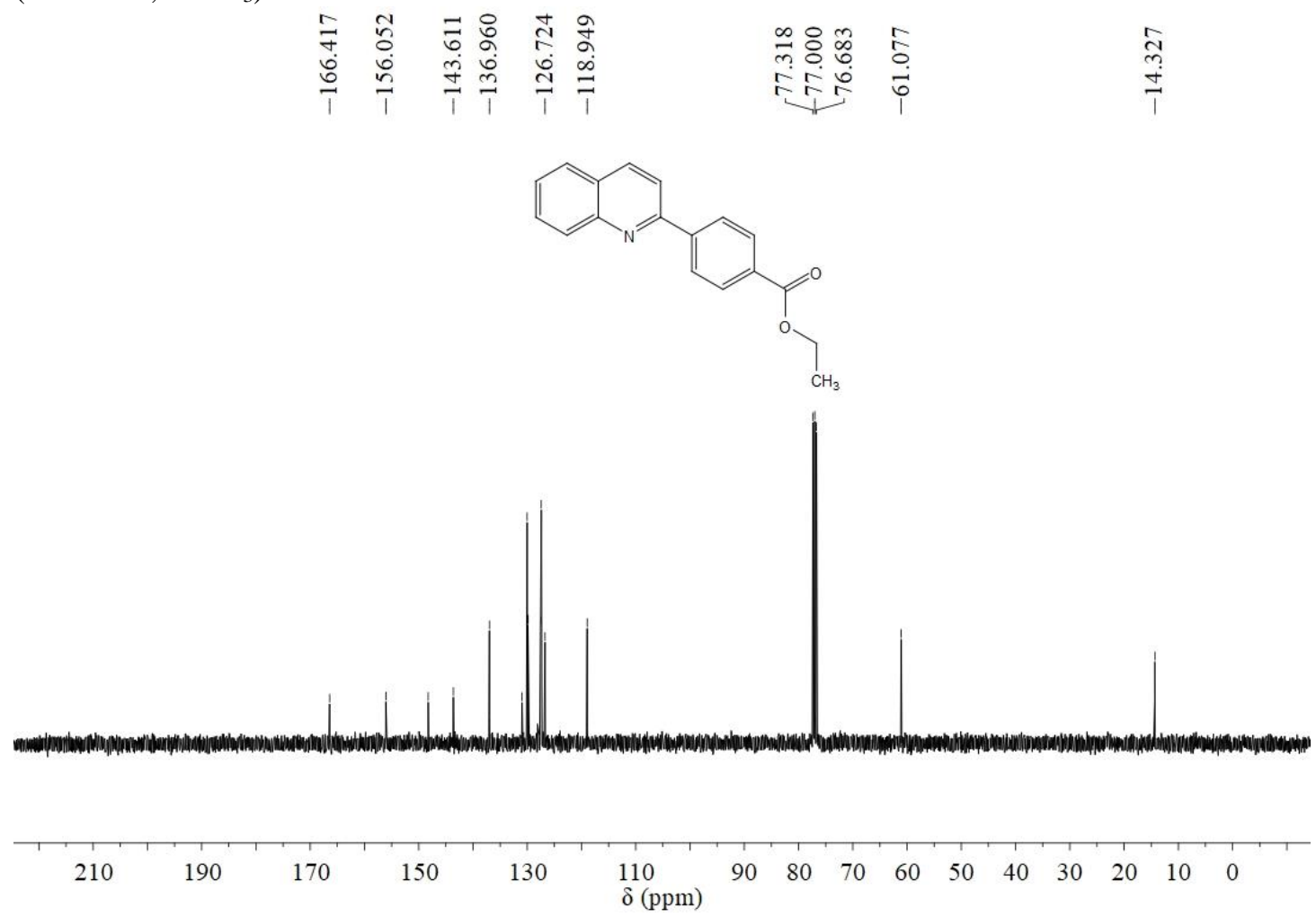
${ }^{1} \mathrm{H}$ NMR (400 MHz, $\left.\mathrm{CDCl}_{3}\right)$

2-(4-(Trifluoromethyl)phenyl)quinolone (3I)

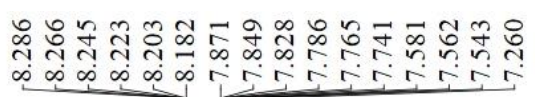
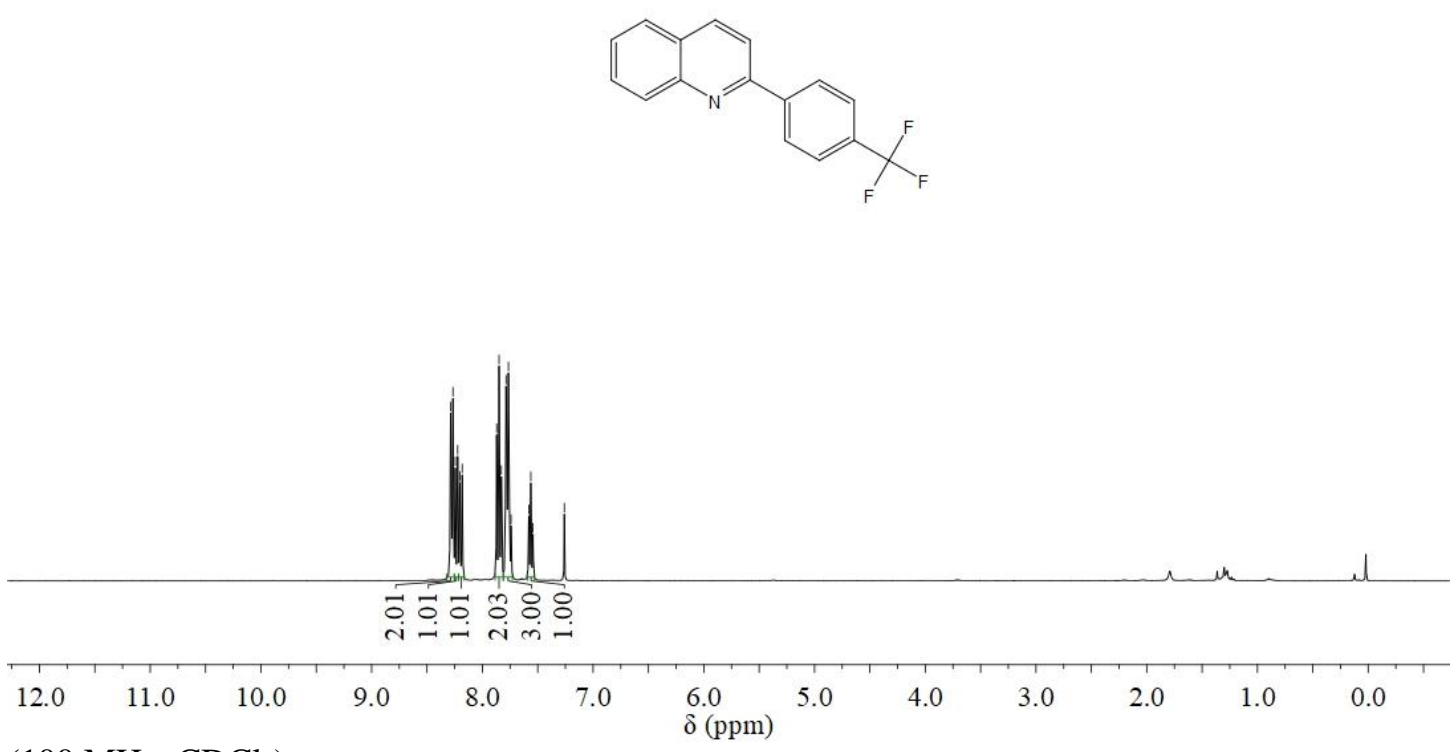

${ }^{13} \mathrm{C} \mathrm{NMR}\left(100 \mathrm{MHz}, \mathrm{CDCl}_{3}\right)$
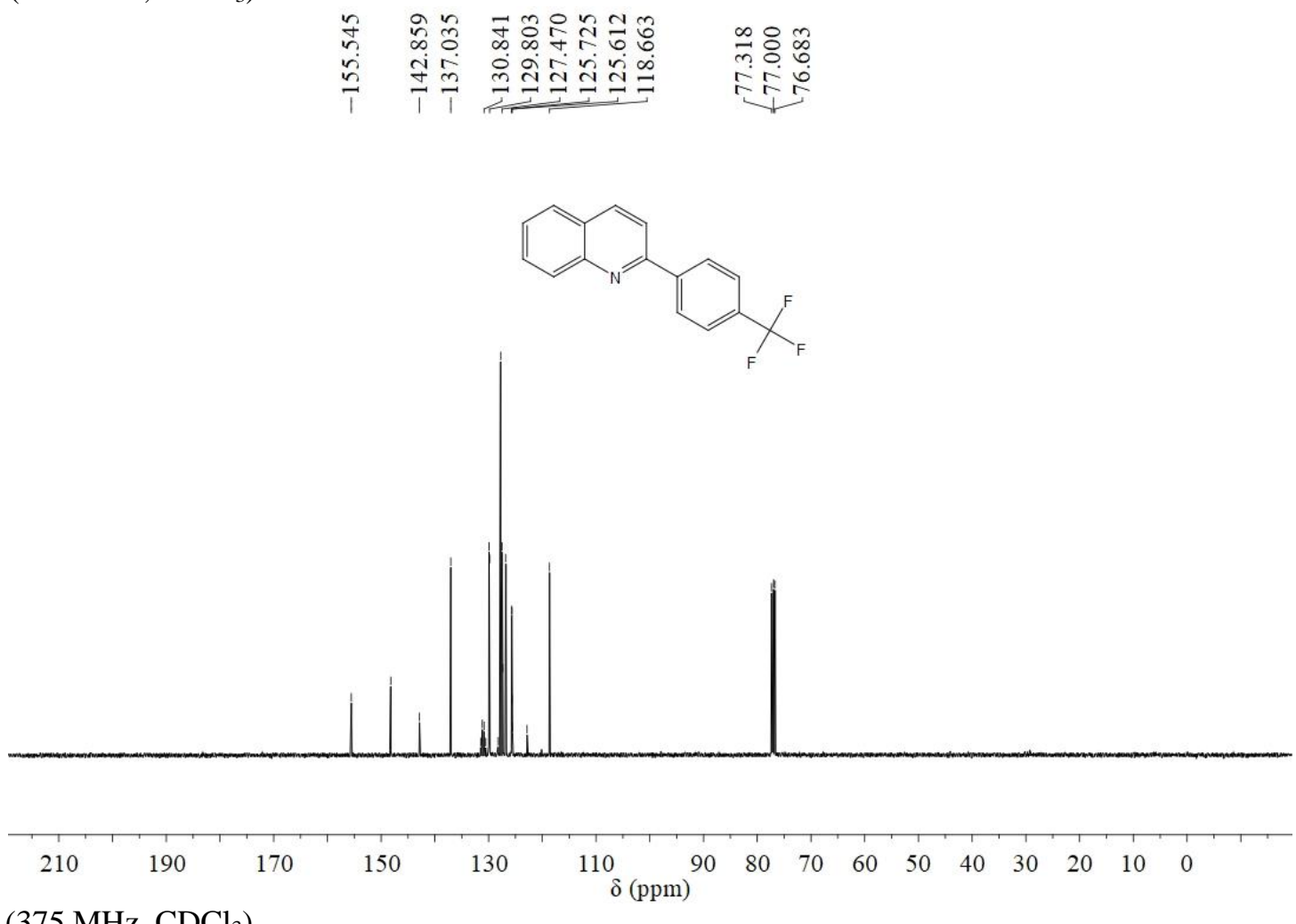

${ }^{19} \mathrm{~F}$ NMR (375 MHz, $\left.\mathrm{CDCl}_{3}\right)$

54 
\pm
กิ
i

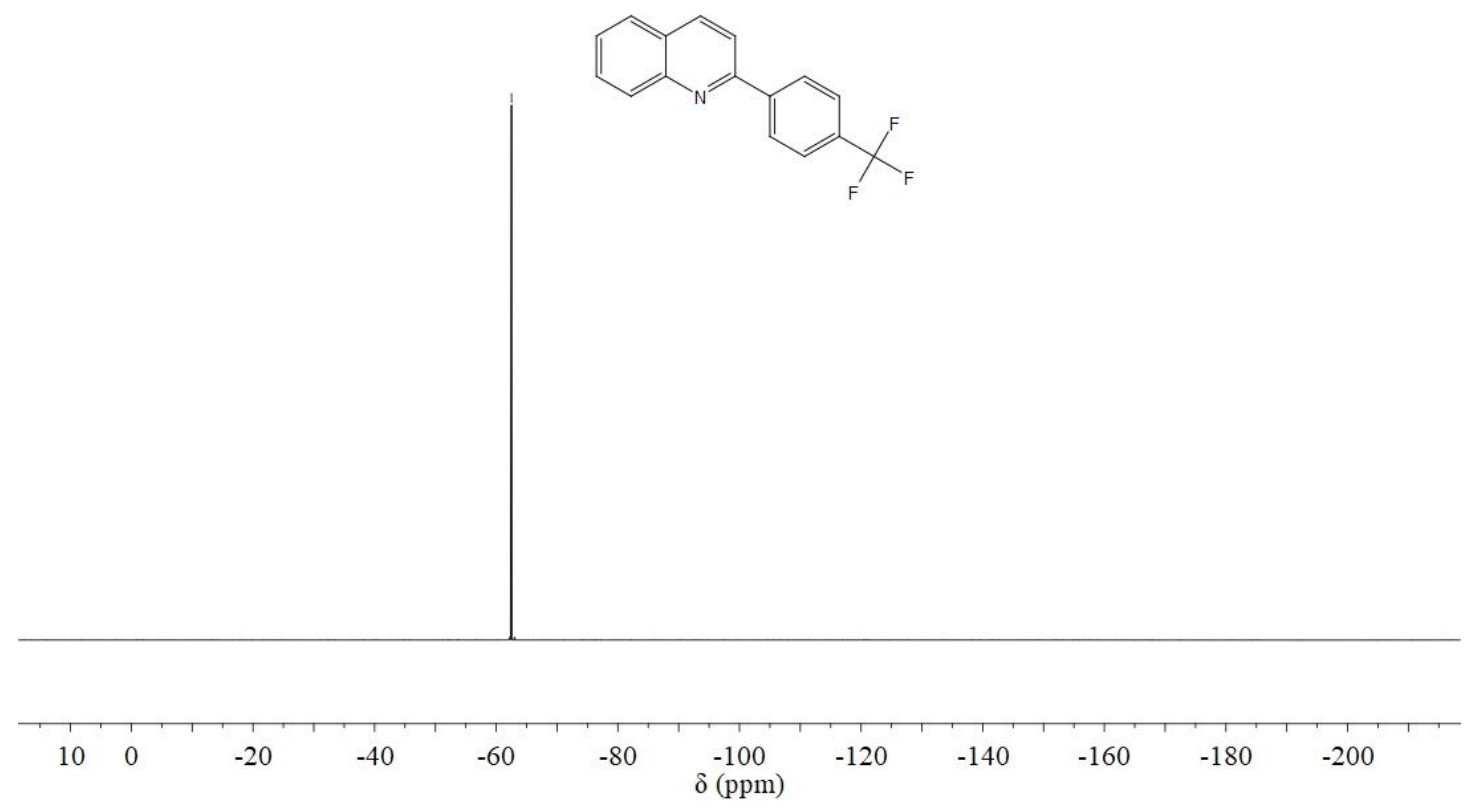


${ }^{1} \mathrm{H}$ NMR (400 MHz, $\left.\mathrm{CDCl}_{3}\right)$

4-(Quinolin-2-yl)benzonitrile (3m)

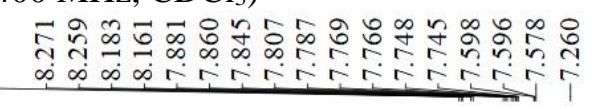
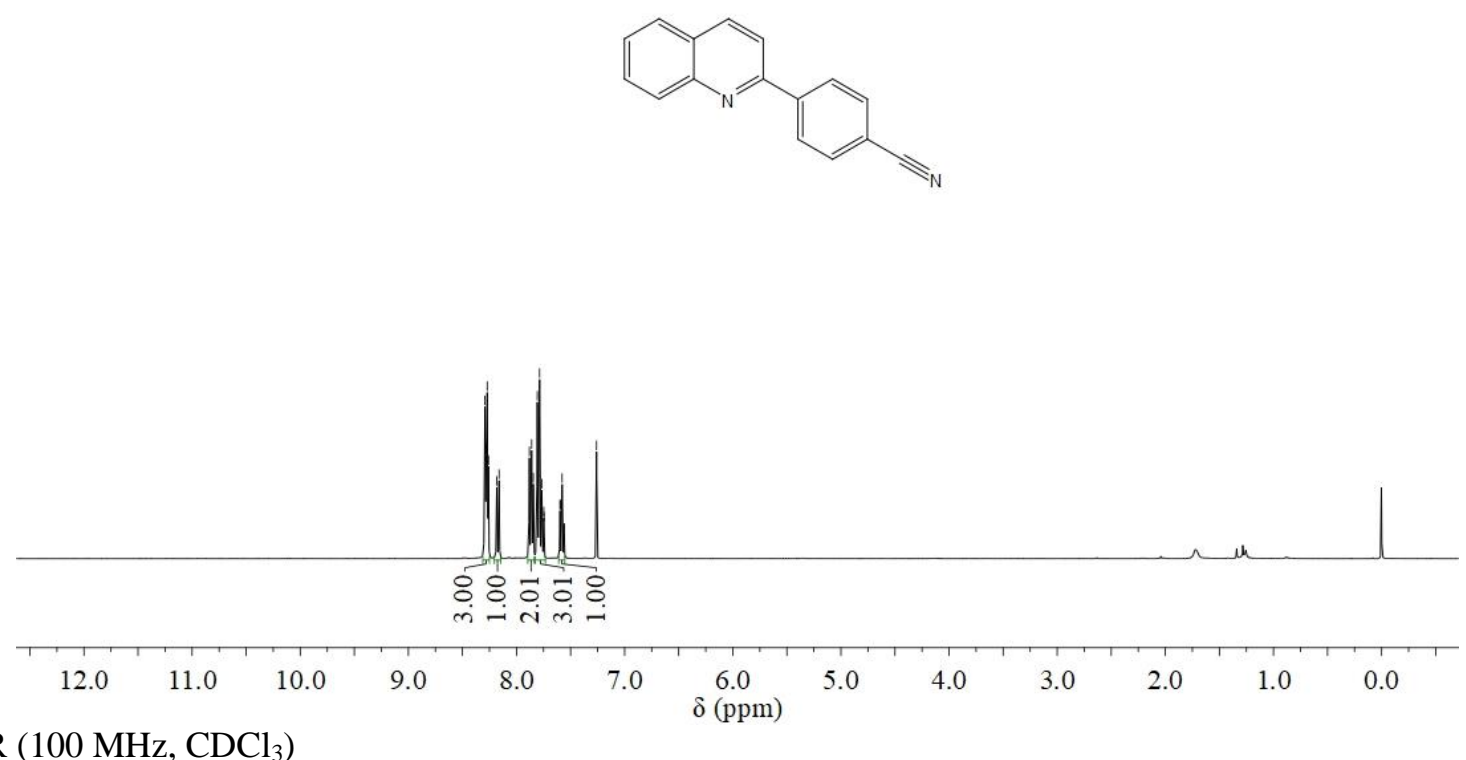

${ }^{13} \mathrm{C}$ NMR (100 MHz, $\left.\mathrm{CDCl}_{3}\right)$

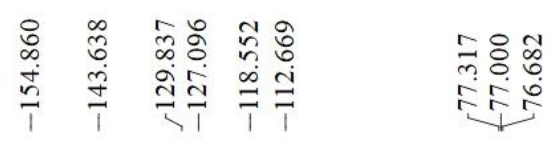
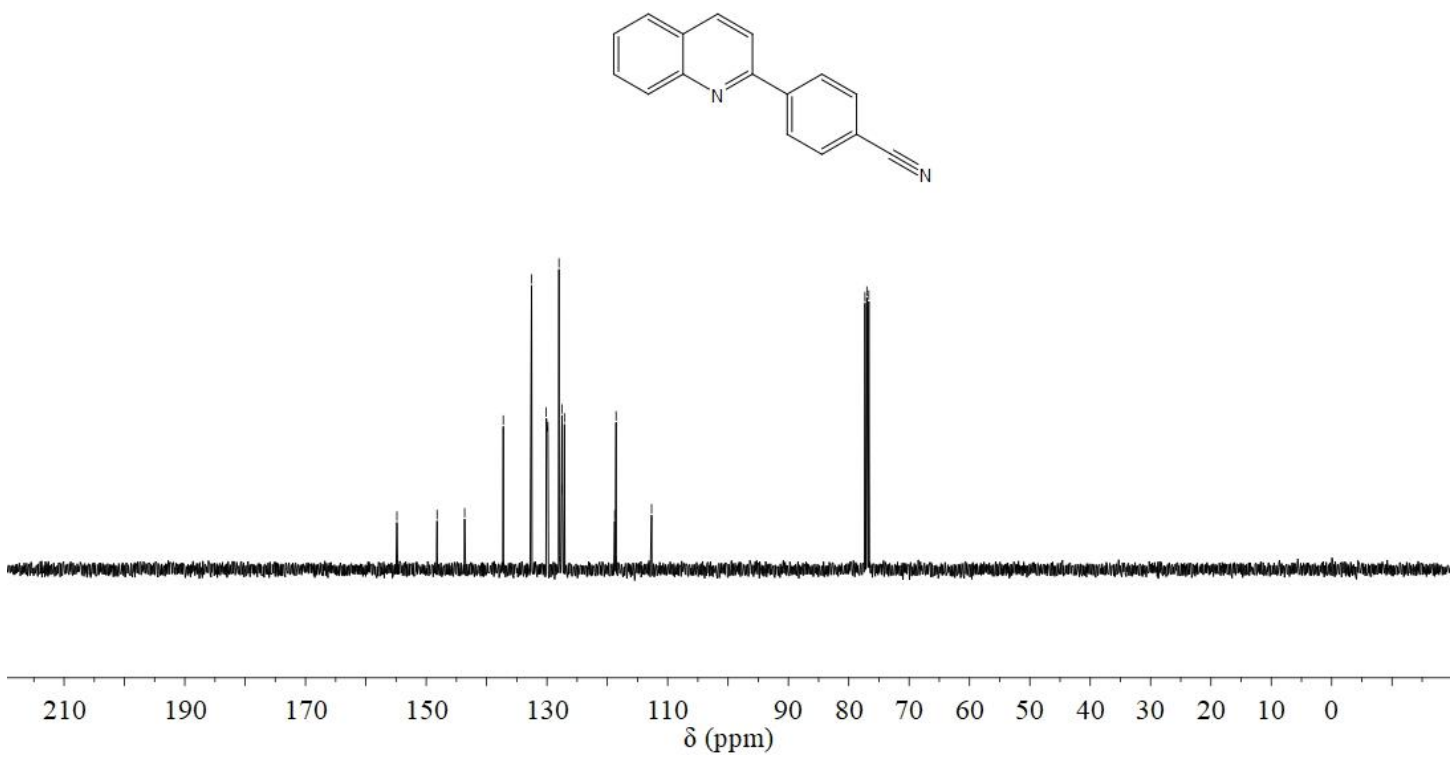

56 
${ }^{1} \mathrm{H}$ NMR (400 MHz, $\mathrm{CDCl}_{3}$ )

2-(4-(Methylsulfonyl)phenyl)quinolone (3n)

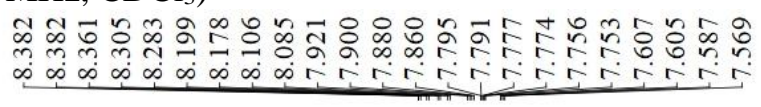

产

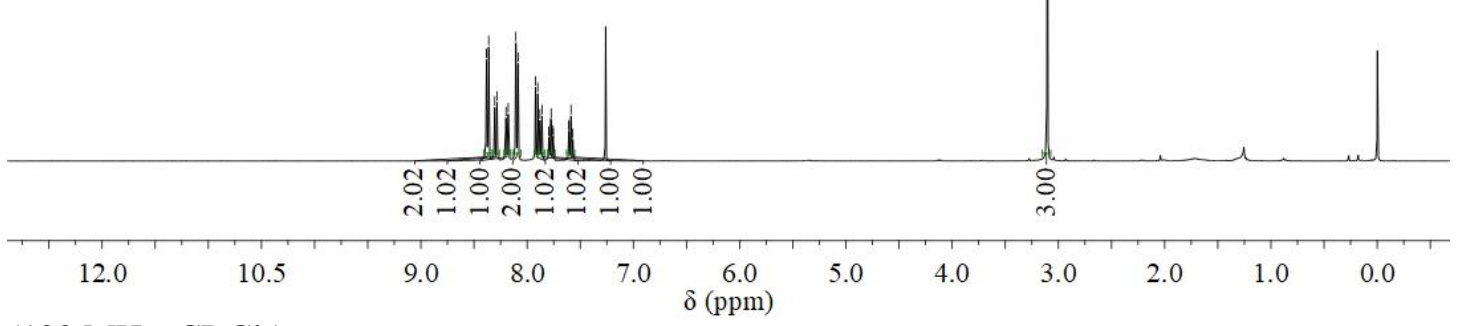

${ }^{13} \mathrm{C} \mathrm{NMR}\left(100 \mathrm{MHz}, \mathrm{CDCl}_{3}\right)$

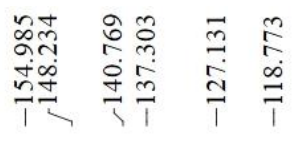

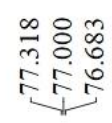

0
$\infty$
$\vdots$
$\vdots$
1
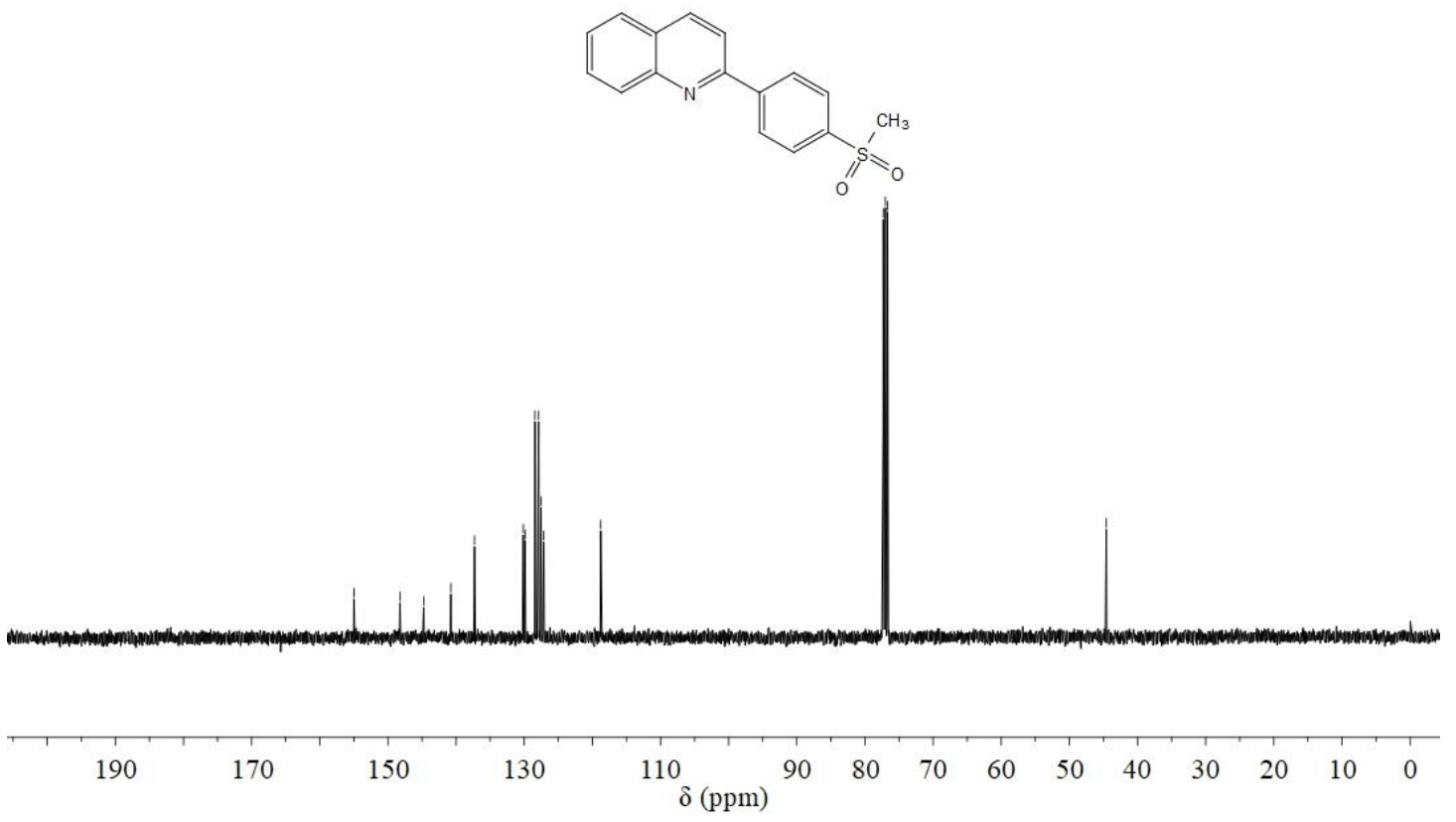

57 
${ }^{1} \mathrm{H}$ NMR (400 MHz, $\mathrm{CDCl}_{3}$ )

\section{2-(Benzo[d][1,3]dioxol-5-yl)quinolone (3o)}

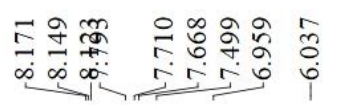
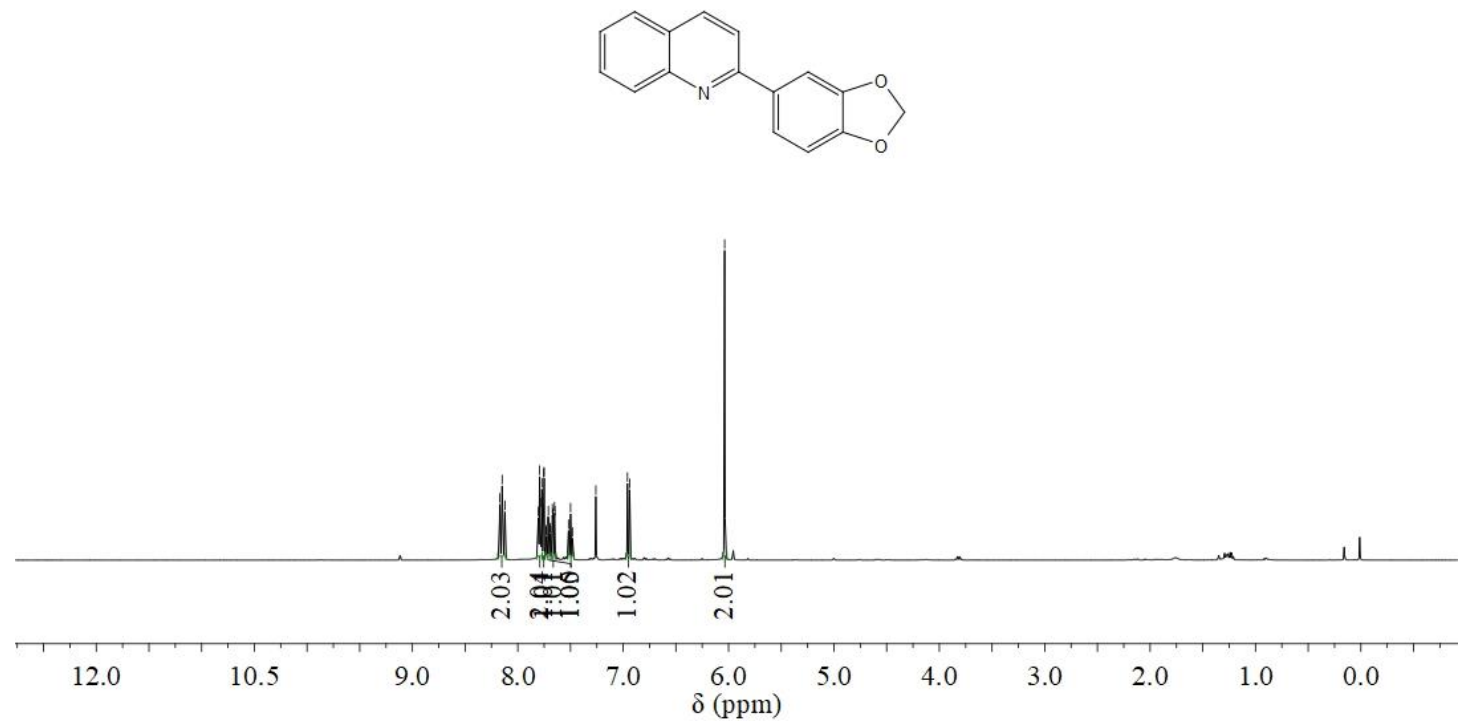

${ }^{13} \mathrm{C}$ NMR $\left(100 \mathrm{MHz}, \mathrm{CDCl}_{3}\right)$

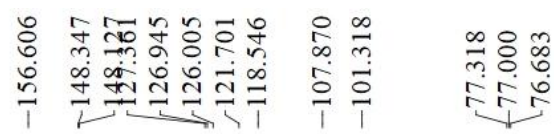
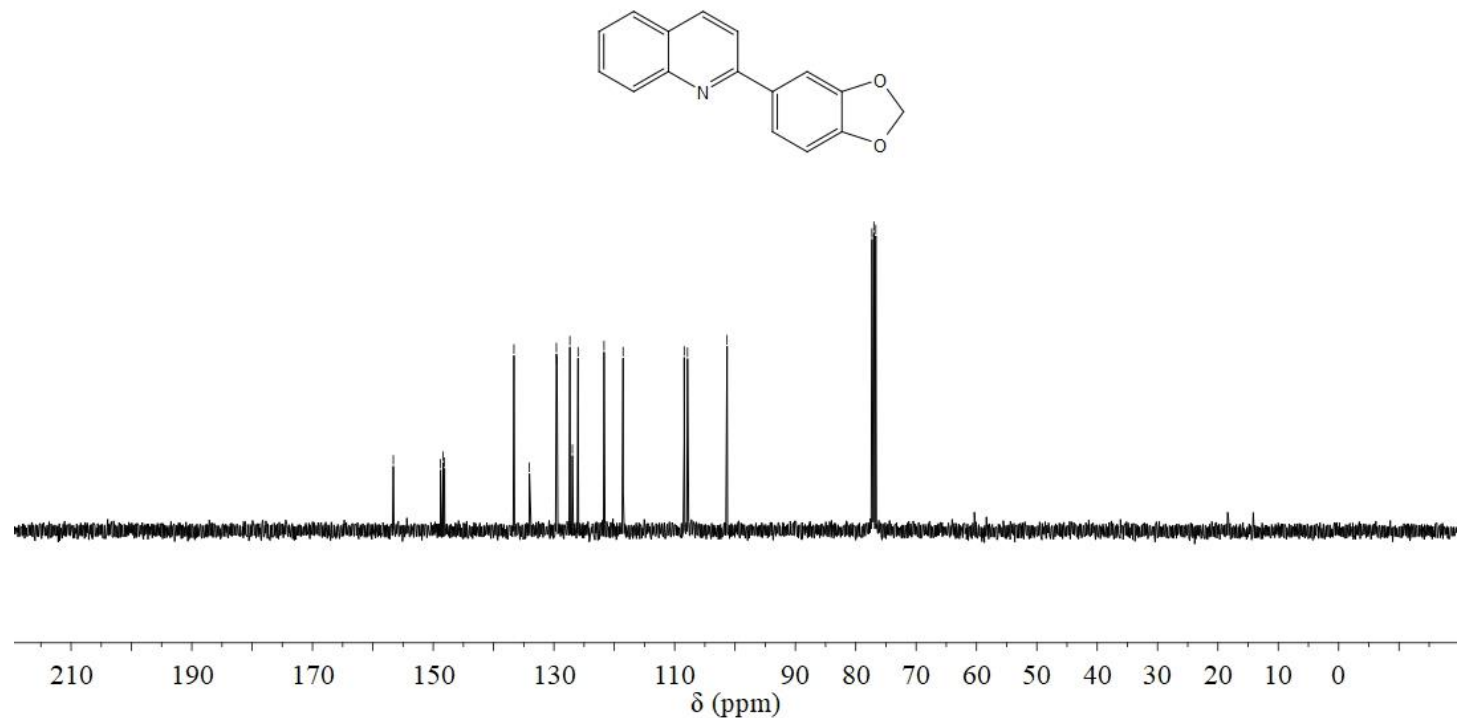
${ }^{1} \mathrm{H}$ NMR (400 MHz, $\mathrm{CDCl}_{3}$ )
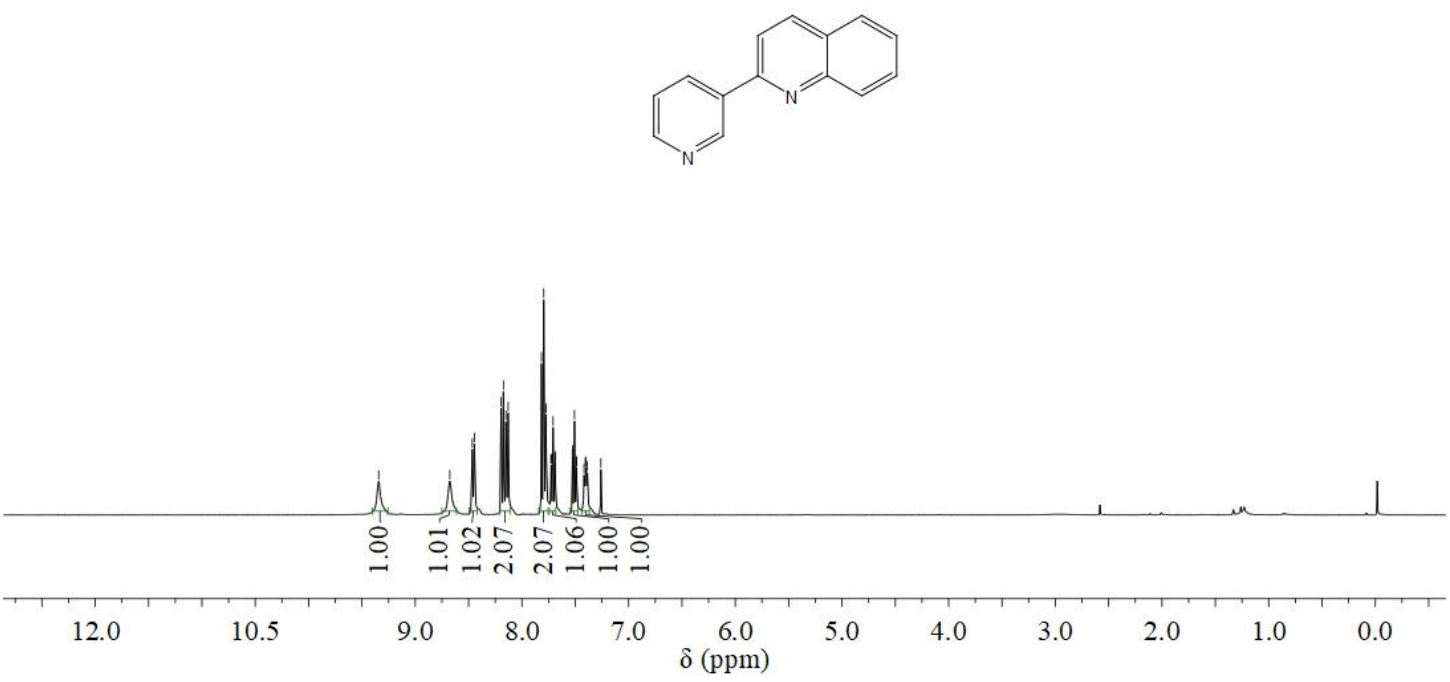

${ }^{13} \mathrm{C} \mathrm{NMR}\left(100 \mathrm{MHz}, \mathrm{CDCl}_{3}\right)$

\begin{tabular}{|c|c|}
\hline 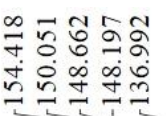 & 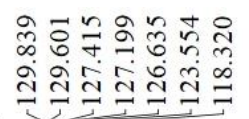 \\
\hline
\end{tabular}<smiles>c1cncc(-c2ccc3ccccc3n2)c1</smiles>
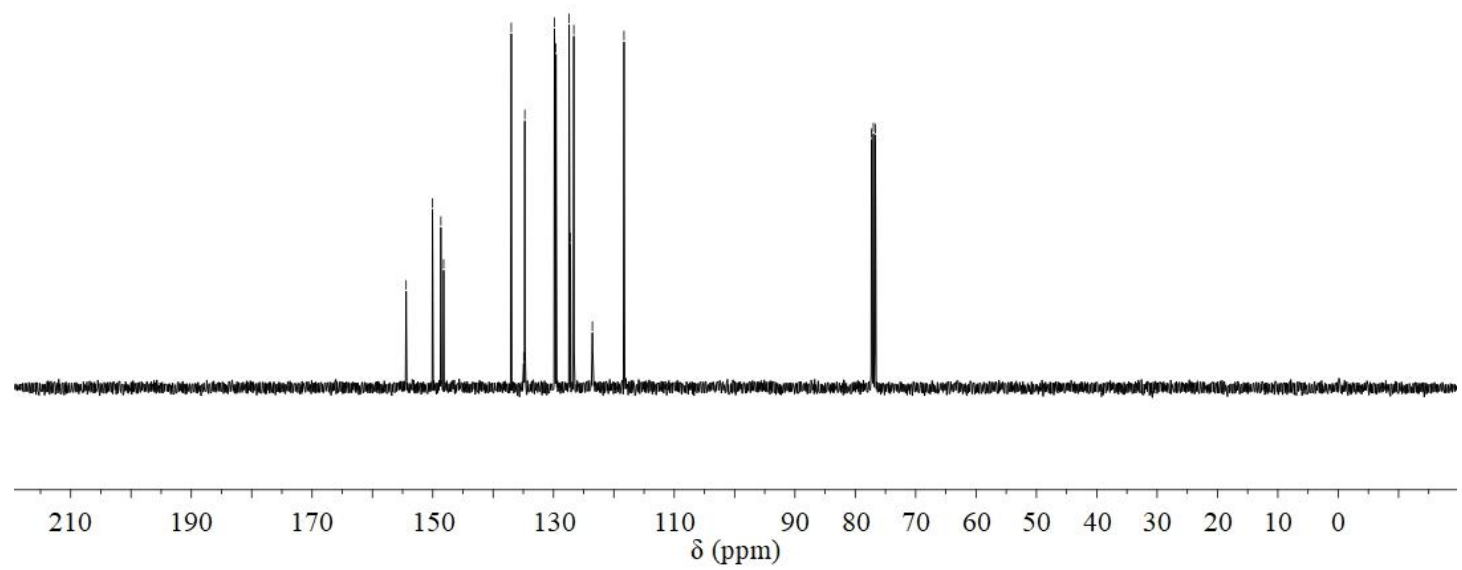
${ }^{1} \mathrm{H}$ NMR (400 MHz, $\mathrm{CDCl}_{3}$ )

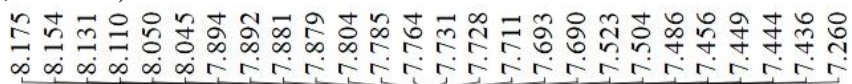
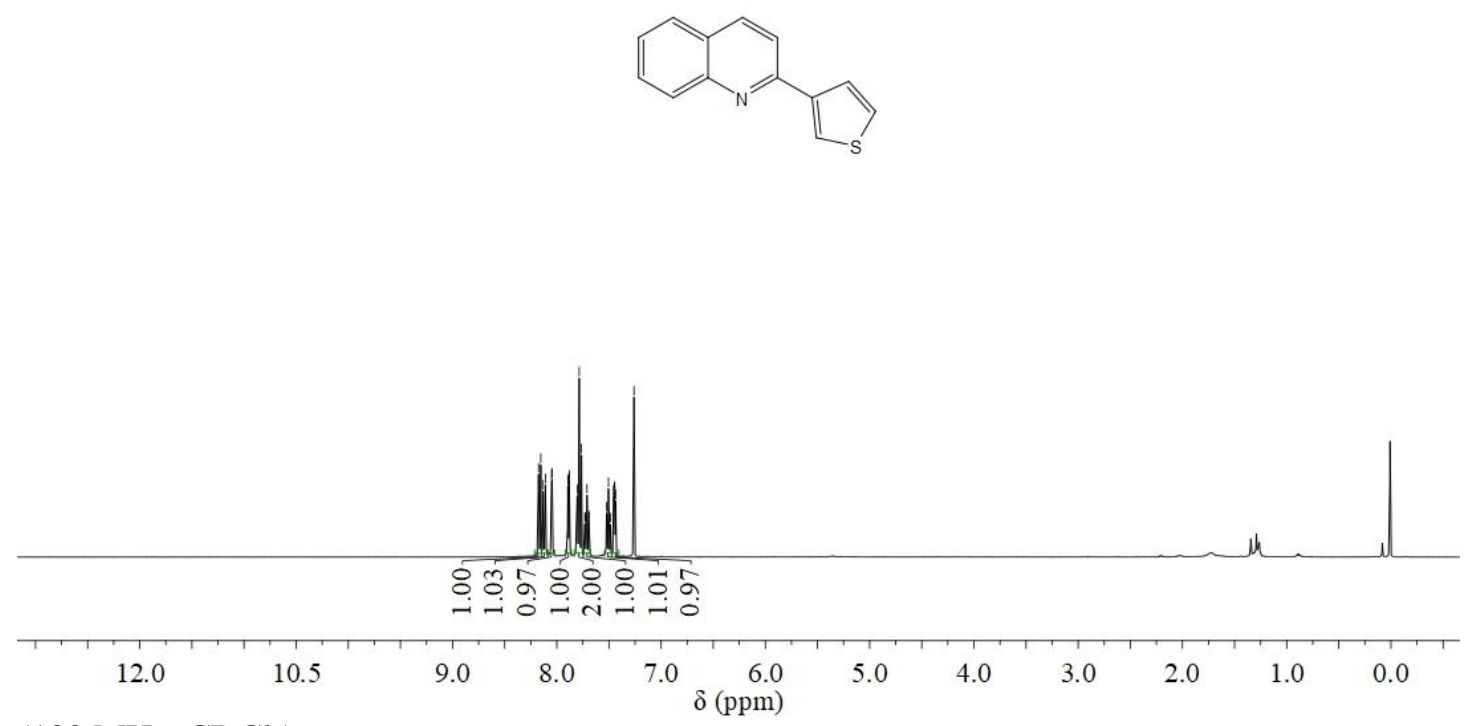

${ }^{13} \mathrm{C} \mathrm{NMR}\left(100 \mathrm{MHz}, \mathrm{CDCl}_{3}\right)$

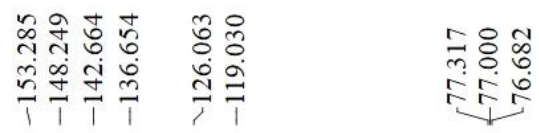
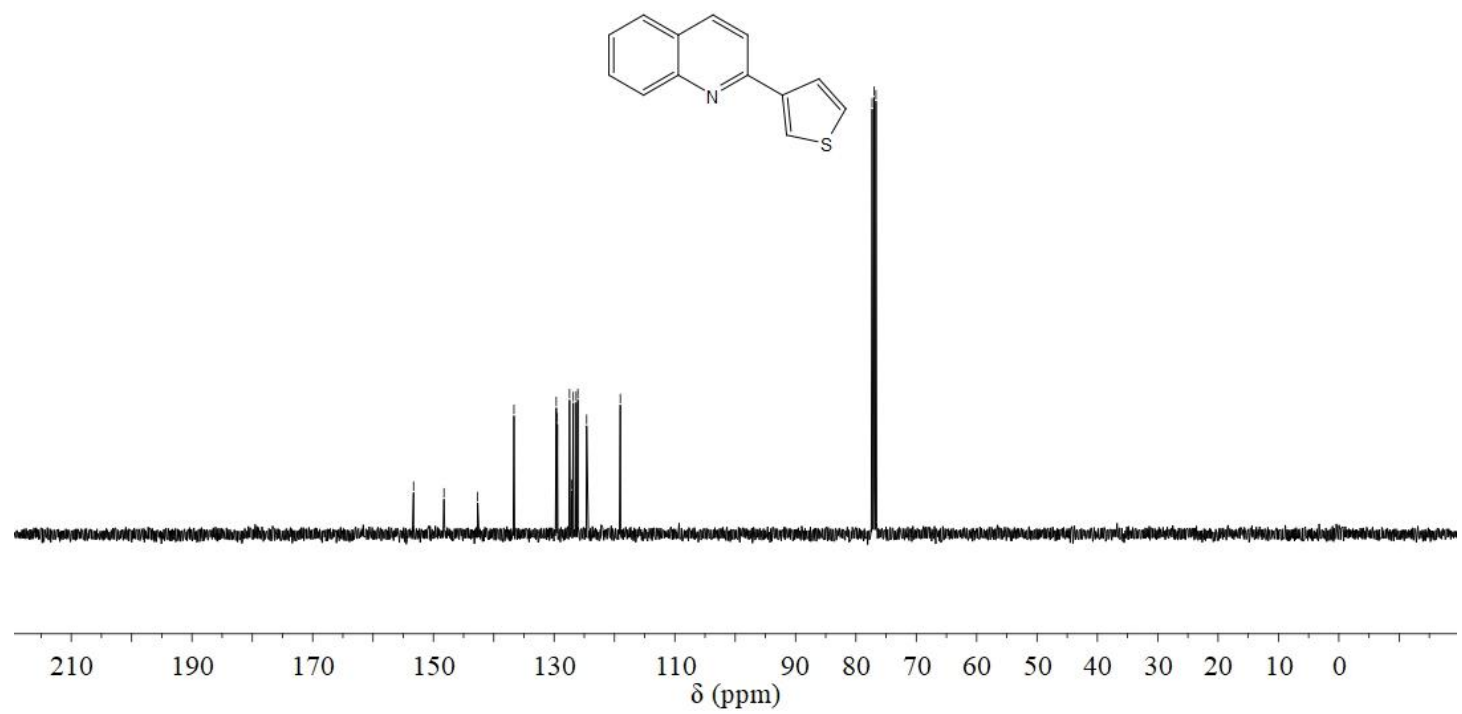
${ }^{1} \mathrm{H}$ NMR (400 MHz, $\left.\mathrm{CDCl}_{3}\right)$
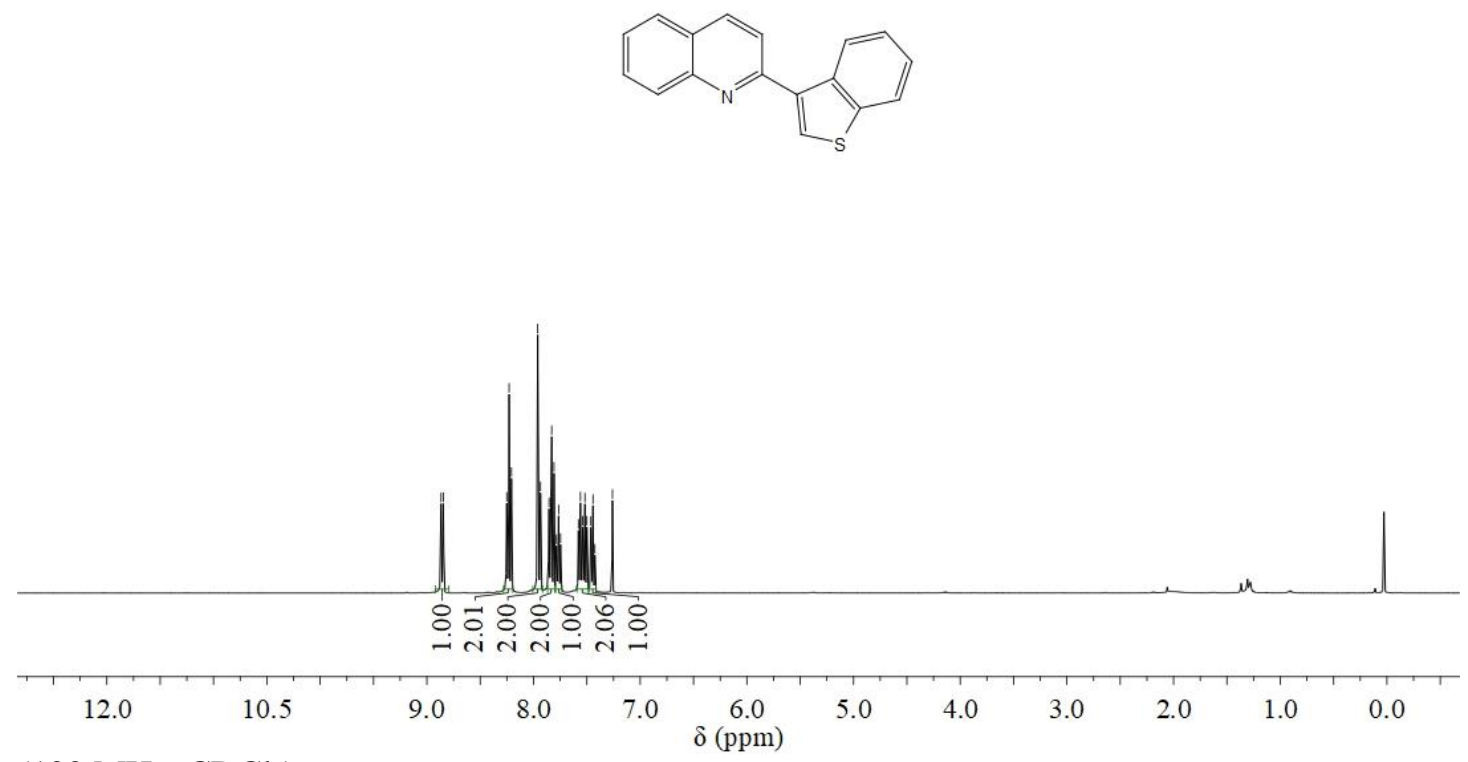

${ }^{13} \mathrm{C} \mathrm{NMR}\left(100 \mathrm{MHz}, \mathrm{CDCl}_{3}\right)$

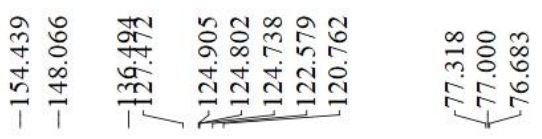
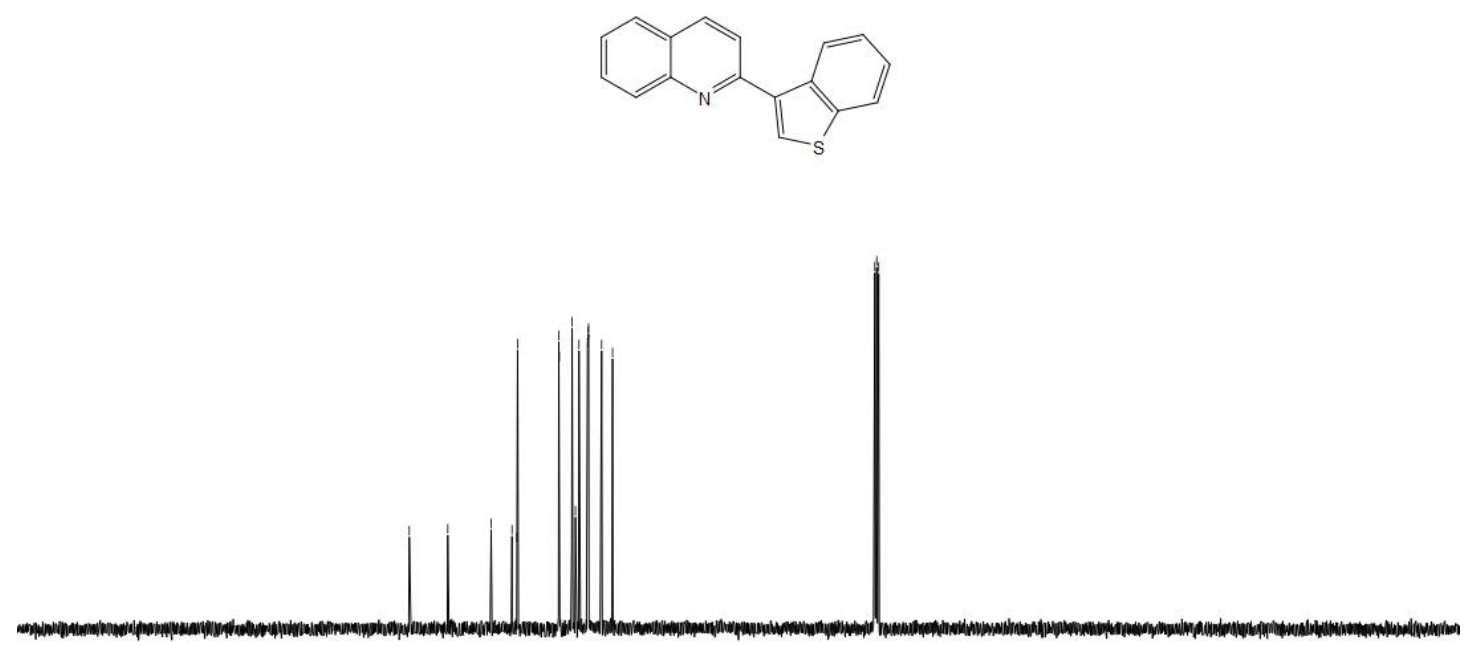

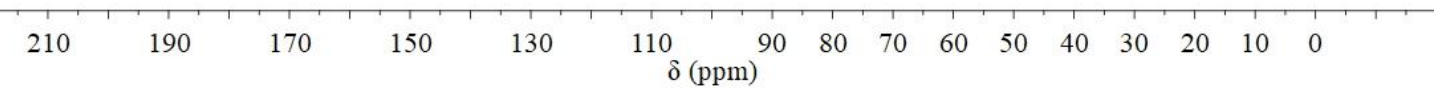


${ }^{1} \mathrm{H}$ NMR (400 MHz, $\left.\mathrm{CDCl}_{3}\right)$

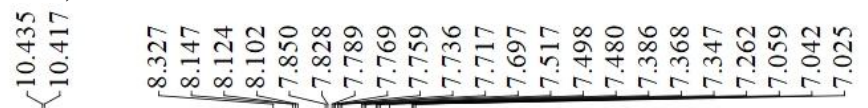
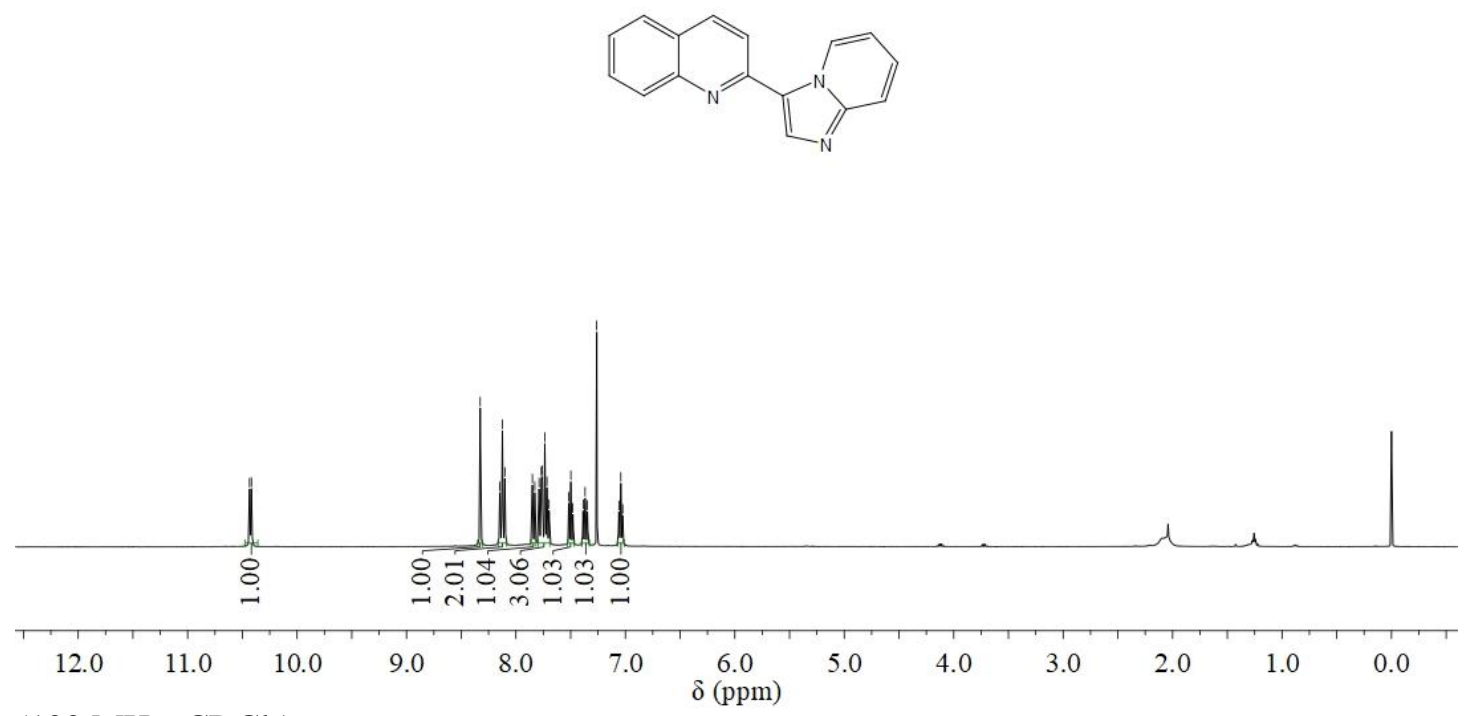

${ }^{13} \mathrm{C}$ NMR $\left(100 \mathrm{MHz}, \mathrm{CDCl}_{3}\right)$

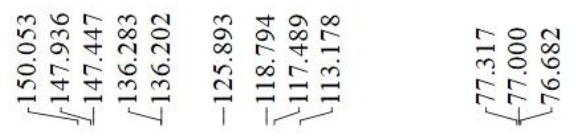
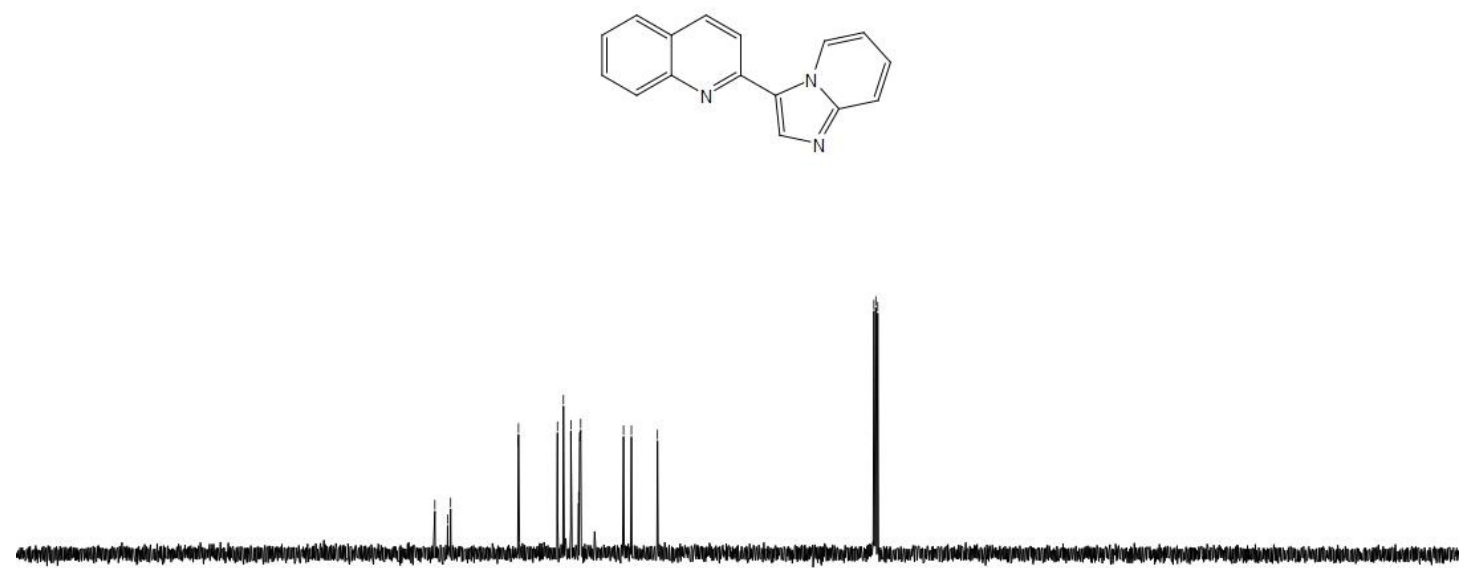

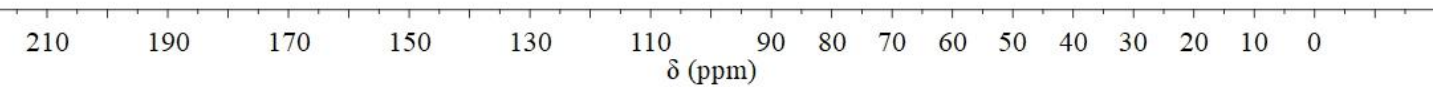




\section{3,5-Dimethyl-4-(quinolin-2-yl)isoxazole (3t)}

${ }^{1} \mathrm{H}$ NMR $\left(400 \mathrm{MHz}, \mathrm{CDCl}_{3}\right)$

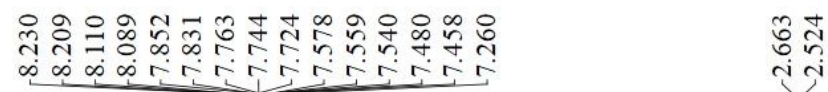

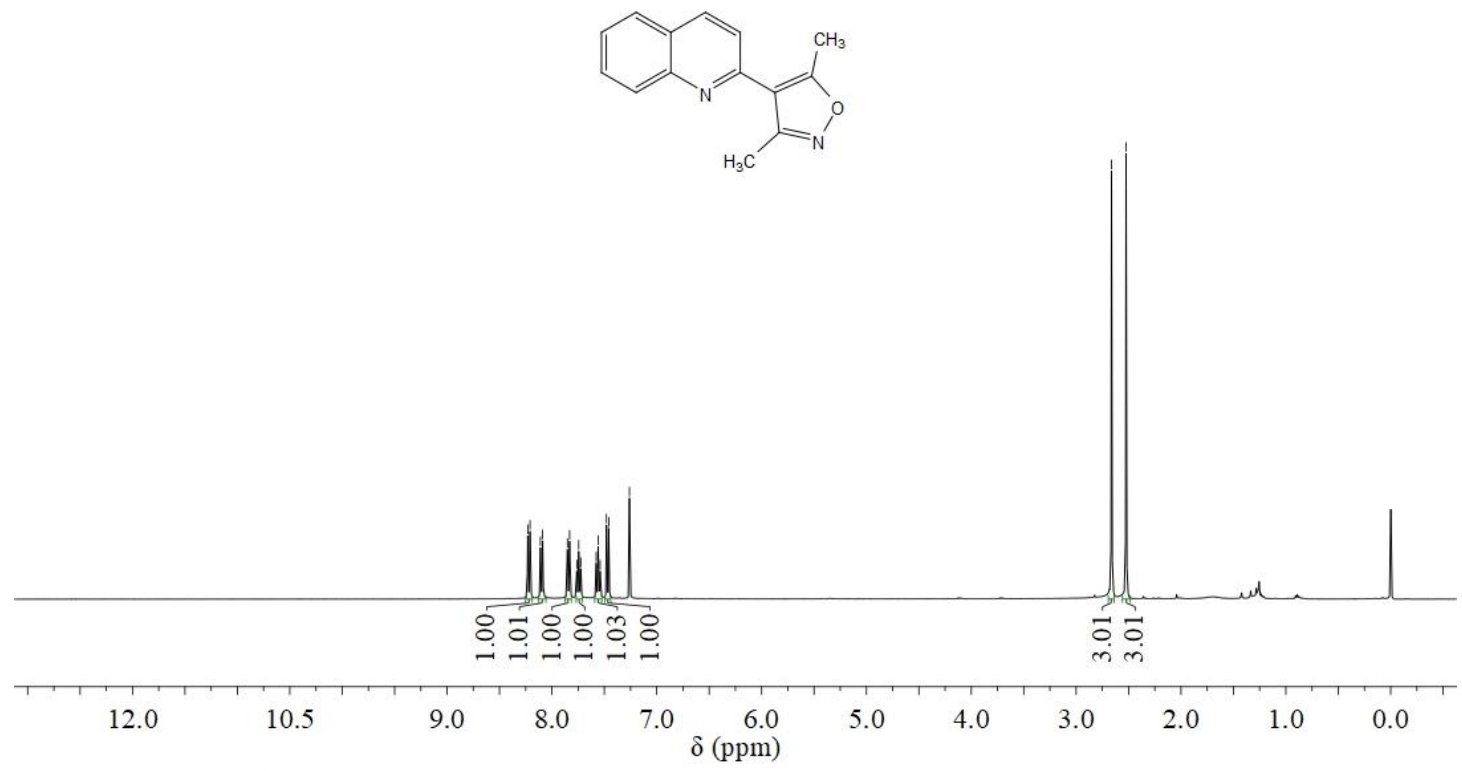

${ }^{13} \mathrm{C}$ NMR $\left(100 \mathrm{MHz}, \mathrm{CDCl}_{3}\right)$

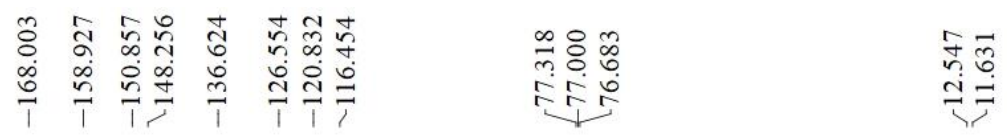<smiles>Cc1nc(-c2ccc3ccccc3n2)c(C)o1</smiles>

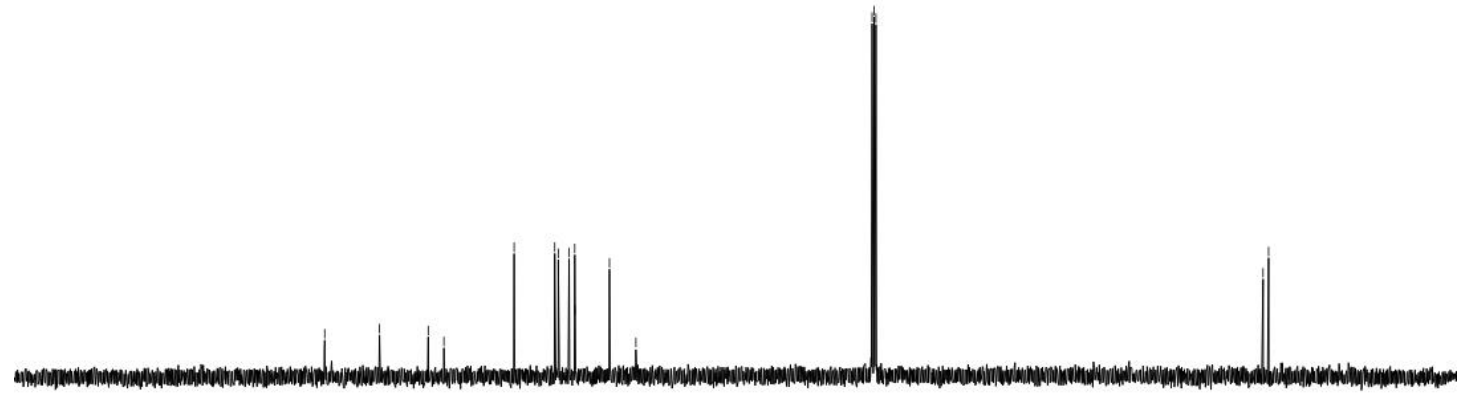

$\begin{array}{lllllllllllllllll}210 & 190 & 170 & 150 & 130 & 110 & 90 & 80 & 70 & 60 & 50 & 40 & 30 & 20 & 10 & 0\end{array}$


${ }^{1} \mathrm{H}$ NMR (400 MHz, $\mathrm{CDCl}_{3}$ )

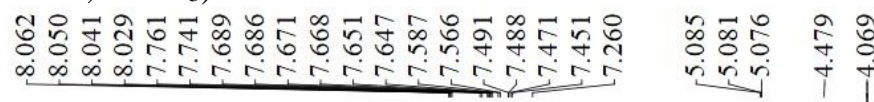

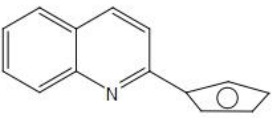

$\mathrm{Fe}$

O

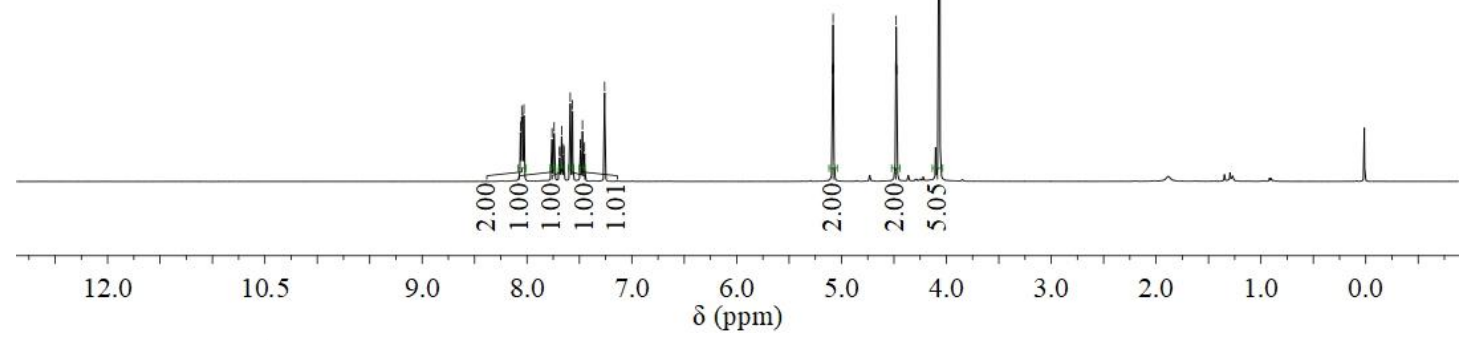

${ }^{13} \mathrm{C}$ NMR $\left(100 \mathrm{MHz}, \mathrm{CDCl}_{3}\right)$

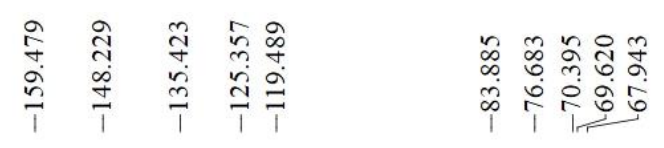

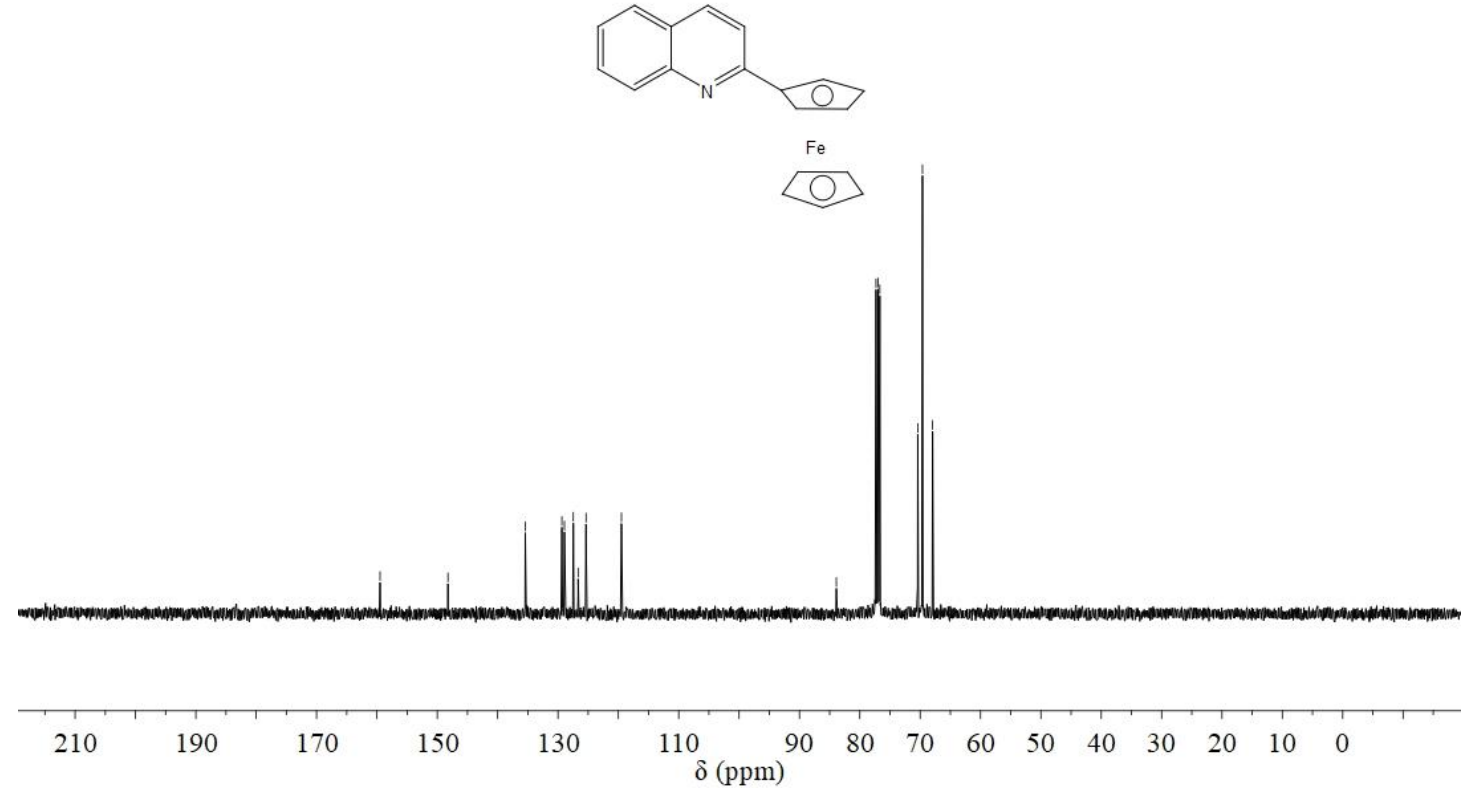


${ }^{1} \mathrm{H} \mathrm{NMR}\left(400 \mathrm{MHz}, \mathrm{CDCl}_{3}\right)$

2-(Naphthalen-2-yl)quinolone (3v)

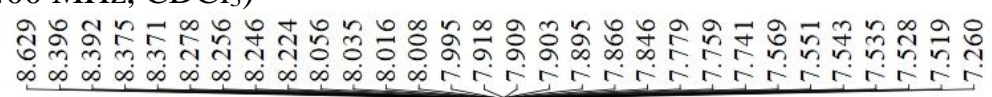
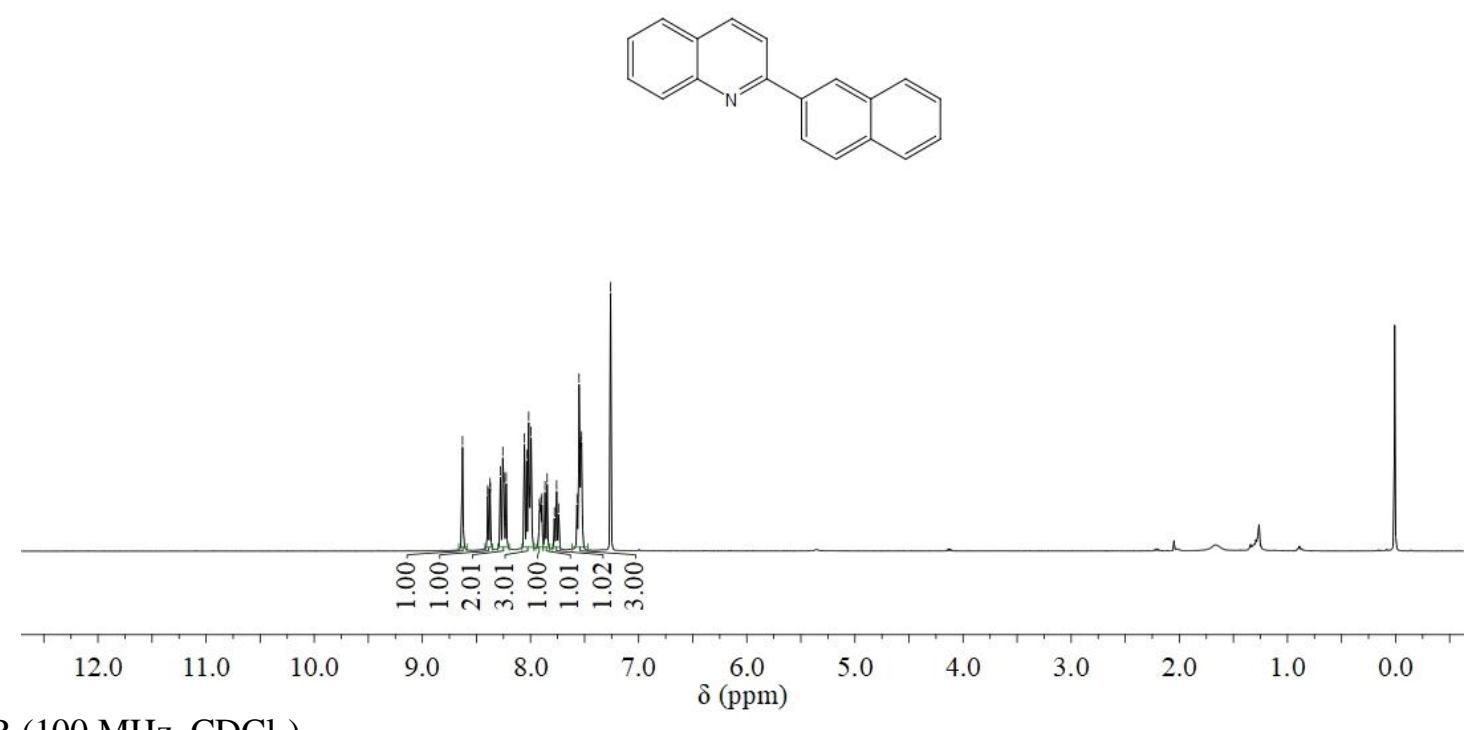

${ }^{13} \mathrm{C} \mathrm{NMR}\left(100 \mathrm{MHz}, \mathrm{CDCl}_{3}\right)$

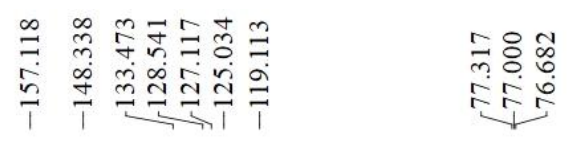
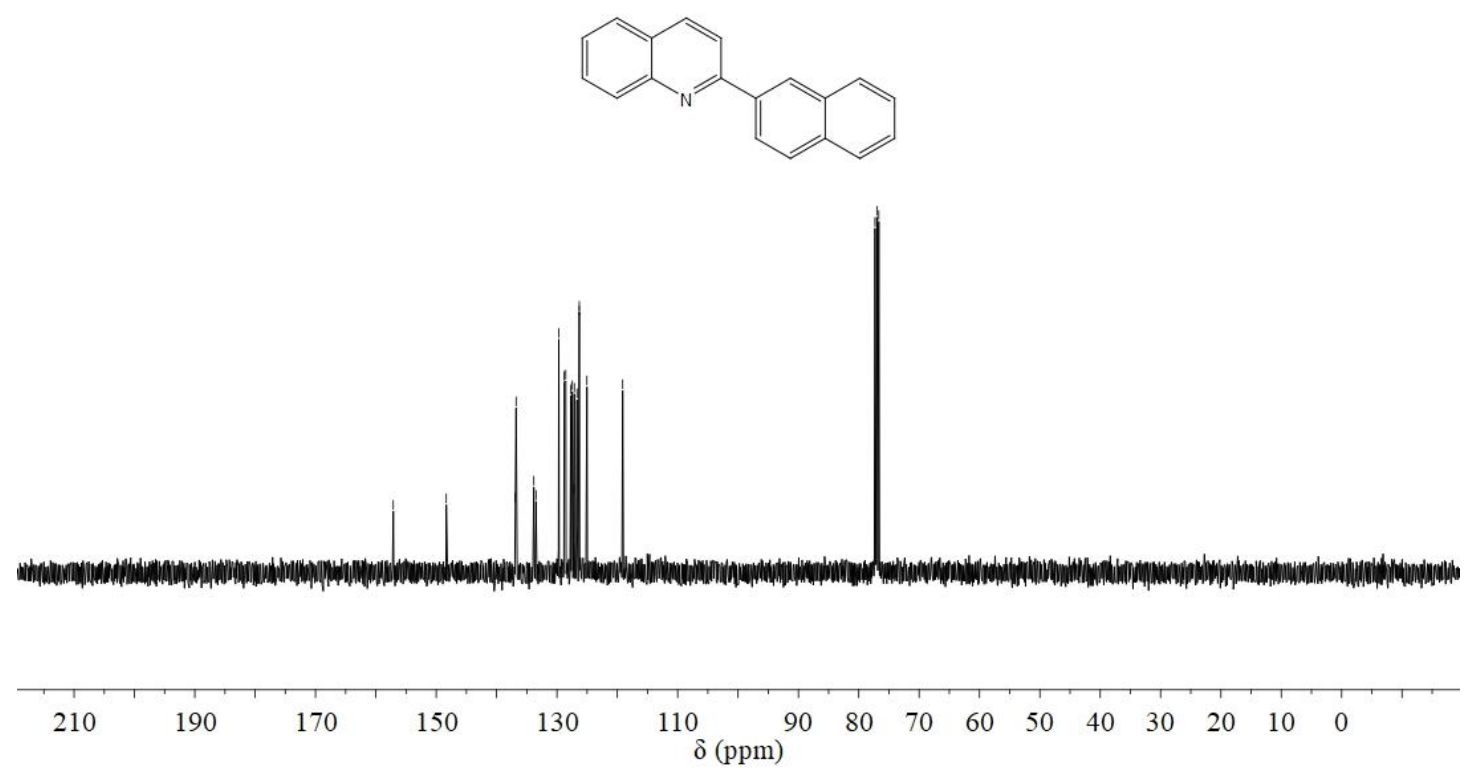

65 
${ }^{1} \mathrm{H}$ NMR (400 MHz, $\mathrm{CDCl}_{3}$ )
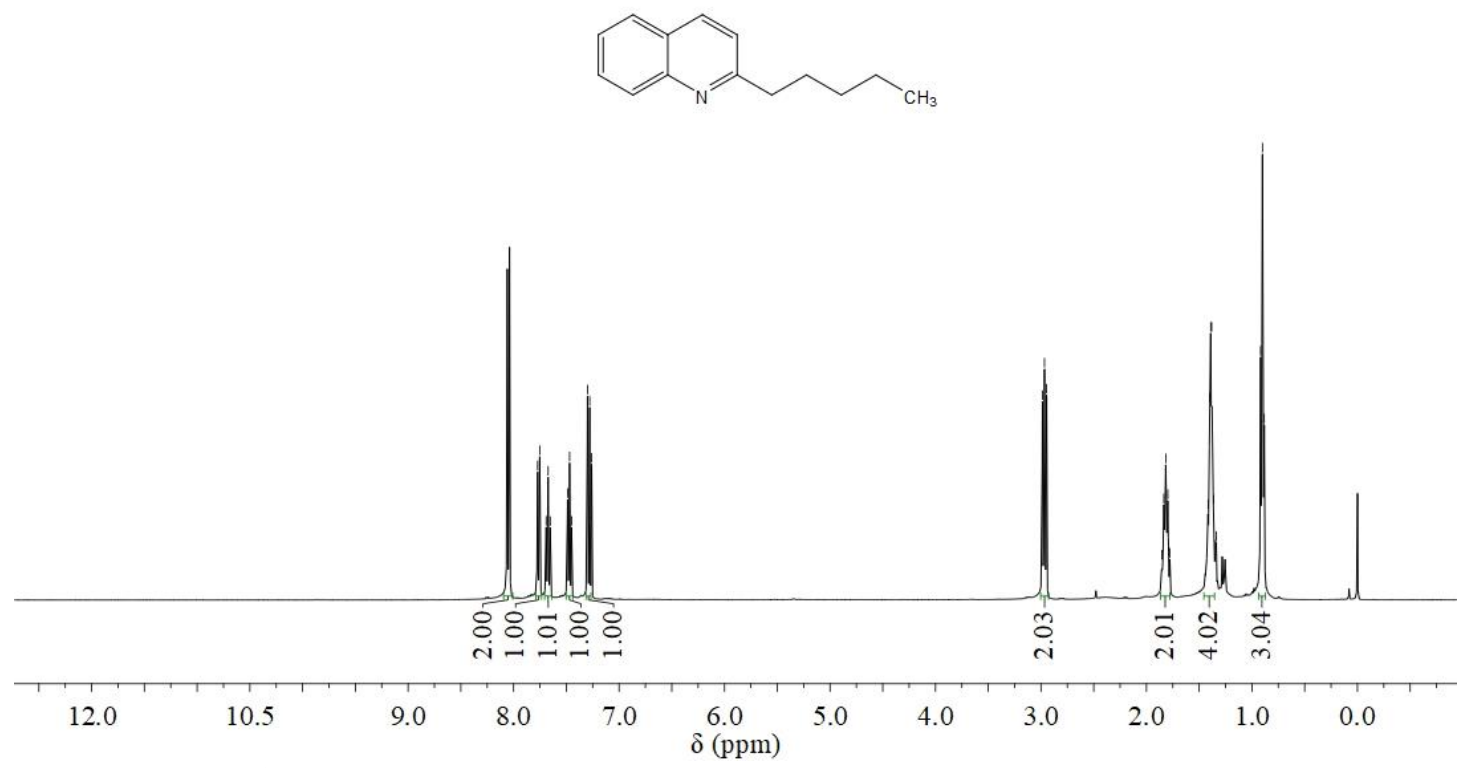

${ }^{13} \mathrm{C}$ NMR (100 MHz, $\left.\mathrm{CDCl}_{3}\right)$

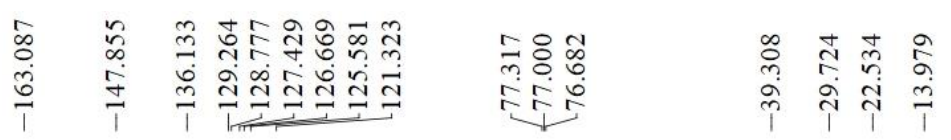
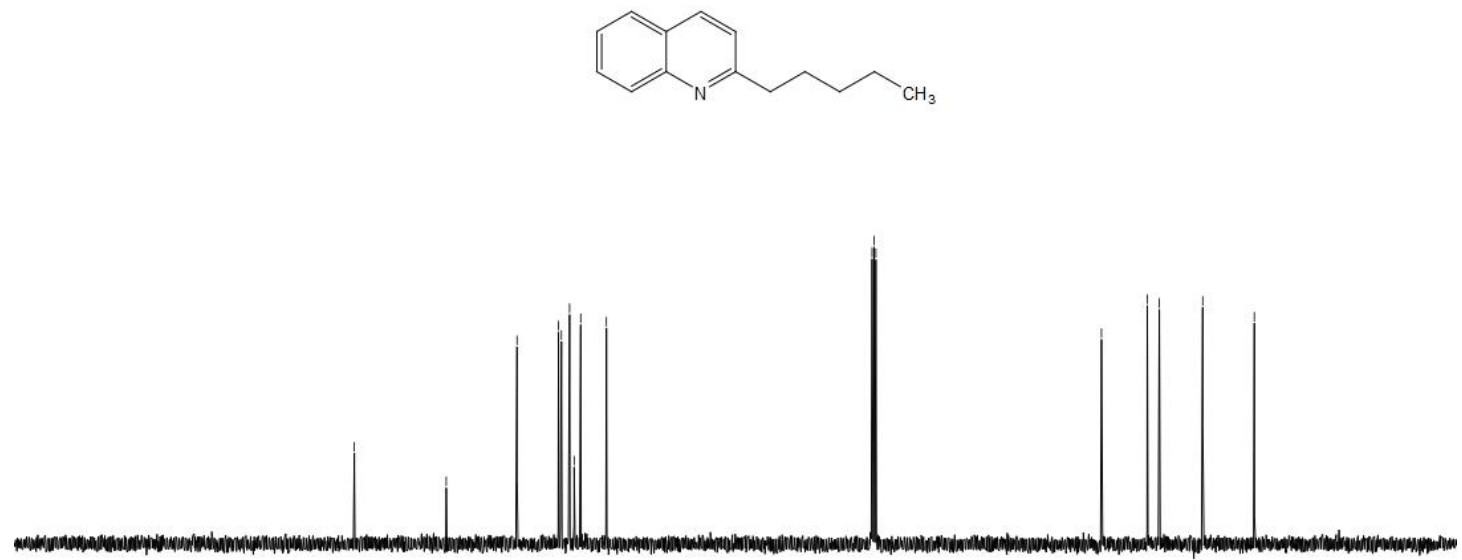

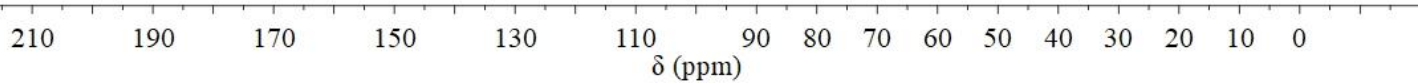


${ }^{1} \mathrm{H}$ NMR (400 MHz, $\mathrm{CDCl}_{3}$ )

\section{2-Butylquinoline (3x)}

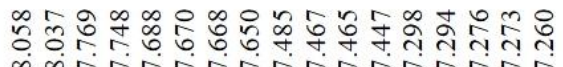

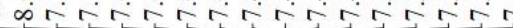

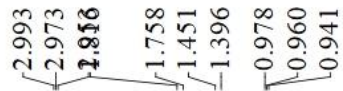
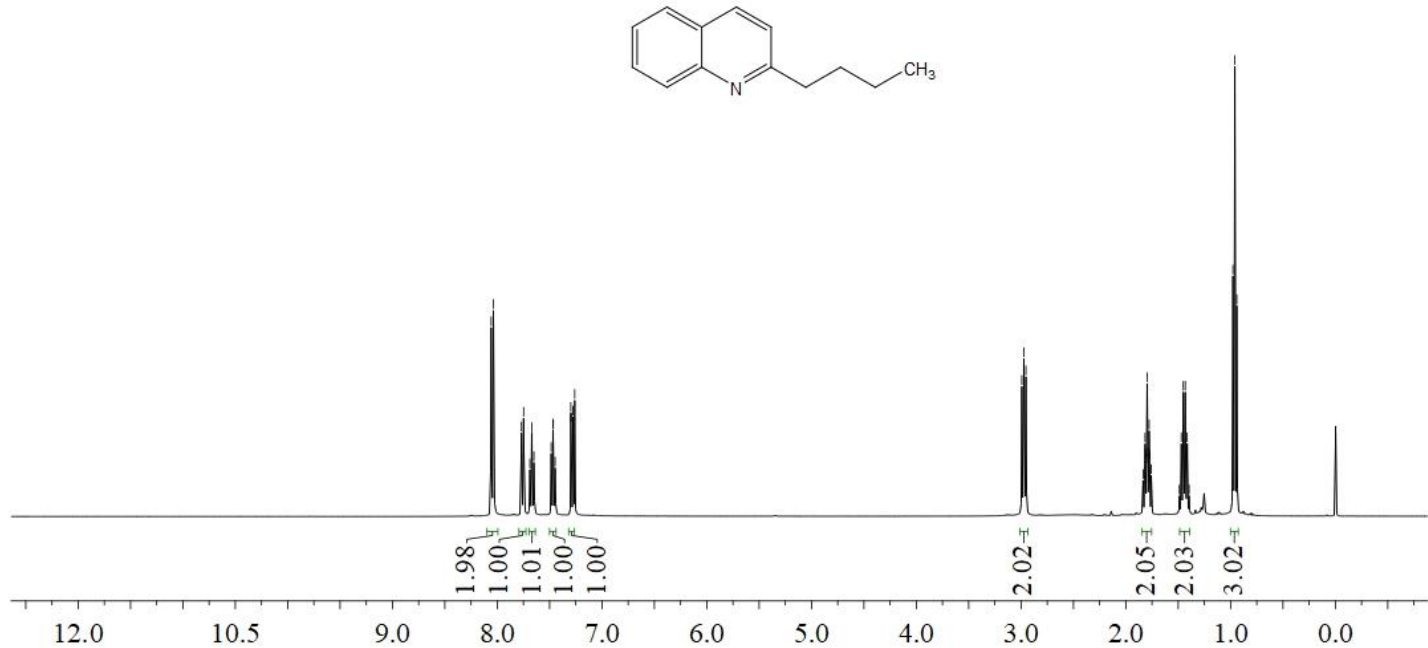

${ }^{13} \mathrm{C} \mathrm{NMR}\left(100 \mathrm{MHz}, \mathrm{CDCl}_{3}\right)$

\begin{tabular}{|c|c|c|c|c|}
\hline$\underset{\substack{n \\
\infty}}{\stackrel{n}{r}}$ & 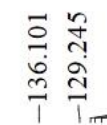 & 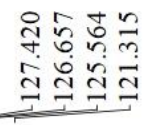 & 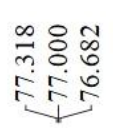 & 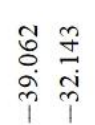 \\
\hline
\end{tabular}
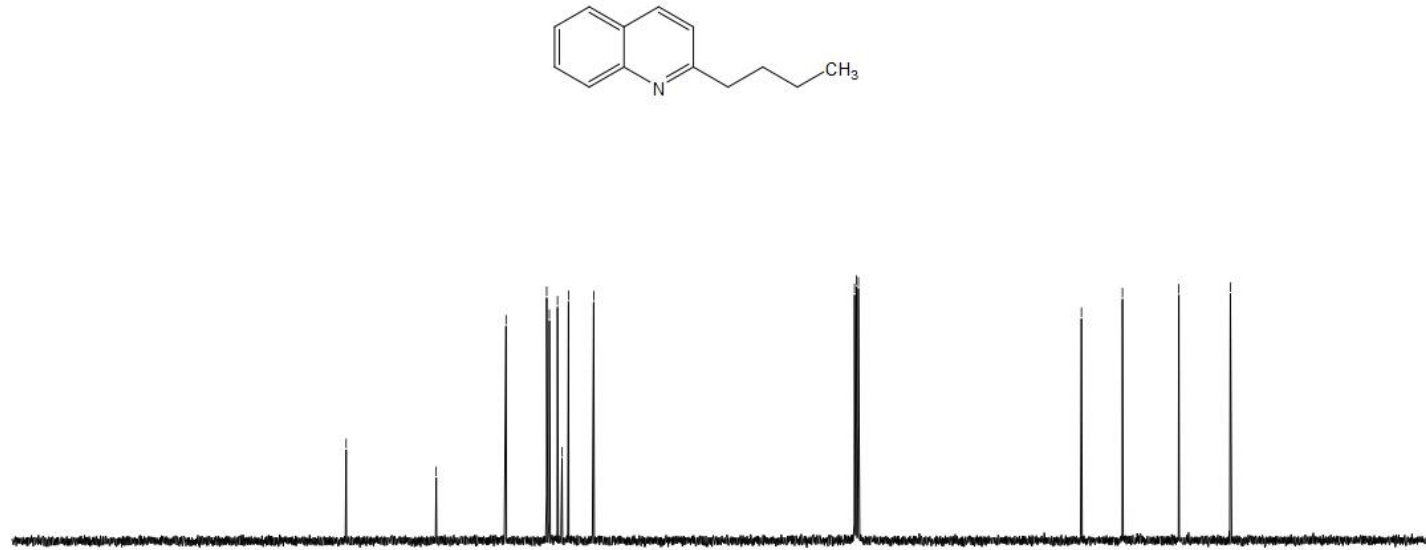

210

190

170

150

130

110

$\begin{array}{llllllllll}90 & 80 & 70 & 60 & 50 & 40 & 30 & 20 & 10 & 0\end{array}$ 
${ }^{1} \mathrm{H}$ NMR $\left(400 \mathrm{MHz}, \mathrm{CDCl}_{3}\right)$

\section{2-Cyclopropylquinoline (3y)}

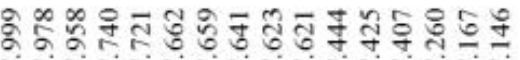

年

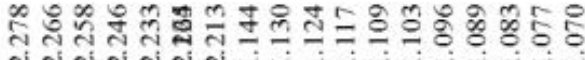
तสतส
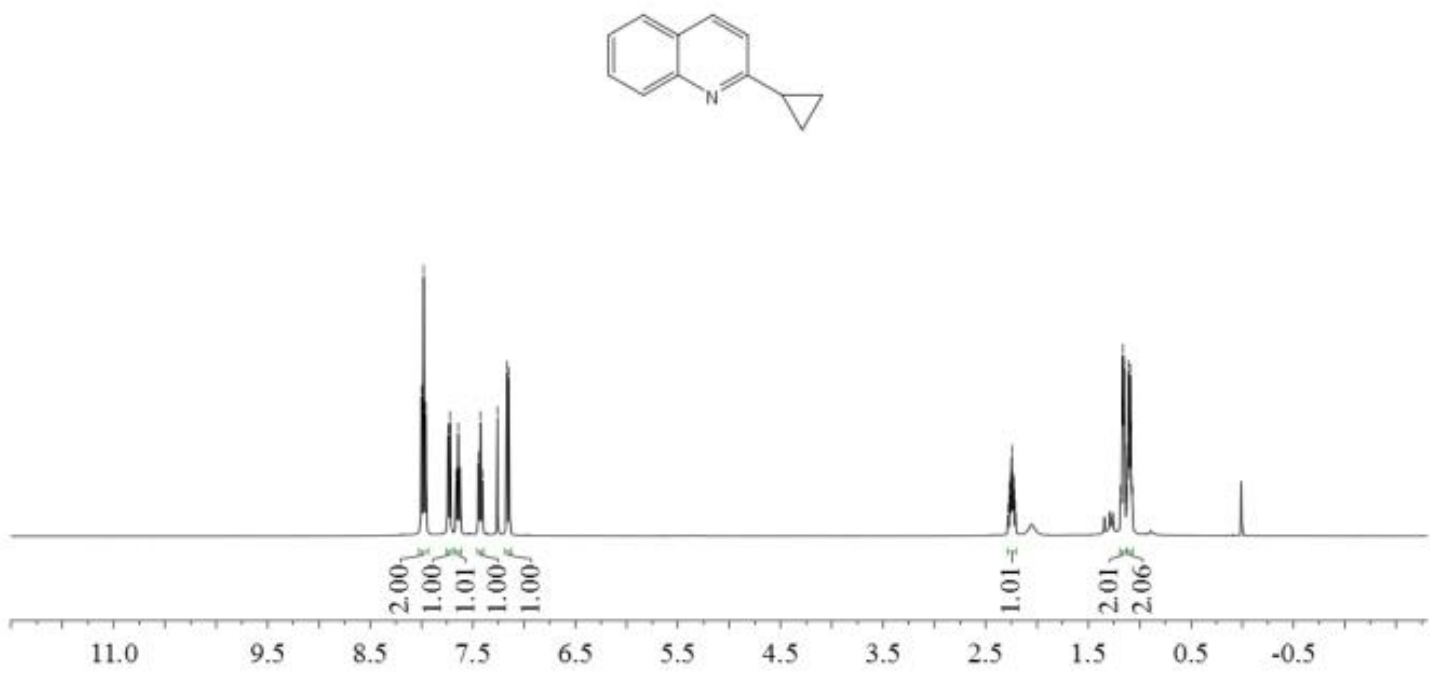

${ }^{13} \mathrm{C}$ NMR (100 MHz, $\mathrm{CDCl}_{3}$ )

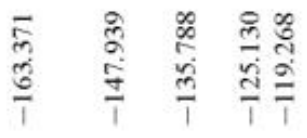
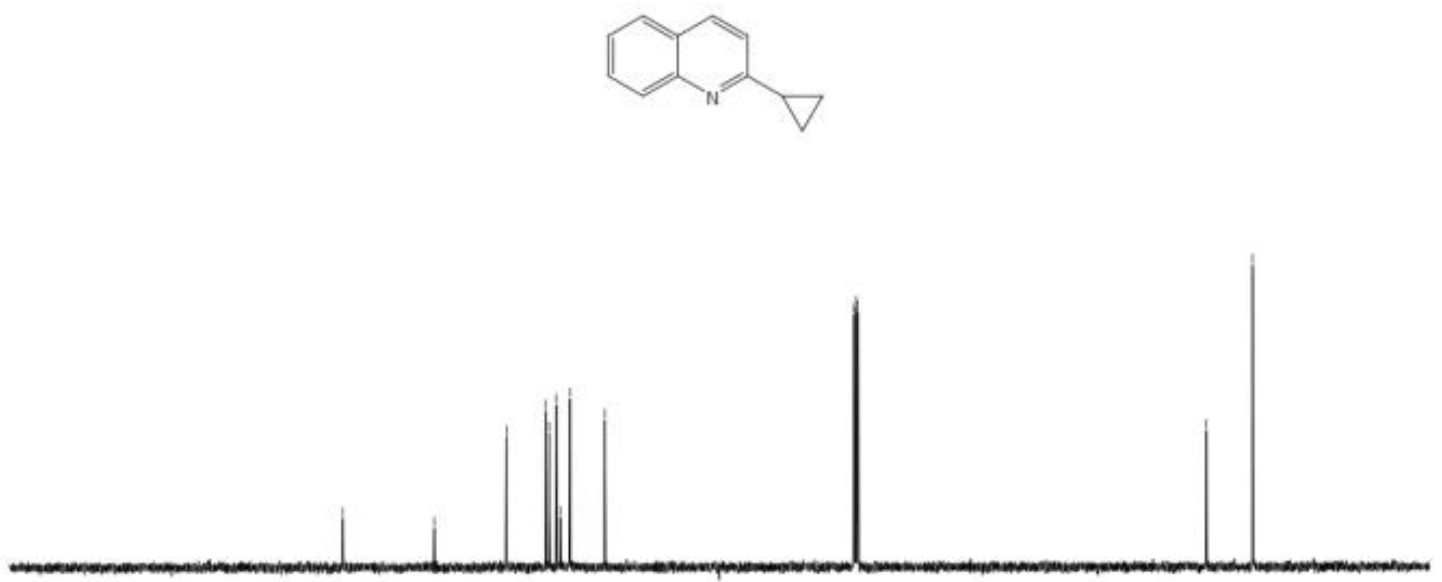

210

190

170

150

130

110

$\begin{array}{llllllllll}90 & 80 & 70 & 60 & 50 & 40 & 30 & 20 & 10 & 0\end{array}$ 
${ }^{1} \mathrm{H}$ NMR (400 MHz, $\left.\mathrm{CDCl}_{3}\right)$

3-(Quinolin-2-yl)propyl benzoate (3z)

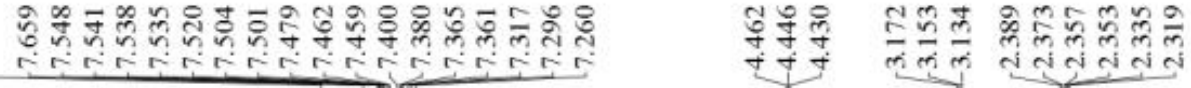
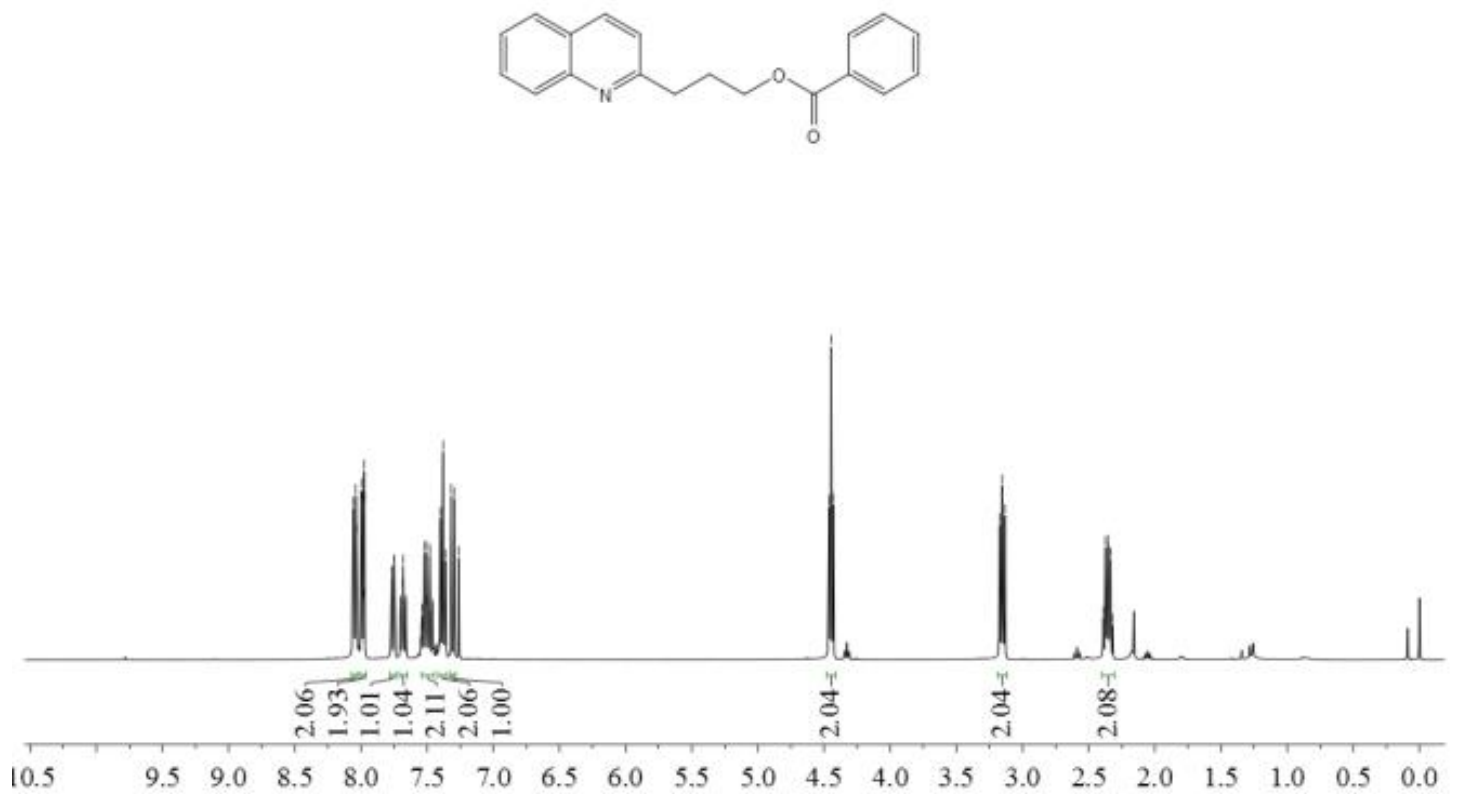

${ }^{13} \mathrm{C}$ NMR $\left(100 \mathrm{MHz}, \mathrm{CDCl}_{3}\right)$
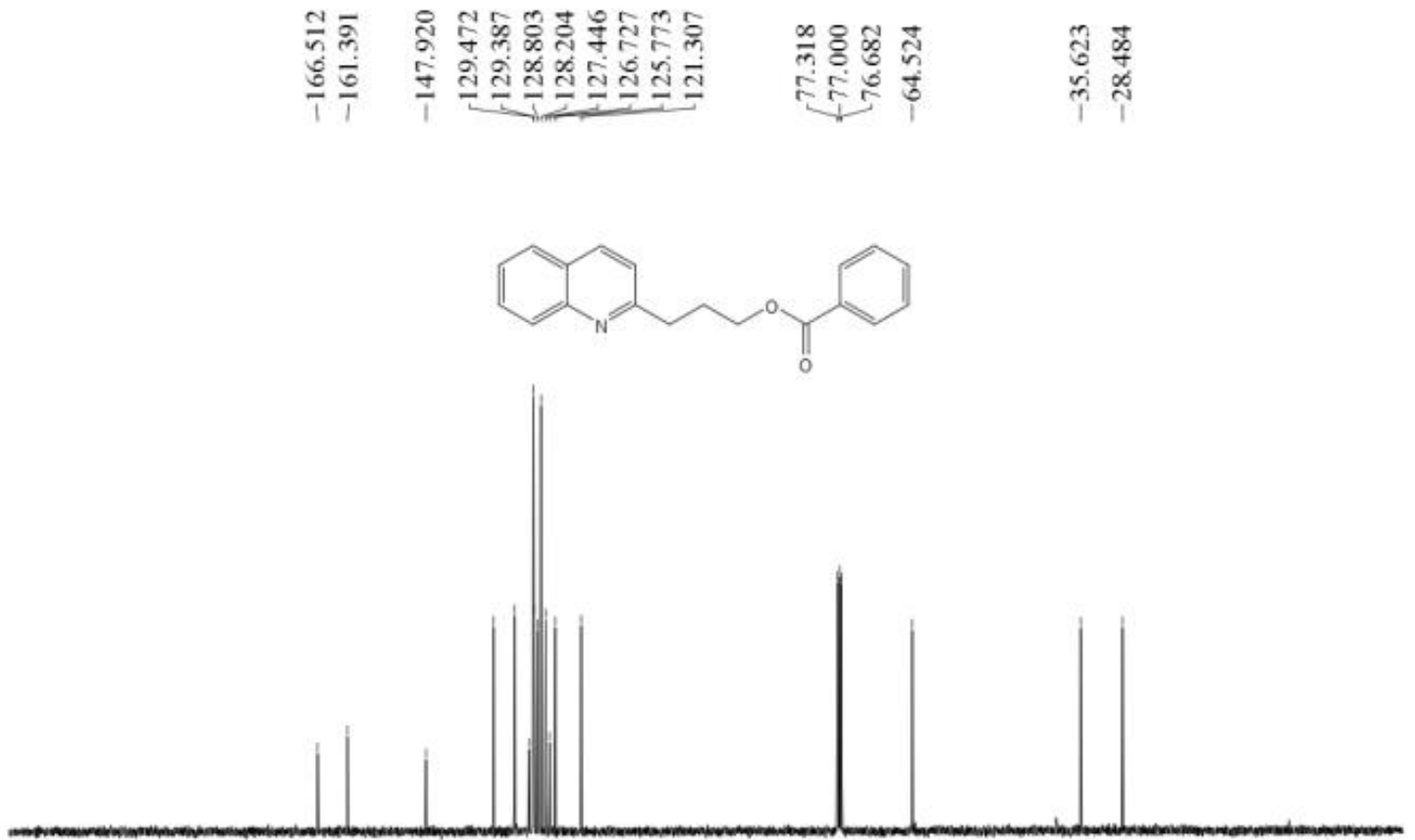

210

190

170

150

130

110

$\begin{array}{llllllllll}90 & 80 & 70 & 60 & 50 & 40 & 30 & 20 & 10 & 0\end{array}$ 
Ethyl quinoline-2-carboxylate (3aa)

${ }^{1} \mathrm{H}$ NMR (400 MHz, $\mathrm{CDCl}_{3}$ )

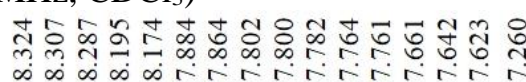

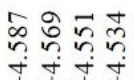

:

$\overbrace{\mathrm{N}}^{\mathrm{O}} \mathrm{N}^{\mathrm{CH}_{3}}$

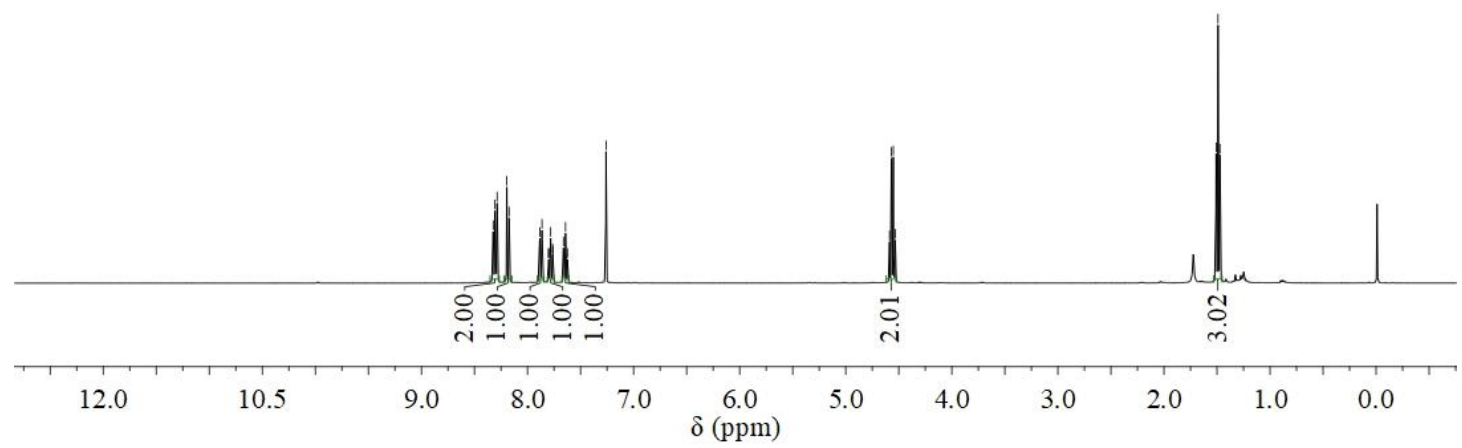

${ }^{13} \mathrm{C}$ NMR $\left(100 \mathrm{MHz}, \mathrm{CDCl}_{3}\right)$

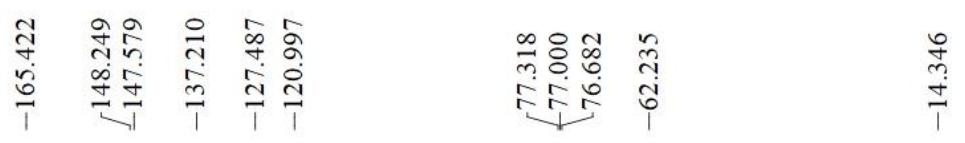
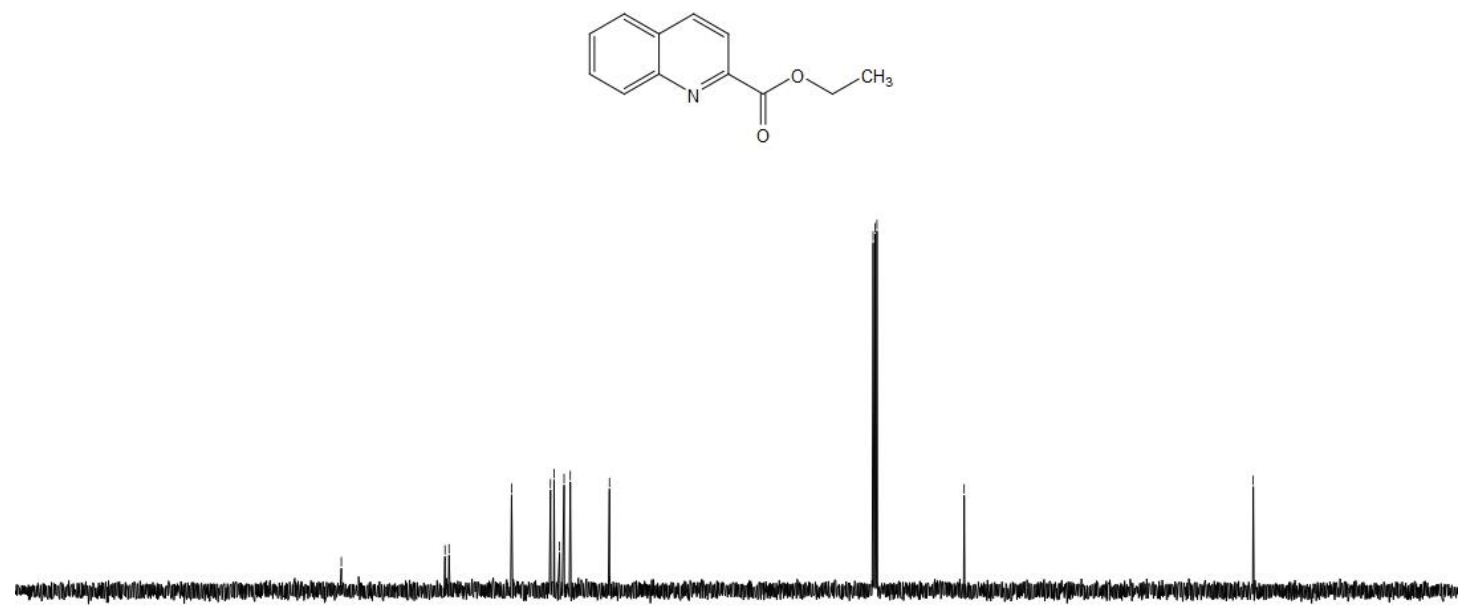

$\begin{array}{lllllllllllllllll}210 & 190 & 170 & 150 & 130 & 110 & 90 & 80 & 70 & 60 & 50 & 40 & 30 & 20 & 10 & 0\end{array}$


${ }^{1} \mathrm{H}$ NMR (400 MHz, $\left.\mathrm{CDCl}_{3}\right)$

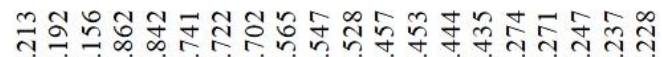

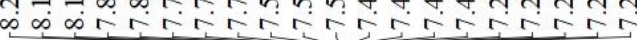
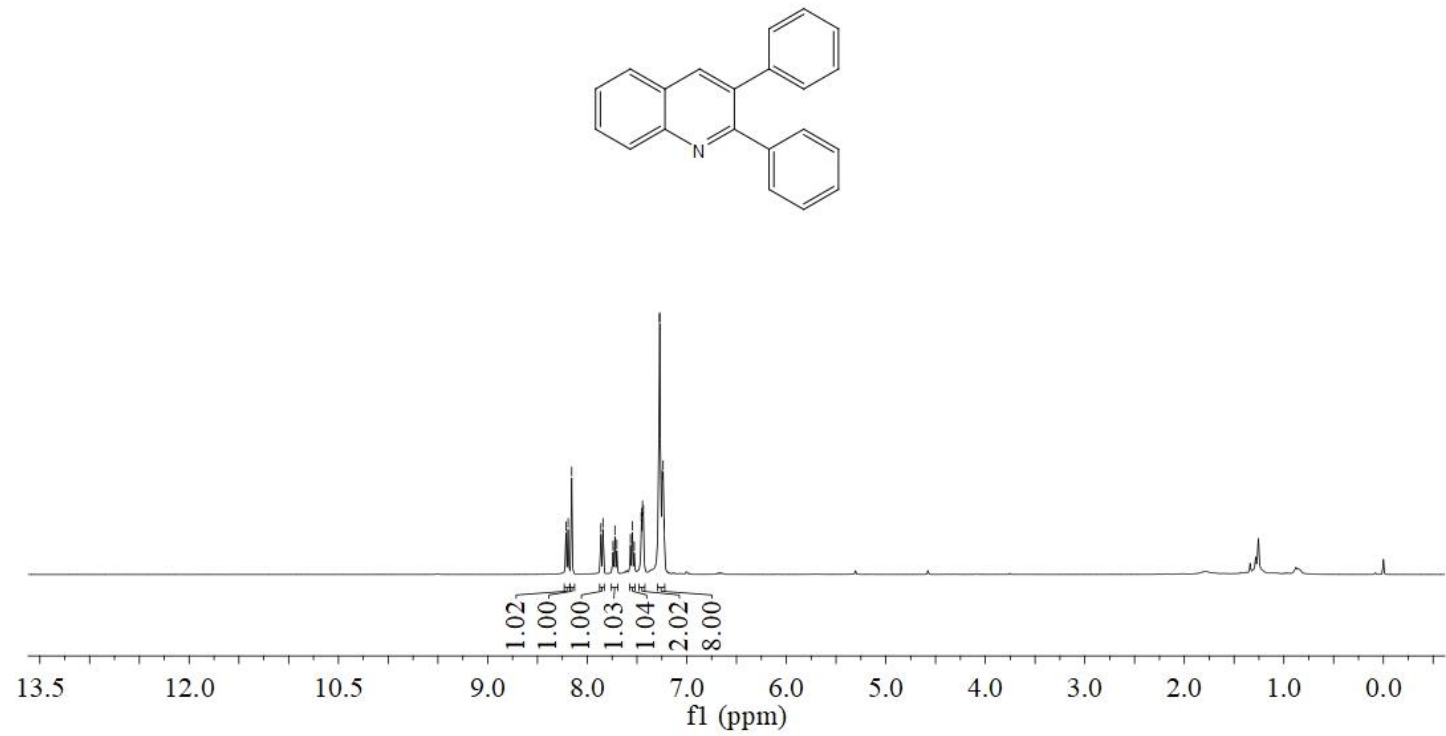

${ }^{13} \mathrm{C}$ NMR $\left(100 \mathrm{MHz}, \mathrm{CDCl}_{3}\right)$

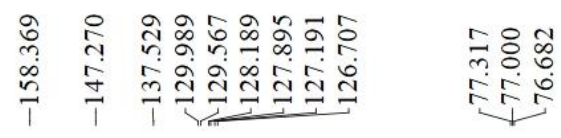
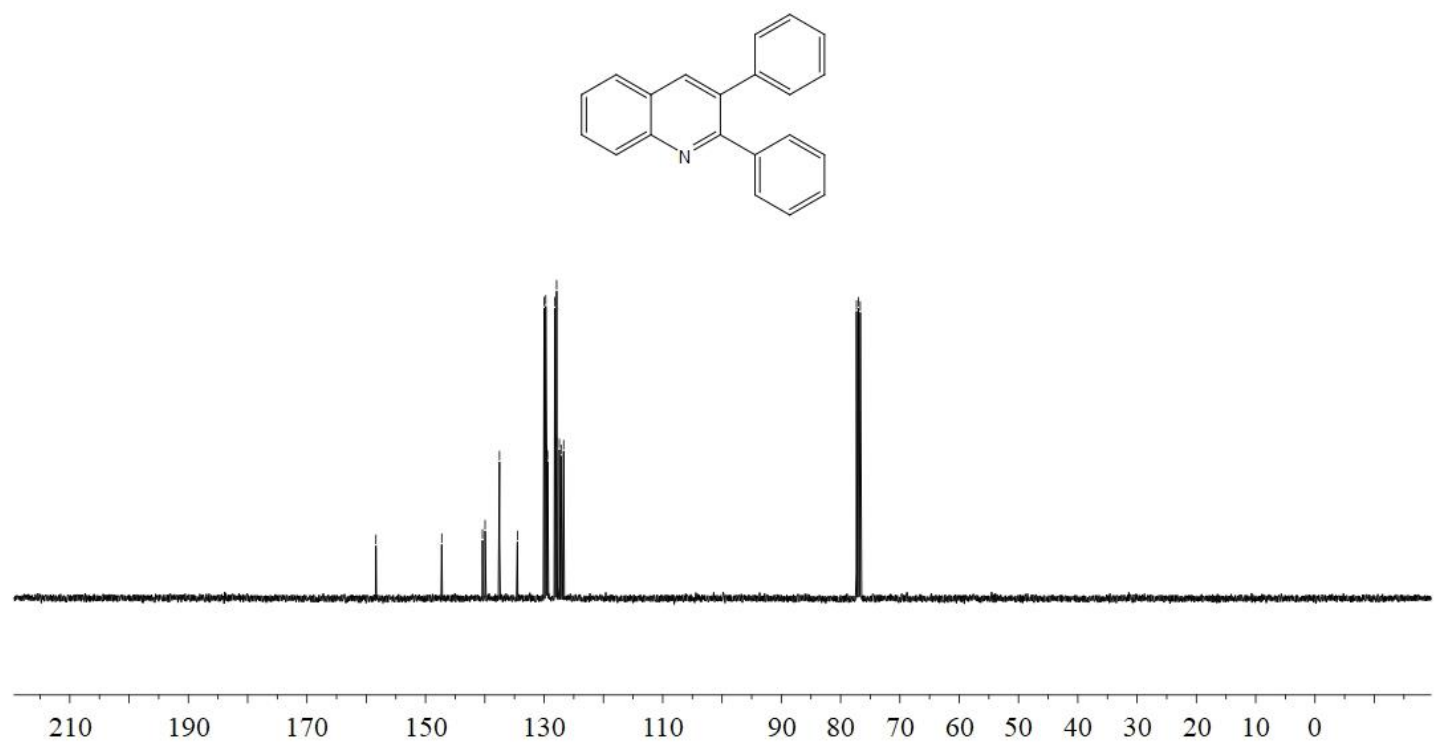
${ }^{1} \mathrm{H}$ NMR (400 MHz, $\left.\mathrm{CDCl}_{3}\right)$

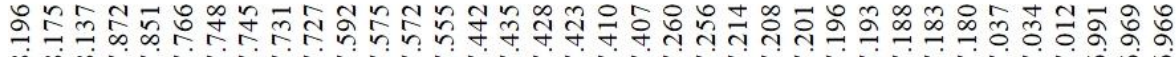

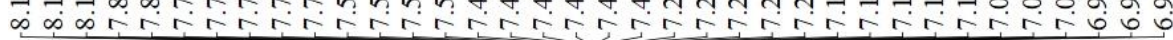
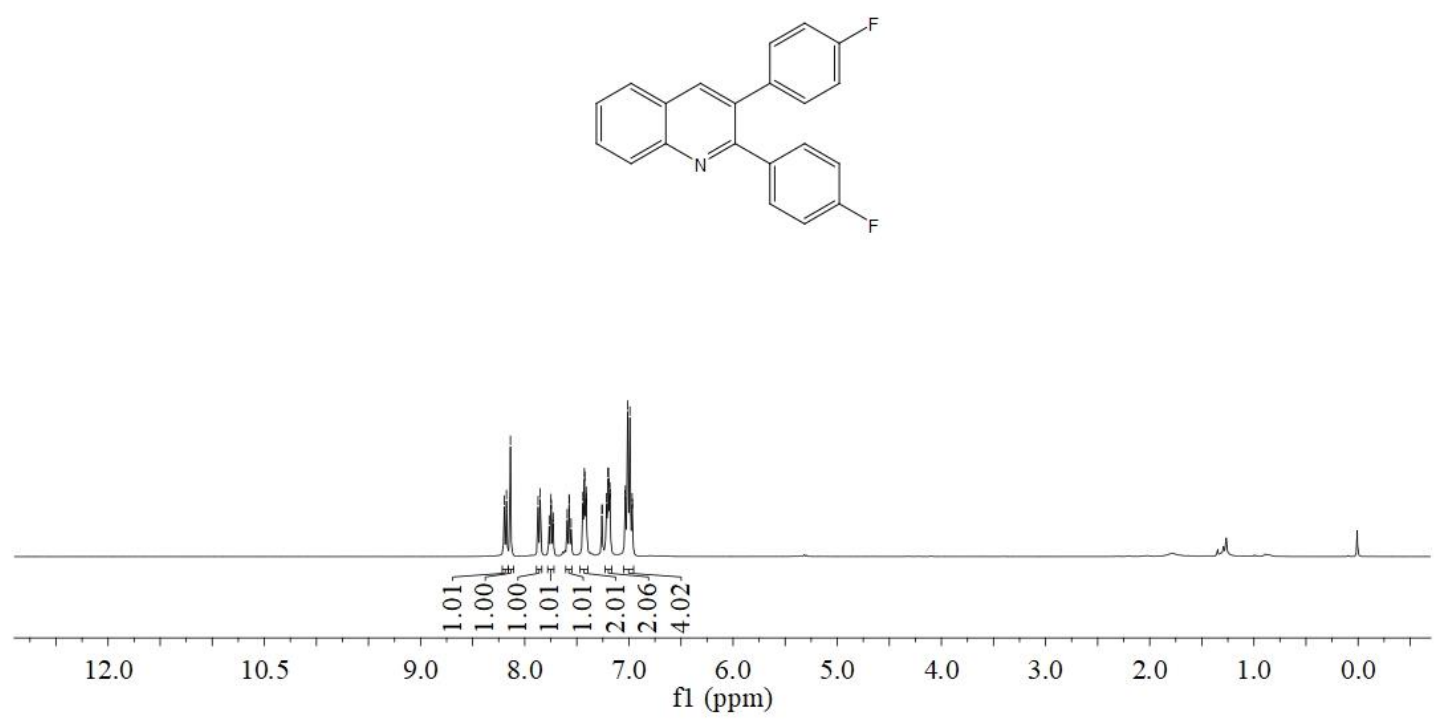

${ }^{13} \mathrm{C}$ NMR (100 MHz, $\left.\mathrm{CDCl}_{3}\right)$
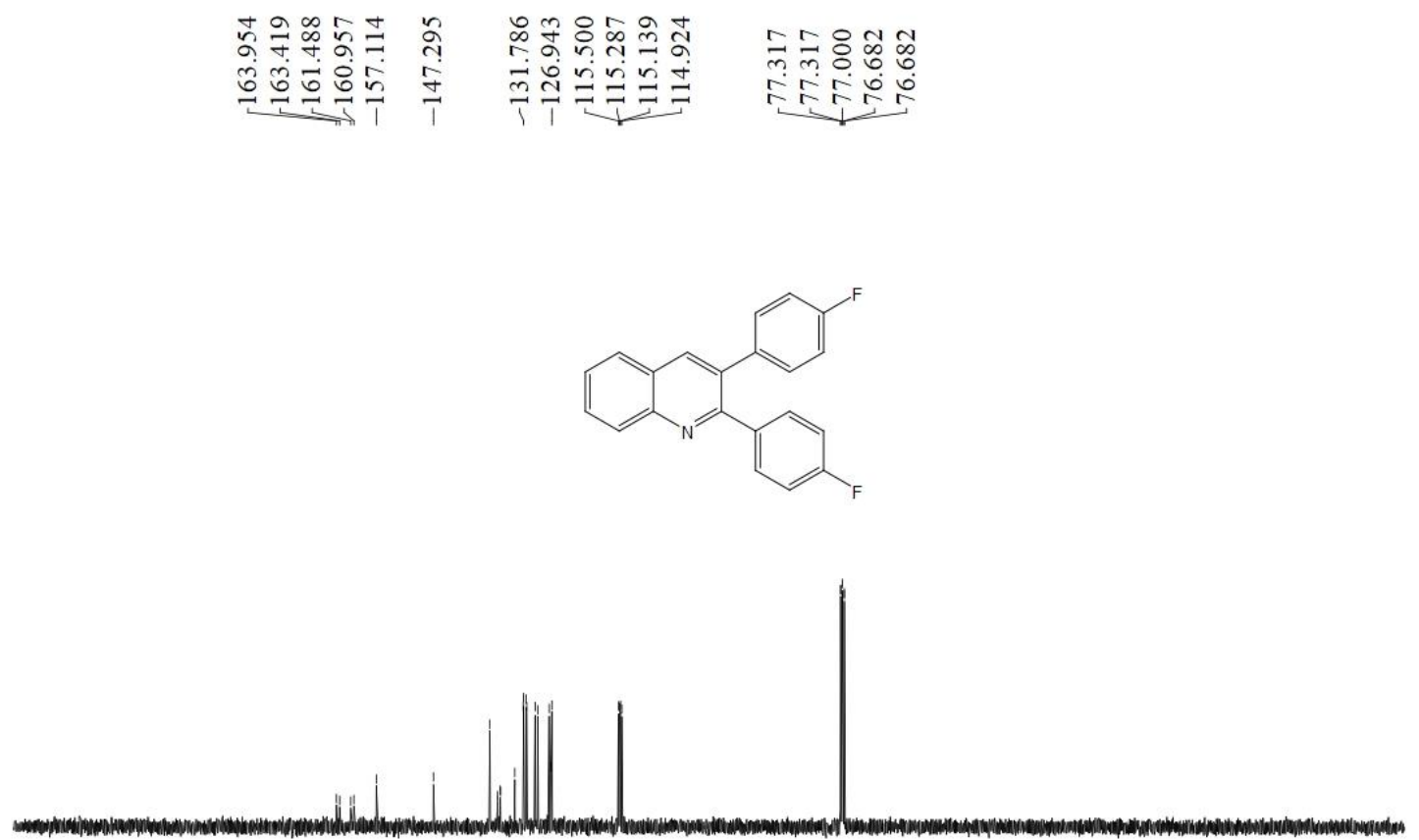

$210 \quad 190 \quad \begin{array}{lllllllllllllll} & 170 & 150 & 130 & 110 & 90 & 80 & 70 & 60 & 50 & 40 & 30 & 20 & 10 & 0\end{array}$


${ }^{19} \mathrm{~F}$ NMR (375 MHz, $\left.\mathrm{CDCl}_{3}\right)$

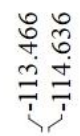
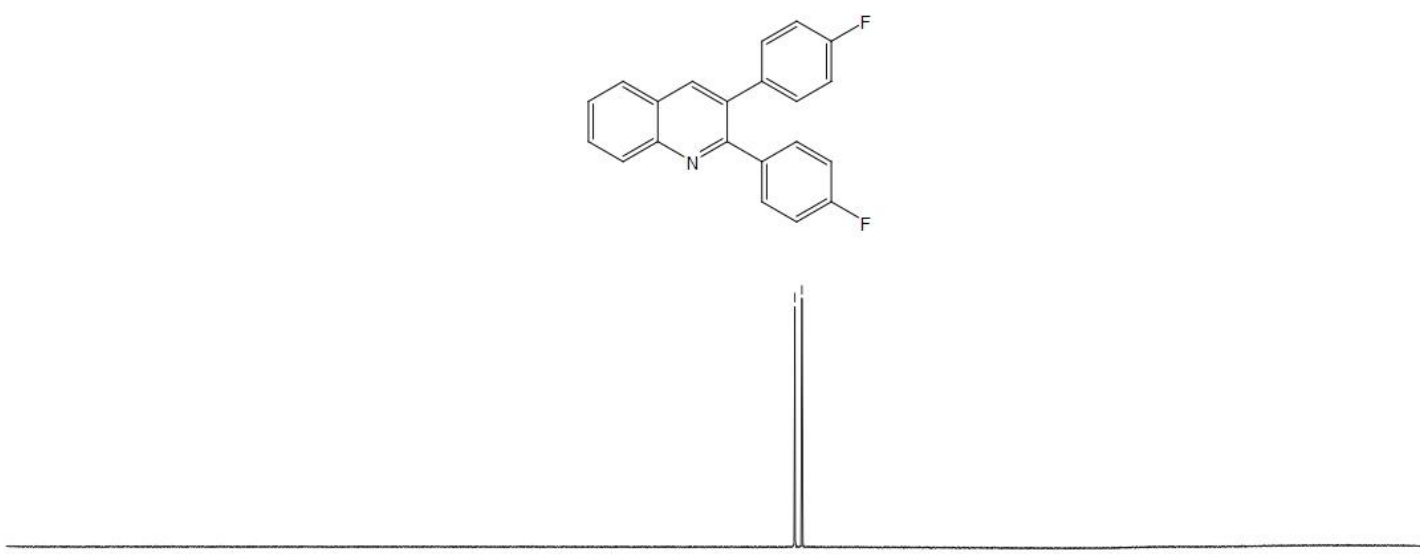

\begin{tabular}{|c|c|c|c|c|c|c|c|c|c|c|}
\hline $10 \quad 0$ & -20 & -40 & -60 & -80 & $\begin{array}{c}-100 \\
\mathrm{fl}(\mathrm{ppm})\end{array}$ & -120 & -140 & -160 & -180 & -200 \\
\hline
\end{tabular}


${ }^{1} \mathrm{H}$ NMR (400 MHz, $\mathrm{CDCl}_{3}$ )

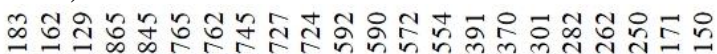

क क क क
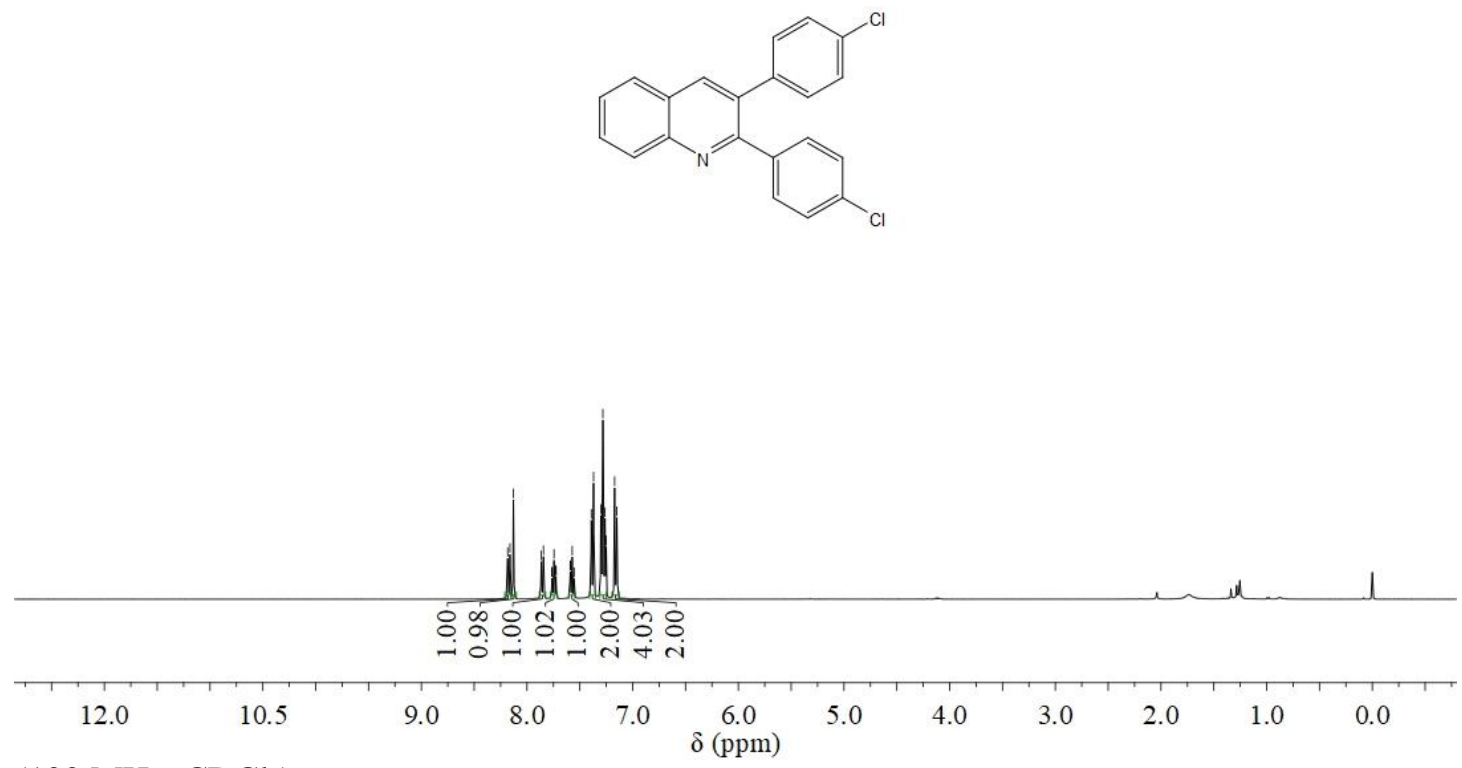

${ }^{13} \mathrm{C}$ NMR $\left(100 \mathrm{MHz}, \mathrm{CDCl}_{3}\right)$

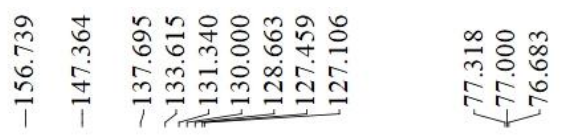
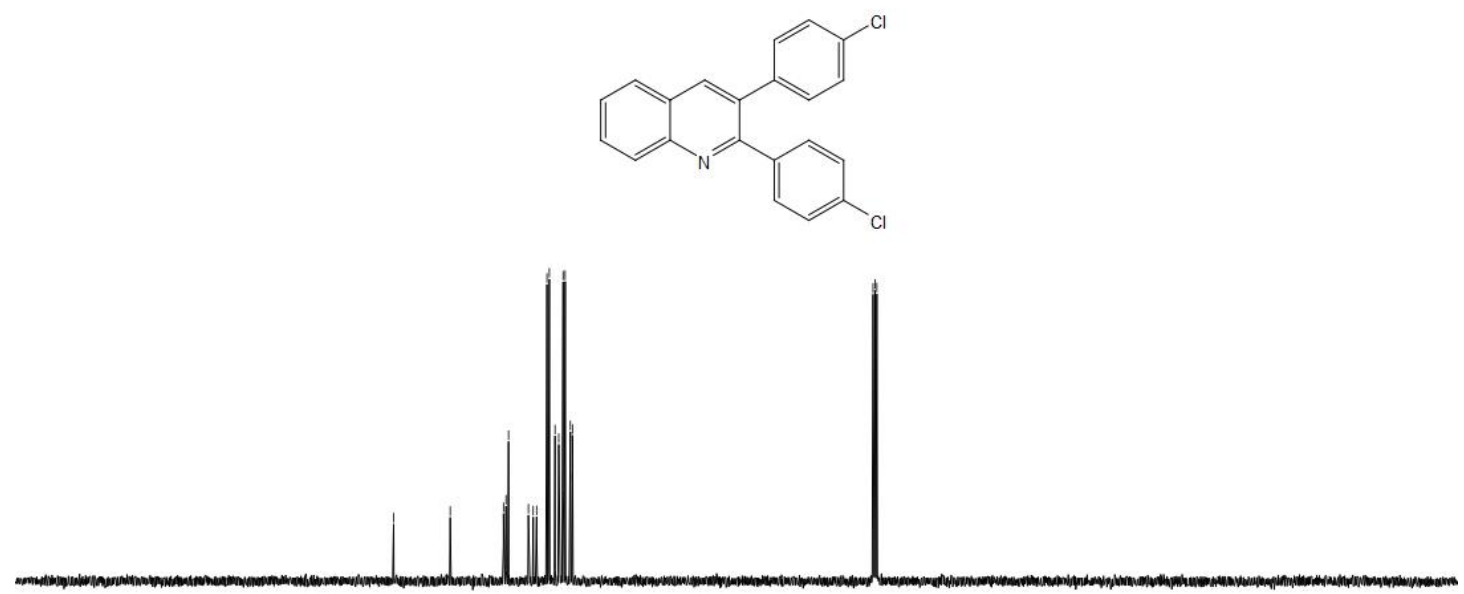

$\begin{array}{lllllllllllllllll}210 & 190 & 170 & 150 & 130 & 110 & 90 & 80 & 70 & 60 & 50 & 40 & 30 & 20 & 10 & 0\end{array}$


${ }^{1} \mathrm{H}$ NMR (400 MHz, $\mathrm{CDCl}_{3}$ )

\section{2,3-bis(4-Bromophenyl) quinolone (3ae)}

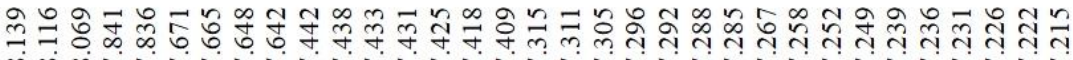

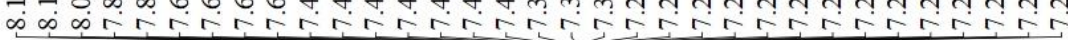
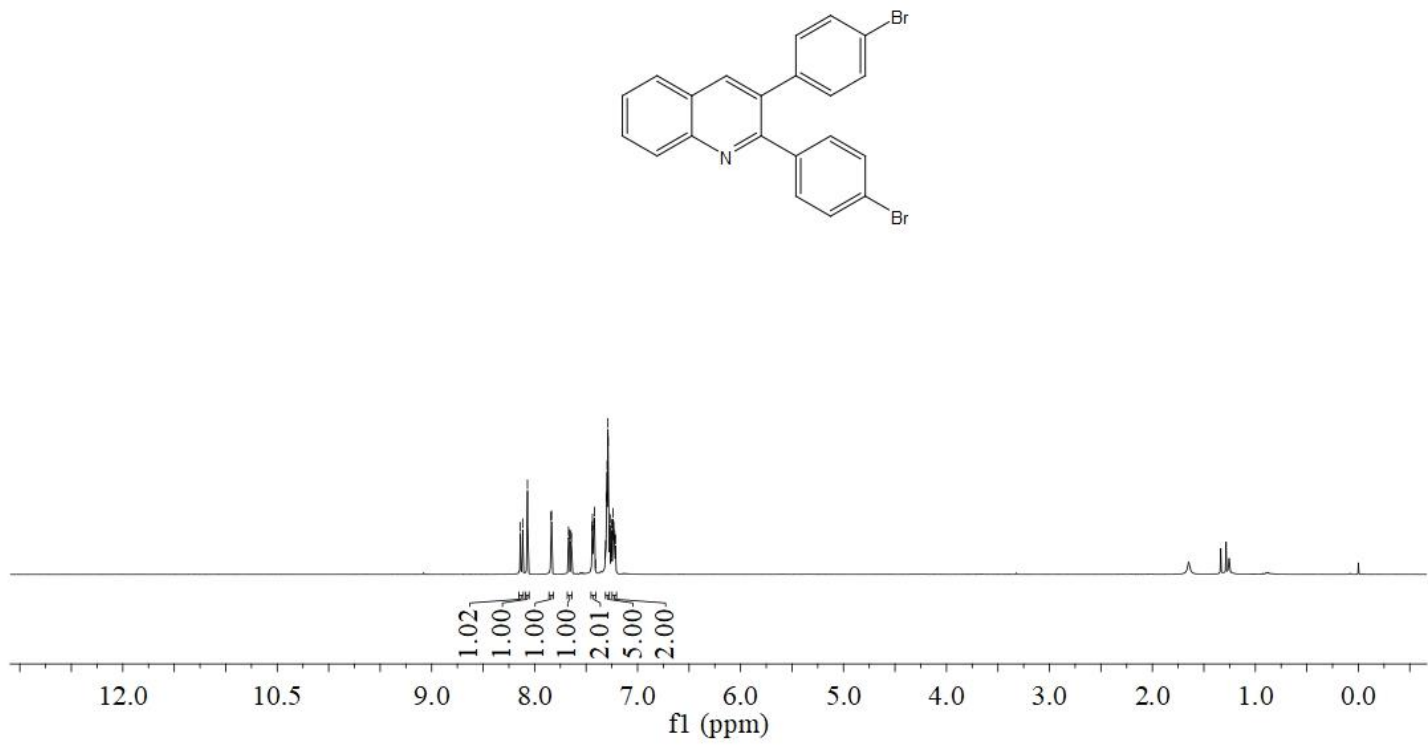

${ }^{13} \mathrm{C}$ NMR $\left(100 \mathrm{MHz}, \mathrm{CDCl}_{3}\right)$
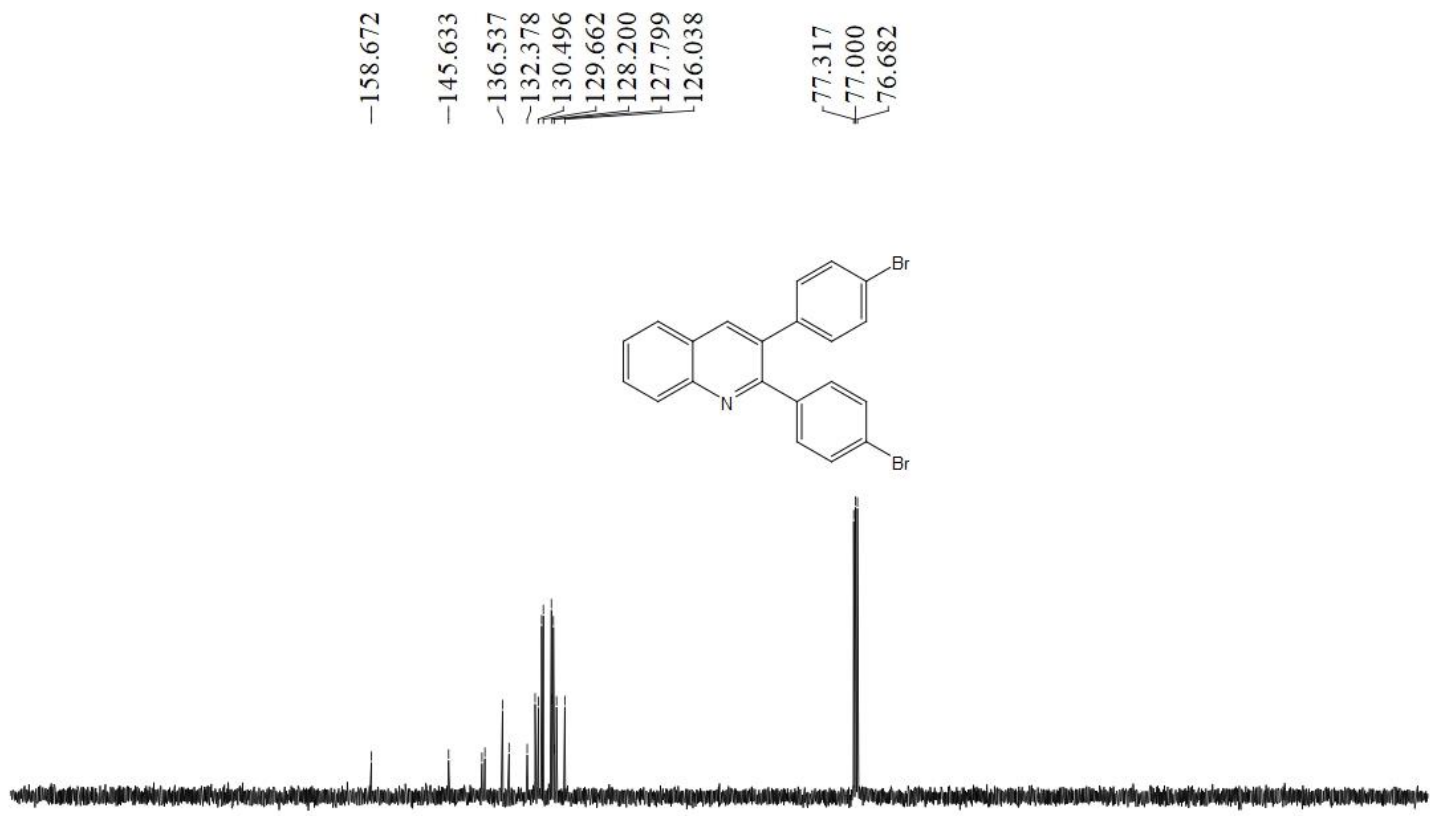

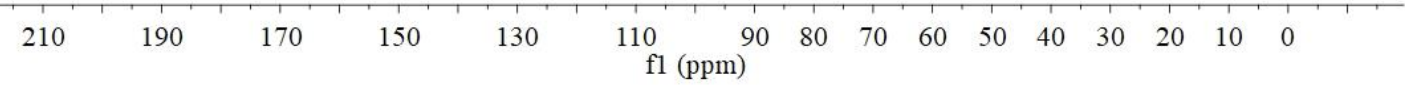


${ }^{1} \mathrm{H}$ NMR $\left(400 \mathrm{MHz}, \mathrm{CDCl}_{3}\right)$

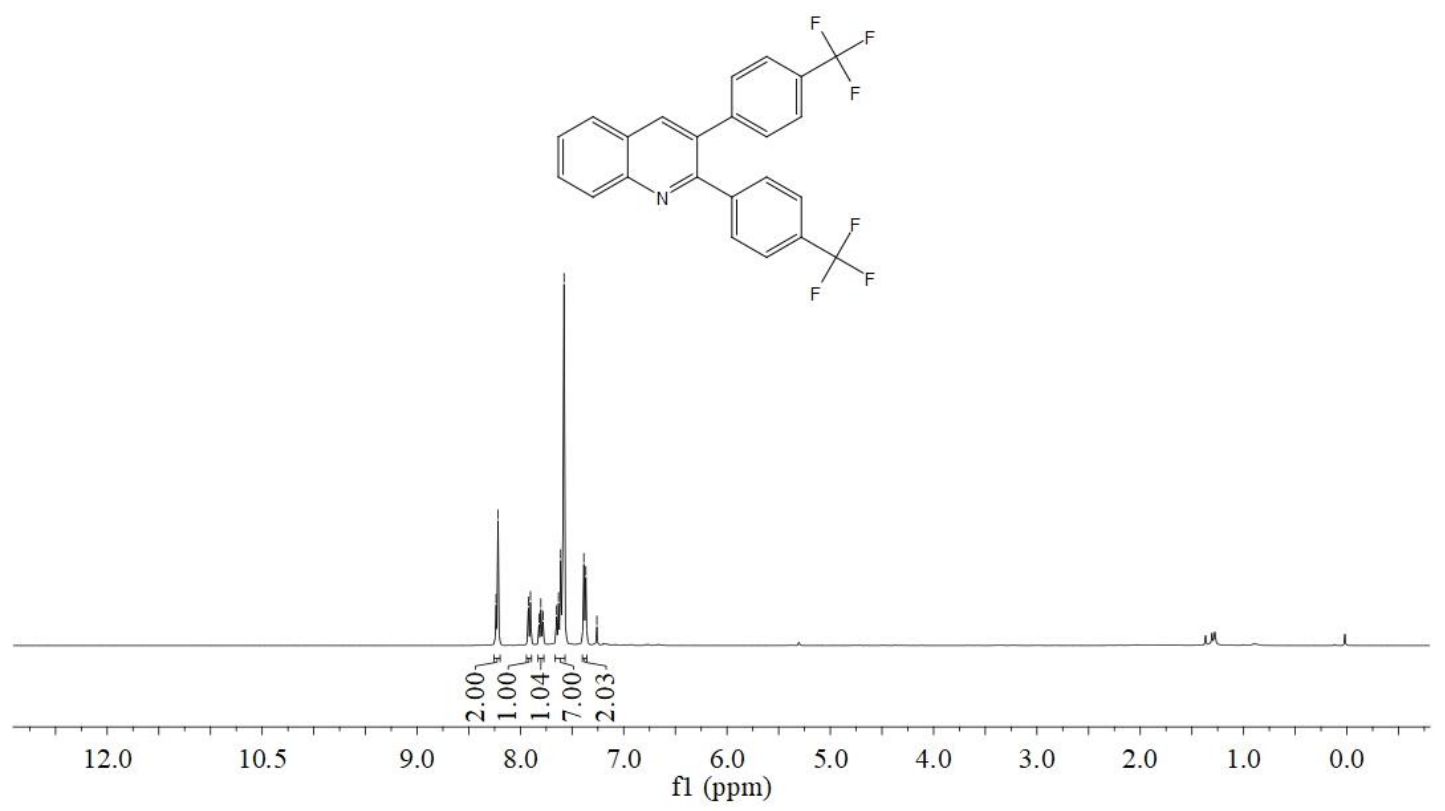

${ }^{13} \mathrm{C}$ NMR (100 MHz, $\left.\mathrm{CDCl}_{3}\right)$

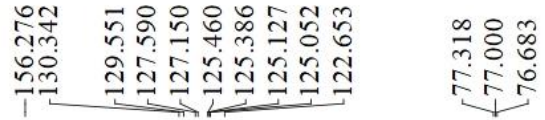

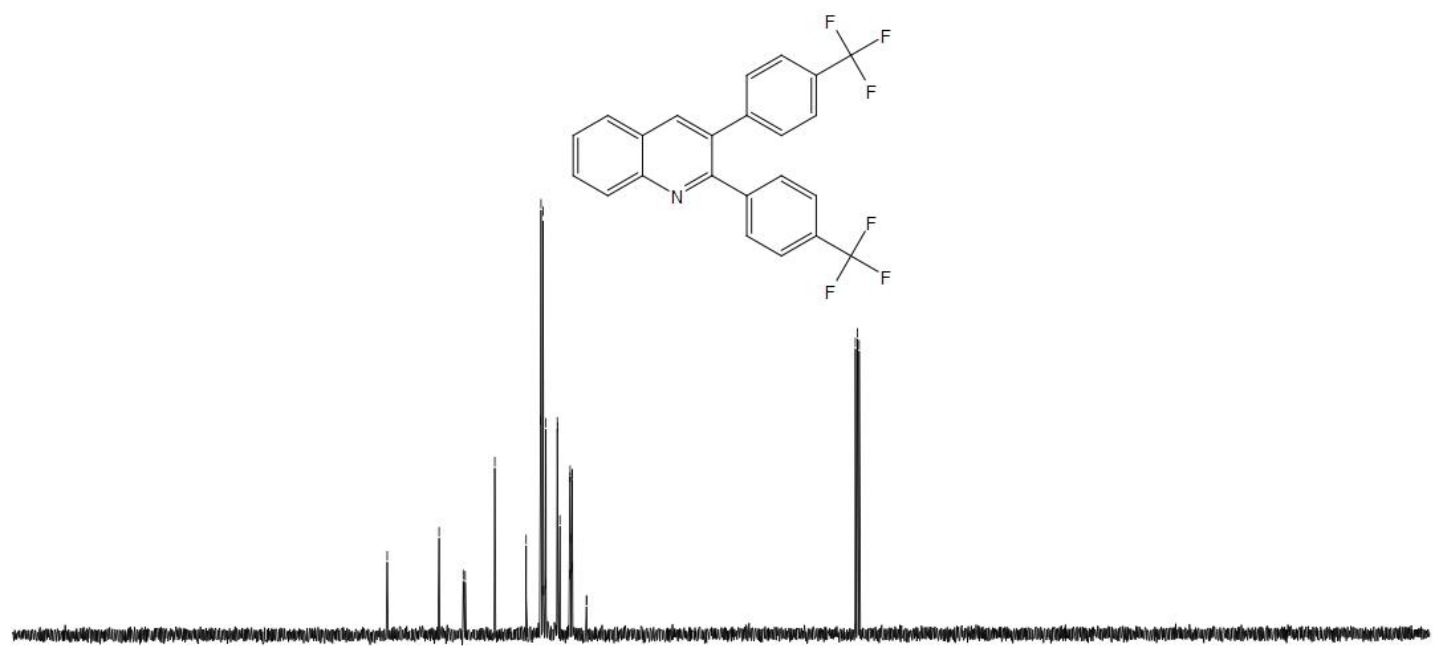

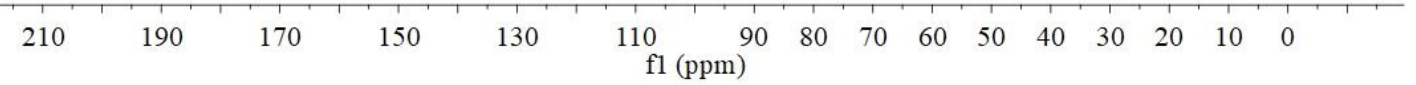


${ }^{19} \mathrm{~F}$ NMR (375 MHz, $\left.\mathrm{CDCl}_{3}\right)$

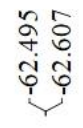

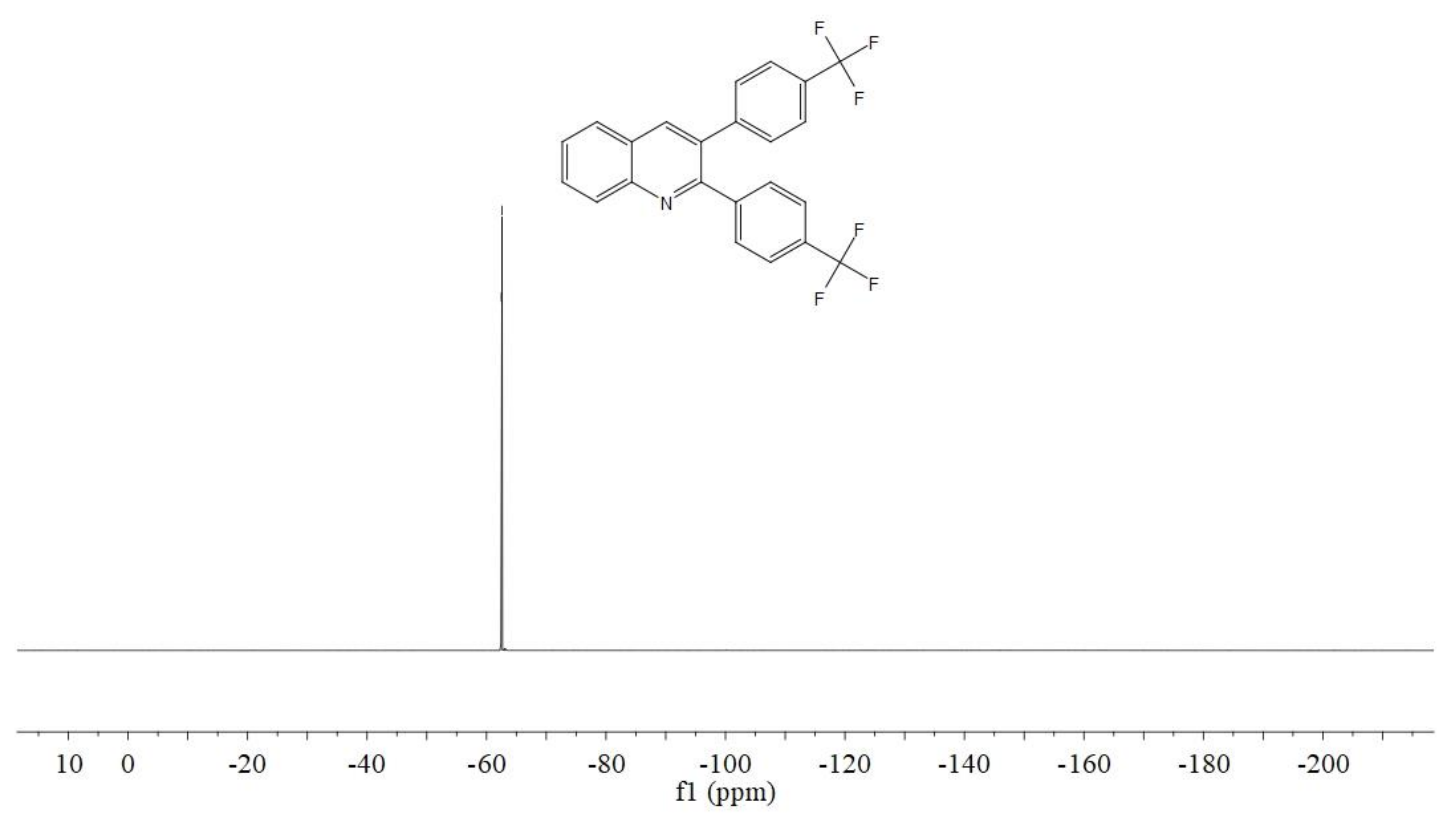


${ }^{1} \mathrm{H}$ NMR (400 MHz, $\left.\mathrm{CDCl}_{3}\right)$

\section{2,3-Di-p-Tolylquinoline (3ag)}

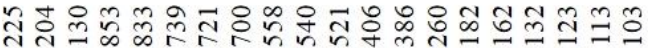

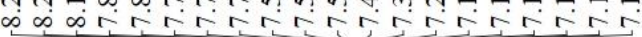

$\underbrace{n}_{\substack{n \\ m}}$
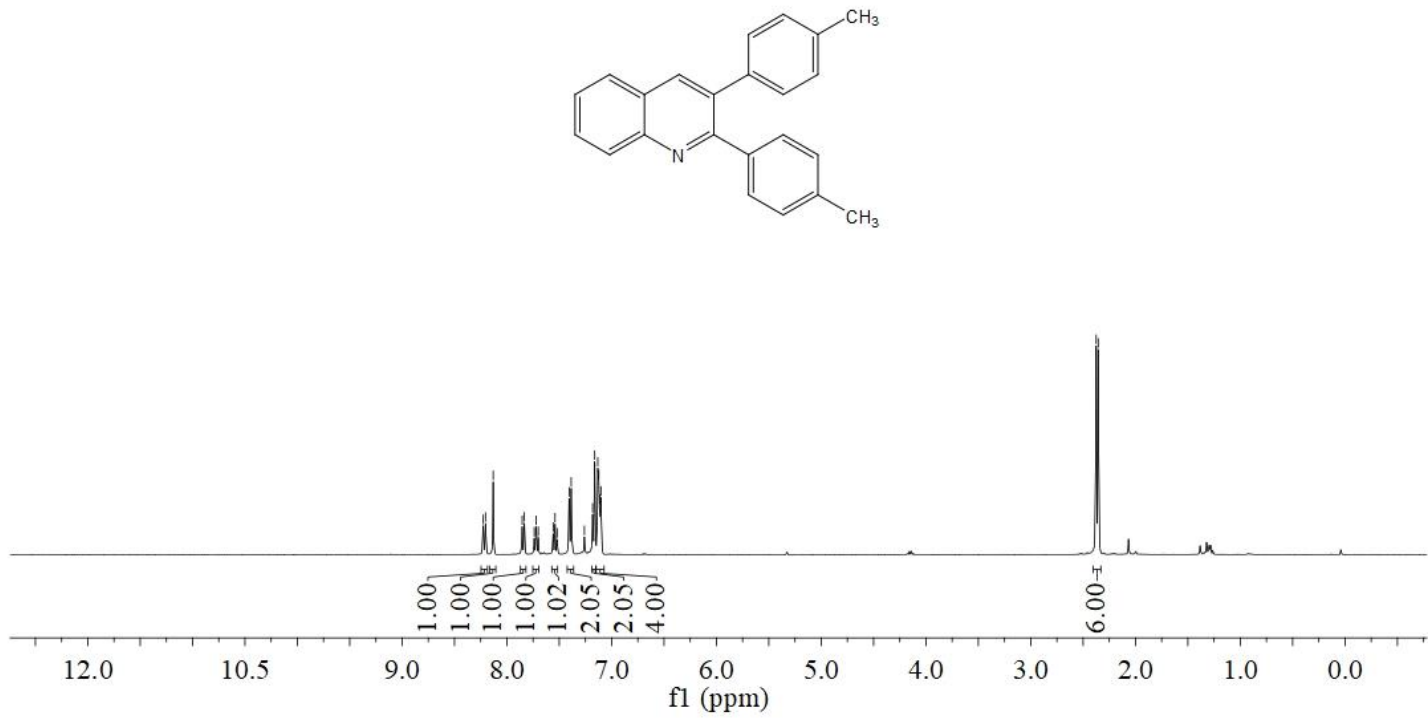

${ }^{13} \mathrm{C}$ NMR $\left(100 \mathrm{MHz}, \mathrm{CDCl}_{3}\right)$

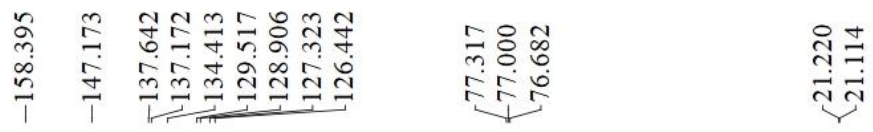

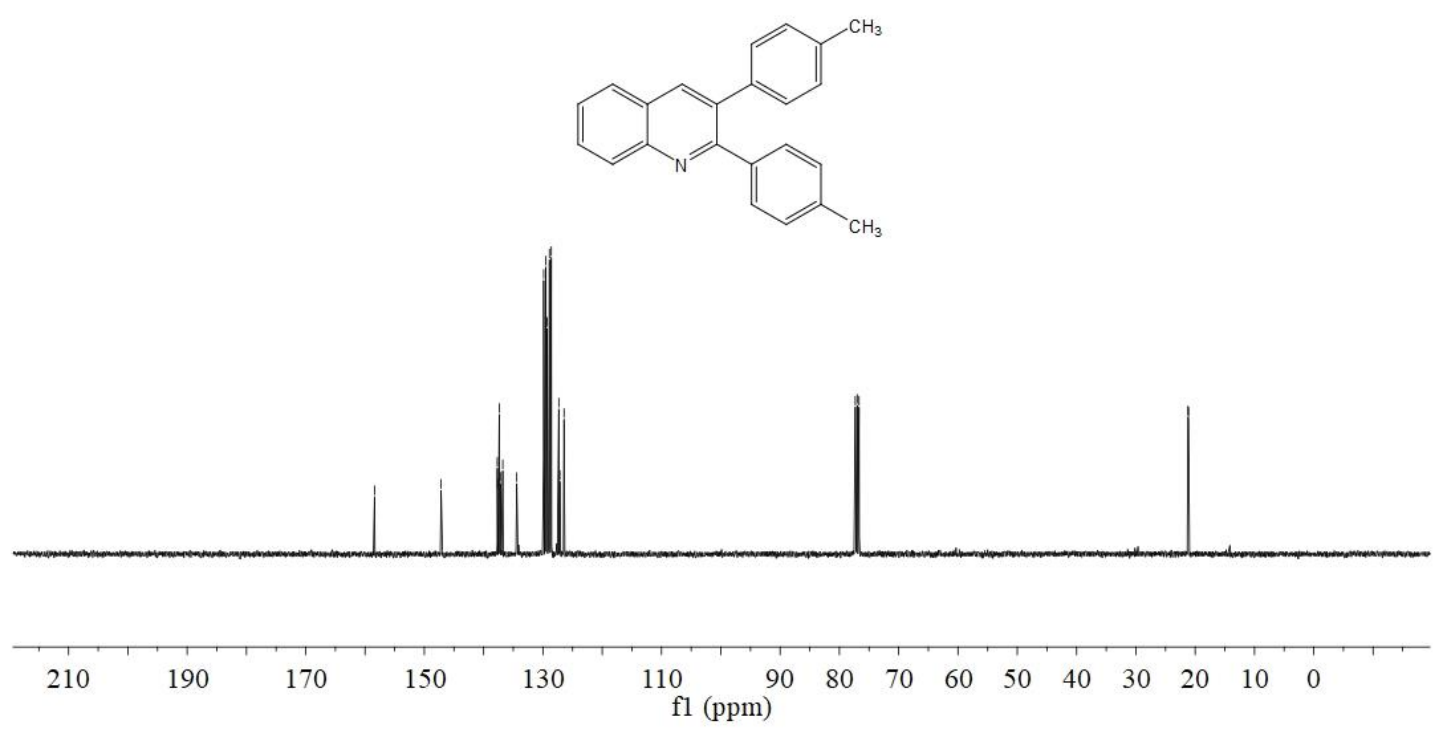


${ }^{1} \mathrm{H}$ NMR (400 MHz, $\mathrm{CDCl}_{3}$ )
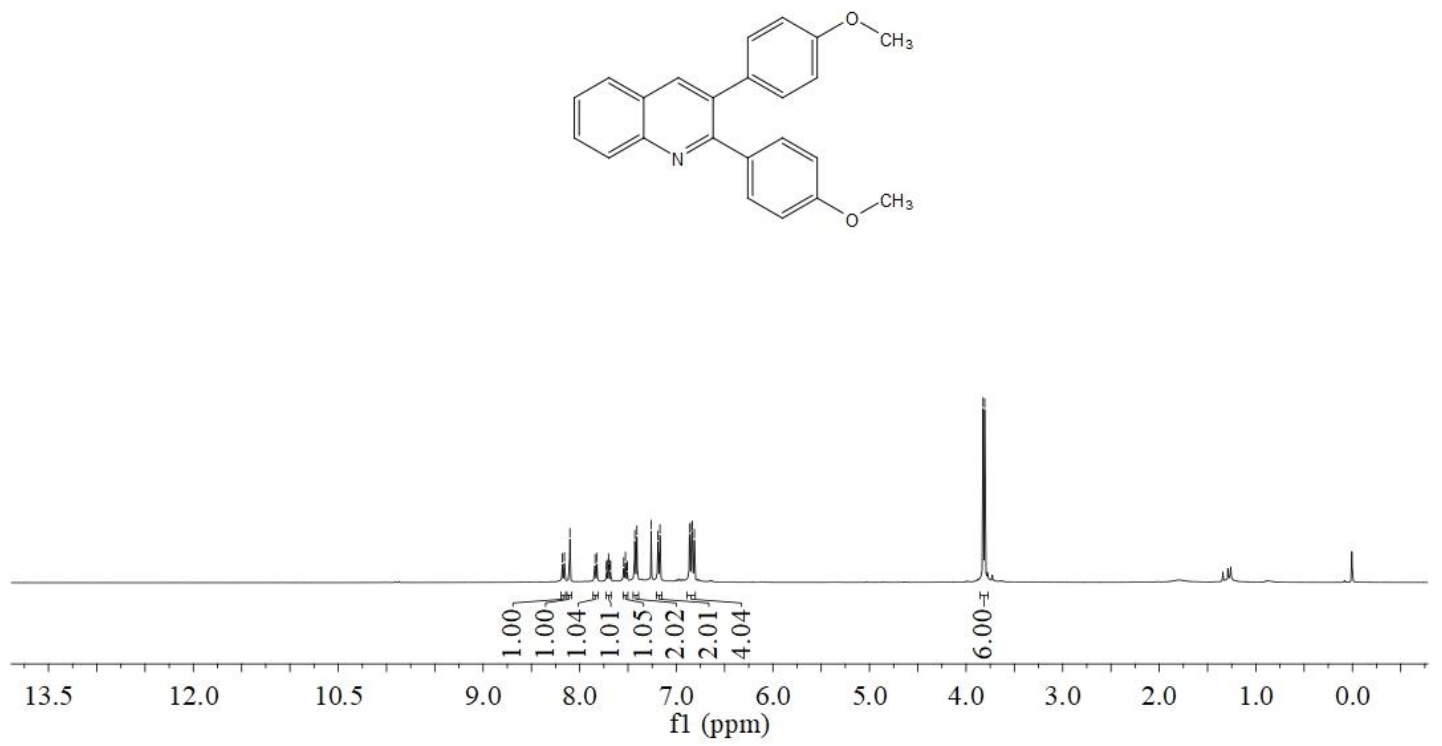

${ }^{13} \mathrm{C} \mathrm{NMR}\left(100 \mathrm{MHz}, \mathrm{CDCl}_{3}\right)$

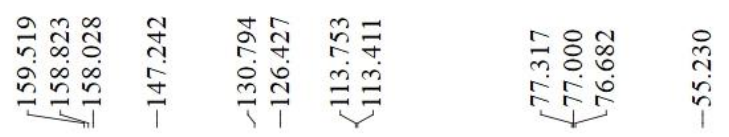
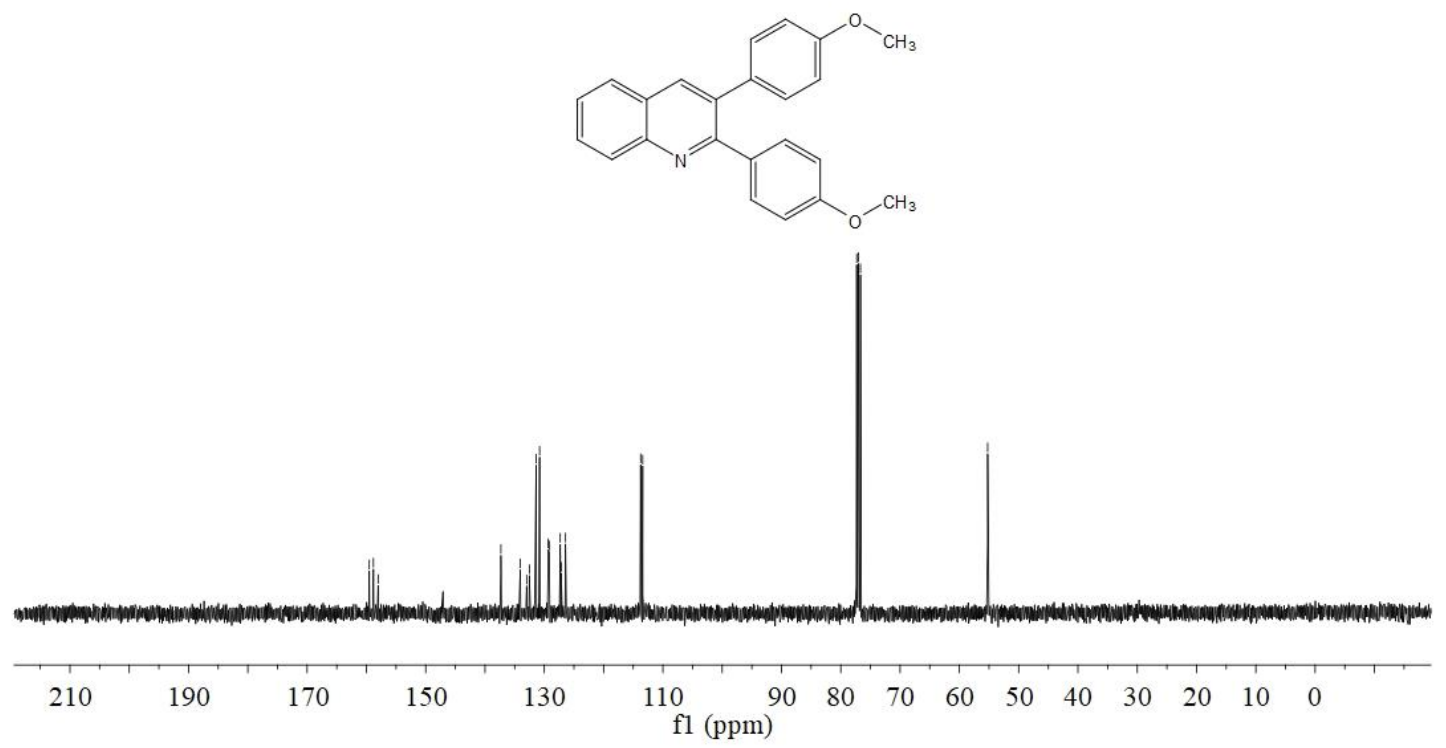
${ }^{1} \mathrm{H}$ NMR (400 MHz, $\mathrm{CDCl}_{3}$ )
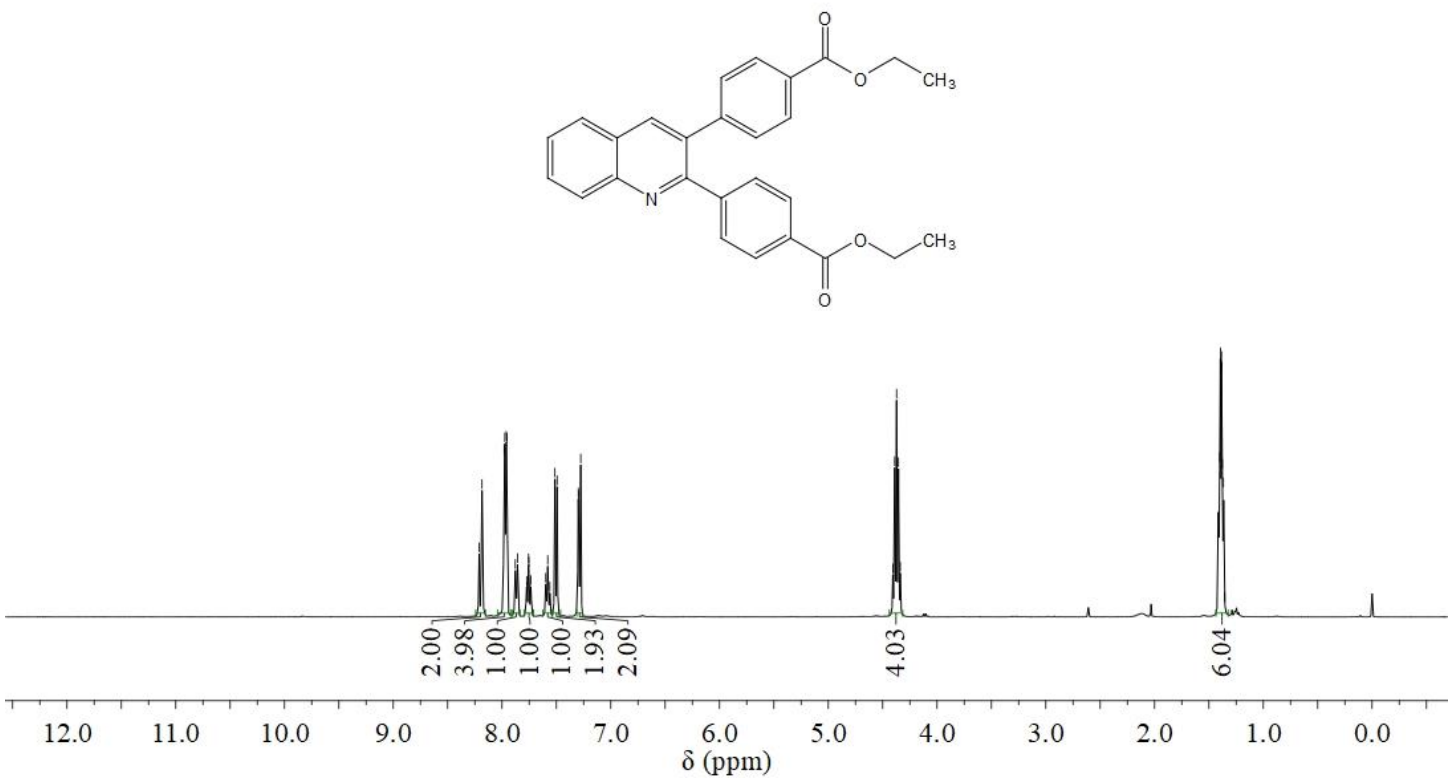

${ }^{13} \mathrm{C} \mathrm{NMR}\left(100 \mathrm{MHz}, \mathrm{CDCl}_{3}\right)$

\begin{tabular}{|c|c|c|c|c|}
\hline 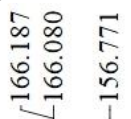 & 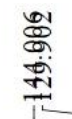 & 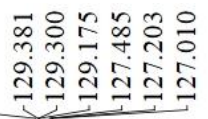 & 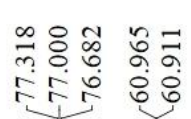 & 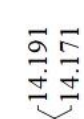 \\
\hline
\end{tabular}
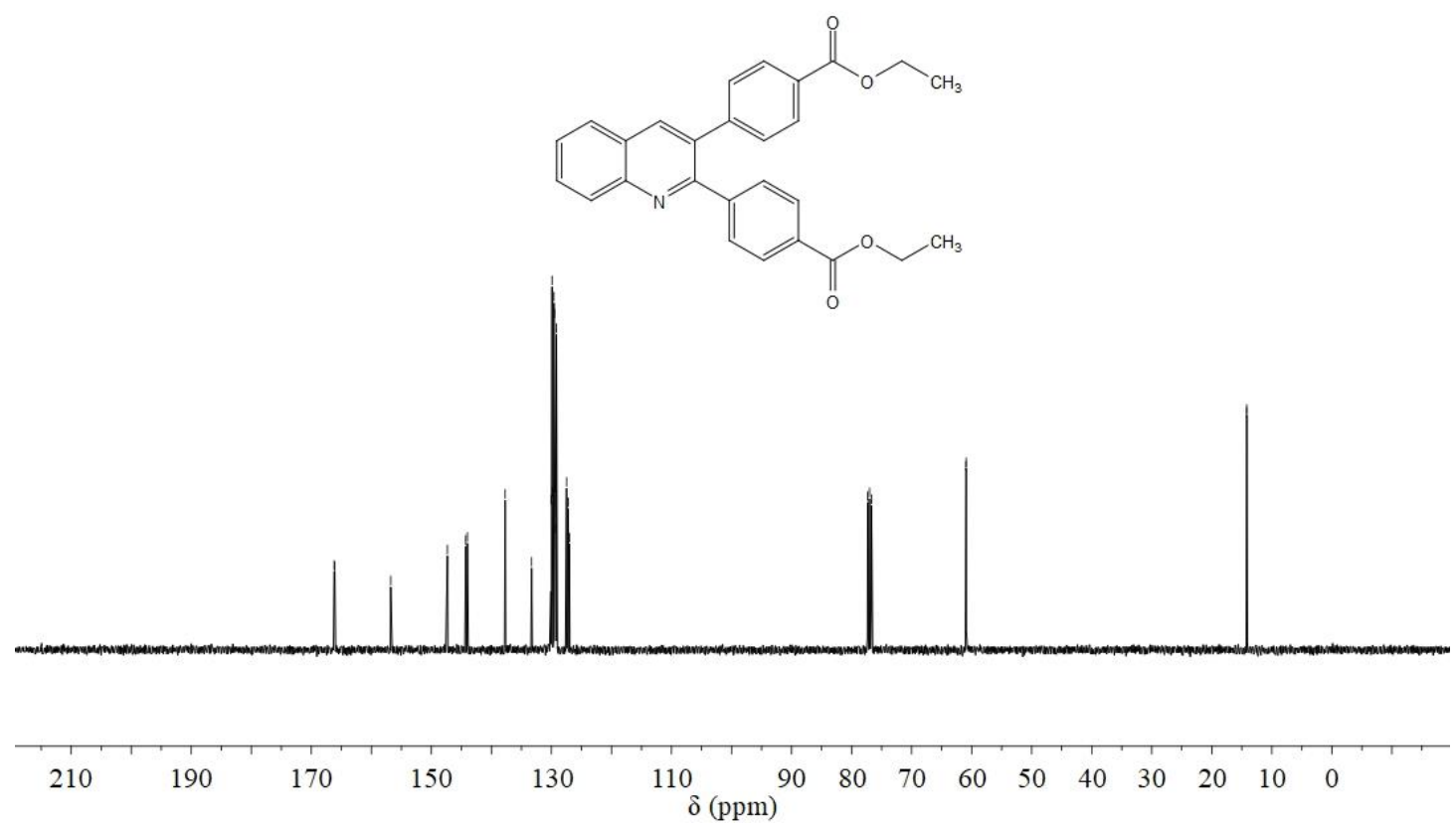
${ }^{1} \mathrm{H}$ NMR (400 MHz, $\left.\mathrm{CDCl}_{3}\right)$

\section{2,3-Di-o-tolylquinoline (3aj)}

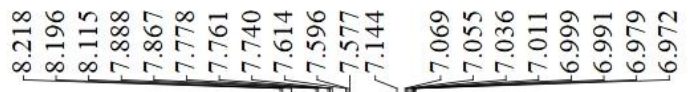

$\overrightarrow{3}$
तु
i
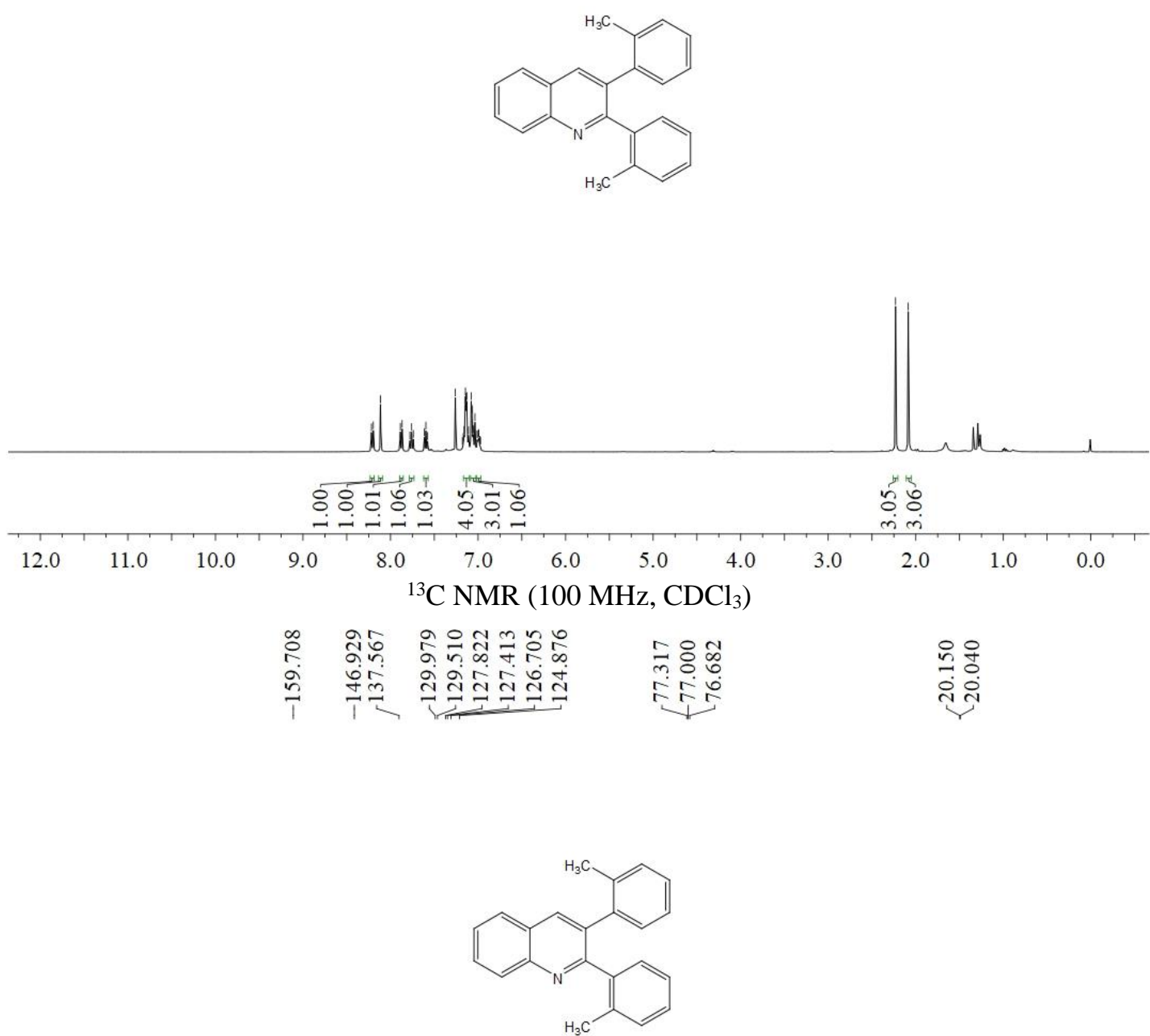

210

190

170

150

130

110

$\begin{array}{lllllllll}90 & 80 & 70 & 60 & 50 & 40 & 30 & 20 & 10\end{array}$ 
${ }^{1} \mathrm{H}$ NMR (400 MHz, $\mathrm{CDCl}_{3}$ )

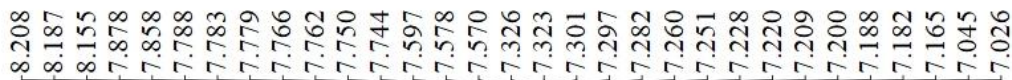
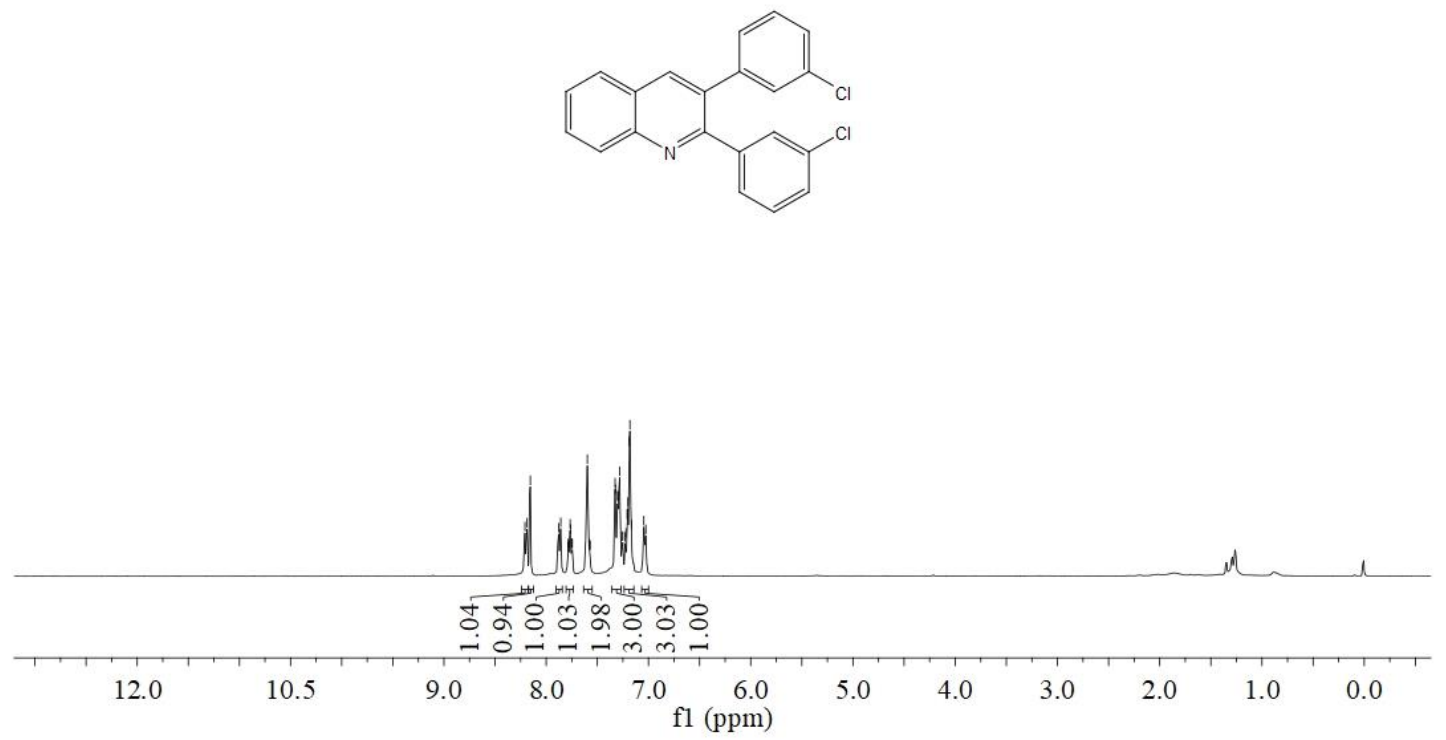

${ }^{13} \mathrm{C}$ NMR $\left(100 \mathrm{MHz}, \mathrm{CDCl}_{3}\right)$
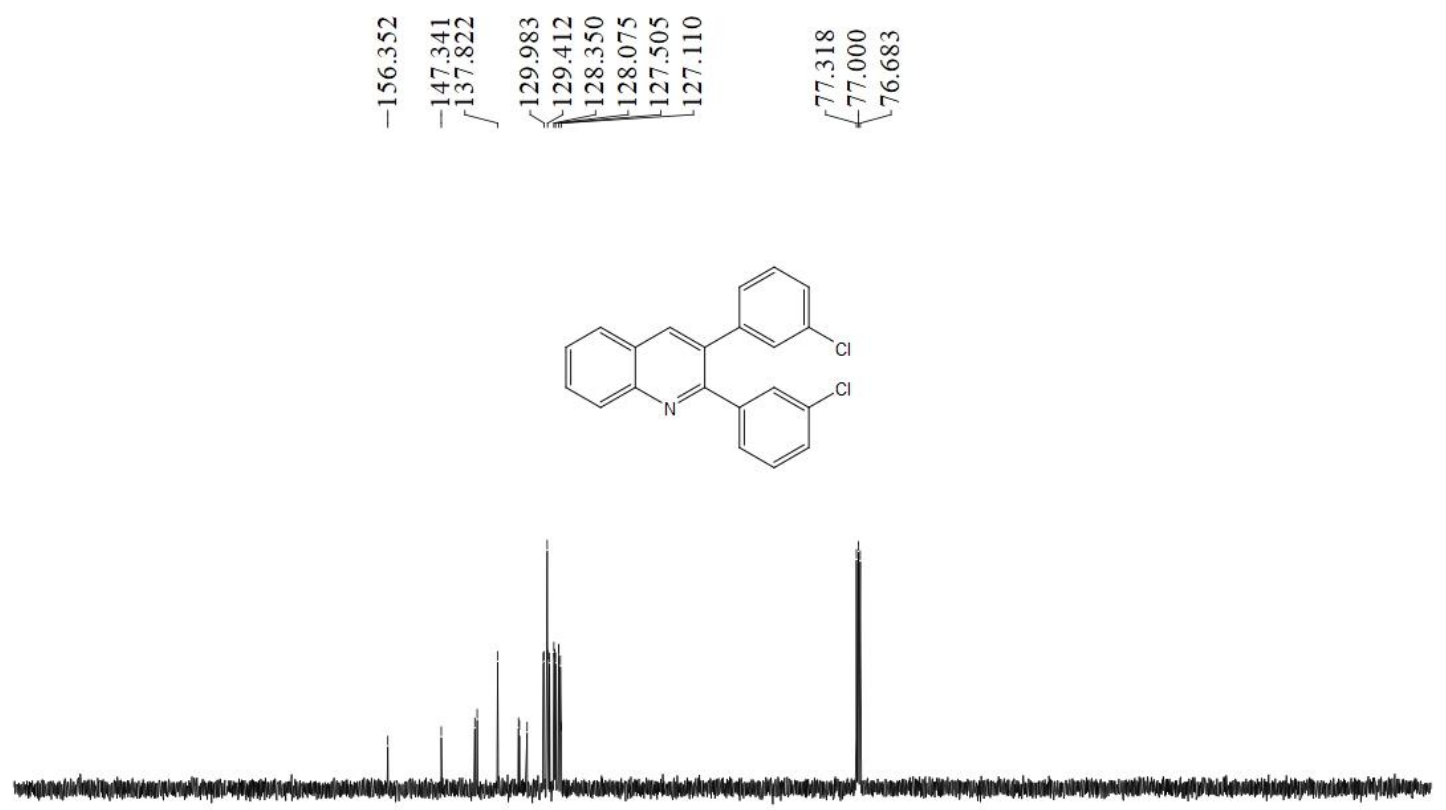

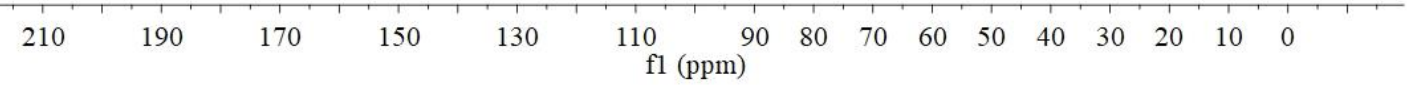


${ }^{1} \mathrm{H}$ NMR (400 MHz, $\left.\mathrm{CDCl}_{3}\right)$

\section{2,3-Di(naphthalen-1-yl) quinolone (3al)}

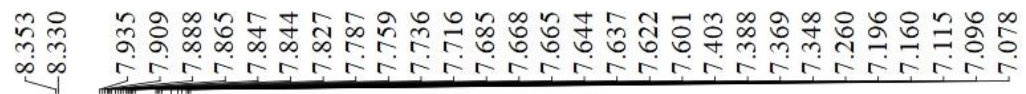<smiles>c1ccc2cc(-c3cc4ccccc4nc3-c3cccc4ccccc34)ccc2c1</smiles>

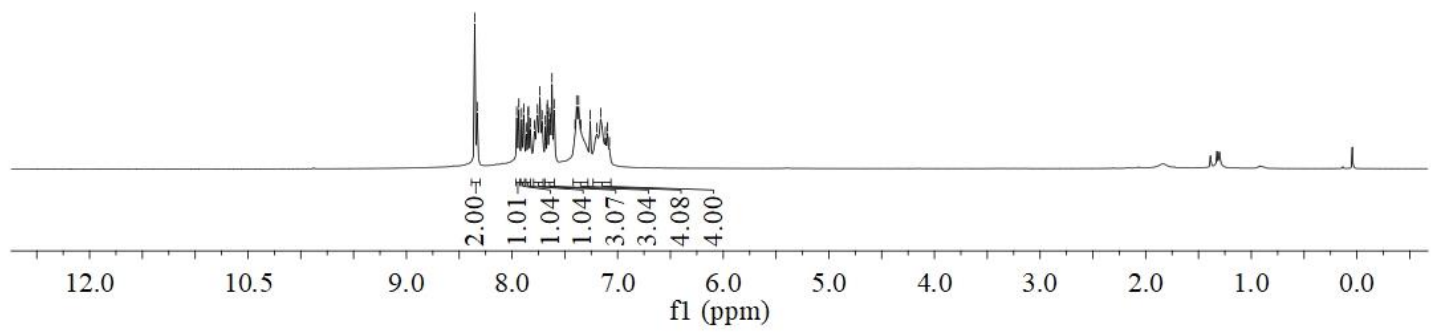

${ }^{13} \mathrm{C}$ NMR (100 MHz, $\left.\mathrm{CDCl}_{3}\right)$

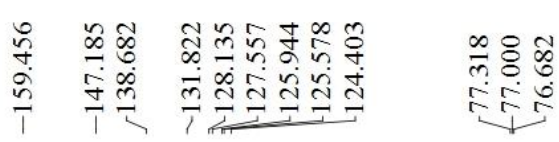

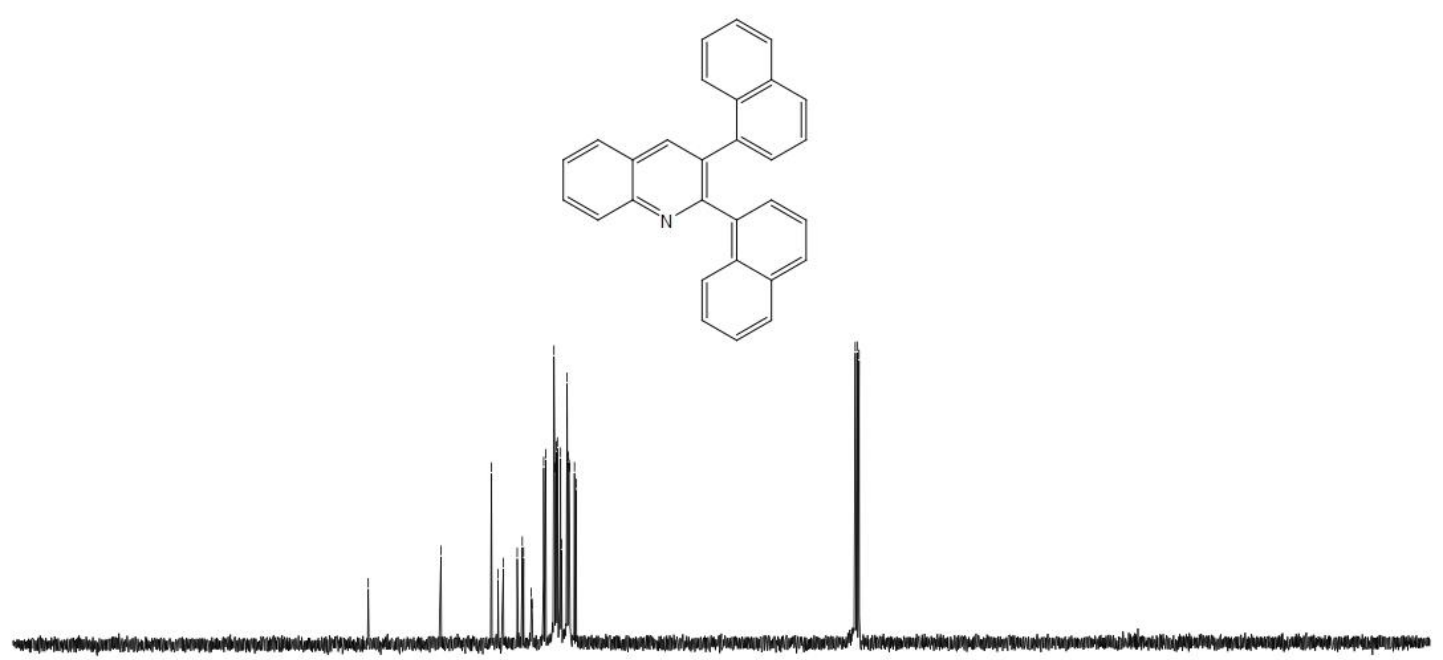

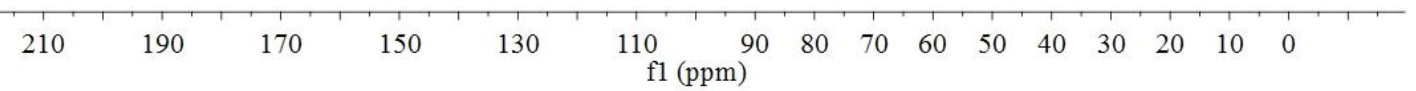


${ }^{1} \mathrm{H}$ NMR (400 MHz, $\left.\mathrm{CDCl}_{3}\right)$

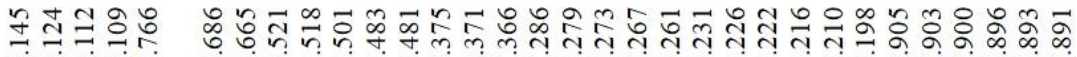

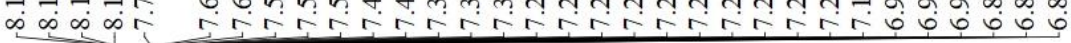
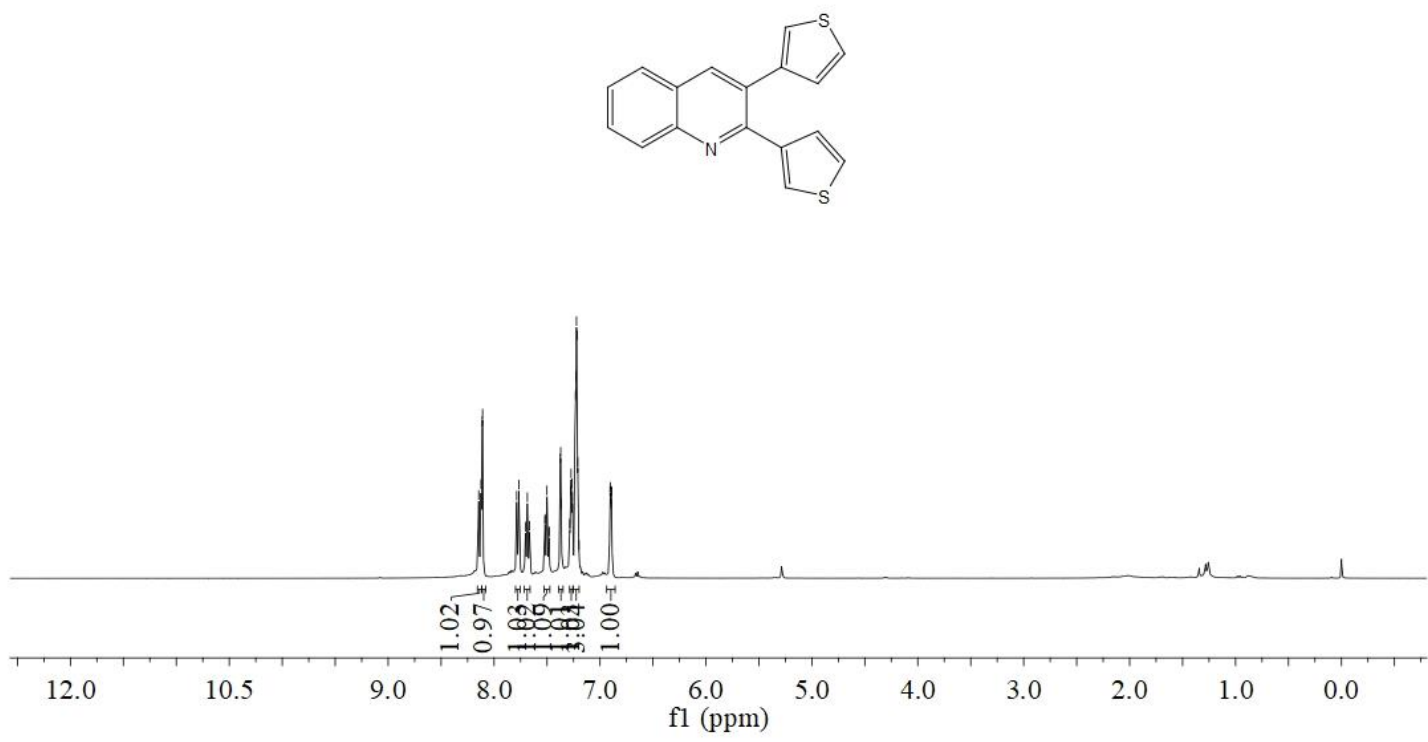

${ }^{13} \mathrm{C}$ NMR (100 MHz, $\left.\mathrm{CDCl}_{3}\right)$

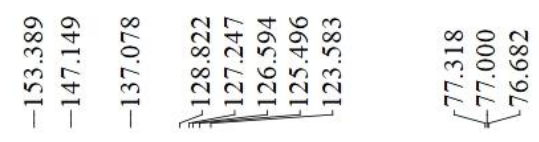<smiles>c1csc(-c2nc3ccccc3cc2-c2ccsc2)c1</smiles>

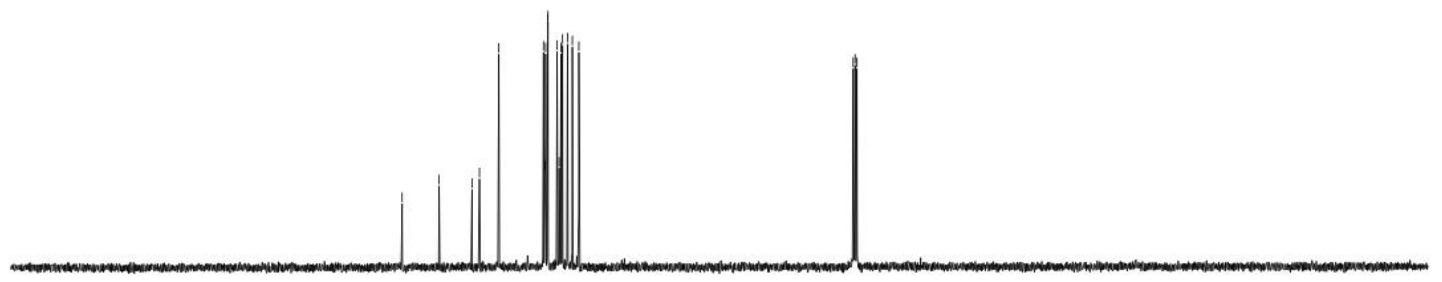

$210 \quad \begin{array}{lllllllllllllll}190 & 170 & 150 & 130 & \begin{array}{c}110 \\ \mathrm{f} 1(\mathrm{ppm})\end{array} & 90 & 70 & 60 & 50 & 40 & 30 & 20 & 10 & 0\end{array}$


${ }^{1} \mathrm{H}$ NMR (400 MHz, $\left.\mathrm{CDCl}_{3}\right)$

\section{2,3-Dipropylquinoline (3an)}

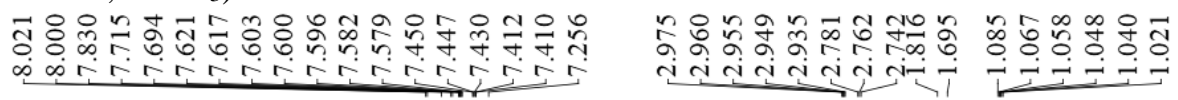

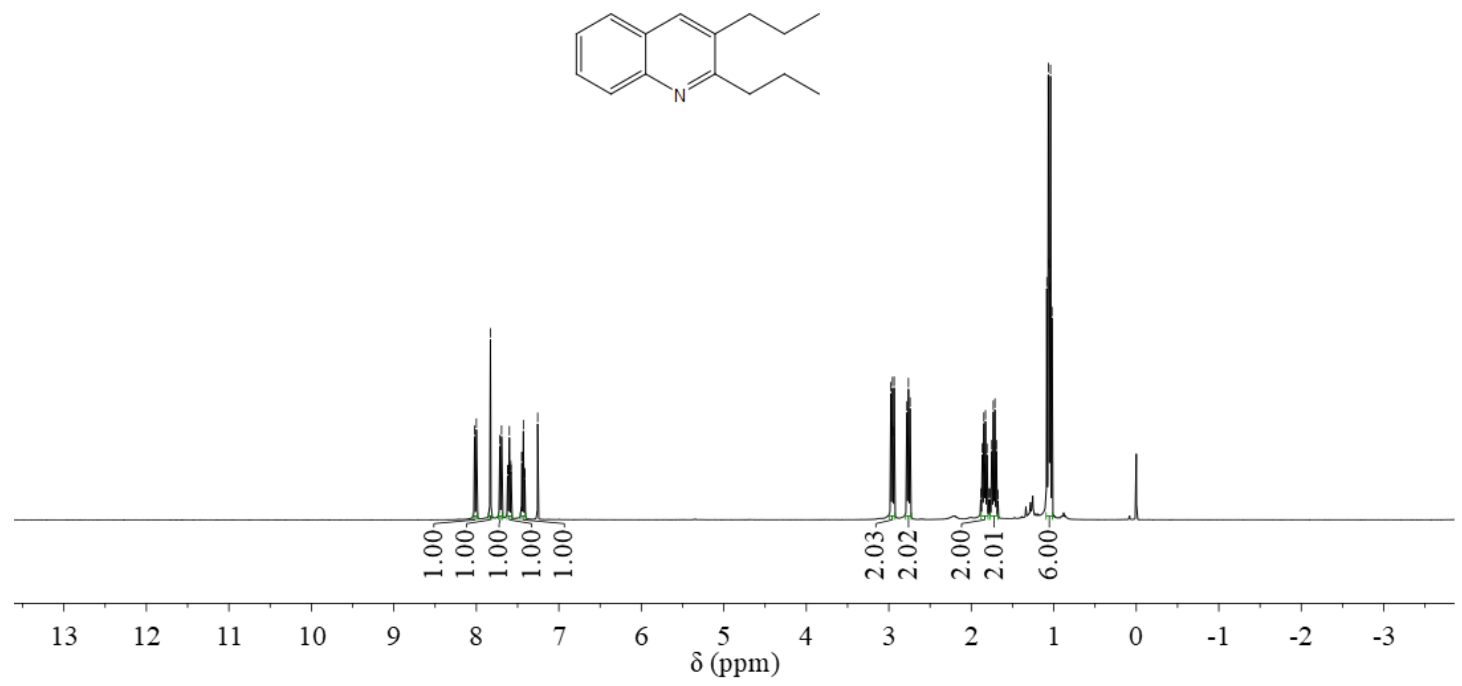

${ }^{13} \mathrm{C}$ NMR (100 MHz, $\left.\mathrm{CDCl}_{3}\right)$

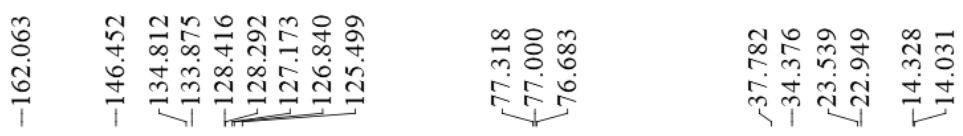
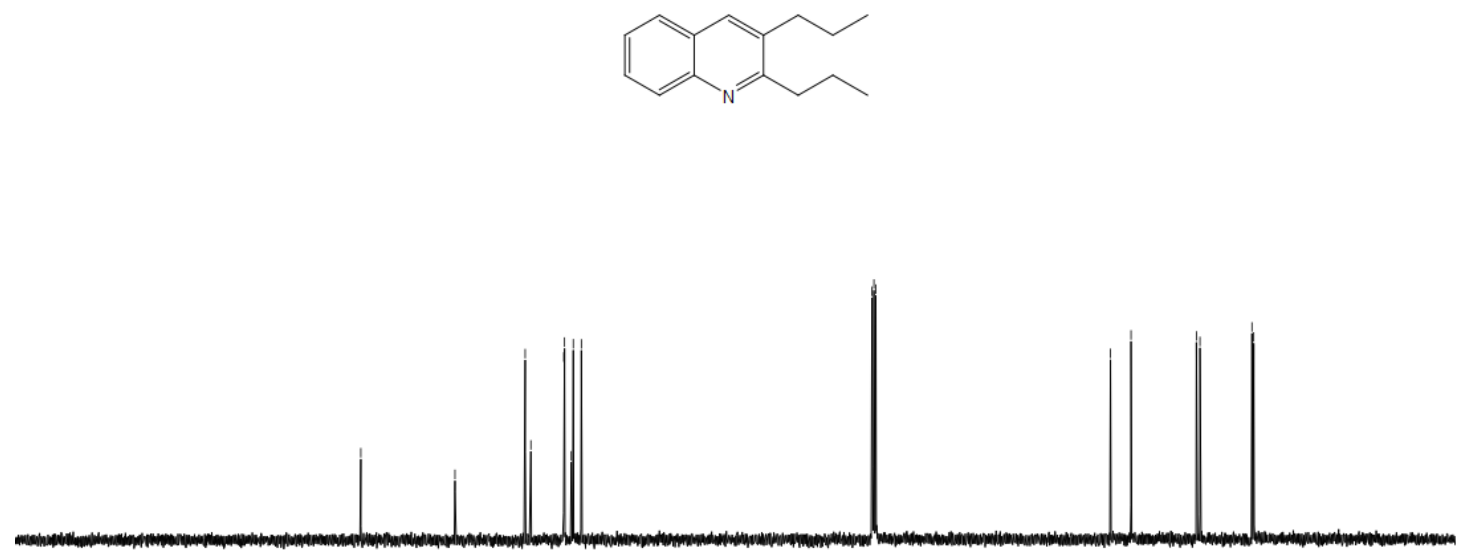

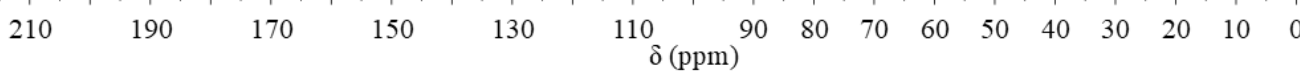


$\mathbf{6 , 7 , 8 , 9 , 1 0 , 1 1 , 1 2 , 1 3 , 1 4 , 1 5 - D e c a h y d r o c y c l o d o d e c a [ b ] q u i n o l i n e ~ ( 3 a o ) ~}$

${ }^{1} \mathrm{H}$ NMR (400 MHz, $\left.\mathrm{CDCl}_{3}\right)$

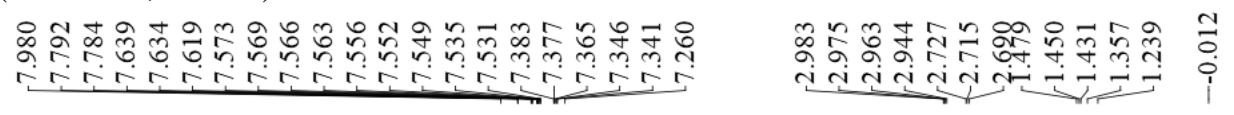

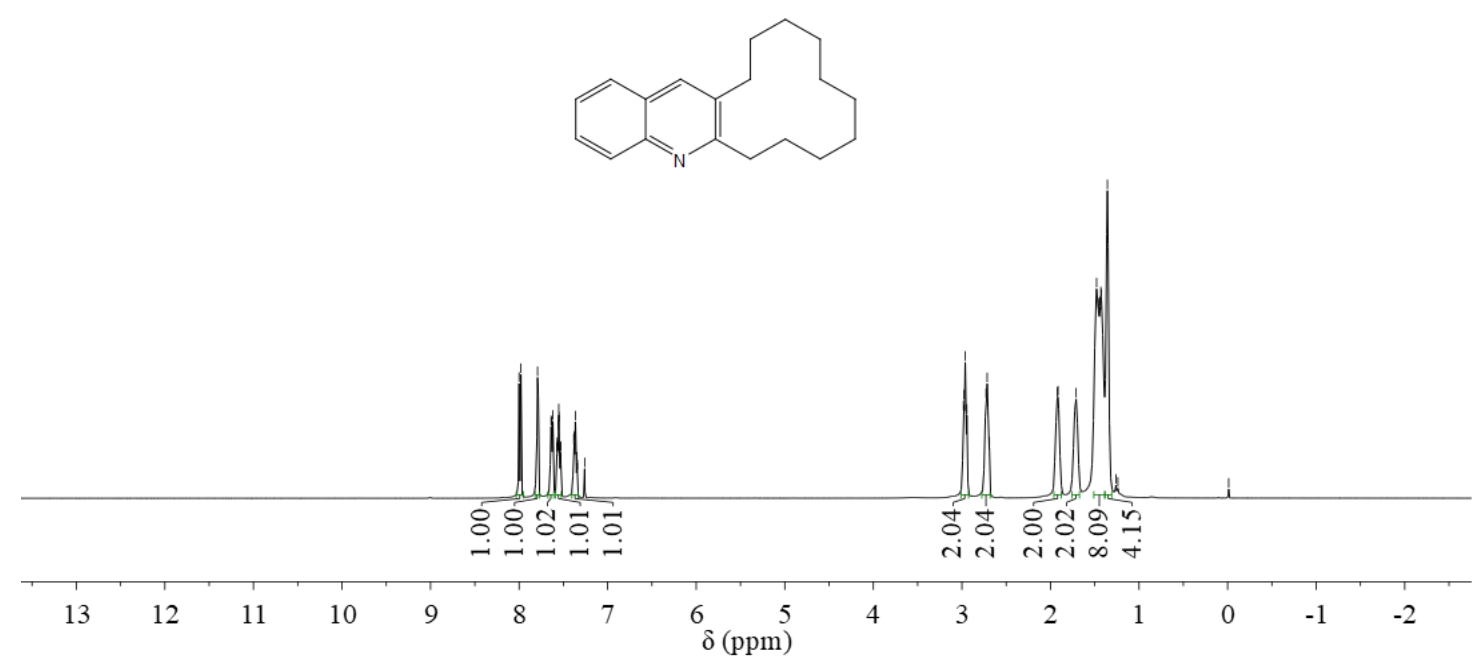

${ }^{13} \mathrm{C}$ NMR $\left(100 \mathrm{MHz}, \mathrm{CDCl}_{3}\right)$

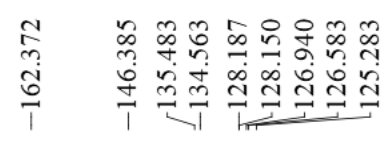

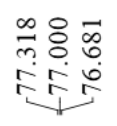

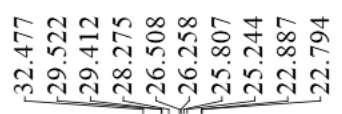
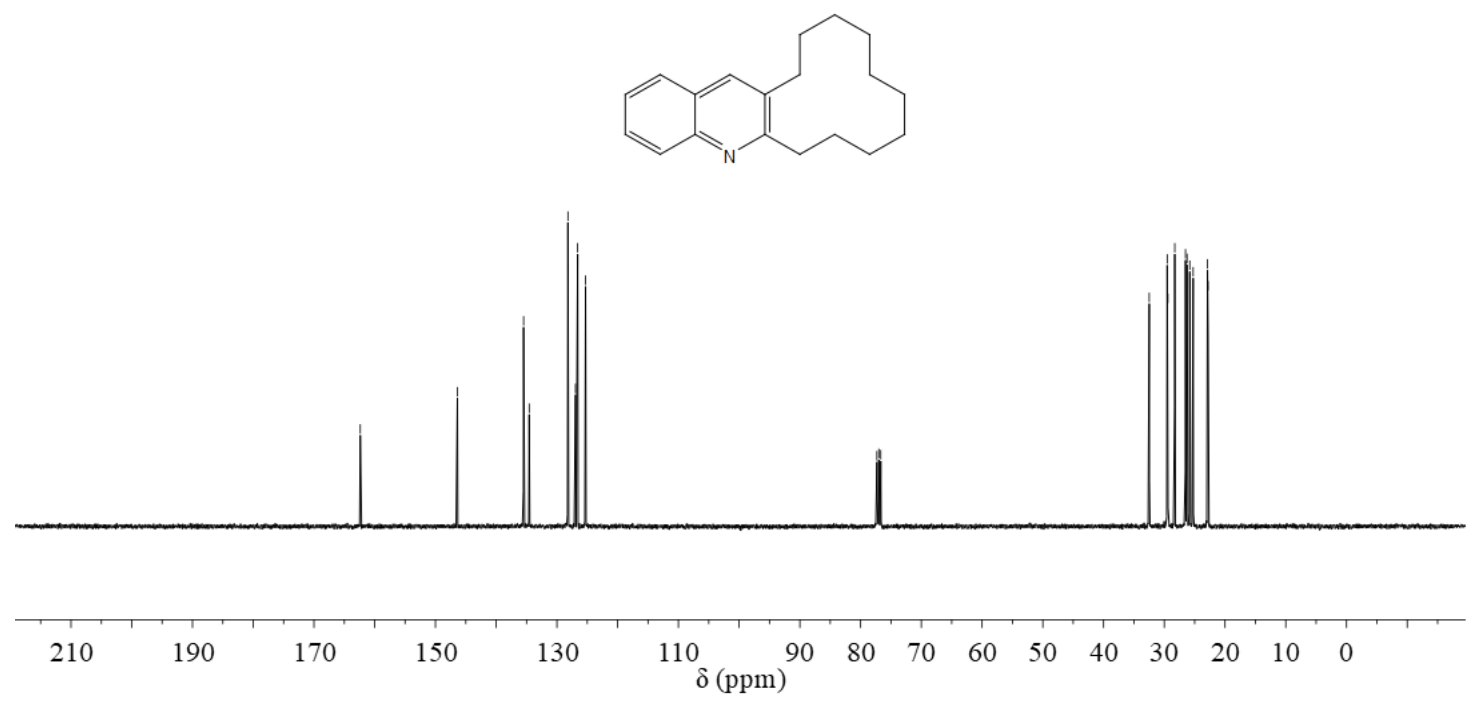

86 
${ }^{1} \mathrm{H}$ NMR (400 MHz, $\left.\mathrm{CDCl}_{3}\right)$
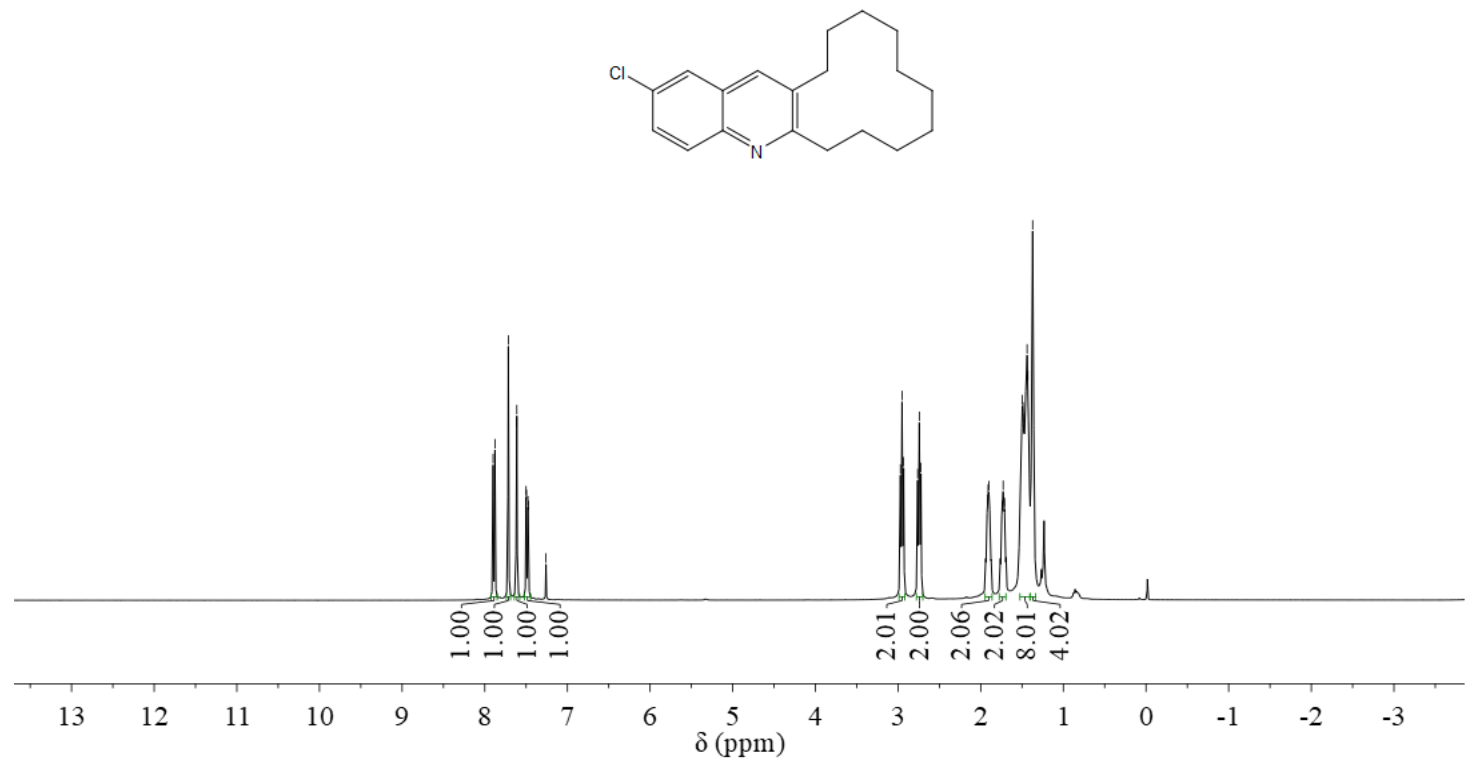

${ }^{13} \mathrm{C} \mathrm{NMR}\left(100 \mathrm{MHz}, \mathrm{CDCl}_{3}\right)$

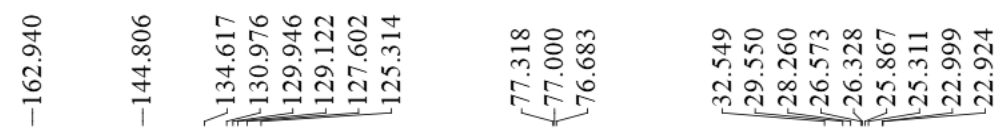

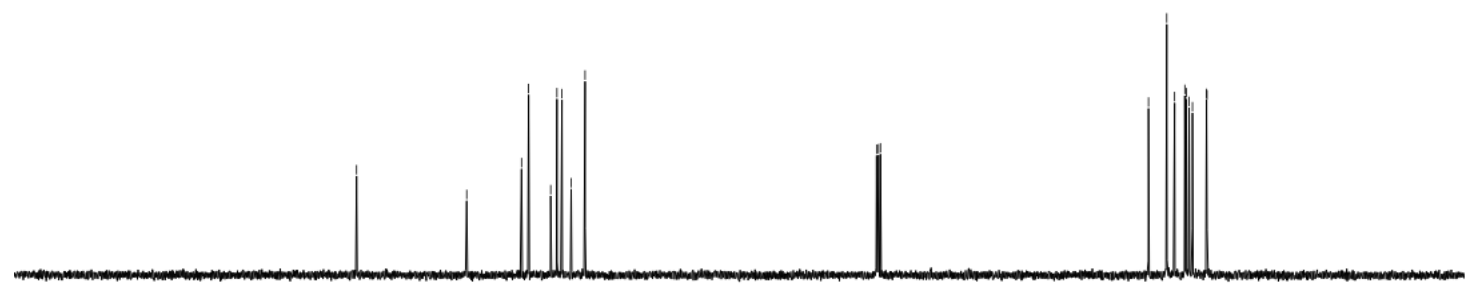

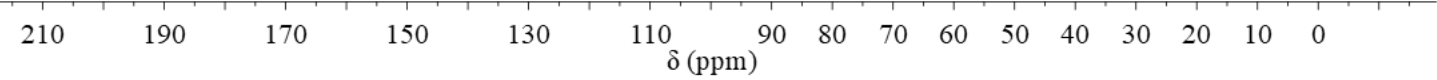


${ }^{1} \mathrm{H}$ NMR (400 MHz, $\left.\mathrm{CDCl}_{3}\right)$

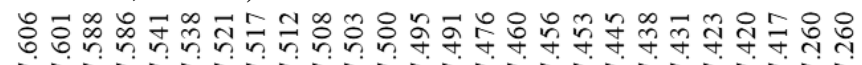

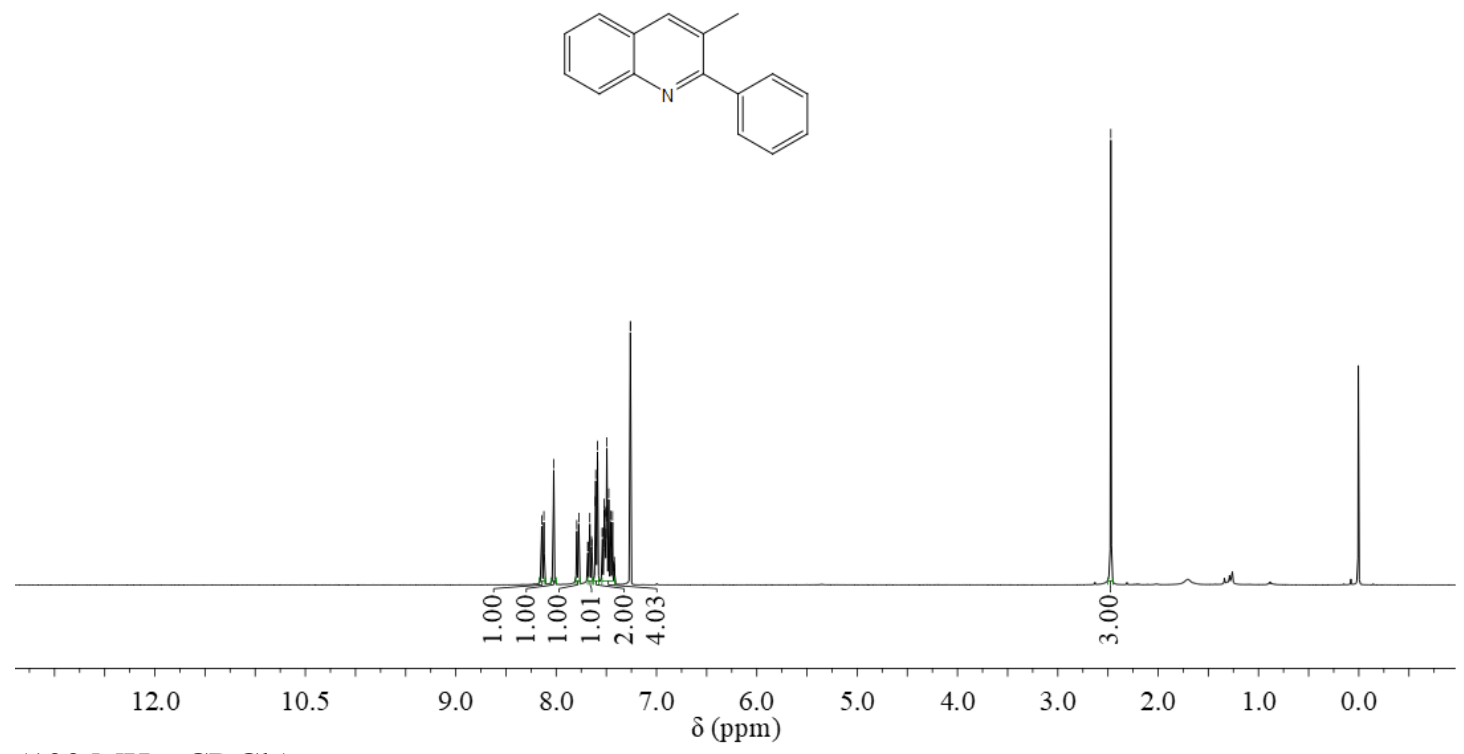

${ }^{13} \mathrm{C} \mathrm{NMR}\left(100 \mathrm{MHz}, \mathrm{CDCl}_{3}\right)$

\begin{tabular}{|c|c|c|c|}
\hline \begin{tabular}{l}
$\infty$ \\
$o$ \\
$n$ \\
0 \\
\multirow{1}{1}{} \\
1
\end{tabular} & 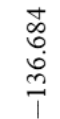 & 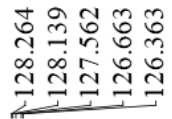 & $\begin{array}{r}=80 \\
m 80 \\
500 \\
5\end{array}$ \\
\hline
\end{tabular}

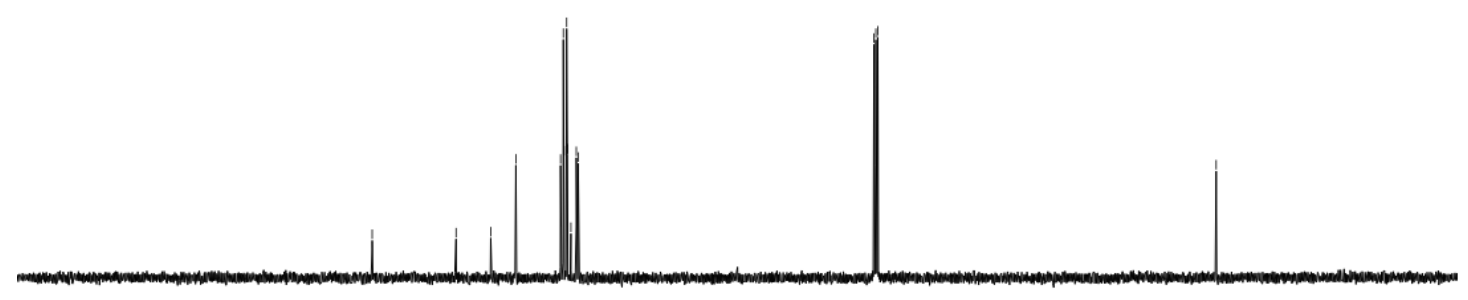

$\begin{array}{lllllllllllllll}210 & 190 & 170 & 150 & 130 & \left.110_{\delta(\mathrm{ppm})}\right) & 80 & 70 & 60 & 50 & 40 & 30 & 20 & 10 & 0\end{array}$ 
${ }^{1} \mathrm{H}$ NMR (400 MHz, $\mathrm{CDCl}_{3}$ )

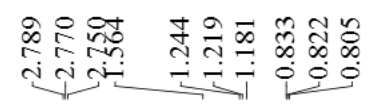

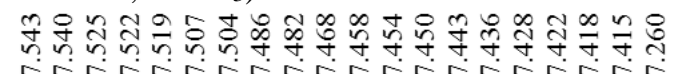

ini
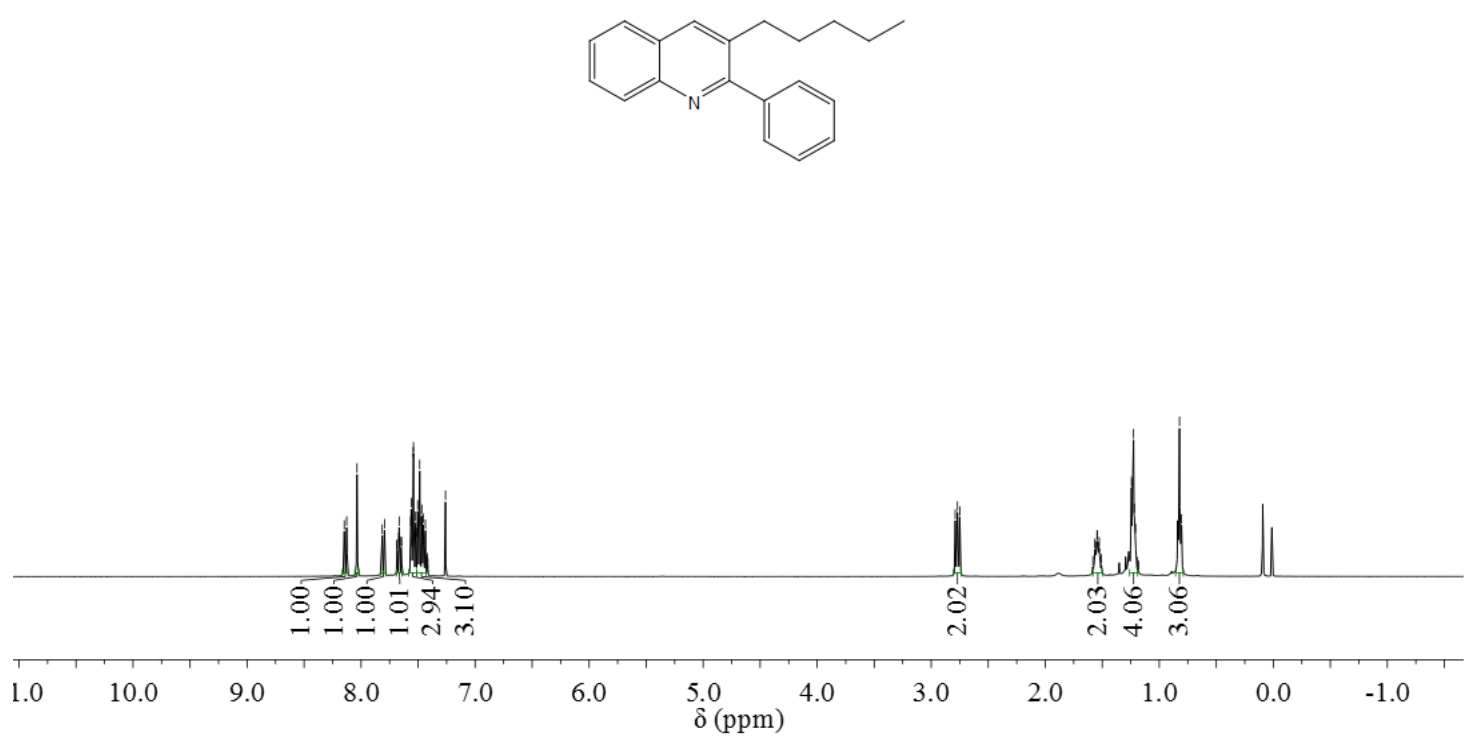

${ }^{13} \mathrm{C}$ NMR (100 MHz, $\left.\mathrm{CDCl}_{3}\right)$

\begin{tabular}{|c|c|c|}
\hline 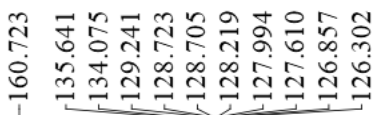 & 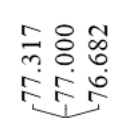 & 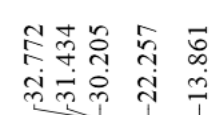 \\
\hline
\end{tabular}<smiles>CCCCCc1cc2ccccc2nc1-c1ccccc1</smiles>

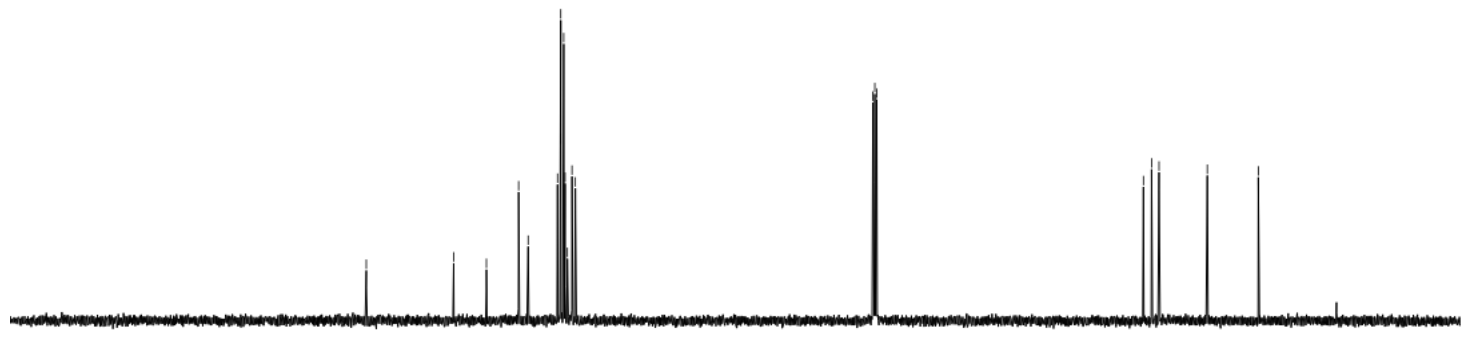

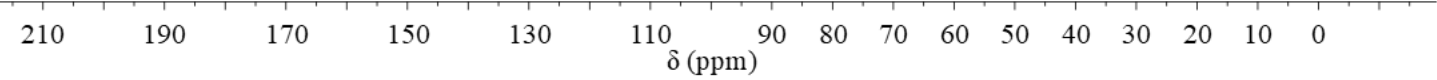




\section{(2-Phenylquinolin-3-yl)methanol (3as)}

${ }^{1} \mathrm{H} \mathrm{NMR}\left(400 \mathrm{MHz}, \mathrm{CDCl}_{3}\right)$

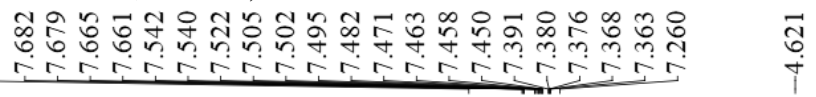
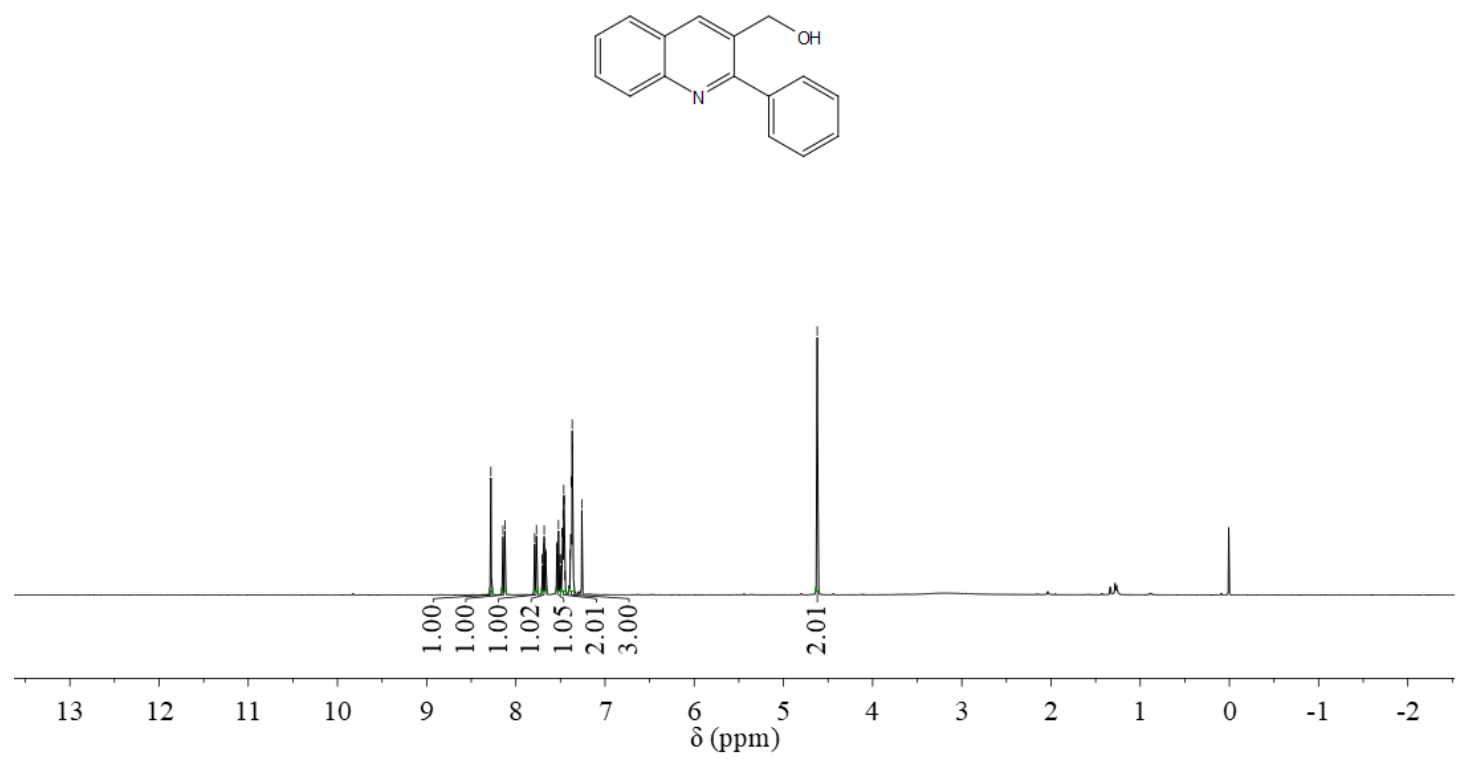

${ }^{13} \mathrm{C}$ NMR (100 MHz, $\mathrm{CDCl}_{3}$ )

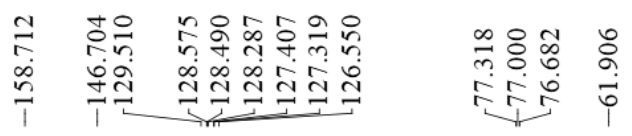
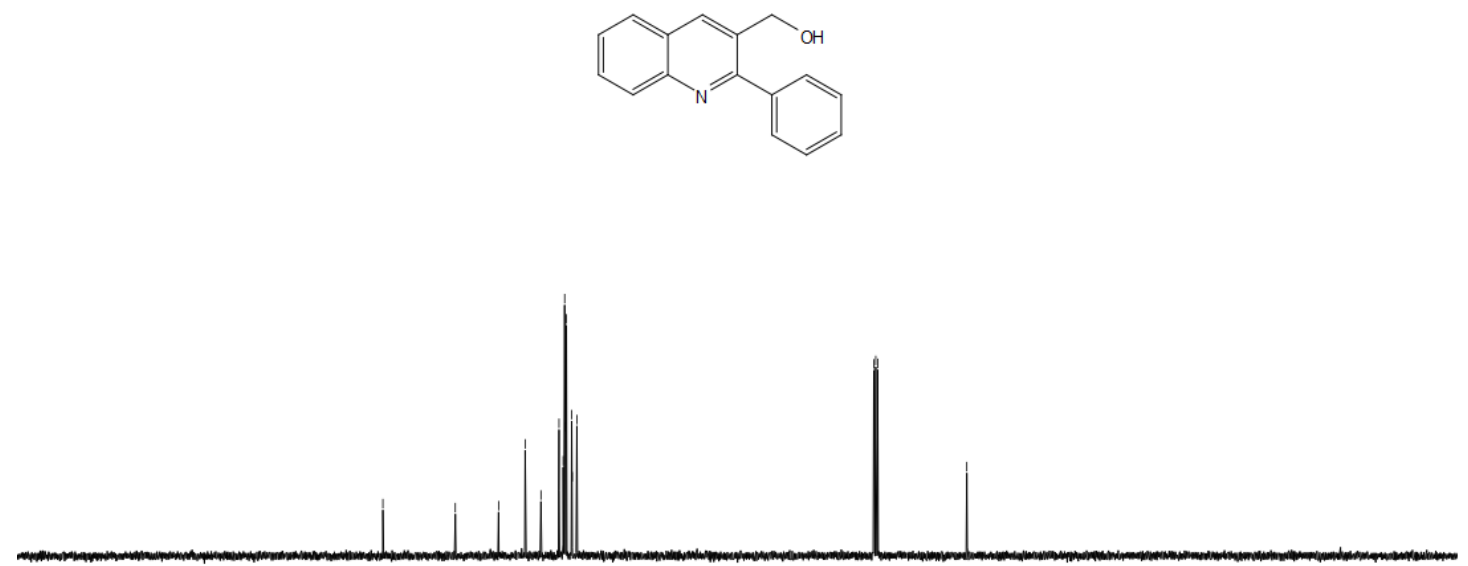

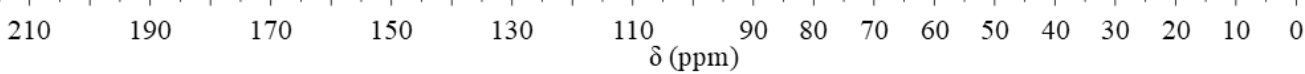



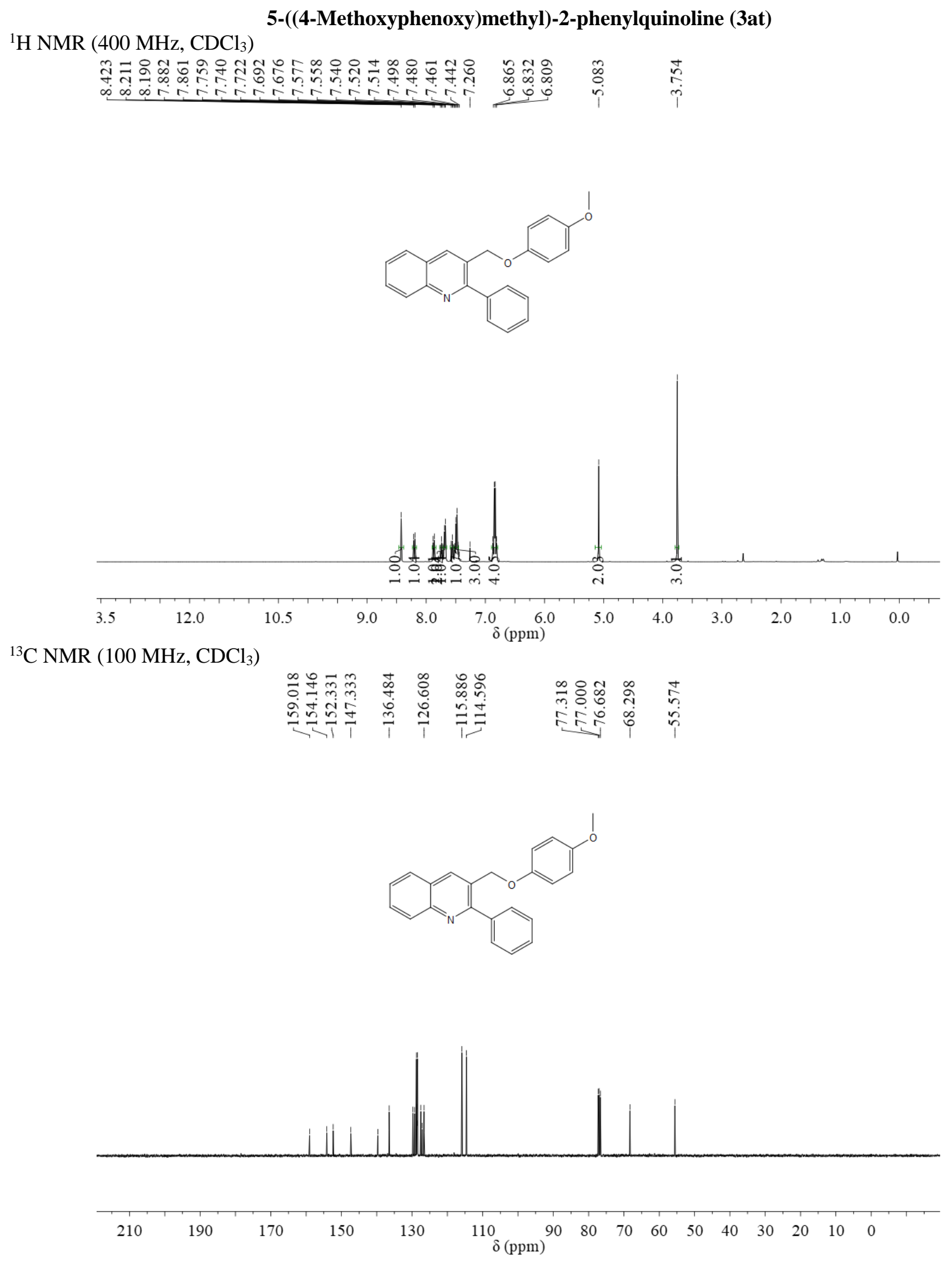

91 
${ }^{1} \mathrm{H}$ NMR (400 MHz, $\left.\mathrm{CDCl}_{3}\right)$

Ethyl 2-phenylquinoline-3-carboxylate (3au)

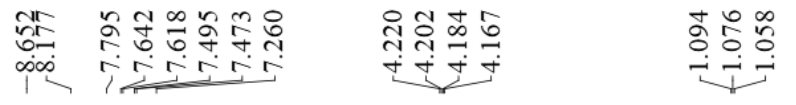
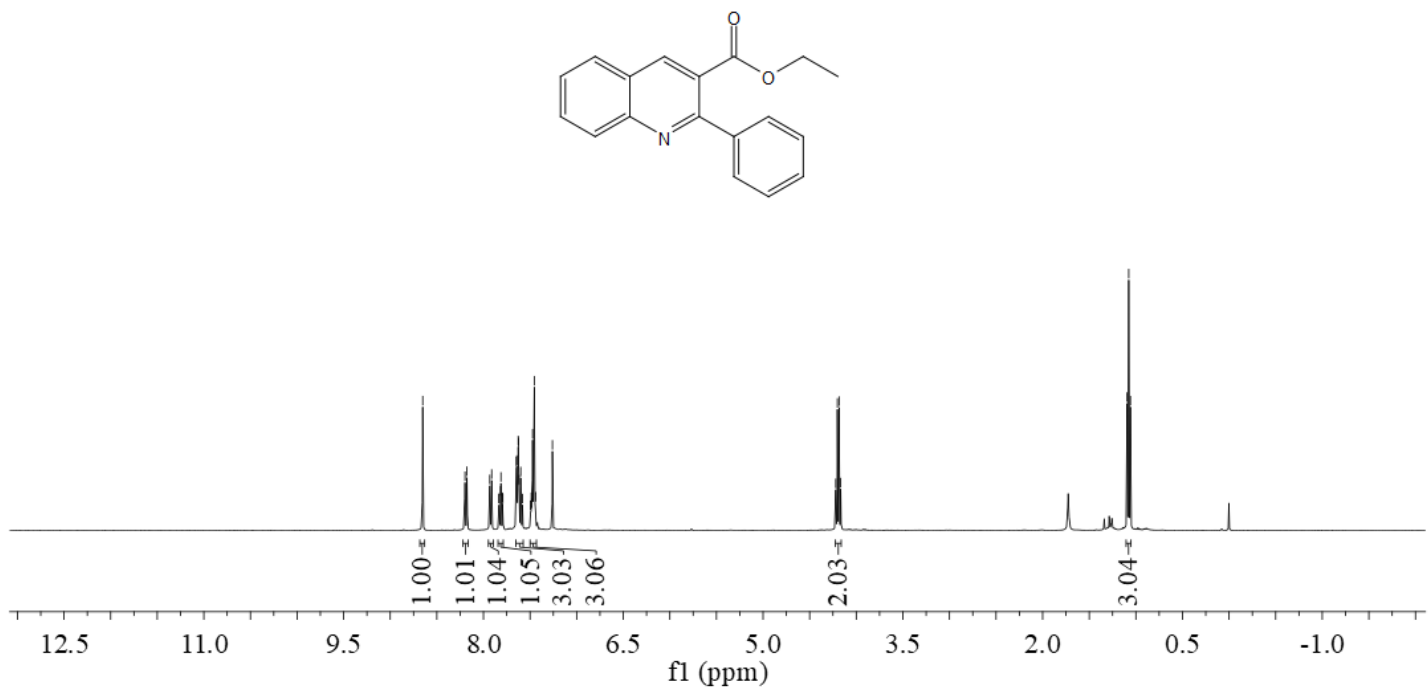

${ }^{13} \mathrm{C}$ NMR $\left(100 \mathrm{MHz}, \mathrm{CDCl}_{3}\right)$

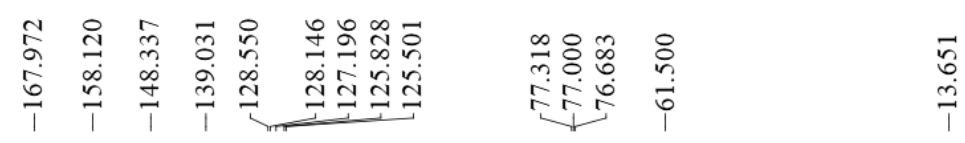<smiles>CCOC(=O)c1cc2ccccc2nc1-c1ccccc1</smiles>

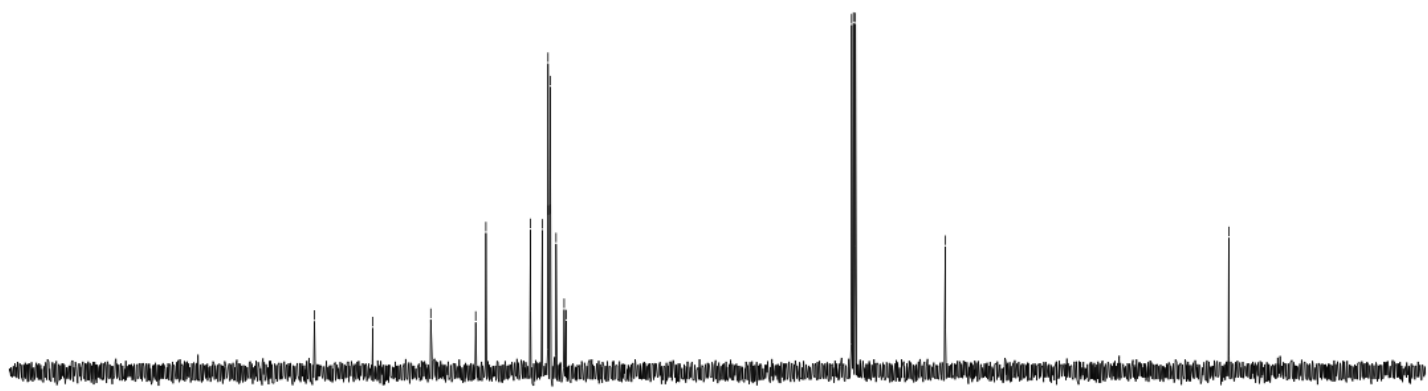

$\begin{array}{cccccccccccccccccc}210 & 190 & 170 & 150 & 130 & \begin{array}{c}110 \\ \mathrm{f} 1(\mathrm{ppm})\end{array} & 90 & 70 & 60 & 50 & 40 & 30 & 20 & 10 & 0 & \end{array}$


${ }^{1} \mathrm{H}$ NMR (400 MHz, $\mathrm{CDCl}_{3}$ )

\section{4-(tert-Butyl)phenyl 2-phenylquinoline-3-carboxylate (3av)}

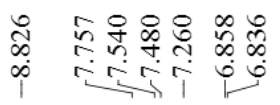
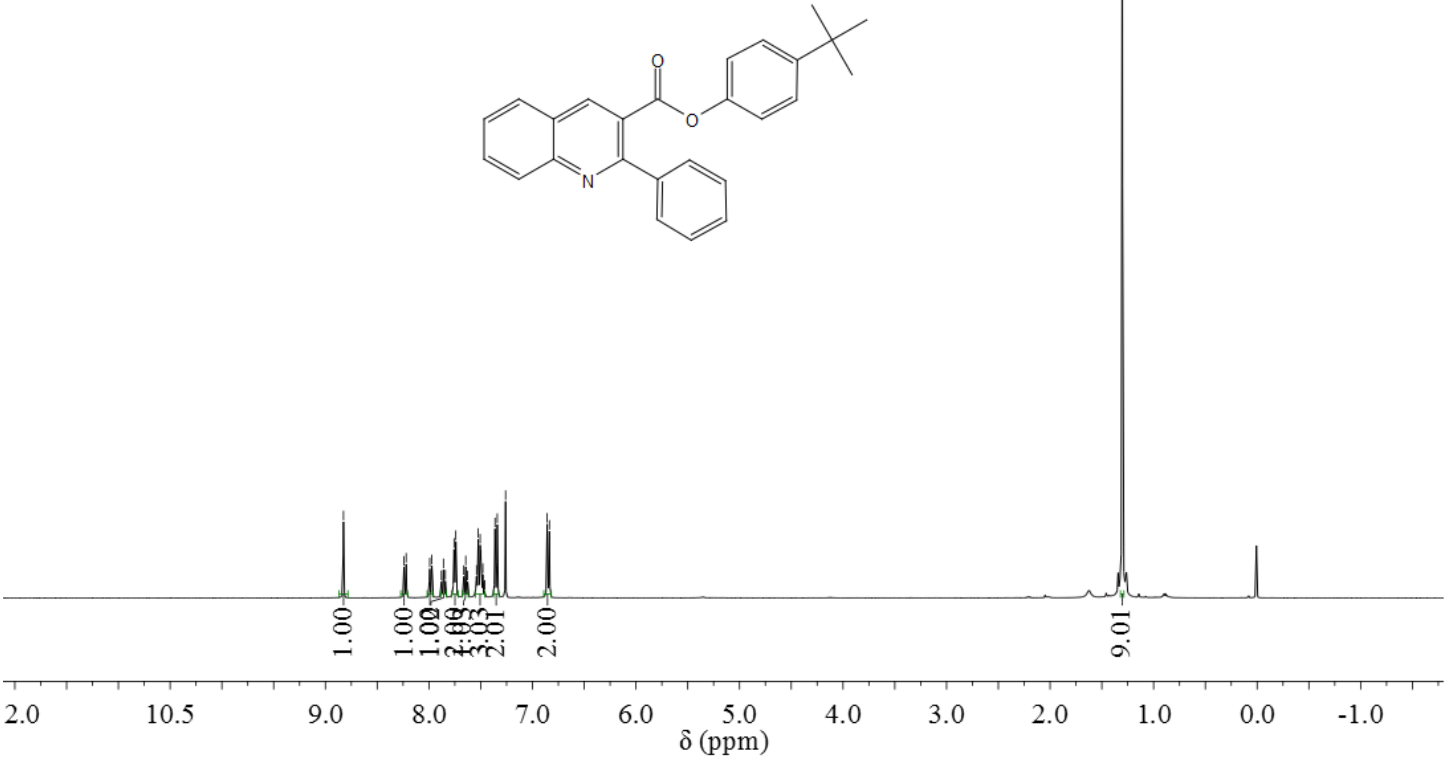

${ }^{13} \mathrm{C} \mathrm{NMR}\left(100 \mathrm{MHz}, \mathrm{CDCl}_{3}\right)$

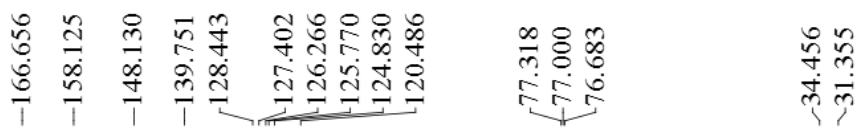

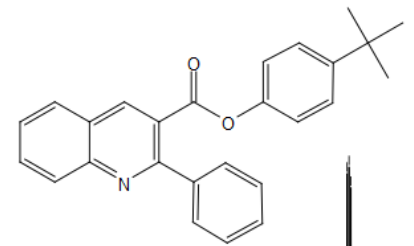

$\frac{n}{m}$

210

190

170

150

130

110

$\begin{array}{llllllllll}90 & 80 & 70 & 60 & 50 & 40 & 30 & 20 & 10 & 0\end{array}$ 


\section{$N$-Methyl- $N$,2-diphenylquinoline-3-carboxamide (3aw)}

${ }^{1} \mathrm{H}$ NMR (400 MHz, $\left.\mathrm{CDCl}_{3}\right)$

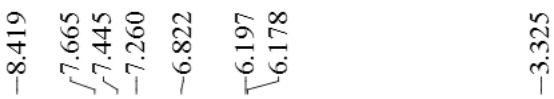

$$
\begin{aligned}
& \stackrel{2}{1}
\end{aligned}
$$

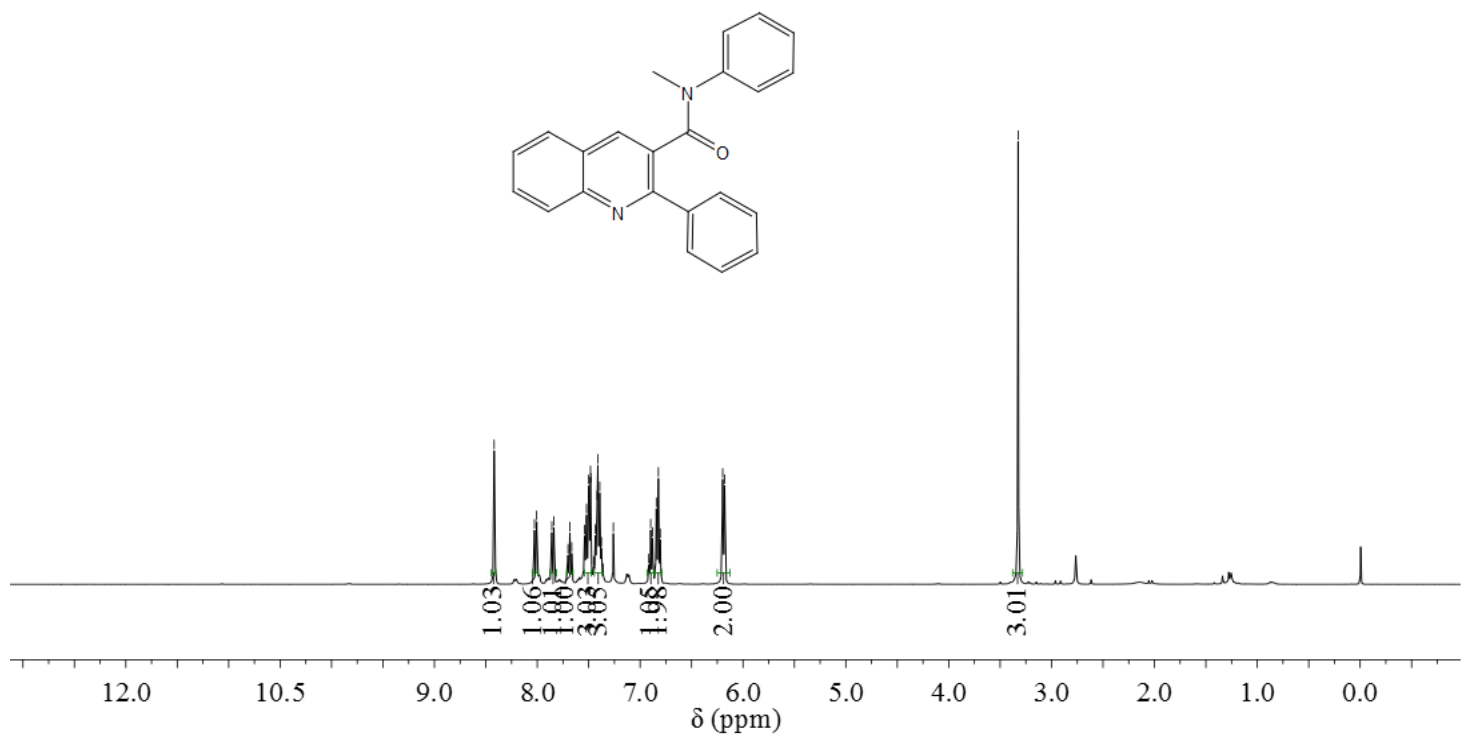

${ }^{13} \mathrm{C}$ NMR $\left(100 \mathrm{MHz}, \mathrm{CDCl}_{3}\right)$

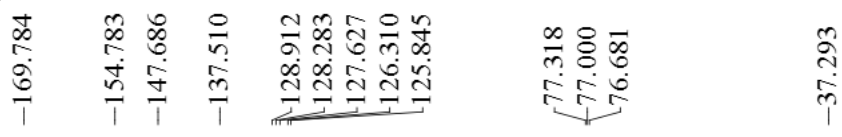
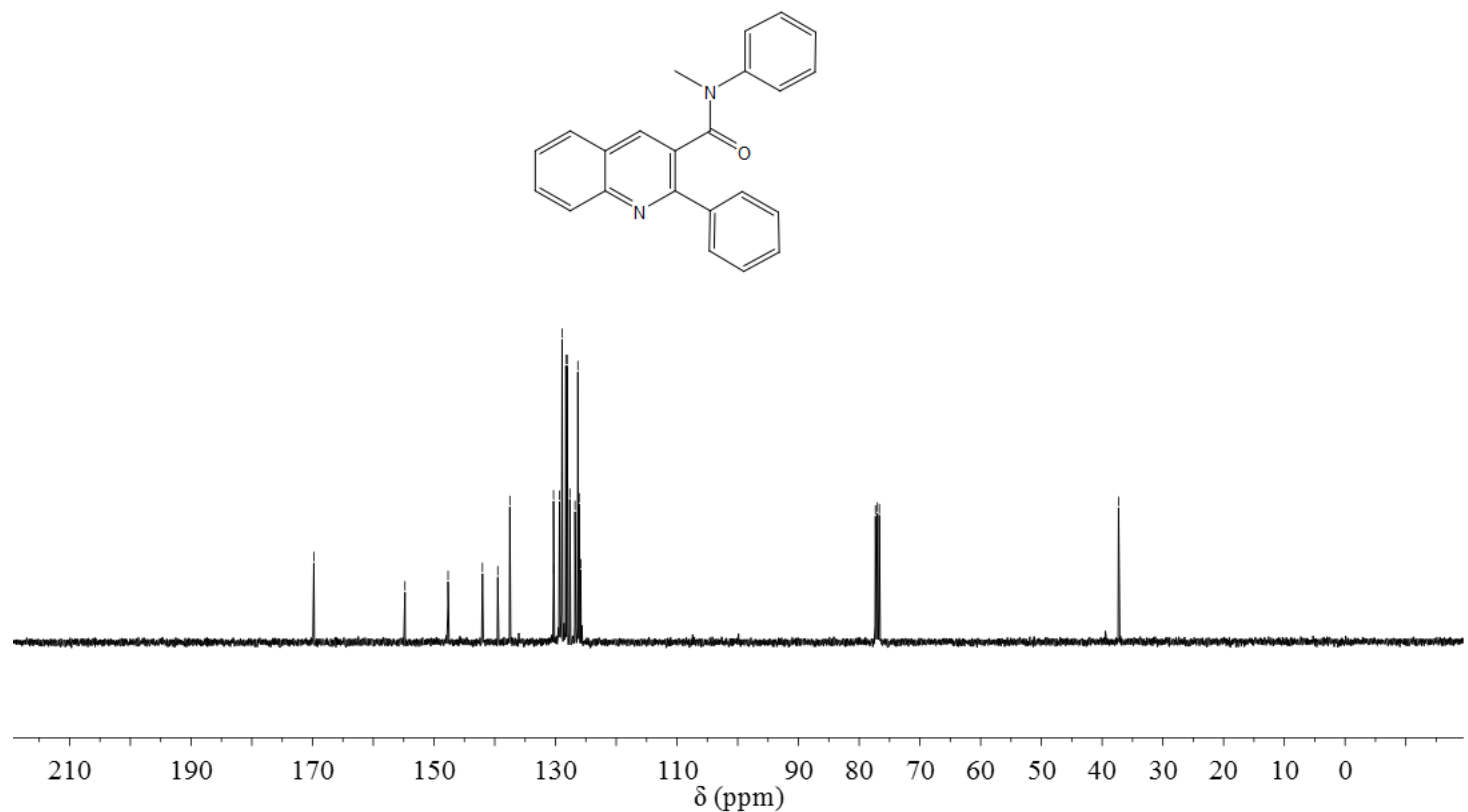
${ }^{1} \mathrm{H}$ NMR $\left(400 \mathrm{MHz}, \mathrm{CDCl}_{3}\right)$

\section{Phenyl(2-phenylquinolin-3-yl)methanone (3ax)}

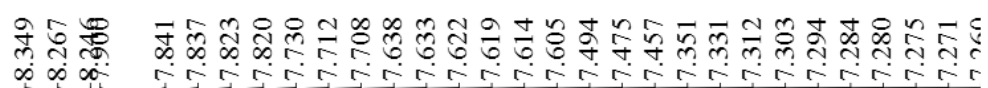
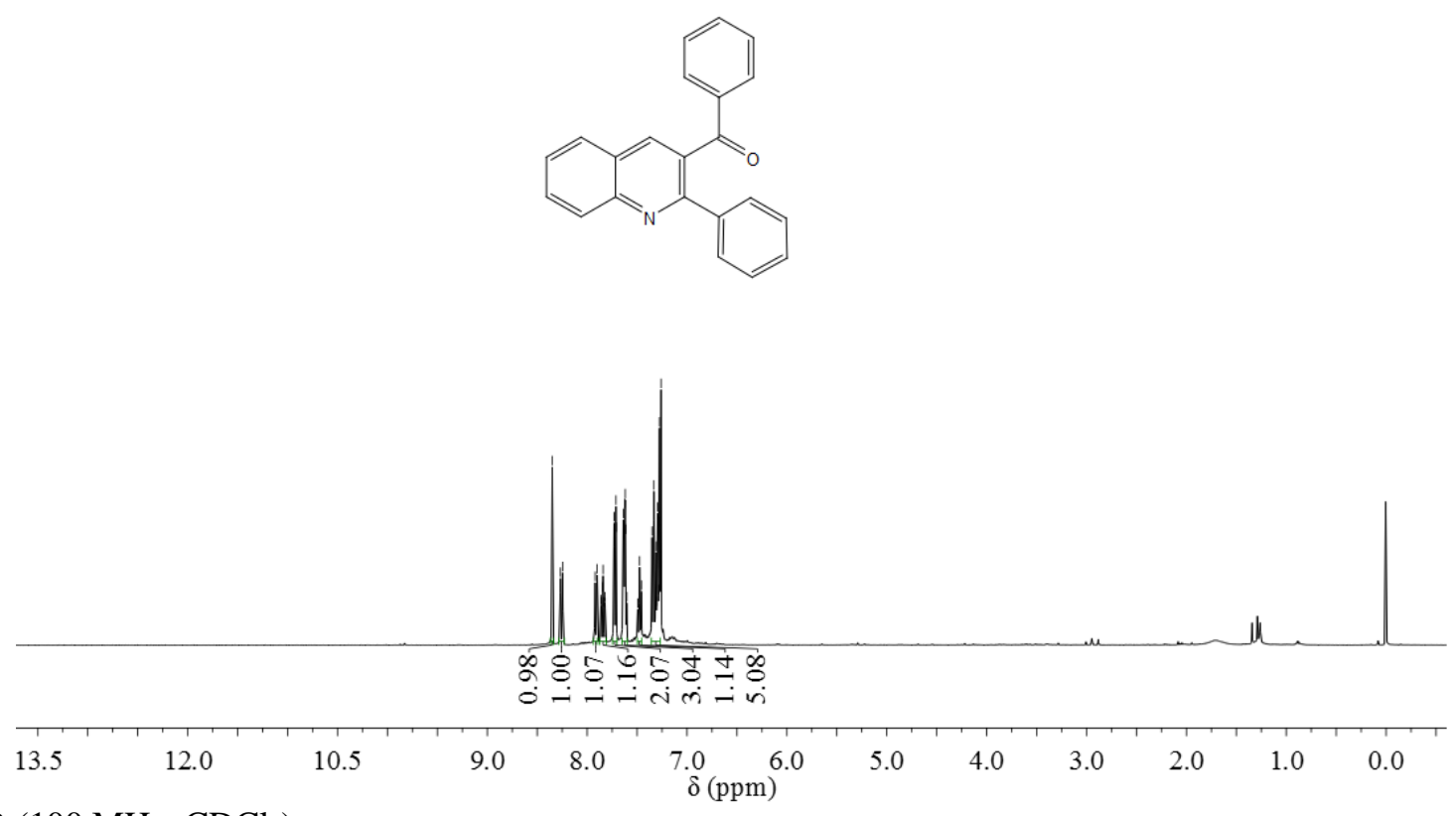

${ }^{13} \mathrm{C}$ NMR (100 MHz, $\mathrm{CDCl}_{3}$ )

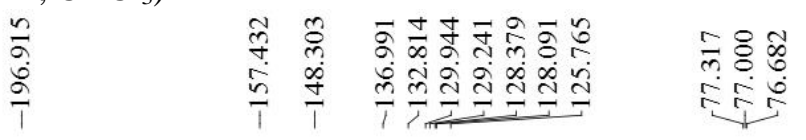
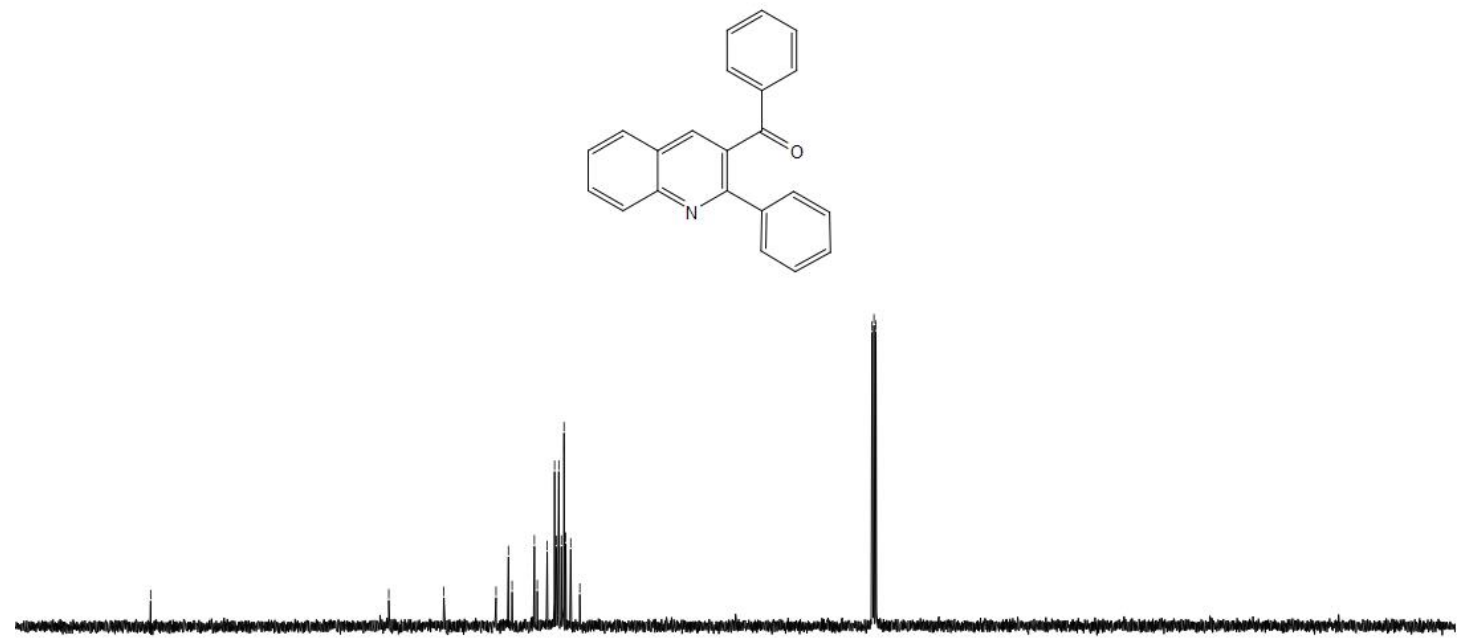

$\begin{array}{llllllllllllllll}210 & 190 & 170 & 150 & 130 & 110_{\delta(\mathrm{ppm})}^{90} & 80 & 70 & 60 & 50 & 40 & 30 & 20 & 10 & 0\end{array}$


${ }^{1} \mathrm{H}$ NMR $\left(400 \mathrm{MHz}, \mathrm{CDCl}_{3}\right)$

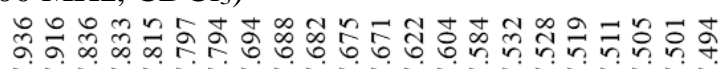

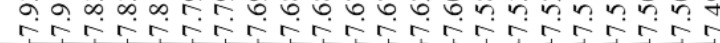
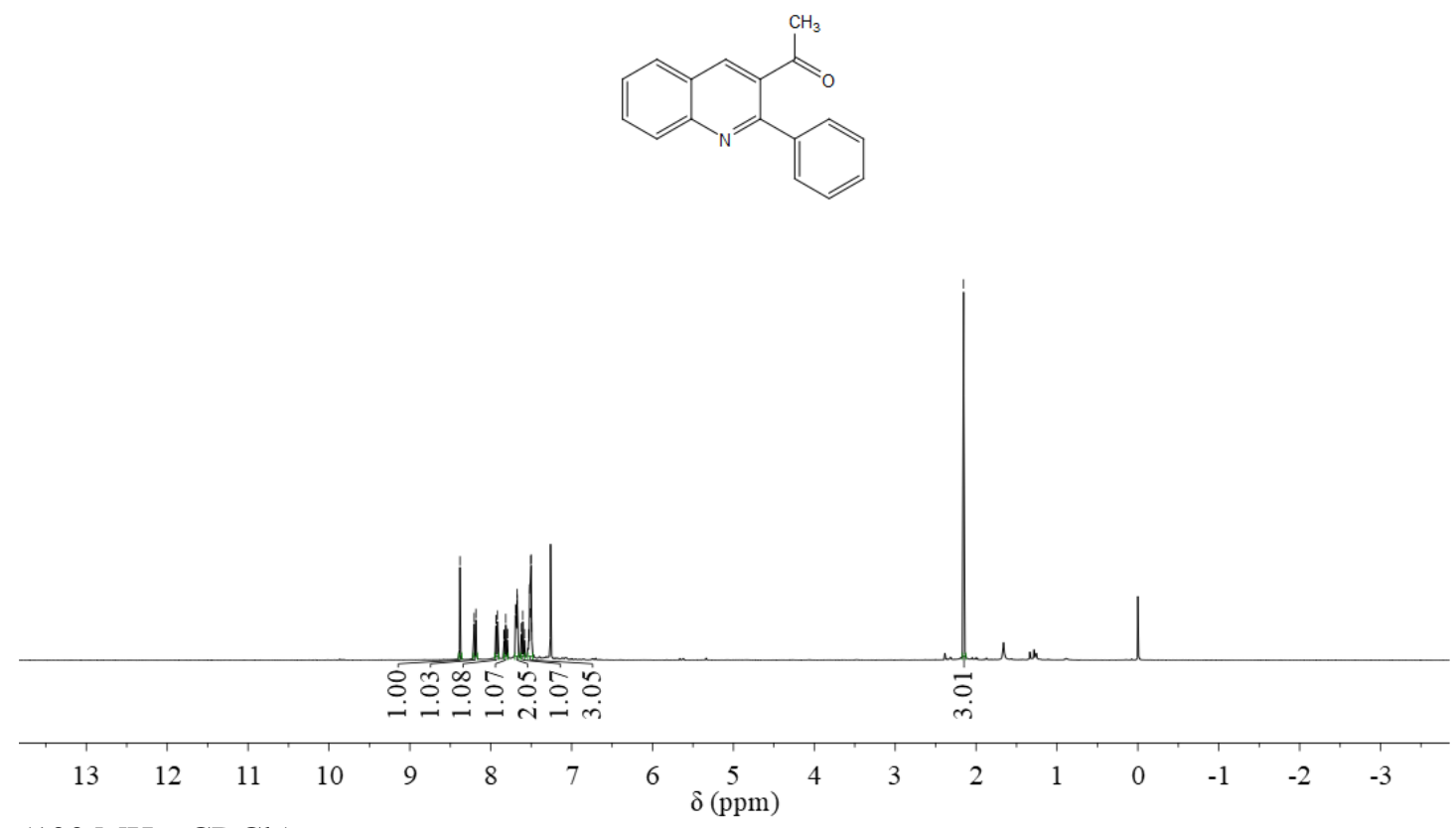

${ }^{13} \mathrm{C}$ NMR (100 MHz, $\mathrm{CDCl}_{3}$ )

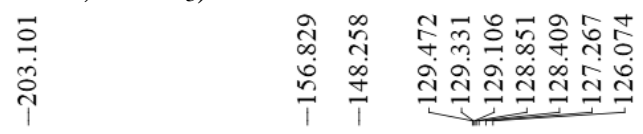

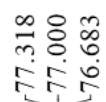

$\underset{\substack{0 \\ \hdashline}}{\substack{1 \\ 0}}$
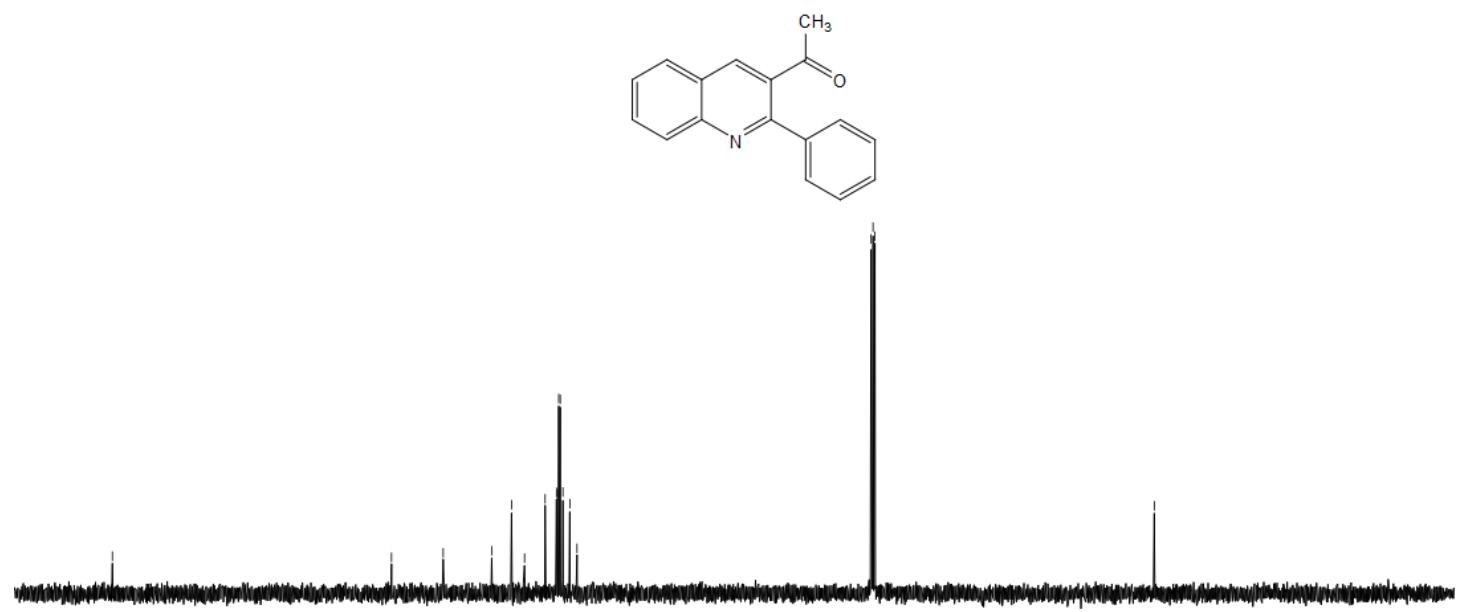

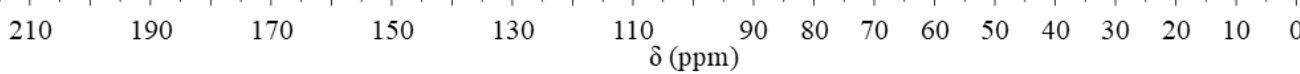


${ }^{1} \mathrm{H}$ NMR (400 MHz, $\mathrm{CDCl}_{3}$ )

2-Phenylquinoline-3-carbaldehyde (3az)

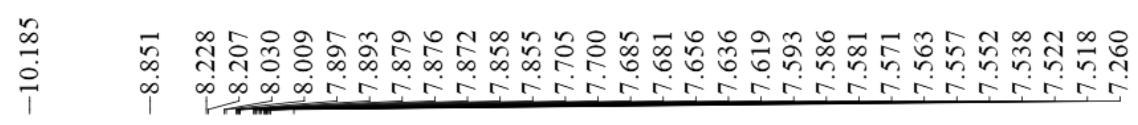
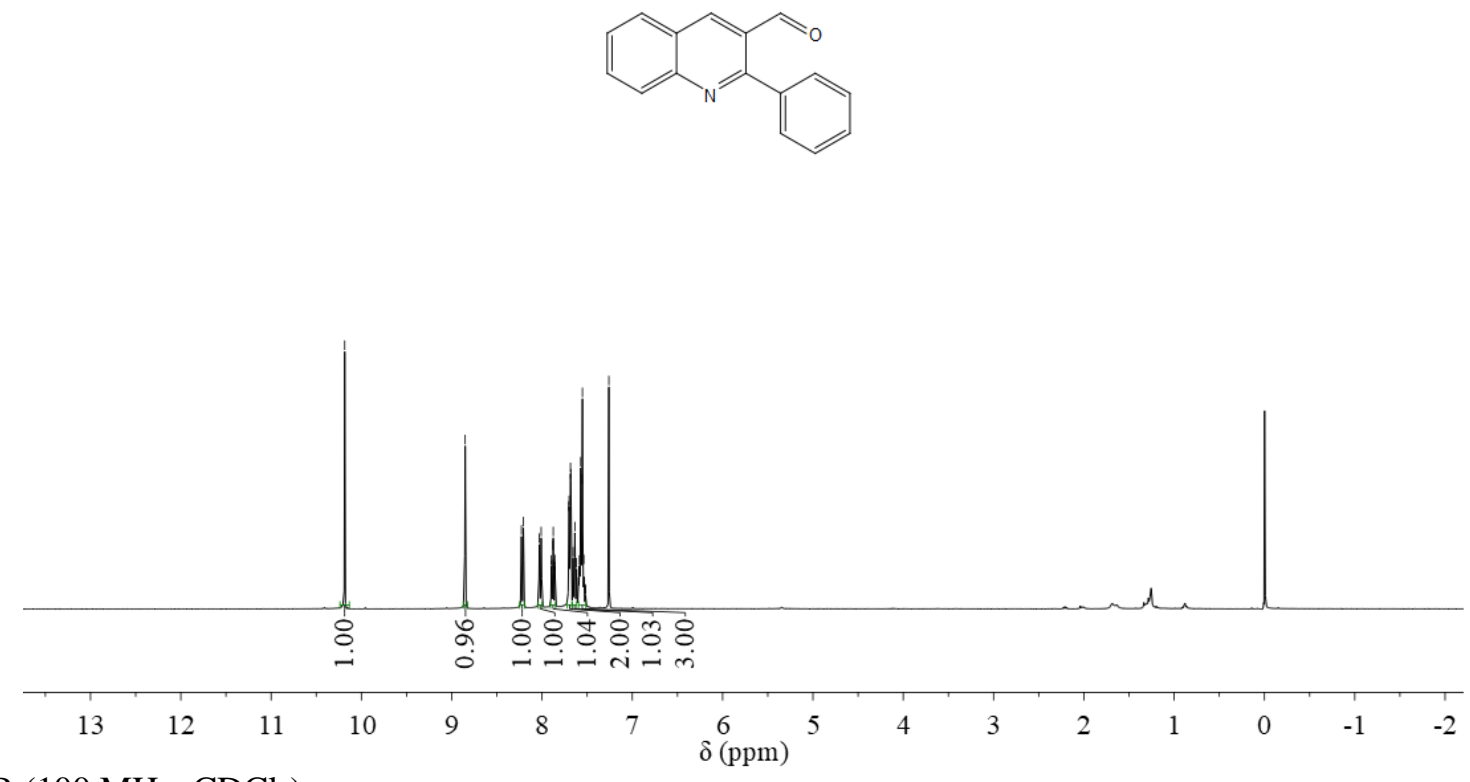

${ }^{13} \mathrm{C}$ NMR (100 MHz, $\left.\mathrm{CDCl}_{3}\right)$
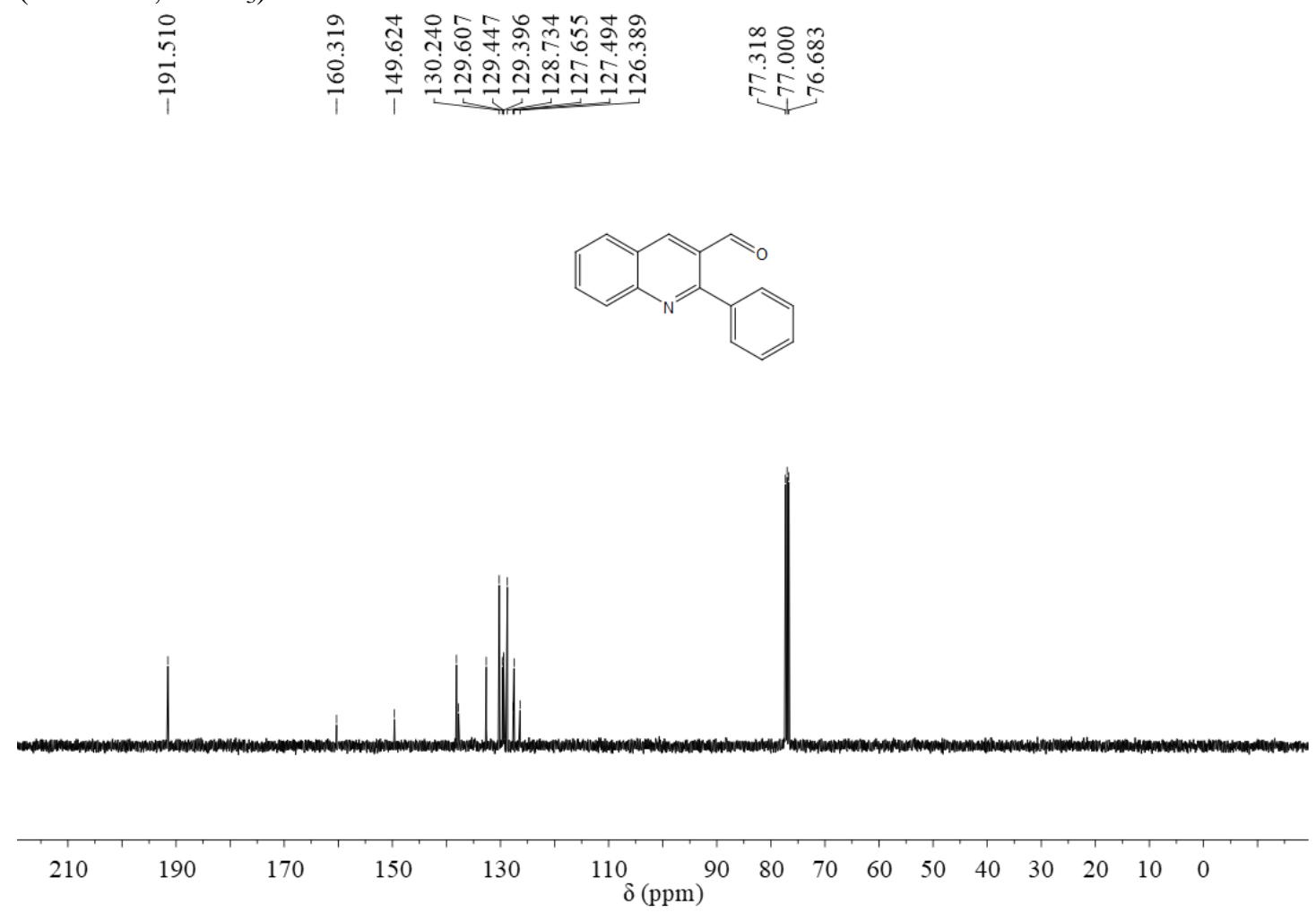

97 


\section{3-Ethoxy-2-phenylquinoline (3ba)}

${ }^{1} \mathrm{H}$ NMR (400 MHz, $\left.\mathrm{CDCl}_{3}\right)$

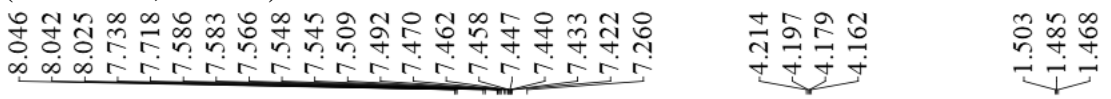
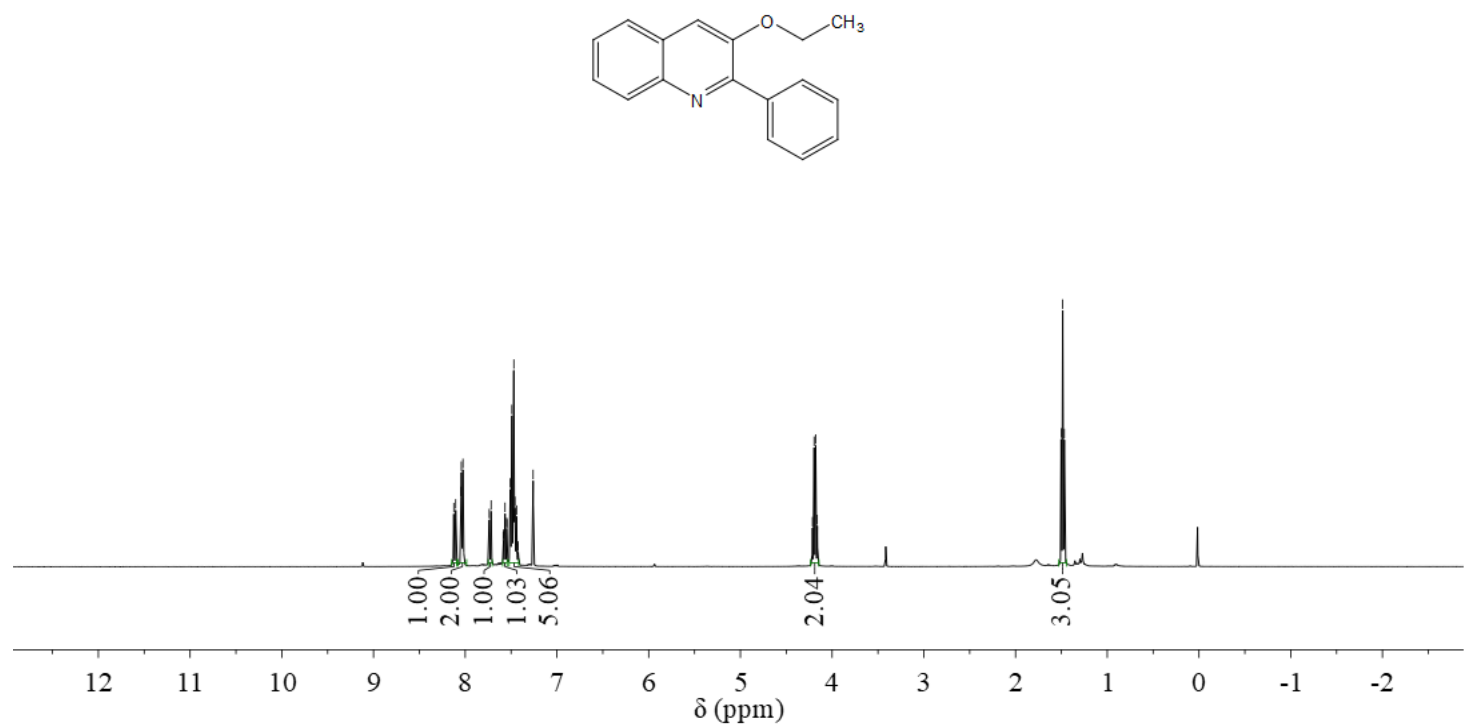

${ }^{13} \mathrm{C}$ NMR (100 MHz, $\mathrm{CDCl}_{3}$ )

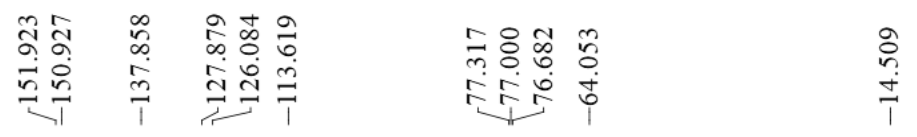
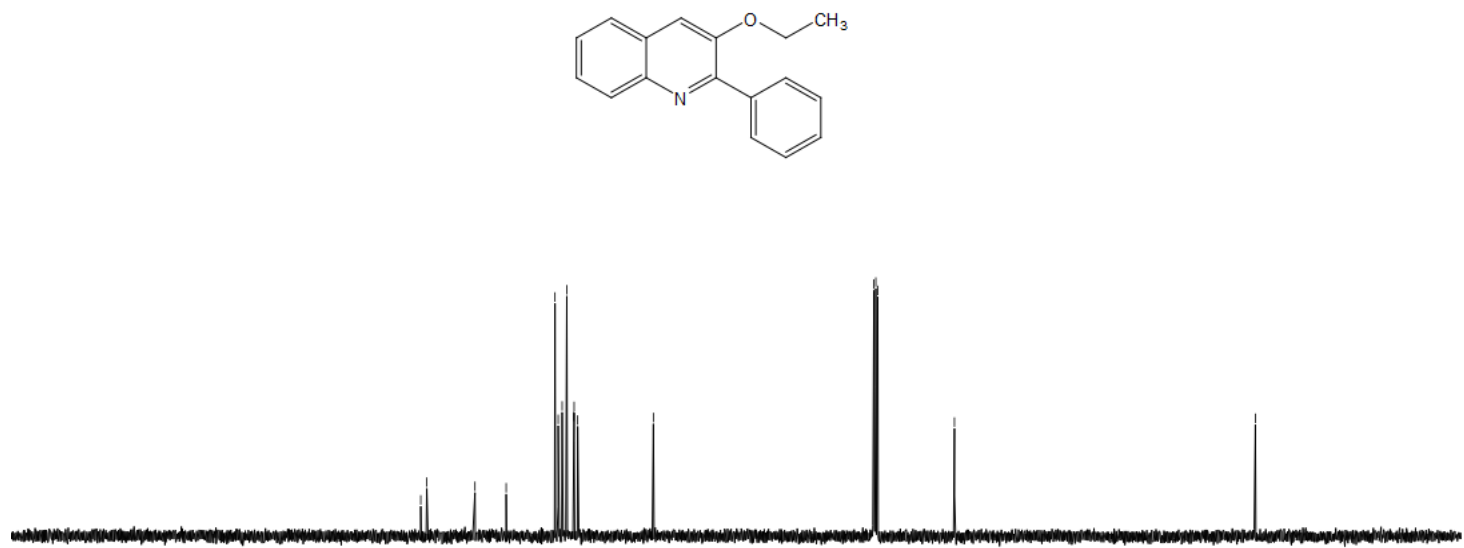

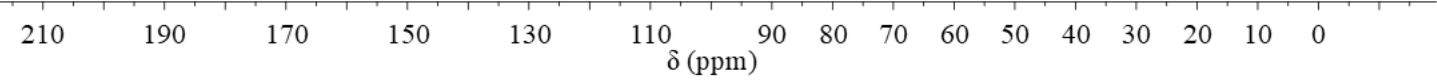


$N$-Butyl-4-methyl- $N$-(2-phenylquinolin-3-yl)benzenesulfonamide (3bb)

${ }^{1} \mathrm{H} \mathrm{NMR}\left(400 \mathrm{MHz}, \mathrm{CDCl}_{3}\right)$

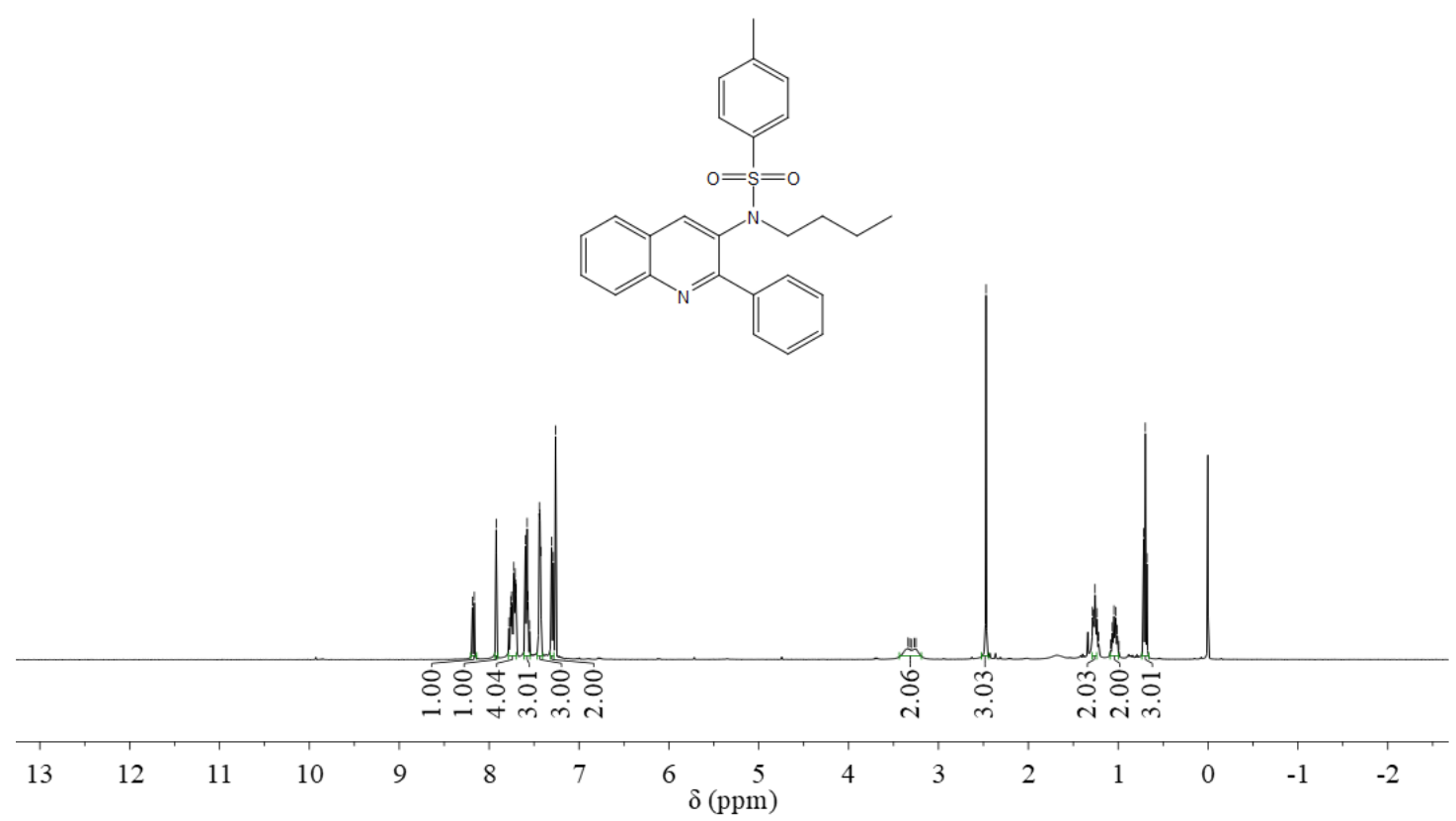

${ }^{13} \mathrm{C} \mathrm{NMR}\left(100 \mathrm{MHz}, \mathrm{CDCl}_{3}\right)$

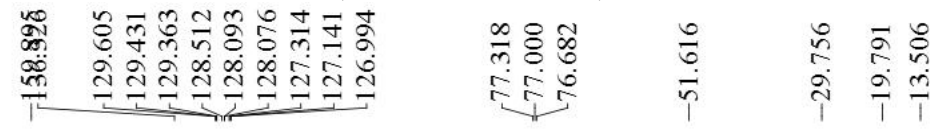

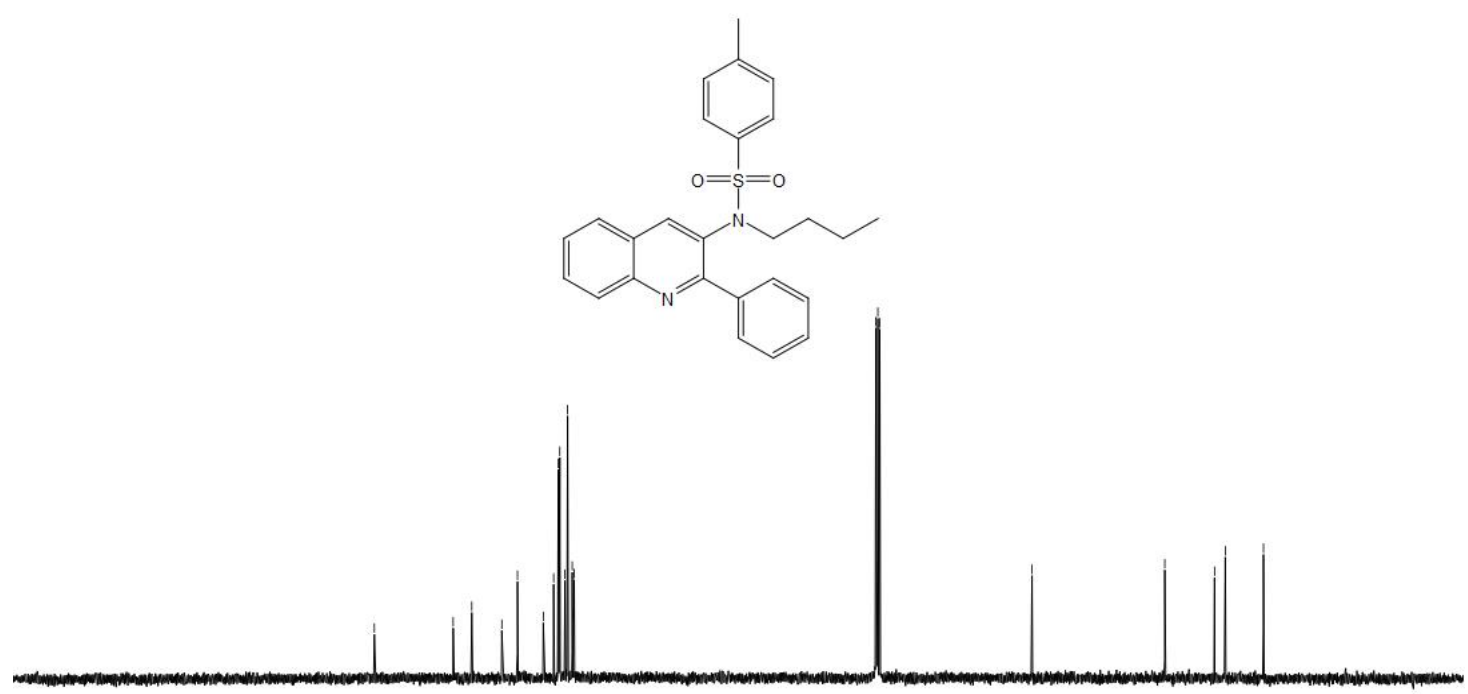

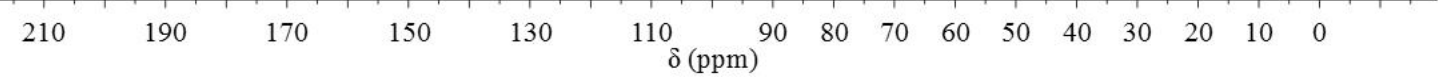


${ }^{1} \mathrm{H}$ NMR (400 MHz, $\mathrm{CDCl}_{3}$ )

1,3-Di(quinolin-2-yl)benzene (3bc)
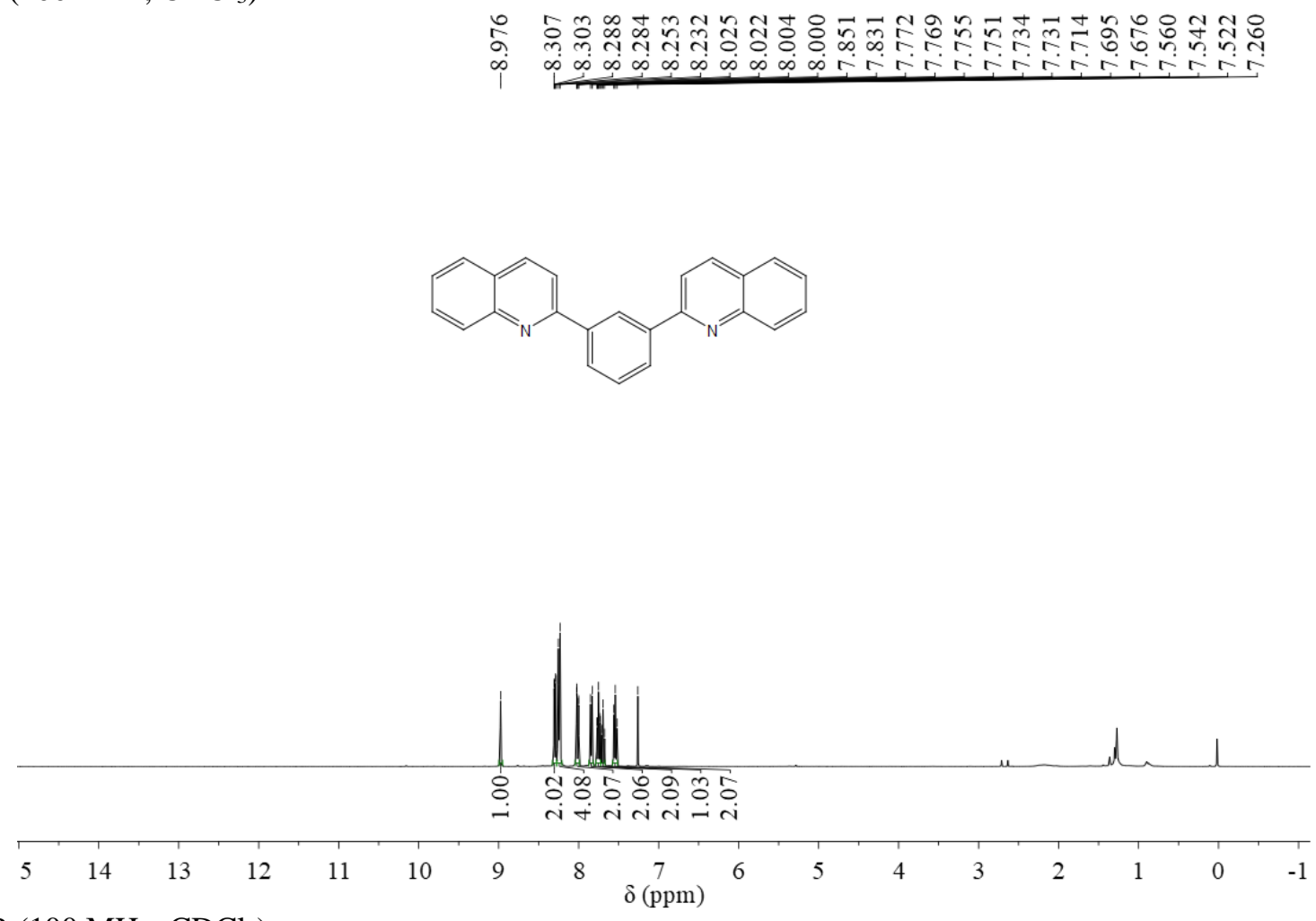

${ }^{13} \mathrm{C}$ NMR (100 MHz, $\left.\mathrm{CDCl}_{3}\right)$

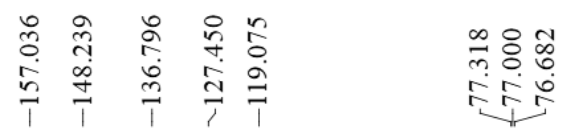
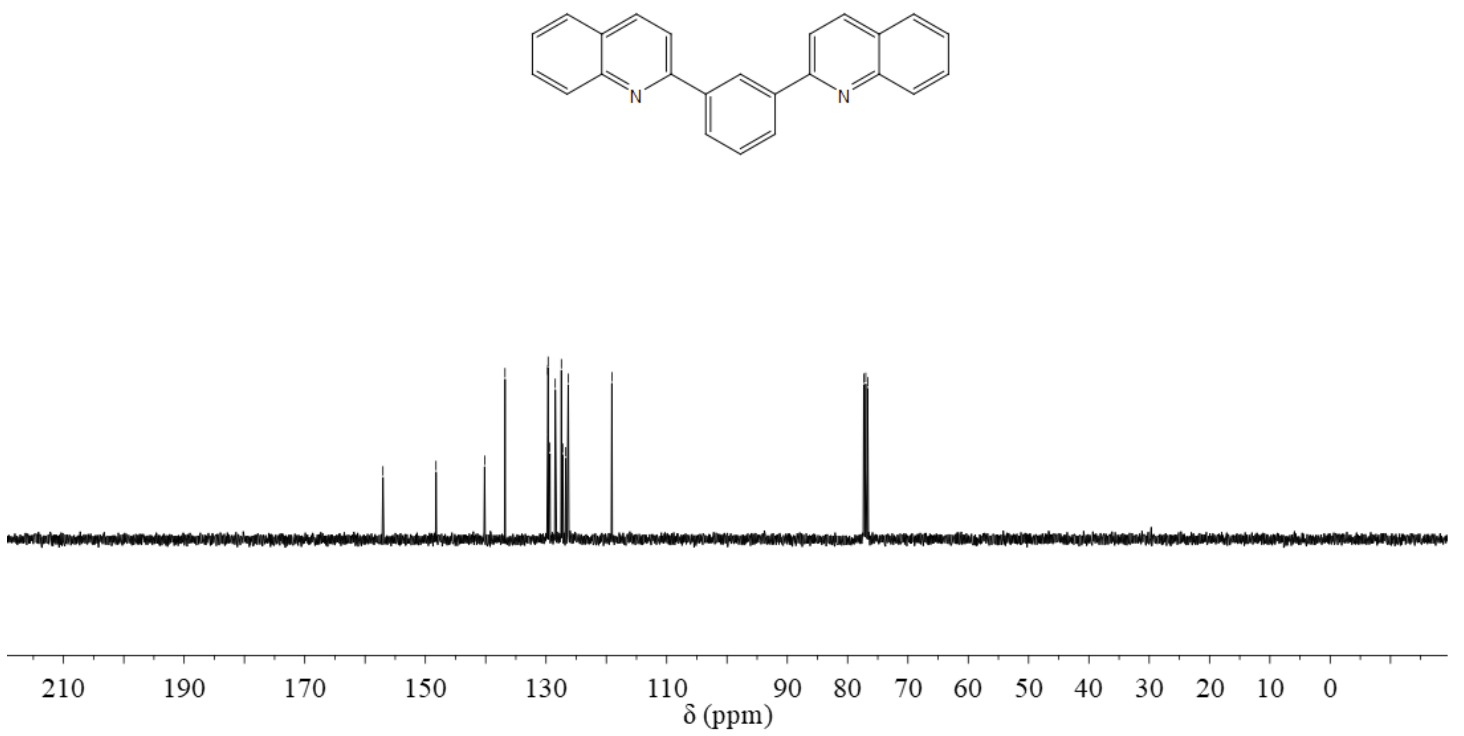

100 
${ }^{1} \mathrm{H}$ NMR (400 MHz, $\left.\mathrm{CDCl}_{3}\right)$

\section{1,3,5-Tri(quinolin-2-yl)benzene (3bd)}

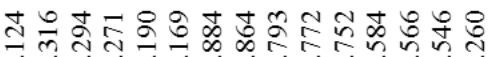

a dis os os
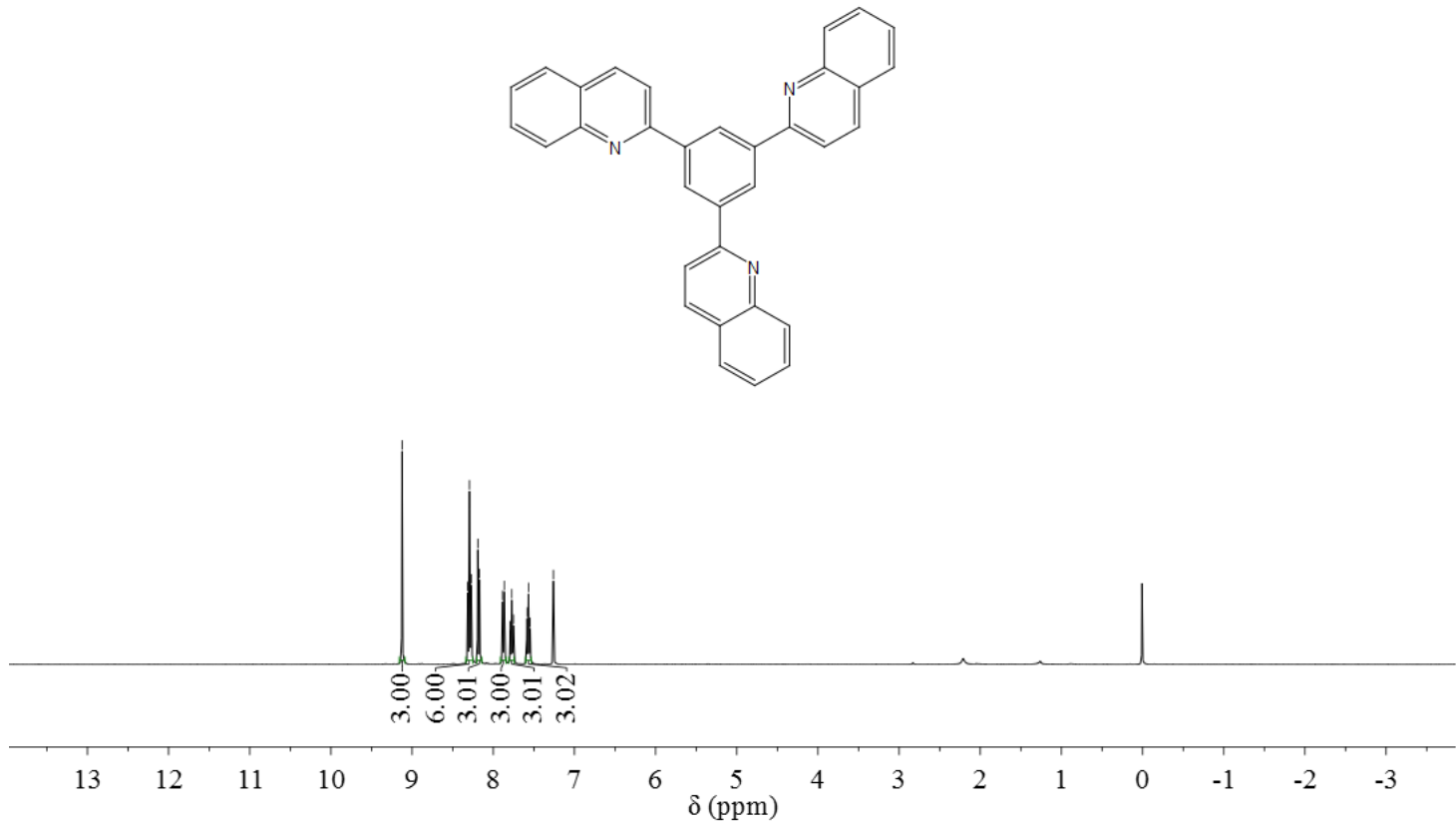

${ }^{13} \mathrm{C}$ NMR $\left(100 \mathrm{MHz}, \mathrm{CDCl}_{3}\right)$

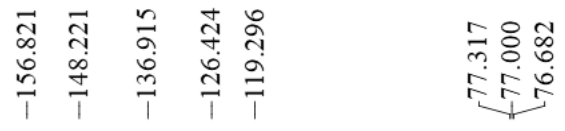
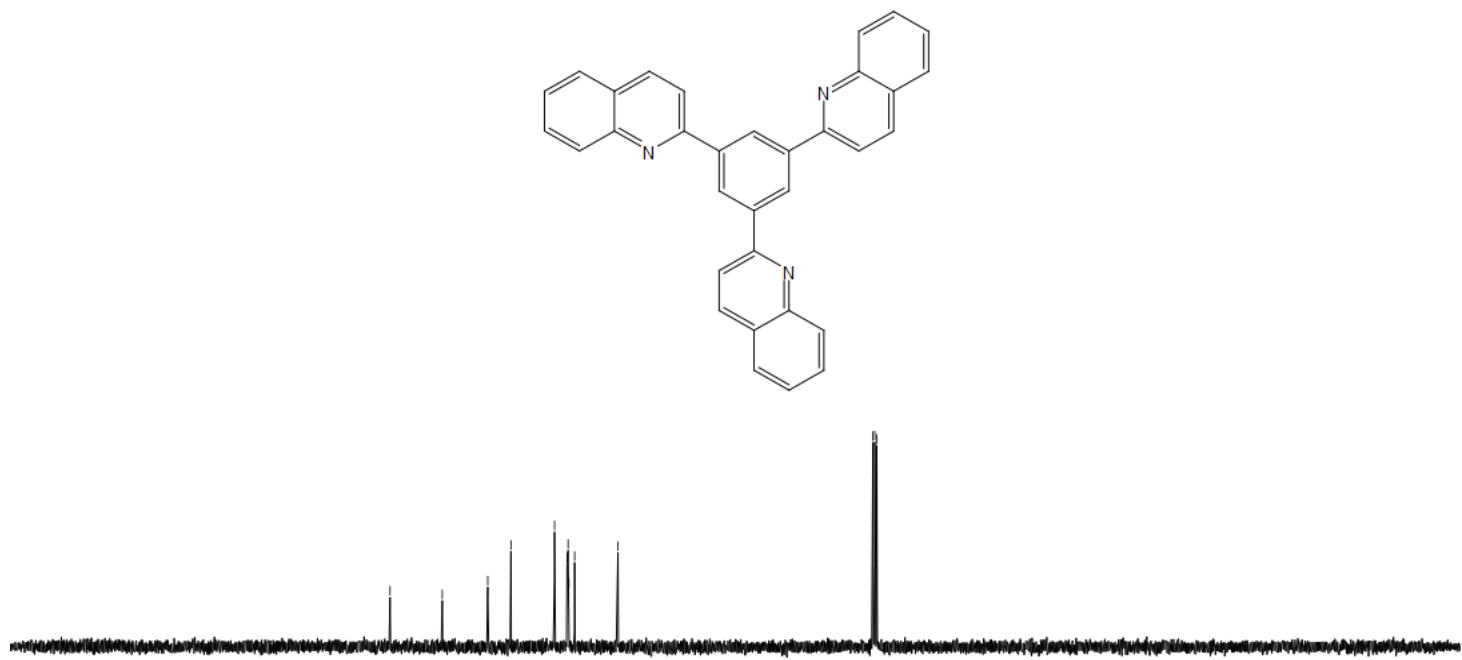

$\begin{array}{llllllllllllllll}210 & 190 & 170 & 150 & 130 & 110_{\delta(\mathrm{ppm})} & 90 & 80 & 70 & 60 & 50 & 40 & 30 & 20 & 10 & 0\end{array}$ 
${ }^{1} \mathrm{H}$ NMR (400 MHz, $\mathrm{CDCl}_{3}$ )
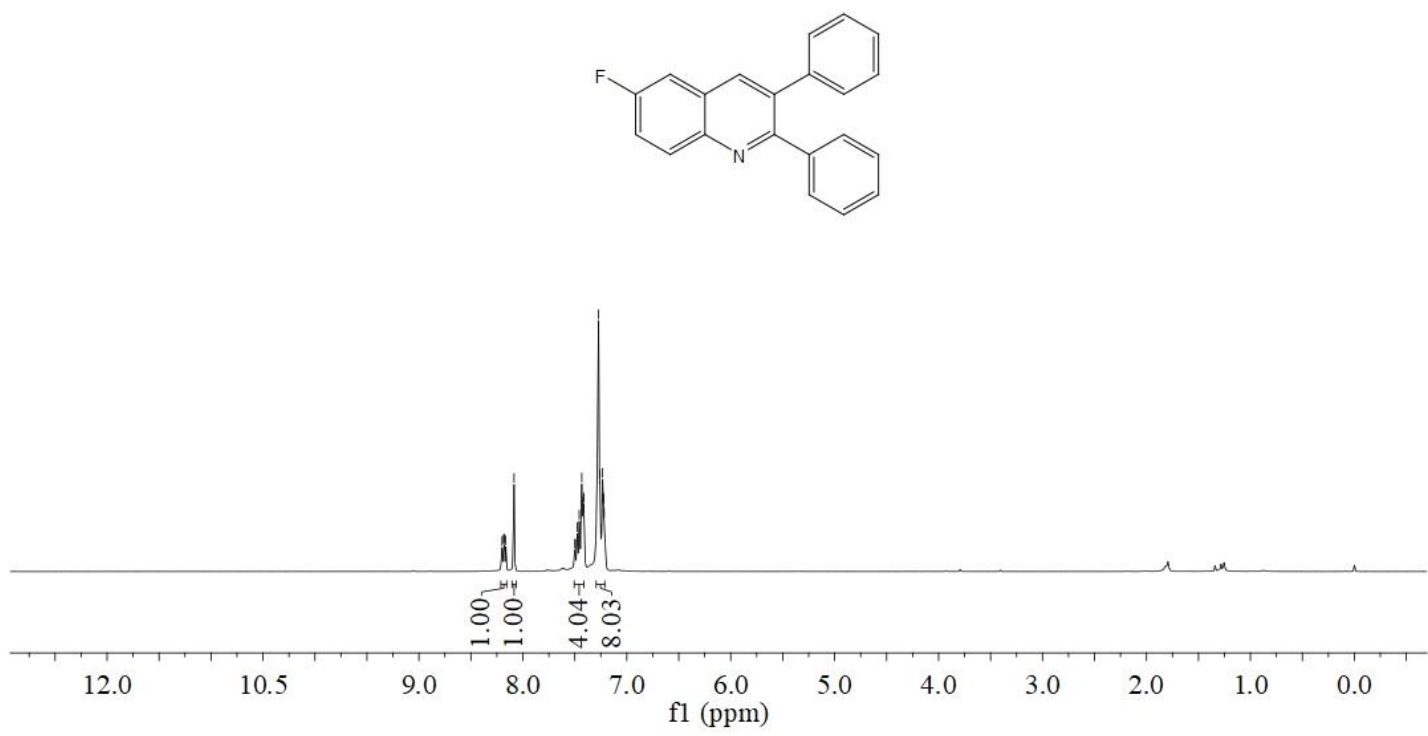

${ }^{13} \mathrm{C}$ NMR (100 MHz, $\left.\mathrm{CDCl}_{3}\right)$
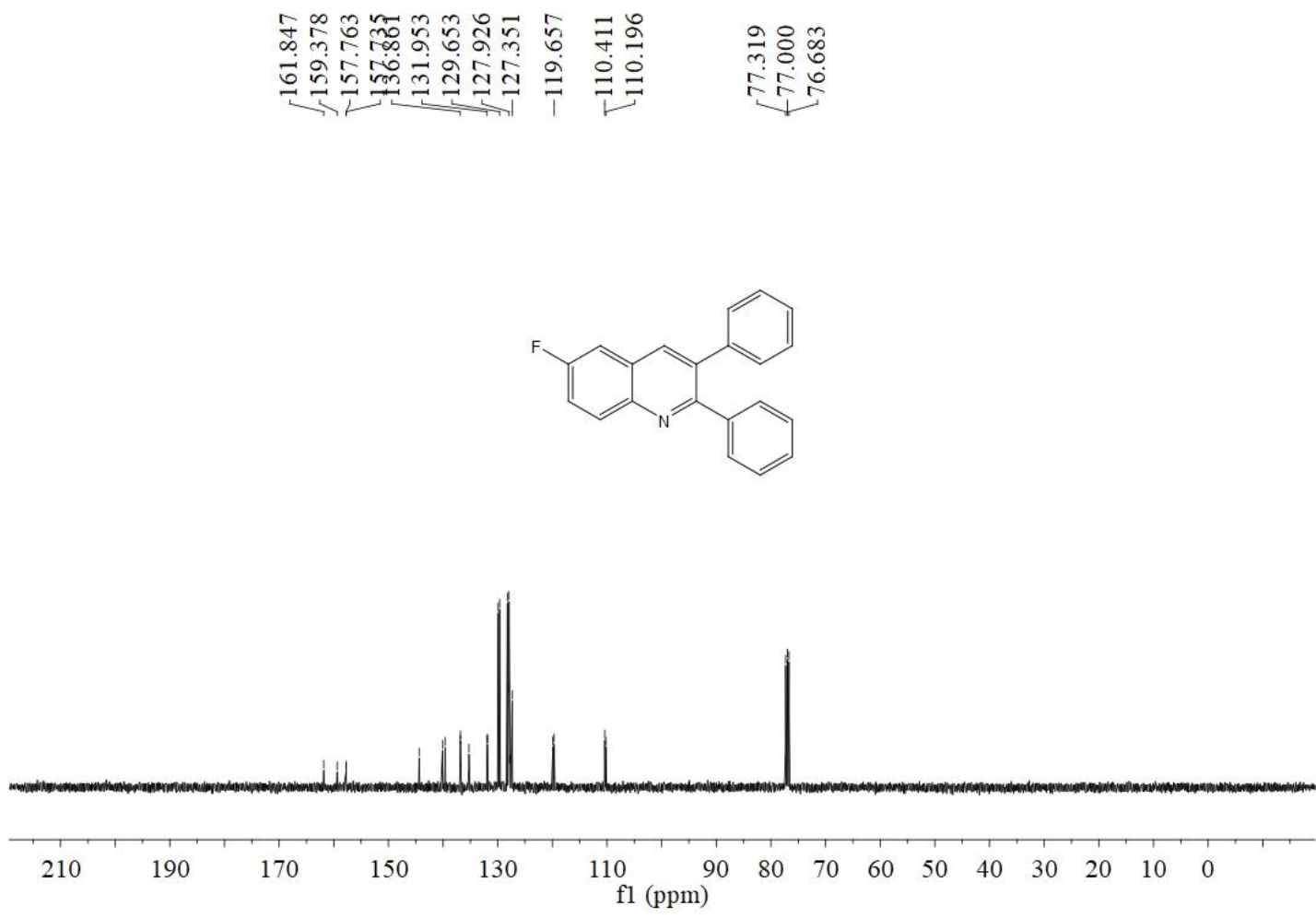
${ }^{19} \mathrm{~F}$ NMR (375 MHz, $\left.\mathrm{CDCl}_{3}\right)$

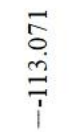
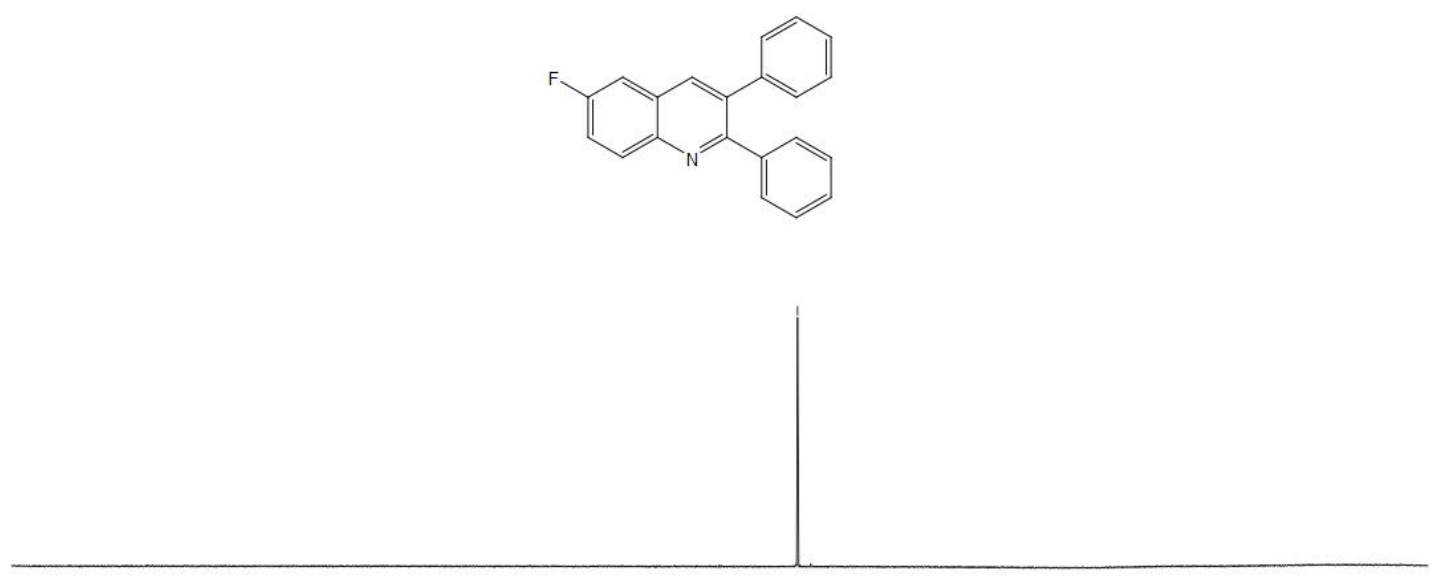

\begin{tabular}{llllllllll|l|l|l|l|}
1 & 0 & -20 & -40 & -60 & -80 & -100 & -120 & -140 & -160 & -180 & -200 & $\mathrm{f} 1 \mathrm{(ppm})$
\end{tabular}


${ }^{1} \mathrm{H}$ NMR (400 MHz, $\mathrm{CDCl}_{3}$ )

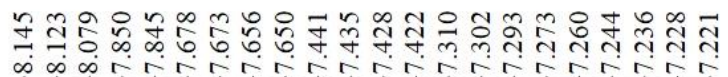
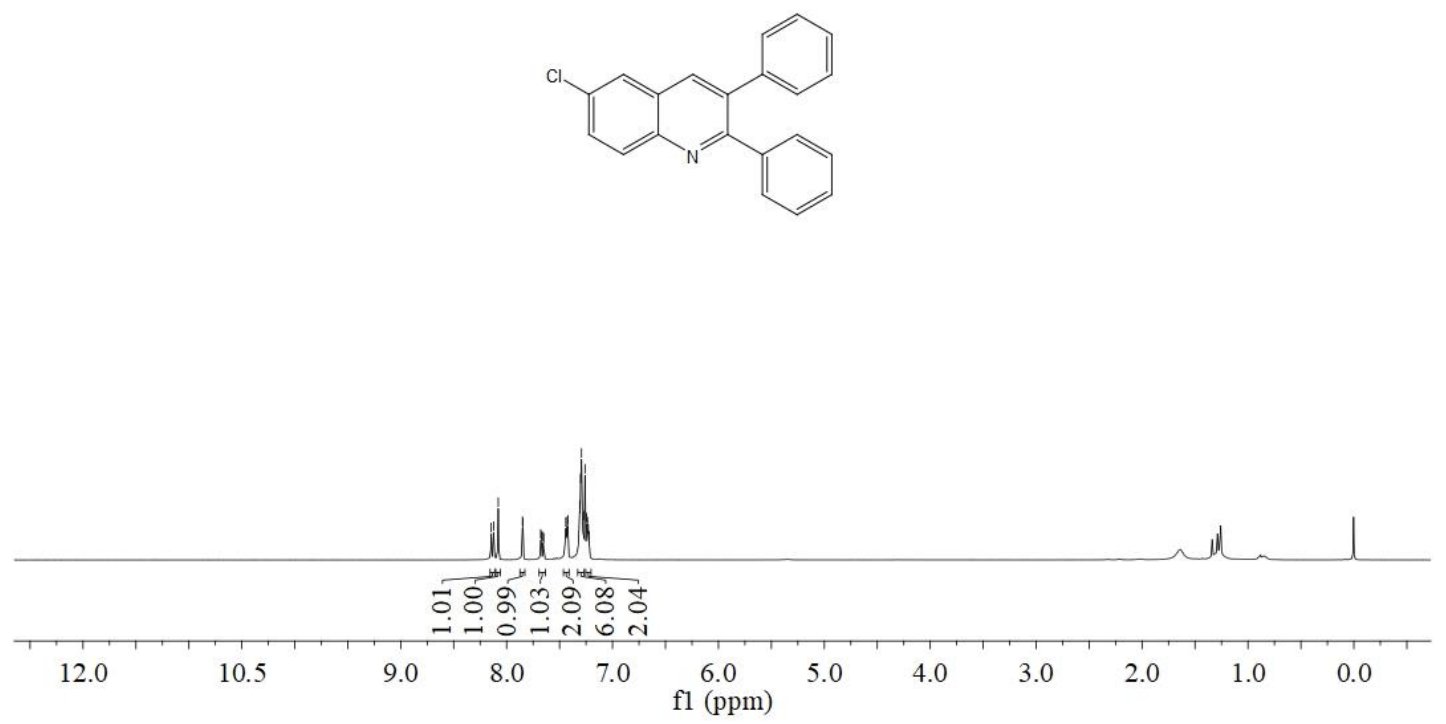

${ }^{13} \mathrm{C}$ NMR (100 MHz, $\left.\mathrm{CDCl}_{3}\right)$

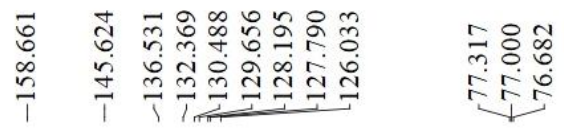
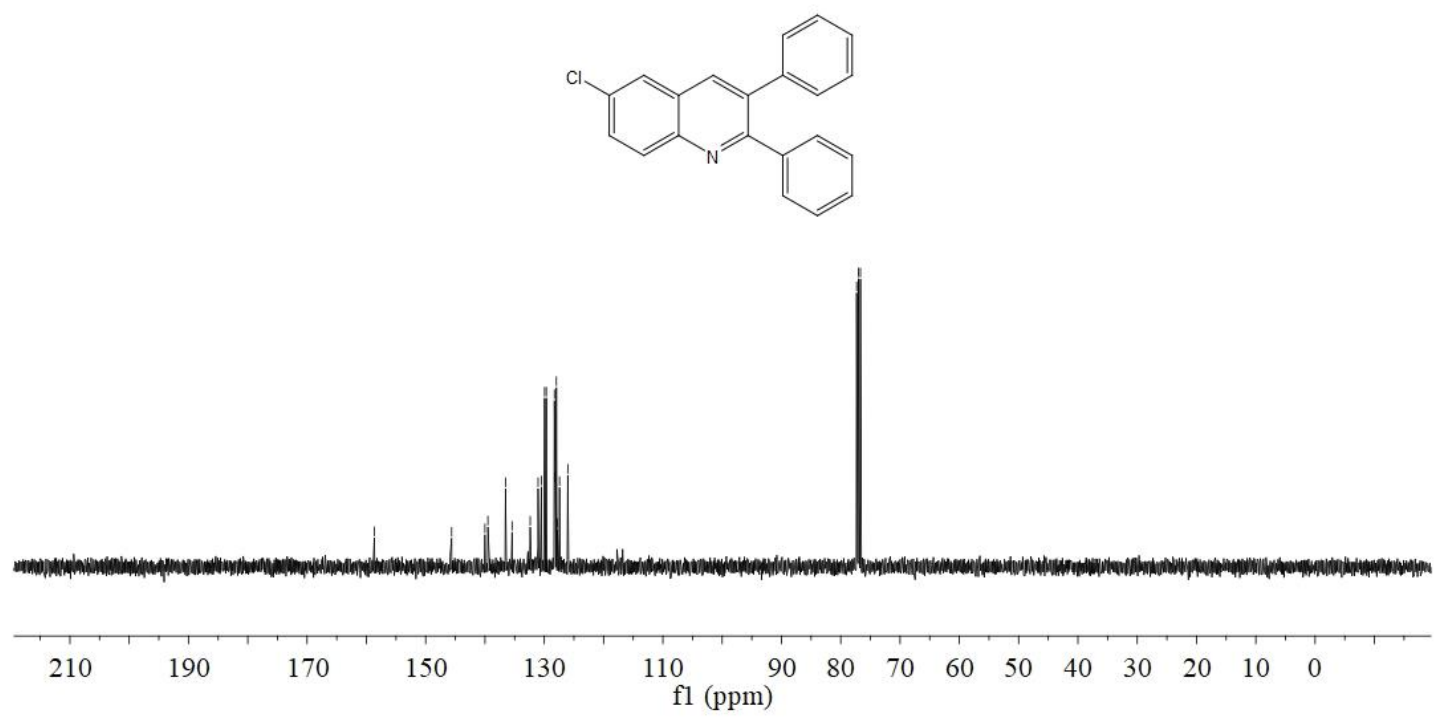
${ }^{1} \mathrm{H}$ NMR (400 MHz, $\left.\mathrm{CDCl}_{3}\right)$
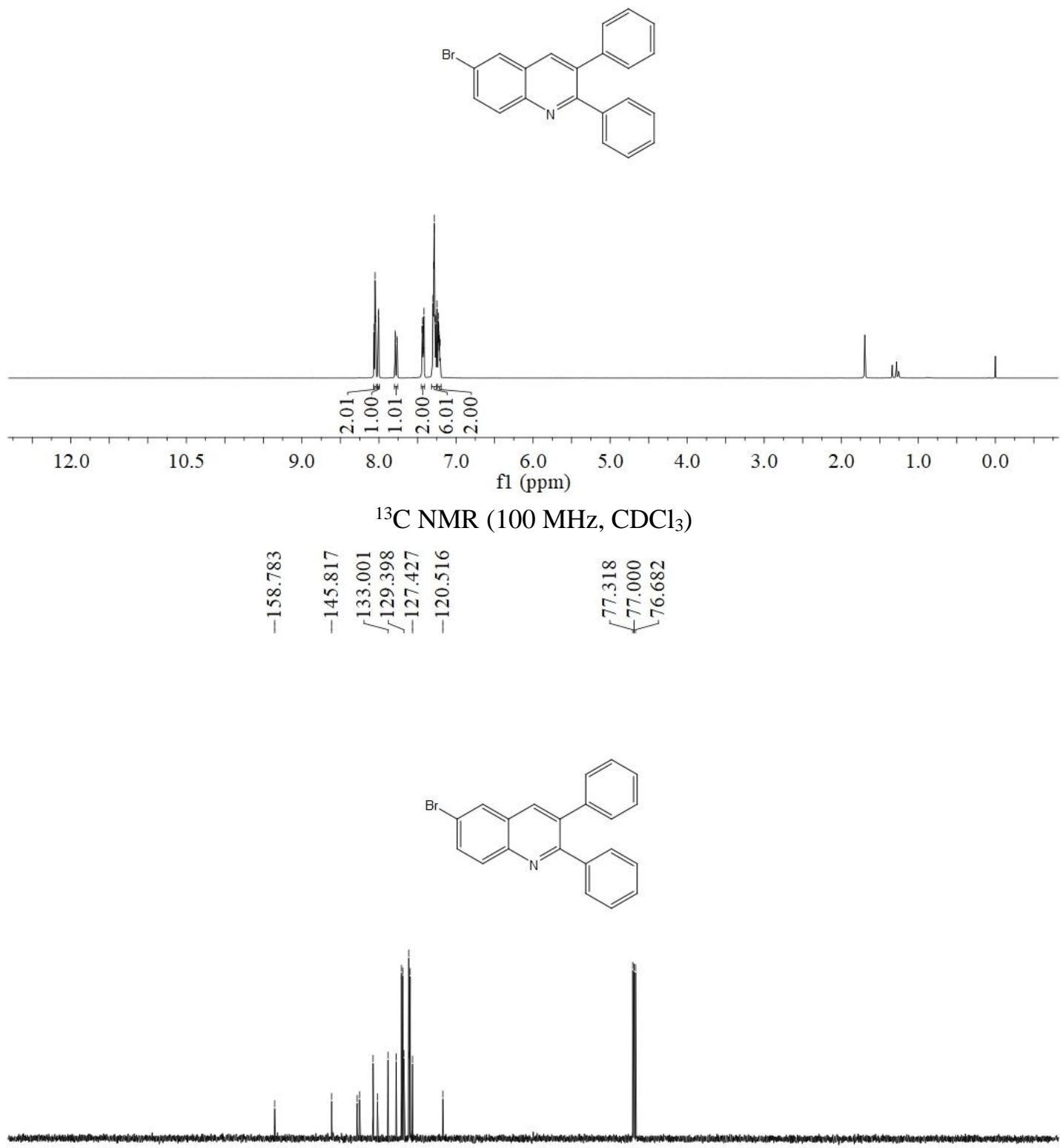

$\begin{array}{lllllllllllllllll}210 & 190 & 170 & 150 & 130 & 110 & 90 & 80 & 70 & 60 & 50 & 40 & 30 & 20 & 10 & 0 & \end{array}$ 
${ }^{1} \mathrm{H}$ NMR (400 MHz, $\mathrm{CDCl}_{3}$ )

\section{6-Methoxy-2,3-diphenylquinoline (3bh)}

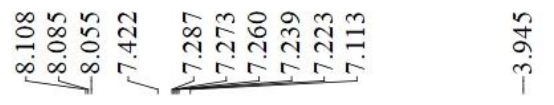
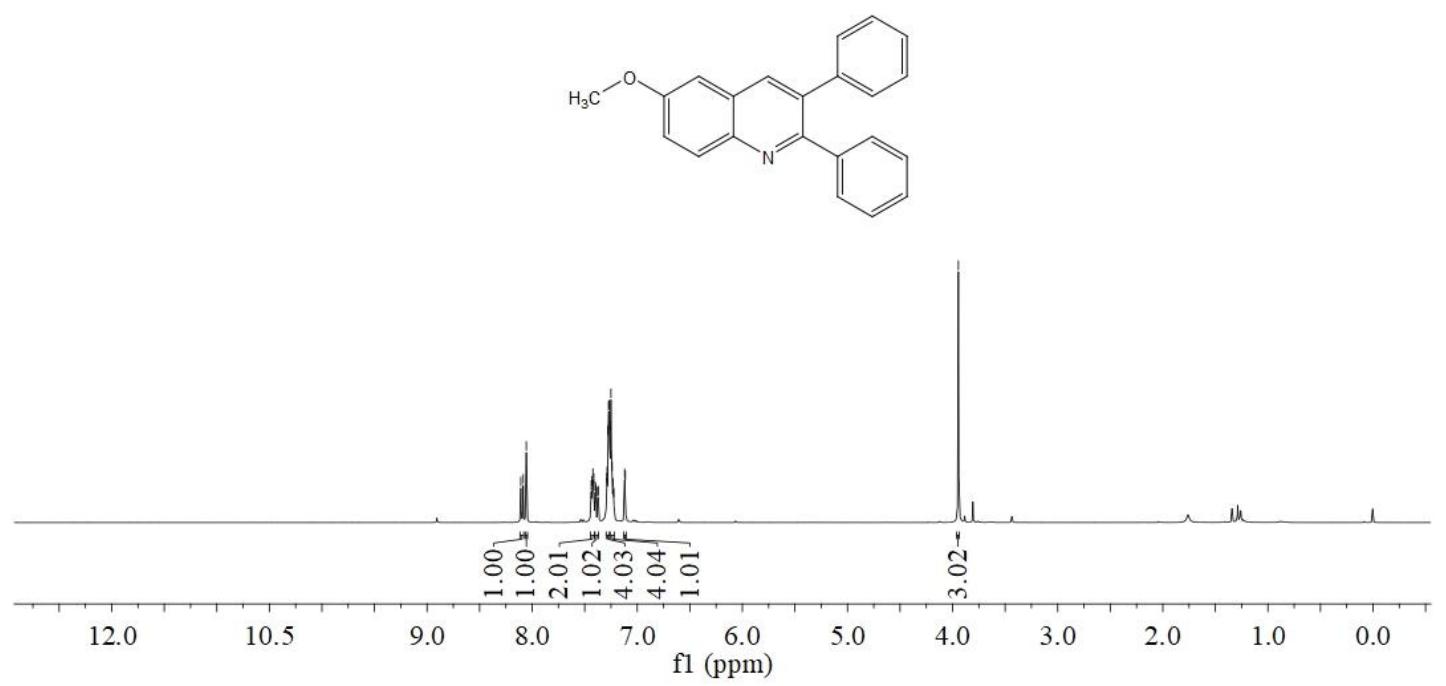

${ }^{13} \mathrm{C} \mathrm{NMR}\left(100 \mathrm{MHz}, \mathrm{CDCl}_{3}\right)$

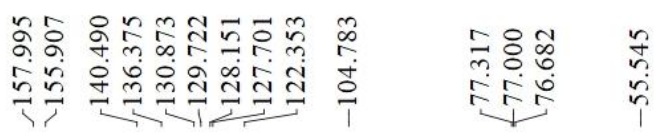
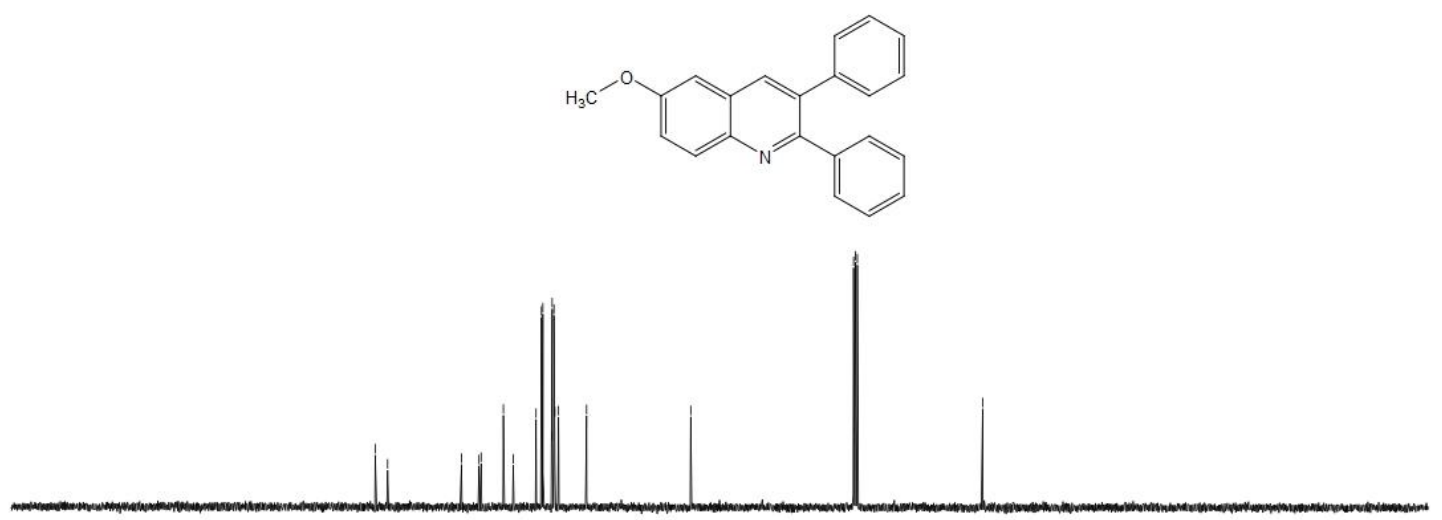

$\begin{array}{lllllllllllllllll}210 & 190 & 170 & 150 & 130 & \begin{array}{c}110 \\ \mathrm{f} 1(\mathrm{ppm})\end{array} & 90 & 80 & 70 & 60 & 50 & 40 & 30 & 20 & 10 & 0\end{array}$


${ }^{1} \mathrm{H}$ NMR (400 MHz, $\mathrm{CDCl}_{3}$ )

\section{6-(Benzyloxy)-2,3-diphenyl (3bi)}

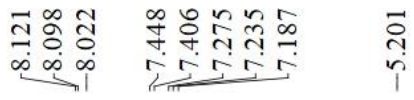
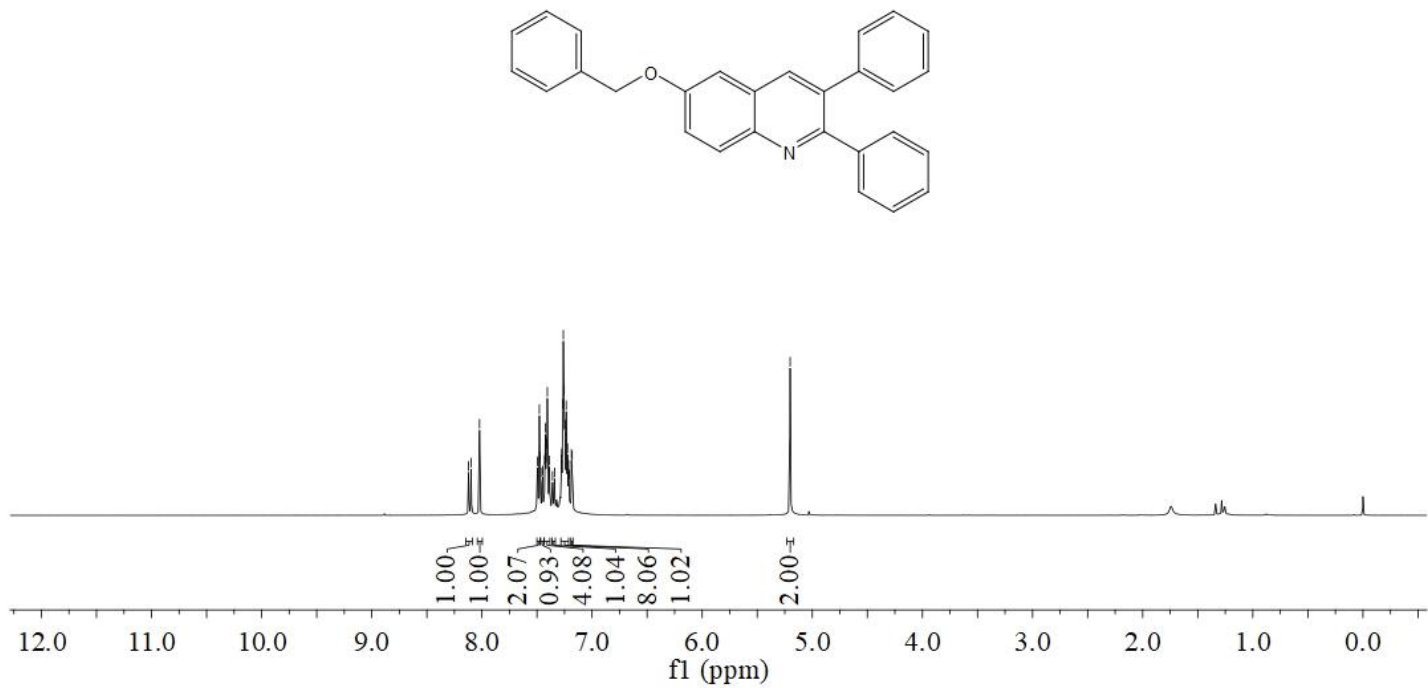

${ }^{13} \mathrm{C} \mathrm{NMR}\left(100 \mathrm{MHz}, \mathrm{CDCl}_{3}\right)$

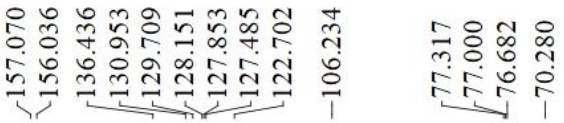
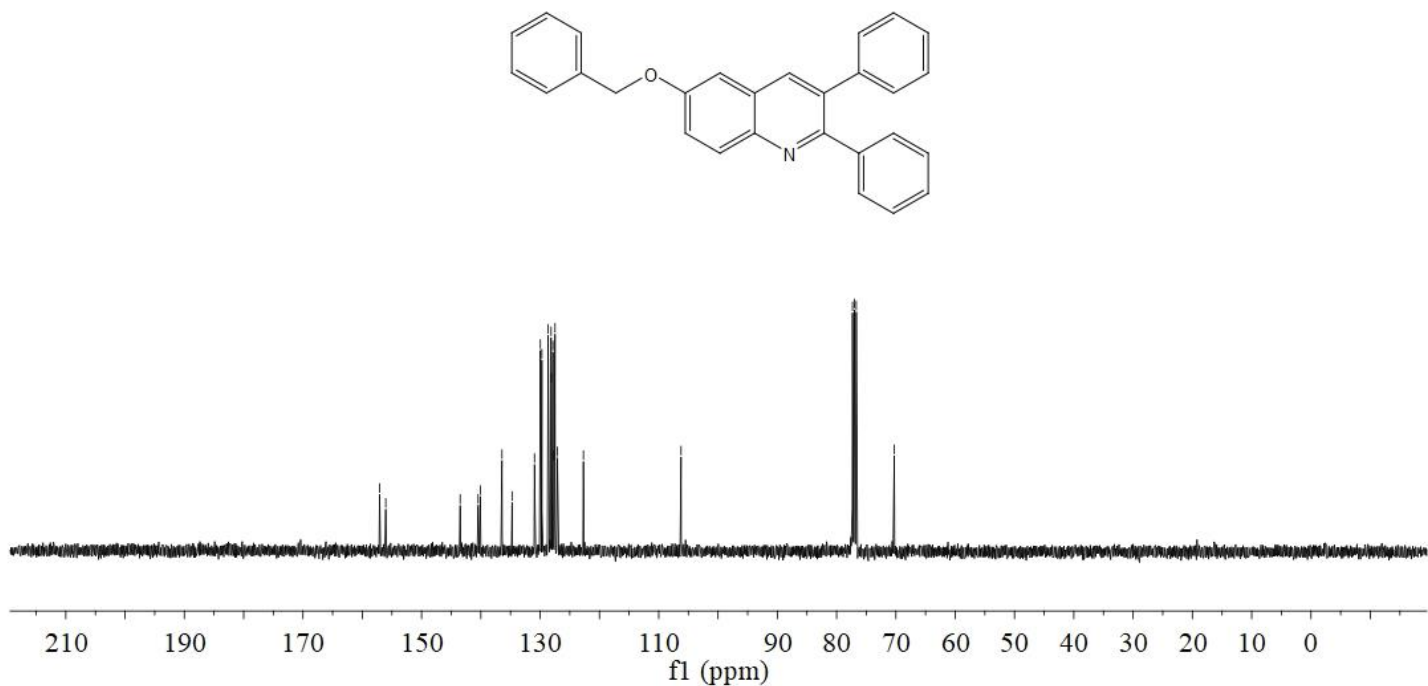
${ }^{1} \mathrm{H}$ NMR (400 MHz, $\mathrm{CDCl}_{3}$ )

\section{2,3-Diphenyl-7-(trifluoromethyl)quinolone (3bj)}

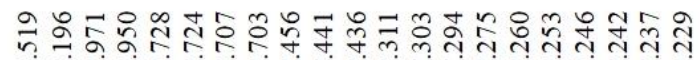

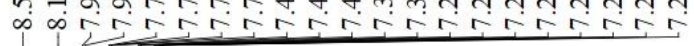
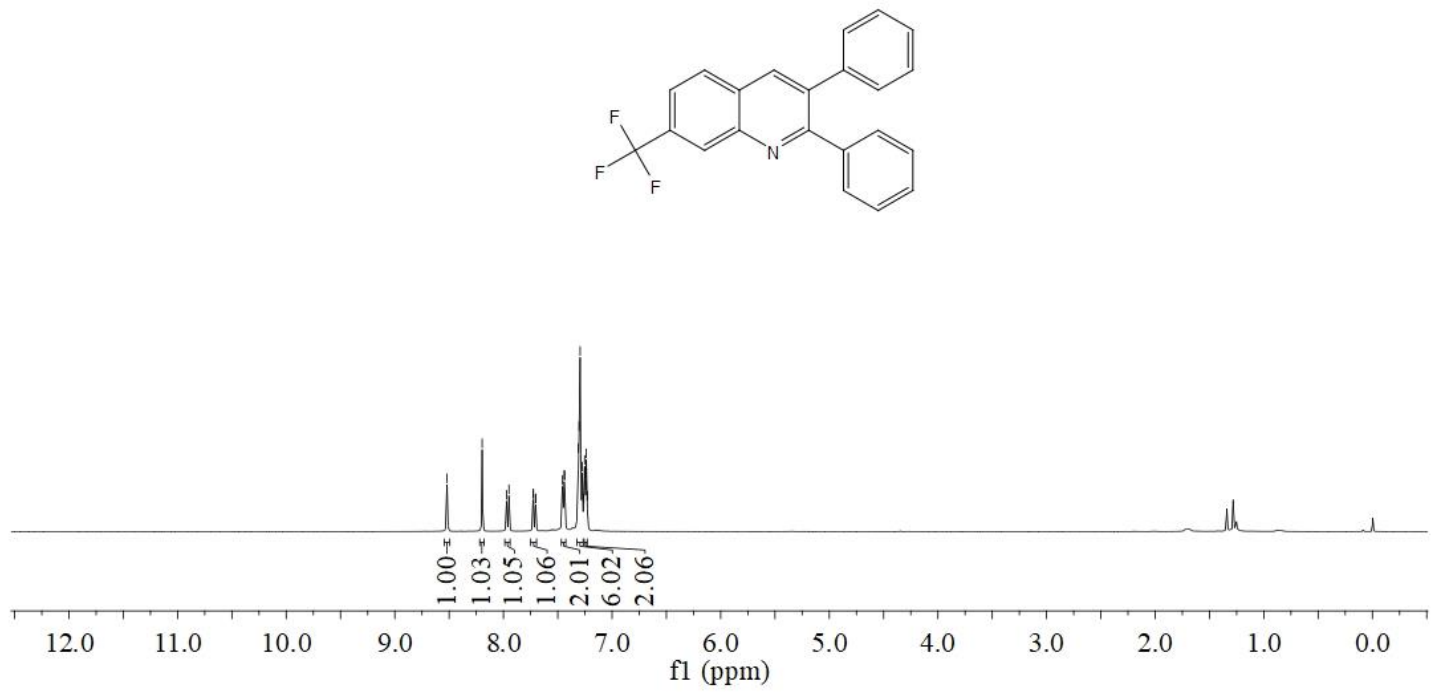

${ }^{13} \mathrm{C} \mathrm{NMR}\left(100 \mathrm{MHz}, \mathrm{CDCl}_{3}\right)$

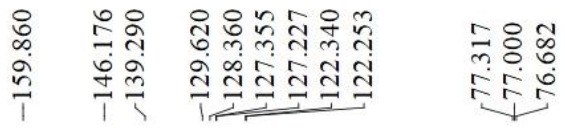
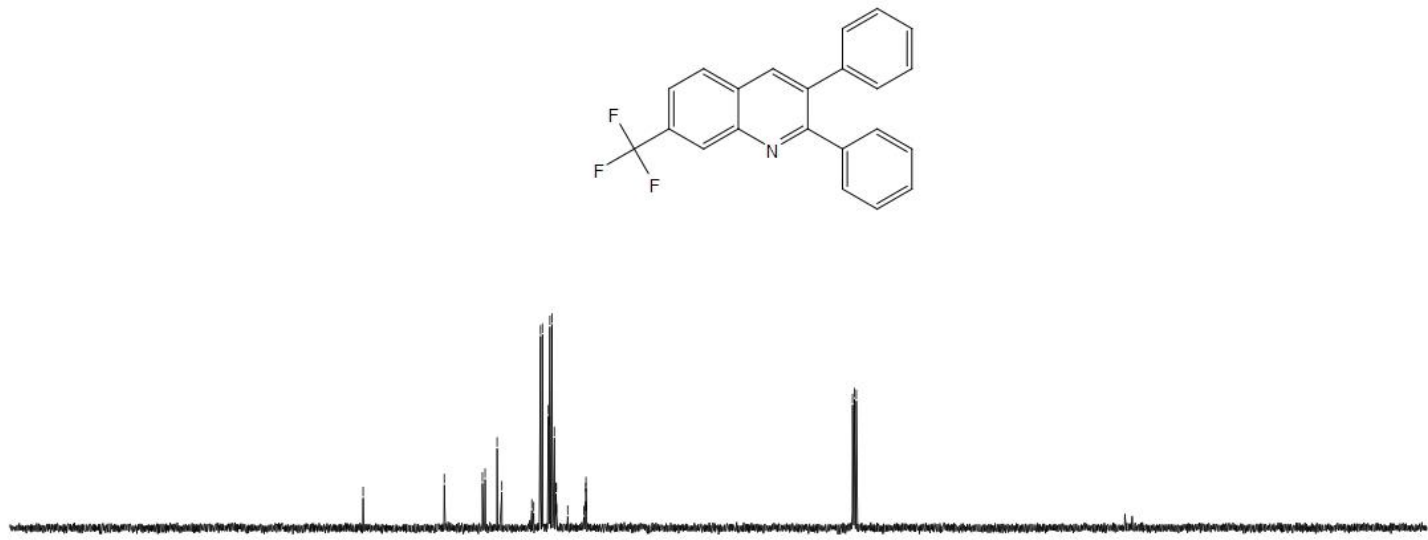

$\begin{array}{lllllllllllllllll}210 & 190 & 170 & 150 & 130 & 110 & 90 & 80 & 70 & 60 & 50 & 40 & 30 & 20 & 10 & 0 & \end{array}$


${ }^{19} \mathrm{~F}$ NMR (375 MHz, $\mathrm{CDCl}_{3}$ )

$$
\begin{aligned}
& 0 \\
& 0 \\
& 1 \\
& 0 \\
& i \\
& i
\end{aligned}
$$

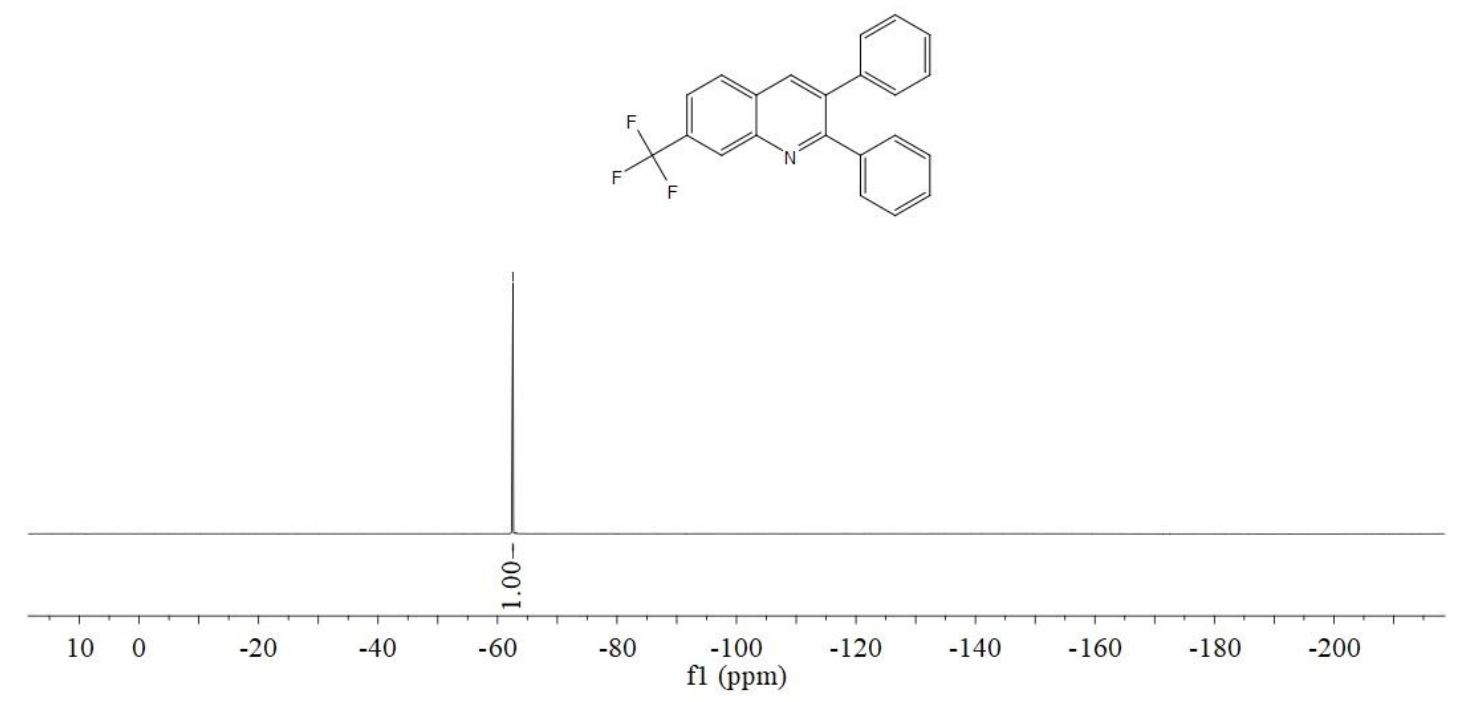


${ }^{1} \mathrm{H}$ NMR (400 MHz, $\left.\mathrm{CDCl}_{3}\right)$
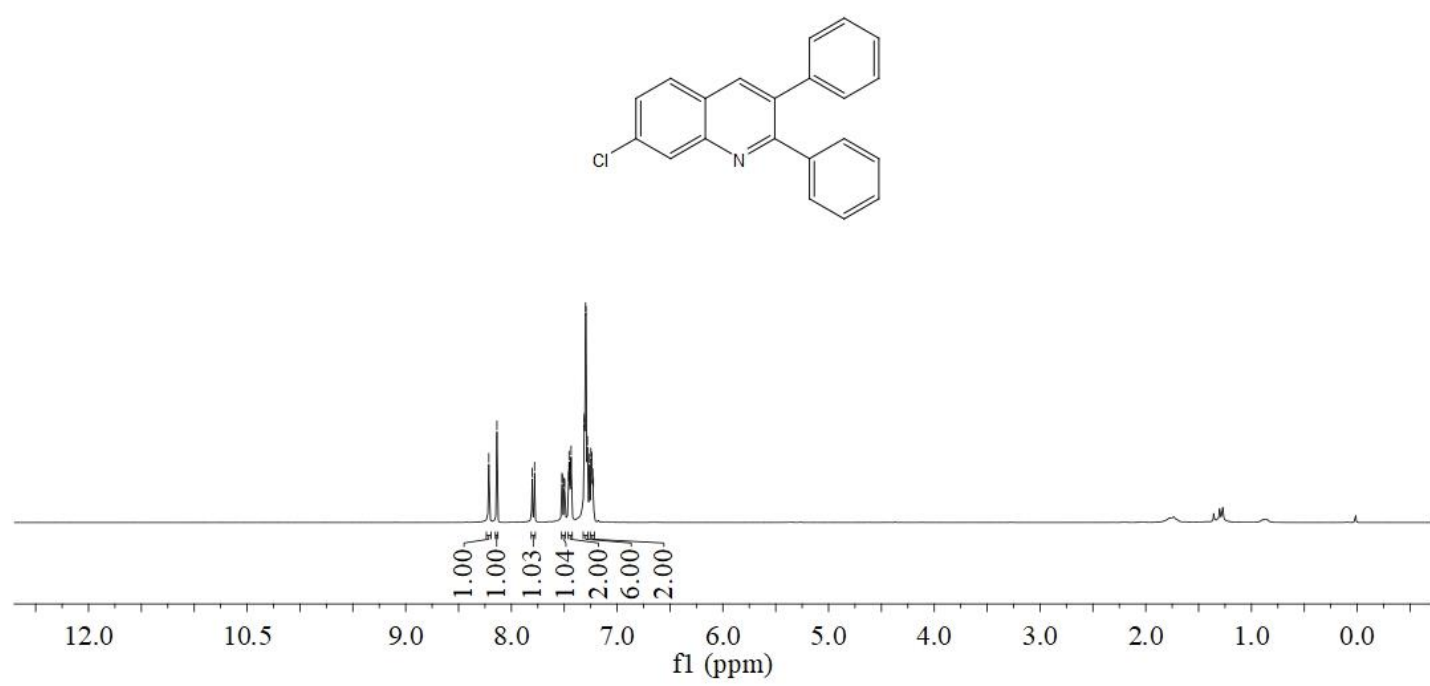

${ }^{13} \mathrm{C}$ NMR (100 MHz, $\left.\mathrm{CDCl}_{3}\right)$

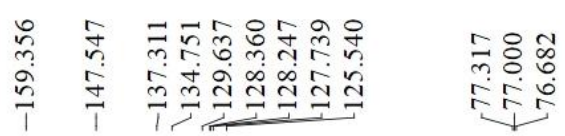<smiles>Clc1ccc2cc(-c3ccccc3)c(-c3ccccc3)nc2c1</smiles>

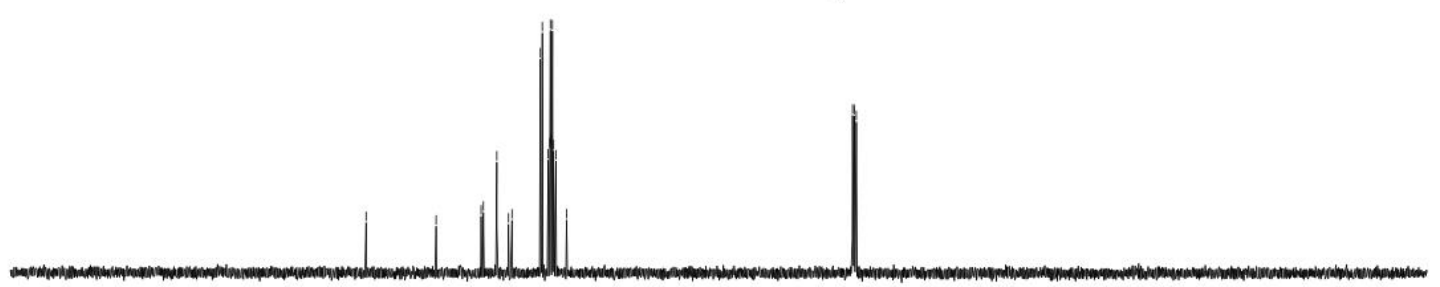

$\begin{array}{lllllllllllllllll}210 & 190 & 170 & 150 & 130 & \begin{array}{c}110 \\ \mathrm{f} 1(\mathrm{ppm})\end{array} & 90 & 70 & 60 & 50 & 40 & 30 & 20 & 10 & 0\end{array}$


${ }^{1} \mathrm{H}$ NMR (400 MHz, $\left.\mathrm{CDCl}_{3}\right)$
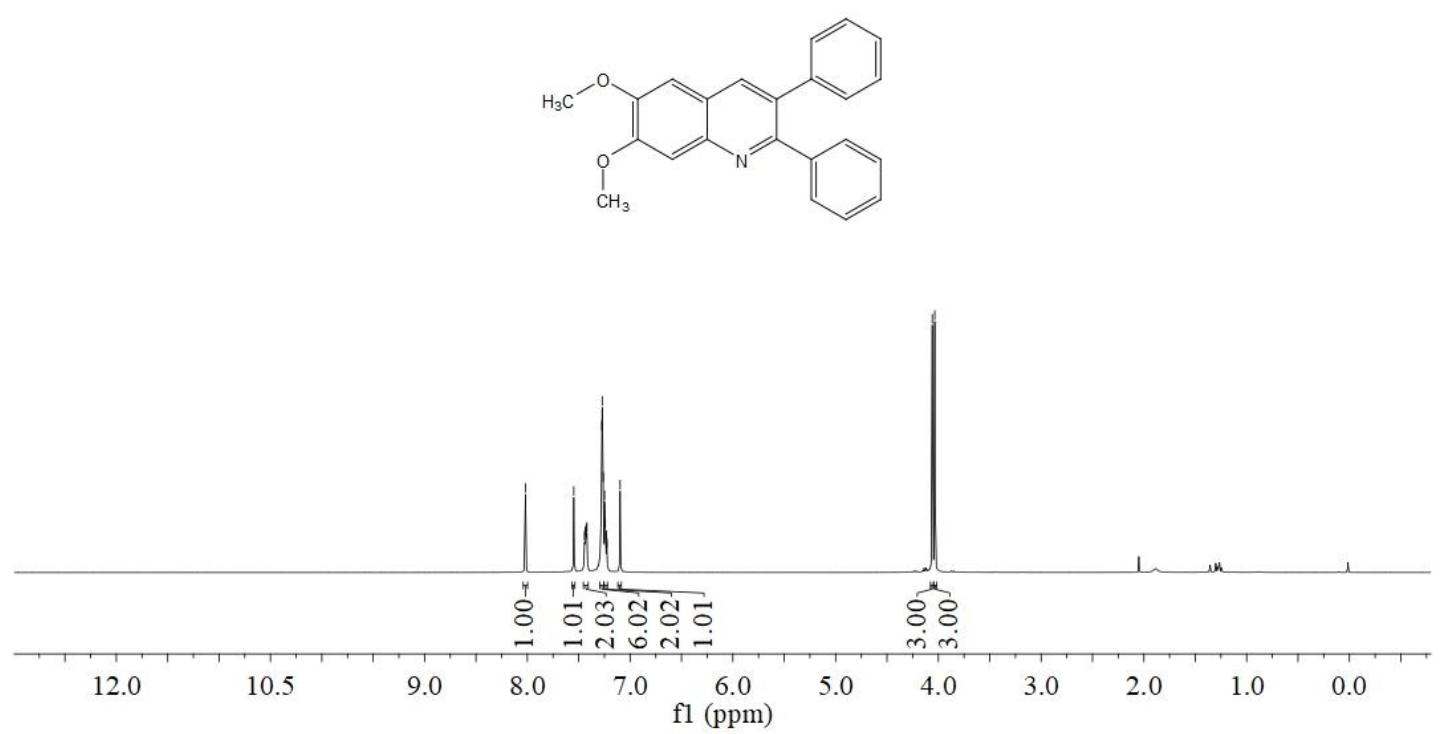

${ }^{13} \mathrm{C} \mathrm{NMR}\left(100 \mathrm{MHz}, \mathrm{CDCl}_{3}\right)$
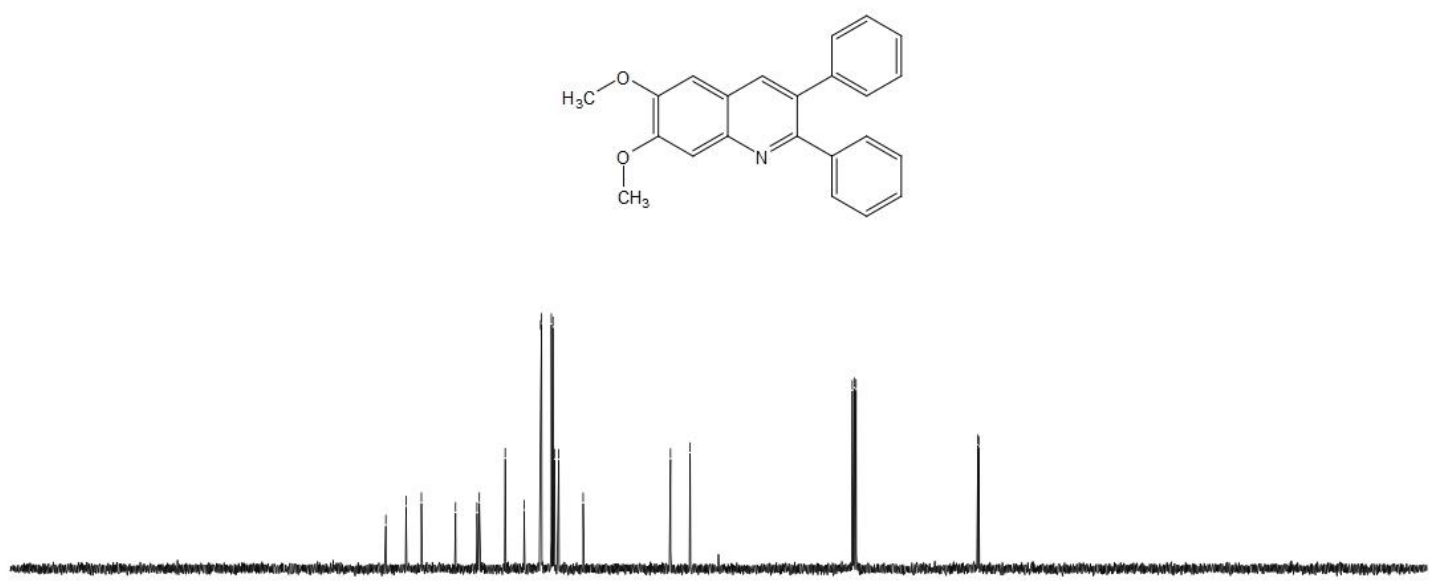

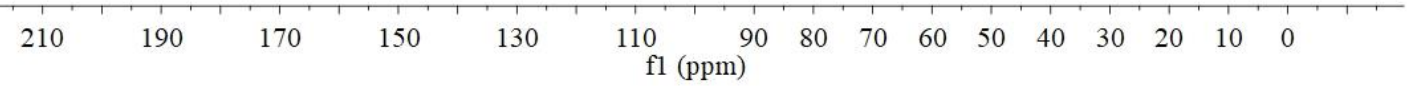


${ }^{1} \mathrm{H}$ NMR (400 MHz, $\left.\mathrm{CDCl}_{3}\right)$

\section{6,7-Diphenyl- $[1,3]$ dioxolo[4,5-g] quinolone (3bm)}

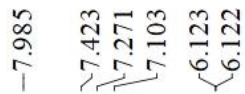
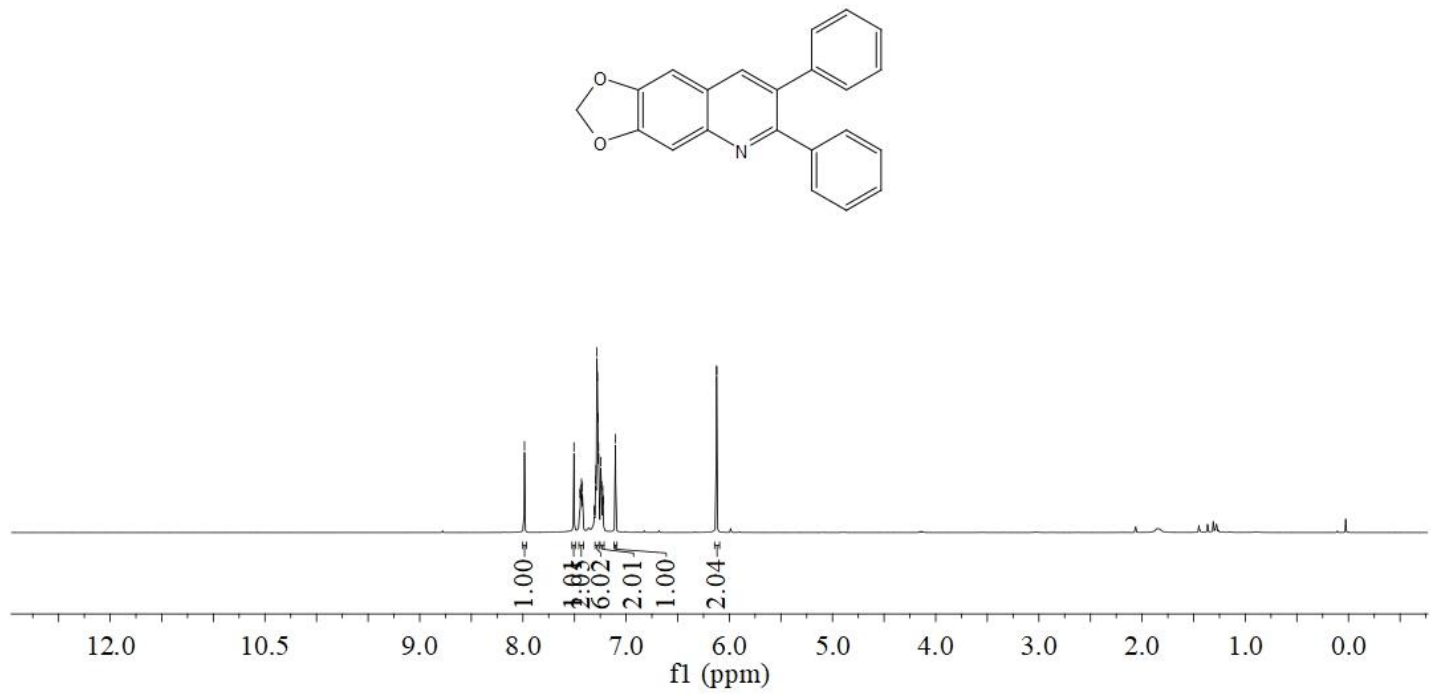

${ }^{13} \mathrm{C}$ NMR (100 MHz, $\left.\mathrm{CDCl}_{3}\right)$
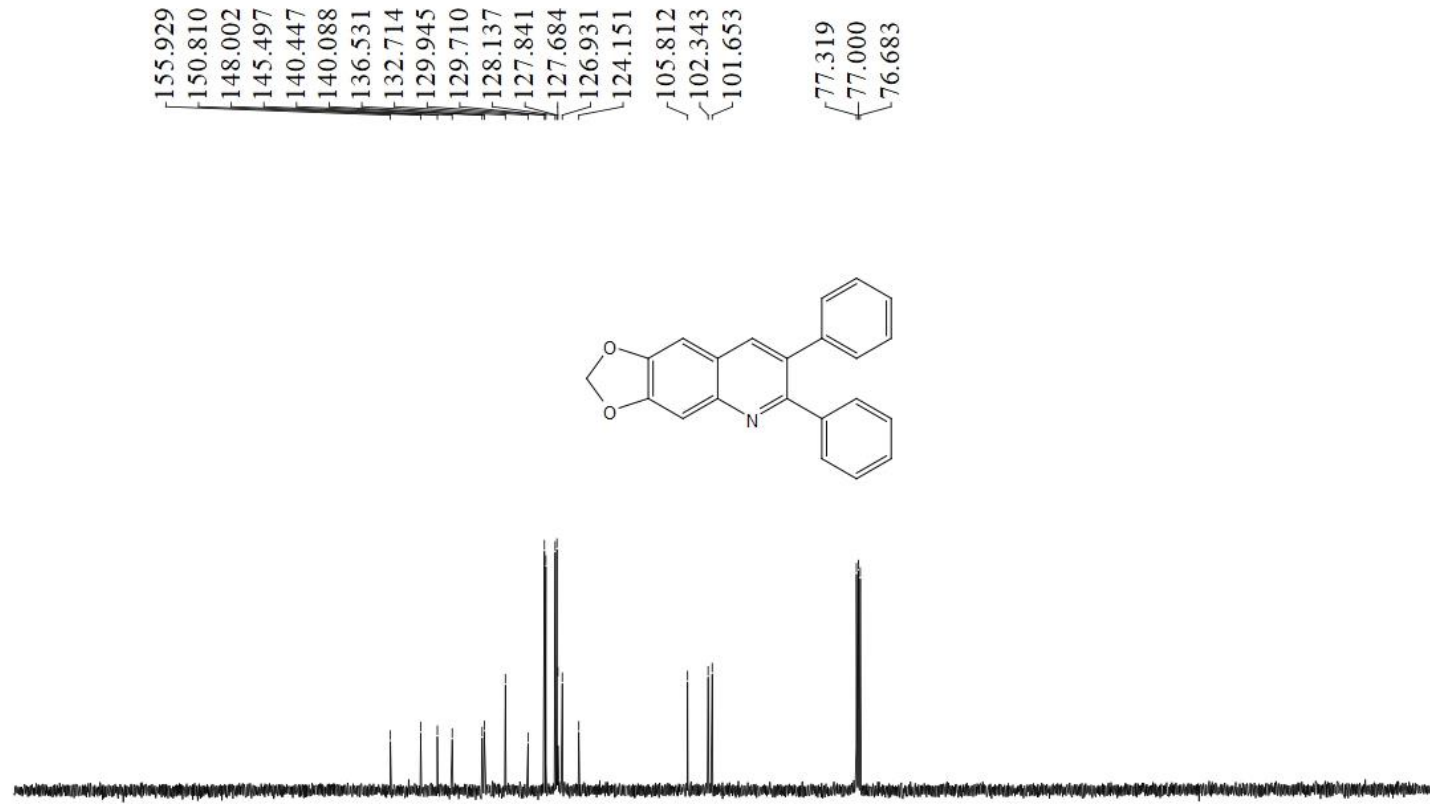

$\begin{array}{llllllllllllllll}210 & 190 & 170 & 150 & 130 & 110 & 90 & 80 & 70 & 60 & 50 & 40 & 30 & 20 & 10 & 0\end{array}$


${ }^{1} \mathrm{H}$ NMR (400 MHz, $\left.\mathrm{CDCl}_{3}\right)$

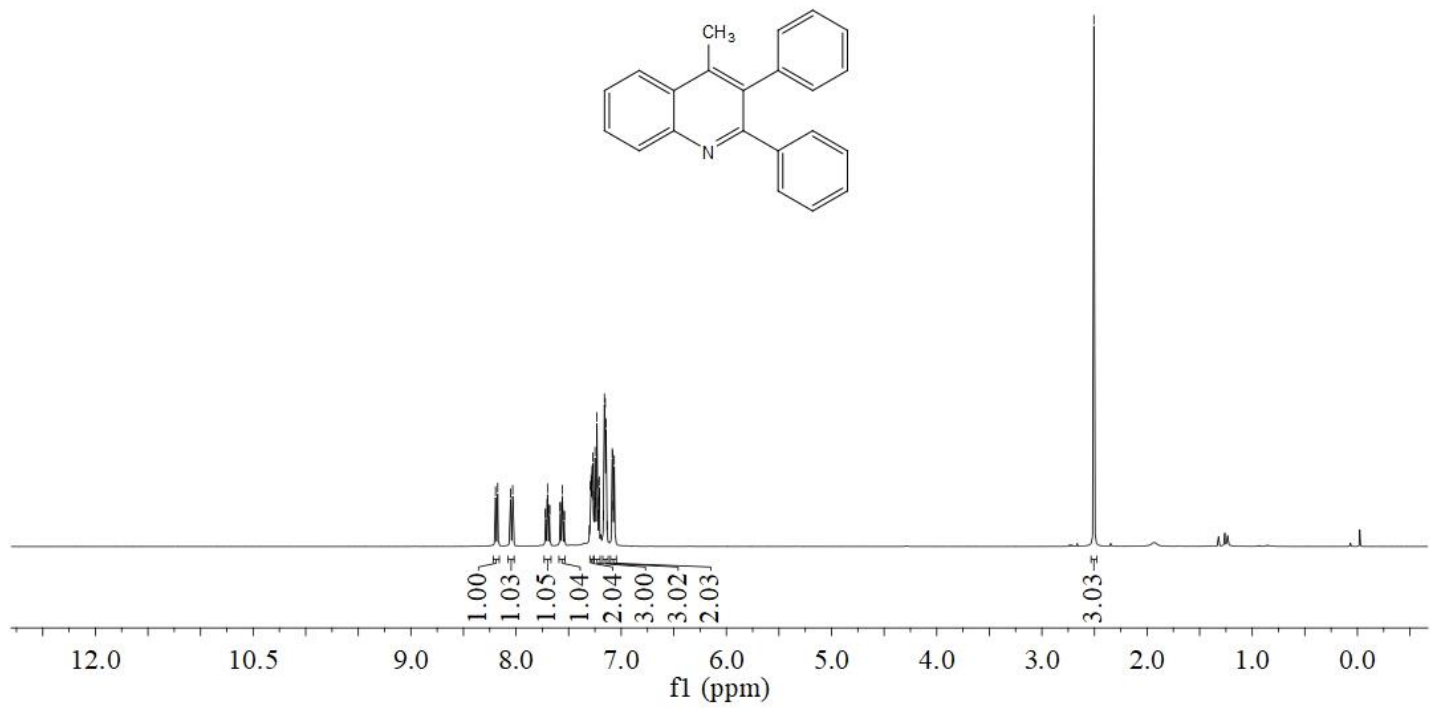

${ }^{13} \mathrm{C} \mathrm{NMR}\left(100 \mathrm{MHz}, \mathrm{CDCl}_{3}\right)$

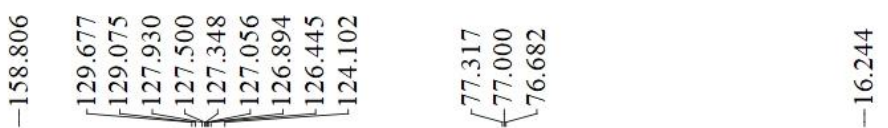
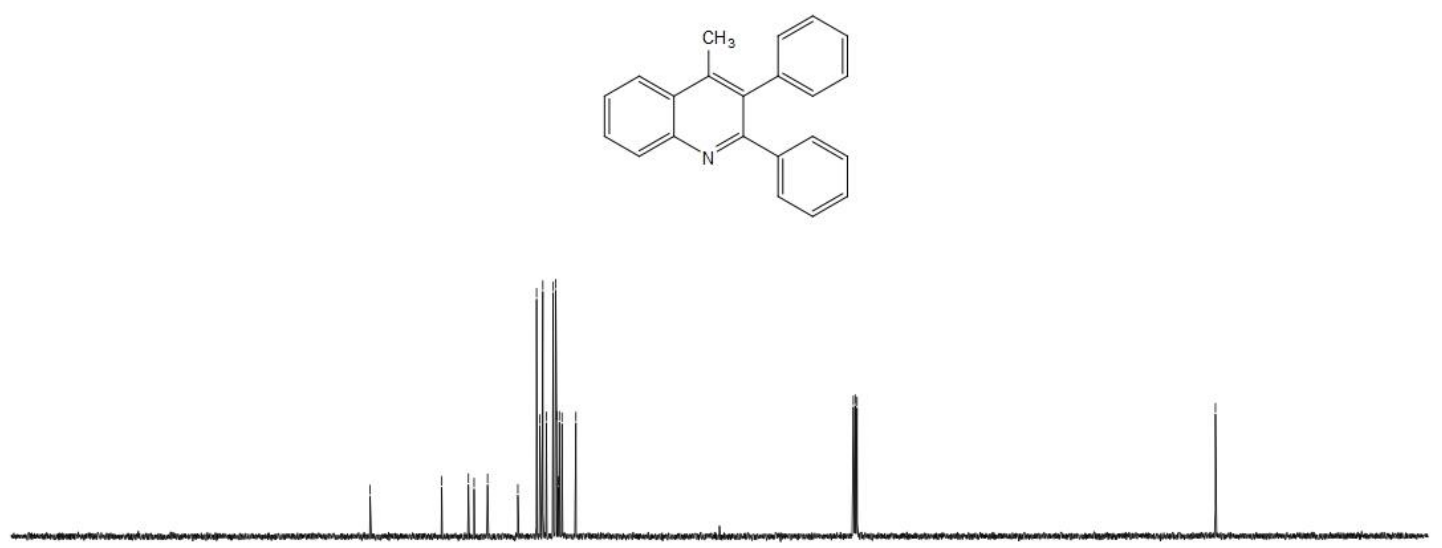

$\begin{array}{llllllllllllllll}210 & 190 & 170 & 150 & 130 & 110 & 90 & 80 & 70 & 60 & 50 & 40 & 30 & 20 & 10 & 0\end{array}$


${ }^{1} \mathrm{H}$ NMR (400 MHz, $\left.\mathrm{CDCl}_{3}\right)$

\section{2,3,4-Triphenylquinoline (3bo)}

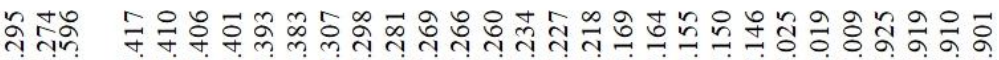

कion
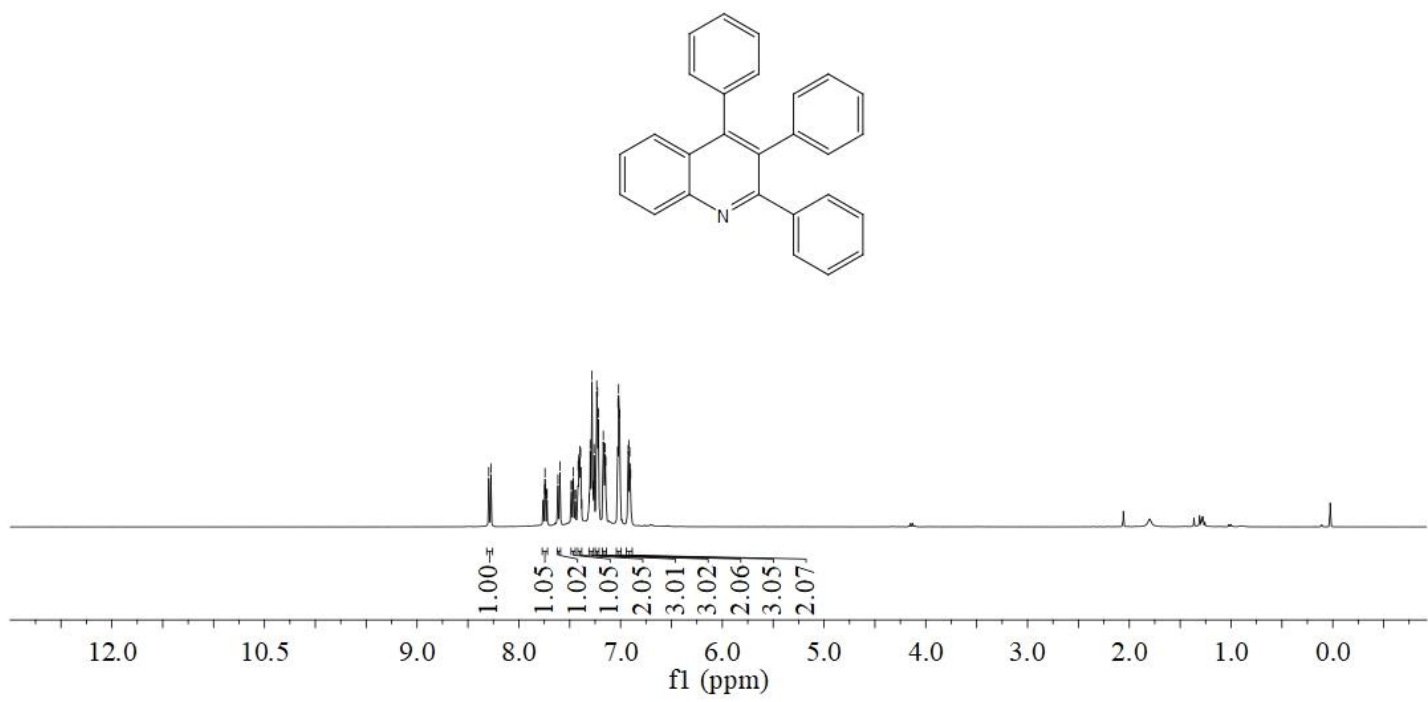

${ }^{13} \mathrm{C}$ NMR (100 MHz, $\left.\mathrm{CDCl}_{3}\right)$
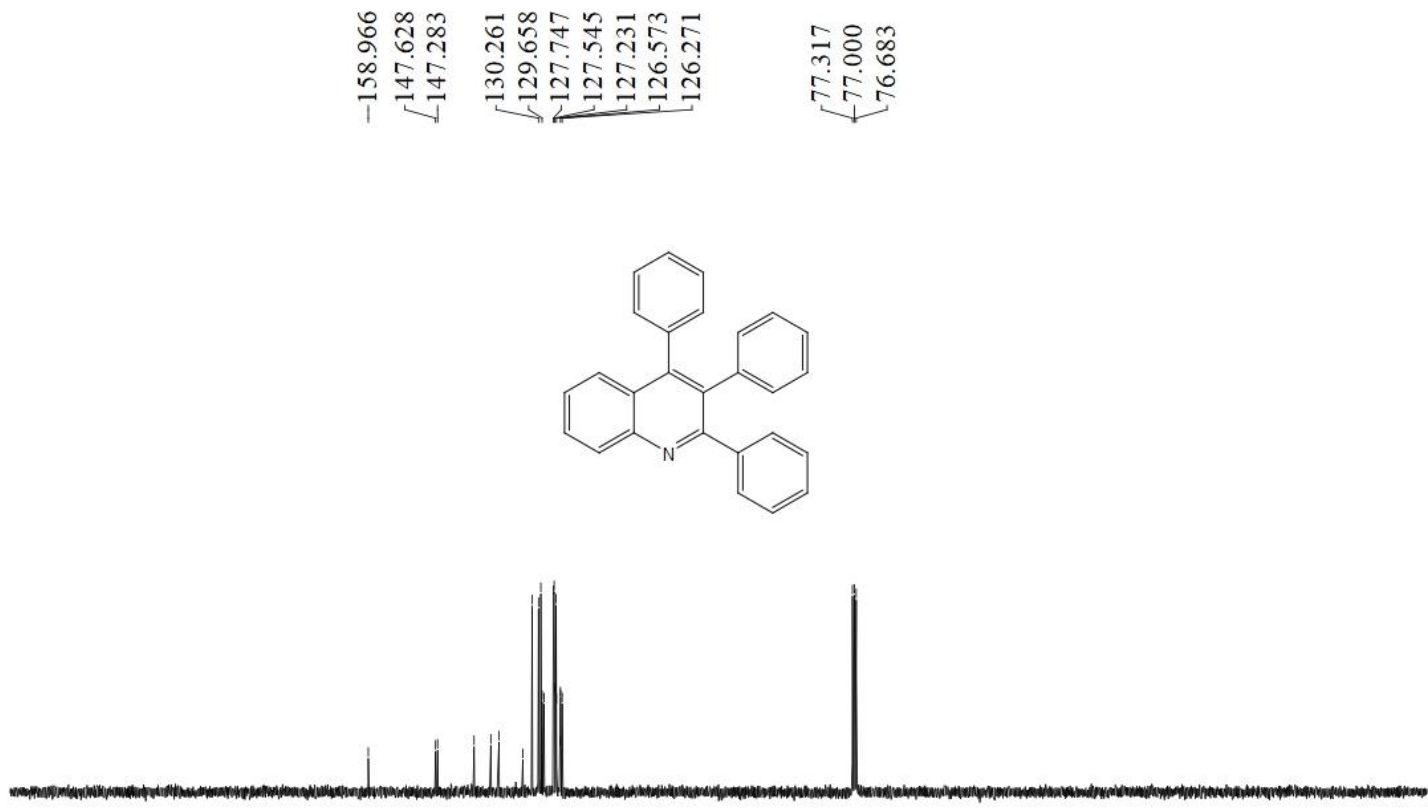

$\begin{array}{lllllllllllllllll}210 & 190 & 170 & 150 & 130 & 110 & 90 & 80 & 70 & 60 & 50 & 40 & 30 & 20 & 10 & 0 & \end{array}$


${ }^{1} \mathrm{H}$ NMR (400 MHz, $\left.\mathrm{CDCl}_{3}\right)$

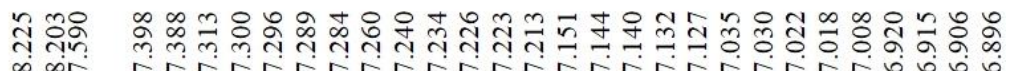

कin
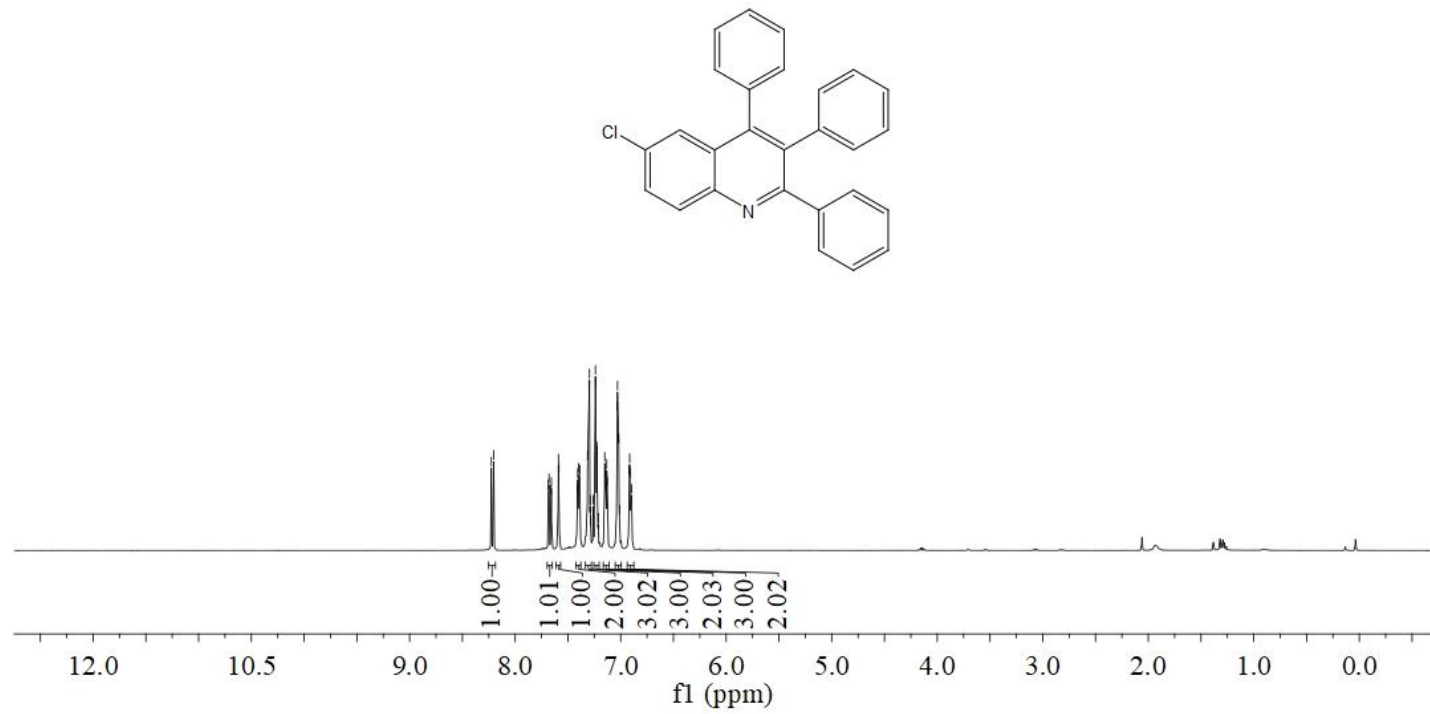

${ }^{13} \mathrm{C}$ NMR (100 MHz, $\left.\mathrm{CDCl}_{3}\right)$
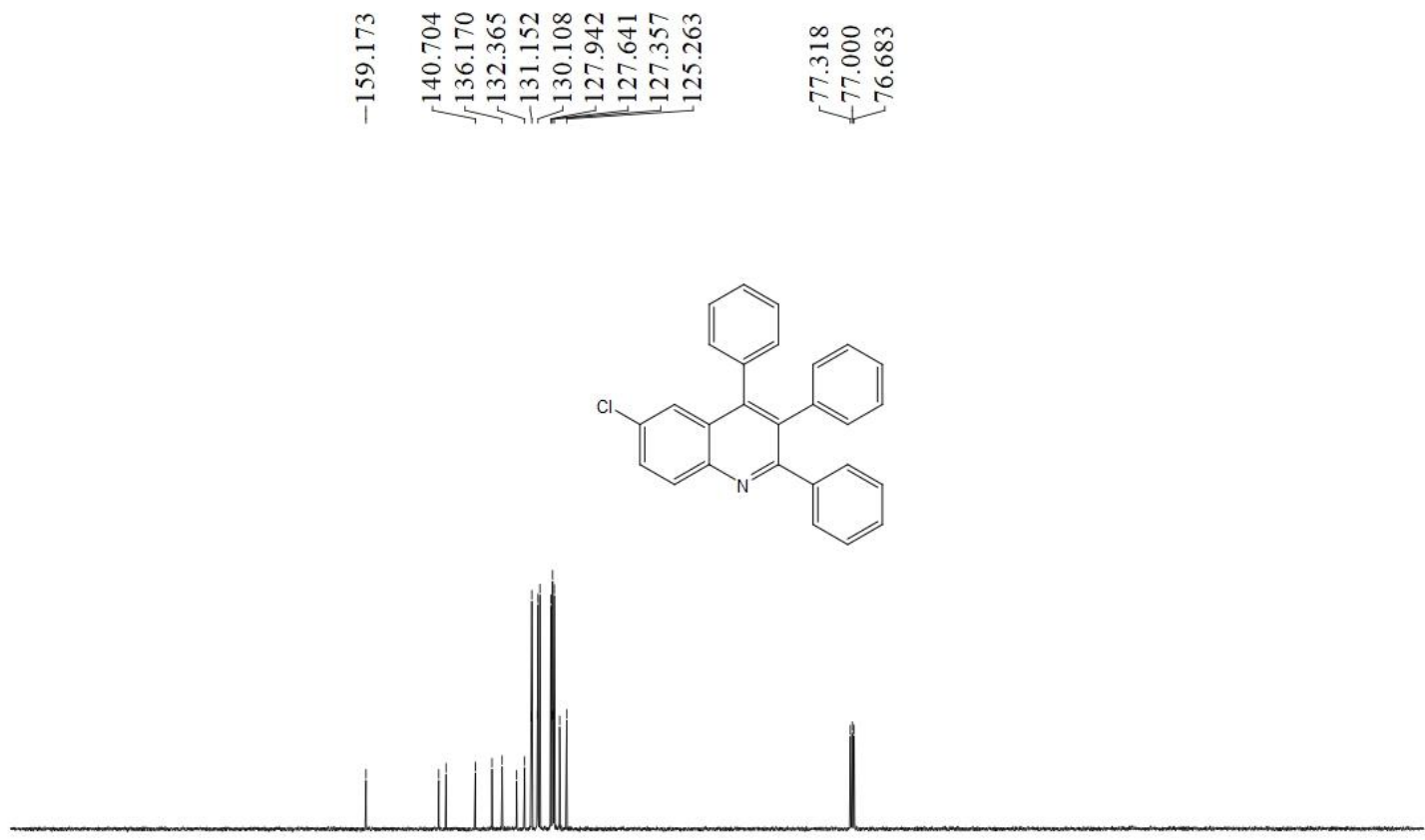

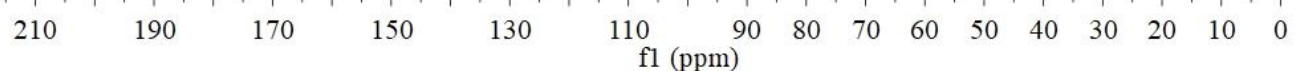


${ }^{1} \mathrm{H}$ NMR (400 MHz, $\mathrm{CDCl}_{3}$ )

3-(Benzyloxy)-2-phenylquinoline (3bq)

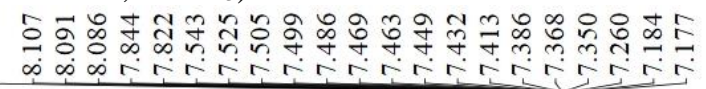

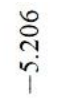
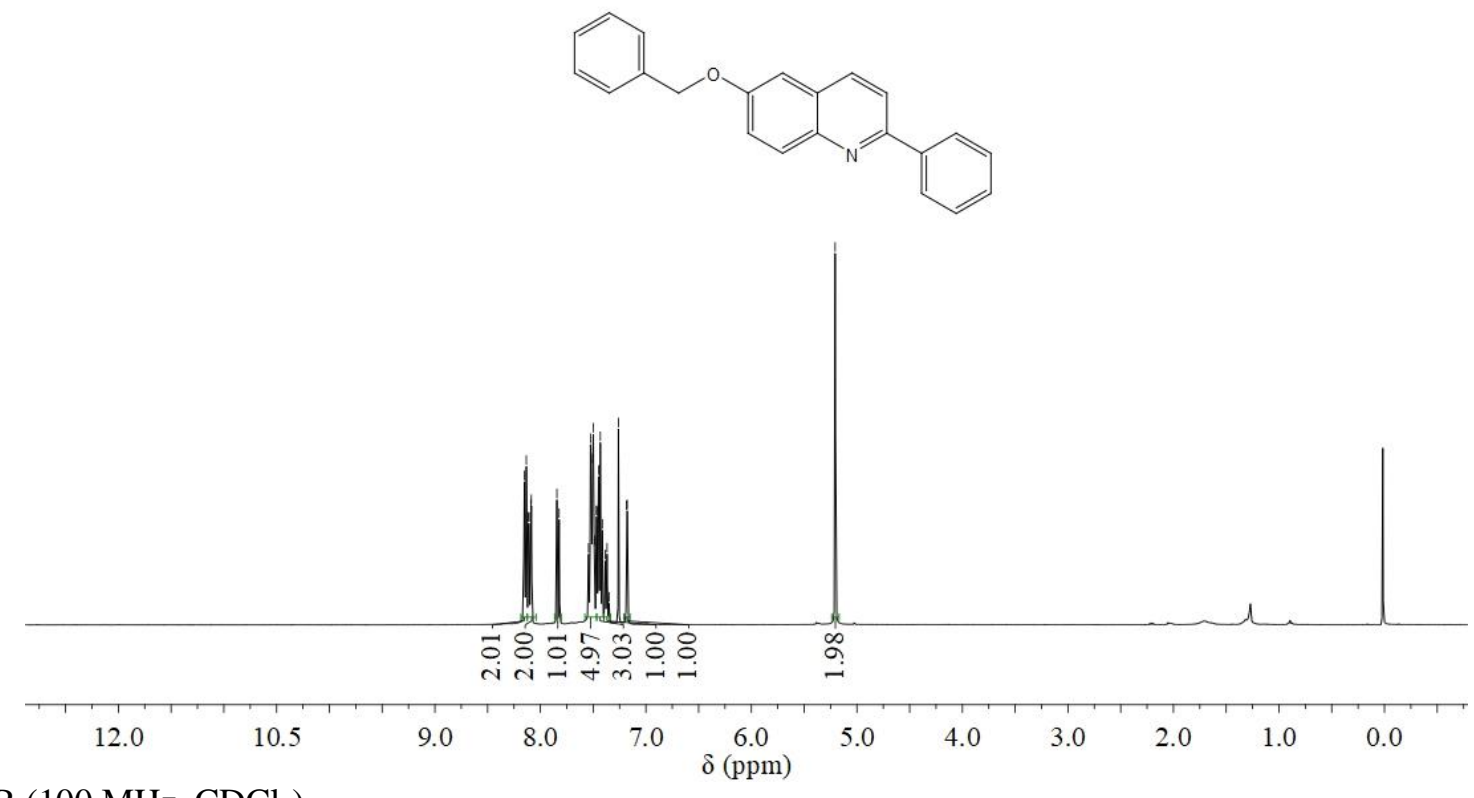

${ }^{13} \mathrm{C}$ NMR (100 MHz, $\left.\mathrm{CDCl}_{3}\right)$

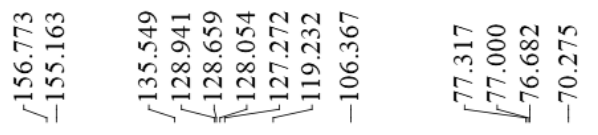
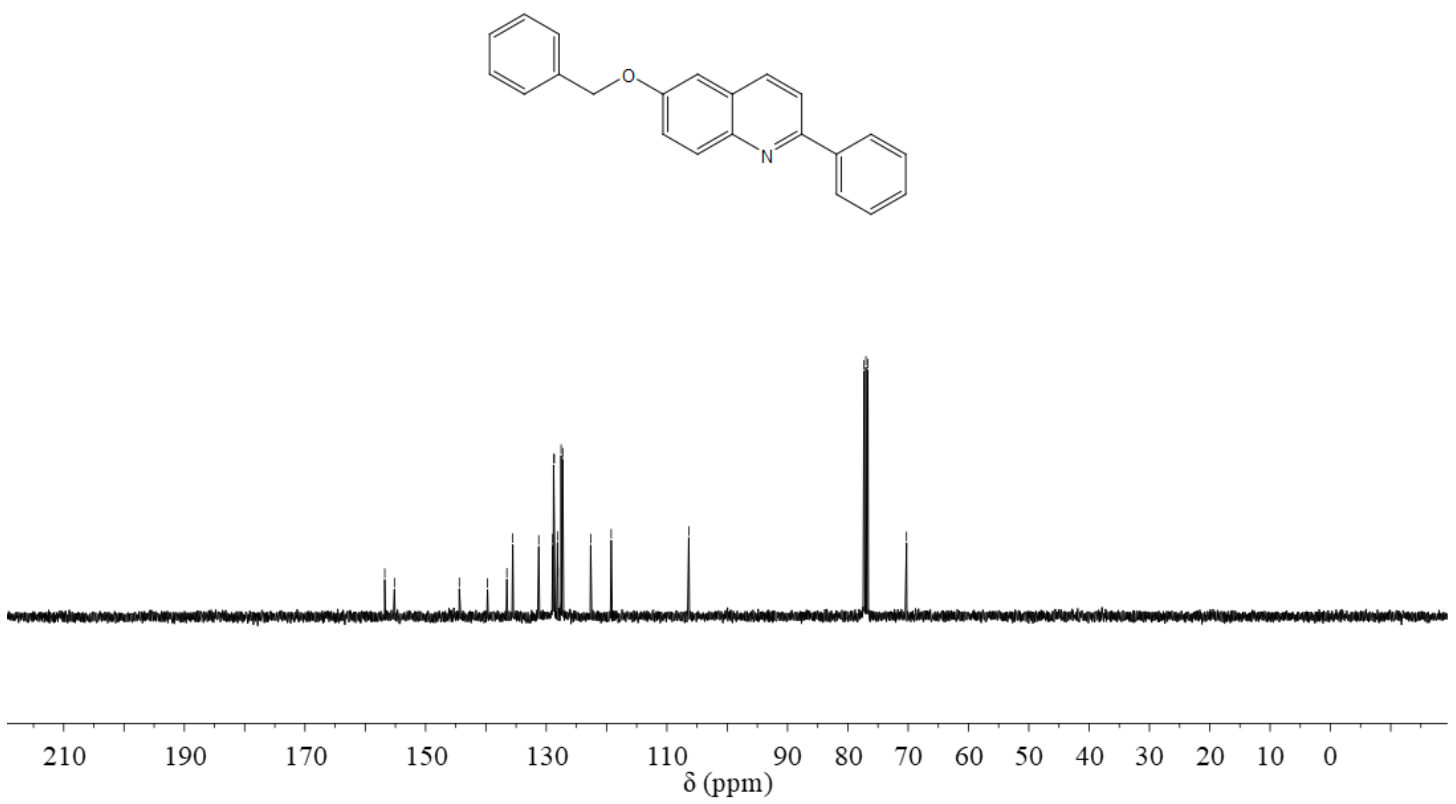

116 
${ }^{1} \mathrm{H}$ NMR (400 MHz, $\mathrm{CDCl}_{3}$ )

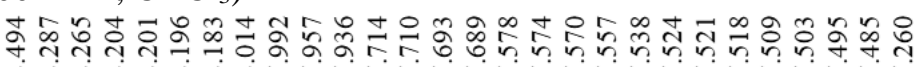

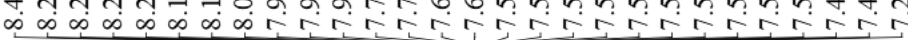
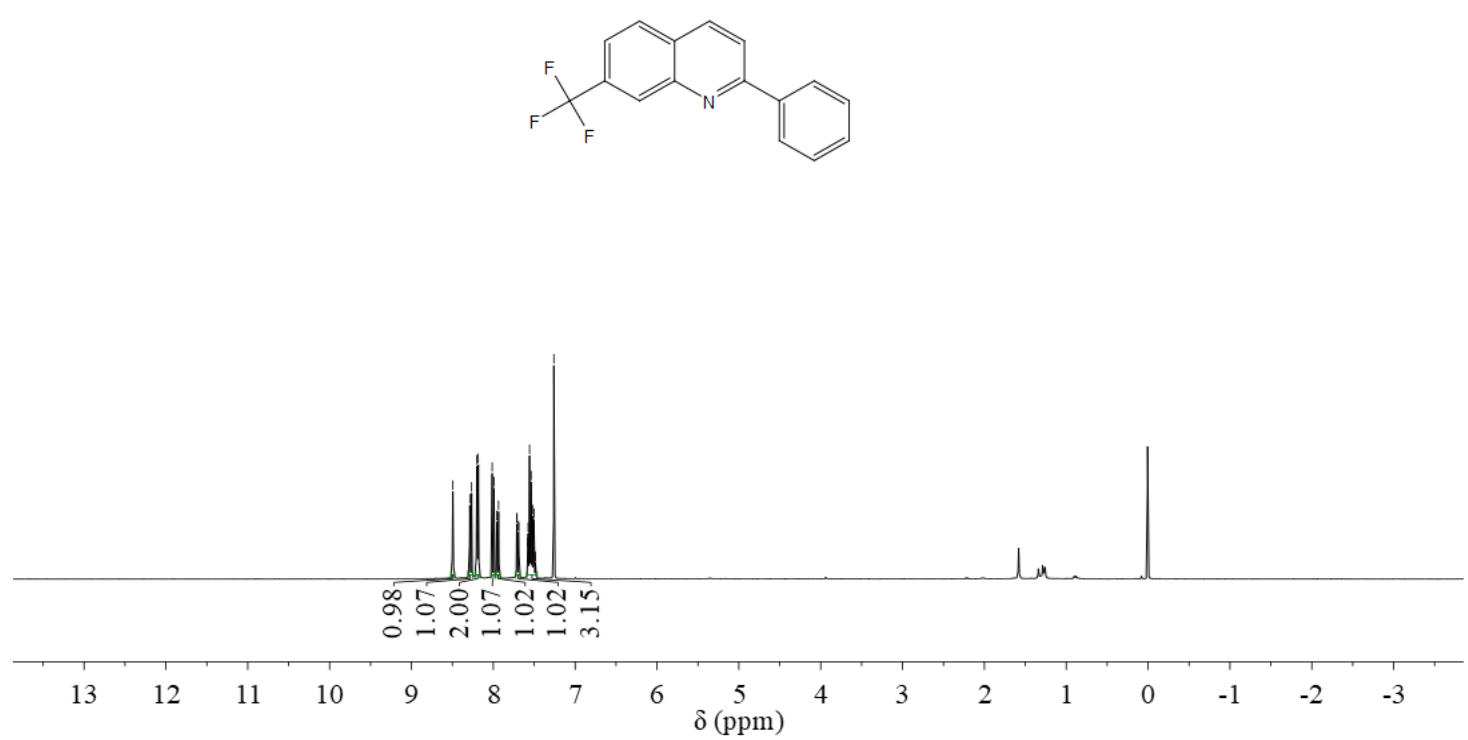

${ }^{13} \mathrm{C}$ NMR (100 MHz, $\left.\mathrm{CDCl}_{3}\right)$

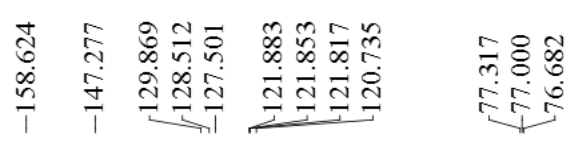
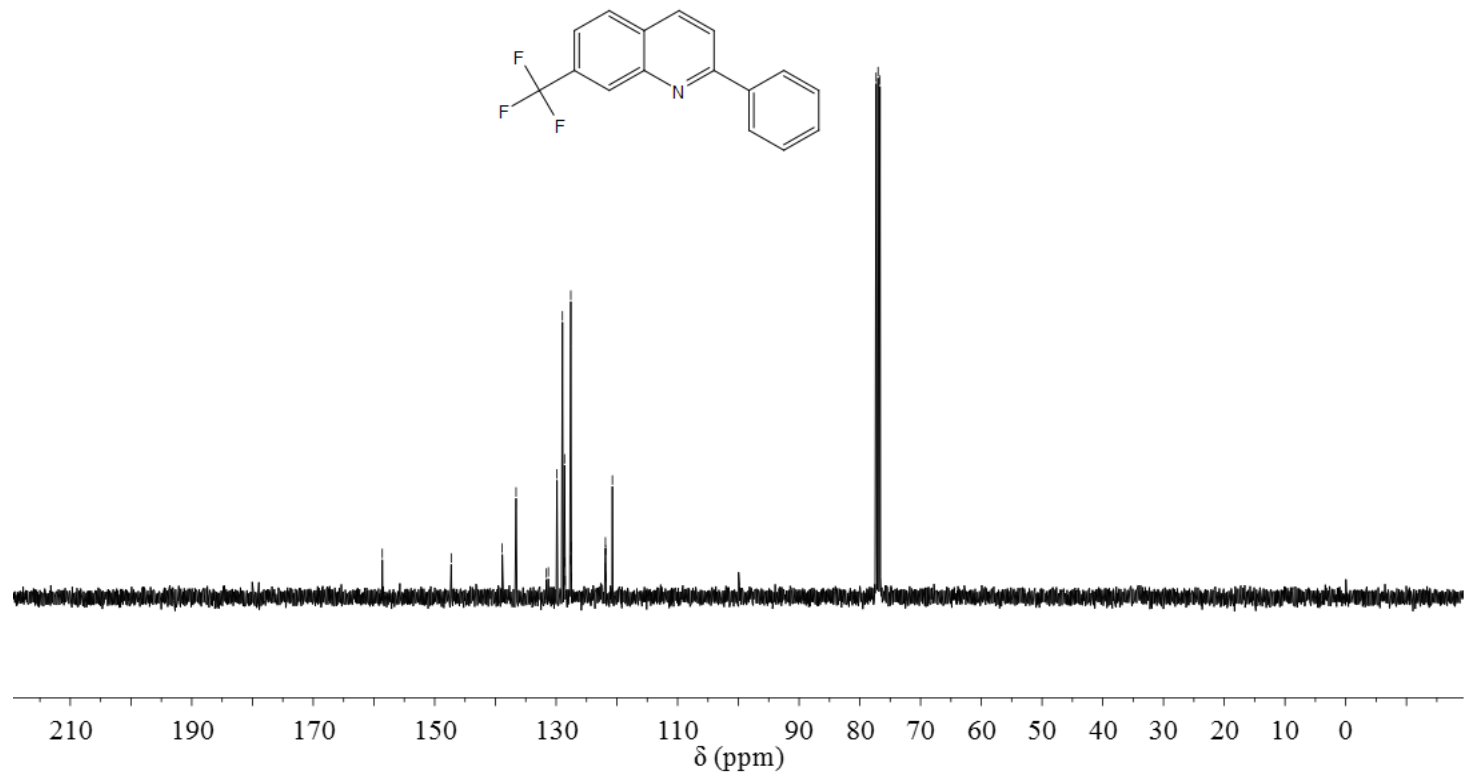
${ }^{19} \mathrm{~F}$ NMR (376 MHz, $\mathrm{CDCl}_{3}$ )

ले
i్
$i$
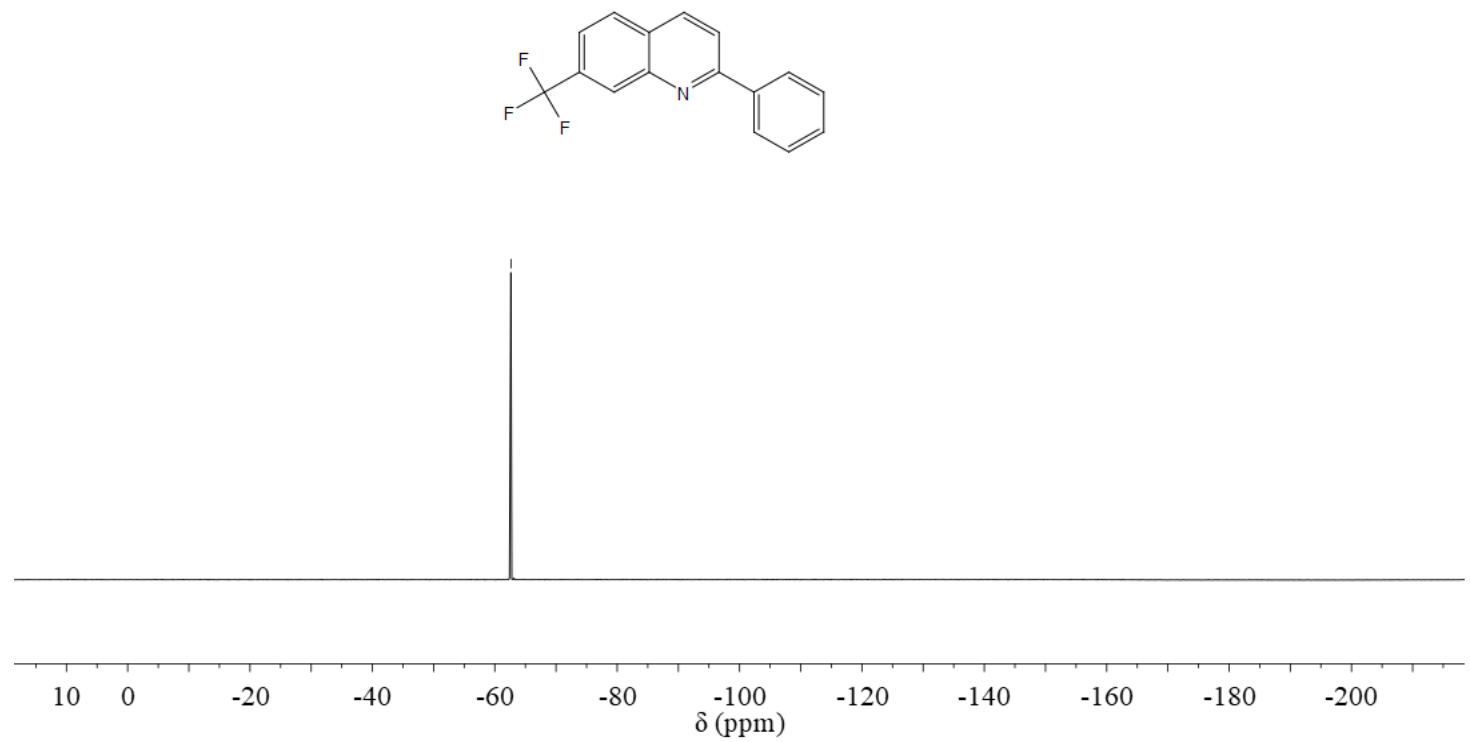
2-Phenylquinolin-6-yl benzoate (Bbs)

${ }^{1} \mathrm{H}$ NMR $\left(400 \mathrm{MHz}, \mathrm{CDCl}_{3}\right)$

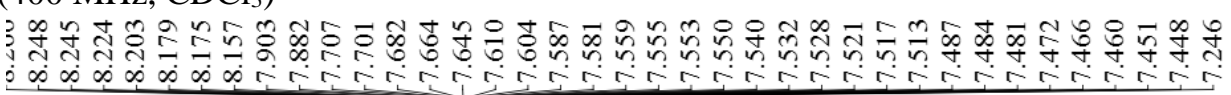
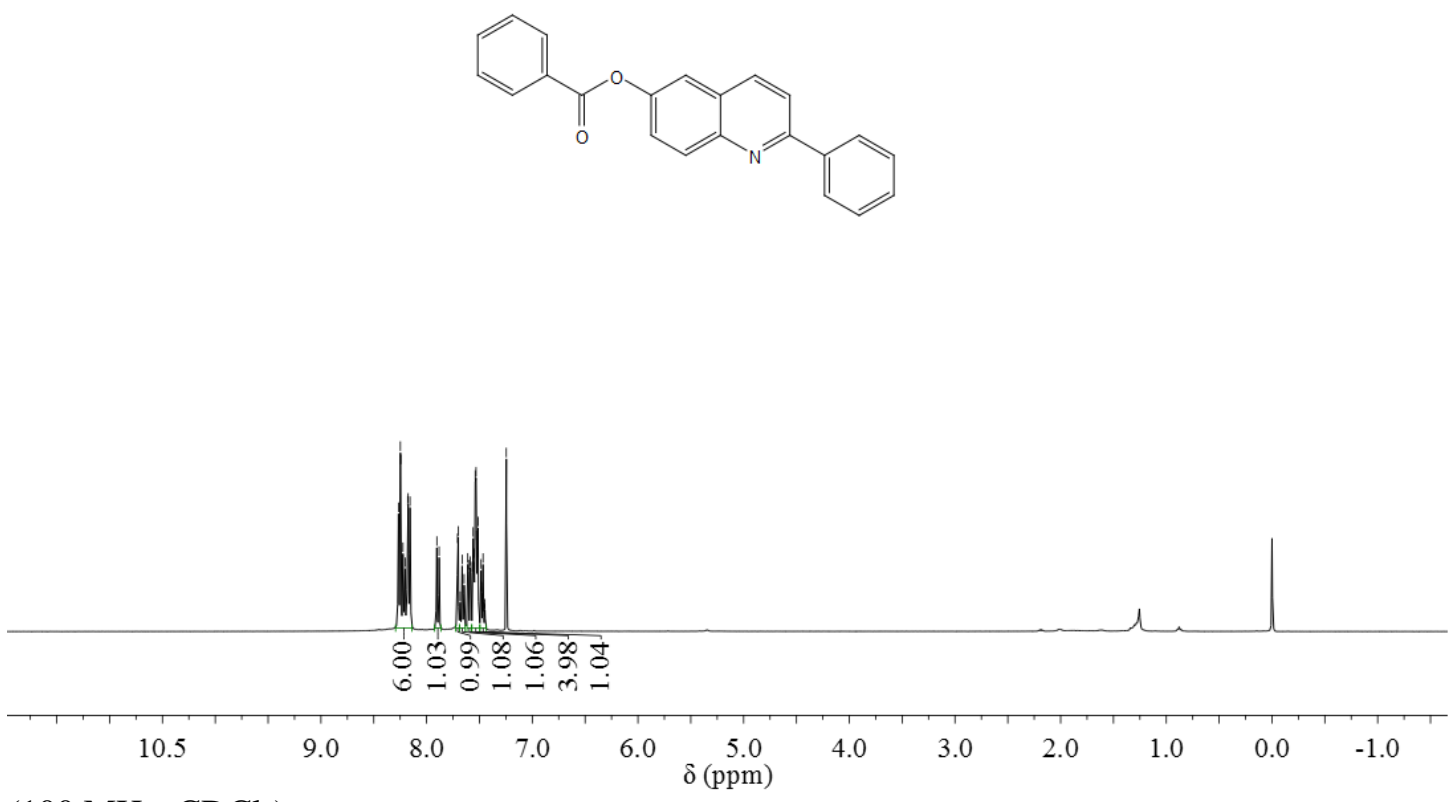

${ }^{13} \mathrm{C}$ NMR (100 MHz, $\left.\mathrm{CDCl}_{3}\right)$
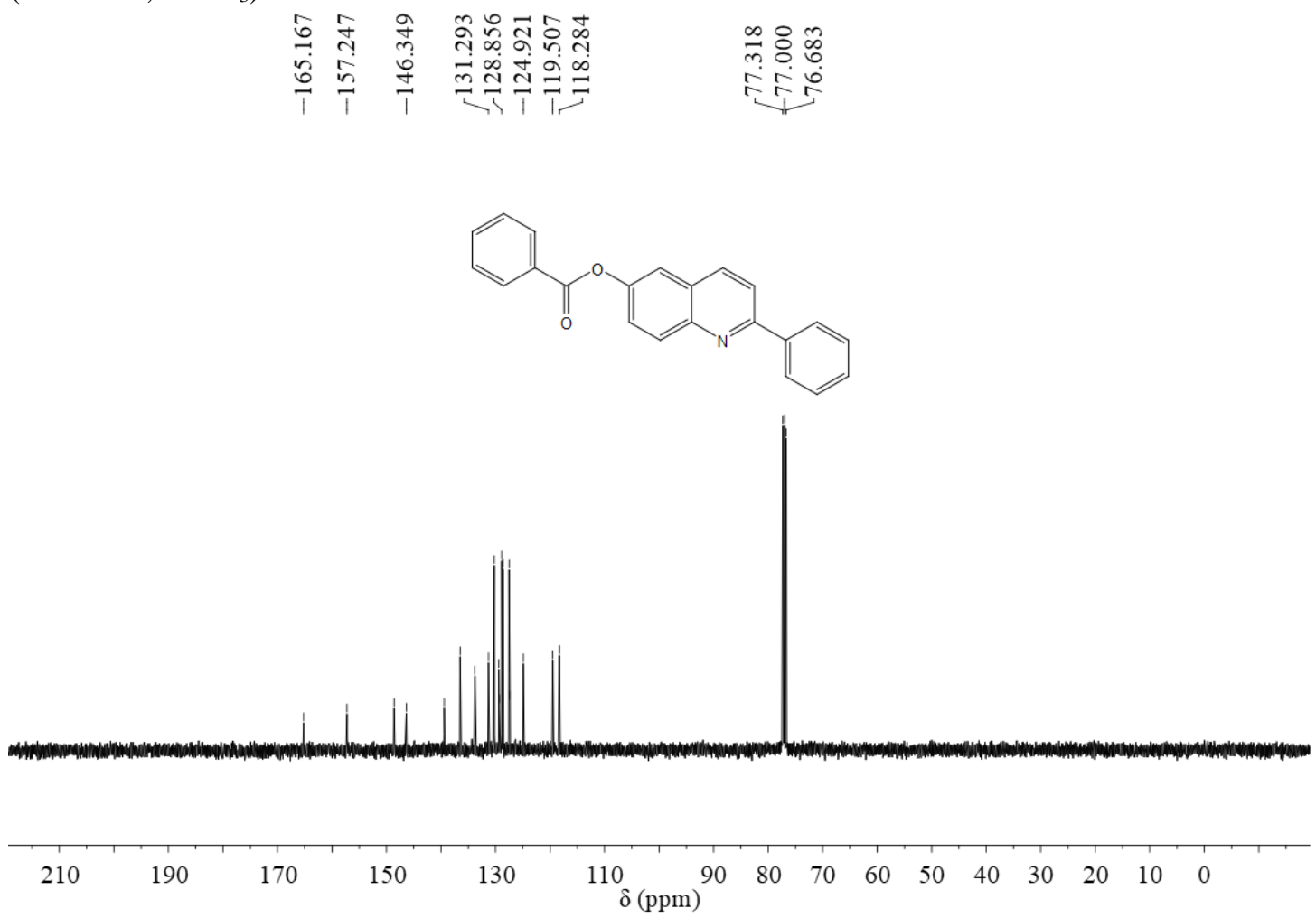

119 
${ }^{1} \mathrm{H}$ NMR (400 MHz, $\left.\mathrm{CDCl}_{3}\right)$

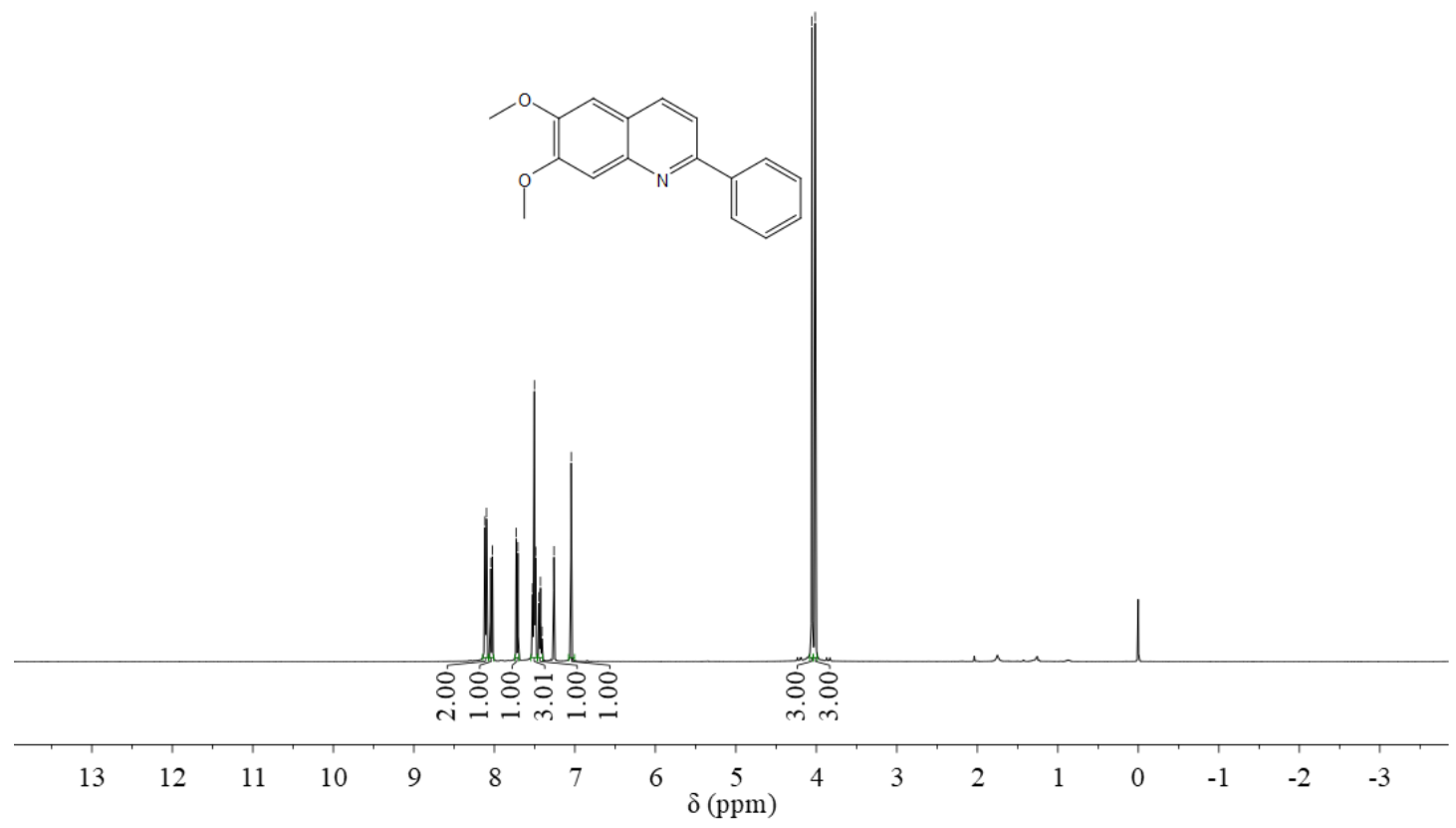

${ }^{13} \mathrm{C} \mathrm{NMR}\left(100 \mathrm{MHz}, \mathrm{CDCl}_{3}\right)$

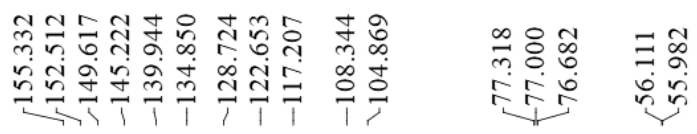<smiles>COc1cc2ccc(-c3ccccc3)nc2cc1OC</smiles>

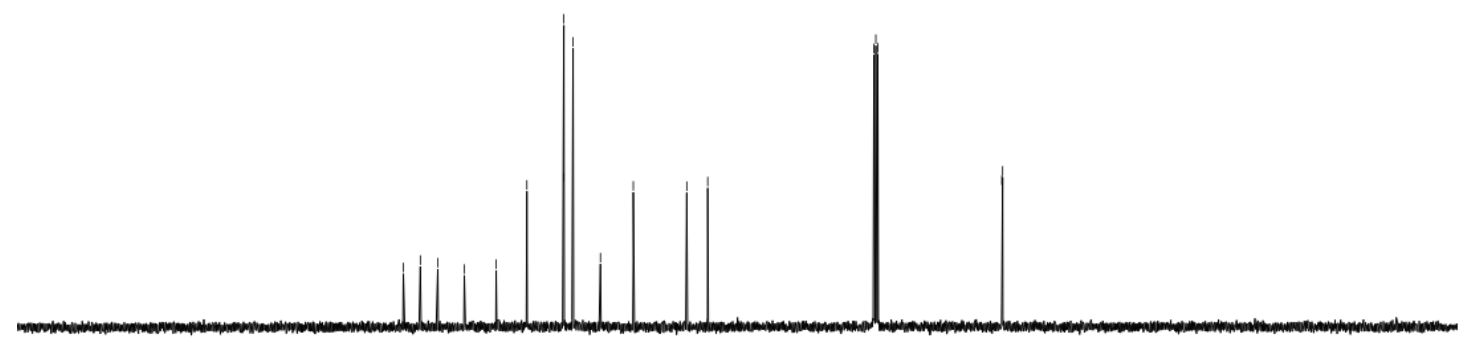

$\begin{array}{llllllllllllllll}210 & 190 & 170 & 150 & 130 & 110 \underset{\delta(\mathrm{ppm})}{90} & 80 & 70 & 60 & 50 & 40 & 30 & 20 & 10 & 0\end{array}$


${ }^{1} \mathrm{H}$ NMR (400 MHz, $\left.\mathrm{CDCl}_{3}\right)$

\section{6-Chloro-2,4-diphenylquinoline (3bu)}

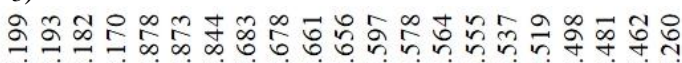

os os os os
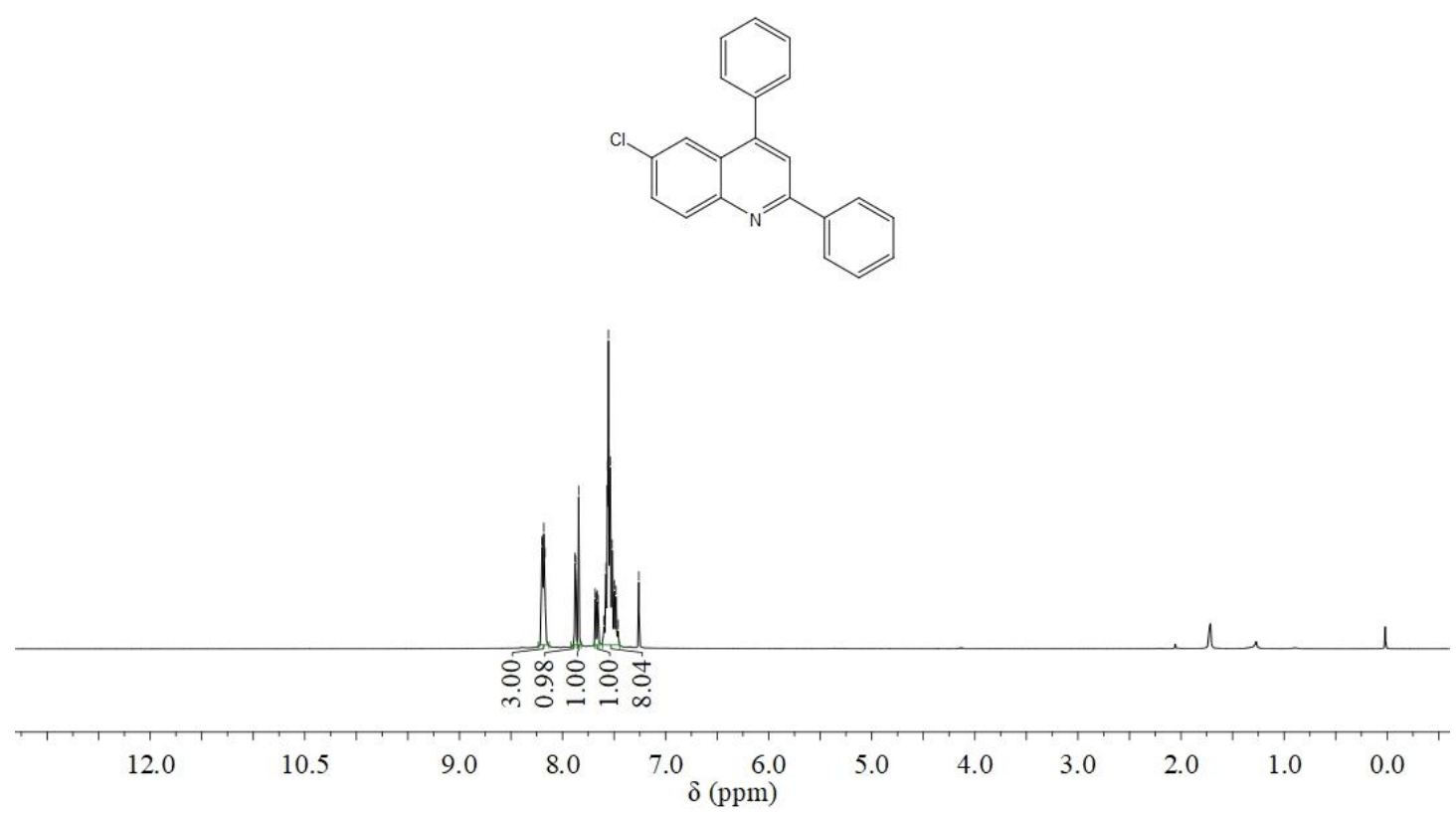

${ }^{13} \mathrm{C}$ NMR $\left(100 \mathrm{MHz}, \mathrm{CDCl}_{3}\right)$

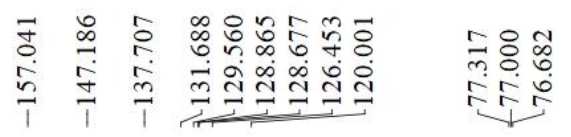
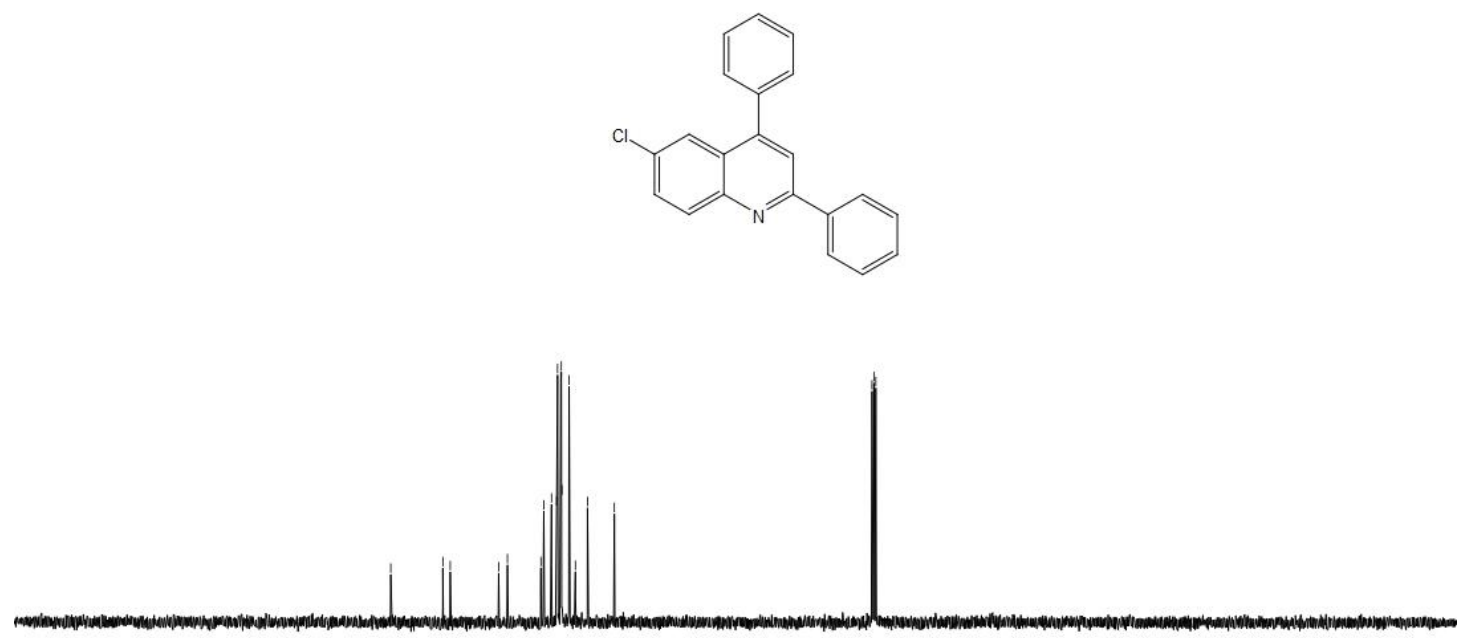

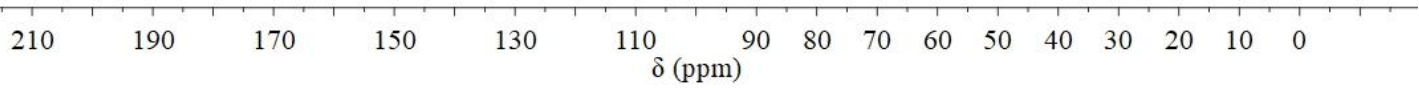


${ }^{1} \mathrm{H} \mathrm{NMR}\left(400 \mathrm{MHz}, \mathrm{CDCl}_{3}\right)$

1-Methyl-2-phenylquinoline (3bv)

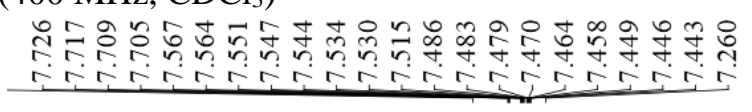

$\underset{\substack{0 \\ i}}{\stackrel{1}{i}}$

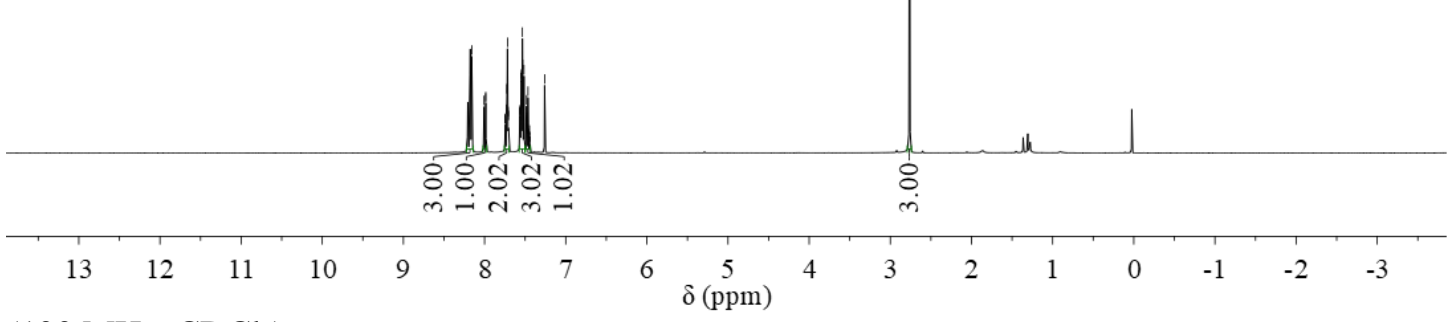

${ }^{13} \mathrm{C} \mathrm{NMR}\left(100 \mathrm{MHz}, \mathrm{CDCl}_{3}\right)$

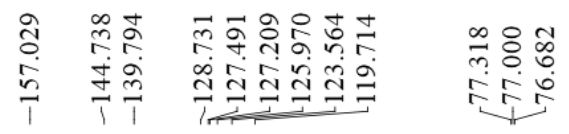

6
$\circ$
$\infty$
1
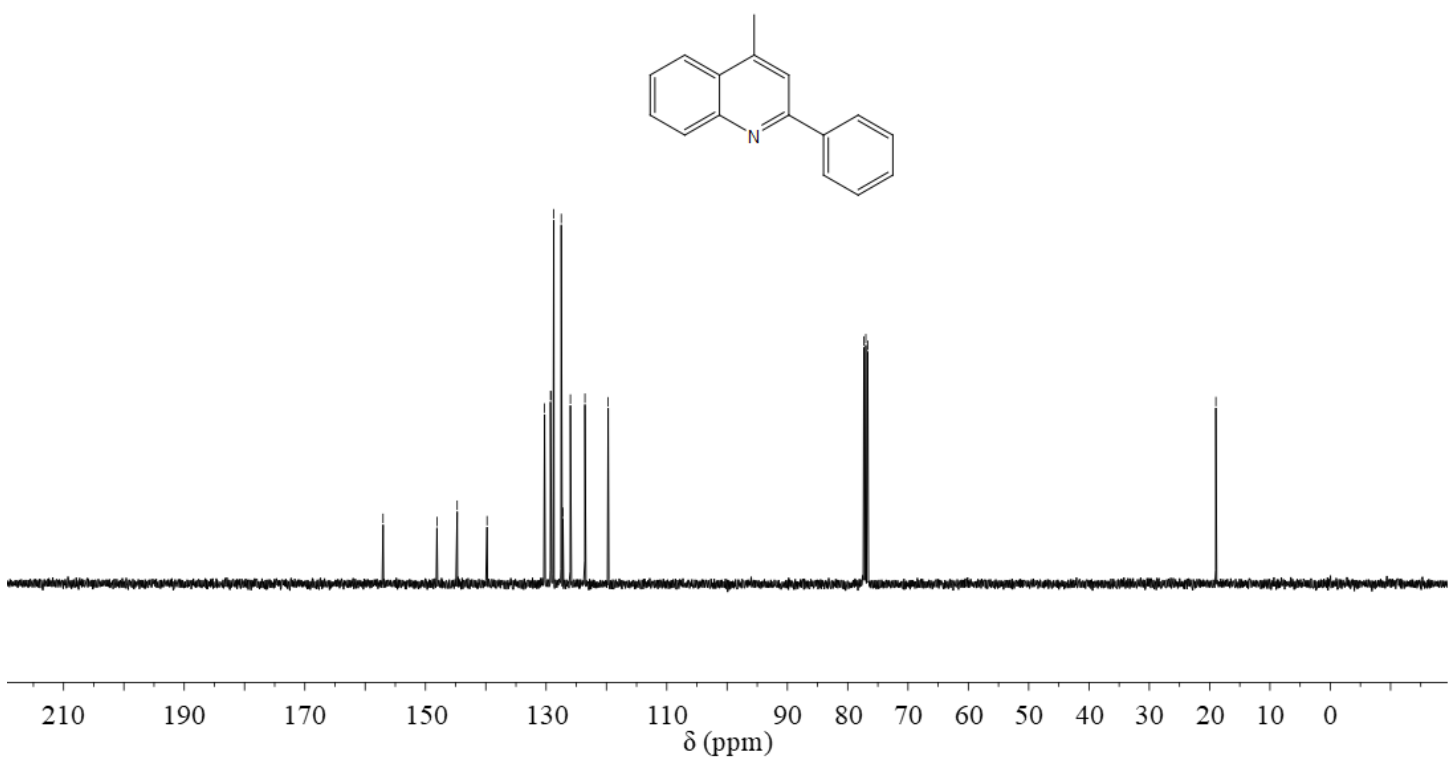

122 
13-Methyl-3-(quinolin-2-yl)-6,7,8,9,11,12,13,14,15,16-decahydro-17H-cyclopenta[a]phenanthren-17-one (3bw) ${ }^{1} \mathrm{H}$ NMR $\left(400 \mathrm{MHz}, \mathrm{CDCl}_{3}\right)$
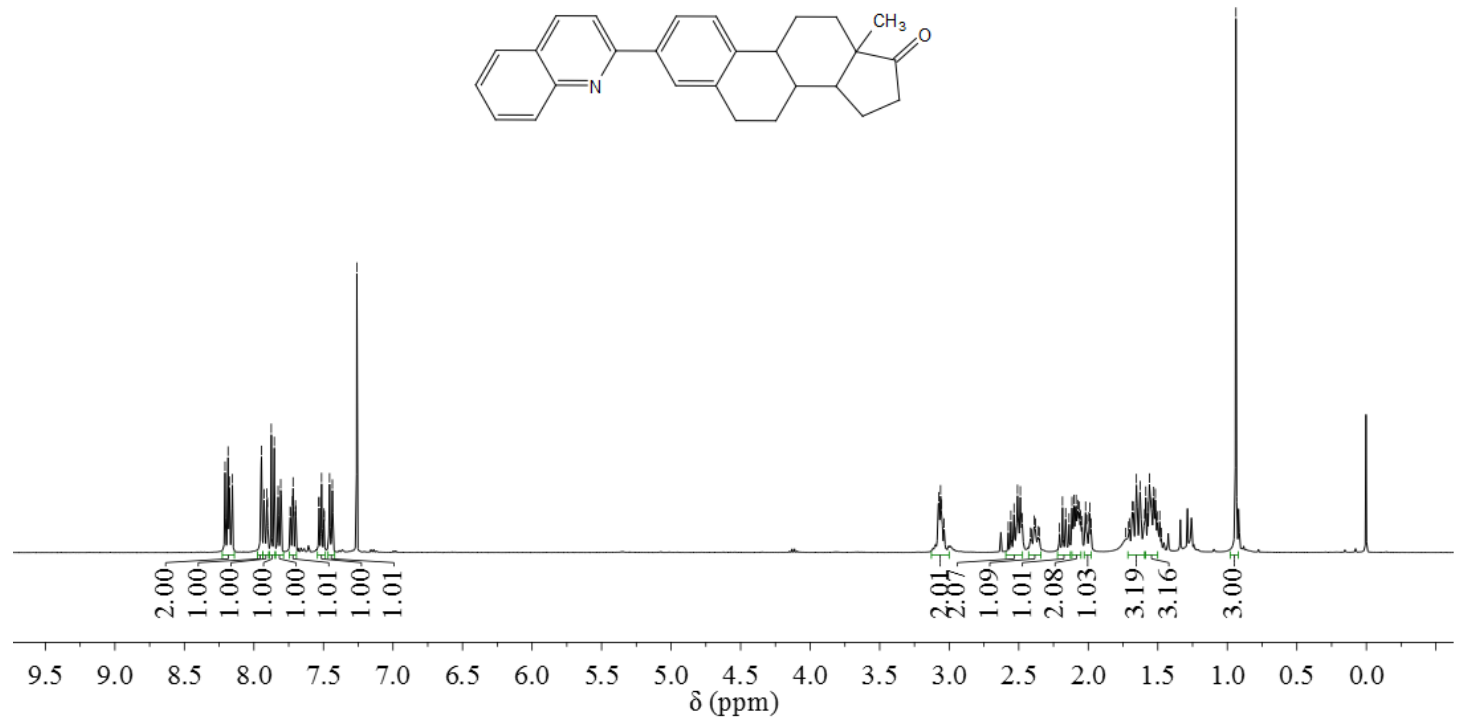

${ }^{13} \mathrm{C} \mathrm{NMR}\left(100 \mathrm{MHz}, \mathrm{CDCl}_{3}\right)$

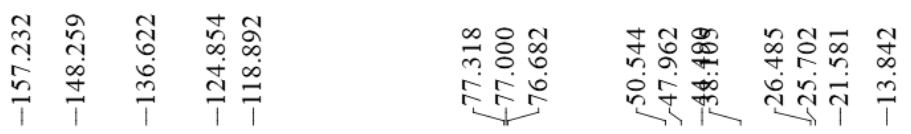
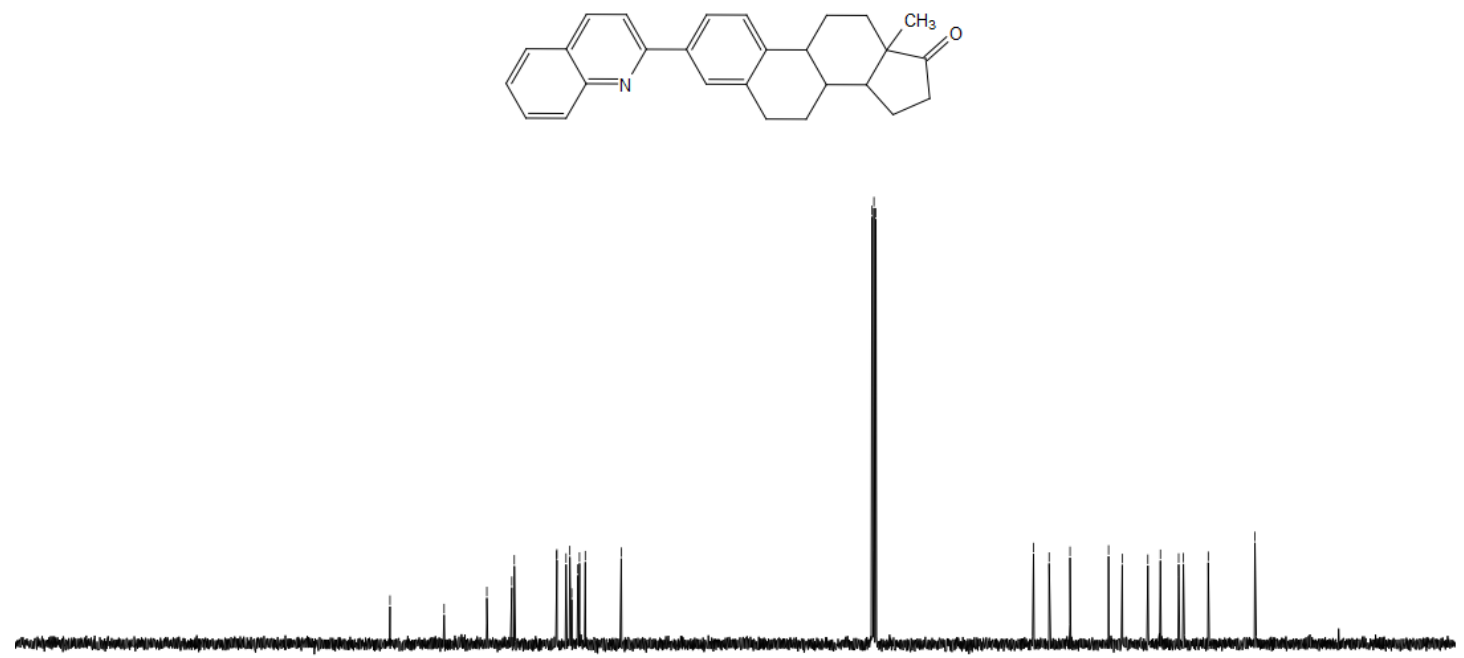

$\begin{array}{llllllllllllllll}210 & 190 & 170 & 150 & 130 & 110 \underset{\delta(\mathrm{ppm})}{90} & 80 & 70 & 60 & 50 & 40 & 30 & 20 & 10 & 0\end{array}$


2-Phenyl-3-(((2,5,7,8-tetramethyl-2-(4,8,12-trimethyltridecyl)chroman-6-yl)oxy)methyl)quinoline (3bx) ${ }^{1} \mathrm{H}$ NMR $\left(400 \mathrm{MHz}, \mathrm{CDCl}_{3}\right)$
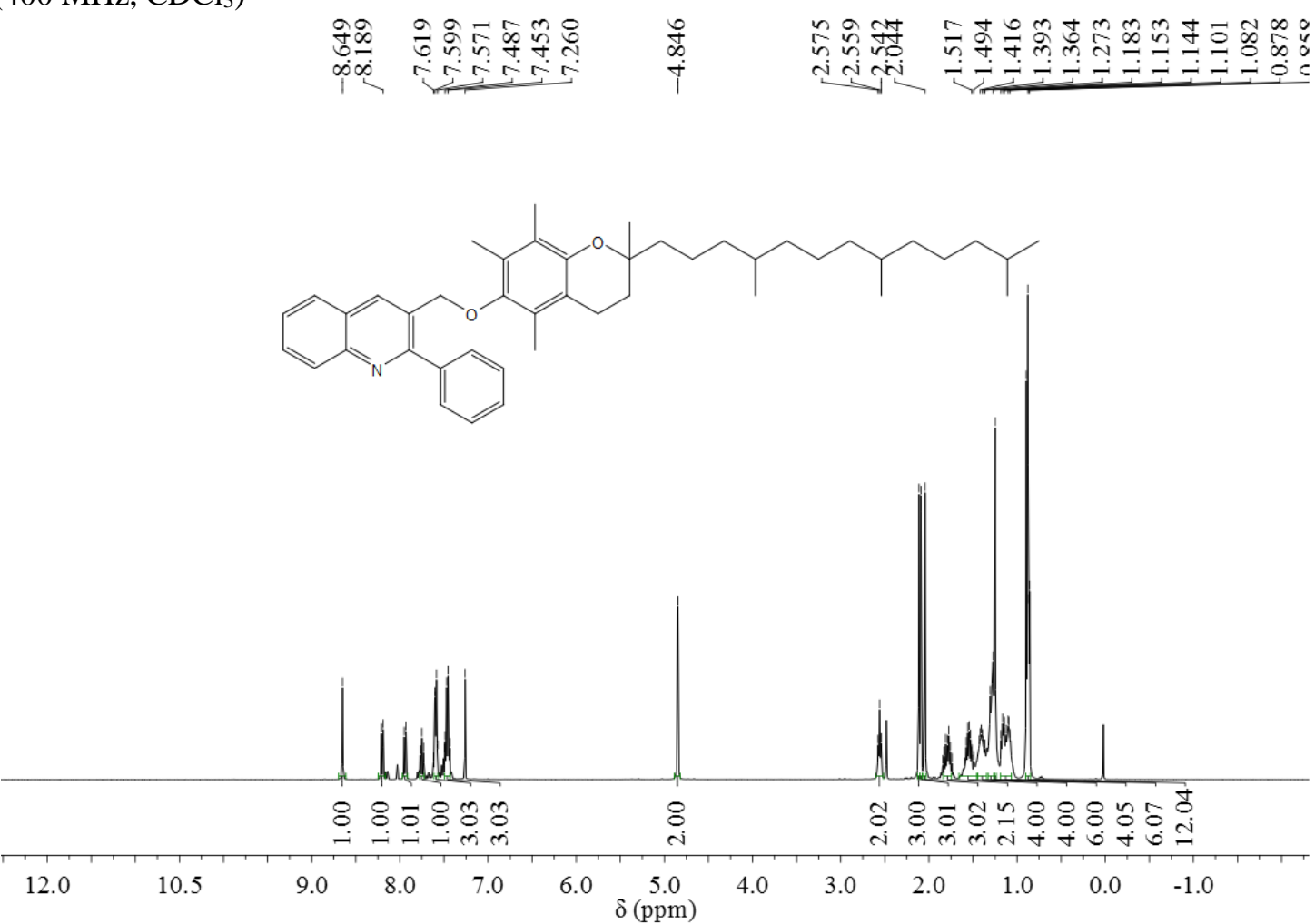

${ }^{13} \mathrm{C}$ NMR $\left(100 \mathrm{MHz}, \mathrm{CDCl}_{3}\right)$

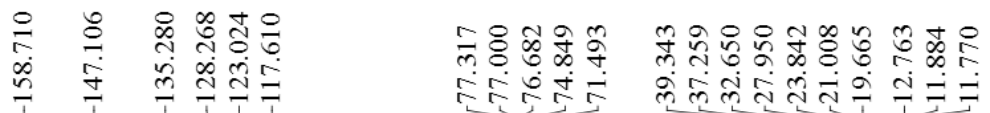
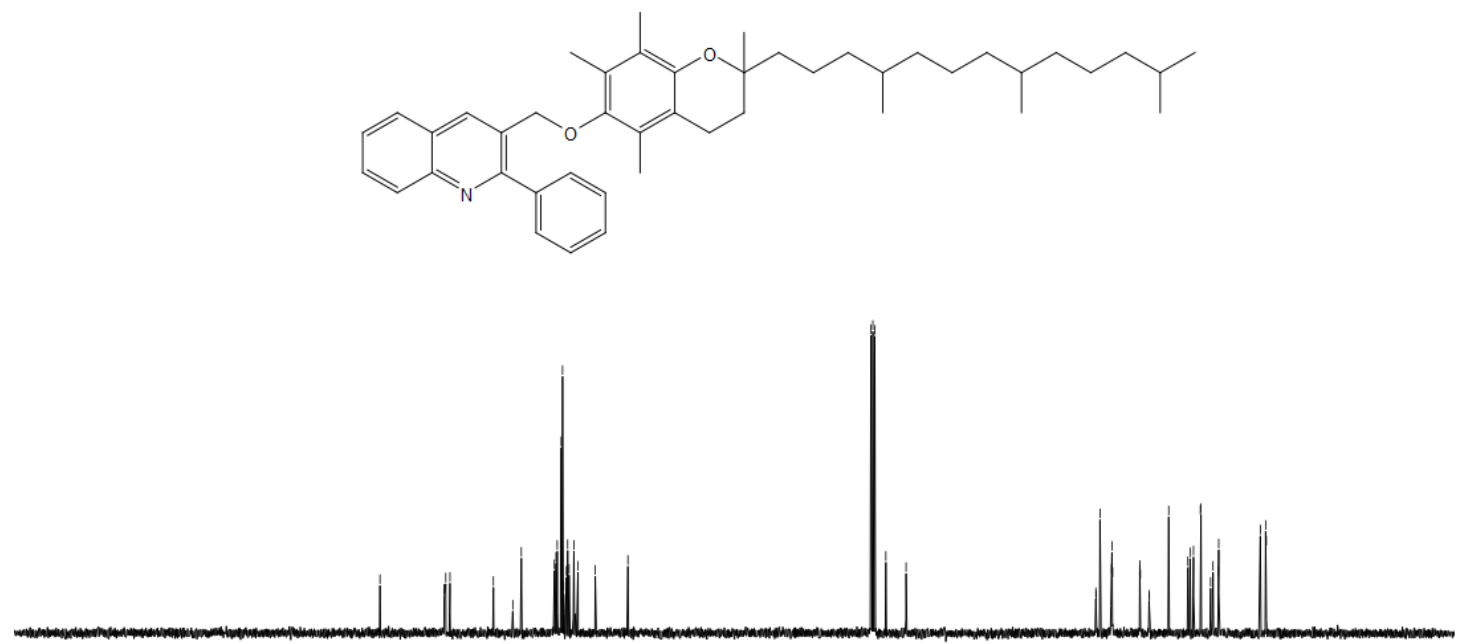

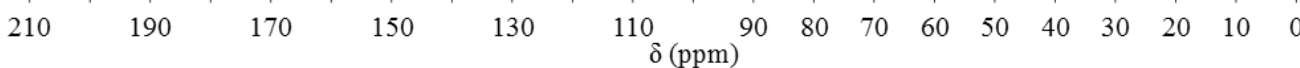


(E)-3,7-Dimethylocta-2,6-dien-1-yl 2-phenylquinoline-3-carboxylate (3by)

${ }^{1} \mathrm{H}$ NMR $\left(400 \mathrm{MHz}, \mathrm{CDCl}_{3}\right)$

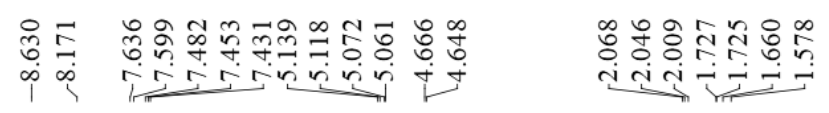
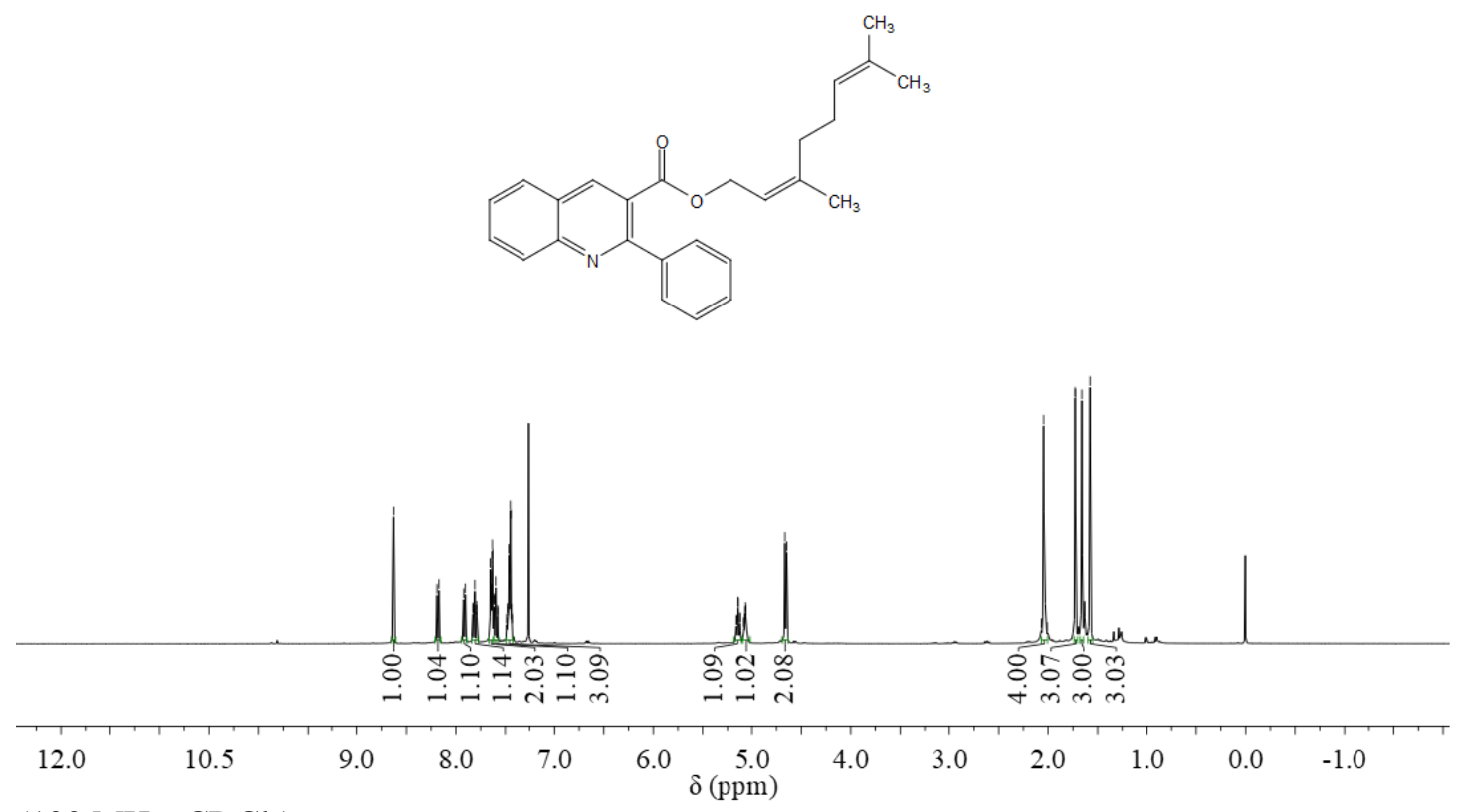

${ }^{13} \mathrm{C}$ NMR $\left(100 \mathrm{MHz}, \mathrm{CDCl}_{3}\right)$

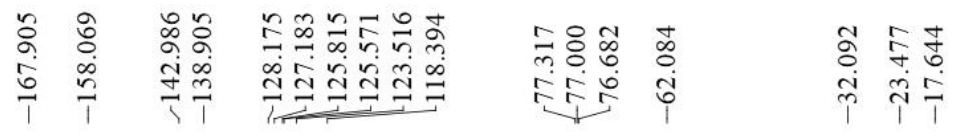<smiles>CC(O)=CCCC=C(C)CCOC(=O)c1cc2ccccc2nc1-c1ccccc1</smiles>

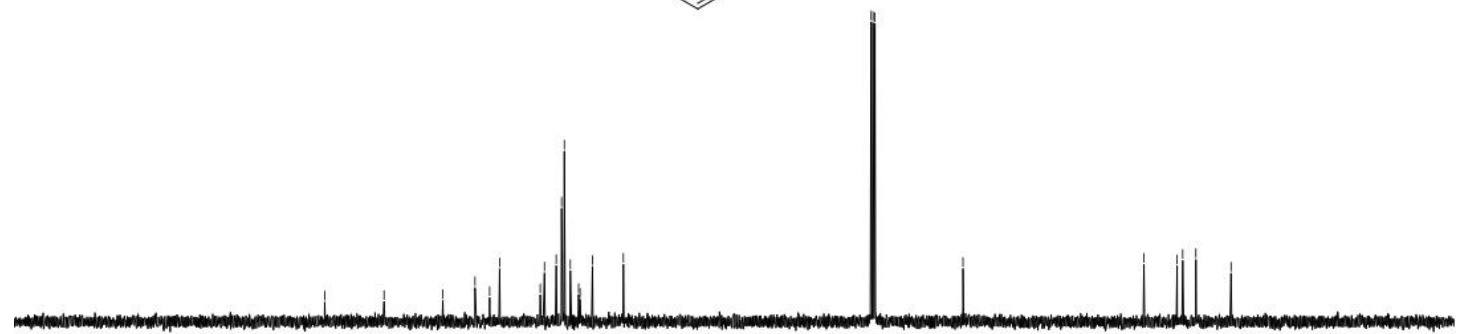

$\begin{array}{llllllllllllllll}210 & 190 & 170 & 150 & 130 & 110 \quad \begin{array}{c}\delta(\mathrm{ppm}) \\ 90\end{array} & 80 & 70 & 60 & 50 & 40 & 30 & 20 & 10 & 0\end{array}$ 
(1R,2S,5R)-2-Isopropyl-5-methylcyclohexyl 2-phenylquinoline-3-carboxylat (3bz)

${ }^{1} \mathrm{H} \mathrm{NMR}\left(400 \mathrm{MHz}, \mathrm{CDCl}_{3}\right)$

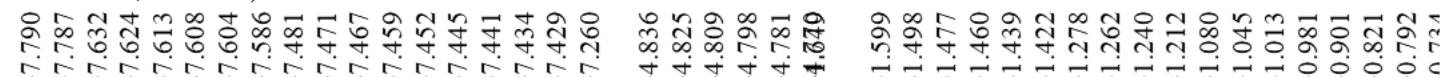
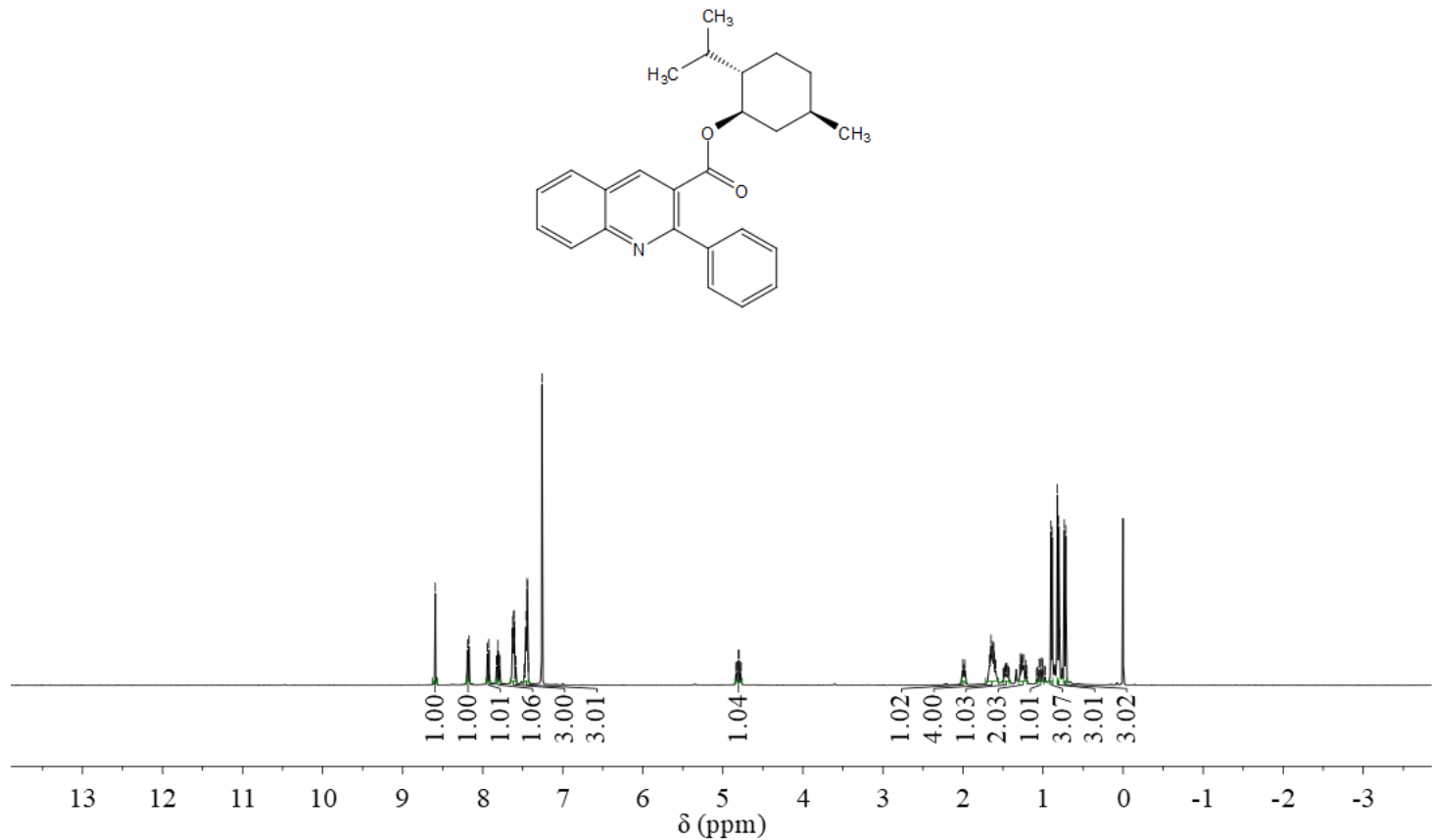

${ }^{13} \mathrm{C}$ NMR $\left(100 \mathrm{MHz}, \mathrm{CDCl}_{3}\right)$

\begin{tabular}{|c|c|c|}
\hline 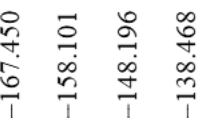 & 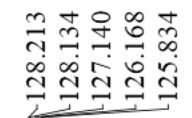 & 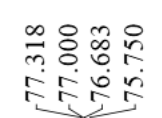 \\
\hline
\end{tabular}
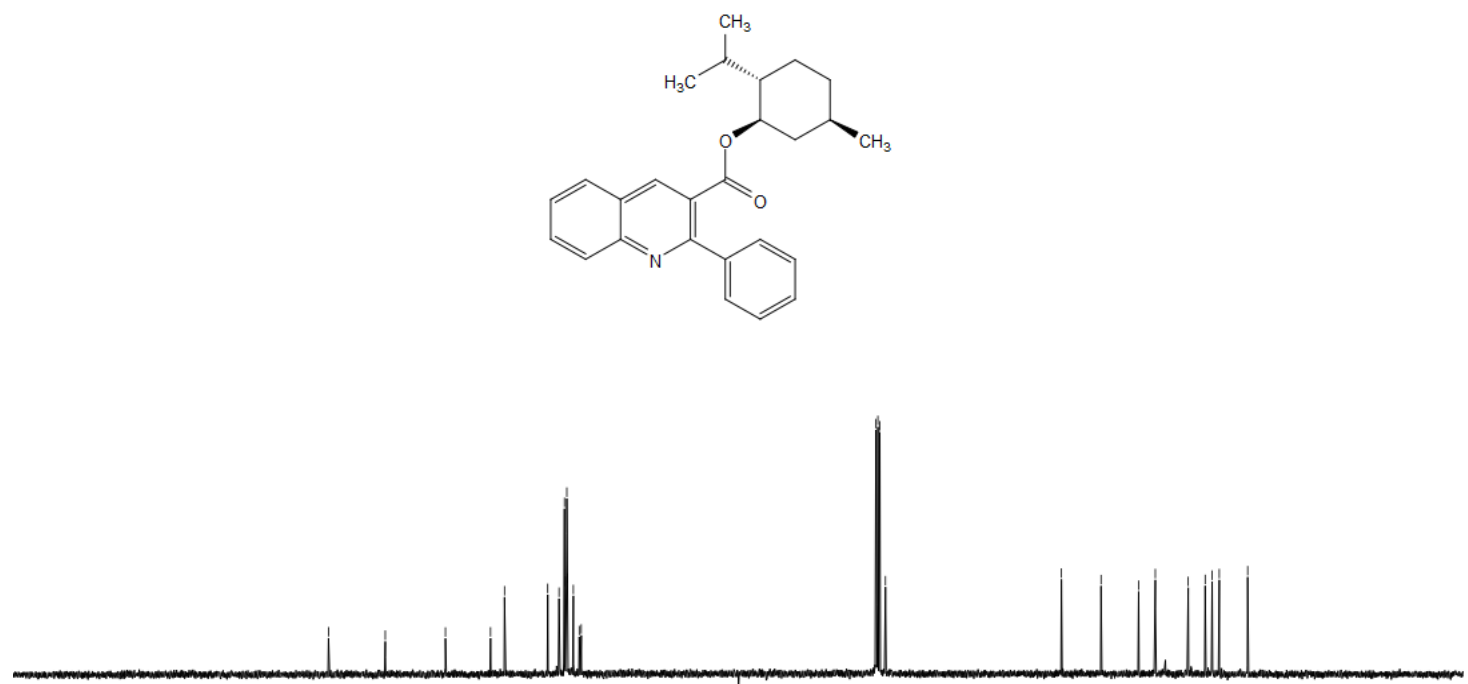

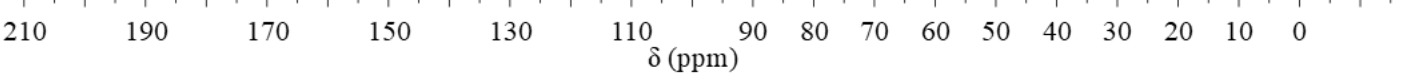


10,13-Dimethyl-17-(6-methylheptan-2-yl)-2,3,4,7,8,9,10,11,12,13,14,15,16,17-tetradecahydro-1Hcyclopenta[a]phenanthren-3-yl 2-phenylquinoline-3-carboxylate (3ca)

${ }^{1} \mathrm{H}$ NMR $\left(400 \mathrm{MHz}, \mathrm{CDCl}_{3}\right)$
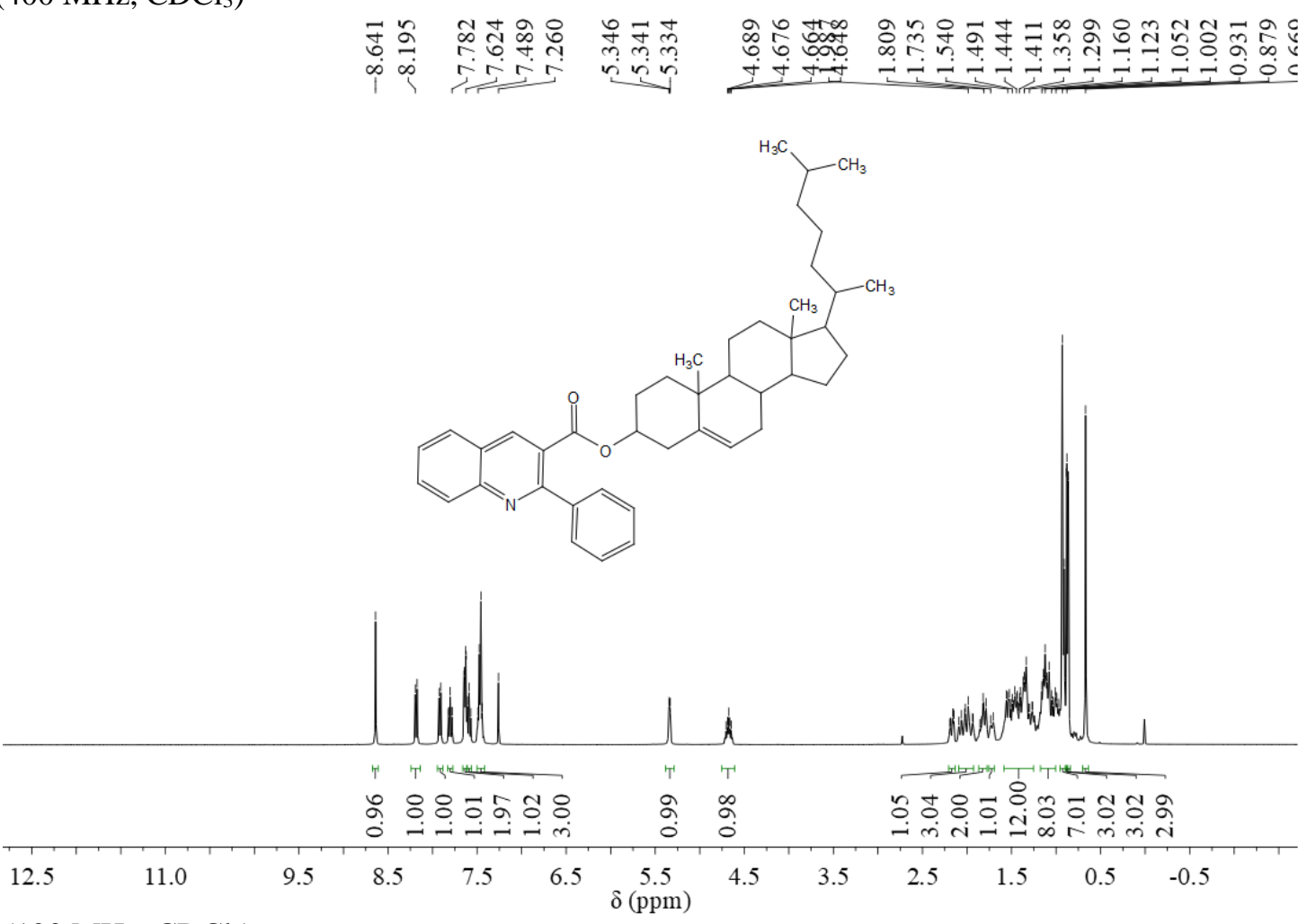

${ }^{13} \mathrm{C}$ NMR (100 MHz, $\left.\mathrm{CDCl}_{3}\right)$
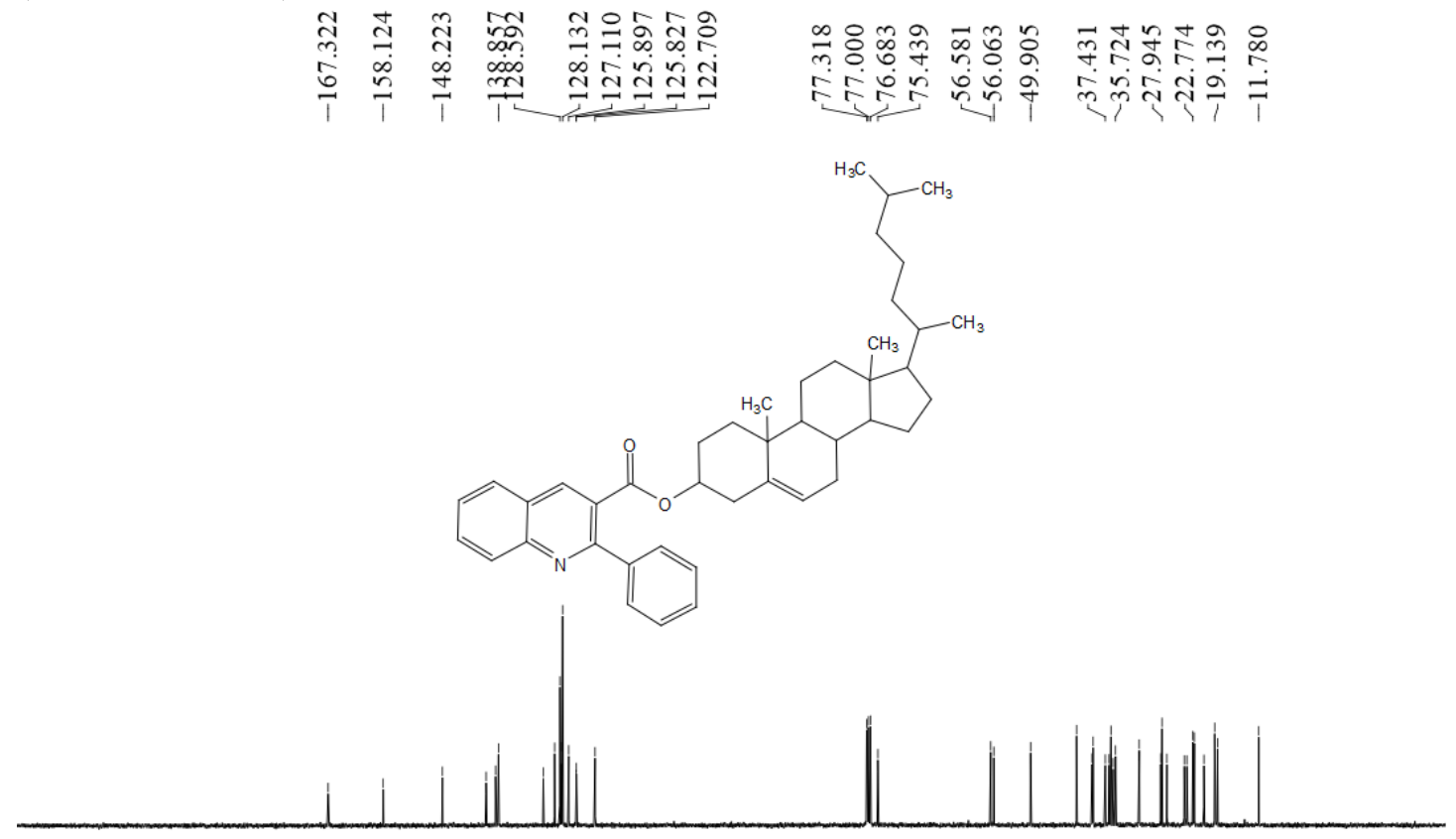

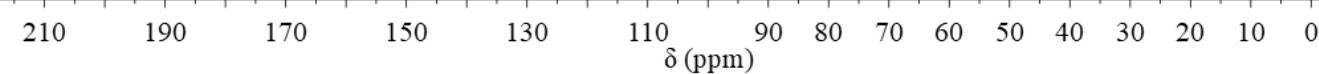


4-(Quinolin-2-yl)phenyl 2-(4-isobutylphenyl)propanoate (3cb)

${ }^{1} \mathrm{H}$ NMR (400 MHz, $\left.\mathrm{CDCl}_{3}\right)$

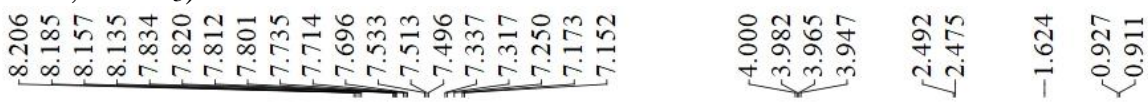
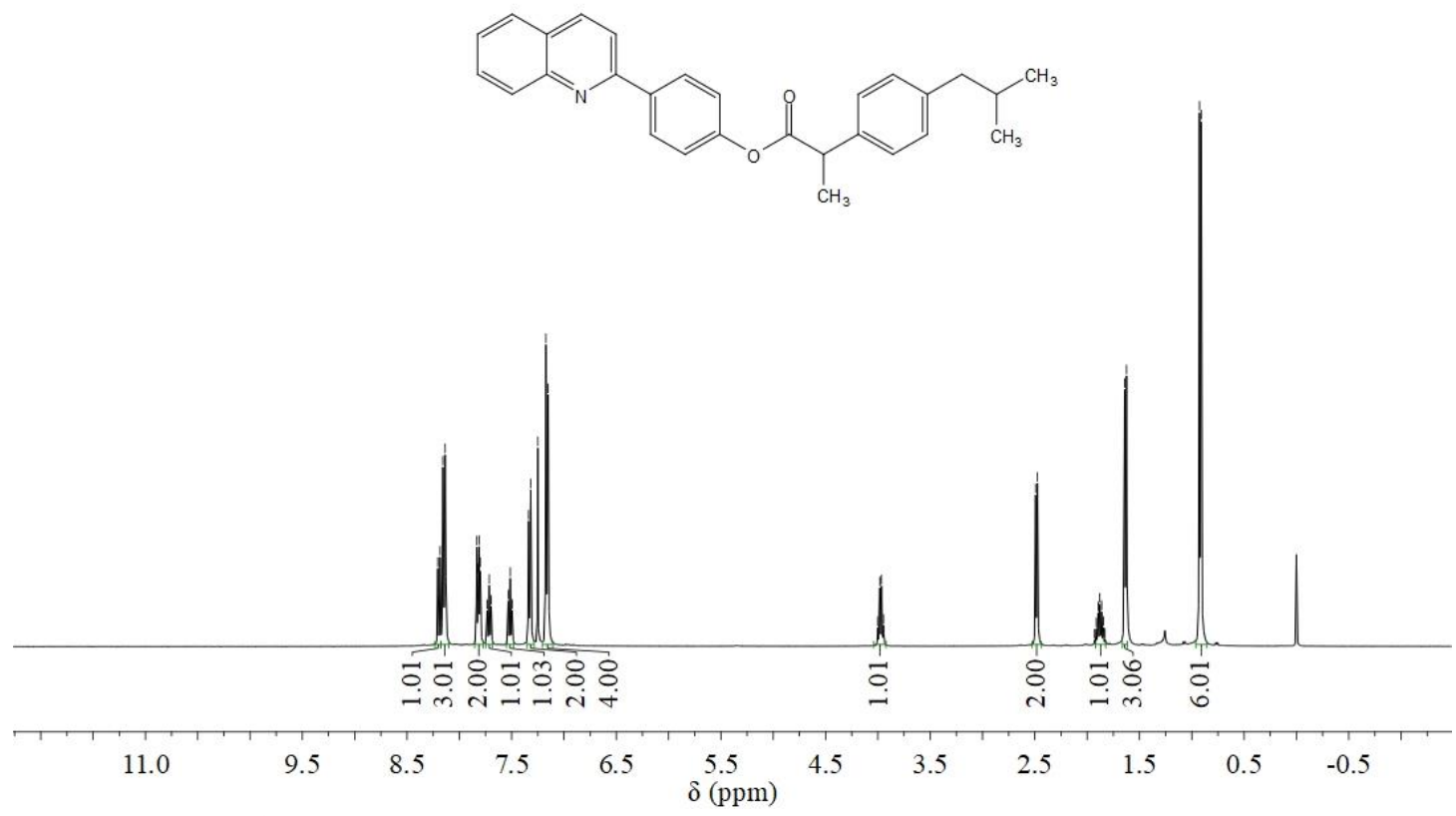

${ }^{13} \mathrm{C}$ NMR (100 MHz, $\left.\mathrm{CDCl}_{3}\right)$

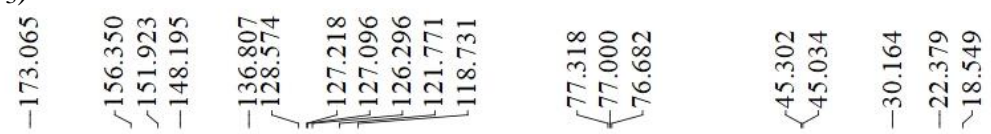
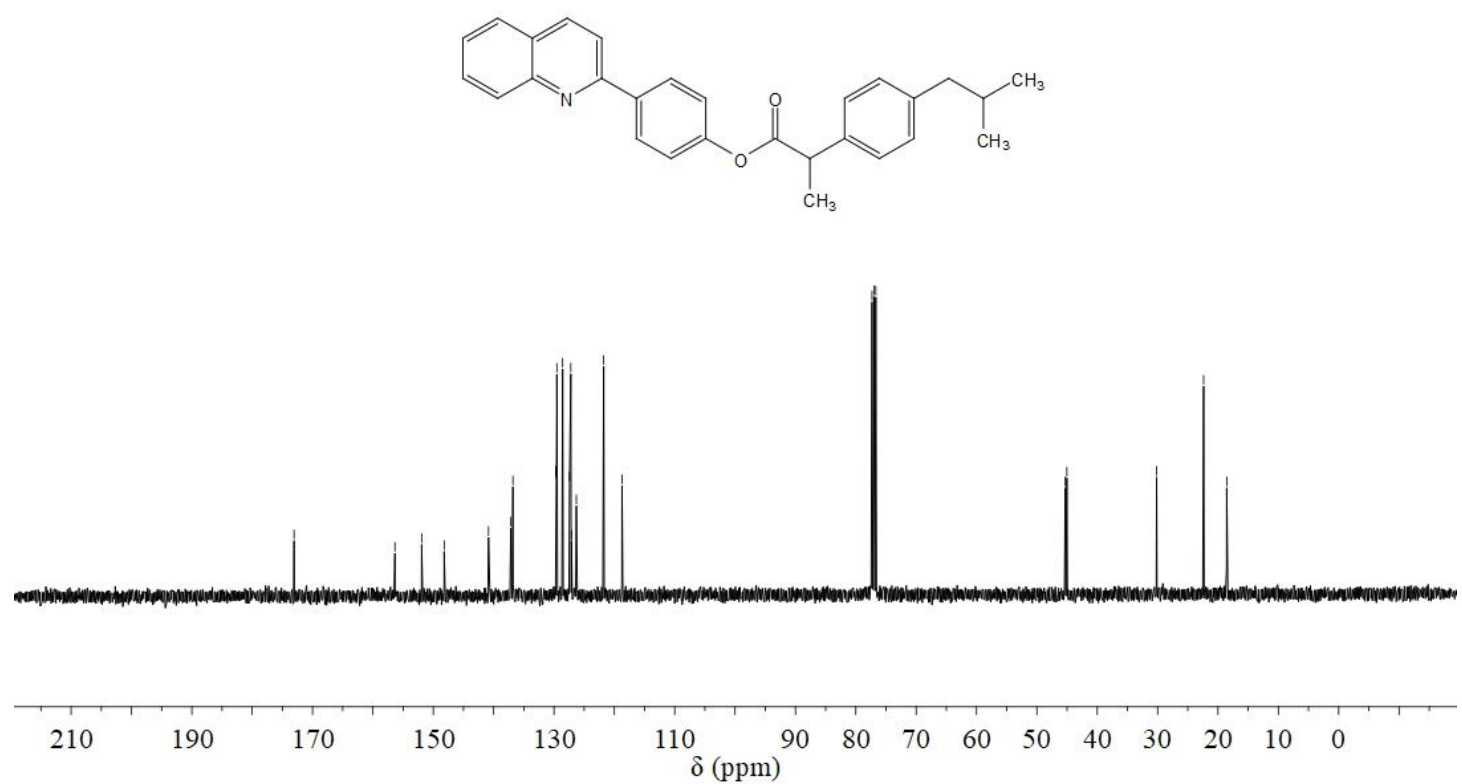
(2,2,7,7-tetra-Methyl-tetra-hydro-5H-bis([1,3]dioxolo)[4,5-b:4',5'-d]pyran-5-yl)methyl 4-(3-butylquinolin-2yl)benzoate (3cc)

${ }^{1} \mathrm{H}$ NMR (400 MHz, $\mathrm{CDCl}_{3}$ )

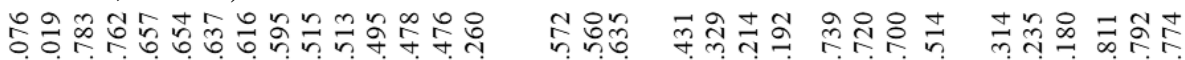

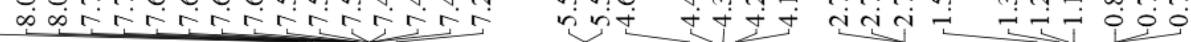
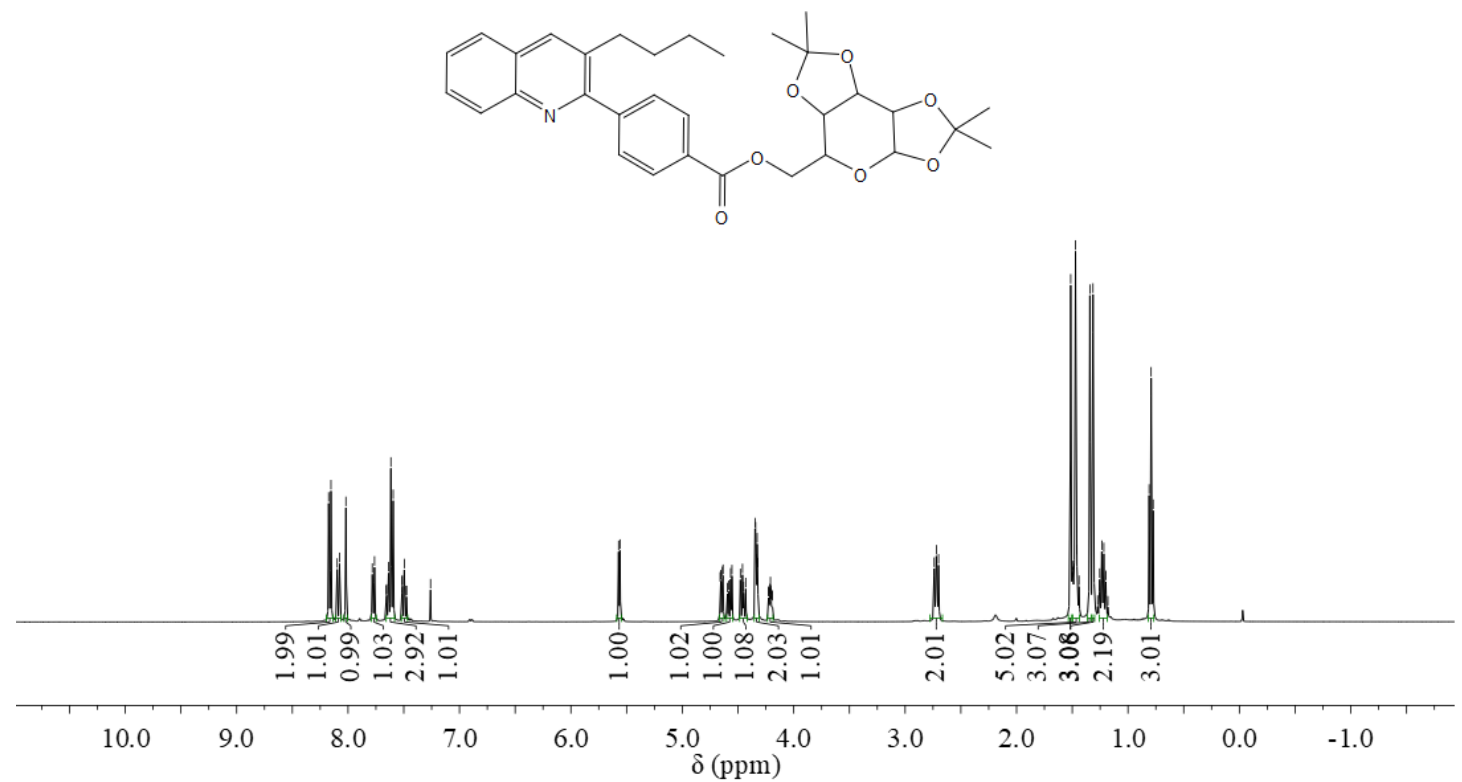

${ }^{13} \mathrm{C}$ NMR $\left(100 \mathrm{MHz}, \mathrm{CDCl}_{3}\right)$

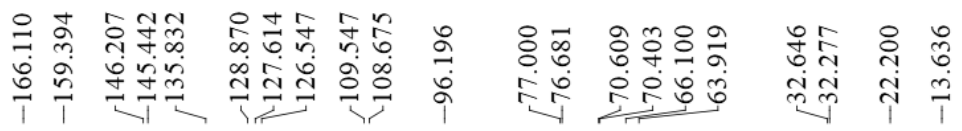
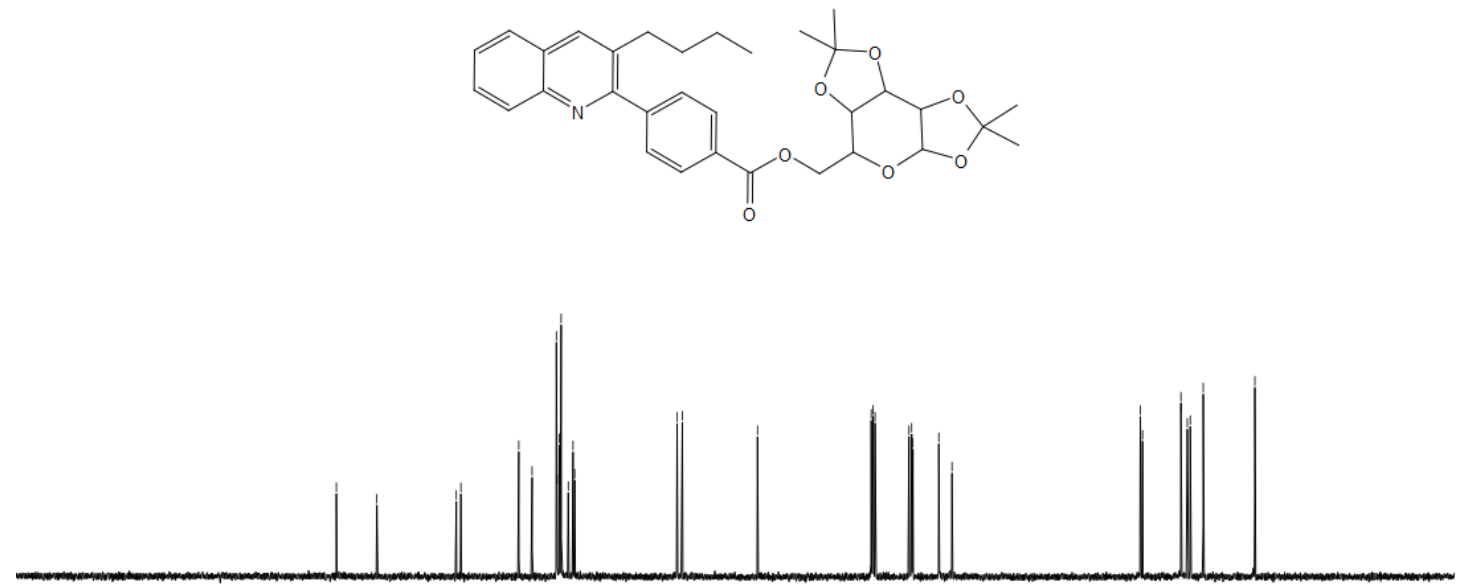

$\begin{array}{llllllllllllllll}210 & 190 & 170 & 150 & 130 & 110 \underset{\delta(\mathrm{ppm})}{90} & 80 & 70 & 60 & 50 & 40 & 30 & 20 & 10 & 0\end{array}$


${ }^{1} \mathrm{H} \mathrm{NMR}\left(400 \mathrm{MHz}, \mathrm{CDCl}_{3}\right)$

2-(Quinolin-2-yl)-9H-fluoren-9-one (3cd)

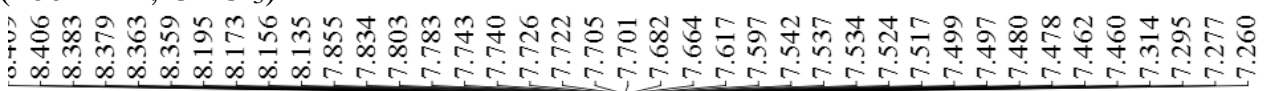
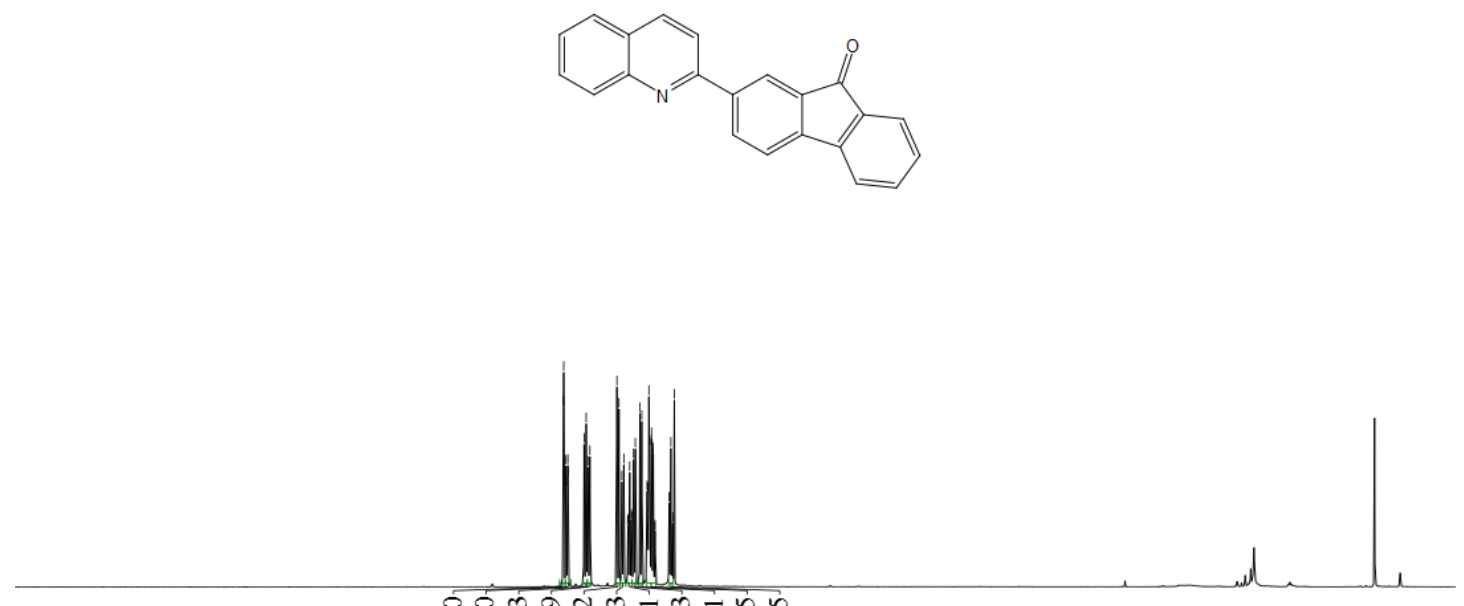

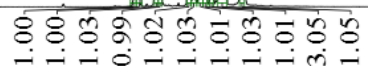

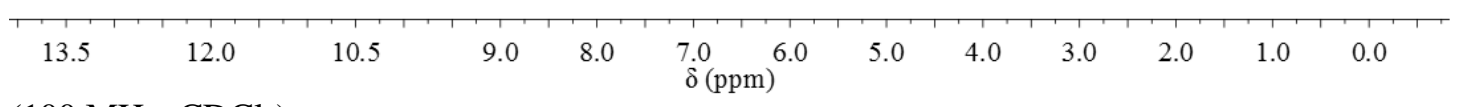

${ }^{13} \mathrm{C}$ NMR $\left(100 \mathrm{MHz}, \mathrm{CDCl}_{3}\right)$
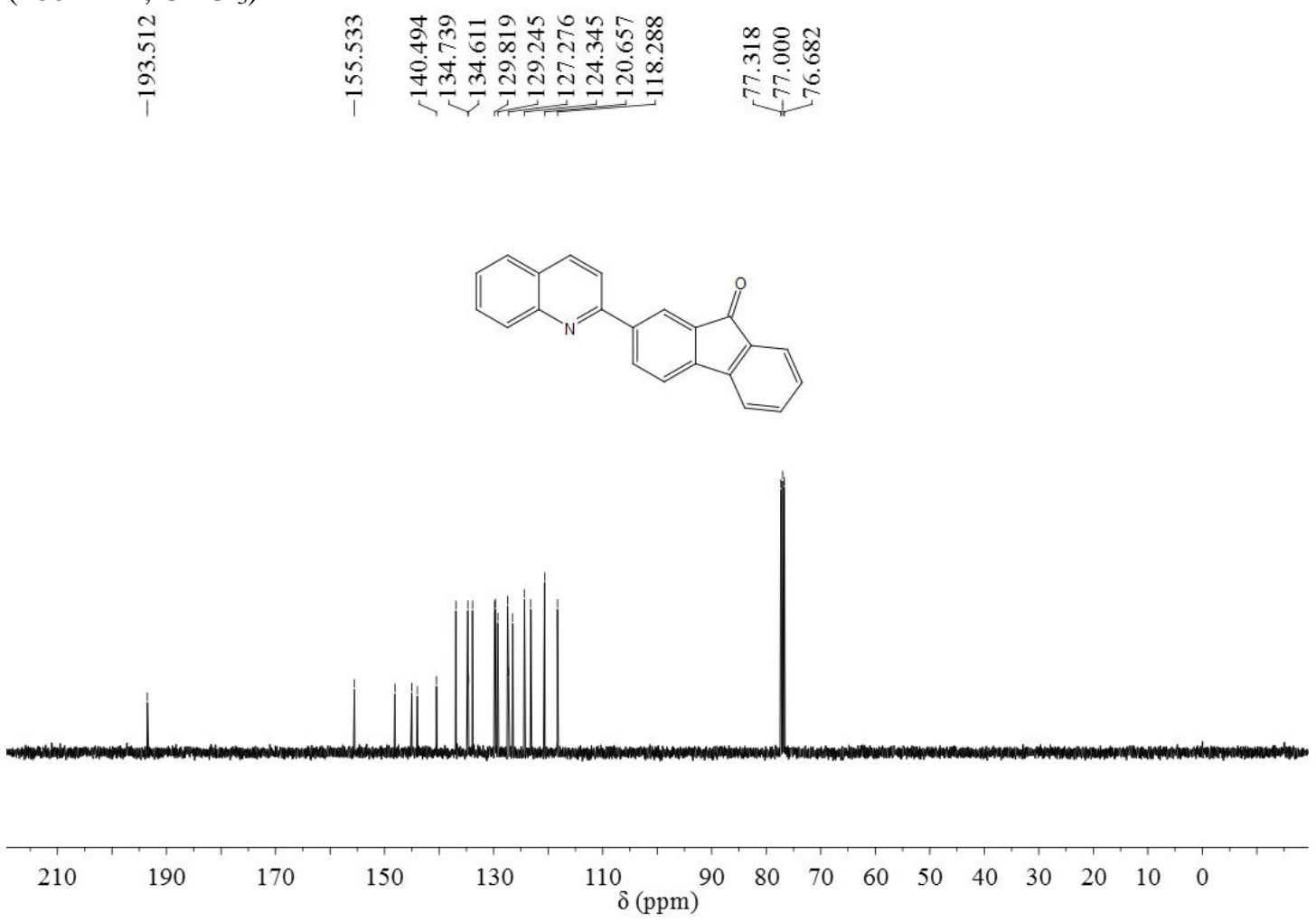

130 
${ }^{1} \mathrm{H}$ NMR (400 MHz, $\left.\mathrm{CDCl}_{3}\right)$
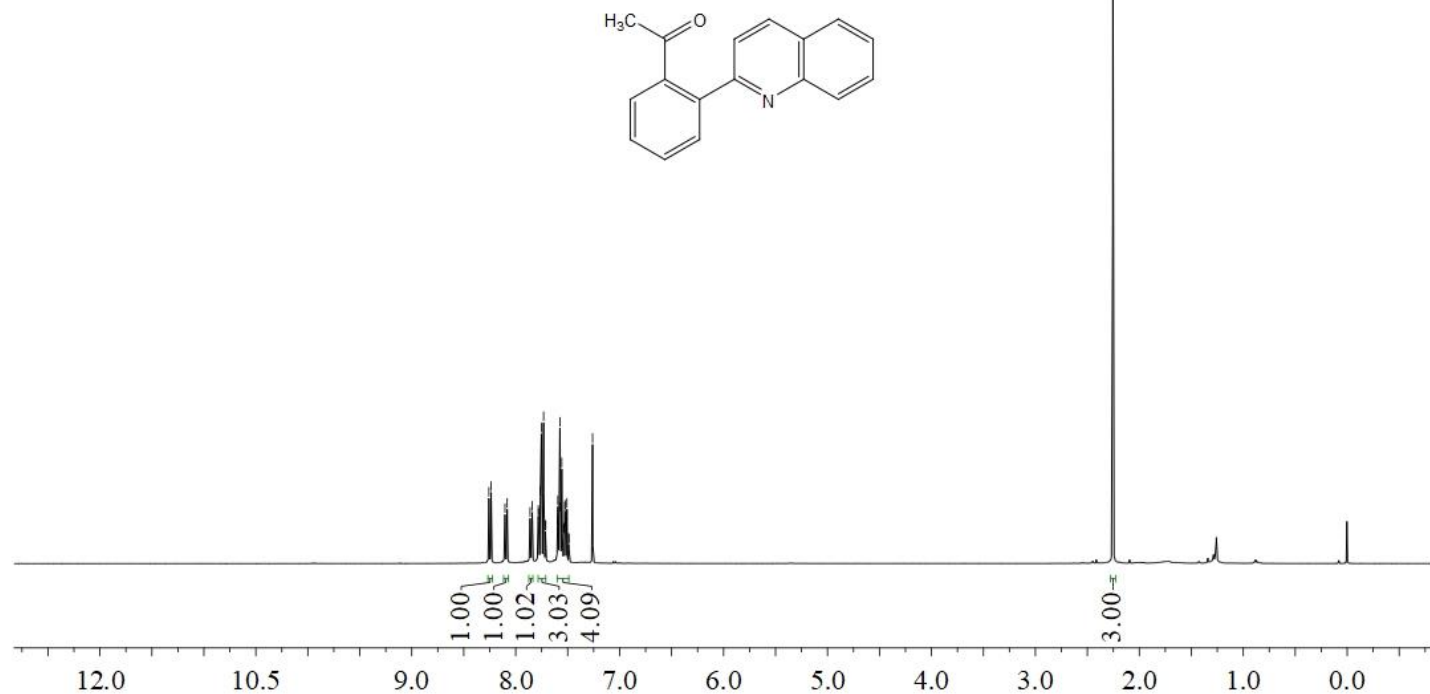

${ }^{13} \mathrm{C} \mathrm{NMR}\left(100 \mathrm{MHz}, \mathrm{CDCl}_{3}\right)$

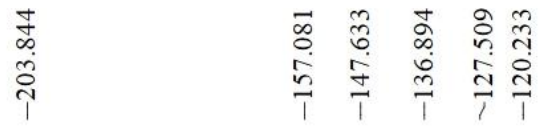
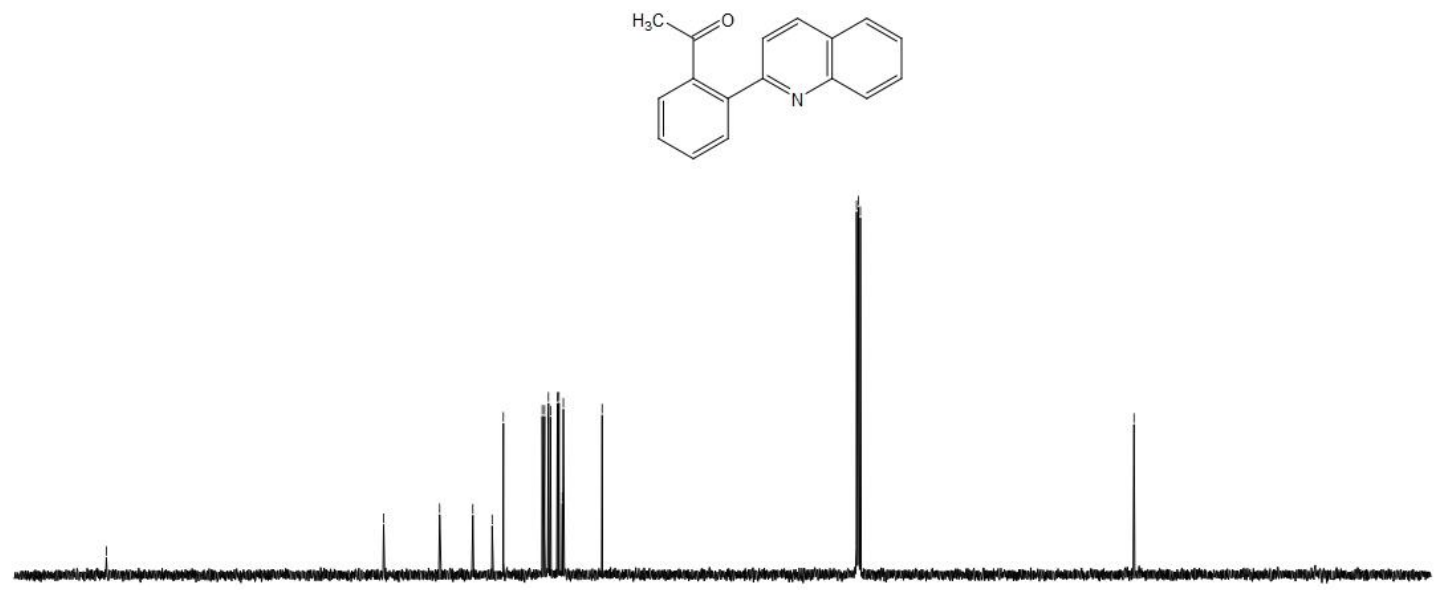

210

190

170

150

130

110

$\begin{array}{llllllllll}90 & 80 & 70 & 60 & 50 & 40 & 30 & 20 & 10 & 0\end{array}$ 
${ }^{1} \mathrm{H}$ NMR (400 MHz, $\mathrm{CDCl}_{3}$ )

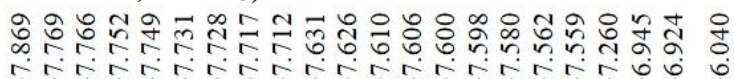

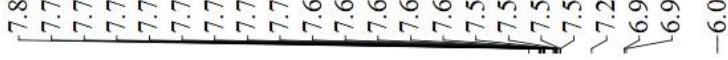

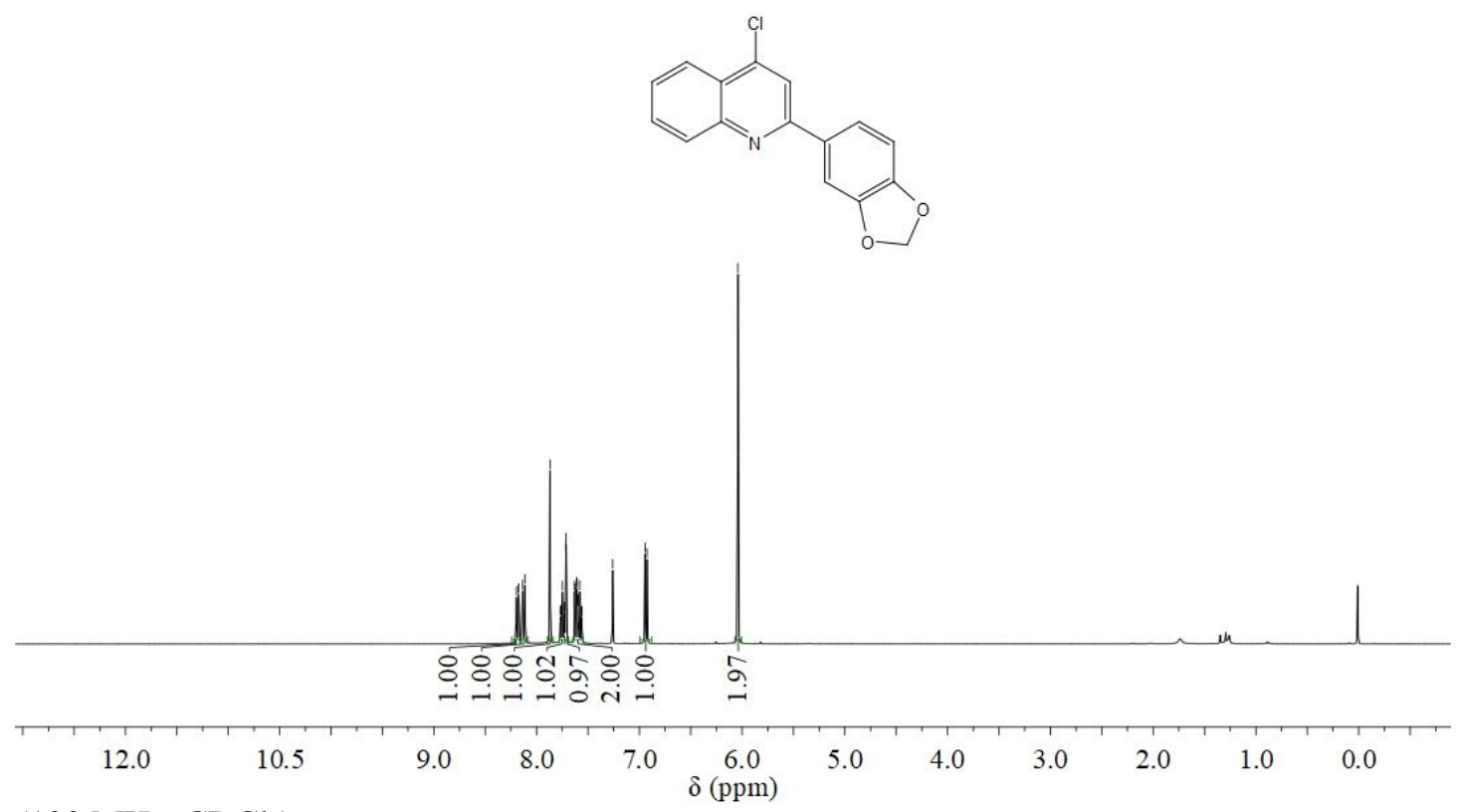

${ }^{13} \mathrm{C} \mathrm{NMR}\left(100 \mathrm{MHz}, \mathrm{CDCl}_{3}\right)$

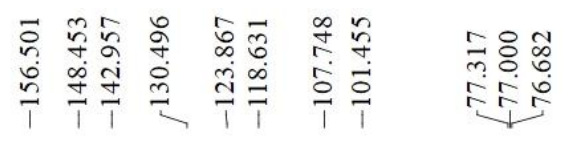
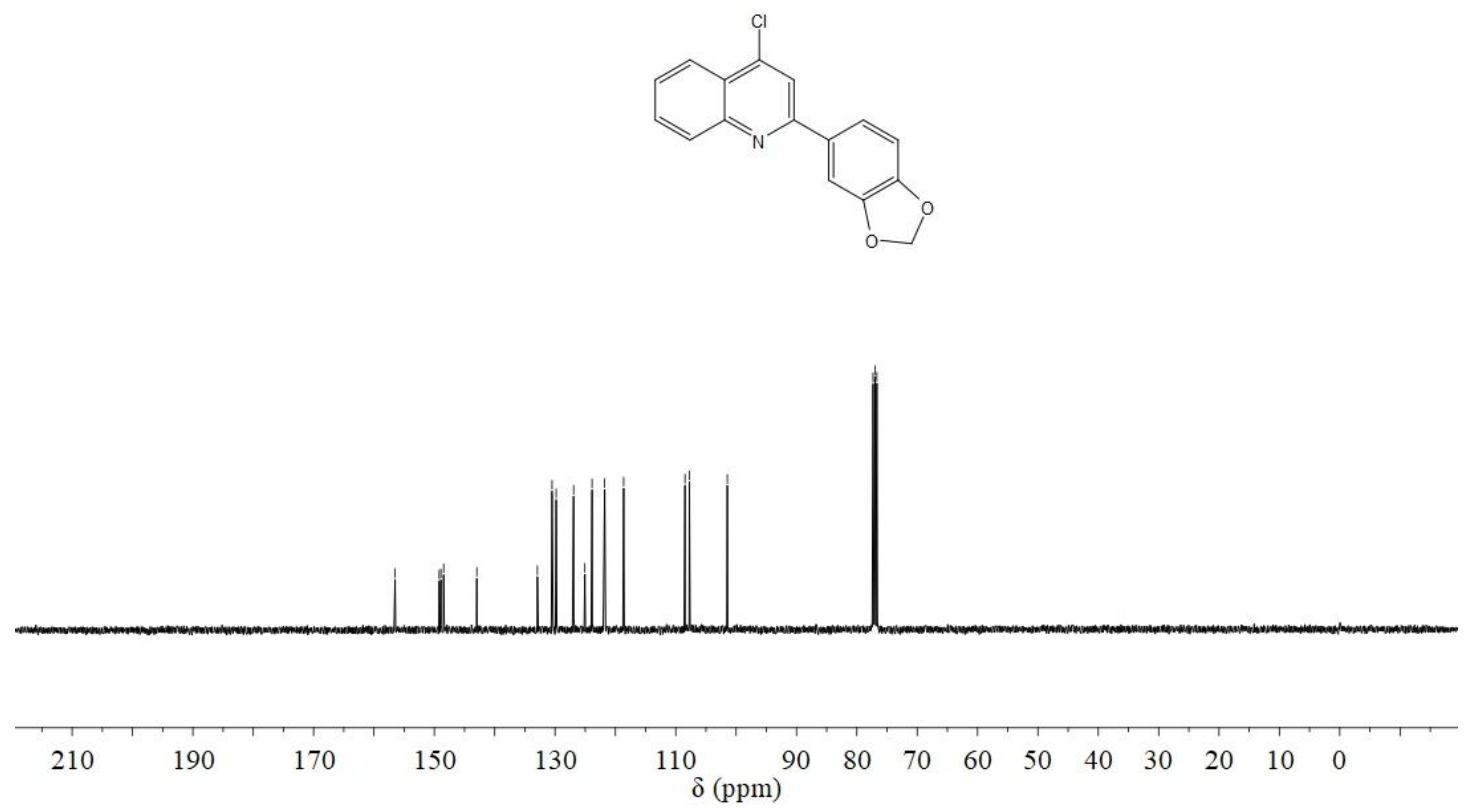


\section{2-(Benzo[d][1,3]dioxol-5-yl)-4-methoxyquinoline (3cg)}

${ }^{1} \mathrm{H}$ NMR $\left(400 \mathrm{MHz}, \mathrm{CDCl}_{3}\right)$

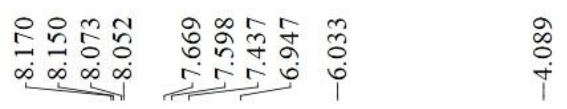

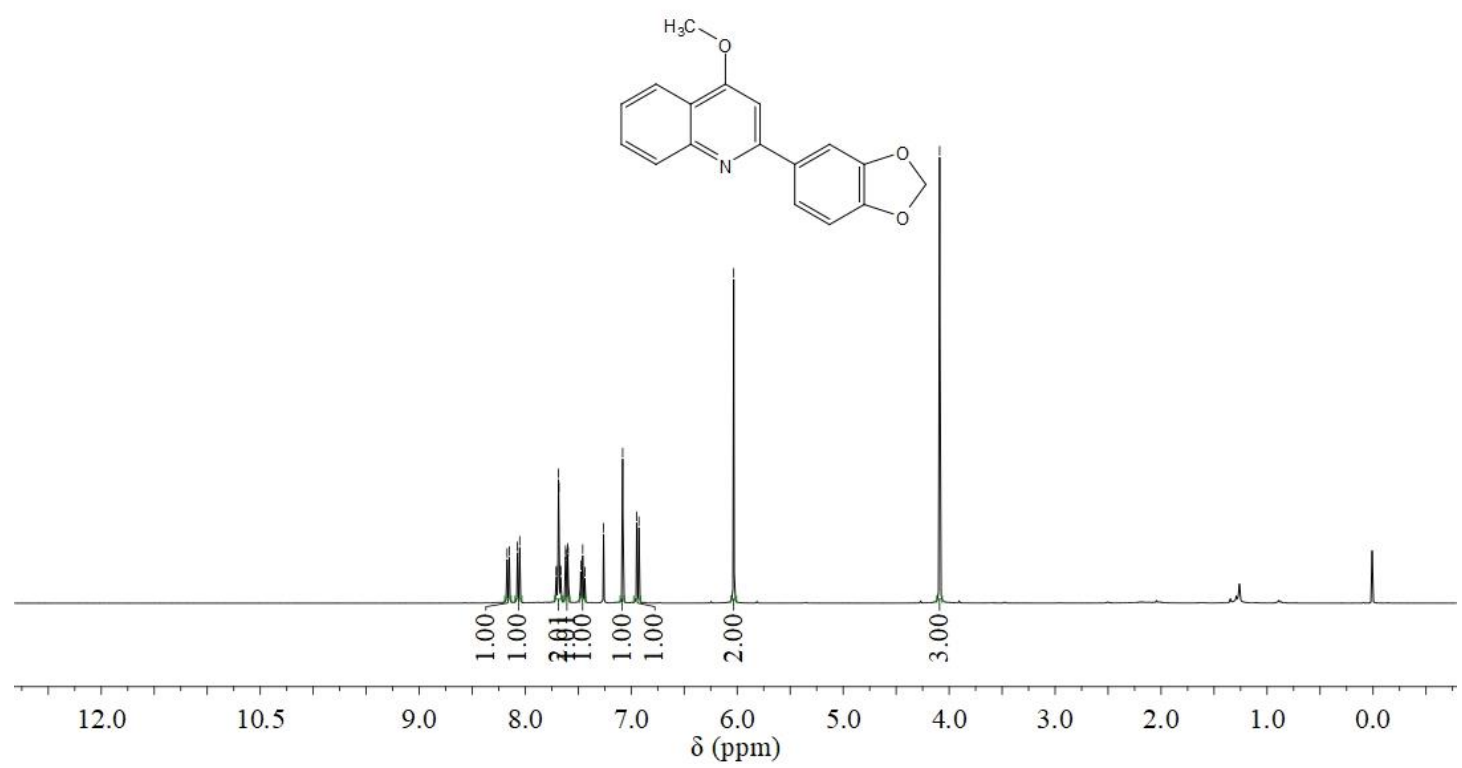

${ }^{13} \mathrm{C}$ NMR (100 MHz, $\left.\mathrm{CDCl}_{3}\right)$

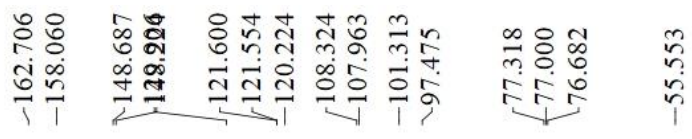
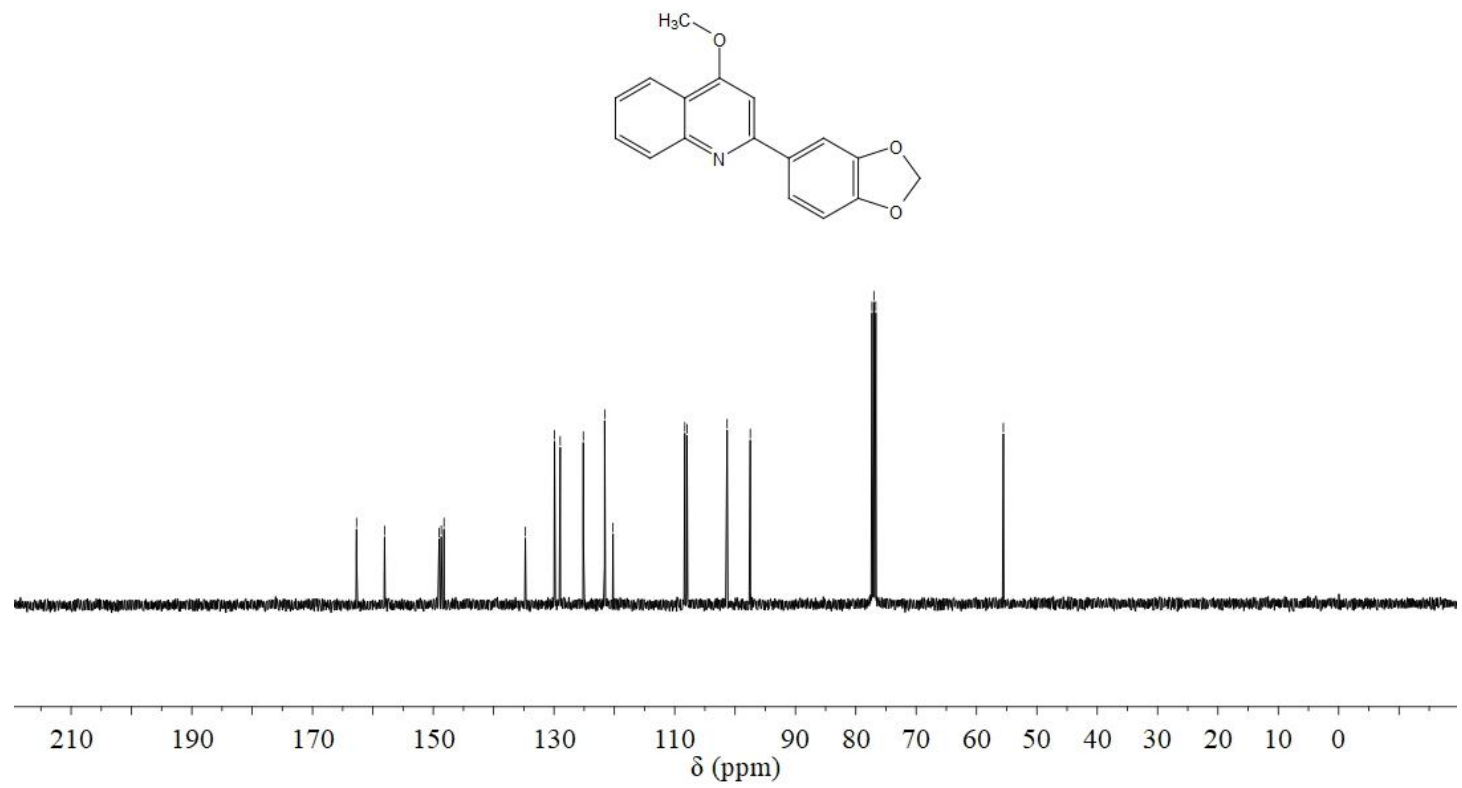
${ }^{1} \mathrm{H}$ NMR (400 MHz, $\left.\mathrm{CDCl}_{3}\right)$

4-Chloro-2-(naphthalen-2-yl)quinoline (3ch)

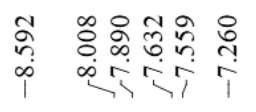
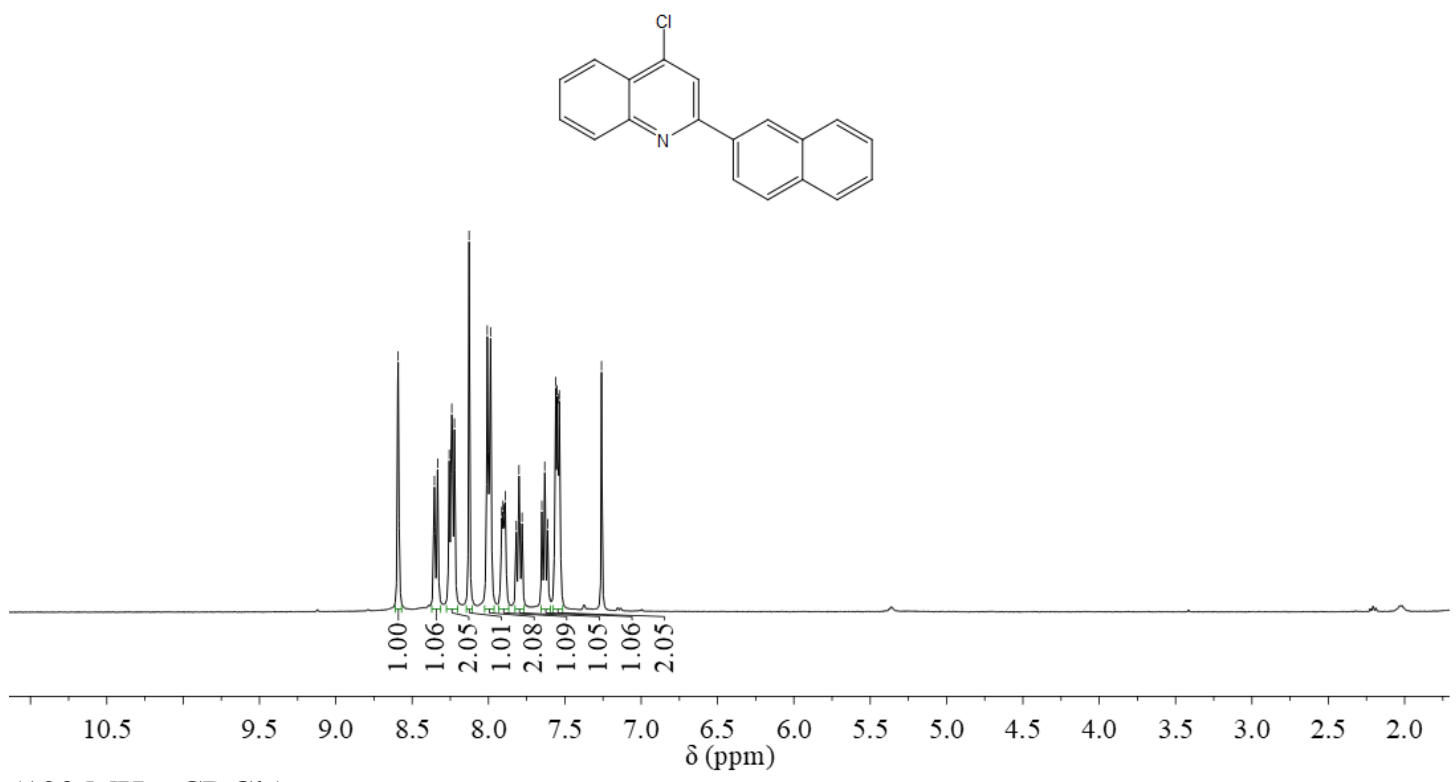

${ }^{13} \mathrm{C}$ NMR $\left(100 \mathrm{MHz}, \mathrm{CDCl}_{3}\right)$

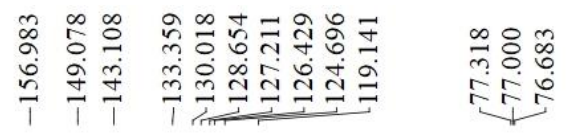
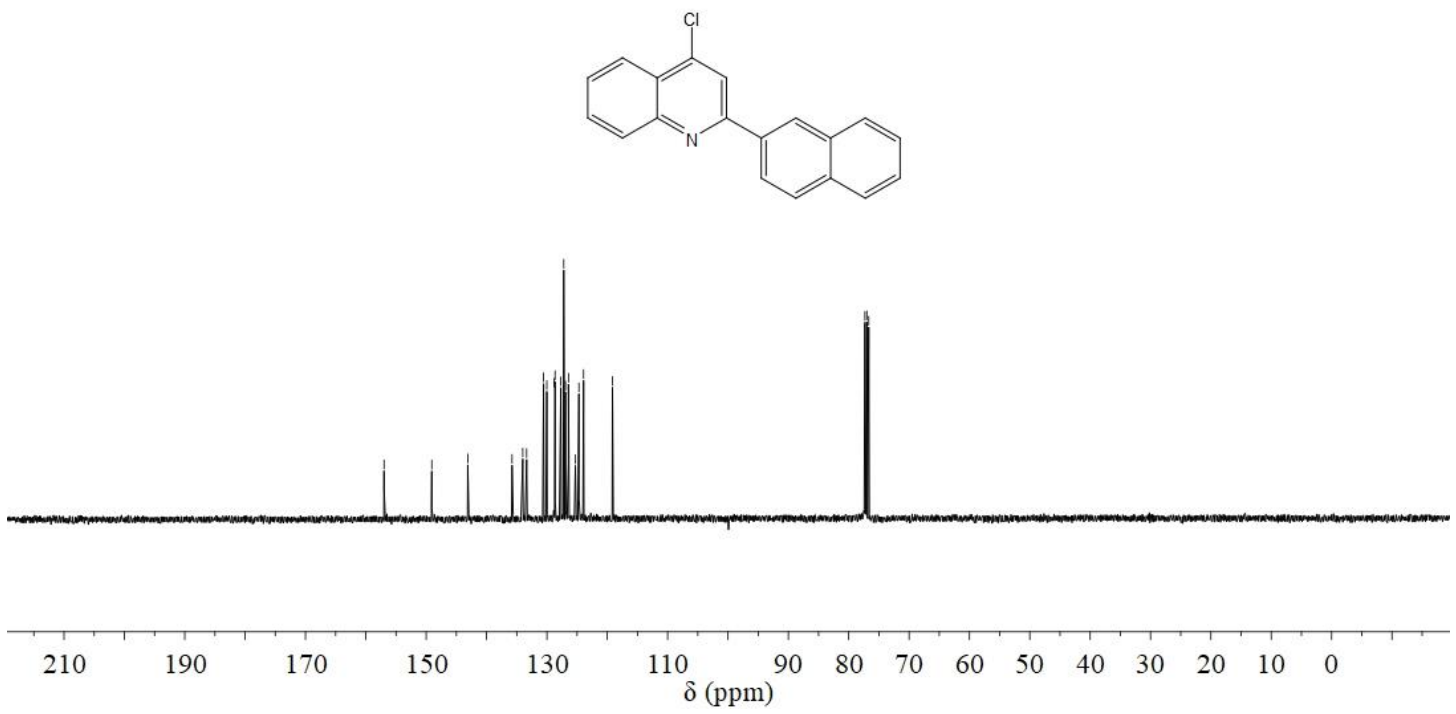

134 
$N^{I}, N^{1}$-Dimethyl- $N^{2}$-(2-(naphthalen-2-yl)quinolin-4-yl)ethane-1,2-diamine (3ci) ${ }^{1} \mathrm{H}$ NMR (400 MHz, $\mathrm{CDCl}_{3}$ )

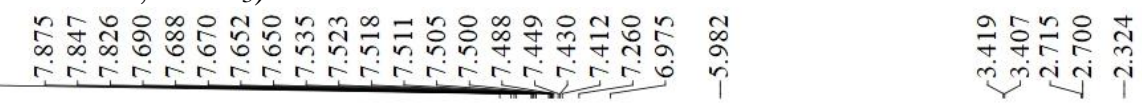
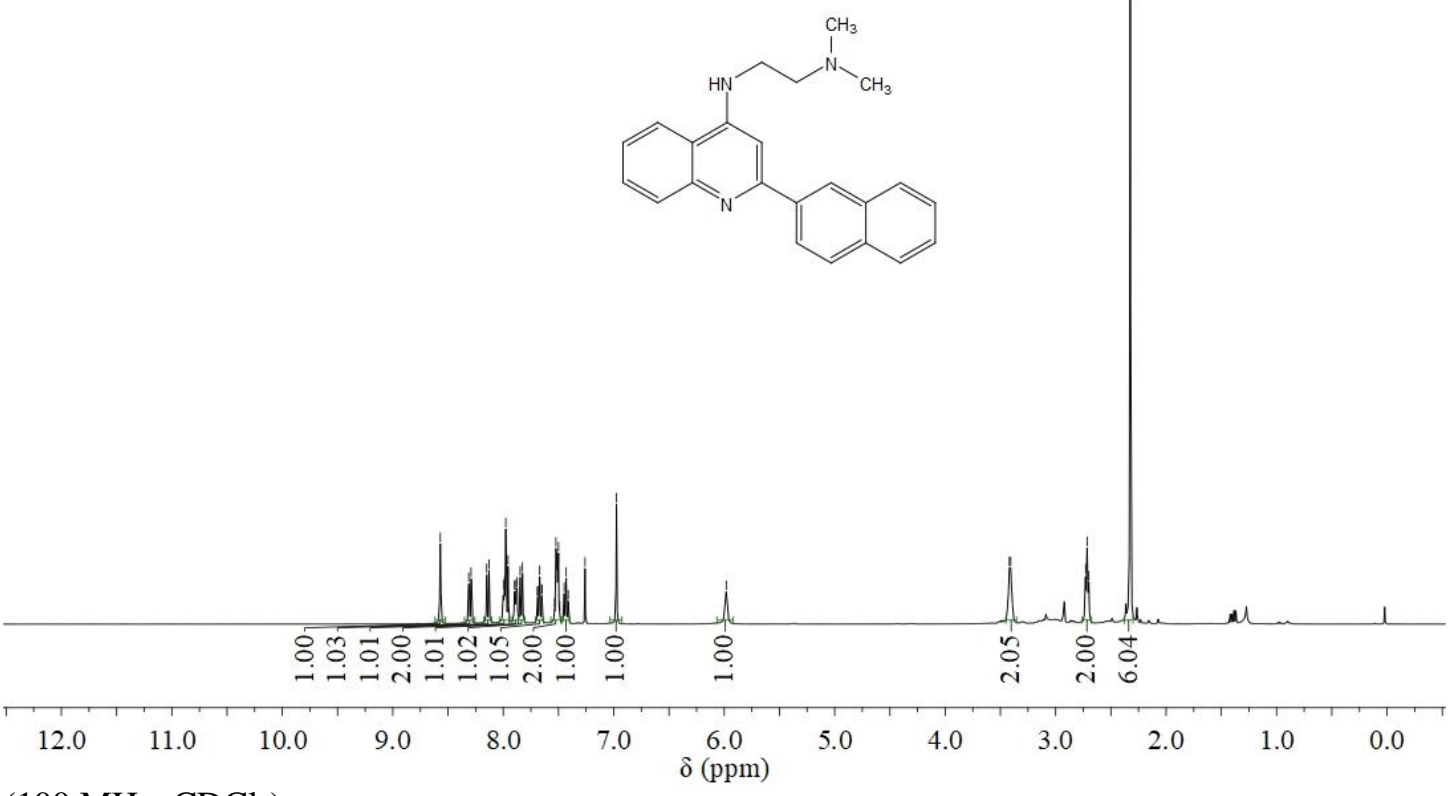

${ }^{13} \mathrm{C}$ NMR $\left(100 \mathrm{MHz}, \mathrm{CDCl}_{3}\right)$

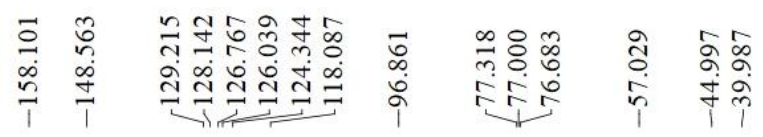
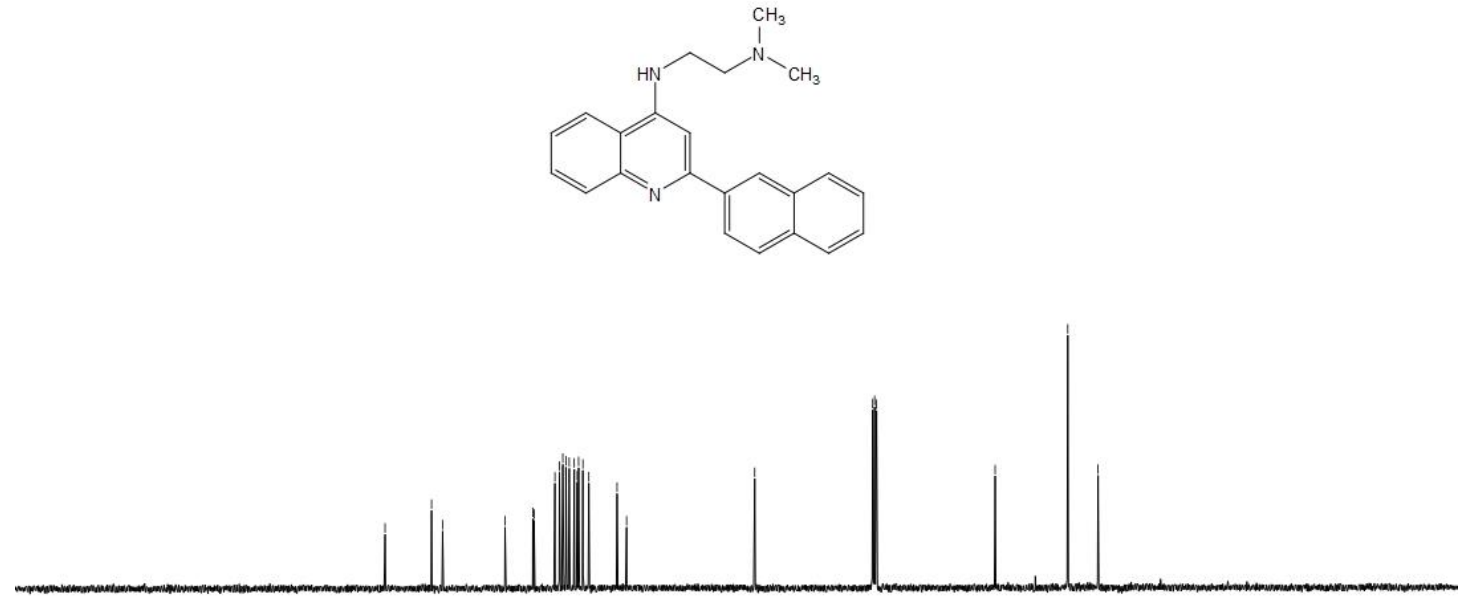

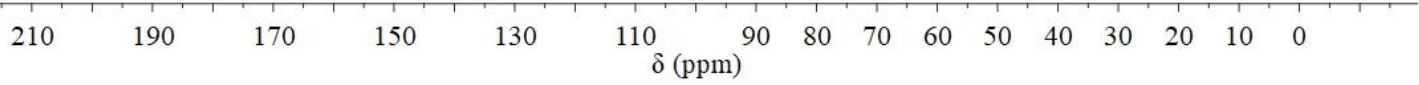


3-((1-(4-(tert-Butyl)phenyl)ethyl)amino)benzaldehyde (8a)

${ }^{1} \mathrm{H} \mathrm{NMR}\left(400 \mathrm{MHz}, \mathrm{CDCl}_{3}\right)$

$$
\text { œ }
$$<smiles>CC(Nc1ccccc1C=O)c1ccc(C(C)(C)C)cc1</smiles>

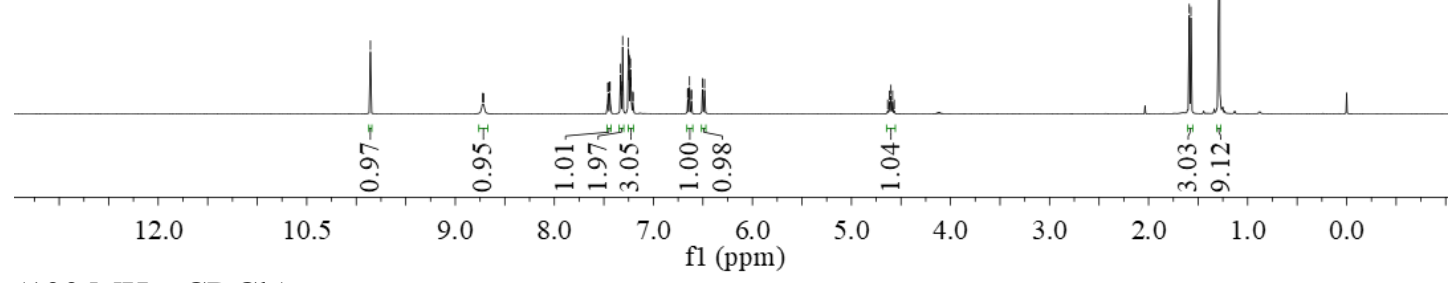

${ }^{13} \mathrm{C}$ NMR $\left(100 \mathrm{MHz}, \mathrm{CDCl}_{3}\right)$

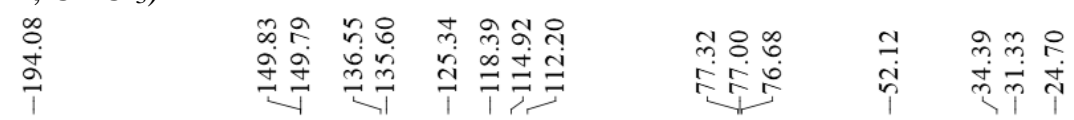
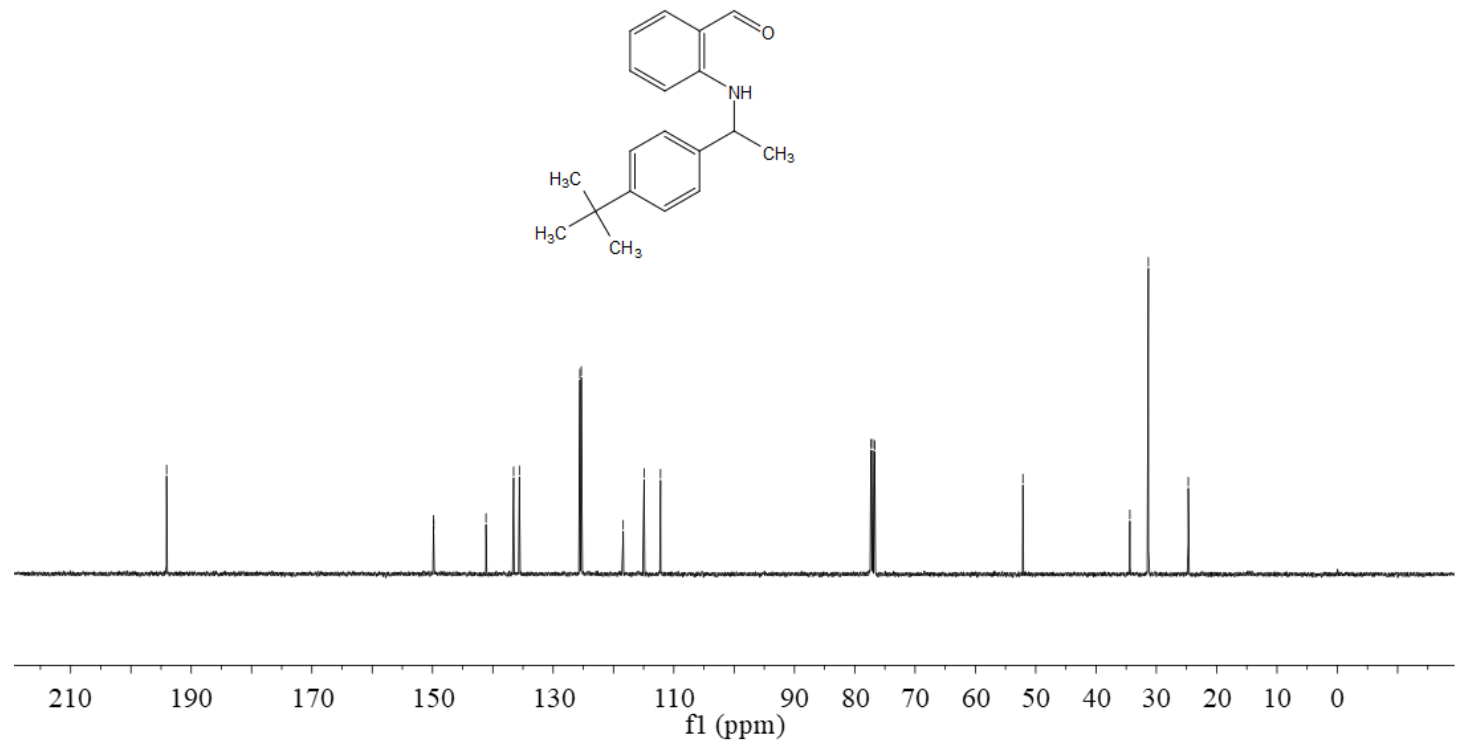